Portland State University

PDXScholar

$1-1-2010$

\title{
The Design of a Novel Tip Enhanced Near-field Scanning Probe Microscope for Ultra-High Resolution Optical Imaging
}

Derek Brant Nowak

Portland State University

Follow this and additional works at: https://pdxscholar.library.pdx.edu/open_access_etds Let us know how access to this document benefits you.

\section{Recommended Citation}

Nowak, Derek Brant, "The Design of a Novel Tip Enhanced Near-field Scanning Probe Microscope for Ultra-High Resolution Optical Imaging" (2010). Dissertations and Theses. Paper 361.

https://doi.org/10.15760/etd.361

This Dissertation is brought to you for free and open access. It has been accepted for inclusion in Dissertations and Theses by an authorized administrator of PDXScholar. Please contact us if we can make this document more accessible: pdxscholar@pdx.edu. 
The Design of a Novel Tip Enhanced Near-field Scanning Probe Microscope for Ultra-High Resolution Optical Imaging

\title{
by
}

Derek Brant Nowak

A dissertation submitted in partial fulfillment of the requirements for the degree of

\author{
Doctor of Philosophy \\ in \\ Applied Physics
}
Dissertation Committee:
Erik J. Sánchez, Chair
Dean B. Atkinson
Andres H. La Rosa
Jonathan J. Abramson
Gwynn R. Johnson

Portland State University

(C) 2010 


\begin{abstract}
Traditional light microscopy suffers from the diffraction limit, which limits the spatial resolution to $\lambda / 2$. The current trend in optical microscopy is the development of techniques to bypass the diffraction limit. Resolutions below $40 \mathrm{~nm}$ will make it possible to probe biological systems by imaging the interactions between single molecules and cell membranes. These resolutions will allow for the development of improved drug delivery mechanisms by increasing our understanding of how chemical communication within a cell occurs. The materials sciences would also benefit from these high resolutions. Nanomaterials can be analyzed with Raman spectroscopy for molecular and atomic bond information, or with fluorescence response to determine bulk optical properties with tens of nanometer resolution.

Near-field optical microscopy is one of the current techniques, which allows for imaging at resolutions beyond the diffraction limit. Using a combination of a shear force microscope (SFM) and an inverted optical microscope, spectroscopic resolutions below $20 \mathrm{~nm}$ have been demonstrated. One technique, in particular, has been named tip enhanced near-field optical microscopy (TENOM). The key to this technique is the use of solid metal probes, which are illuminated in the far field by the excitation wavelength of interest. These probes are custom-designed using finite difference time domain (FDTD) modeling techniques, then fabricated with the use of a focused ion beam (FIB) microscope. The measure of the quality of probe design is based directly on the field enhancement obtainable. The greater the field enhancement of the probe, the more the ratio of near-field to far-field background contribution will increase. The elimination of
\end{abstract}


the far-field signal by a decrease of illumination power will provide the best signal-tonoise ratio in the near-field images. Furthermore, a design that facilitates the delocalization of the near-field imaging from the far-field will be beneficial.

Developed is a novel microscope design that employs two-photon non-linear excitation to allow the imaging of the fluorescence from almost any visible fluorophore at resolutions below $30 \mathrm{~nm}$ without changing filters or excitation wavelength. The ability of the microscope to image samples at atmospheric pressure, room temperature, and in solution makes it a very promising tool for the biological and materials science communities. The microscope demonstrates the ability to image topographical, optical, and electronic state information for single-molecule identification. A single computer, simple custom control circuits, field programmable gate array (FPGA) data acquisition, and a simplified custom optical system controls the microscope are thoroughly outlined and documented. This versatility enables the end user to custom-design experiments from confocal far-field single molecule imaging to high resolution scanning probe microscopy imaging.

Presented are the current capabilities of the microscope, most importantly, highresolution near-field images of J-aggregates with PIC dye. Single molecules of Rhodamine 6G dye and quantum dots imaged in the far-field are presented to demonstrate the sensitivity of the microscope. A comparison is made with the use of a mode-locked 50 fs pulsed laser source verses a continuous wave laser source on single molecules and J-aggregates in the near-field and far-field. Integration of an intensified CCD camera with a high-resolution monochromator allows for spectral information about the sample. The system will be disseminated as an open system design. 
For my wife Valerie, her holistic support made this effort a reality.

To her I owe my sincerest love and respect. 


\section{ACKNOWLEDGMENTS}

The journey portrayed by the beauty of scientific discovery typically leaves out the hard work and dedication that is required by anyone who ventures the path. While the sacrifices are great, the reward can overshine the sacrifices of the individual and those who were supportive during the tough times. In my life, there have been many who have offered their hand without hesitation in helping my expectations become reality. I would like to personally thank my parents, Gernot and Elizabeth Nowak, for all their support over the last 8 years. It has meant more than I could possibly express. A lot of my work would not have been obtainable in a more structured environment. Both my mother and father have given me the space to think freely and discover my potential, without judgment but with support. For this, I am truly grateful.

My sincerest thanks goes out to two individuals at Mesa Community College, Barron Arenson and Mathew Wozniak, for giving me a chance to work in the science stockroom and introducing me to the world of academia and science. They would pave the way for my undergraduate education at Northern Arizona University. It was at NAU that I would meet William Delinger, a wise and insightful man, whose true character is extremely patient and compassionate. William took me under his wing and further developed my interested in hardware design and programming. His colleague, Kathleen Eastwood, would become my mentor for a NASA space grant. Kathy is a truly amazing person who would become a second mother, introducing me to the world of astronomy research. As with all of the professors that I had at NAU, in particular, Gary Bowman, Dave Cole and Barry Lutz, I would like to thank you for your compassion and 
commitment to teaching.

It wasn't until I came to Portland State University two years ago, when I realized that my potential was much greater than previously thought. Finding a love for teaching, interacting with students, and sharing a love for the sciences was realized. The physics department has been very supportive in my endeavors, and for this, I am truly thankful. However, the my sincerest thanks goes to my advisor Erik Sánchez, he has singlehandedly opened doors that I could not have even dreamt existed. I have a sincere admiration for his talents, motivation, and character. His selfless sacrifice for research, education, and students is unparalleled. In many respects, I would not consider Erik as an advisor, but more of a friend and mentor. Thank you for all of your support.

The microscope that was developed as the main component of this dissertation would not have been possible without the members of the Sánchez Nano-Development Lab. In particular, I would like to thank A.J. Lawrence for his help with 3D CAD design and the physical microscope design, Zechariah Dzegede for his pivotal role in the initial programming tests of the microscope, Justin Hiester for his role in beta testing every hairbrained idea that I had in the lab, and Cliff Kim for his assistance in programming. I would also like to give special thanks to the following lab colleagues for their constructive discussions during the design process: Mike DeArmond, Mohan Vattipalli, Deepak Vedhachalam, Richard Swinford, Randy Smith, Jeff Doughty, Amber Lauer, Kelvin Pittman, and Amen Kester. Josh Smith, Marcus Oswald, and Amy Sibal have also provided incredible emotional support.

I am very grateful to Matt Spiegelberg from National Instruments for his FPGA expertise, Analog Devices for their help with the IC designs, and Sunstone Circuits for 
their generous sponsorship that aided in the PCB designs. I would also like to thank Leroy Laush for circuit analysis, Marc Nisenfeld and Chuck Heino for machining assistance, and Richard Young of FEI Company for his suggestions on tip fabrication.

This research was funded by National Science Foundation through award DBI0500812 for Biological Sciences Instrument Development. Tip fabrication was assisted through NSF support ECCS-NSF-0520891, with additional support from PSU and ONAMI and ONR awards \#N00014-07-1-0457, \#N00014-08-1-1237, \#N00014-10-10082, NSF DMR REU site grant DMR-NSF-REU-\#069280, Western Institute of Nanoelectronics (WIN), and the National Research Initiative (NRI). 


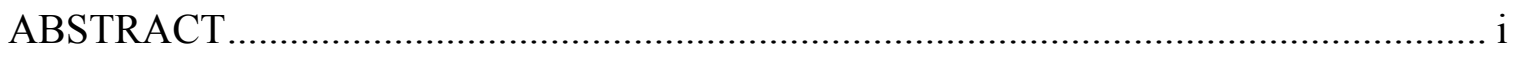

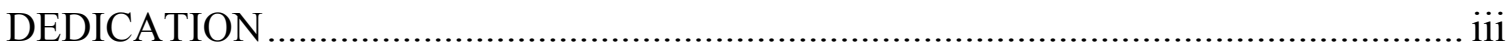

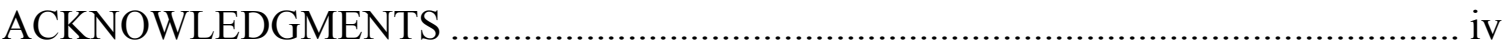

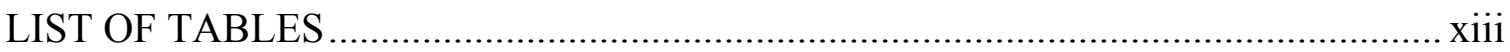

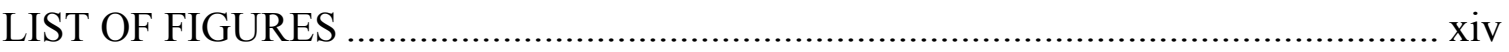

LIST OF ACROYNMS AND UNITS OF MEASURE................................................ xxviii

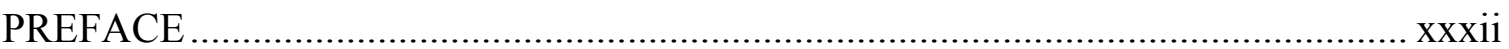

\section{CHAPTERS}

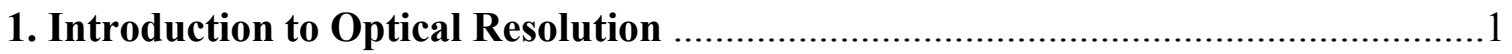

1.1 Historical Prospective ………………………………......................................

1.2 Far-Field Imaging and the Diffraction Limit ..........................................................2

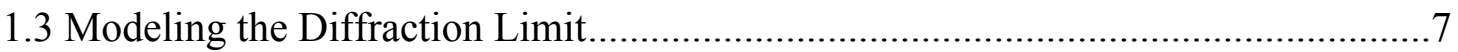

1.4 The Diffraction Limit and Non-Optical Techniques..............................................

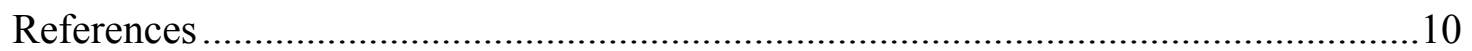

2. Introduction to Nonlinear Optical Microscopy .....................................................11

2.1 Multiphoton Fluorescence Microscopy ……………......................................12

2.2 Other Nonlinear Optical Imaging Techniques .....................................................14

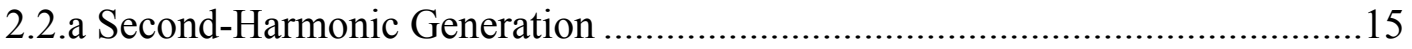

2.2.b Coherent Anti-Stokes Raman Scattering ........................................................15

2.2.c Förster Resonance Energy Transfer ..............................................................16

2.3 Advances in Sub Diffraction Limit Far-field Nonlinear Optical Imaging ...............16

2.3.a STimulated Emission Depletion (STED) .......................................................17

2.3.b Using Photo-Switchable Fluorophores for Temporal Localization .................17

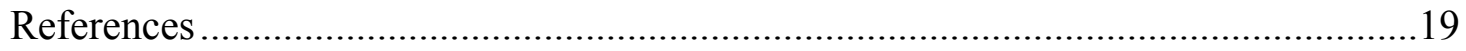


3. Introduction to Near-Field Optical Imaging .......................................................21

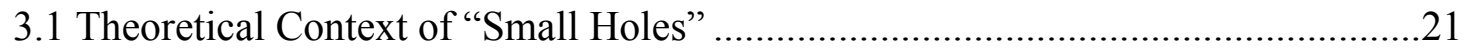

3.2 Near-Field Optical Imaging with Aperture Probes ................................................23

3.3 Near-Field Optical Imaging with Apertureless Probes ............................................27

3.4 Scientific Motivation for the Development of a TENOM System .........................32

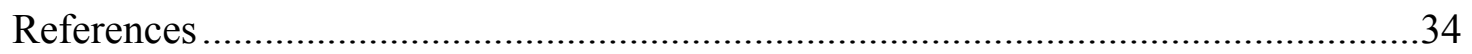

4. Modeling Nanophotonic Geometries for Near-Field Imaging …………...............38

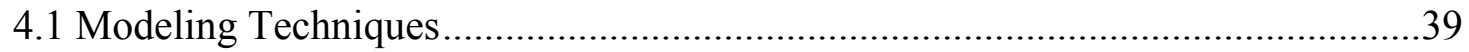

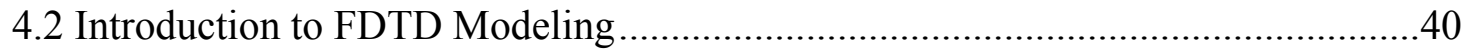

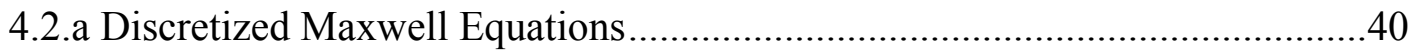

4.2.b Material Parameters for Modified Debye-Drude Model...................................42

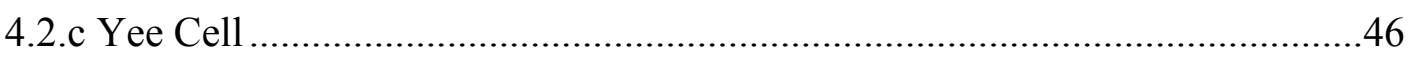

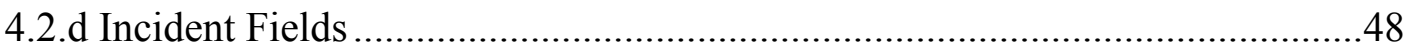

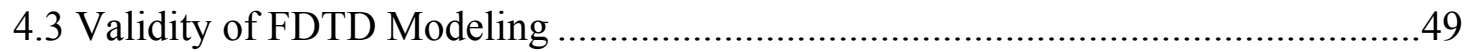

4.4 Implementing MPI Communication for Parallel Computing ................................57

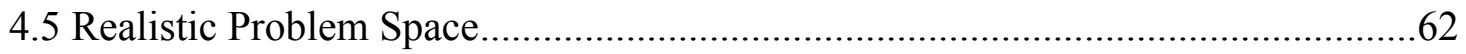

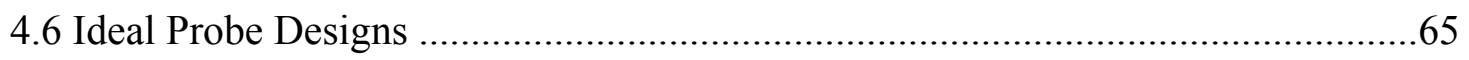

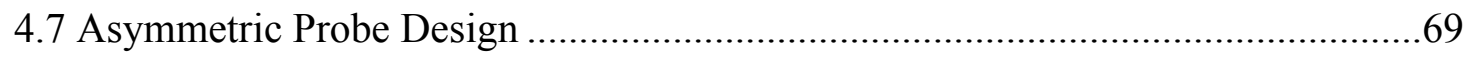

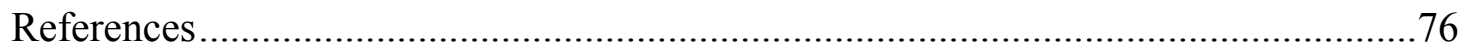

5. Introduction to the Design of a TENOM-based Microscope ………………….......79

5.1 Technical Motivation for Developing a TENOM System ......................................80

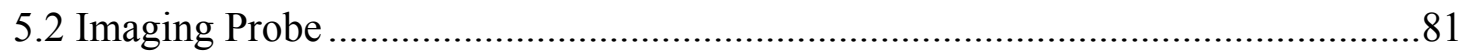

5.3 Nano-Scale Movements: Piezo Electric Crystals....................................................84

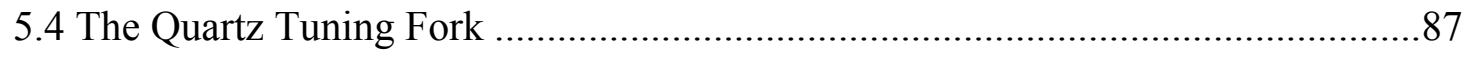

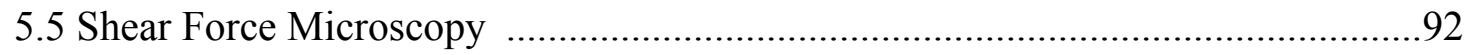

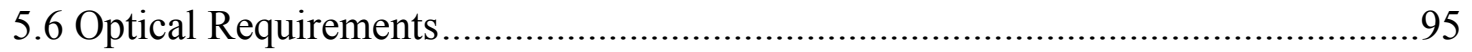

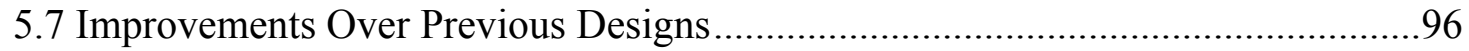

5.8 Flexibility in Design: Modifying for Other Applications .......................................97

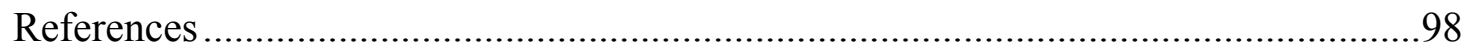


6. The Physical TENOM Microscope ...................................................................101

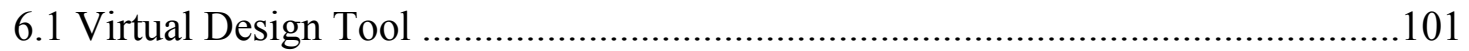

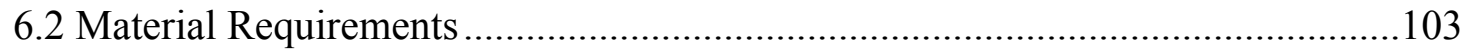

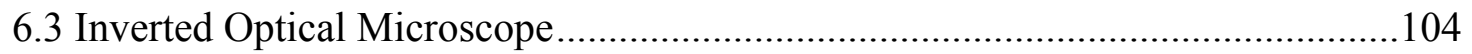

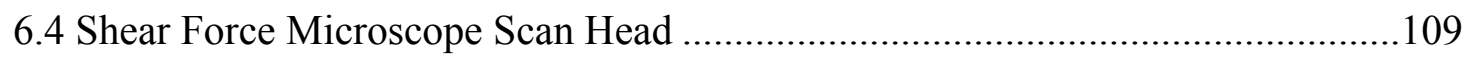

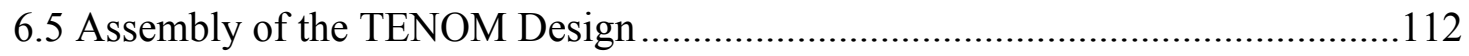

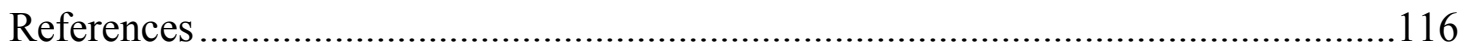

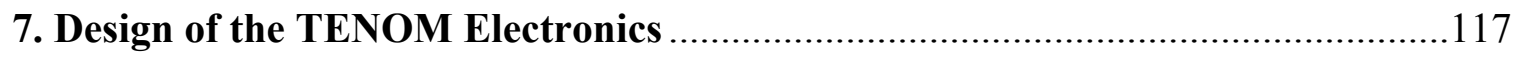

7.1 Field Programmable Gate Array (FPGA) Microcontroller....................................118

7.2 National Instruments Reconfigurable I/O .....................................................120

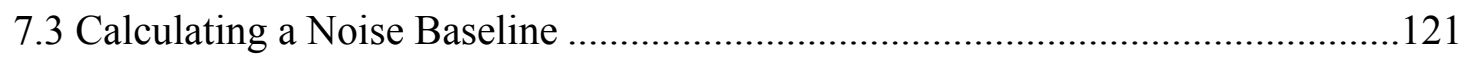

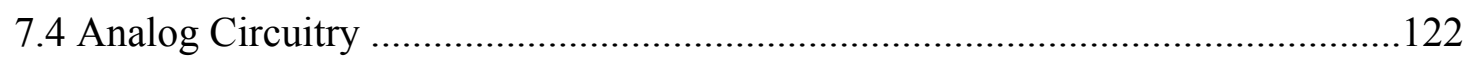

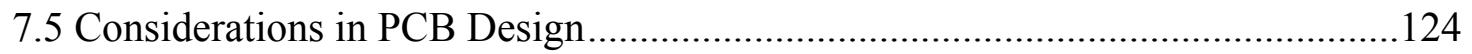

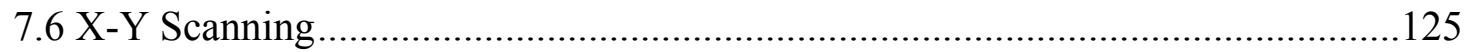

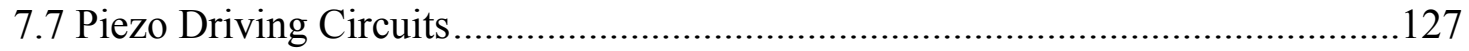

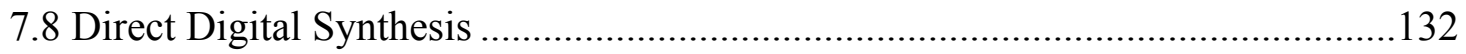

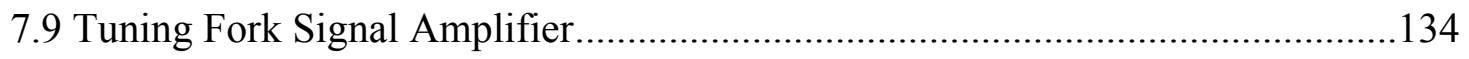

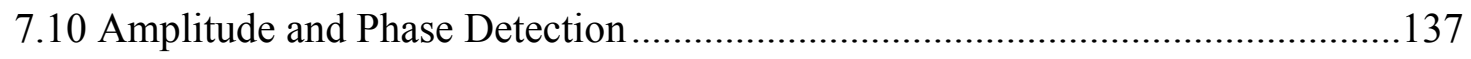

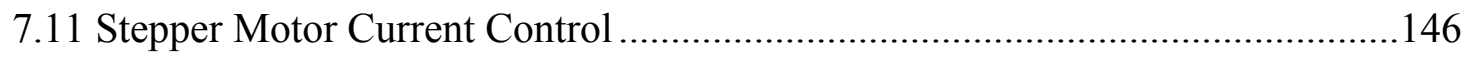

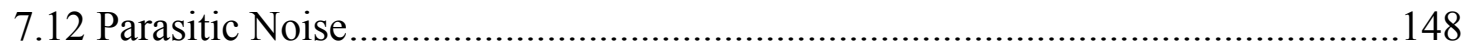

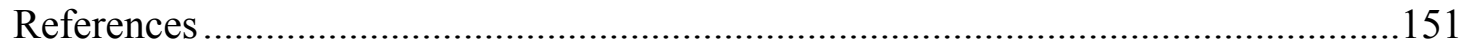

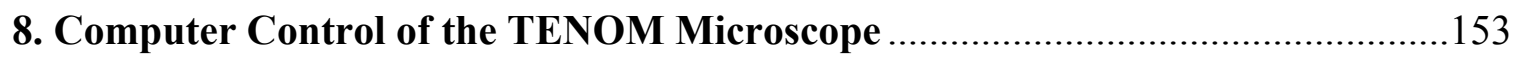

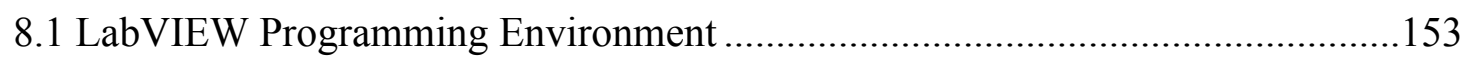

8.2 FPGA Real Time Programming ...................................................................155

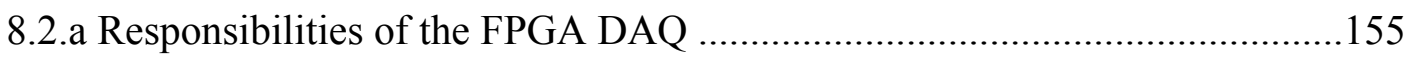

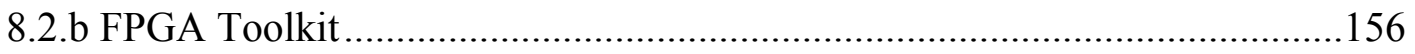

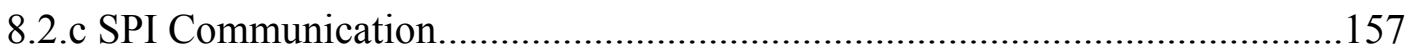

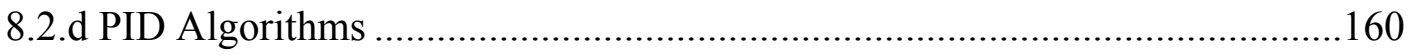

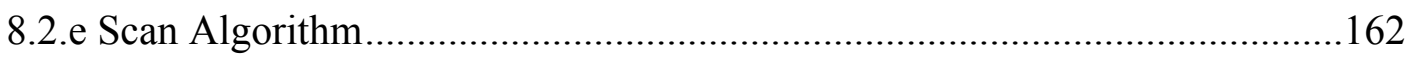

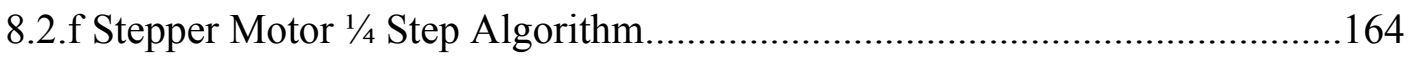




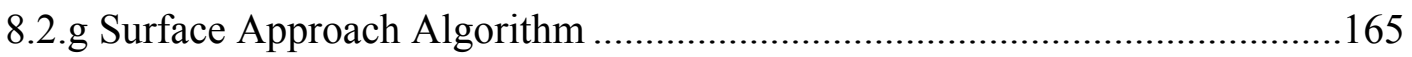

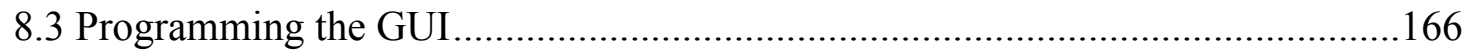

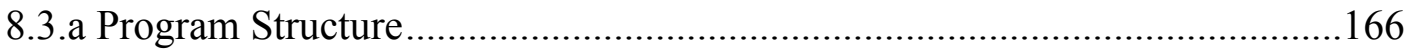

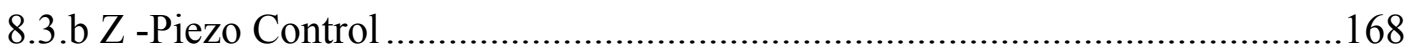

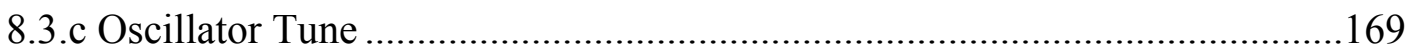

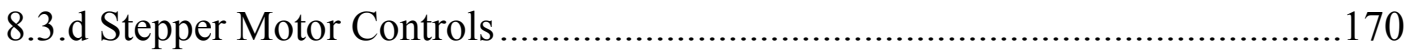

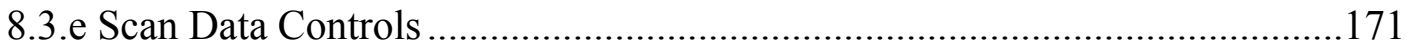

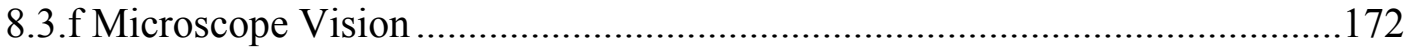

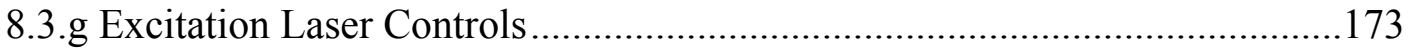

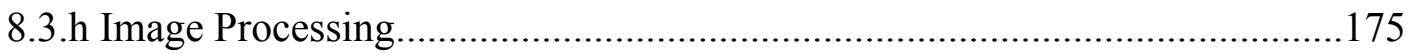

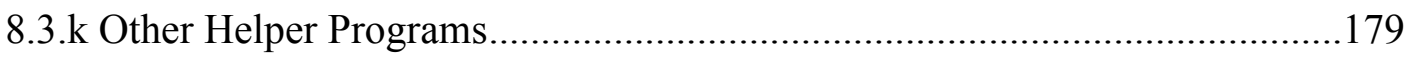

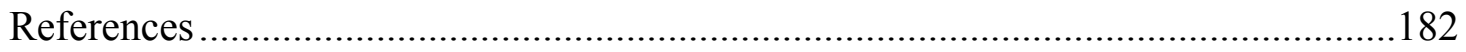

9. Nonlinear Far-Field Diffraction Limited Imaging...............................................183

9.1 Diffraction Limited Imaging of Polymers …………..........................................183

9.2 Single Molecule Sensitivity .......................................................................188

9.3 Biological Application: Bovine Pulmonary Artery Endothelial Cells ...................189

9.4 Biological Application: Imaging of Caenorhabditis Elegans..............................189

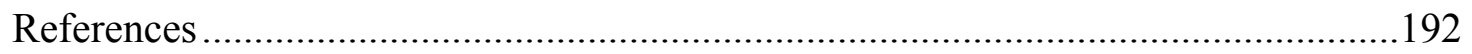

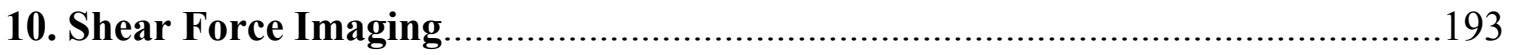

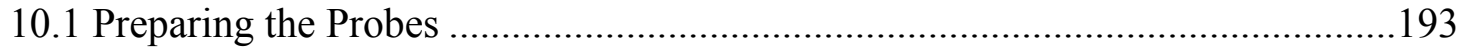

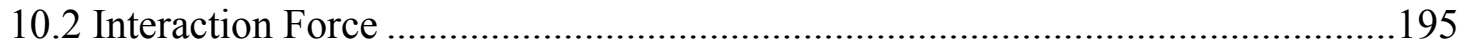

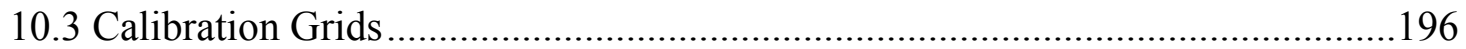

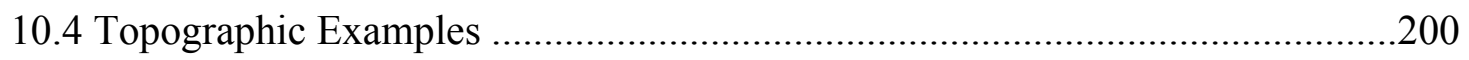

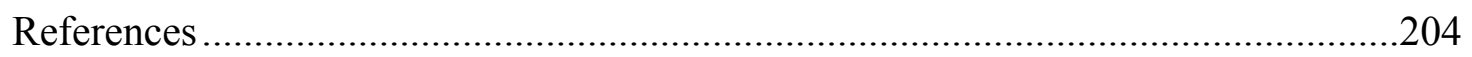

11. Fabrication of Probes for TENOM Imaging .....................................................205

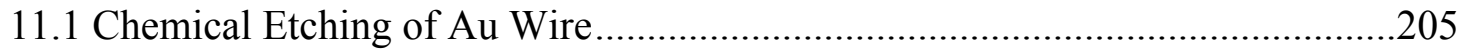

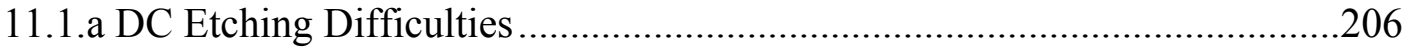

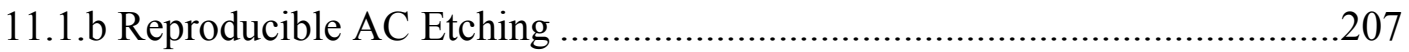

11.2 Mounting Probes to Tuning Forks ………………......................................209 
11.3 Milling Probes with a Focused Ion Beam ....................................................211

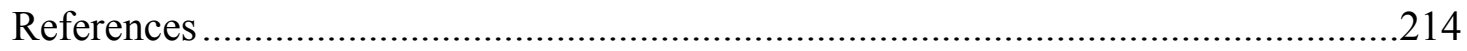

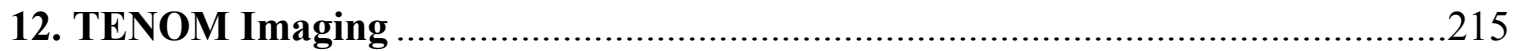

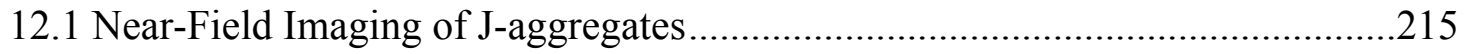

12.1.a Probe Interaction and Scattering Effects .............................................216

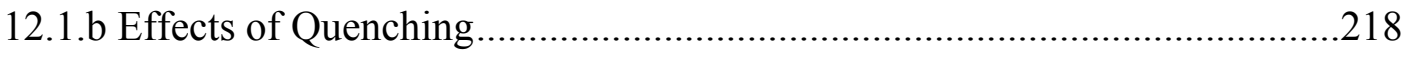

12.1.c Kerr-Mode Locked Pulsed Laser Source ...............................................219

12.1.d Continuous Wave Coherent Laser Source ...............................................224

12.2 Biological Application: Imaging of Thylakoid Membranes .............................227

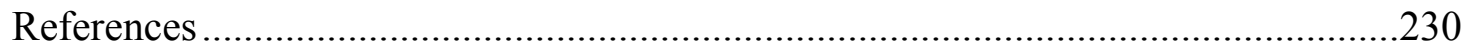

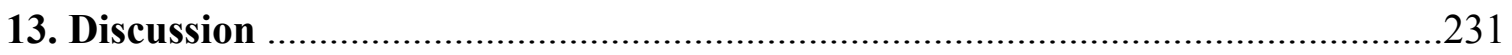

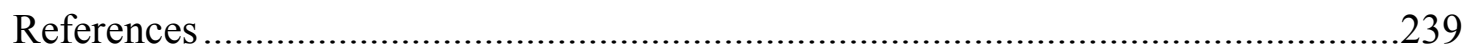

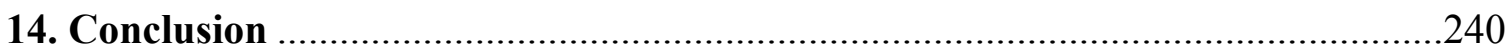

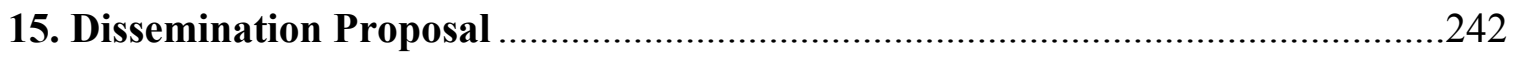

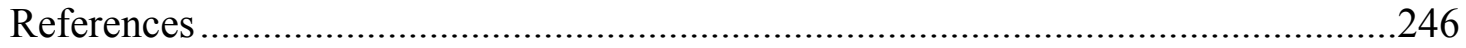

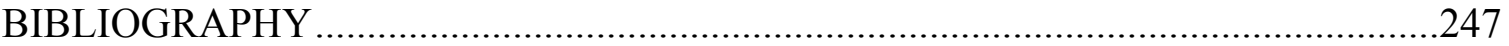

\section{APPENDICES}

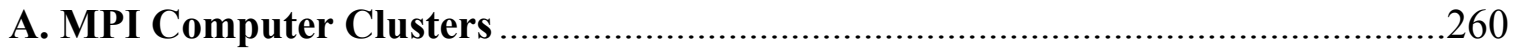

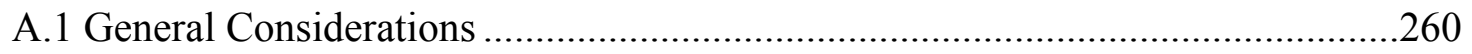

A.2 Installation Notes for an MPI Based Cluster.....................................................261

A.3 Execution Routine for an MPI xFDTD Calculation .........................................266

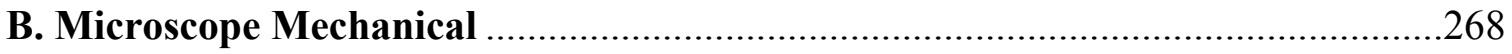

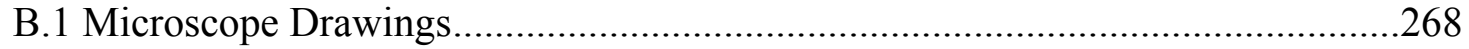

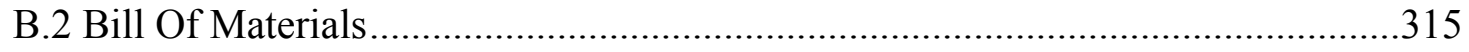

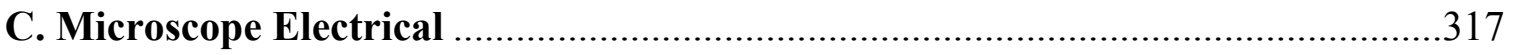

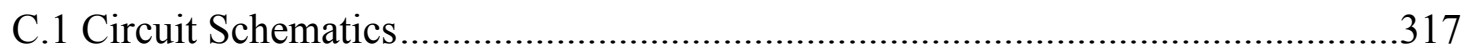




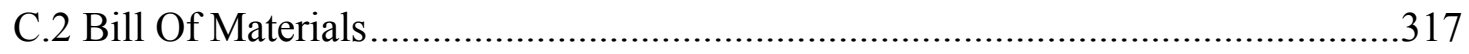

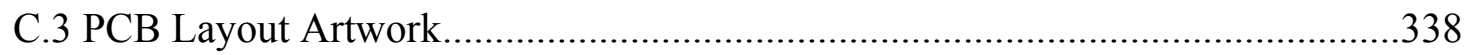

C.2 Assembled Microscope Controller Prototype ..................................................353

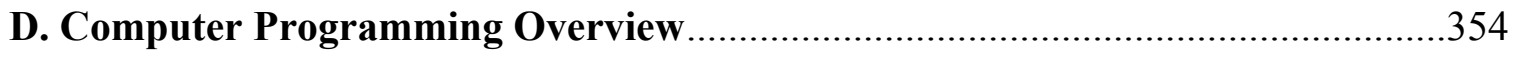

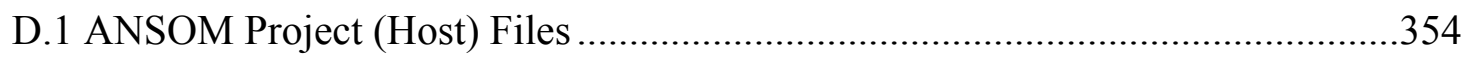

D.2 FPGA (Embedded Target) Files and Dependencies ........................................355

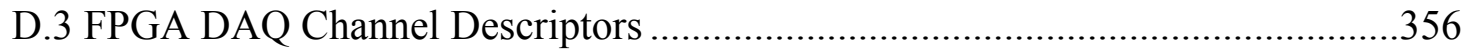

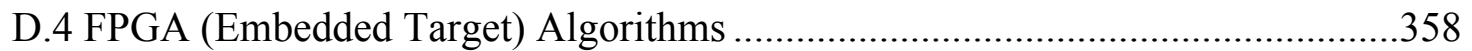

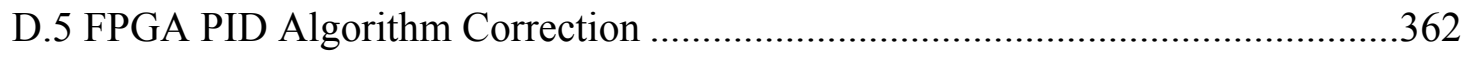

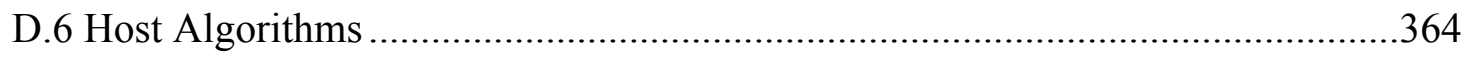

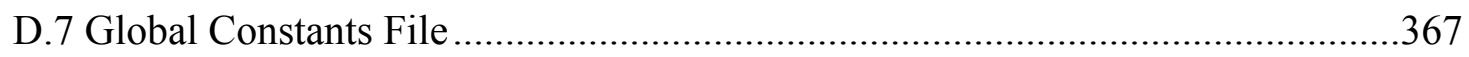

E. Fabrication of Substrates for Nanometer Relocation of Samples........................368 


\section{LIST OF TABLES}

Table 6.1 Comparison of coefficients of linear thermal expansion for commonly used materials. Note the negative value for Zerodur..............104 


\section{LIST OF FIGURES}

Figure 1.1 Diffraction pattern generated by diffraction of light by a small circular hole in a screen.

Figure 1.2 Effect of numerical aperture (NA) of focusing lens on the size of an Airy disk.

Figure 1.3 Diffraction patterns for different overlapping Airy disks: (a) the two different airy disk patterns are easily seen with a large separation, (b) the limit by which the points are just resolvable, reaching the resolvable limit of the overlap, and (c) the two airy disks are not resolvable when overlapped.

Figure 1.4 Bessel function of the first kind and zero order. The graph shows a minimum at $\sim 3.85$ which when divided by $\pi$ is $\sim 1.22$.

Figure 1.5 Finite difference time domain modeling (FDTD) of a $5.0 \mu \mathrm{m} 3 \mathrm{D}$ lens focusing $800 \mathrm{~nm}$ light. The modeling shows the finite size of the focal region. ${ }^{*}$

Figure 2.1 Energy diagram for two-photon transition, based on wavelength of the incoming photon(s).

Figure 2.2 Two-photon excitation cross section compared to one photon excitation.

Figure 3.1 Electric field distributions for an aperture smaller than the wavelength in an infinite conduction sheet.

Figure 3.2 Near-field intensity pattern of 3 different polarization orientations (a) the horizontal (x-direction), (b) $90^{\circ}$ to the horizontal (y-direction), and (c) vertical polarizations ( $\mathrm{x}-\mathrm{y}$ plane).

Figure 3.3 Side profile of a near-field aperture probe scanning over fluorescing chromophores. ${ }^{*}$

Figure 3.4 Conceptual drawing of a side illuminated TENOM probe. Spectroscopic signals are excited in the near-field by a high localized field at the end of the tip.

\footnotetext{
* Abridged version of the figure caption.
} 
Figure 3.5 Conceptual drawing of an epi-lluminated TENOM probe. Fluorescent molecules are excited in the near-field by a high localized field at the end of the tip. ${ }^{*}$

Figure 3.6 FDTD modeling of an Au TENOM probe, $800 \mathrm{~nm}$ incident light polarized in the horizontal direction, incident from the top-down. The FDTD algorithm reported a field enhancement with an increase of two orders of magnitude over that of the excitation source.

Figure 4.1 Yee Cell. The six calculation points for the electric field components and the magnetic field components are shown.

Figure 4.2 Coordinate system used in XFDTD. To define the incident field direction, both $\theta$ and $\phi$ angle need to be specified [Remcom Inc., 2003].

Figure 4.3 Defining the incident field parameters, the user is allowed to define the frequency, field magnitude and number of time steps required to complete 10 wavelengths of radiation. The upper graph shows the incident field as a function of time. The lower graph gives the FFT of the incident field.

Figure 4.4 Electric field intensity due to Mie scattering. The equator locations are areas of highest enhancement. The Yee cell size is $3 \mathrm{~nm}$, with a sphere of radius of $75 \mathrm{~nm}^{*}$.

Figure 4.5 Electric field intensity due to Mie scattering near the equator of the sphere.

Figure 4.6 Mie Scattering workspace. Locations of data collection in the $x-y$ plane at cell 76 in the $\mathrm{z}$ direction for gold sphere with $15 \mathrm{~nm}$ cell size representation (linear scale).

Figure 4.7 Total fields as a function of cell size, in a semi log scale. Data points show the corner artifacts that are present on the sphere.

Figure 4.8 Total electric field amplitude as a function of number of cells away the $150 \mathrm{~nm}$ sphere is from the Liao boundary in the xFDTD simulation.

Figure 4.9 Total electric field amplitude as a function of distance away the 150 $\mathrm{nm}$ sphere is from the Liao boundary in the XFDTD simulation.

\footnotetext{
* Abridged version of the figure caption.
} 
Figure 4.10 Corrected: Total fields as a function of cell size, a semi log scale. Any cell size that is not divisible by the object size was removed, and adequate spacing from the boundaries was applied for each model.

Figure 4.11 Number of compute nodes used vs the calculation time required. Times for a Windows and a Linux-based system calculation of $\pi$ to the same level of precision.

Figure 4.12 Shows a comparision of the number of compute nodes used vs. the calculation time required. Times for various computer and networking types are shown.

Figure 4.13 Comparison of a Pentium III computer cluster versus a Pentium 4 computer cluster.

Figure 4.14 The effect of network latency on MPI calculations. Both a Pentium 4 cluster and Pentium III cluster were used to demostrate the effect of latency. ${ }^{*}$

Figure 4.15 A semi-realistic modeling space for focusing the incident light on the TENOM probes. Color scale is logarithmic.

Figure 4.16 3D workspace for the semi-realistic modeling conditions, showing 2D planes of interest. Lens is green colored and TENOM probe yellow. 2D sample plane is the electric-field intensities a few nanometers above the lens glass. The $2 \mathrm{D}$ cross section plane is mid probe along the asymmetric axis.

Figure 4.17 G-T cone workspace, $\mathrm{x}-\mathrm{y}$ plane at cell 137 in the $\mathrm{z}$ direction with 3 $\mathrm{nm}$ cell size representation. (a) G-T cone geometry showing a maximum electric field of $180 \mathrm{~V} / \mathrm{m}$. (b) The effect of an elongated apex on the G-T cone, giving a maximum electric field of $650 \mathrm{~V} / \mathrm{m}$.

Figure 4.18 High aspect ratio $\mathrm{Au}$ probe, illuminated from below with $800 \mathrm{~nm}$ light.

Figure 4.19 Sample imaging plane showing electric field magnitude distribution with the high aspect ratio $\mathrm{Au}$ probe, illuminated from below using $800 \mathrm{~nm}$ light. One half of an optical cycle is shown in four divisions.

* Abridged version of the figure caption. 
Figure 4.20 Electric field intensity as a function of incident field direction. The fields are shown by their $\mathrm{x}, \mathrm{y}, \mathrm{z}$, and total field components.

Figure 4.21 Dimensions of asymmetric probe.

Figure 4.22 Asymmetric Au probe, illuminated from below with $800 \mathrm{~nm}$ light, focused at the sample surface.

Figure 4.23 Imaging plane electric field magnitude distribution with the asymmetric Au probe and focus at sample surface. One half of an optical cycle over four divisions.

Figure 4.24 Asymmetric Au probe, illuminated from below with $800 \mathrm{~nm}$ light, focused at bulk surface on probe.

Figure 4.25 Imaging plane electric field magnitude distribution with the asymmetric Au probe and focus on probe bulk surface. One half of an optical cycle over four divisions.

Figure 4.26 Imaging plane electric field magnitude distribution with the asymmetric $\mathrm{Au}$ probe and focus on probe bulk surface. Model differs from Figure 4.25, due to a rotation of incident electric field polarization by $90^{\circ}$. One half of an optical cycle over four divisions.

Figure 4.27 Imaging plane electric field components (X, Y, and Z) at the nearfield imaging region. (Same area as zoomed feature in Figure 4.26) The same asymmetric Au probe and focus on probe bulk surface as model for Figure 4.25 .

Figure 4.28 Asymmetric Au probe, illuminated from below with $800 \mathrm{~nm}$ light, focused at end of protrusion cone. The highest electric field intensities are measured a few hundred nanometers above the end of the protrusion cone.

Figure 4.29 Imaging plane electric field magnitude distribution with the asymmetric Au probe and focus at protrusion cone. One half of an optical cycle over four divisions.

Figure 5.1 Effect of imaging probe aspect ratio on topographical imaging. (a) Represents the actual sample surface, (b) and (c) are the recorded sample surface for the high aspect ratio probe and low aspect ratio probe, respectively....

\footnotetext{
* Abridged version of the figure caption.
} 
Figure 5.2 Effect of quenching and topographical features on image resolution. I Photons is the photon emission intensity from the sample surface (colored regions). It is important to note the peaks on before and after the high topography surface.

Figure 5.3 Lattice structure of a piezoelectric crystal, where black is $\mathrm{O}^{2-}$, green is $\mathrm{Pb}$ and red is Ti or $\mathrm{Zr}$. (a) Is an unpoled lattice cube with no net electric field. (b) is the similar lattice cube with a poled dipole moment.

Figure 5.4 Hysteresis curve for open loop piezo positioners. The expansion and retraction of the piezo do not follow the same paths.

Figure 5.5 Electrode configuration for a tuning fork oscillator. In the case of an electrically driven oscillator, one of the electrodes $(\mathrm{X})$ is oscillated and the piezo-induced electrical charge in $(\mathrm{Y})$ is detected. .88

Figure 5.6 The mechanical and electrical models of a quartz tuning fork oscillator. $\mathrm{C}_{\mathrm{s}}$ is the parasitic capacitance of the tuning fork.

Figure 5.7 Measure of quality factor "Q" based on the amplitude and frequency response of the tuning fork. $\Delta \mathrm{f}$ is measured at the FWHM of the waveform.

Figure 5.8 (a) Optical image of the quartz tuning fork exposed by the partial removal of the protective canister. (b) An electron micrograph of the same tuning fork. In both, an Au probe is mounted to the very end of the tuning fork. The size of the probe helps maintain high $Q$ factors $(\sim 6000)$

Figure 5.9 Conceptual drawing of the tuning fork SFM. The tuning fork is held by a tube-piezo stack comprised of a $\mathrm{Z}$-axis piezo and a separate $\mathrm{X}$ $\mathrm{Y}$ axis segmented piezo.

Figure 5.10 Conceptual drawing of a tuning fork SFM used in a TENOM system. The sample is raster scanned to compose a spatial near-field optical image.

Figure 5.11 Optical arrangement for the TENOM experimental apparatus. Note the upper location of the stepper motor, which is a remnant of a previous design.

* Abridged version of the figure caption. 
Figure 6.1 SolidWorks 2009 3D CAD software main GUI. The design concept for an asymmetric tip design for TENOM imaging, shown in four different perspectives.

Figure 6.2 Optical pathways of the TENOM system. ${ }^{*}$

Figure 6.3 3D representation of the inverted microscope. 108

Figure 6.4 Piezo stack: (a) is a 3D assembly of all the parts for the piezo stack generated in SolidWorks, (b) is photograph of the parts before physical assembly.

Figure 6.5 3D representations of the SFM scan head. Including the amplifier section within the scan head helps remove noise from the feedback signal.

Figure 6.6 3D representation of the alignment flexibility needed to place the probe within the focused laser spot.

Figure 6.7 Views from the three alignment CCDs: (A) is from the CCD mounted after the Bertrand Lens, (B) is from the CCD focused on the sample surface with probe side illuminated with LEDs, $(\mathrm{C})$ is from the CCD mounted to the scan head or microscope base plate, focusing on the tuning fork and imaging probe.

Figure 6.8 TENOM system layout: The system is placed on silicon vibration dampening material to reduce high frequency vibrations. The optical table dampens the lower frequency floor vibrations. Lasers, camera, and monochromator are mounted to the table for ease of alignment....

Figure 6.9 3D representation of the TENOM system.

Figure 7.1 A block diagram of the TENOM control system. The arrows indicate direction of data signal flow.

Figure 7.2 A block diagram of the analog circuitry layout on multiple PCBs to allow for modularity in design. The arrows indicate direction of data flow throughout the controller.

Figure 7.3 (a) Gerber file image from the PCB123 layout program, showing all four layers of the PCB stacked together. (b) Main controller board after production with all components placed.

* Abridged version of the figure caption.

xix 
Figure 7.4 Circuit logic for the summing of the two analog outputs for the scanning offset and raster scan signals. The microscope implements two summing circuits for independent control of the $\mathrm{X}$ and $\mathrm{Y}$ axis.

Figure 7.5 Scanning circuit on PCB, OP471 (U1, U2) operational amplifiers sum and amplify the signals to the users specifications.

Figure 7.6 X (upper), Y (lower) scan waveforms for the TENOM system. Both channels displayed at $5.0 \mathrm{~V} / \mathrm{div}$ with a $1.0 \mathrm{sec} / \mathrm{div}$ time base.

Figure 7.7 A resistor diagram of a bridge circuit configuration of two PA88 high voltage operational amplifiers.

Figure 7.8 PCB of bridge circuit piezo driver with components. The two amplifiers are Apex PA88 chips with heat sinks. The remaining components configure the gain and slew rates with phase compensation.

Figure 7.9 Noise output of both $+\mathrm{HV}$ (upper) and - HV (lower) outputs with a shorted input. Both channels displayed at $50 \mathrm{mV} / \mathrm{div}$ with a 2.0 $\mathrm{ms} /$ div time base.

Figure 7.10 Positive output of bridge circuit and its response to an input step function. Channel one (upper) $2.0 \mathrm{~V} / \mathrm{div}$, channel two (lower) 20 V/div, $10 \mu \mathrm{s} /$ div time base.

Figure 7.11 Block Diagram of the internal function of an AD9835 DDS, adapted from Analog Devices data sheet [Analog Devices, 1998].

Figure 7.12 DDS Oscillator circuit on PCB, U5 is the AD9835, X1 is a $50 \mathrm{MHz}$ clock oscillator, B1 is the ferrite bead, and U7 is the line driver (74HCT244) to convert from low voltage TTL to CMOS logic voltages.

Figure 7.13 The DDS signal is used to directly drive the tuning fork, the signal is voltage divided in close proximity to the tuning fork to minimize signal degradation from the DDS chip, which is generated in the controller over a meter away from the tuning fork.

Figure 7.14 Response of the tuning fork with a sweep of frequencies, passing the tuning fork through resonance. 5.0 V/div, $200 \mathrm{~ms} /$ div time base.

\footnotetext{
* Abridged version of the figure caption.
} 
Figure 7.15 (a) The dual stage AD8599 (U30) amplifier circuit board, which is also placed in the scan head. U29 is the precision voltage source for the MESFET. (b) Small MESFET board and holder used to obtain the high gain of $10^{6}$ required to amplify the signal from the tuning fork.

Figure 7.16 Inverting Active band-pass filter.

Figure 7.17 Tuning fork oscillation and detection scheme.

Figure 7.18 2-pole Sallen-Key filter design.

Figure 7.19 $\mathrm{PCB}$ implementation of AD637 RMS to DC converter. U14 is the AD637 and U15 is an OP27 to aid in conditioning the return signal from the amplifier stages in the scan head.

Figure 7.20 Response of tuning fork with a sweep of frequencies, passing the tuning fork through resonance (upper). RMS calculated response of the upper signal with the AD637 (lower). Channel one (upper) 10 V/div, channel two (lower) $5.0 \mathrm{~V} / \mathrm{div}$, with a $200 \mathrm{~ms} / \mathrm{div}$ time base.

Figure 7.21 Four quadrant multiplier configured with a scaling factor.*

Figure 7.22 Phase detector logic: (a) Input signals pre-conditioned for comparators, (b) signals after comparison, (c) signals after multiplication. Waveforms (i), (ii), (iii) are example conditions and the effect of integration on those waveforms.

Figure 7.23 PCB implementation of phase detection circuitry.

Figure 7.24 Amplitude and phase relationship for the quartz-tuning fork recorded by the feedback electronics.

Figure 7.25 DDS reference oscillator signal, conditioned for the comparator IC (upper). Tuning fork signal after amplification, before reaching the phase detection board (lower). Channel one (upper) $5.0 \mathrm{~V} /$ div, channel two (lower) $10 \mathrm{~V} / \mathrm{div}$, with a $10 \mu \mathrm{s} / \mathrm{div}$ time base.

Figure 7.26 DDS reference oscillator signal after comparator IC measured at R140 (upper). Tuning fork signal after comparator IC measured at R138 (lower). Channel one (upper) and two (lower) are $2.0 \mathrm{~V} / \mathrm{div}$, with a $10 \mu \mathrm{s} /$ div time base.

* Abridged version of the figure caption. 
Figure 7.27 Result of the $-55^{\circ}$ relative phase difference and the variable transfer function of the four-quadrant multiplier (square wave), measured at R122. The constant voltage line represents the DC output of the low-pass filter, measured at R136. Both channel one and channel two are set at $2.0 \mathrm{~V} / \mathrm{div}$, with a $5.0 \mu \mathrm{s} / \mathrm{div}$ time base.

Figure 7.28 Result of the $55^{\circ}$ relative phase difference and the variable transfer function of the four-quadrant multiplier (square wave), measured at $\mathrm{R} 122$. The constant voltage line represents the DC output of the low pass filter, measured at R136. Both channel one and channel two are set at $2.0 \mathrm{~V} / \mathrm{div}$, with a $5.0 \mu \mathrm{s} / \mathrm{div}$ time base.

Figure 7.29 Oscilloscope trace of the four phases (a-d) of the stepper motor.

Figure 7.30 PCB implementation of stepper motor circuitry. ${ }^{*}$

Figure 7.31 Noise output of both $+\mathrm{HV}$ (upper) and $-\mathrm{HV}$ (lower) outputs when connected to 7833R DAQ card. Both channels $100 \mathrm{mV} /$ div with a $2.0 \mathrm{~ms} / \mathrm{div}$ time base.

Figure 7.32 Noise output of both $+\mathrm{HV}$ (upper) and $-\mathrm{HV}$ (lower) outputs when connected to 7833R DAQ card, showing the $50 \mathrm{MHz}$ oscillations from the clock oscillator. Both channels $100 \mathrm{mV} / \mathrm{div}$ with a 10 ns/div time base.

Figure 8.1 Demonstration of LabVIEW programming environment. DAQ voltage converter takes the raw 16-bit integer and converts it to a double precision floating point value, representing the recorded voltage. DAQ phase converter does the same but scales the value to represent a predefined phase value.

Figure 8.2 Three-signal communication of SPI. The CS signal opens the SDATA for communication, while the SDATA is read with the rising of the clock signal (SCLK).

Figure 8.3 FPGA communication and timing algorithm for the SPI protocol in the TENOM system. ${ }^{*}$

Figure 8.4 PID algorithm flow chart: The difference between the desired setpoint and the feedback signal is parallel processed by a proportional, integral, and differential gain equations, then summed together as the correction needed to maintain the setpoint.

\footnotetext{
* Abridged version of the figure caption.
} 
Figure 8.5 Scan algorithm for a four by four pixel scan area. The dashed line represents the path of the probe as it creates a trace and retrace line for each y-axis pixel.

Figure 8.6 Stepper motor four-phase logic for $1 / 4$ steps.

Figure 8.7 Sample approach algorithm: (a) is the approach swing of the z-piezo, followed by (b) which is a quick retract swing, after (b), the stepper motor is pulsed a predetermined distance, (c) is the delay time to allow for the stepper motor movement before the next approach swing. The process is repeated until the surface is located.

Figure 8.8 Main Entry VI for the TENOM microscope uses a combination of action buttons and drop down menus. ANSOM project is the dissemination name for the microscope design and documentation as an open system design.

Figure 8.9 Windows program tree overview

Figure 8.10 Z-Piezo control GUI. ${ }^{*}$ 168

Figure 8.11 Oscillator Tune GUI. The oscillator tune shows the simultaneous real-time measurement of phase (red) and amplitude (white).

Figure 8.12 Motor Control GUI.

Figure 8.13 Scan Data Controls GUI. The images are multi-photon induced fluorescence of chlorophyll; both images are false color look-uptables.

Figure 8.14 Line Analyzer GUI invoked from the Scan Data Controls GUI.

Figure 8.15 ANSOM Vision GUI. Image shows a sample surface imaged from below with a high numerical aperture objective lens. Both the tip and diffraction limited focused laser spot is shown

Figure 8.16 Excitation Laser Power GUI.

Figure 8.17 Image Processing GUI, displays the raw unprocessed image, image header information, and the processed image. The program flags the user if the image has been mathematically modified.....

Figure 8.18 Cross section GUI, invoked from the Image Processing VI. .

* Abridged version of the figure caption.

xxiii 
Figure 8.19 Photon Counter GUI, displays live photon counts rates (\# of photons per second) in both a graphic indicator and a numeric indicator. ${ }^{*}$

Figure 8.20 X-Y Piezo Offset GUI, allows the user to directly interface with the segmented alignment tube piezo in the scan head.

Figure 9.1 J-Aggregates, where the same region of interest was imaged at increasing (from right to left) excitation power levels.

Figure 9.2 The quadratic nature of multi-photon fluorescence.

Figure 9.3 (a) CCD optical image of J-Aggregates. (b) CW Far-field Multiphoton fluorescence image of the same region acquired by point-bypoint raster scan with a PMT.

Figure 9.4 (a) CW Far-field Multi-photon fluorescence point-by-point raster scanned image of J-Aggregates (b) is a magnified image of the same area showing near diffraction limited imaging.

Figure 9.5 J-aggregates on glass, imaged with $\mathrm{CW}$ laser light at $850 \mathrm{~nm}$. (a) Using an excitation power at the sample of $1.3 \mathrm{~mW}$. (b) using an excitation power at the sample of $350 \mu \mathrm{W}$.

Figure 9.6 Single molecule sensitivity - Images $(a, b)$ are far-field optical responses of two photon excitation of single Rhodamine 6G molecules. (c) Shows two photon excited Si quantum dots."

Figure 9.7 Two-photon far-field fluorescence mapping of BPAE cells mounted on a cover glass slip. The spectrum of MitoTracker red CMXRos (a) and Alexa Fluor 488 (Phalloidin) (b) was acquired with a $1 \mathrm{sec}$ integration time at the area marked in the image.

Figure 9.8 Two-photon far-field fluorescence mapping of Caenorhabditis Elegans mounted on a cover glass slip. (a) The lower part of the nematode on a glass slide in agar. (b) The focus was increased in $\mathrm{z}$ and another image taken. Due to the highly confined $\mathrm{Z}$-focal range, little out-of-focus fluorescence is seen.

Figure 10.1 Relative phase as a function of distance from the surface of the sample. The shear force interaction begins about $20 \mathrm{~nm}$ away from the sample surface.

\footnotetext{
* Abridged version of the figure caption.
} 
Figure 10.2 Shear force closed-loop images of a calibration grid (Digital Instruments, Veeco, PG platinum coated calibration grid $1.0 \mu \mathrm{m} x$ $1.0 \mu \mathrm{m}$ period), (a) The topographic image in the trace direction (left to right). (b) The error in the phase feedback signal in the retrace direction (right to left). Both images were acquired at a scan rate of $0.3 \mathrm{~Hz}$

Figure 10.3 Shear force closed-loop imaging of a calibration grid with a larger field of view (Digital Instruments, Veeco, PG platinum coated calibration grid $1.0 \mu \mathrm{m} \times 1.0 \mu \mathrm{m}$ period). (a) The topographic image in the trace direction (left to right). (b) The error in the phase feedback signal engaged at $11^{\circ}, 14^{\circ}$, and $17^{\circ}$

Figure 10.4 Shear force open-loop images of calibration grid (Digital Instruments, Veeco, PG platinum coated calibration grid $1.0 \mu \mathrm{m} x$ $1.0 \mu \mathrm{m}$ period). ${ }^{*}$

Figure 10.5 Topographic shear force image of $5.0 \mu \mathrm{m}$ x $5.0 \mu \mathrm{m}$ periodicity, 119 $\mathrm{nm}$ high pillars. Scan noise is a result of an overly sensitive integral gain term in the PID feedback loop.

Figure 10.6 Topographic shear force image of an unknown contamination layer on mica.

Figure 10.7 Topographic shear force image of DVD.* .202

Figure 10.8 Topographic shear force image of a rewritable DVD. 203

Figure 11.1 Five different gold probes etched with DC etch circuit. ${ }^{*}$ .206

Figure 11.2 Electrical schematic of AC circuit for electrochemically etching $\mathrm{Au}$ wire. The circuit consists of a laboratory Variac (a) and a step down transformer (b)

Figure 11.3 Scanning electron micrographs of Au wire after AC etching procedure. These two examples are very typical results.

Figure 11.4 Tuning fork in can, in preparation of opening with Thorlabs laser diode opener.

* Abridged version of the figure caption. 
Figure 11.5 Sample stubs with gold probes mounted on tuning forks for milling. (a) Flat aluminum sample stub that has a notch milled to allow space for the remaining portion of the tuning fork can. (b) Aluminum stub milled at $52^{\circ}$.

Figure 11.6 Milling cuts for the asymmetric TENOM probe design as outlined in the text. Shown in order from first cut to last (a) to (g).

Figure 12.1 Scattering effects from a near-field probe on two-photon imaging of J-aggregates with PIC dye. (a) Far-field diffraction limited optical image without tip present. (b) Diffraction limited resolution optical image with tip present. (c) Surface topography of imaging ROI. (d) Shows a total offset of $640 \mathrm{~nm}$ between the optical image and the topographic image.

Figure 12.2 Quenching effects from the metallic near-field probe on two-photon imaging of R6G dye crystals. (a) $\mathrm{CW}$ excited near-field optical image, demonstrating below $50 \mathrm{~nm}$ resolution, measured from edgecontrast. The dark regions are areas of optical quenching. (b) Topography of the sample surface, acquired simultaneously. The regions of high topography correspond with the quenching regions.

Figure 12.3 Two-photon imaging of J-aggregates with PIC dye excited at $833 \mathrm{~nm}$ with a mode-locked laser operating at 52 fs pulses. (a) Far-field diffraction limited at $10.1 \mu \mathrm{W}$. (b) Near-field with average excitation power of $9.8 \mu \mathrm{W}$, same ROI as (a). (c) Phase feedback image generated by the error signal for the topography scan, simultaneously acquired with (b).

Figure 12.4 Proposed displacement between the near-field and far-field images presented in Figure 12.2, due to focal delocalization. Both images (a) and (b) are of the same ROI, one with the TENOM imaging probe (a) and one with the probe removed (b). The green contour maps were generated with ImageJ and Photoshop from image (b).

(c) Shows the amount of displacement is roughly $1.0 \mu \mathrm{m}$

Figure 12.5 Fluorescence spectrum from the emission of the PIC dye.* .222

Figure 12.6 Near-field two-photon excitation fluorescence image of J-aggregates of PIC dye in a PVS film on a glass substrate (c), $\lambda_{e}=833 \mathrm{~nm}$ at 10.2 $\mu \mathrm{W}$ mode locked at 52 fs. (d) Diffraction limited far-field twophoton excitation fluorescence of same ROI and excitation source as (c) at $31.5 \mu \mathrm{W} .^{*}$

* Abridged version of the figure caption. 
Figure 12.7 Near-field two-photon excitation fluorescence image of J-aggregates of PIC dye in a PVS film on a glass substrate. (a) $\lambda_{e}=834 \mathrm{~nm}$ at 1.3 $\mathrm{mW} \mathrm{CW}$. (b) Topography of J-aggregates taken simultaneously with an optical image.

Figure 12.8 Two-photon imaging of J-aggregates with PIC dye excited at $817 \mathrm{~nm}$ with a Ti:sapphire operating in $\mathrm{CW}$ mode with $1.18 \mathrm{~mW}$ of excitation power at the sample surface. Resolutions of $40 \mathrm{~nm}$ and less are demonstrated.

Figure 12.9 Near-field two-photon excitation fluorescence image of-thylakoid membranes from Spinacea Oleracea on a glass substrate. (a) Nearfield image with $\lambda_{e}=833 \mathrm{~nm}$ at $16.5 \mu \mathrm{W}$ mode locked at $52 \mathrm{fs}$. (b) Diffraction limited far-field image of same ROI. (c) Demonstrating the offset between the near-field and far-field images

* Abridged version of the figure caption. 


\section{ACROYNMS}

\begin{tabular}{|c|c|}
\hline $1 \mathrm{D}$ & One Dimensional \\
\hline $2 \mathrm{D}$ & Two Dimensional \\
\hline $3 \mathrm{D}$ & Three Dimensional \\
\hline $\mathrm{AC}$ & Alternating Current \\
\hline $\mathrm{ADC}$ & Analog to Digital Converter \\
\hline AFM & Atomic Force Microscopy \\
\hline ANSOM & Apertureless Near-field Optical Microscopy \\
\hline APD & Avalanche Photodiode \\
\hline B.C. & Before Christ \\
\hline CA & Cyanoacrylate (glue) \\
\hline CAD & Computer-Aided Design \\
\hline CARS & Coherent Anti-Stokes Raman Spectroscopy \\
\hline CCD & Charged Coupled Device \\
\hline CLSM & Confocal Laser Scanning Microscopy \\
\hline CMOS & Complementary Metal-Oxide-Semiconductor \\
\hline CMS & Content Management System \\
\hline $\mathrm{CNC}$ & Computer Numerically Control \\
\hline CPU & Central Processing Unit \\
\hline $\mathrm{CS}$ & Chip Select \\
\hline $\mathrm{CW}$ & Continuous Wave \\
\hline $\mathrm{DAC}$ & Digital to Analog Converter \\
\hline DAQ & Data Acquisition \\
\hline $\mathrm{DC}$ & Direct Current \\
\hline DDS & Direct Digital Synthesis \\
\hline DIO & Digital Input / Output \\
\hline DMA & Direct Memory Access \\
\hline DSP & Digital Signal Processor \\
\hline DVD & Digital Video Disk \\
\hline FDTD & Finite Difference Time Domain \\
\hline FEL & Finite Element \\
\hline FFT & Fast Fourier Transform \\
\hline FIB & Focused Ion Beam \\
\hline FIFO & First In First Out \\
\hline FPALM & Fluorescence Photoactivation Localization Microscopy \\
\hline FPGA & Field Programmable Gate Array \\
\hline FRET & Förster Resonance Energy Transfer \\
\hline FWHM & Full Width at Half Maximum \\
\hline G-T & Gilbert Taylor Cone \\
\hline GUI & Graphic User Interface \\
\hline HV & High Voltage \\
\hline
\end{tabular}




\begin{tabular}{|c|c|}
\hline IC & Integrated Circuit \\
\hline ICCD & Intensified Charged Coupled Detector \\
\hline IEEE & Institute of Electrical and Electronics Engineers \\
\hline IMAQ & Image Acquisition \\
\hline IR & Infrared \\
\hline IRQ & Interrupt Request \\
\hline $\mathrm{I} / \mathrm{O}$ & Input / Output \\
\hline KE & Kinetic Energy \\
\hline LED & Light Emitting Diode \\
\hline LMIS & Liquid Metal Ion Source \\
\hline MESFET & Metal Semiconductor Field Effect Transistor \\
\hline MPE & Multi-Photon Excitation \\
\hline MPI & Message Passing Interface \\
\hline NA & Numerical Aperture \\
\hline N.D. & Neutral Density \\
\hline NI & National Instruments \\
\hline NIH & National Institute of Health \\
\hline NSOM & Near-field Scanning Optical Microscopy \\
\hline O.D. & Optical Density \\
\hline Op-amp & Operational Amplifier \\
\hline OPE & One-Photon Excitation \\
\hline OS & Operating System \\
\hline PALM & Photo-Activated Localization Microscopy \\
\hline $\mathrm{PC}$ & Personal Computer \\
\hline PCB & Printed Circuit Board \\
\hline PCI & Peripheral Component Interconnect \\
\hline PCI-express & Peripheral Component Interconnect - Express \\
\hline PIC & Pseudo-Isocyanine \\
\hline PID & Proportional Integral Differential \\
\hline PIII & Pentium Three \\
\hline PMT & Photomultiplier Tube \\
\hline PVS & Poly Vinyl Sulfate \\
\hline PZT & Lead Zirconate Titanate (Piezo Crystal) \\
\hline Q-(factor) & Quality Factor \\
\hline $\mathrm{R} 6 \mathrm{G}$ & Rhodamine $6 \mathrm{G}$ \\
\hline RAM & Random Access Memory \\
\hline RF & Radio Frequency \\
\hline RMS & Root Mean Squared \\
\hline ROI & Region of Interest \\
\hline RPM & Revolutions Per Minute \\
\hline SCLK & Serial Clock \\
\hline SDATA & Serial Data \\
\hline SEM & Scanning Electron Microscopy \\
\hline SFM & Shear Force Microscopy \\
\hline SHG & Second Harmonic Generation \\
\hline
\end{tabular}




$\begin{array}{ll}\text { SNOM } & \text { Scanning Near-field Optical Microscopy } \\ \text { SPAPD } & \text { Single Photon Avalanche Photodiode } \\ \text { SPI } & \text { Serial Peripheral Interface } \\ \text { SPM } & \text { Scanning Probe Microscopy } \\ \text { STED } & \text { Stimulated Emission Depletion } \\ \text { STM } & \text { Scanning Tunneling Microscopy } \\ \text { STORM } & \text { Stochastic Optical Reconstruction Microcopy } \\ \text { SW } & \text { SolidWorks } \\ \text { TCSPC } & \text { Time Correlated Single Photon Counting } \\ \text { TEM } & \text { Transmission Electron Microscopy } \\ \text { TEM(mode) } & \text { Transverse Electromagnetic } \\ \text { TENOM } & \text { Tip Enhanced Near-field Optical Microscopy } \\ \text { TPE } & \text { Two-Photon Excitation } \\ \text { TTL } & \text { Transistor-Transistor Logic } \\ \text { USB } & \text { Universal Serial Bus } \\ \text { UV } & \text { Ultra Violet } \\ \text { VHDL } & \text { VHSIC Hardware Description Language } \\ \text { VHSIC } & \text { Very-High-Speed Integrated Circuit } \\ \text { VI } & \text { Virtual Instruments }\end{array}$

UNITS OF MEASURE

$\begin{array}{ll}\AA & \text { Angstrom } \\ \mathrm{C} & \text { Celsius } \\ \mathrm{cm} & \text { Centimeter } \\ \mathrm{fs} & \text { Femtosecond } \\ \mathrm{GB} & \text { Gigabyte } \\ \mathrm{GM} & \text { Göppert-Mayer } \\ \mathrm{Hz} & \text { Hertz } \\ \mathrm{kHz} & \text { Kilohertz } \\ \mathrm{kV} & \text { Kilovolts } \\ \mu \mathrm{m} & \text { Micrometer } \\ \mu \mathrm{M} & \text { Micromolar } \\ \mu \mathrm{V}_{\text {rms }} & \text { Microvolt RMS } \\ \mu \mathrm{W} & \text { Microwatt } \\ \mathrm{MB} & \text { Megabyte } \\ \mathrm{MeV} & \text { Mega-Electron Volts } \\ \mathrm{MHz} & \text { Megahertz } \\ \mathrm{mL} & \text { Milliliter } \\ \mathrm{mm} & \text { Millimeter } \\ \mathrm{mM} & \text { Millimolar } \\ \mathrm{mV} & \text { Millivolt } \\ \mathrm{m} / \mathrm{V} & \text { Meters per Volt } \\ \mathrm{mV} & \text { Millivolt RMS }\end{array}$




$\begin{array}{ll}\mathrm{mW} & \text { Milliwatt } \\ \mathrm{nm} & \text { Nanometer } \\ \mathrm{nm}_{\mathrm{rms}} & \text { Nanometer RMS } \\ \mathrm{ns} & \text { Nanosecond } \\ \mathrm{V} & \text { Volt } \\ \mathrm{V} / \mathrm{div} & \text { Volt per Division } \\ \mathrm{V} / \mu \mathrm{s} & \text { Volt per Microsecond } \\ \mathrm{V} / \mathrm{m} & \text { Volt per Meter } \\ \mathrm{nF} & \text { NanoFarad } \\ \mathrm{nm} / \mathrm{V} & \text { Nanometer per Volt } \\ \mu \mathrm{s} & \text { Microsecond } \\ \mu \mathrm{s} / \mathrm{div} & \text { Microsecond per Division }\end{array}$




\section{PREFACE}

The focus of this dissertation is to outline the development of a microscope that breaks the diffraction limit, while remaining obtainable and reproducible by the average experimental science and engineering research laboratory. With this motivation, a lot of time was devoted to designing and testing many different aspects of the apparatus to help keep future users from running into the kind of experimental difficulties that have been found in previous near-field microscope design efforts. Many details about the nuts and bolts of the microscope will be discussed throughout the dissertation, along with the difficulties found. I hope that attempts to reproduce these designs are a fruitful endeavor, but I also hope the user has a little patience as well. I have found elements of the microscope I would change and redesign if time had allowed and have noted in a few places where these design changes would be beneficial to future designs.

The dissertation is laid out with the following logic, starting with the diffraction limit. Chapters 1-3 analyze many different approaches to imaging below than the diffraction limit, including a historical and mathematical examination. The introduction topics provide the basis to understand the current state of technology, with the advantages and limitations of various microscopy techniques. The near-field microscope design that is being presented builds upon various microscopy techniques, as discussed in these introductory chapters. These chapters should be used as a starting point for further study. Chapter 3 concludes with the scientific justification for the presented microscope design, as a comparison to other subdiffraction limit imaging methods. Chapter 4 examines nanophotonics with numerical calculations using finite difference time domain modeling 
as a guide for the technique. These models can be used as a basis for the construction of ideal imaging probes for near-field, in either aperture or apertureless near-field microscopy. Chapter 5 discusses the overall requirements for near-field microscopy, the hybrid of shear force microscopy technologies and inverted optical microscopes, and the complicated dance of the many different pieces of hardware. It is this complicated combination of hardware and knowledge with probe design that has prevented near-field imaging at ultra-high resolutions from becoming a common day piece of commercial hardware. Chapter 6 discusses the design of the physical microscope. This includes topics from material selection to optical filters and detectors. A detailed discussion shows that by simplifying a microscope to its most basic optical components, i.e., objective lens and detectors placed at the imaging plane, one can create an optical microscope with high collection efficiency that in many ways rivals commercial products. Chapter 7 delves into the driving components of the project with detailed accounts of the electrical circuits, from high voltage bridge circuits for piezo driving to analog and digital circuit design for frequency detection and feedback. I also comment on many of the tips and tricks for successfully laying out printed circuit boards. Chapter 8 covers the application of computer software. A majority of the computer code has been written in a graphical programming environment that will make it difficult to show in great detail, but I will strive to be a descriptive as possible. Chapter 9 demonstrates the nonlinear far-field imaging capabilities of the microscope to image just beyond the diffraction limit. Furthermore, the imaging can be accomplished with low cost CW laser systems, greatly reducing the cost of traditional nonlinear light sources. Chapter 10 shows the shear force imaging capabilities of the microscope. Chapter 11 outlines the xxxiii 
procedure to manufacture apertureless near-field imaging probes. It turns out that the design of the probe, determined in Chapter 4, is the key to obtaining high-resolution optical images. Chapter 12 showcases the data obtained with these probes. And Chapter 13 gives a detailed discussion about the complications with the microscope.

Finally, I would like take the opportunity to mention that all documentation, i.e., electrical schematics, mechanical drawings, bill of materials, and design notes, has been checked many times for errors, but please do not assume they are 100\% correct. All of the presented documentation can be found in the Appendix sections. The reader may find that after skimming through this material, there are a lot of details, and a misplaced number, incorrect dimension, or missing dimension can occur. I've also designed the microscope with the intent to use as many off the shelf components as possible. Please use these resources, i.e., application notes, component data sheets, and manufactures drawings, to aid in the building process. I've placed over three years of effort into the development of the microscope described within this dissertation, so I guarantee the subject may not be completely comprehensible overnight, especially if the reader is new to the field. However, given a little time, it is all very possible. 


\section{CHAPTER 1}

\section{Introduction to Optical Resolution}

We are a visually observant species in a world of complex images, colors, and visual effects. From the time of birth, we try to quantify our natural environment, find rational meaning in these patterns, and make them, in some way, tangible. It's commonly said that a picture is worth a thousand words. In many ways, this is an understatement. Our visual training since birth has tied words, emotions, and sensations to visual images. Artists try to capture and recreate these images to generate an emotional response and convey ideas. Scientists rely heavily on the use of visual observation to formulate opinions, justifications, and conclusions from experimentation. One could easily argue that visual observation is the most critical and important tool in the sciences. It should be no big surprise that the study of optical phenomenon has a long and rich history. An historical perspective will be shown in terms of the fundamental limitations of spatial resolution in optical microscopy.

\subsection{Historical Perspective}

Early documented accounts put the study of optics and development of optical technologies at $3200-4000$ years in the making, from the time of the ancient Egypt [Hecht, 1998]. Mirrors have been uncovered from ancient Egypt. Exodus 38:8 also referenced the use of mirrors as an aid in personal hygiene. Most notably, among the many Greek philosophers who studied optical phenomenon, Euclid (300 B.C.) demonstrated the geometrical properties of the law of reflection in the book Catoptrics, 
the Greek word for "specular". During this time, it was common to use a mirrored lens, archaically known as a "burning glass", to ignite fires. It wasn't until about two hundred years later, in 130 B.C., that Ptolemy attempted to determine the angular relationship of refraction. In $14^{\text {th }}$ century Italy, the art of lens grinding was developed and used to help correct vision problems [Ilardi, 1976]. The development of a compound microscope (containing multiple lenses) would not be accomplished for another two centuries.

The invention of the first compound microscope is credited to a pair of Dutch brothers and lens grinders, Hans and Zacharias Janssen. In 1590, they built a microscope, simply comprised of two lenses. In 1665, Robert Hooke published works detailing the microscopic features of insects, blood, and various other biological specimens [Hooke, 1665]. Around the same time, Anton van Leeuwenhoek cataloged a vast number of microscopic organisms [Leeuwenhoek, 1678; Dobell, 1932]. These studies would spawn the microscopic study of the natural world. In 1621, Willebrord Snellius would derive the theoretical understanding of the law of refraction, well known as Snell's law, roughly 1700 years after Ptolemy. In 1690, Christiaan Huygens would show that light slows down inside materials of high index of refraction, using wave theory [Huygens, 1690]. Huygens would also realize the phenomenon of polarization in his work.

\subsection{Far-Field Imaging and the Diffraction Limit}

The basic understanding of the compound microscope would continue with centuries of improvement in lens design. Currently, the production of optical systems is aided by the use of computer-aided numerical calculations. Numerical calculations have aided in reaching the theoretical limit of light collection and the removal of spherical and 
chromatic aberrations. Even with these improvements, optical microscopy has one distinct limitation: the spatial resolution is limited by the wavelength being used. The diffraction limit is a result of the wave nature of light. Light waves, converging with each other in a focus, create interference patterns. Oftentimes, geometrical optic analysis (ray tracing, in particular) ignores the diffraction effect of light by imposing two conditions: first, that the wavelength must be much smaller than the size of the optical system, and second, that the light used in the system is paraxial to the optical plane of the lens system. While these conditions approximate most lens conditions with a high degree of accuracy, they cannot accurately describe image information in the near-field with a resolution better than the diffraction limit.
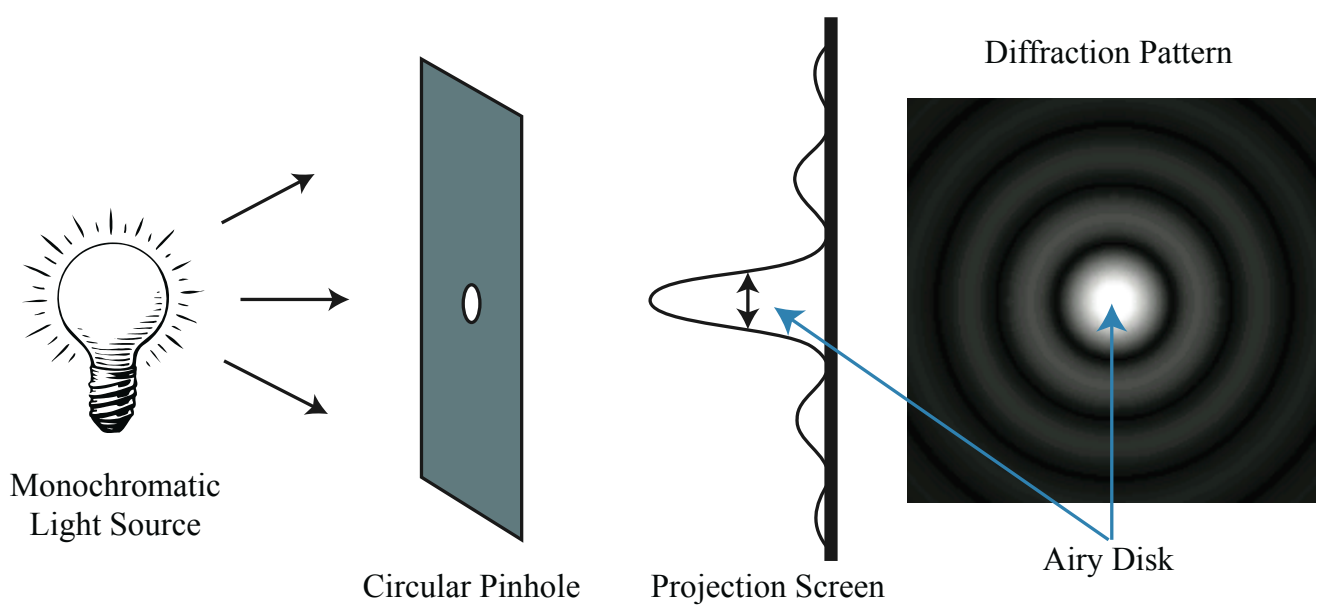

Figure 1.1 Diffraction pattern generated by diffraction of light by a small circular hole in a screen. Before the advent of monochromatic laser light sources, a lamp source and a dispersion prism could be used.

To explore the diffraction concept further, a simple case can be made. Consider a monochromatic light source that illuminates a very small circular aperture. The light that passes through the hole is projected onto a screen. Huygens Principle proposes that the aperture can be equally divided into many points, where each point acts as a point source 
of wavefronts. After passing through the aperture, the wavefronts generate an interference pattern, which has been experimentally shown, similar to Figure 1.1. Thomas Young performed many of the classical diffraction and interferences experiments in the early 1800's [Young, 1804]. The diffraction pattern resulting from the circular aperture is commonly known as an Airy disk. In 1835, George Biddell Airy published the theoretical basis of the circular diffraction pattern, with the combination of an aperture and lens [Airy, 1835]. His work justifies that the smallest image simply reduces to an Airy disk pattern, an effect of diffraction.

Assuming that all optical systems are diffraction limited, the practical resolution of any optical system is known. It can be calculated, based on the wavelength of light used and the numerical aperture $(N A)$ of the lens system. In 1873, Ernst Abbe discovered that the minimum spot diameter obtainable with a focusing lens is given by,

$$
D=\frac{\lambda}{N A}
$$

where $\lambda$ is the wavelength of light used and $N A$ is the numerical aperture of the lens [Abbe, 1873]. The $N A$ is given by,

$$
N A=n \sin \alpha,
$$

where $\alpha$ is defined by the half-angle subtended by the focusing light and $n$ the index of refraction of the material. Figure 1.2 shows the effect that $N A$ has on the width and intensity of the Airy disk. The distance between two objects, or the separation of two overlapping Airy disks, is used to define the resolution of an optical system. If one minimizes the Airy disk pattern by creating a smaller focusing radius, two objects can be 
resolved at a smaller distance. A practical application of separation distance is demonstrated in Figure 1.3.

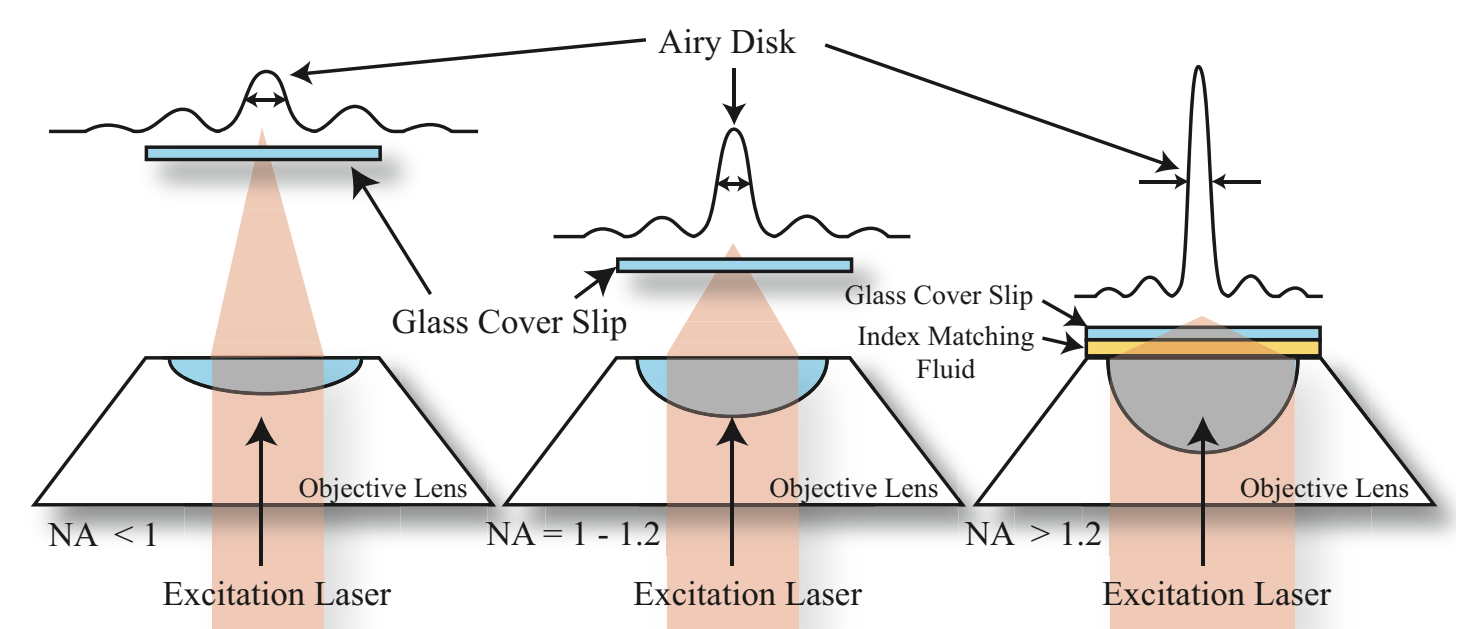

Figure 1.2 Effect of numerical aperture $(N A)$ of focusing lens on the size of an Airy disk. Note for a $N A$ greater that 1.2, an index matching fluid between the lens and the glass substrate supporting the sample is used.

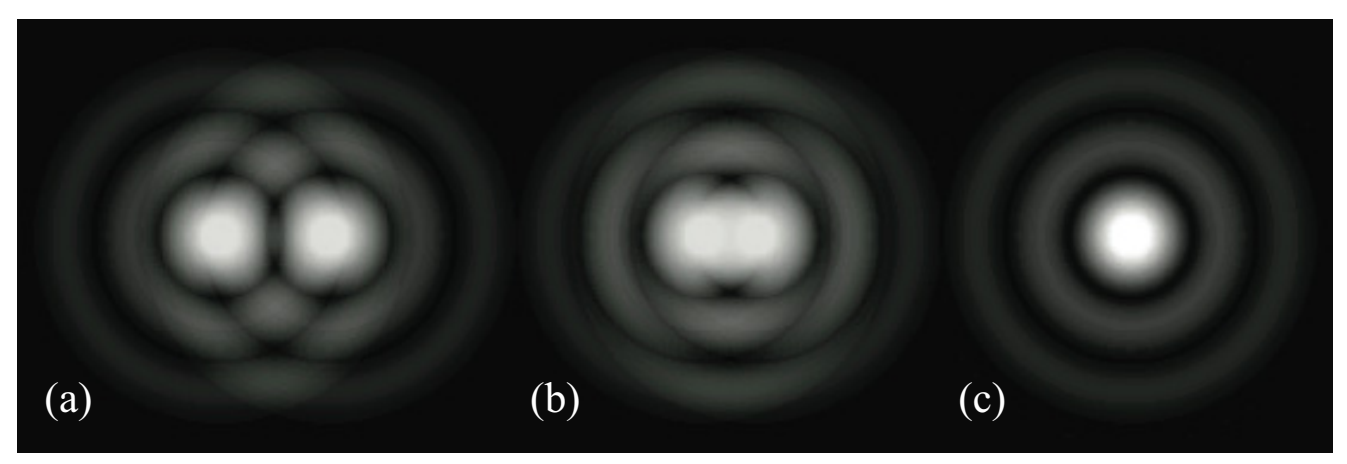

Figure 1.3 Diffraction patterns for different overlapping Airy disks: (a) the two different airy disk patterns are easily seen with a large separation, (b) the limit by which the points are just resolvable, reaching the resolvable limit of the overlap, and (c) the two airy disks are not resolvable when overlapped.

The observable resolution is formally defined as the separation distance for two objects, where the Airy disks overlap at no more than the full-width at half-maximum (FWHM). The limit of resolution is defined by the minimum separation distance between two 
objects in order to resolve them in the far field, known as Rayleigh's criterion. In 1879, Lord Rayleigh determined through empirical methods that the minimal angular separation is defined by,

$$
\sin \theta=1.22 \frac{\lambda}{2 R},
$$

where $\theta$ is the angular resolution in radians, and $R$ the radius of the aperture of the lens [Rayleigh, 1879]. This same relationship is derivable with a Bessel function of the first kind and zero order, and has a minimum at approximately 1.22, shown in Figure 1.4.

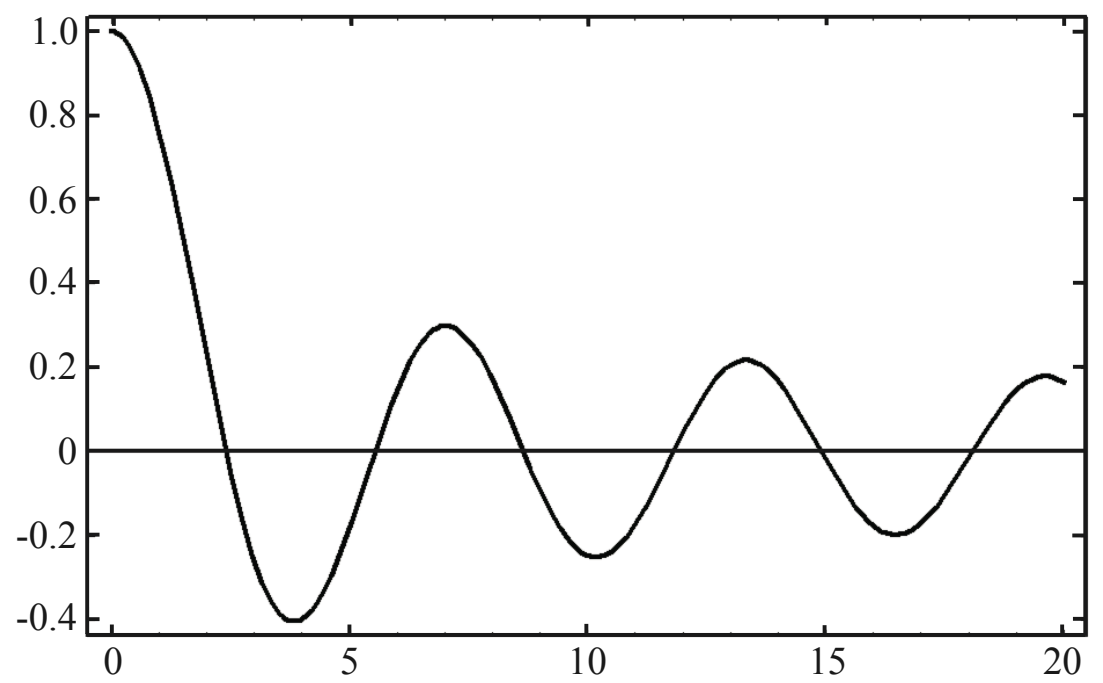

Figure 1.4 Bessel function of the first kind and zero order. The graph shows a minimum at $\sim 3.85$ which when divided by $\pi$ is $\sim 1.22$.

With these relationships, it is possible to define resolution as the separation distance $(d)$ in a microscope given by,

$$
d=\frac{1.22 \lambda}{2 N A} .
$$

For a commercially available $1.4 \mathrm{NA}$ objective lens, the highest practical imaging resolution with $800 \mathrm{~nm}$ light is $348 \mathrm{~nm}$. 


\subsection{Modeling the Diffraction Limit}

Today's available computational power provides the ability to approximate complex analytical problems via intensive numerical calculations. Applying this ability to optical systems, a visualization of an electromagnetic wave passing through a lens can be obtained. Many of these calculations are processed by finite difference methods, starting with a $3 \mathrm{D}$ problem space, then dividing the problem into discrete cubical units. Each of the units is given as set of parameters based on a known physical constant for the material. Finite difference modeling shows the diffraction effect of a monochromatic light source passing through a focusing lens. Figure 1.5 shows the propagation of a linearly polarized monochromatic electromagnetic wave with a wavelength of $800 \mathrm{~nm}$ passing through a lens with an index of refraction of 1.514 .

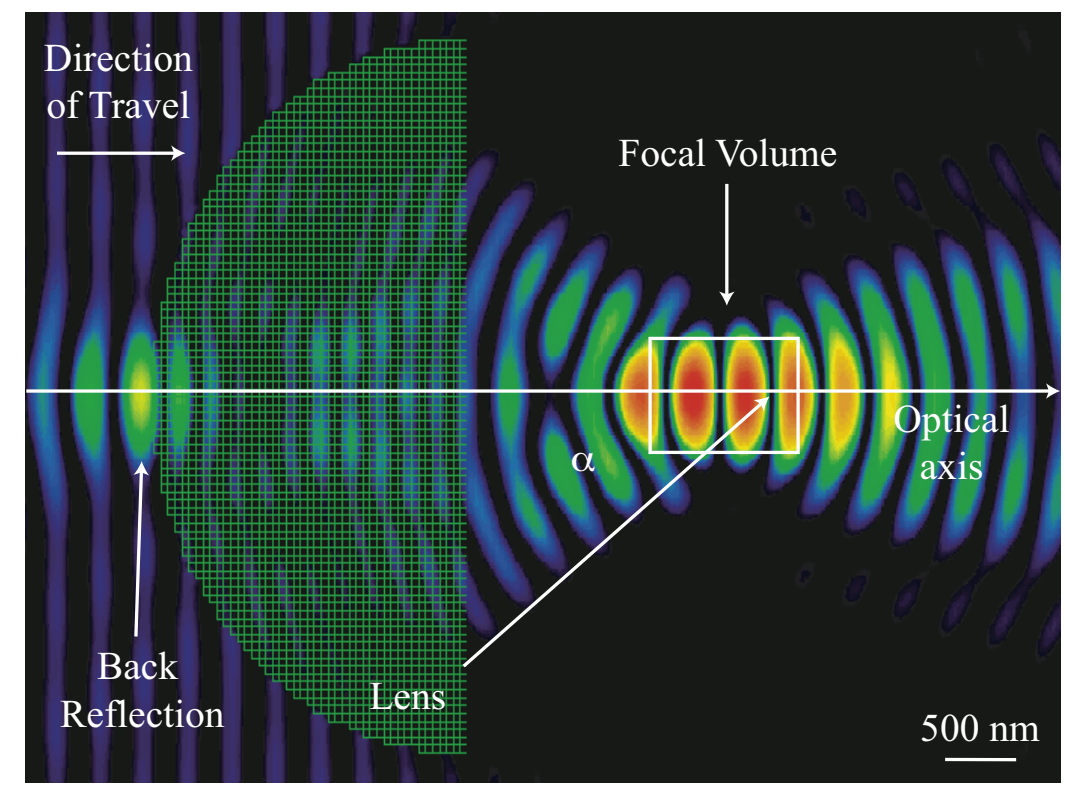

Figure 1.5 Finite difference time domain modeling (FDTD) of a $5.0 \mu \mathrm{m}$ 3D lens focusing $800 \mathrm{~nm}$ light. The modeling shows the finite size of the focal region. FDTD calculations presented were performed on Remcom's xFDTD (version 6.1). In-depth studies of FDTD calculations, as applied to nanophotonics, are presented in Chapter 4. 
Since it has been shown that the numerical aperture of the focusing lens governs the diffraction-limited resolution, it is possible to determine the accuracy of the model. From the model, one could visually estimate that $\alpha$ is approximately $40^{\circ}$ which makes the $N A$ of the lens 0.97 . Then by Equation 1.4, it would follow that the best resolution obtainable for the modeled lens is $504 \mathrm{~nm}$. Based on the result of the FDTD model shown in Figure 1.5 , it can be concluded that the FWHM of the focal waist demonstrates this resolution.

\subsection{The Diffraction Limit and Non-Optical Techniques}

Charged beam microscopies offer many different techniques to image and analyze features in microscopic and nanoscopic scales. Scanning electron microscopy (SEM), transmission electron microscopy (TEM), and focused ion beam (FIB) systems are commonly used charged beam microscopes. In terms of resolution, the TEM provides the highest, on the orders of angstroms. However, there are several constraints on the samples that a TEM can image. The sample must be conductive, or coated with a conductive material, to prevent the build up of electrons, otherwise known as "charging". For the TEM, the sample must fit on a copper support grid that is roughly three millimeters in diameter and a tenth of a millimeter thick. Both the SEM and FIB have less constraints on size, although the larger the object, the lower the obtainable resolution. This decrease in resolution is a function of the working distance away from the end of the charged particle gun [Goldstein et al., 1992]. The primary limitation of all three charged beam methods is the need for the imaging to be accomplished under vacuum. The constraint of a vacuum system makes it difficult to image biological dynamics in ambient conditions. The physiology of the sample will be affected, since one must dry and coat 
the samples before imaging. It is this rationale that motivates the need to determine methods that break the diffraction limit of light in the visible spectrum.

The charged beam imaging methods also have a fundamental diffraction limit. While outside the context of this dissertation, as a microscopist, it is interesting to note that the resolutions for electron and ion beam optics have yet to reach the diffraction limit and there is still room for improvement in charged beam technologies before this limit becomes an experimental reality. Using the wave formulism for particles, the diffraction limit for either an electron or ion source can be determined. In 1924, Louis de Broglie determined the relationship between the wavelength and momentum of a particle, and the relationship between the frequency and kinetic energy of a particle, ultimately earning him a Noble Prize in Physics in 1929 [de Broglie, 1924]. DeBroglie's relations are given by the following,

$$
\lambda=\frac{h}{p} \quad \text { and } \quad f=\frac{E}{h},
$$

where $h$ is Planck's constant. As an example, the wavelength of a $20 \mathrm{kV}$ electron beam, commonly found in an electron microscope, can be calculated with DeBroglie's wavelength expressed in electron volts. Equation 1.6 gives this expression,

$$
\lambda=\frac{h c}{p c} \quad \text { where } \quad p c \approx \sqrt{2 \cdot K E \cdot m_{0} c^{2}},
$$

neglecting a completely relativistic treatment of the problem. The electron has a rest mass $\left(m_{0}\right)$ of $0.511 \mathrm{MeV}$ and $K E$ of $20 \mathrm{kV}$. The wavelength of the electron is $0.08 \AA$, about an order of magnitude smaller than current day electron microscopes [Erni et al., 2009]. Limitations in current charge beam microscopes result from lens aberrations and the lack of monochromatic charge emission points. 


\section{CHAPTER 1 References}

Abbe E., "Beiträge zur Theorie des Mikroskops und der mikroskopischen Wahrnehmung," Archiv f. Miroskop. Anat. 9, 413 (1873).

Airy G. B., "On the Diffraction of an Object-glass with Circular Aperture," Transactions of the Cambridge Philosophical Society 5, 283-291 (1835).

De Broglie L., Ph.D. dissertation. "Recherches sur la théorie des quanta" (Researches on the quantum theory), (Paris, 1924).

Dobell C., Antony van Leeuwenhoek and His 'Little Animals'. (Dover Publications, New York, 1932).

Erni Rolf, Rossell M. D., Kisielowski C., and Dahmen U., “Atomic-Resolution Imaging with a Sub-50-pm Electron Probe," Phys. Rev. Lett. 102, 096101 (2009).

Goldstein J. I., Newbury D. E., Echlin P., Joy D. C., Romig Jr. A. D., Lyman C. E., Fiori C., and Lifshin E., Scanning Electron Microscopy and X-Ray Microanalysis, $2^{\text {nd }}$ ed. (Plenum Press, New York, 1992) pp. 51.

Hecht E., Optics, $3^{\text {rd }}$ ed. (Addison Wesley, New York, 1998) pp. 1-2.

Hooke R., Micrographia: or, Some physiological descriptions of minute bodies made by magnifying glasses (J. Martyn and J. Allestry, London, 1665).

Huygens C., Traité de la Lumière (Pieter van der Aa, Leiden, 1690).

Ilardi V., "Eyeglasses and Concave Lenses in Fifteenth-Century Florence and Milan: New Documents," Renaissance Quarterly 29, 341-360 (1976).

Leeuwenhoek A., "De natis è semine genitali animalculis". R Soc (London) Philos Trans. 12, $1040-1043$ (1678).

Rayleigh L., "Investigations in optics, with special reference to the spectroscope," Phil. Mag. 8, 261-274/403-411/477-486 (1879).

Young T., "The Bakerian Lecture: Experiments and Calculations Relative to Physical Optics," Philosophical Transactions of the Royal Society of London 94, 1-16 (1804). 


\section{CHAPTER 2}

\section{Nonlinear Optical Microscopy}

The term nonlinear describes an effect that does not follow a linear response function to an external perturbation. Nonlinear systems violate the superposition principle because of the lack of these linear relationships. In optical microscopy, there are several forms of nonlinear imaging: optical second-harmonic generation [Franken et al., 1961], multiphoton fluorescence [Denk, Strickler, \& Webb, 1990], surface enhanced Raman scattering ${ }^{*}$ [Fleischmann, Hendra, \& McQuillan, 1974], and coherent anti stokes Raman spectroscopy (CARS) [Maker \& Terhune, 1965]. The nonlinear response of the sample differs from linear techniques such as one-photon excitation (OPE) and reflection (Rayleigh scattering). For linear methods, the response of the sample is proportional to the intensity of the illumination light used. In two-photon nonlinear imaging, for example, the emission of the sample has a quadratic dependence on the illumination intensity. The nonlinear effects provide methods to increase spatial resolution, improve imaging contrast, and explore timing dynamics, vibrational, and electronic states. Practical use requires an understanding of the effects, requirements, experimental complexity, and artifacts that correlate to nonlinear excitation. Our primary focus here will be two-photon excitation (TPE) as a primer for this dissertation; other related techniques and tricks are included for context, including current "super-resolution" farfield methods of optical imaging.

\footnotetext{
* The Raman effect [Raman \& Krishan, 1928] is a weak linear response to light scattering from a surface. Nonlinear techniques are typically used to amplify this weak linear response.
} 


\subsection{Multi-Photon Fluorescence Microscopy}

Using two or more photons to stimulate a single fluorophore absorption in a single quantized event is the basis for multi-photon excitation. An incoming photon of half the required excitation energy excites the fluorophore to a virtual state when the second photon, also of half the required excitation energy, arrives and adds to the virtual state, completing the excitation transition. In 1931, Maria Göppert-Mayer published the theoretical bases for TPE transitions and emission processes in atomic quantum states [Göppert-Mayer, 1931]. Her Nobel Prize winning research becomes the basis for twophoton nonlinear imaging techniques. Figure 2.1 shows an energy diagram describing a two-photon transition.

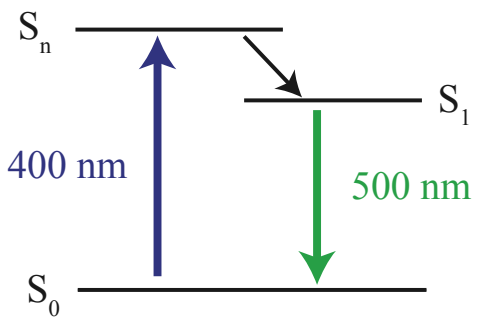

Single Photon Excitation

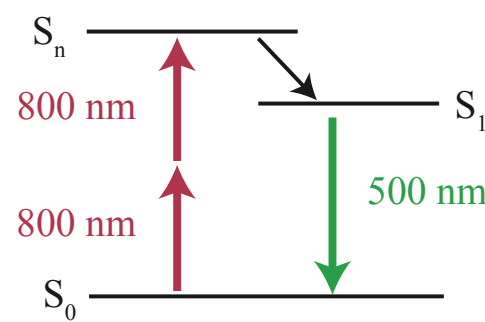

Two Photon Excitation

Figure 2.1 Energy diagram for two-photon transition, based on wavelength of the incoming photon(s). Values chosen for simplicity, a range of wavelengths can generate TPE, dependent on the specific dye's absorption cross section.

With the advent of laser technologies and, in particular, mode-locked pulsed laser systems, Walter Webb has published articles outlining the optical TPE characteristics of various dyes [Xu \& Webb, 1996]. The important measure for two-photon excitation is the absorption cross section for a particular dye. The absorption cross section is a function of wavelength given in units of GM (Göppert-Mayer). 
Multi-photon excitation (MPE) limits fluorescence to a small region of the focal plane, eliminating the need for apertures as used in confocal microscopy [Denk et al., 1990]. Figure 2.2 shows an example of this improved resolution (roughly $\lambda / 3$ ) caused by MPE over one-photon excitation (OPE).

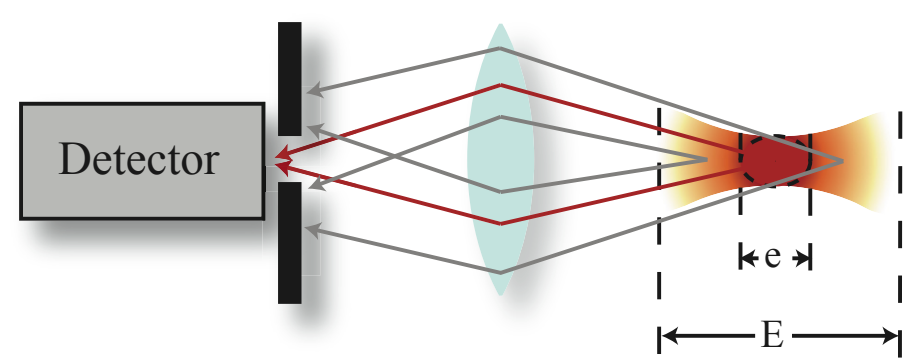

Figure 2.2 TPE cross section compared to OPE. "E" is the cross sectional area for OPE, and "e" is the cross sectional area for TPE.

For a two-photon process to occur, two photons must arrive and be absorbed by the fluorescent molecule within approximately an attosecond. To stimulate a two-photon process, significant photon density is required. Typically, mode-locked pulsed lasers with high pulse power are used to aid in the generation of high photon densities. Multiphoton imaging also benefits from the use of high numerical aperture objective lenses to ensure a high density of photons. The time-averaged two-photon emission from the fluorophore is given by

$$
\langle F(t)\rangle \approx \frac{1}{2} g \phi \eta_{2} C \delta \frac{8 n\langle P(t)\rangle^{2}}{\pi \lambda},
$$

where,

$$
\begin{array}{ll}
g=2^{\text {nd }} \text { order temporal coherence } & \eta_{2}=\text { Fluorescence quantum efficiency } \\
\phi=\text { Fluorescence collection efficiency } & C=\text { Fluorophore concentration } \\
\delta=\text { Two-photon absorption cross-section } & P(t)=\text { Instantaneous incident power } \\
n=\text { Index of refraction of the sample } & \lambda=\text { wavelength in vacuum. }
\end{array}
$$


Another look at the equation shows the quadratic nature of photon emission from TPE of the fluorophore, given by the following relationship,

$$
\langle F(t)\rangle \propto\langle P(t)\rangle^{2} .
$$

It has been experimentally shown that TPE of a single molecule follows a quadratic increase in intensity in response to a doubling of excitation power [Sánchez et al., 1997].

Stephen Hell and associates have shown TPE imaging with continuous wave (CW) laser sources [Hell et al., 1998]. CW laser sources offer a simplification of the optical system design and a substantial reduction in cost. Analyzing the power levels required for two-photon emission (TPE), one could demonstrate the ability for a $\mathrm{CW}$ laser to work as well as a mode-locked pulsed Ti:sapphire laser. The $2^{\text {nd }}$ order temporal coherence factor in Equation 2.1 proves the feasibility of replacing the Ti:sapphire with a diode laser as the TPE source. This requires the average power to be roughly 100 times that of a typical, mode-locked Ti:sapphire. Caution should be used when imaging with the $\mathrm{CW}$ laser, due to the higher power requirement, which may cause localized heating of the sample.

\subsection{Other Nonlinear Optical Imaging Techniques}

Of the numerous types of nonlinear optical imaging techniques, there are a few that offer interesting advantages over others. It is important to mention a few different nonlinear imaging techniques to give context beyond two-photon excitation microscopy. Brief descriptions of these are discussed to give a larger scope to different optical techniques. It is possible to integrate these ideas into near-field imaging. 


\section{2.a Second-Harmonic Generation}

Second-harmonic generation (SHG) is one type of nonlinear optical imaging that takes advantage of the interaction of photons with nonlinear materials [Hellwarth \& Christensen, 1974; Sheppard et al., 1977]. When the photon interacts with this type of material, a frequency doubling occurs that in return generates a photon with twice the frequency. The benefits of SHG are used in laser technologies and high-resolution imaging of biological specimens. Collagen in biological tissues is a nonlinear material. The nonlinear imaging of collagen ultimately gives resolutions comparable to confocal and two-photon microscopy techniques [Fine \& Hansen, 1971; Freund, Deutsch, \& Sprecher, 1986]. The large spectral difference between the excitation (typically near-IR) and emission (UV) gives SHG high signal to background noise. The penetration depth for near-IR illumination sources and lack of photobleaching adds to SHG benefits, assuming the sample material exhibits this nonlinear effect.

\section{2.b Coherent Anti-Stokes Raman Scattering}

Coherent anti-stokes Raman scattering (CARS) is another form of nonlinear spectroscopy and imaging [Duncan, Reintjes, \& Manuccia, 1982]. The core motivation of CARS is to image live biological samples without the need for dye labels. CARS amplifies anti-Stokes Raman vibrational frequencies by tuning the difference of two laser beams in order to coherently drive the Raman vibrational mode. This is accomplished with a pump laser beam and a Stokes frequency beam. The response is measured at twice the pump laser frequency minus the Stokes frequency. Since the lasers can be tuned to take advantage of specific molecular bonds, selectivity in the response marker of a 
sample is possible. CARS also benefits from a small focal region similar to two-photon excitation.

\section{2.c Förster Resonance Energy Transfer}

Förster resonance energy transfer (FRET) is another form of nonlinear imaging that utilizes the transfer of energy between two fluorophores [Förster, 1948]. During excitation of the donor fluorophore, energy is transferred to the acceptor molecule that then produces a photon that is red shifted from the excitation wavelength. The transfer of energy requires that the donor and acceptor molecules (FRET pair) be within $10 \mathrm{~nm}$ or less of each other. This spatial dependency makes FRET pairs ideal for conformational change in protein binding interactions for molecular dynamic experiments. FRET pairs can be used to determine the transport mechanisms of ion channels in biological membranes.

\subsection{Advances in Sub Diffraction Limit Far-field Nonlinear Optical Imaging}

Currently, many advances are being made in the reconstruction and or modification of far-field focusing of light, allowing researchers to obtain far-field spatial resolutions that are much less than the diffraction limit. The most important of these starts with the confocal laser scanning microscopy (CLSM) [Minsky, 1957; Denk, Piston \& Webb, 1995]. CLSM uses a diffraction limited laser spot, focused on the surface of the sample. The spot is then raster scanned by either moving the laser beam or moving the sample. A computer correlates the fluorescence signal returning from the sample with the known location of the laser beam and constructs a two dimensional intensity graph of fluorescence signal vs. $x-y$ spatial location. A CLSM system improves upon the 
traditional $\lambda / 2$ resolution by obtaining resolutions closer to $\lambda / 3$. This improvement is due to the placement of a small aperture at the illumination source and the detector. This is similar to the effect obtained with multi-photon methods, as shown in Figure 2.2. The aperture rejects stray light from outside of the focal plane that contributes to the edges of the airy disk. By moving the focal plane, one can compile multiple images at different zheights, providing the ability for 3D imaging.

\section{3.a STimulated Emission Depletion (STED)}

In 1994, Stefan Hell published the concept of stimulated emission depletion (STED) [Hell \& Wichmann 1994]. Klar and Hell would publish the experimental realization of STED a couple of years later [Klar \& Hell, 1999]. STED uses a diffraction limited excitation laser pulse followed by a depletion laser pulse. The second pulse is configured to generate stimulated emission from dye molecules, forcing them to return to the ground state. Using higher order laser propagation modes, in particular doughnut mode, only the dark region in the middle of the secondary pulse will allow spontaneous fluorescent emission to return from the excitation laser pulse. The STED method has shown resolutions of $\lambda / 25$ [Westphal, Kastrup, \& Hell, 2003]. STED requires emission and depletion lasers that are tuned to the dyes of interest, combined with high-speed laser pulse timing and laser optics for mode generation.

\section{3.b Using Photo-Switchable Fluorophores for Temporal Localization}

In 2006, Eric Betzig and colleagues developed a method that uses photoactivatable fluorescent molecules separated by several nanometers [Betzig et al., 2006]. They call the method photo-activated localization microscopy or PALM, for 
short. At roughly the same time, Xiaowei Zhuang's group developed stochastic optical reconstruction microcopy or STORM [Rust, Bates \& Zhuang, 2006]. Sam Hess independently developed the same technique that he named FPLAM [Hess, Girirajan \& Mason, 2006]. All of these methods work under very similar principles. These methods use a light pulse that activates the molecules. Since spontaneous fluorescence is a stochastic event, only a few of the molecules emit a fluorescent photon. An image of these molecules is captured for every light pulse. Images are acquired until the molecules photobleach. The images are then compiled together. The Gaussian profile of the fluorescence is fitted to a point spread function. Since the fluorophore is physically smaller than the diffraction limit, a statistical determination of the spatial location of the fluorophore can be inferred. These methods require functionalized dye molecules that have significant spacing between neighbors to differentiate the spatial location. Excitation laser wavelengths are tuned to optimize the dye's functionality. Multiple image captures and the time to compile the final images are also requirements of this technique. Currently, acquisition time is too slow to determine cellular dynamics, although resolutions of $20 \mathrm{~nm}$ have been demonstrated [Betzig, 2006; Rust, 2006]. In 2008, Bo Huang of Xiaowei Zhuang's group has extended STORM into threedimensional imaging, showing $20 \mathrm{~nm}$ lateral and $50 \mathrm{~nm}$ axial imaging resolutions [Huang et al., 2008]. 


\section{CHAPTER 2 References}

Albrecht G. M. and Creighton A. J., "Anomalously Intense Raman Spectra of Pyridine at a Silver Electrode," Journal of the American Chemical Society 99, 5215-5219 (1977).

Betzig E., Patterson G. H., Sougrat R., Lindwasser O. W., Olenych S., Bonifacino J. S., Davidson M. W., Lippincott-Schwartz J., and Hess H. F., "Imaging Intracellular Fluorescent Proteins at Nanometer Resolution," Science 313, 1642-1645 (2006).

Denk, W., Piston, D. W., and Webb, W. W., Handbook of Biological Confocal Microscopy, ed. J. Pawley (Plenum Press: New York, 1995), pp 445-458.

Denk W., Strickler J., and Webb W., "Two-Photon Laser Scanning Fluorescence Microscopy," Science 248, 73-6 (1990).

Duncan M., Reintjes J., and Manuccia T. J., "Scanning Coherent Anti-Stokes Raman Microscope," Opt. Lett. 7, 350-352 (1982).

Fine S. and Hansen W. P., "Optical Second Harmonic Generation in Biological Systems," Appl. Opt. 10, 2350-2353 (1971).

Fleischmann M., Hendra P. J. and McQuillan A. J., "Raman Spectra of Pyridine Adsorbed at a Silver Electrode," Chemical Physics Letters 26, 163-166 (1974).

Förster T., "Zwischenmolekulare Energiewanderung und Fluoreszenz,” Ann. Physik 437, 55 (1948).

Franken P. A., Hill A. E., Peters C. W., and G. Weinreich, "Generation of Optical Harmonics," Phys. Rev. Lett. 7, 118-121 (1961).

Freund I., Deutsch M., and Sprecher A., "Connective Tissue Polarity,” Biophys. J. 50, 693-712 (1986).

Goeppert-Mayer M., “Über Elementarakte mit zwei Quantensprüngen,” Ann Phys 9, 273-95 (1931).

Hell S. W., Booth M., Wilms S., Schnetter C. M., Kirsch A. K., Arndt-Jovin D. J., and Jovin T. M., "Two-photon Near- and Far-field Fluorescence Microscopy with Continuous-Wave Excitation," Opt. Lett. 23, 1238 (1998).

Hell S. W. and Wichmann J. "Breaking the Diffraction Resolution Limit by Stimulated Emission: Stimulated-Emission-Depletion Fluorescence Microscopy,” Optics Letters 19, 780-782 (1994). 
Hellwarth R. and Christensen P., "Nonlinear Optical Microscopic Examination of Structure in Polycrystalline ZnSe," Optics Comm. 12, 318-322 (1974).

Hess S. T., Girirajan T. P. K., and Mason M. D., "Ultra-High Resolution Imaging by Fluorescence Photoactivation Localization Microscopy," Biophysical Journal 91, 42584272 (2006).

Jeanmaire, D. L. and Van Duyne R. P., "Surface Raman Electrochemistry Part I. Heterocyclic, Aromatic and Aliphatic Amines Adsorbed on the Anodized Silver Electrode," Journal of Electroanalytical Chemistry 84, 1-20 (1977).

Klar T. A. and Hell S. W., "Subdiffraction Resolution in Far-Field Fluorescence Microscopy," Optics Letters 24, 954-956 (1999).

Maker P. D. and Terhune R. W., "Study of Optical Effects Due to an Induced Polarization Third Order in the Electric Field Strength," Phys. Rev. 148, 990 (1966).

Minsky M., U. S. Patent No. 3,013,467 (19 December, 1961).

Raman C. V. and Krishnan K. S., “A New Type of Secondary Radiation,” Nature (London) 121, 501 (1928).

Rust M. J., Bates M. and Zhuang X., "Sub-Diffraction-Limit Imaging by Stochastic Optical Reconstruction Microscopy (STORM)" Nature Meth. 3, 793-796 (2006).

Sánchez E. J., Novotny L., Holtom G. R., and Xie X. S., "Room-Temperature Fluorescence Imaging and Spectroscopy of Single Molecules by Two-Photon Excitation," J. Phys. Chem. A 101, 7020 (1997).

Sheppard C. J. R., Kompfner R., Gannaway, and Walsh D., "Scanning Harmonic Optical Microscope,” IEEE J. Quantum Electronics 13, 100 (1977).

Westphal V., Kastrup L. and Hell S. W., "Lateral Resolution of $28 \mathrm{~nm}(\lambda / 25)$ in Far-Field Fluorescence Microscopy,” Appl. Phys. B 77, 377-380 (2003).

Xu C. and Webb W. W., "Measurement of Two-Photon Excitation Cross Sections of Molecular Fluorophores with Data from 690 to 1050 nm," J. Opt. Soc. Am. B 13, 481491 (1996). 


\section{CHAPTER 3}

\section{Introduction to Near-Field Optical Imaging}

Near-field is defined as the area that is "very close" to the emission of a signal, normally within one wavelength of the source at the frequency of interest. Any distance that is greater than one wavelength away is considered to be in the far-field. Near-field optical imaging is simply the ability to generate optical responses in the near-field that are controlled at known spatial locations in order to generate a mapping of the specimen. These responses are generated from fluorophores, molecular bonds, or any conformational information inherent to the sample that was excited within one wavelength of the incident radiation. The near-field response of the sample is normally detected in the far-field by a detector that is sensitive to the emitted radiation. To give a general understanding of near-field excitation and near-field imaging, a short theoretical context is given followed by experimental realization methods. A motivation for using near-field imaging to obtain high-resolution optical images in comparison to other highresolution optical imaging methods is discussed toward the end of the chapter.

\subsection{Theoretical Context of "Small Holes"}

In 1944, Bethe, and in 1950, Bouwkamp theoretically investigated the propagation of electromagnetic radiation impinging on an infinite conducting plane containing a small aperture hole that was smaller in radius than the wavelength of the incident radiation [Bethe, 1944; Bouwkamp, 1950]. Bethe's work looked at microwave wavelengths and the effect of a small hole in the cavity of microwave radiation. His 
results showed the power dissipation in the near-field was proportional to $1 / r^{6}$ in comparison to the far-field dissipation that is proportional to $1 / r^{2}$. The importance of this finding is that it demonstrates that electromagnetic radiation can be confined to a region that is smaller than the diffraction limit. Moving from the microwave scale to the nanometer scale of optical light should follow the same basic relationship. Figure 3.1 shows the electric field distribution of the Bethe - Bouwkamp calculations.

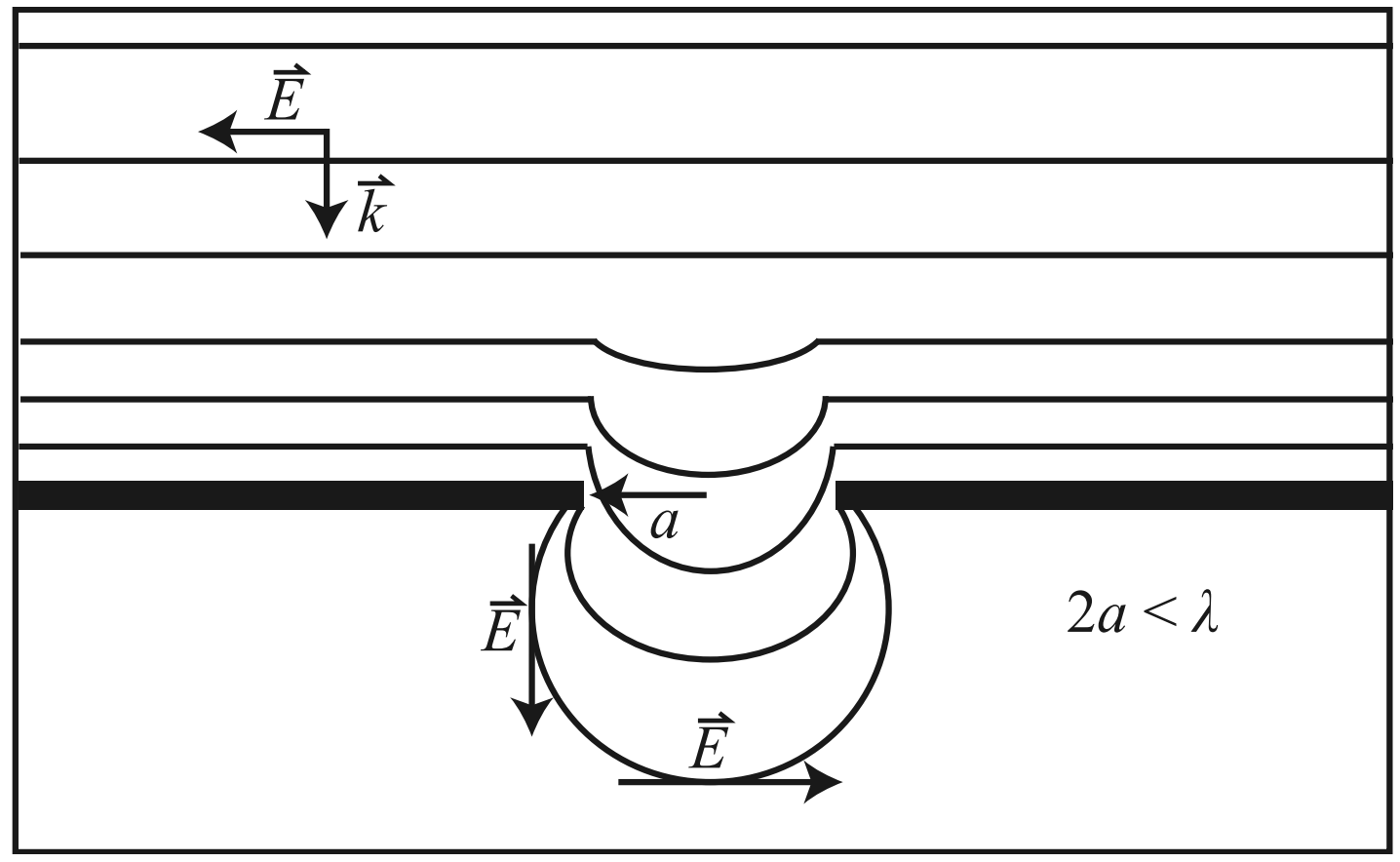

Figure 3.1 Electric field distributions for an aperture smaller than the wavelength in an infinite conduction sheet.

Figure 3.1 shows a monochromatic, polarized light source with an incident field that is polarized in the $\mathrm{x}$ direction. Once the light passes the small aperture, the field develops components in both the $\mathrm{y}$ and $\mathrm{z}$ directions, in addition to $\mathrm{x}$. Bethe's calculation showed that the incident field polarization maintains the highest magnitude, while the $\mathrm{z}$ polarization is an order of magnitude weaker and the y polarization a couple orders of 
magnitude weaker. Figure 3.2 shows the patterns for the field distributions in the nearfield of the three different polarizations.

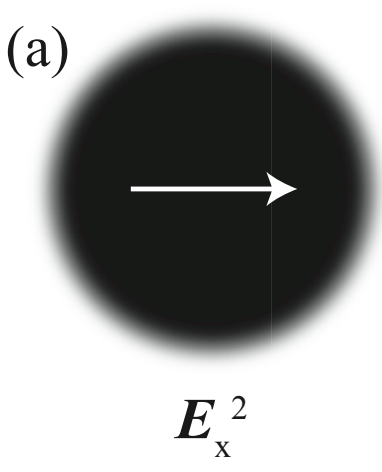

(b)

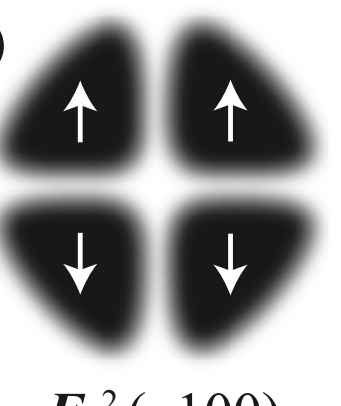

(c)
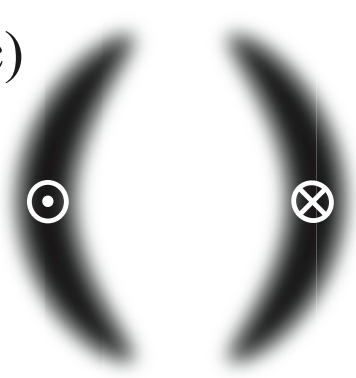

$\boldsymbol{E}_{\mathrm{z}}^{2}(\mathrm{x} 10)$

Figure 3.2 Near-field intensity pattern of 3 different polarization orientations (a) the horizontal (x-direction), (b) $90^{\circ}$ to the horizontal (y-direction), and (c) vertical polarizations ( $\mathrm{x}-\mathrm{y}$ plane).

\subsection{Near-Field Optical Imaging with Aperture Probes}

The fundamental goal of near-field microscopy is to create a sub-diffraction limited field distribution in a location favorable for use in a scanning probe microscope. The original idea for a near-field microscope came about before Bethe and Bouwkamp's theoretical calculations. In 1928, Synge postulated an instrument composed of a thin metal film and an aperture much smaller than the wavelength of illumination [Synge, 1928]. Synge further pointed to the use of piezo electrical devices to move the imaging source [Synge, 1932]. Ultimately, he would not be able to make a functional microscope with the available technologies. In 1956, John O'Keefe published a theoretical construct for the development of a near-field microscope, concluding that movement of the pinhole would be too technologically challenging [O'Keefe, 1956]. The first demonstration of sub-diffraction limited imaging would be in 1972, when the diffraction limit was broken using microwave radiation [Ash \& Nichols, 1972]. 
The experimental reality of using optical light for near-field scanning optical microscopy NSOM/SNOM* became successful in the 1980's. Lewis and Pohl published some of the first papers showing applications of SNOM [Lewis et al., 1984; Pohl, Denk, \& Lanz, 1984]. The first creation of a NSOM system in the United States was by Eric Betzig, using a coated micropipette [Betzig et al., 1991; Betzig, Finn \& Weiner, 1992]. Although this method was successful, it wasn't the optimal waveguide for power throughput. Later, this design was improved with the use of an optical fiber [Betzig et al., 1993]. This design uses a single mode optical fiber as a waveguide, which is pulled down to a sub $\lambda / 2$ end diameter via simultaneous heating and pulling (micropipette puller). The fiber probe is then coated with a light confining material, usually a good conductor. When coated properly, it is left with a small aperture at the end of the probe. Figure 3.3 shows a diagram of the aperture probe in a typical imaging environment. Other groups have made improvements and modifications to this basic design, although the fundamental concepts are still employed [Garcia-Parajo, Cambril \& Chen, 1994; Ambrose et al., 1994; Biefefeldt et al., 1994; Valaskovic, Holton \& Morrison, 1995; Yakobson, Moyer \& Paesler, 1994]. Many groups use a modified pipette puller with a built-in $\mathrm{CO}_{2}$ laser for the heat source. Others have used a chemical etching technique in a hydrofluoric (HF) solution to shape the fiber [Schoch, Jones \& Franks, 1994; Courjon, Banier, \& Spajer, 1992; Marchman, Griffith \& Filas, 1994]. The HF method allows for high taper angle fiber probes, which have much higher light throughput [Novotny, Pohl,

\footnotetext{
${ }^{*}$ NSOM is the American convention, where SNOM is the European convention. Due to the near simultaneous development of the techniques, both conventions are used interchangeably.
} 
\& Hecht, 1995]. Some have implemented focused ion beam milling to create aperture probes [Veerman et al., 1998].

The application of the aperture probe can be used in two distinct modes, either as an excitation probe or as a combination of excitation and detection. For excitation mode, a light is guided down the fiber in a propagating $\mathrm{TEM}_{00}$ mode. This involves a neutral density (N.D.) filter, half and quarter wave plate, and fiber coupler lens system. The quarter wave plate is added for removal of ellipticity induced by the fiber. The half wave plate is used to control the linear polarization orientation. The probe is attached to a scanning probe microscope, which is then applied carefully to a sample surface.

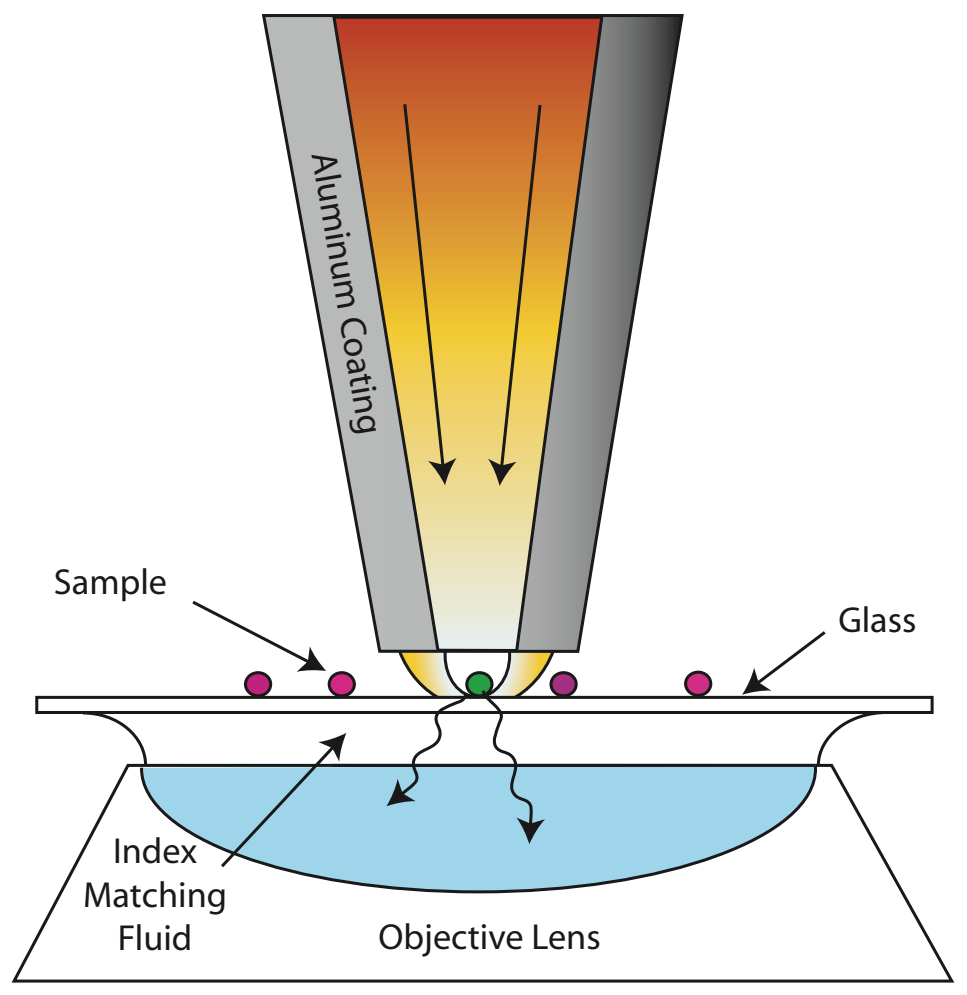

Figure 3.3 Side profile of a near-field aperture probe scanning over fluorescing chromophores. The light can be collected from below by the high numerical aperture lens and directed to a detector in the far field, or the light can be detected back up through the fiber. 
The sample is illuminated in the near-field and the fluorescence emission from the sample is collected through an objective lens and miscellaneous optics and then inevitably reaches $\mathrm{n}$-blocking filters. Typically, a single photon avalanche photodiode (SPAPD) is used for collection, due to its high quantum efficiency (typically $>80 \%$ ) and very low dark counts. It is different in its operation from a basic APD in that the detector has single digit background counts due to its design and fabrication. This low background allows for very accurate time-correlated single-photon counting (TCSPC) measurements involving fast instrument responses since these detectors also tend to possess low jitter from pulse to pulse. Although the SPAPD is the preferred detector, there exist the dangers of high sensitivity to damage from high-count rates of photons. Typically, going higher than $15 \mathrm{Mhz}$ can damage the SPAPD due to the high voltages and currents present during longer exposure times to these high counts. In the latest developments of optical detection, photomultiplier tubes (PMTs) have shown great promise as more robust single photon counters with increasing quantum efficiencies (QEs). Although the normal collection mode for photons is through the sample and collection objective below, a popular method employed for non-transmissive samples involves collecting light back through the excitation fiber. In fiber collection mode, fluorescence emission is collected back through the fiber, then diverted by a $45^{\circ}$ splitter to an SPAPD. The advantages of this method include the ability to image nontransmissive substrates as well as simple collection optics, although the collection efficiency back through the fiber is typically lower than collection efficiency of a objective lens below the sample. 
Although these techniques have the ability to image with spatial resolutions less than $\lambda / 2$, serious issues exists with this technique. One issue is that the angle of the fiber pull is less than ideal and the tips often will form very high aspect ratio geometries. Although great for topographic imaging, they have extremely low throughput, typically on the order of nanowatts. HF chemical etching has allowed the researchers to make better aspect ratio tips. However, the use of HF is quite hazardous and requires special hoods during the etching process. Whether the user makes etched or pulled methods, making tips the same from run to run is a large problem. Making fibers reproducible at the level of sub $100 \mathrm{~nm}$ has not demonstrated a very high reproducibility. Coatings also suffer from a lack of reproducibility. Coatings should be made at vacuum pressures below $5.0 \times 10^{-7}$ torr to obtain coatings with minimal contamination [Dr. Erik Sánchez, Portland State University, Private Conversation]. Oftentimes, nucleation issues of thermal evaporation techniques will result in residual metal on the end apertures. This nucleation can create problems for throughput. Another coating issue involves miniature pinholes where light can escape the confinement of the fiber. Topographically, the tip is usually very wide (>350 nm), leading to poor surface imaging features. For a more complete review, an informative primer written by Robert Dunn on SNOM/NSOM is available [Dunn, 1999].

\subsection{Near-Field Optical Imaging with Apertureless Probes}

In order to obtain sub $20 \mathrm{~nm}$ resolutions reproducibly in the near-field, a technique known as apertureless near-field scanning optical microscopy (ANSOM) can be used. John Wessel first proposed the idea of apertureless scanning optical microscopy 
in 1985 [Wessel, 1985]. ANSOM gains resolution over traditional NSOM by decreasing the size of the near-field interaction volume. A two-fold benefit results one is improved topographic imaging over the aperture method, and two, the apertureless probes are easier to manufacture. Initial ANSOM probe designs showed the ability to scatter incident light, which generates images in the far-field of the light's interaction with the surface [Inouye \& Kawata, 1994; Zenhausern, Martin \& Wickramasinghe, 1995]. Scattering images are typically difficult to quantify due to the complexity of the scattering effects.

A subset of the more general ANSOM technique is tip enhanced near-field optical microscopy (TENOM) [Sánchez, Novotny, \& Xie, 1999; Sánchez, 1999]. The principle of TENOM is to use excitation light in the proper polarization to induce a strong localized enhanced field at the end of the tip [Novotny, Sánchez \& Xie, 1998]. The enhanced field consists mainly of non-propagating (evanescent) components and is, thus, strongly confined to the end of the metal tip. The enhanced fields locally interact with the sample surface and generate a spectroscopic response that can be detected in the farfield. Furthermore, the tips can be illuminated by a two-photon excitation source in order to improve contrast, due to a quadratic emission profile. This experimental arrangement can be used for the excitation of molecular florescence, surface enhanced Raman scattering, and other forms of linear and non-linear imaging [Sánchez et al., 1999; Hartschuh, 2003; Ichimura, 2004]. Field enhancements of TENOM probes are several orders of magnitude greater than the background excitation light. With this increased field, the time required for imaging of the chromophores with a good signal-to-noise ratio is reduced. The ultimate resolution obtainable by TENOM is purely dependent on the geometry of the tip [Krug, Sánchez \& Xie, 2002; Nowak, 2005]. 
TENOM's dependency on proper polarization to maximize the enhanced field has resulted in multiple experimental configurations. Side illumination of the tip is one of these configurations [Pettinger et al., 2004]. A sharp metal probe absorbs the incident field, which has a polarization along the tip length to maximize the localized field enhancement [Martin \& Girard, 1997]. This configuration allows for imaging of opaque samples lending itself to tip-enhanced Raman spectroscopy. Imaging usually suffers from poor signal to noise ratios and collection efficiencies (low $N A$ lens). Side illuminated tips are relatively easy to manufacture, usually requiring a basic etching circuit. A drawback is that high resolutions need sharp tips on the order of $30 \mathrm{~nm}$ end diameters; the basic chemical etching methods lack reproducibility at this size. More complex etching methods have been developed to improve this reproducibility, including modifications to the probe itself [Ren, 2004; Neacsu, 2010]. Moreover, the small cross sectional area present at the end of the tip creates difficulty with side illumination, resulting in poor field enhancements. Figure 3.4 demonstrates the field enhancement effect of a side illuminated metal tip.

Ideally, epi-illumination ${ }^{\dagger}$ would give the highest collection efficiency for a TENOM system, but some difficulties have to be overcome. Epi-illumination would rule out opaque samples. While this would be acceptable for biological samples, bulk material samples would not be possible. It would still be possible to analyze nanoparticle objects such as carbon nanotubes, quantum dots, and nanocrystalline structures from below, assuming care was taken in the preparation of the samples on glass substrates. The benefits of epi-illumination include much higher collection efficiency due the use of high 
N.A. lenses and existing optical microscopes to provide optical pathways. The biggest drawback to the epi-illumination is that the polarization will be rotated 90 degrees, making it useless with high aspect ration sharp metal probes. A new probe design will be needed to take advantage of epi-illumination.

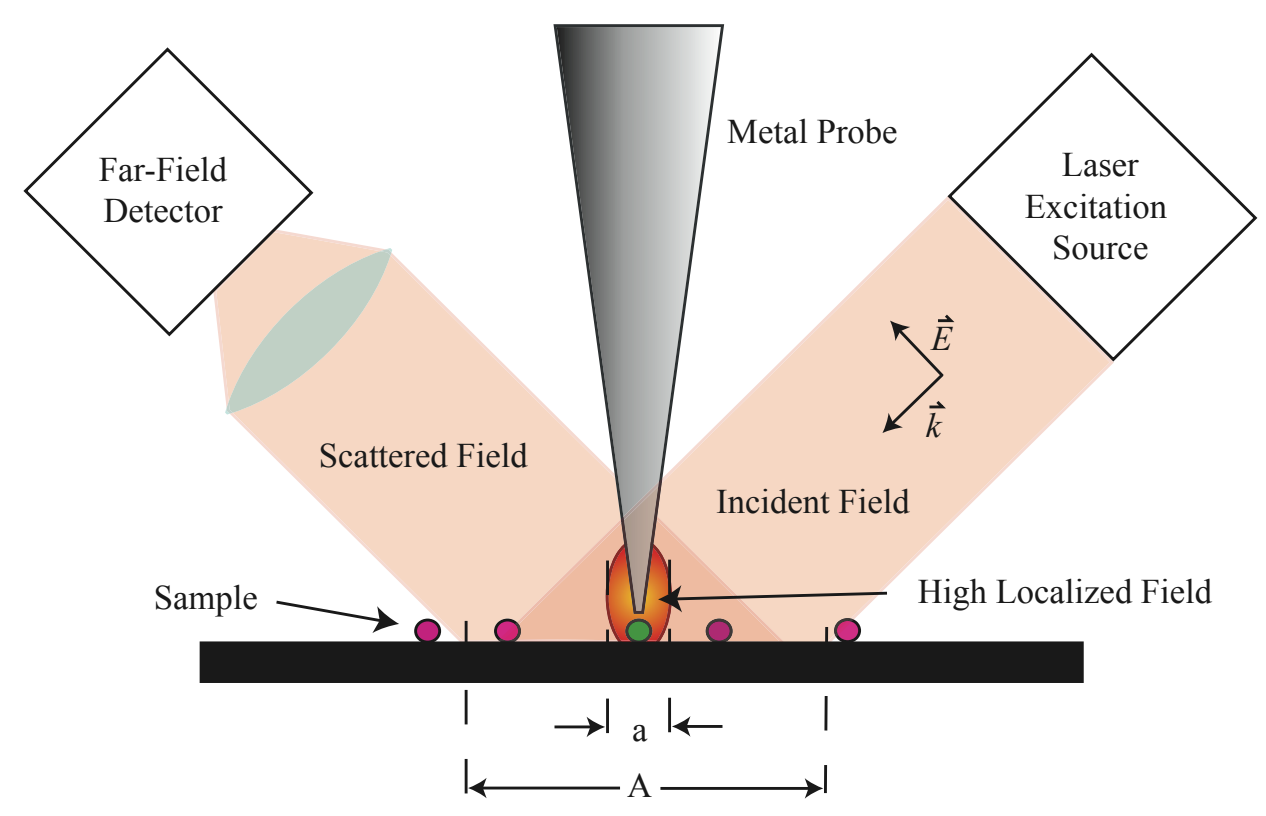

Figure 3.4 Conceptual drawing of a side illuminated TENOM probe. Spectroscopic signals are excited in the near-field by a high localized field at the end of the tip. "a" is the effective area of localized field and "A" is the diffraction limited incident field spot area. In experimental settings, usually a long focal length objective lens provides the focus of the laser source and the collection pathways. Functionalized surfaces to help amplify the localized field can also be used.

The use of nanoparticles attached to the end of an imaging probe has demonstrated successful apertureless near-field imaging in an epi-illumination configuration [Kalkbrenner et al., 2001; Palomba, Danckwerts \& Novotny, 2009]. The overall field enhancement is typically limited to the finite number of electrons available

\footnotetext{
$\dagger$ Epi-illumination is using the objective lens as both a condenser and objective lens.
} 
in a confined particle. If the size and shape of the geometry can be tailored to generate a resonance condition with the incident radiation, the enhancement can be further increased [Jensen, 2000]. The use of bow-tie geometries has also demonstrated feasibility, but the fabrication of near-perfect atomic termination corners of the triangles needs refinement [Grober, Schoelkopf, \& Prober, 1997; Farahani, 2005]. Ultimately, the use of nanoparticles for near-field imaging requires a lot of control and manufacturing precision, which currently lacks reproducibility.

Creating a metal probe that has a sharp aspect ratio, followed by a larger low aspect ratio bulk, will allow for the incident light to be absorbed in the bulk and propagated to the end of the high aspect sharp section of the probe. Figure 3.5 shows a possible design that could take advantage of epi-illumination.

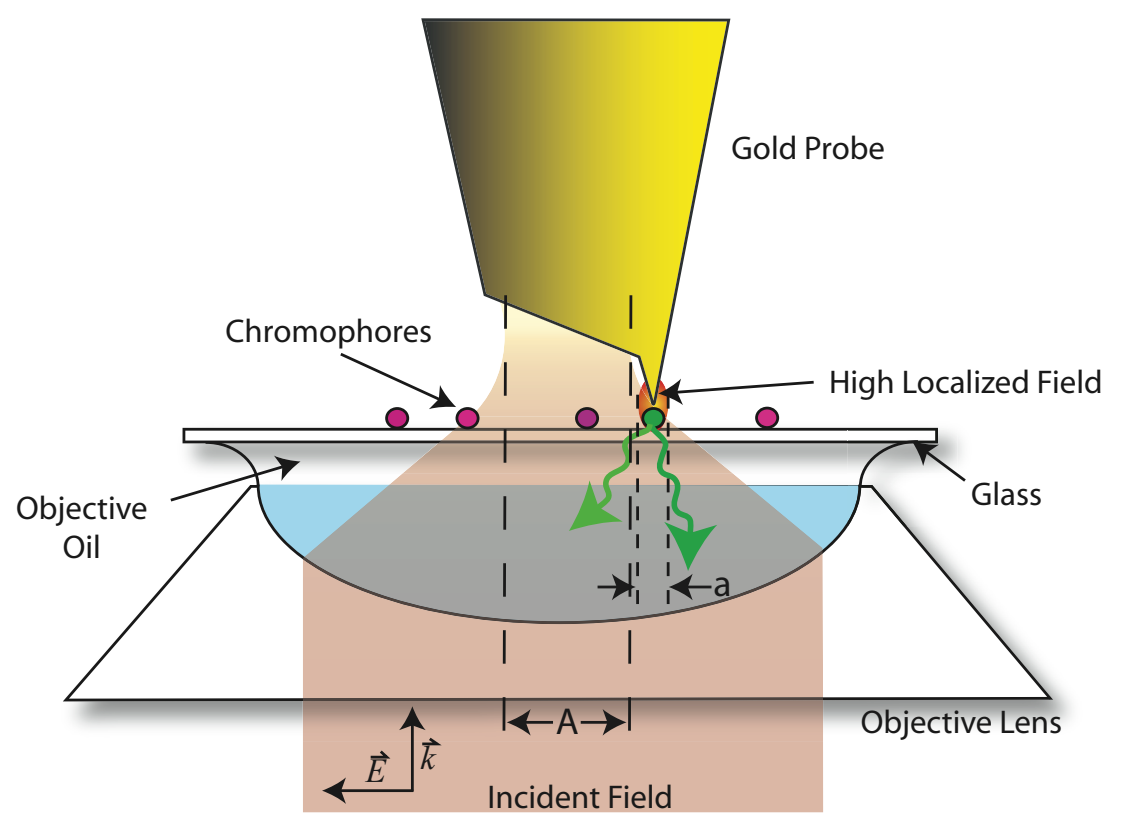

Figure 3.5 Conceptual drawing of an epi-lluminated TENOM probe. Fluorescent molecules are excited in the near-field by a high localized field at the end of the tip. "A" is the diffraction limited incident field spot area and "a" is the effective area of localized field. 
Figure 3.6 validates the design with preliminary finite difference time domain (FDTD) modeling of the geometry. This design idea for TENOM in an epi-illuminated configuration using Au probes will be the primary focus of this dissertation.

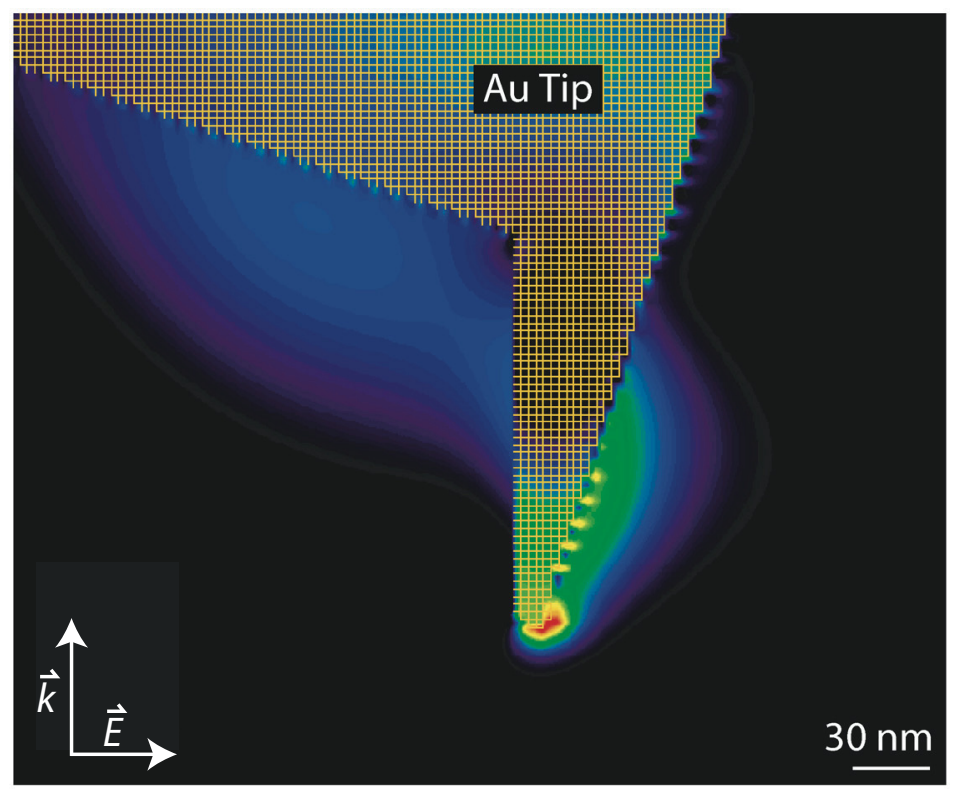

Figure 3.6 FDTD modeling of an Au TENOM probe, $800 \mathrm{~nm}$ incident light polarized in the horizontal direction, incident from the top-down. The FDTD algorithm reported a field enhancement with an increase of two orders of magnitude over that of the excitation source.

The detection of the near-field response in TENOM imaging will use similar far-field detectors as well as aperture probes, SPAPDs, PMTs, and intensified CCDs for spectral imaging. Two-photon excitation can be used to minimize the background contribution from the far-field signal that will be mixed with the near-field signal.

\subsection{Scientific Motivation for the Development of a TENOM System}

There are two main scientific motivations for the development of a TENOM system: subdiffraction limited instantaneous measurements with the ability to measure molecular time dynamics and the integration of $\mathrm{CW}$ laser sources. From a scientific 
standpoint, a TENOM system has the ability to image spectroscopic unknowns at highresolutions with minimal sample interaction, maintaining comparably high-speed imaging. While the "super-resolution" methods offer high-resolution, they are dependent on known dye-labels and long acquisition times. These methods also require complex laser systems with correlated timing of pulses and emission detection. In general, most nonlinear optical microscopy methods are destructive to the sample due to heating or photobleaching of the labeling mechanism. TENOM is also affected by photobleaching and quenching in fluorescence imaging, but the lower power levels needed aid in sample longevity. TENOM's reach into Raman spectroscopy is another real benefit of the technique. Raman's instantaneous spectroscopic response does not allow for time correlation of an emission response. "Super-resolution" methods require a stochastic emission from a sample to statistically determine molecular locations; this would not be possible with a Raman spectrum.

A demonstration of the use of a $\mathrm{CW}$ laser system in contrast to a mode-locked pulsed laser system for TENOM imaging will greatly simplify optical pathways for TENOM. Of course, the damage threshold of both the sample and the imaging probe will have to be investigated, due to the higher power levels required with the $\mathrm{CW}$ excitation. Using two-photon excitation, a single set of excitation and emission filters can be used for a wide range of fluorescence molecules with the benefit of a diminished background signal. This simplification will help lower the overall cost of a TENOM system, placing it with in reach of many academic labs. 


\section{CHAPTER 3 References}

Ambrose, W. P., Goodwin, P. M., Martin, J. C., and Keller, R. A., "Alterations of Single Molecule Fluorescence Lifetimes in Near-Field Optical Microscopy," Science 265, 364 (1994).

Ash E. A. and Nichols G., "Super-resolution aperture scanning microscope," Nature 237, 510 (1972).

Bethe, H. A., “Theory of Diffraction by Small Holes,” Phys. Rev. 66, 163 (1944).

Betzig E., Finn P. L., and Weiner S. J., "Combined shear force and near-field scanning optical microscopy,” Appl. Phys. Lett. 60, 2484-2486, (1992).

Betzig E., Grubb S. G., Chichester R. J., DiGiovanni D. J., and Weiner J. S., "Fiber Laser Probe for Near-Field Scanning Optical Microscopy," Appl. Phys. Lett. 63, 3550 (1993).

Betzig E., Trautman J. K., Harris T. D., Weiner J. S., and Kostelar R. L., "Breaking the diffraction barrier: optical microscopy on a nanometric scale," Science 251, 1468-1470, (1991).

Bielefeldt, H., Hörsch I., Krausch G., Lux-Steiner M., Mlynek J. and Marti O., "Reflection-scanning near-field optical microscopy and spectroscopy of opaque samples," Appl. Phys. Lett. 59, 103 (1994).

Bouwkamp, C. J., “On Bethe's Theory of diffraction by Small Holes,” Phillips. Res. Rep. 5, 401, (1950).

Courjon, D., Banier, C., and Spajer, M., "Imaging of submicrion index variations by scanning optical tunneling,” J. Vac. Sci. Tech. B 10, 2436 (1992).

Dunn, R. R., “Near-field scanning optical microscopy,” Chem. Rev. 99, 2891-2927 (1999).

Farahani, J. N., Pohl D. W., Eisler, H. J., and Hecht B., "Single quantum dot coupled to a scanning optical antenna: A tunable super emitter," Phys. Rev. Lett. 95, 017402 (2005).

Garcia-Parajo, M. F., Cambril, E., and Chen Y., "Simultaneous scanning tunneling microscope and collection mode scanning near-field optical microscope using gold coated optical fiber probes," Appl. Phys. Lett. 65, 1498 (1994).

Grober R. D., Schoelkopf R. J., and Prober D. E., “Optical Antenna: Towards a Unity Efficiency Near-Field Optical Probe,” Appl. Phys. Lett. 70, 1354 (1997). 
Hartschuh A., Sánchez, E. J., Xie, X. S., and Novotny L., "Near-field Raman Spectroscopy of Single-Walled Carbon Nanotubes," Phys. Rev. Lett. 90, 9 (2003).

Ichimura T., Hayazawa N., Hashimoto M., Inouye Y. and Kawata S. et. al. "Tip Enhanced Coherent Anti-Stokes Raman Scattering for Vibrational Nanoimaging”, Phys. Rev. Lett. 92, 220801 (2004).

Inouye Y. and Kawata S., "Near-field Scanning Optical Microsocpe with a Metallic Probe Tip,” Opt. Lett. 19, 159 (1994).

Jensen T. R., Malinsky M. D., Haynes C. L., and Van Duyne R. P., "Nanosphere Lithography: Tunable Localized Surface Plasmon Resonance Spectra of Silver Nanoparticles," J. Phys. Chem. B 104, 10549 (2000).

Kalkbrenner T., Ramstein M., Mlynek J., and Sandoghdar V., "A Single Gold Particle as a Probe for Apertureless Scanning Near-Field Optical Microscopy," J. Microscopy 202, $72-76$ (2001).

Krug, J. T., Sánchez, E. J., and Xie, X. S., "Design of near-field optical probes with optimal field enhancement by finite difference time domain electromagnetic simulation," J. Chem. Phys. 116, 10895 (2002).

Lewis A., Isaacson M., Harootunian A. and Murray A., "Development of a $500 \AA$ spatial resolution light microscope. I. Light is efficiently transmitted through $\lambda / 16$ diameter apertures," Ultramicroscopy 13, 227 (1984).

Marchman, H. M., Griffith, J. E., and Filas, R. W., "Fabrication of optical fiber probes for nanometer-scale dimensional metrology," Rev. Sci. Instrum. 65, 2538-2541 (1994).

Martin O. J. F., and Girard C., "Controlling and Tuning Strong Optical Field Gradients at a Local Probe Microscope Tip Apex,” Appl. Phys. Lett. 70, 705 (1997).

Neacsu C. C., Berweger S., Olmon R. L., Saraf L. V., Ropers C. and Raschke M. B., "Near-Field Localization in Plasmonic Superfocusing: A Nanoemitter on a Tip," Nano Lett, 10, 592 (2010).

Novotny L., Pohl D. W. and Hecht B., "Light Confinement in Scanning Near-field Optical Microscopy," Ultramicroscopy 61, 1-9 (1995).

Novotny L., Sánchez E. J., and Xie X. S., "Near-field Optical Imaging Using Metal Tips Illuminated by Higher-Order Hermite-Gaussian Beams," Ultramicroscopy 71, 21 (1998).

Nowak D. B., Krug J. T., Xie X. S., and Sánchez E. J., "Pushing the Limits of Near-field Microscopy," World Multi-conference on Systemics, Cybernetics and Informatics, WMSCII Proceedings, Orlando, Florida, 2005. 
O'Keefe J. A., "Resolving power of visible light," J. of the Opt. Soc. of Am. 46, 359 (1956).

Palomba S., Danckwerts M., and Novotny L., "Nonlinear Plasmonics with Gold Nanoparticle Antennas,” J. Opt. A.: Pure Appl. Opt. 11, 114030 (2009).

Pettinger B., Ren B., Picardi G., Schuster R., and Ertl G., "Nanoscale Probing of Absorbed Species by Tip-Enhanced Raman Spectroscopy," Phy. Rev. Lett. 92, 096101 (2004).

Pohl D. W., Denk W., and Lanz M., "Optical stethoscopy: Image recording with resolution $\lambda / 20$," Appl. Phys. Lett. 44, 651 (1984).

Ren B., Picardi G., and Pettinger B., "Preparation of Gold Tips Suitable for TipEnhanced Raman Spectroscopy and Light Emission by Electrochemical Etching," Rev. Sci. Inst. 75, 837 (2004).

Sánchez, E. J., Ph.D. dissertation, "A Novel Scheme for High Resolution Near-Field Fluorescence Microscopy”, Portland State University, 1999 (UMI No. 3018652).

Sánchez E. J., Novotny L., and Xie X. S., "Near-field Fluorescence Microscopy Based on Two-Photon Excitation With Metal Tips," Phys. Rev. Lett. 82, 20 (1999).

Schoch, B., Jones, B. E., and Franks, A., "A simple technique for the manufacture of optical probes for scanning near-field optical microscopes," Meas. Sci. Tech. 5, 663 (1994).

Synge E. H., "A suggested method for extending the microscopic resolution into the ultramicroscopic region.” Phil. Mag. 6, 356 (1928).

Synge E. H., “An application of piezoelectricity to microscopy,” Phil. Mag. 13, 297 (1932).

Valaskovic, G. A., Holton, M., and Morrison, G. H., "Parameter control, characterization, and optimization in the fabrication of optical fiber near-field probes," Applied Optics 34, 1215 (1995).

Veerman, J. A., Otter, A. M., Kuipers, L., and Van Hulst, N. F., "High definition aperture probes for near-field optical microscopy fabricated by focused ion beam milling," Appl. Phys. Lett. 72, 3115-3117 (1998).

Wessel J., “Surface-Enhanced Optical Microscopy,” J. Opt. Soc. Am. B 2, 1538 (1985).

Yakobson, B. I., Moyer, P. J., and Paesler, M. A., "Kinetic limits for sensing tip morphology in near-field scanning optical microscopes," J Appl. Phys. 73, 7984 (1994). 
Zenhausern F., Martin Y., and Wickramsinghe H. K., "Scanning Interferometric

Apertureless Microscopy: Optical Imaging at 10 Angstrom Resolution,” Science 269, 1083 (1995). 


\section{CHAPTER 4}

\section{Modeling Nanophotonic Geometries for Near-Field Imaging}

Classically, bulk materials observe well-characterized functions when analyzed at the macroscale. These rules change when those materials are reduced down to the nanoscale; the materials begin to exhibit behavior far from their bulk counter part. Both gold and silver are good examples of this concept. The ancient Romans were the first to use gold nanoparticles to color glass ${ }^{*}$ [Barber \& Freestone, 1990]. Scientist Michael Faraday would be the first to make gold nanoparticles through quantifiable techniques [Faraday, 1857]. When gold nanoparticles are suspended in solution, the appearance of gold changes from the familiar yellow luster to a red appearance as its size is reduced [Frens, 1973; Sau et al., 2001], while silver nanotriangles exhibit a blue color [Rongchao, 2001]. Moreover, the interaction of light with particles becomes dependent on the specific size and shape. Changing the particle size by just a few nanometers can yield different results [Eustis \& El-Sayed, 2006]. Mie scattering dominates the interaction of optical frequencies with the nanoparticles. This is assuming that the particles are not smaller than roughly $1 / 10^{\text {th }}$ of the incident wavelength. Smaller particles will require treatment as Rayleigh scattering [Rayleigh, 1871]. Analytical Mie scattering solutions to spherical nanoparticles predict these effects [Mie, 1908]. Unfortunately, there are only a limited number of geometries that can be analyzed. In order to determine the effects of light on nanoparticles that cannot be analyzed due to complex geometries, numerical modeling is

\footnotetext{
* The Romans did not know what physical mechanism colored the glass.
} 
required [Kelly, 2002; Krug, Sánchez \& Xie, 2002; Zhang et al., 2009]. Simulating nearfield imaging probes also requires numerical modeling. The lack of symmetry and geometric complexity of the near-field probes would make analytical modeling improbable. Using qualitative measurements, the imaging mechanisms for near-field imaging and the creation of geometries that are beneficial for tip enhanced near-field optical microscopy (TENOM) imaging are presented.

\subsection{Modeling Techniques}

With the advent of microprocessors in the mid-seventies, scientific research has been able to reasonably approximate complex analytical problems via intensive numerical calculations on computers. Today's available computational power gives scientists the ability to calculate a large array of problems that would have been impossible just thirty years ago. These problems include mapping human genes [Luedi, 2007] and modeling weather patterns with resolutions of less than a square mile [Baer \& Zhang, 1998]. Modeling electromagnetic fields as they interact with dispersion materials have also developed, as they require a lot of computational power [Luebbers et al., 1990]. Fortunately, in recent years, the analysis of military radar frequencies on objects has spurred the development of modeling software that allows for complex geometries. Utilizing these modeling techniques and applying them to nanoscale and optical frequencies rather than the radio frequency regimen, researchers are able to obtain numerical solutions to nanoparticles. These computer simulations of optical fields can be performed on model nanogeometries under ideal conditions, in order to quantify electric field intensities and field distributions of near-field probe geometries that are to be used in TENOM imaging. 
There are many software packages and algorithms that can provide information on the field distribution characteristics of the TENOM probe geometries. Multimultipole (MMP), Maxwell, finite difference time domain (FDTD), and finite element (FEL), can all model the passing of transient, oscillating electric fields using optical frequencies at the nanoscale. Some of these modeling techniques are very complex, requiring analytical expertise, normally attributed to theoretical disciplines. Others are easier to use but may sacrifice some degree of accuracy. There is also the question of the overall monetary cost that may limit one's access to more complex modeling programs. Recent open-source packages offer monetary relief, but in general, these only include the modeling calculation engine, offering little ability to build 3D workspaces or import CAD models [MEEP, 2010; JFDTD, 2010]. However, continued development will most likely alleviate this complication.

\subsection{Introduction to FDTD Modeling}

In order to determine the relative electric field enhancement and qualitative response of the TENOM probes, FDTD was chosen as the modeling technique. FDTD was chosen because of its availability and user interface, which allows for the creation of 3D nano-objects of real materials and CAD importing capabilities. All FDTD calculations presented were calculated on either Remcom xFDTD version 6.1 and 6.3 software or Remcom MPI-xFDTD clustering software version 6.3.

\section{2.a Discretized Maxwell Equations}

The core of the simulation is based on differential time domain Maxwell equations (4.1 - 4.4). These equations are discretized for use in FDTD (4.5 - 4.8), 


$$
\begin{aligned}
& \nabla \times E=-\frac{\partial B}{\partial t}, \\
& \nabla \times H=\frac{\partial D}{\partial t}+J, \\
& \nabla \cdot D=\rho, \\
& \nabla \cdot B=0, \\
& \frac{\partial H}{\partial t}=-\frac{1}{\mu}(\nabla \times E)-\frac{\sigma^{m}}{\mu} H, \\
& \frac{\partial E}{\partial t}=\frac{-\sigma}{\varepsilon} E+\frac{1}{\varepsilon}(\nabla \times H), \\
& \nabla \cdot D-\rho=0, \\
& \nabla \cdot B=0,
\end{aligned}
$$

where the magnetic conductivity term $\sigma^{m}$ is included in the calculation to take care of any magnetic loss [Kunz \& Luebbers, 1993]. For the FDTD calculations, only the curl equations are necessary, since the fields at time zero will be zero. The two curl $(4.1,4.2)$ equations are discretized to obtain a total field calculation in FDTD shown in Equation 4.9 .

$$
\begin{aligned}
& E_{x}^{s}(I, J, K)^{n}=E_{x}^{s}(I, J, K)^{n-1}\left(\frac{\varepsilon}{\varepsilon+\sigma \Delta t}\right) \\
& -\left(\frac{\sigma \Delta t}{\varepsilon+\sigma \Delta t}\right) E_{x}^{i}(I, J, K)^{n}-\left(\frac{\left(\varepsilon-\varepsilon_{0}\right) \Delta t}{\varepsilon+\sigma \Delta t}\right) \dot{E}_{x}^{i}(I, J, K)^{n} \\
& +\frac{H_{z}^{s}(I, J, K)^{n-\frac{1}{2}}-H_{z}^{s}(I, J-1, K)^{n-\frac{1}{2}}}{\Delta y}\left(\frac{\Delta t}{\varepsilon+\sigma \Delta t}\right) \\
& +\frac{H_{y}^{s}(I, J, K)^{n-\frac{1}{2}}-H_{y}^{s}(I, J-1, K)^{n-\frac{1}{2}}}{\Delta y}\left(\frac{\Delta t}{\varepsilon+\sigma \Delta t}\right)
\end{aligned}
$$


Then, by the superposition principle, the calculation for the scattered field from an object can be determined. Equations 4.10 and 4.11 show the field superposition methodology used in FDTD,

$$
\begin{aligned}
& E^{\text {Total }}=E^{\text {incident }}+E^{\text {scattered }}, \\
& H^{\text {Total }}=H^{\text {incident }}+H^{\text {scattered }},
\end{aligned}
$$

where the scattered field is the most revealing element in the FDTD calculation. When calculating the field enhancement of geometries for TENOM probes the scattered field is the primary source for molecular excitation, since it is usually a much larger field than the incident field.

\section{2.b Material Parameters for Modified Debye-Drude Model}

Modeling of electric fields interacting on basic shapes is fairly straightforward, when the objects are perfectly conducting materials. However, modeling of perfect conductors will not reveal the underlying material resonances that might exist, nor accurately predict the material-dependant effects. In order to model real conductors, one must be aware of various issues before identifying pertinent material parameters. The proper material property values of the geometries to be modeled must be defined and applied by the modeling software. The modeling of the Au material for the TENOM probes utilizes the Drude free electron model, in the form of a modified Debye model formalism. The classical Drude model assumes that the conduction electrons are the transport mechanism for the thermal current [Drude, 1900]. In the Debye model, the electrons are treated as free electrons, and a relaxation time is used to describe the electrons collision frequency within the phonon lattice of the metal [Debye, 1912]. The 
formulation of the Drude model consists of: $\tilde{\varepsilon}(\omega)$ is the complex permittivity in frequency domain, $\omega_{p}$ the material dependent plasma frequency, $\omega$ the frequency of radiation, and $\tau$ the relaxation time for the material. $\mathcal{E}^{\prime}(\omega)$ represents the real portion of the complex permittivity and $\varepsilon^{\prime \prime}(\omega)$ is the complex. Starting with the complex permittivity in frequency domain, as shown in 4.12 , one must obtain time domain defined permittivity values where,

$$
\tilde{\varepsilon}(\omega)=\varepsilon^{\prime}(\omega)+i \varepsilon^{\prime \prime}(\omega),
$$

with the real and complex components,

$$
\begin{aligned}
& \varepsilon^{\prime}(\omega)=1-\frac{\omega_{\rho}^{2} \tau^{2}}{1+\omega^{2} \tau^{2}}=\operatorname{Re} \tilde{\varepsilon}(\omega), \\
& \varepsilon^{\prime \prime}(\omega)=\frac{\omega \omega_{\rho}^{2} \tau}{\omega^{2}+\omega^{4} \tau^{2}}=\operatorname{Im} \tilde{\varepsilon}(\omega) .
\end{aligned}
$$

The FDTD formulation of the modified Debye model is given by,

$$
\begin{aligned}
& \varepsilon^{\prime}(\omega)=\varepsilon_{\infty}+\frac{\left(\varepsilon_{s}-\varepsilon_{\infty}\right)}{1+\omega^{2} \tau_{0}^{2}}=\operatorname{Re} \tilde{\varepsilon}(\omega), \\
& \varepsilon^{\prime \prime}(\omega)=-\frac{\omega t_{0}\left(\varepsilon_{s}-\varepsilon_{\infty}\right)}{1+\omega^{2} \tau_{0}^{2}}=\operatorname{Im} \tilde{\varepsilon}(\omega),
\end{aligned}
$$

where $\varepsilon_{s}$ is the static permittivity and $\varepsilon_{\infty}$ is the relative infinite permittivity. By combining the Drude model and the Debye model, FDTD can model material parameters reasonably. Typically, $\varepsilon_{\infty}$ is defined as unity; this variable is defined as the optical response parameter at very high frequencies. Equations 4.17 thru 4.21 give the material parameters used to define free electron dispersive materials in (real conductors) xFDTD [Kunz \& Luebbers, 1993]. The relationships for the time domain parameters are: 


$$
\begin{aligned}
& \varepsilon_{\infty}=1, \\
& \sigma=\varepsilon_{0} \frac{\omega_{\rho}^{2}}{v_{c}}, \\
& \varepsilon(\omega)=\varepsilon_{\infty}+\chi(\omega)+\frac{\sigma}{i \omega \varepsilon_{0}}, \\
& \tau_{0}=\frac{1}{v_{c}}, \\
& \left(\varepsilon_{s}-\varepsilon_{\infty}\right)=-\left(\frac{\omega_{p}}{v_{c}}\right)^{2},
\end{aligned}
$$

where $\sigma$ is the conductivity term, $\chi(\omega)$ is the electric susceptibility as a function of angular frequency, $\varepsilon_{0}$ vacuum permittivity, and $v_{c}$ is the collision frequency with $\tau_{0}$ the relaxation time.

The complexity of materials cannot be easily modeled, analytically, but rather determined, experimentally. The optical properties determined in a material are the real index of refraction $(n)$ and the imaginary index of refraction, or extinction coefficient, $(k)$. One must verify that the modeling will give the proper steady state values (after ten optical cycles); verifying that the frequency domain values equal the time domain values satisfies this requirement. Remcom's xFDTD does not have a material parameter calculator, which would allow the user to define a material, based on the incident frequency used, and calculate the four parameters needed by the modified Debye model. Thus, it is up to the user to define and verify these parameters. Therefore, a program was developed to make a comparison between the time domain values and the experimental values for $n$ and $k$ [Nowak et al., 2005]. The derivation for this comparison is presented, 
starting with Equation 4.12 and the frequency domain formulas for the real and imaginary parts of the complex permittivity given by,

$$
\begin{aligned}
& \operatorname{Re} \tilde{\varepsilon}(\omega)=n^{2}-k^{2}, \\
& \operatorname{Im} \tilde{\varepsilon}(\omega)=2 n k .
\end{aligned}
$$

Substituting Equation 4.21 into 4.21 and solving for $n$ and $k$ the following result,

$$
\begin{aligned}
& n=\left[\frac{1}{2}\left(\varepsilon_{R}+\left(\varepsilon_{I}^{2}+\varepsilon_{R}^{2}\right)^{\frac{1}{2}}\right)\right]^{\frac{1}{2}}, \\
& k=\left[\frac{1}{2}\left(-\varepsilon_{R}+\left(\varepsilon_{I}^{2}+\varepsilon_{R}^{2}\right)^{\frac{1}{2}}\right)\right]^{\frac{1}{2}} .
\end{aligned}
$$

With further substitution of the real and complex permittivities from the modified Debye formalism, the determination of $n$ and $k$ can be made. These are shown in Equations 4.26 and 4.27, which were elucidated by Sánchez and colleagues,

$$
n=\left[\frac{1}{2}\left(\varepsilon_{\infty}+\frac{\varepsilon_{s}-\varepsilon_{\infty}}{1+\omega^{2} \tau_{0}^{2}}+\left(\left[\frac{\left(\varepsilon_{s}-\varepsilon_{\infty}\right) \omega \tau_{0}}{1+\omega^{2} \tau_{0}^{2}}+\frac{\sigma}{\omega \varepsilon_{0}}\right]^{2}+\left[\varepsilon_{\infty}+\frac{\varepsilon_{s}-\varepsilon_{\infty}}{1+\omega^{2} \tau_{0}^{2}}\right]^{2}\right)^{\frac{1}{2}}\right)^{\frac{1}{2}}\right],
$$

and

$$
k=\left[\frac{1}{2}\left(-\varepsilon_{\infty}-\frac{\varepsilon_{s}+\varepsilon_{\infty}}{1+\omega^{2} \tau_{0}^{2}}+\left(\left[\frac{\left(\varepsilon_{s}-\varepsilon_{\infty}\right) \omega \tau_{0}}{1+\omega^{2} \tau_{0}^{2}}+\frac{\sigma}{\omega \varepsilon_{0}}\right]^{2}+\left[\varepsilon_{\infty}+\frac{\varepsilon_{s}-\varepsilon_{\infty}}{1+\omega^{2} \tau_{0}^{2}}\right]^{2}\right)^{\frac{1}{2}}\right)^{\frac{1}{2}}\right]
$$

These formulas allow for the verification of the frequency and time domain steady state solutions with the experimental data available for the optical properties of materials. Determination of the correct values requires tuning the frequency domain parameters 
until a match is made with the time domain parameters. If the parameters do not agree in the steady state, at the frequency $(\omega)$ in question, then the model of material used in the FDTD program will provide undesirable singularities. Many times, chosen values with the appropriate $\omega$ can still lead to a negative real permittivity, which will lead to instabilities. Recent publications have questioned this presented method of the conversion from time domain to frequency domain constants [Zhu, Wang \& Jin, 2005; Gai, Wang \& Tian, 2007; Goncharenko, Chang \& Wang, 2007]. While still debatable in terms of the overall accuracy of the models, the newly suggested frequency domain values offer an improvement when compared to Mie scattering. The models presented for possible TENOM probe designs are discussed qualitatively and, in the worst case, underestimate the full potential of the probes based on these new parameters. Modeling artifacts could also contribute to these complications; an analysis is presented in previous work and is reiterated later in this chapter in section 4.4 [Nowak, 2004].

\section{2.c Yee Cell}

The modeling space in FDTD is defined by a three dimensional volume which is divided into smaller Yee cells of equal cubic proportions [Yee, 1966]. Maxwell's discretized curl equations are solved in a leapfrog fashion; that is, the electric field is solved at a given instant in time, then the magnetic field is solved at the next time instant. The process is then repeated in the direction of propagation. Figure 4.1 shows the Yee cell and the locations of calculations. There are six calculations performed per cell, one for each of the electric field components in the $\mathrm{x}, \mathrm{y}$, and $\mathrm{z}$ directions, and one for magnetic field components in the $\mathrm{x}, \mathrm{y}$, and $\mathrm{z}$ directions. 
The determination of the correct Yee cell size in FDTD modeling must be taken under careful consideration. As the number of cells increases in three dimensions to represent a workspace, the amount of computer RAM needed to run the simulation increases, cubically. This increase in the number of cells will also mean more intensive calculations, resulting in longer run times; at the same time, the cell size must be much smaller than the wavelength of electromagnetic radiation used. A rule of thumb is to start with a cell size of at least one-tenth of the wavelength of radiation. If any geometry in the workspace contains details smaller than this cell size, the cell size will have to be decreased to a size that allows for this detail to be detectable. For the modeling of TENOM probes, which have typical geometric features of less then $20 \mathrm{~nm}$, the cell size will be $3.0 \mathrm{~nm}$ or roughly $1 / 250^{\text {th }}$ of the wavelength of incident radiation.

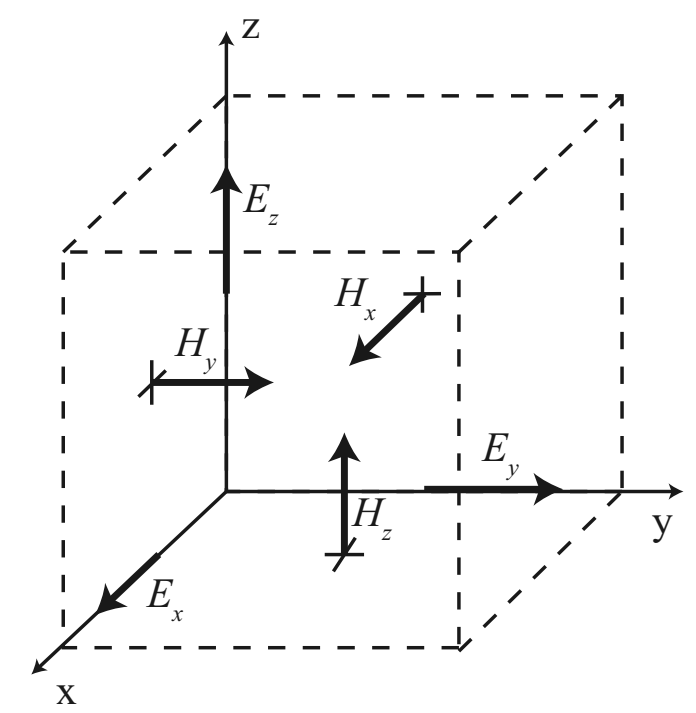

Figure 4.1 Yee Cell. The six calculation points for the electric field components and the magnetic field components are shown.

The correct cell size and the overall workspace size is always a compromise, in order to generate a good model. Ideally, a large workspace with a large amount of detail from 
very small cell sizes would be desired. Unfortunately, even today's computer technology is somewhat limited in this regard.

\section{2.d Incident Fields}

The final step required in preparing a FDTD run is the specification of the incident field. Remcom's xFDTD package allows the user flexibility in incident fields, such as Gaussian, modified Gaussians, and sinusoidal fields. The sinusoidal fields will be used with the TENOM probe modeling. xFDTD allows the user to define the frequency of the sinusoidal radiation and the number of time steps which will be used to complete a run. Because the FDTD workspace is three dimensional, the programmer must define the direction of the incident field in spherical coordinates. xFDTD requires that the programmer defines the incident fields direction, by pointing to the direction of the source. Figure 4.2 shows the Cartesian coordinate system used in the xFDTD program.

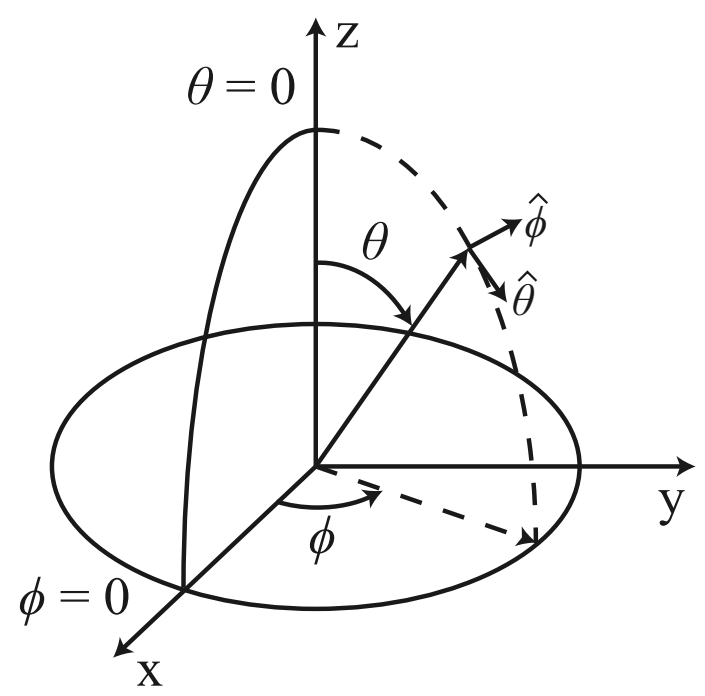

Figure 4.2 Coordinate system used in xFDTD. To define the incident field direction, both $\theta$ and $\phi$ angle need to be specified [Remcom Inc., 2003]. 
Since Maxwell's curl equations are used under the assumption that the incident field is a zero at time zero, an interesting condition during the modeling occurs. The modeling will start with a transient field, then over time, stabilize to a steady state solution. Here, it is assumed that roughly ten wavelengths of the incident field are needed to reach the steady state oscillatory condition. The need to wait for ten wavelengths to pass by greatly increases the computational time of the modeling. As the number of Yee cells in the workspace increases, the time for ten wavelengths to pass thru the workspace also increases, cubically.

\subsection{Validity of FDTD Modeling}

Modeling programs give numerical answers to complex physical systems based on analytical formalisms of the physical laws governing the system. For the modeling system to work, various approximations are typically made to integrate the analytical formalisms. The most important question to ask is, how accurate are these approximations and with what accuracy can the modeling software predict the real world condition?

To determine the accuracy of Remcom's xFDTD software package, a few calculations were made based on the analytical solutions of simple geometries, obtainable with Mie scattering theory. The first test consists of an Au sphere with a diameter of 150 $\mathrm{nm}$ and an incident field of $1.0 \mathrm{~V} / \mathrm{m}$ with a frequency of $3.75 \times 10^{14} \mathrm{~Hz}(800 \mathrm{~nm}$ wavelength). The polarization direction for this particular problem is along the horizontal direction and the field will be incident on the southern pole of the sphere. Figure 4.3 shows the waveform and frequency components for incident field. The final parameters needed before running the Mie scattering calculation are defined by the material 
parameters for the Au sphere. Based on previous work by Krug and Sánchez, using the modified Debye model, the following optical parameters for light of $800 \mathrm{~nm}$ incident on the Au material were chosen $\left(\varepsilon_{s}=-12987, \varepsilon_{\infty}=9.012, \sigma=1.464 \times 10^{7} \mathrm{~S} / \mathrm{m}\right.$, and $\tau_{0}=$ $8.017 \times 10^{-15}$ s) [Krug, Sánchez \& Xie, 2002].
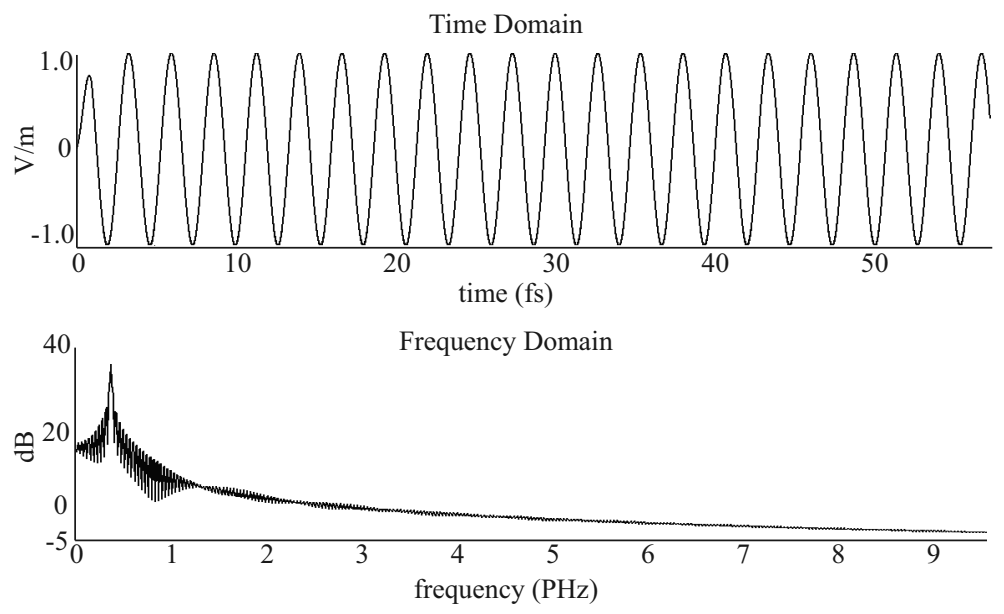

Figure 4.3 Defining the incident field parameters, the user is allowed to define the frequency, field magnitude and number of time steps required to complete 10 wavelengths of radiation. The upper graph shows the incident field as a function of time. The lower graph gives the FFT of the incident field.

The calculated material parameters are entered directly into XFDTD as a Debye model.

The FDTD calculation is then run on a dual Pentium III $933 \mathrm{MHz}$ computer with 512 MB's of RAM. With an overall work space of $151 \times 151$ x 151 cells with 11,000 time steps, the Mie scattering simulation requires roughly $14 \mathrm{hrs}$ to complete.

The Remcom software has the capacity to save any of the calculations at any cell location(s) during the execution of the program. To minimize space, only the last thousand time steps are recorded for the equatorial plane, which lies on $76^{\text {th }}$ cell in the $\mathrm{z}$ direction. Figure 4.4 shows the total field interaction with the sphere; the locations of 
highest field intensity are in the sides of the sphere at the equator, perpendicular to the incident field.
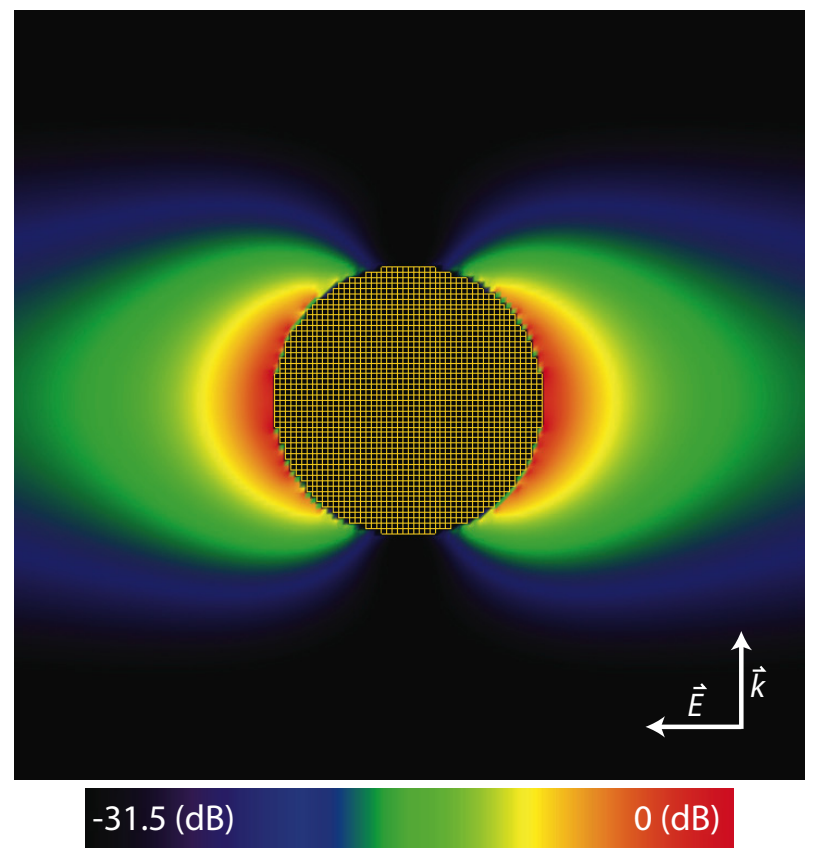

$0 \mathrm{~dB}=4.122 \mathrm{~V} / \mathrm{m}(\mathrm{Mag} \mathrm{E})$

Figure 4.4 Electric field intensity due to Mie scattering. The equator locations are areas of highest enhancement. The calculation is performed in $3 \mathrm{D}$; a slice along the $\mathrm{z}$ direction on the equatorial plane of the sphere gives a $2 \mathrm{D}$ cross section. The Yee cell size is $3.0 \mathrm{~nm}$, with a sphere of radius of $75 \mathrm{~nm}$.

This location is in agreement with the locations of field enhancement due to Mie scattering theory. The highest recorded electric field on the equator of the sphere was equal to $3.45 \mathrm{~V} / \mathrm{m}$. By squaring this intensity, a field enhancement factor of $11.9(\mathrm{~V} / \mathrm{m})^{2}$ is calculated for the sphere. This value is an anomalous underestimation of the value calculated by Mie scattering. Mie scattering predicts a field enhancement factor of roughly $17.5(\mathrm{~V} / \mathrm{m})^{2}$ for $800 \mathrm{~nm}$ light. [Krug, Sánchez \& Xie, 2002] With close evaluation, it becomes evident that the location of highest field enhancement is not at the equator, as was predicted in theory. FDTD numerical calculations suffer from these 
issues due to the discretization of objects. FDTD shows the highest field five cells above the equator location in this particular run. A perfectly smooth sphere would not exhibit such behavior. The sides of the sphere appear as straight lines. The high field location is around a sharp edge where these lines fall off to create the circular object. Figure 4.5 shows the workspace of the run, zoomed in on the cells around the equator. The edge of the plane, created by the side of the sphere, shows a field enhancement of $18.06(\mathrm{~V} / \mathrm{m})^{2}$, slightly higher than what theory would predict, but still fairly consistent. Due to the discrepancy, this problem deserves proper attention.

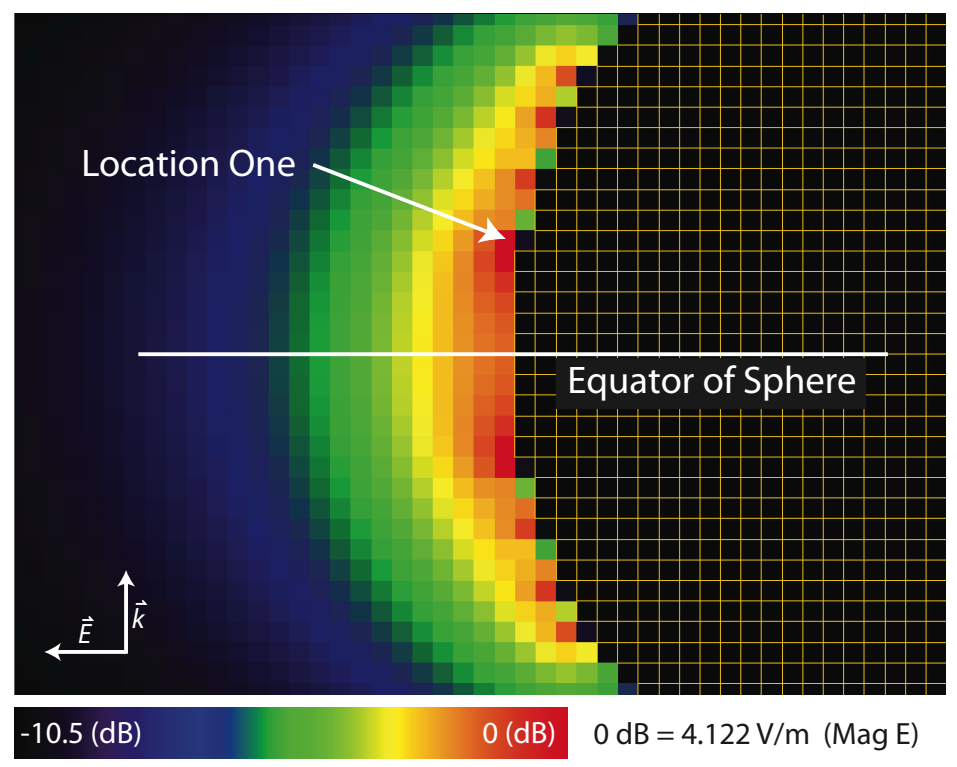

Figure 4.5 Electric field intensity due to Mie scattering near the equator of the sphere. Location one had the highest field intensity during the calculation.

To investigate this problem further, the effect of changing the cell sizes of the 150 nm diameter Au sphere is considered. The following cell sizes are investigated: 50, 25, $15,10,8.0,6.0,5.0,4.0,3.0,2.0$, and $1.0 \mathrm{~nm}$. There are two motivations: to determine the optimum cell size for the workspace and to optimize the size of the workspace, in 
order to minimize run time. The total field intensity is measured from four locations on the workspace. All four locations are on the equatorial plane, two of which fall on the east and west equatorial points and two of which the highest field is recorded on the east and west sides. Figure 4.7 shows the data obtained for these four locations given the variations in cell sizes, in a normal graph and a semi-logarithmic graph. The data was obtained during the last period of a wavelength of a ten-wavelength calculation.

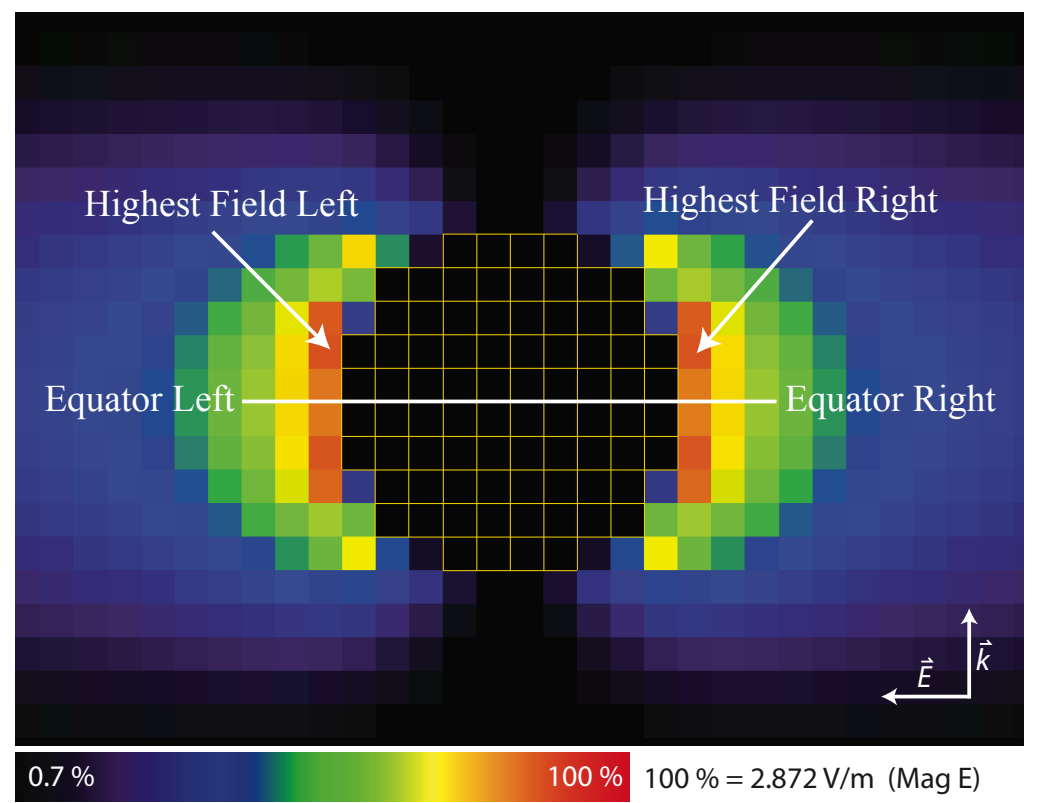

Figure 4.6 Mie Scattering workspace. Locations of data collection in the $\mathrm{x}-\mathrm{y}$ plane at cell 76 in the $\mathrm{z}$ direction for Au sphere with $15 \mathrm{~nm}$ cell size representation (linear scale).

Upon inspection, complications were detected with the method in which the data was collected. By default, the Remcom software package saves the transient wave data, once per ten time steps. Potentially, this will distort the overall uniformity of the data, since the highest field may fall between the 10 steps, between recordings. To correct for this issue, the experiment was recalculated, recording the data at every time step for the last wavelength of the transient field. 


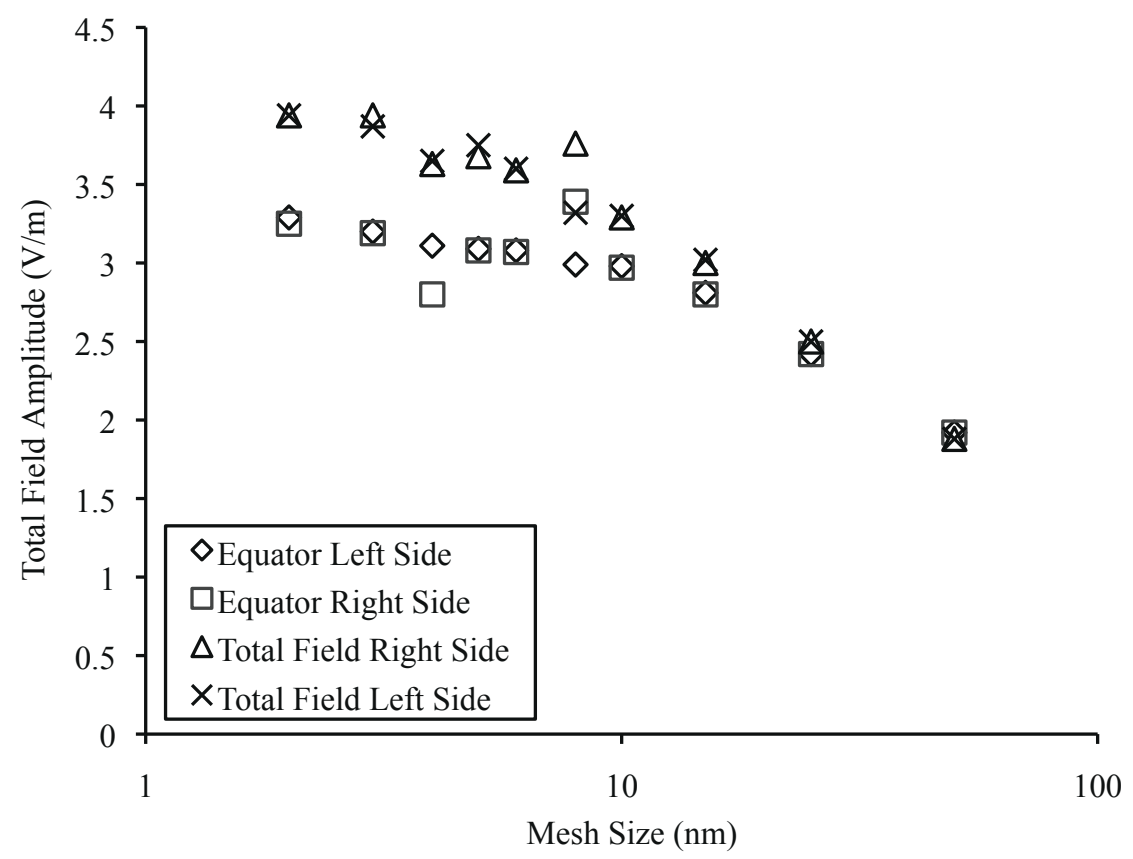

Figure 4.7 Total fields as a function of cell size, in a semi log scale. Data points show the corner artifacts that are present on the sphere.

It becomes evident that there are other parameters that become important, which need to be taken into consideration. One is how the object interacts with the boundary conditions. In the xFDTD program, Liao boundaries are used to dissipate the transient field in the far field regions of the workspace. To determine the effects of the boundary on the total field intensity measured on the sphere, eight different scenarios are tested. Using the same $150 \mathrm{~nm}$ sphere, a $10 \mathrm{~nm}$ and $5.0 \mathrm{~nm}$ cell size is used and the distance between the sphere and the boundary is varied. Figure 4.8 shows the results of these tests by plotting the field intensity at the equator for different number of cells away from the boundaries. The $10 \mathrm{~nm}$ run shows that the optimum distance the sphere needs to be from the boundary is greater than 15 cells. Moreover, the $5.0 \mathrm{~nm}$ run shows that the sphere needs to be more than 25 cells from the boundary. Further investigation of the actual distance of the object from the boundary is shown in Figure 4.9. 


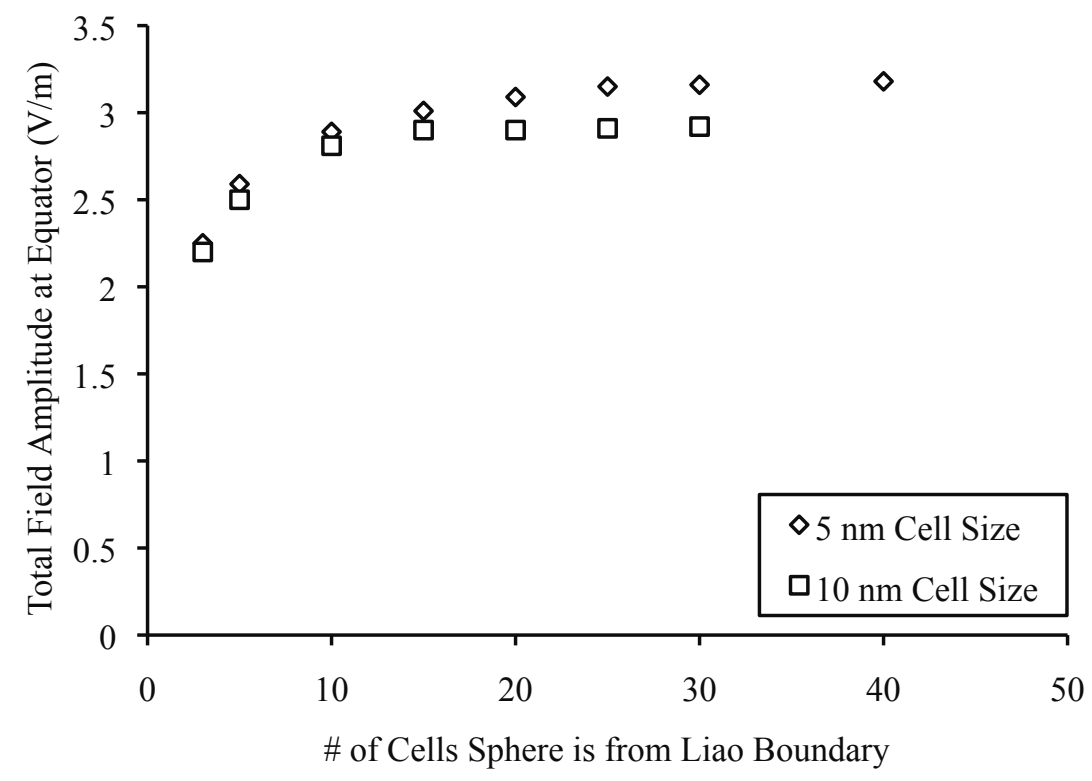

Figure 4.8 Total electric field amplitude as a function of number of cells away the $150 \mathrm{~nm}$ sphere is from the Liao boundary in the xFDTD simulation.

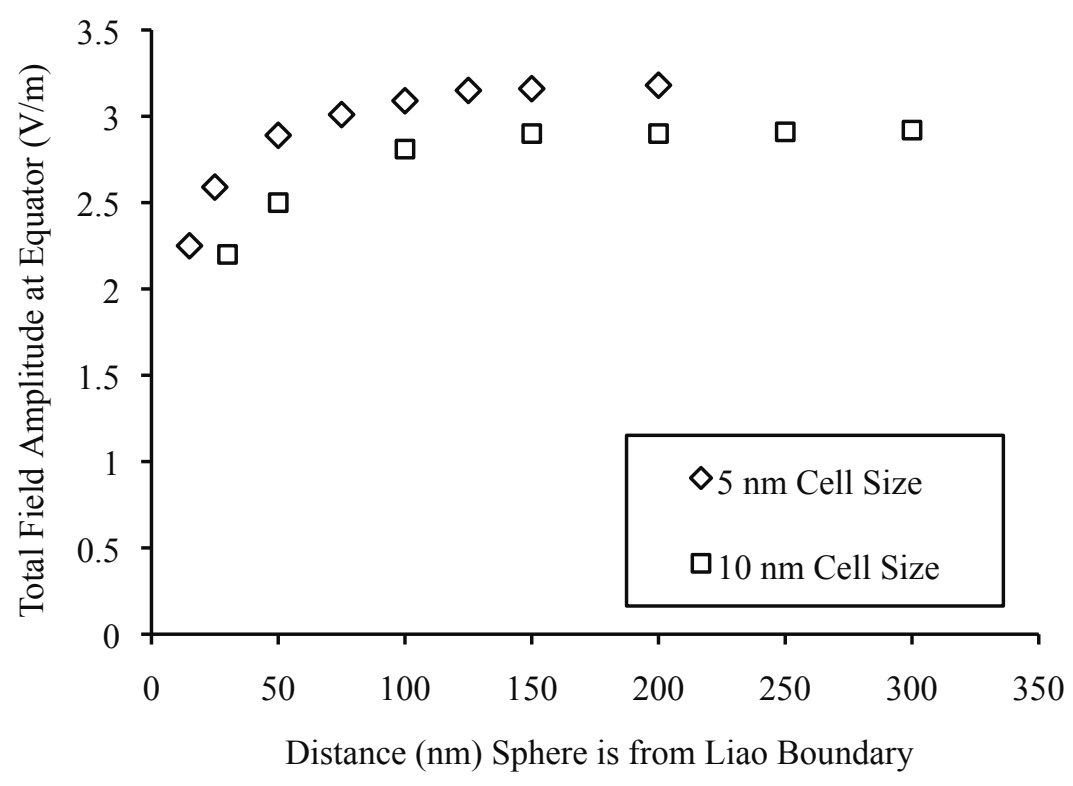

Figure 4.9 Total electric field amplitude as a function of distance away the $150 \mathrm{~nm}$ sphere is from the Liao boundary in the XFDTD simulation. 
It becomes obvious that as more detail is needed in the simulation, an increase in the overall size of the workspace is needed to create an accurate model. Otherwise, the model will underestimate the actual field intensity. This can be seen as a benefit since quick initial runs can be made to test basic geometries, then placed under more detailed modeling schemes, if necessary.

The second consideration is the effect of subdividing the object in a symmetric manner. To obtain symmetry in the sphere problem, a dividing factor is used to define the cell size parameters for the object of interest. Using this assumption, data was obtained using $50,25,15,10,5.0$, and $3.0 \mathrm{~nm}$, at the four previously defined locations, while maintaining proper spacing from the boundaries as determined by Figures 4.8 and 4.9. Recording data every time step, the total field intensity is shown in Figure 4.10.

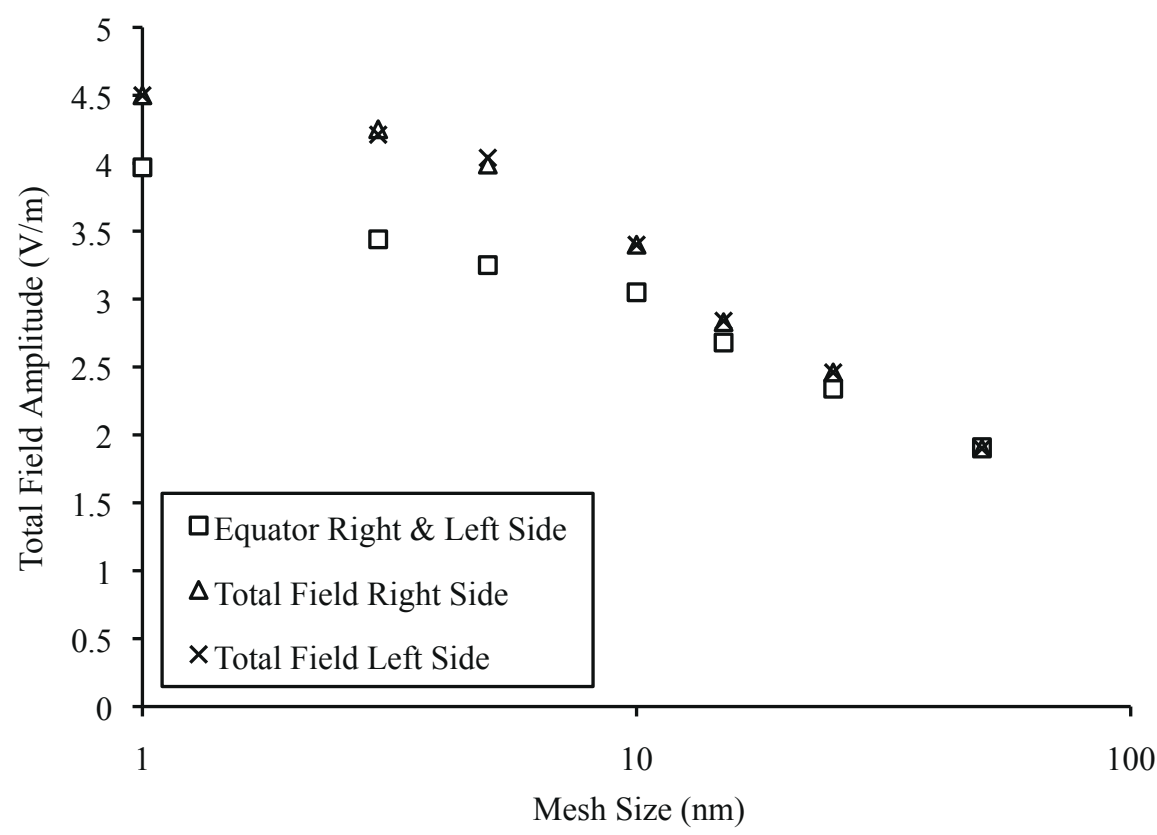

Figure 4.10 Corrected: Total field as a function of cell size, a semi log scale. Any cell size that is not divisible by the object size was removed, and adequate spacing from the boundaries was applied for each model. 
The physics of the modeling program gives an accurate interpretation of the real physical system. Careful consideration must be made to determine the optimum way in which to analyze the model with FDTD. The way that the program is utilized can cause unforeseen complications to arise. A very positive aspect of the modeling software is that most unforeseen complications will provide an underestimate of the real world system. The most important parameters in the modeling are found by choosing a cell size that will accurately represent the object to be modeled. Also, attempt to use symmetry arguments to find a dividing factor for the cell size, based on one of the dimensions of the object. Finally, one must be aware of the location of interest in the modeling, with respect to the closest Liao boundary in the overall workspace.

\subsection{Implementing MPI Communication for Parallel Computing}

The rule of thumb for using FDTD calculations for the microwave regime is to use cell sizes that are one-tenth of the wavelength of electromagnetic field used. However, looking at the validity of applying FDTD modeling techniques to the electromagnetic fields of wavelengths in the visible region, this rule of thumb fails. The application of FDTD is still applicable, assuming that the cell sizes used are much smaller than the wavelength of light being modeled. As demonstrated in the previous section, the cell size should really be one-hundredth or less of the wavelength of illumination. Of course, this assumes a simple spherical case; any complicated geometries possessing asymmetries will need to be assessed with caution. One practical solution is to use smaller cell sizes and a larger number of cells, but this will require more memory and computational power. A typical workstation computer will have difficulty with these computations, mostly caused by limited resources of RAM and CPU 
calculation power. In this case, a general alternative is to distribute the calculation load onto several computers, where the RAM of all the systems can be combined with the parallel processing of the CPUs to efficiently calculate the larger workspace.

Distributing calculations onto multiple computers requires a communication protocol, which coordinates the instructions from a server computer and the supporting node computers. Message passing interface (MPI) is a type of communication protocol that allows the coordination of multiple computers in a language-independent application-programming interface. Application-programming interfaces allow the software programs to interact with each other through the sharing of libraries and programs. [William, Ewing \& Anthony, 1999] A useable implementation of MPI is a program called MPICH. MPICH has been developed for many different operating systems, including Microsoft Windows, Linux distributions and UNIX systems.

It is possible to build a MPI based computer cluster with a couple of computers, an open source Linux distribution and the freely available MPICH program. It is important to note that MPICH does not require homogenous computer configurations. Homogenous computers can be advantageous, since the installations of Linux and MPICH, using cloning techniques, speeds up the installation process. Moreover, complications from different driver requirements between different computers are mitigated. Appendix A has a detailed account of the installation of software for a computer cluster, including all the permissions needed for file sharing and seamless communication between the server and the node computers.

Variables that are important to understand when building a MPI cluster include the number of computers and types, network bandwidth, and problem space RAM 
requirements. A few benchmarks were run on various computer configurations, network configurations, and different problems to determine and quantify the best cluster configurations for calculating MPI-based FDTD modeling routines. The FDTD benchmarks were run using Remcom's 32-bit MPI CalcFDTD engine version 6.3. Starting with a generic MPICH example program, Figure 4.11 demonstrates the calculation execution time benefit from breaking up the problem space over multiple compute nodes. The calculation is of a simple example program provided with the MPICH distribution, where $\pi$ is calculated to a user requested precision. While the example program nicely demonstrates a decrease in time with the addition of more compute nodes, it fails to demonstrate the effects of network latency, which becomes a factor when nodes need to exchange a large amount of time dynamic information.

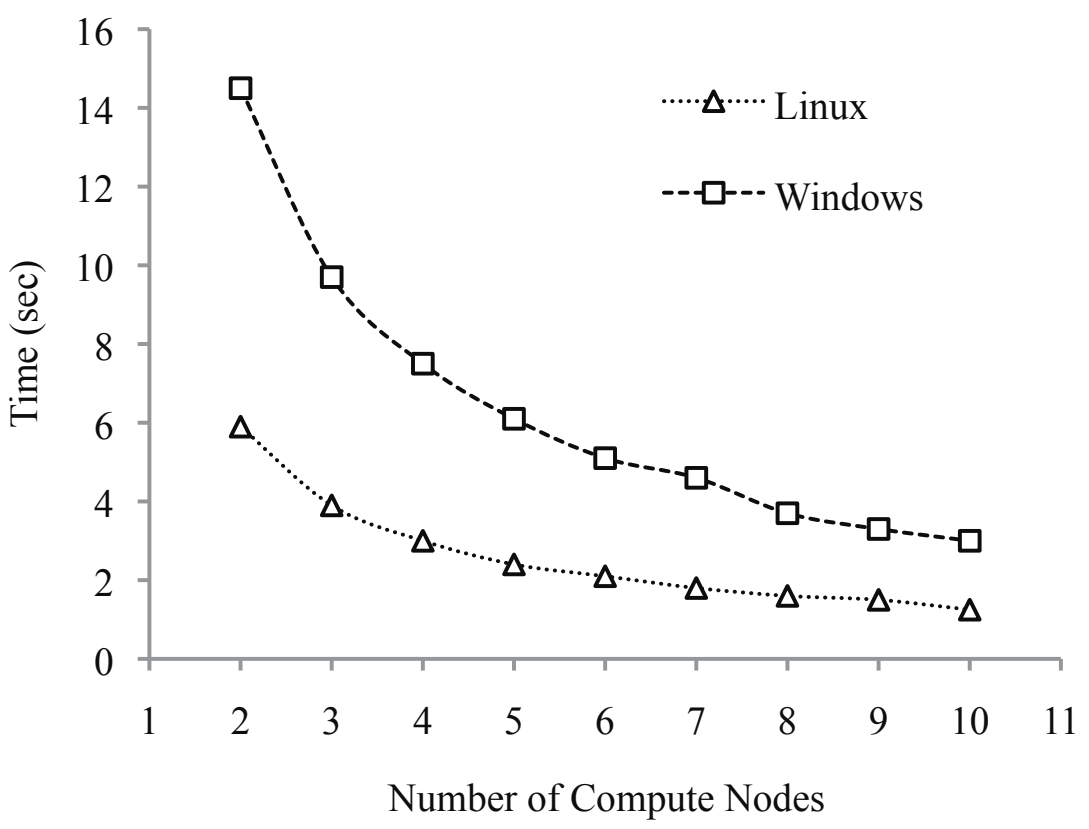

Figure 4.11 Number of compute nodes used vs the calculation time required. Times for a Windows and a Linux-based system calculation of $\pi$ to the same level of precision. 


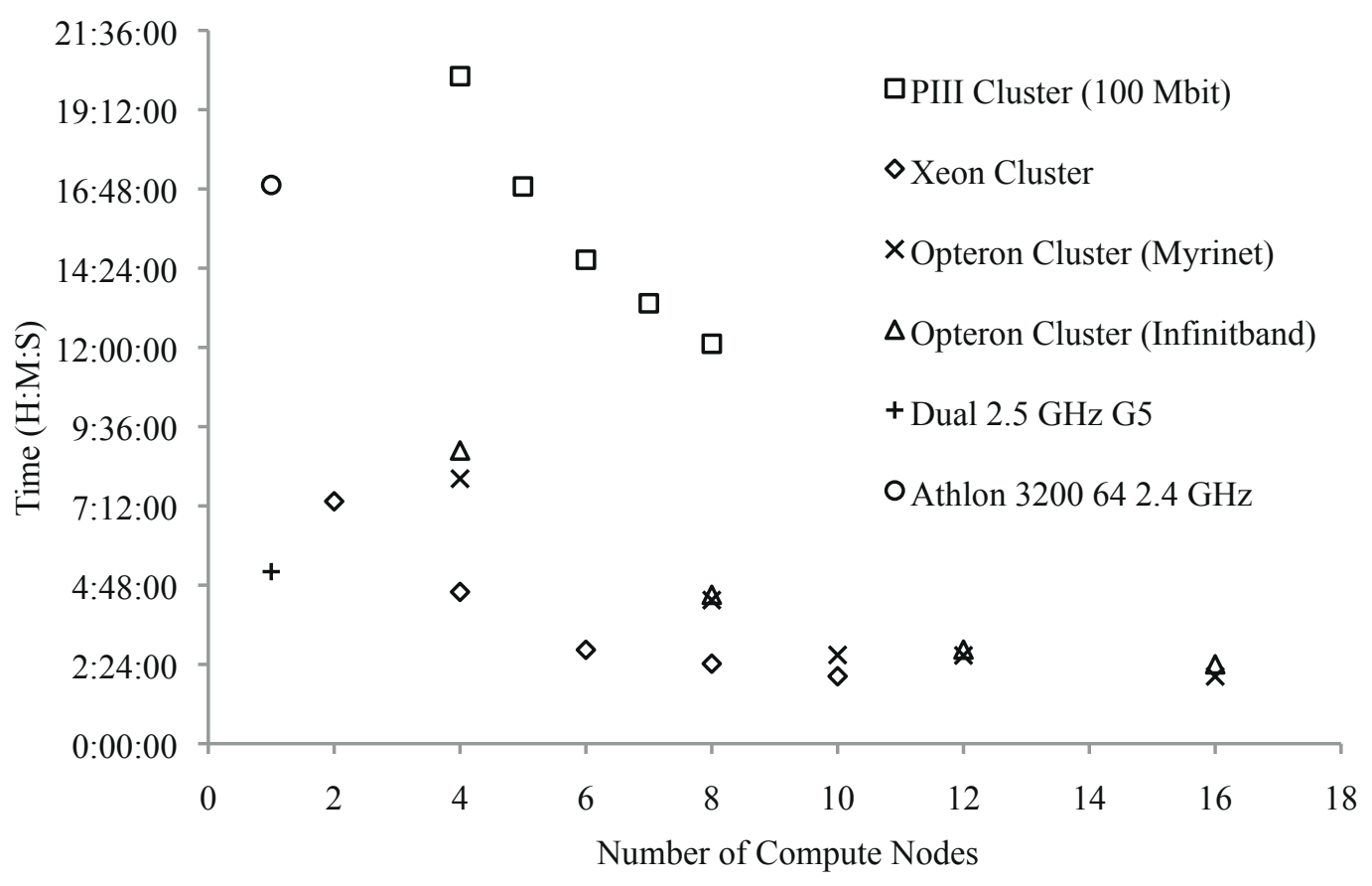

Figure 4.12 Shows a comparision of the number of compute nodes used vs. the calculation time required. Times for various computer and networking types are shown.

For FDTD calculations, the compute nodes will have to store and exchange data on a dedicated NFS (network file system) folder on the server computer. This requirement will put stress on the network bandwidth. Figure 4.12 gives the benchmarks for various cluster systems and network configurations. These were generated with a Remcom test file that was $1.2 \mathrm{~GB}$ executed for 6000 time-steps. This comparison demonstrates the feasibility of relying on $100 \mathrm{Mbit} / \mathrm{s}$ network bandwidth over the faster, more exotic and expensive Infiniband and Myrinet networking solutions. Moreover, as gigabit networking becomes more standard, these benchmarks will only improve. Of course, the size of the model will play a role in these benchmarks as well. Figure 4.13 analyzes the times for a $725 \mathrm{MB}$ workspace executed over 2300 time-steps. It shows that 
after roughly 7 compute nodes the calculation time hits a lower limit. This lower limit can be attributed to network latency. Figure 4.14 amplifies the effect of network latency by processing a very small model (16.96 MB and 2000 timesteps) over many computers. Examination of the graph shows that the Pentium 4s have no benefit of running the model over multiple compute nodes.

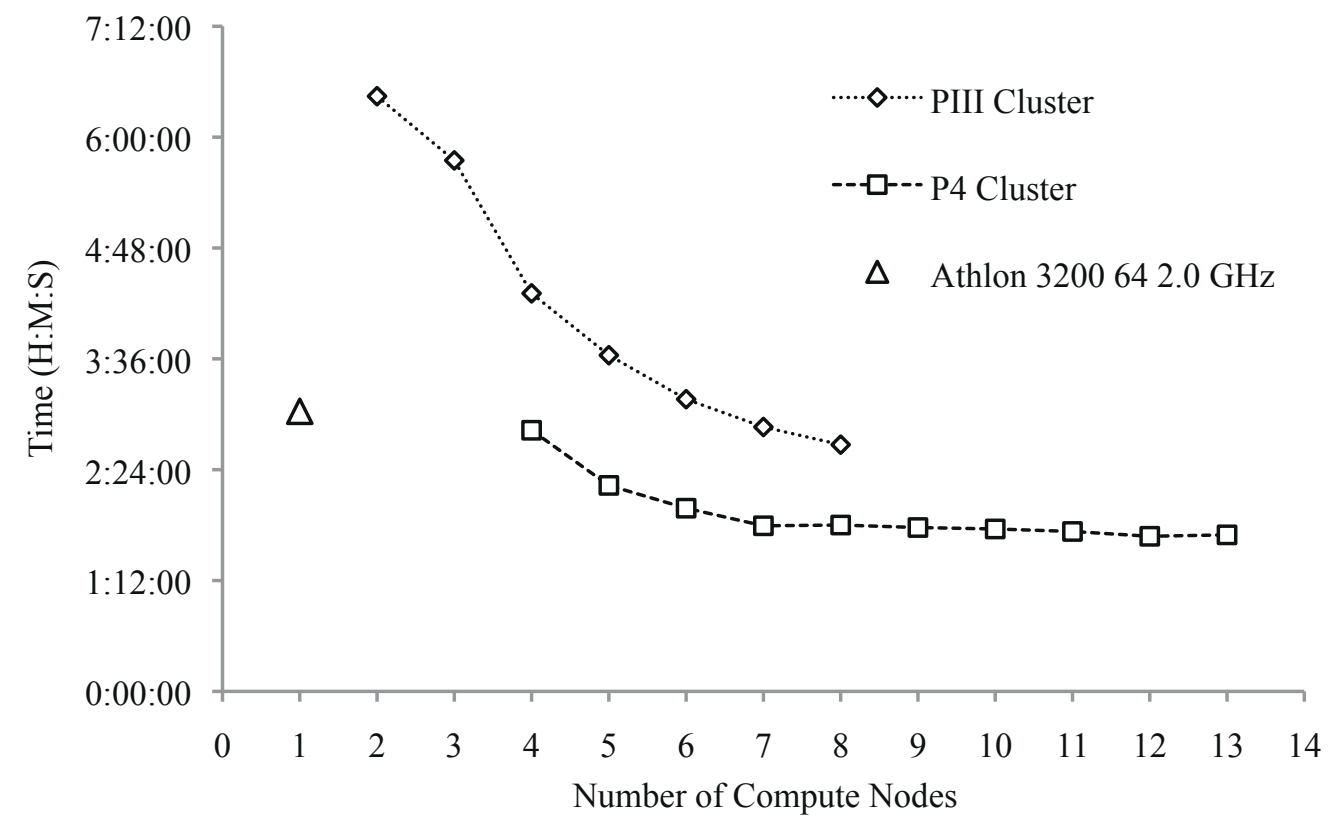

Figure 4.13 Comparison of a Pentium III computer cluster versus a Pentium 4 computer cluster. Model used for comparison was $725 \mathrm{MB}$ executed for 2300 timestamps.

Using these benchmarks as justification, a simple 32-bit calculation cluster can be built using basic, built-in networking hardware. Computers, a few generations old, can be acquired for low cost. The benefits to the cluster will not be speed, but rather the ability to run models greater than $4 \mathrm{~GB}^{\dagger}$. Newer 64-bit computer systems are removing this 4 GB. Even with newer 64-bit computer systems, the scaling principle of MPI

\footnotetext{
${ }^{\dagger}$ Addressable RAM limit for 32-bit computer systems.
} 
clustering still remains the same, independent of the configuration hardware. Many of the models presented here were calculated on a PIII cluster of 15 compute nodes with combined memory resources of $30 \mathrm{GBs}$.

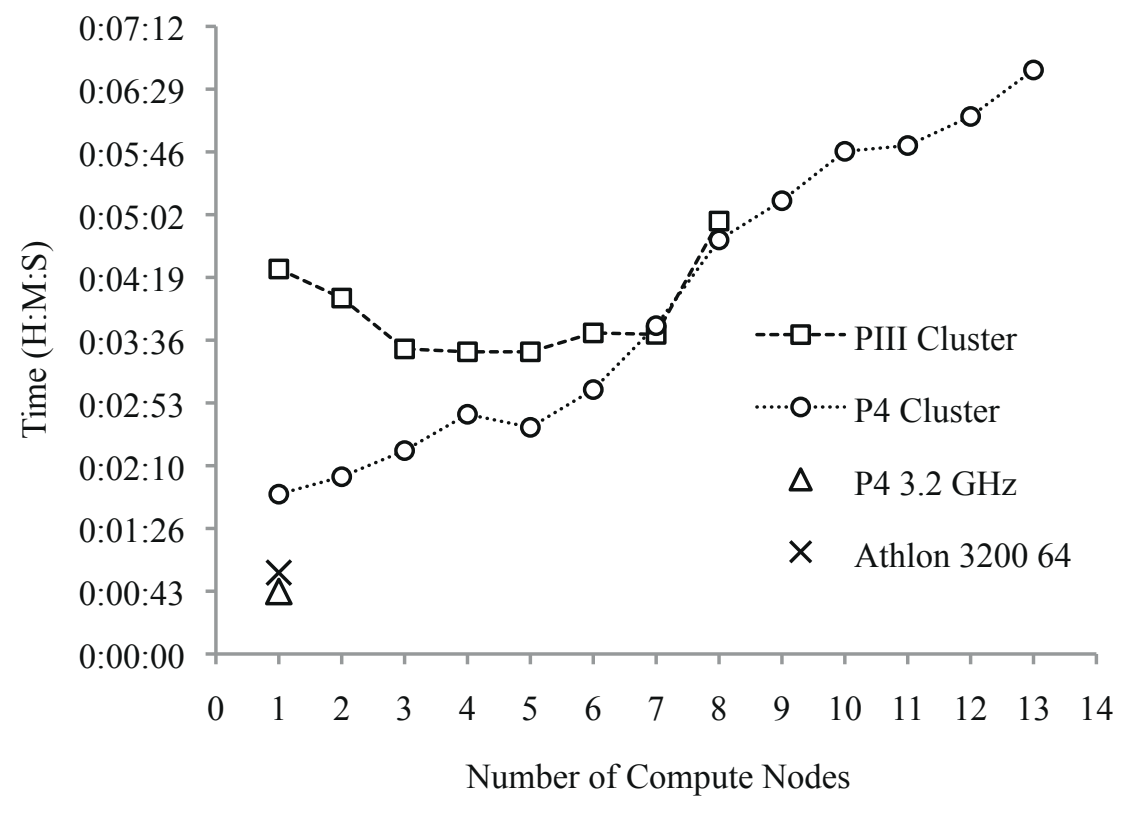

Figure 4.14 The effect of network latency on MPI calculations (Remcom provided dipole test file). The problem space was $16.96 \mathrm{MB}$ and was executed for 2000 timesteps. Both a Pentium 4 cluster and Pentium III cluster were used to demostrate the effect of latency.

\subsection{Realistic Problem Space}

Typically, modeling makes some assumptions in order to simply the complexity of the problem space. A majority of the FDTD near-field studies use an incident plane wave, where the propagation direction has been optimized to the probe design. For epiillumination of the TENOM probes, this is less than ideal. The TENOM probe is placed within a focal spot that has many different polarization attributes, which cannot be modeled with a simple plane wave. Due to the size and complexity of a 1.4 NA (Numerical Aperture) objective lens, it is rarely included in the problem space. 
A growing trend is to use adaptive meshing for the discretization of models [Berger \& Colella, 1989]. Adaptive meshing allows for small cell sizes in regions of importance and larger cell size in regions of less importance. Using adaptive meshing, a simplified focusing lens can be created with the probe interaction areas defined in high detail.

The modeling space for TENOM probe design comparisons is comprised of 440 by 325 by 310 total cells, which requires roughly $1.5 \mathrm{~GB}$ of memory. The baseline dimension for the cells is $80 \mathrm{~nm}$, which represents $\lambda / 10$ for $800 \mathrm{~nm}$ light. The adaptive meshing reduces the dimension of the cells to $3.0 \mathrm{~nm}$ for the area around the imaging location of the probe that has the highest localized electric-field enhancement. The length of the adaptive meshing region is $\sim 620 \mathrm{~nm}$ in $\mathrm{x}, \mathrm{y}$, and $\mathrm{z}$ dimensions. The models are comprised of a focusing lens and the imaging probe. The focusing lens is $10 \mu \mathrm{m}$ in diameter and maintains a high NA (1.1 NA estimated), as shown in Figure 4.15. It is still technically challenging to obtain a $1.4 \mathrm{NA}^{\ddagger}$ due to the reduced problem space. The lens is defined in the Remcom software as a "Normal Material Type" with a conductivity of 0.0 $\mathrm{S} / \mathrm{m}$ and a relative permittivity of 2.375 . Relative permittivity is obtained by squaring the index of refraction of the glass of the lens, which is 1.541. The Au TENOM imaging probe uses the same Debye modeling constants that were presented previously $\left(\varepsilon_{s}=-\right.$ 12987, $\varepsilon_{\infty}=9.012, \sigma=1.464 \times 10^{7} \mathrm{~S} / \mathrm{m}$, and $\left.\tau_{0}=8.017 \times 10^{-15} \mathrm{~s}\right)$. The edge of the modeling workspace that crosses the probe is defined as a perfect conducting boundary.

\footnotetext{
$\$$ A typical commercial 1.4 N.A. objective lens is designed with more than 16 pieces of glass with the final exit aperture roughly a millimeter across, not something easily modeled on a desktop PC.
} 
Figure 4.16 shows a 3D representation of the modeling workspace, including the planes of interest for the various models to be presented.

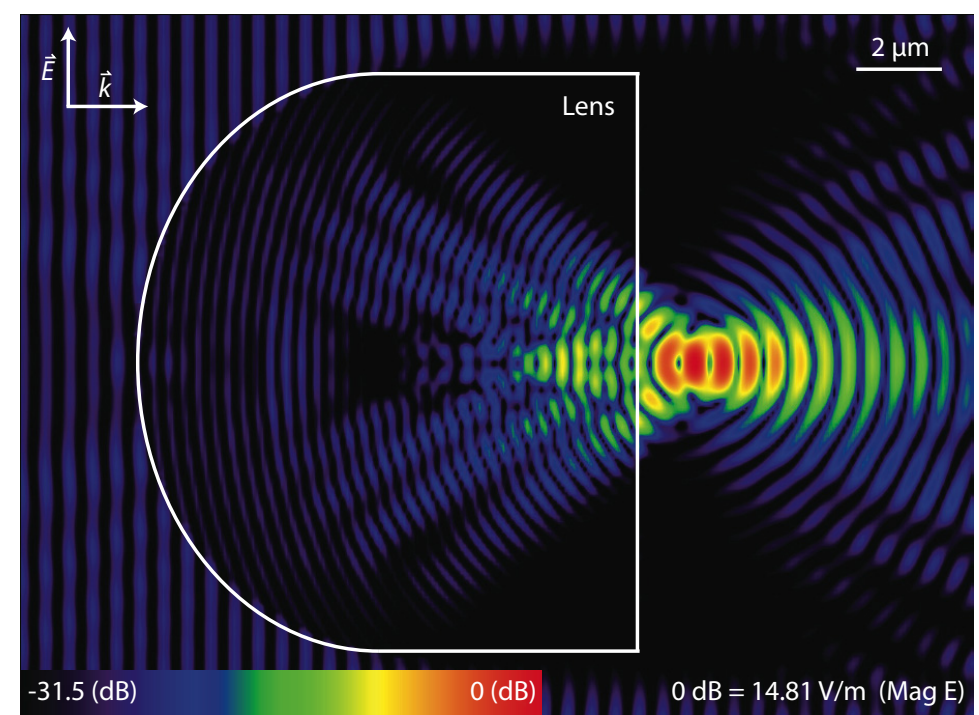

Figure 4.15 A semi-realistic modeling space for focusing the incident light on the TENOM probes. The light has vertical E-field polarization, propagating from left to right. Color scale is logarithmic, with maximum electric field strength of $14.81 \mathrm{~V} / \mathrm{m}$ in the focus.

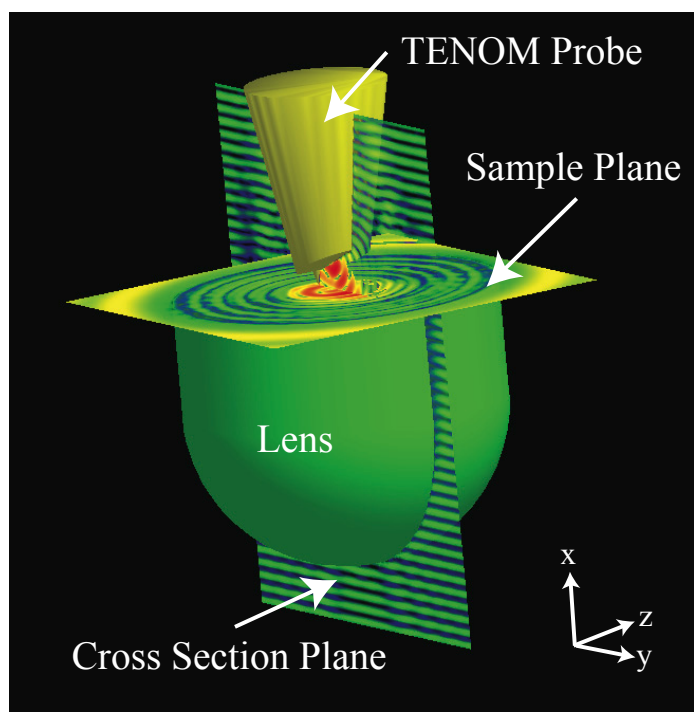

Figure 4.16 3D workspace for the semi-realistic modeling conditions, showing 2D planes of interest. Lens is green colored and TENOM probe yellow. 2D sample plane is the electric-field intensities a few nanometers above the lens glass. The $2 \mathrm{D}$ cross section plane is mid probe along the asymmetric axis. 


\subsection{Ideal Probe Designs}

The ideal TENOM probe provides a high near-field to far-field contribution ratio. There are two possibilities that could be explored to generate good near-field imaging probes. First, the electric field could be made many orders of magnitude stronger at the imaging probe apex than the focusing far-field incident field strength. This could be accomplished with small perturbations at the end of the imaging probe. Acting like a lightning rod, a strong confined electric field will result within tens of nanometers of the perturbation. A carbon nanotube extending from the Au probe could generate just this condition. Figure 4.17 shows the effect of placing a $3.0 \mathrm{~nm}$ in diameter, $15.0 \mathrm{~nm}$ in length, multi-walled carbon nanotube at the end of a low aspect ratio probe, adapted from Nowak's thesis [Nowak, 2004]. The carbon nanotube increased the localized field by $400 \%$.
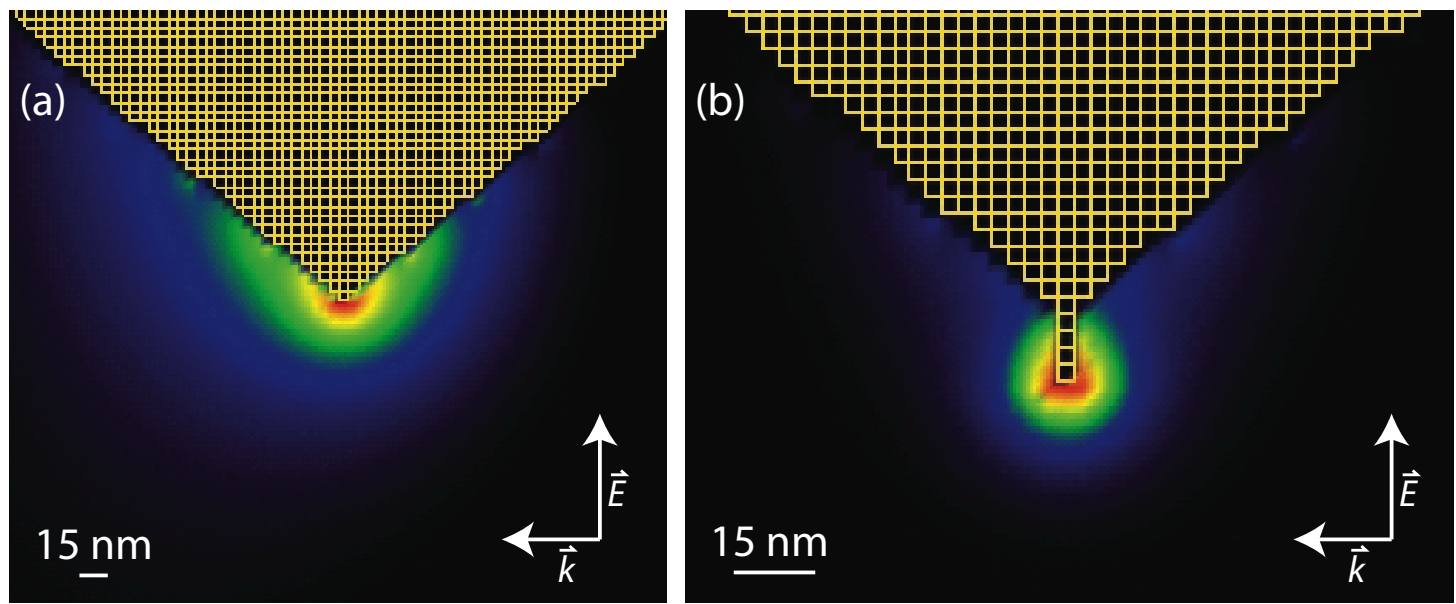

Figure 4.17 G-T cone workspace, $\mathrm{x}-\mathrm{y}$ plane at cell 137 in the $\mathrm{z}$ direction with $3 \mathrm{~nm}$ cell size representation. (a) G-T cone geometry showing a maximum electric field of $180 \mathrm{~V} / \mathrm{m}$. (b) The effect of an elongated apex on the G-T cone, giving a maximum electric field of $650 \mathrm{~V} / \mathrm{m}$. 
Another possibility for far-field background suppression is to delocalize the near-field imaging location from the far-field focal region. This method is worth serious consideration, since the development, and more importantly, the manufacture of probes that exhibit high field enhancement factors is difficult and oftentimes suffers from irreproducible nanoscale geometries.

Modeling shows that polarization of the incident electric field is paramount to near-field imaging. Novotny presented electric-field enhancement of sharp metal probes that required phase delayed, higher-order Hermite-Gaussian illumination to generate a electric-field polarization in the propagation direction through superposition of the electric-fields [Novotny, Sánchez \& Xie, 1995]. Using a sharp metal tip placed nine nanometers $^{\S}$ away from the lens surface, Figure 4.18 shows the illumination of a sharp metal probe with first-order Gaussian epi-illumination, further justifying Novotny's calculations. Figure 4.19 shows the electric field intensities in the sample plane. Analysis of one half of an optical cycle shows a large far-field contribution with a maximum field intensity of $10.9 \mathrm{~V} / \mathrm{m}$. According to Figure 4.15 , the electric field intensity in the far-field diffraction limited focus is $14.81 \mathrm{~V} / \mathrm{m}$. Even though the maximum electric field demonstrated in Figure 4.18 is $33.25 \mathrm{~V} / \mathrm{m}$, this intensity is found further up the probe, away from the imaging plane. Thus, a sharp metal tip without polarization in the axis of the tip length is not favorable for near-field imaging.

\footnotetext{
$\S$ The imaging distance of $9 \mathrm{~nm}$ is to minimize optical quenching. Chapter 5 discusses quenching and other near-field imaging artifacts.
} 

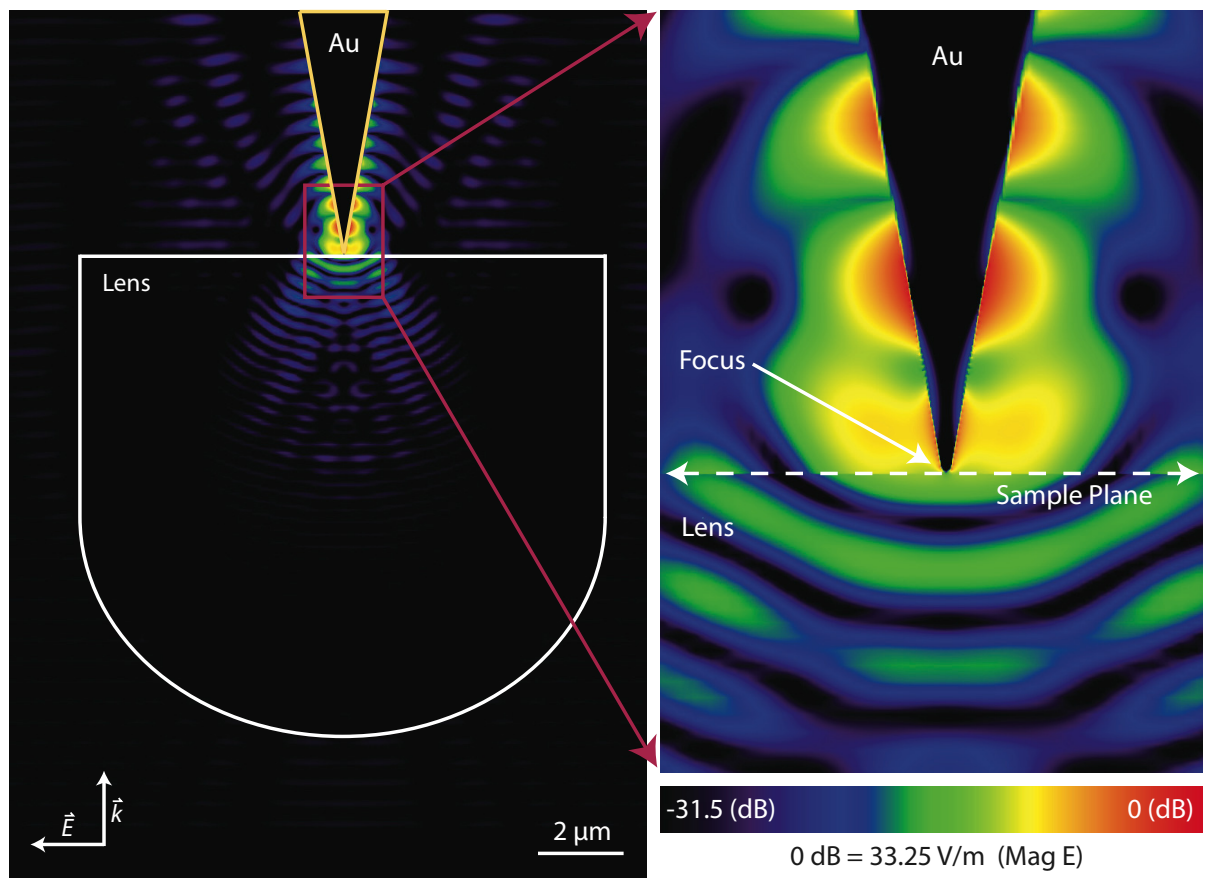

Figure 4.18 High aspect ratio $\mathrm{Au}$ probe, illuminated from below with 800 $\mathrm{nm}$ light. The highest electric field intensities are measured $750 \mathrm{~nm}$ away from the focus, along the tip shank. Electric field intensities are given in a logarithmic scale.

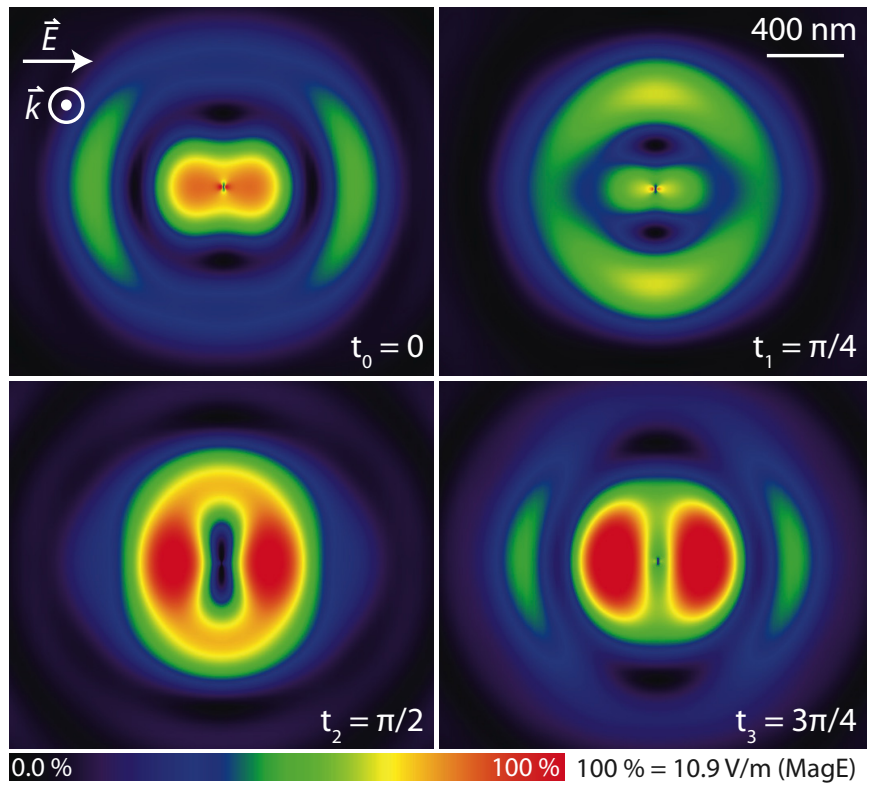

Figure 4.19 Sample imaging plane showing electric field magnitude distribution with the high aspect ratio Au probe, illuminated from below using $800 \mathrm{~nm}$ light. One half of an optical cycle is shown in four divisions. Electric field intensities are given in a logarithmic scale. 
A reasonable question that can be asked is, what polarization would be optimal? The Gilbert-Taylor (G-T) cone geometry proposed in earlier work was used to analyze the effect of incident polarization on the probe, shown in 4.17 [Nowak, 2004]. A GilbertTaylor cone is generated by a static electric field on liquids, which produces a half angle of $49.3^{\circ}$ [Taylor, 1964]. Forbes investigated the effect of these fields on liquid metals in several publications, specifically as they would pertain to liquid metal ion sources (LMISs) [Forbes, 1976; 1981; 1999]. Using a plane wave (i.e., no lens) the G-T cone is illuminated from various incident field angles, given in Figure 4.20.

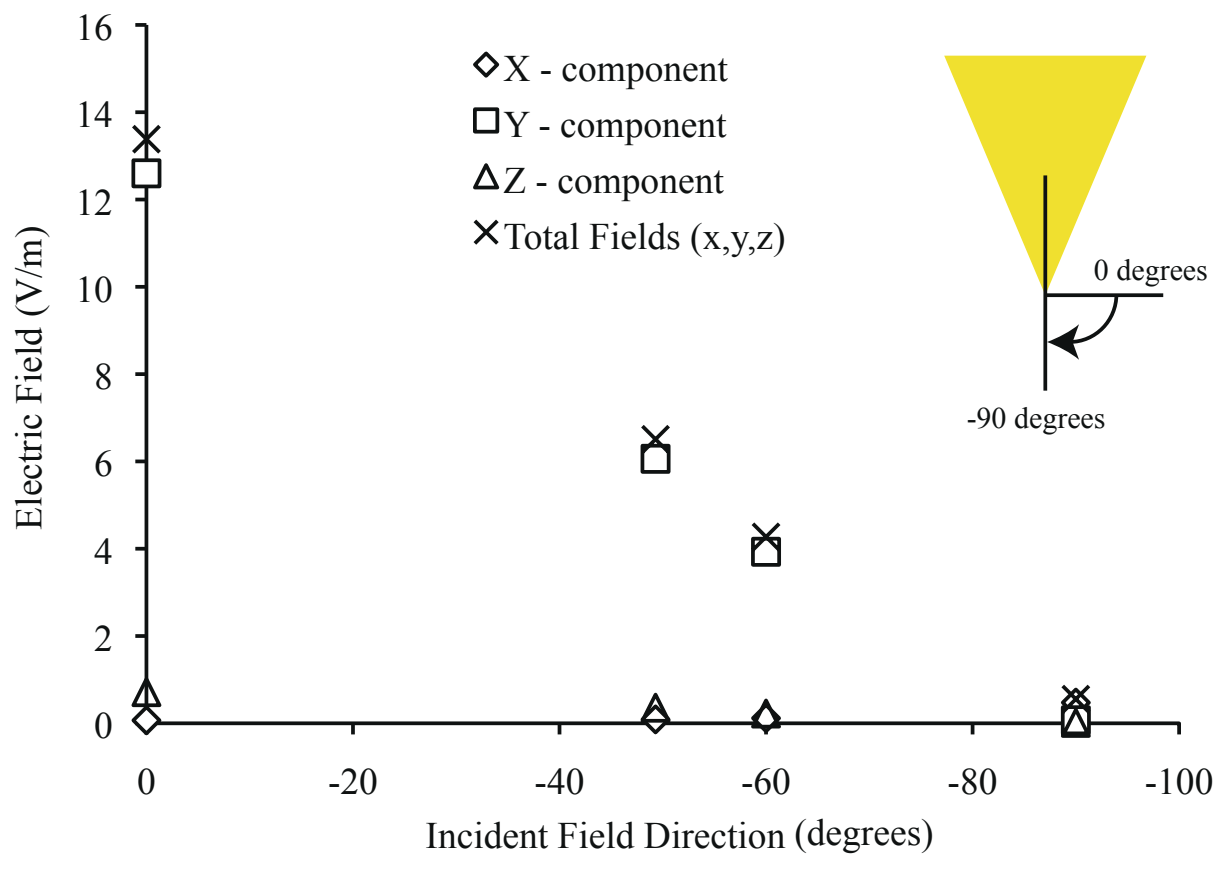

Figure 4.20 Electric field intensity as a function of incident field direction. The fields are shown by their $\mathrm{x}, \mathrm{y}, \mathrm{z}$, and total field components (total field $\left.=\left(\mathrm{x}^{2}+\mathrm{y}^{2}+\mathrm{z}^{2}\right)^{1 / 2}\right)$.

Figure 4.20 demonstrates that the optimal polarization is not directly related to the angle of the cone but rather to the vertical direction of charge oscillation. Moreover, illumination orthogonal to the probe length provides zero electric field enhancements, as 
expected. Being able to drive charge from the large upper bulk of the tip down to a smaller region is the most favorable for a high electric field enhancement at the imaging point of the probe.

\subsection{Asymmetric Probe Design}

Developing a TENOM probe that has a large bulk area that contains a reservoir of electrons that can be driven to a small sharp region is the basis for the asymmetric probe geometry. The asymmetric geometry is designed for epi-illumination and ease of manufacture in a focused ion beam (FIB) system (discussed in Chapter 11). One further benefit of the asymmetric design is the potential to delocalize the near-field imaging location from the far-field focal region. Figure 4.21 outlines the basic dimensions for the asymmetric probe. Figures 4.22 through 4.29 present different modeling scenarios for the asymmetric probe.

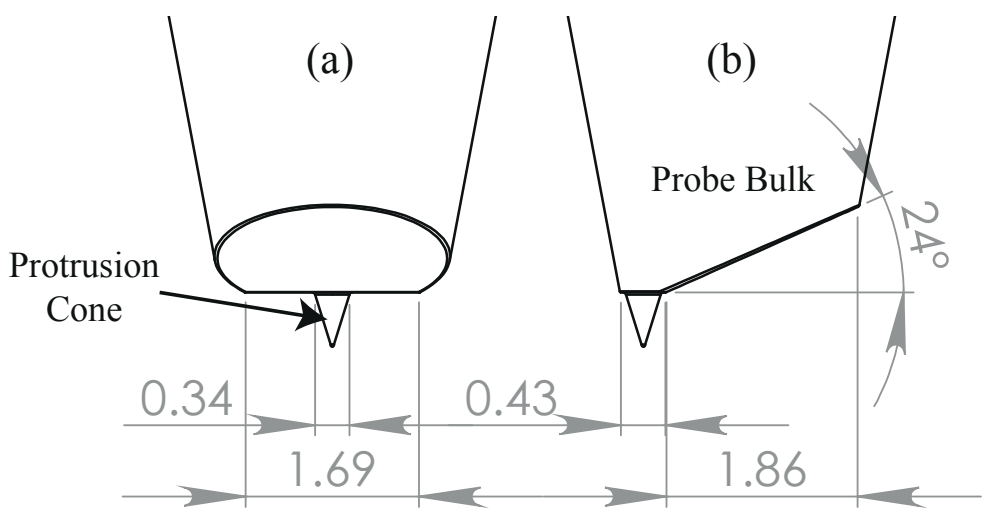

Figure 4.21 Dimensions of asymmetric probe. All values except the 24 degrees are in microns.

The different models examine the effect of focal location on the probe. Figure 4.22 shows the focus at the sample plane. This focal location would typically be advantageous for sensitive, diffraction limited, far-field, two-photon imaging. 

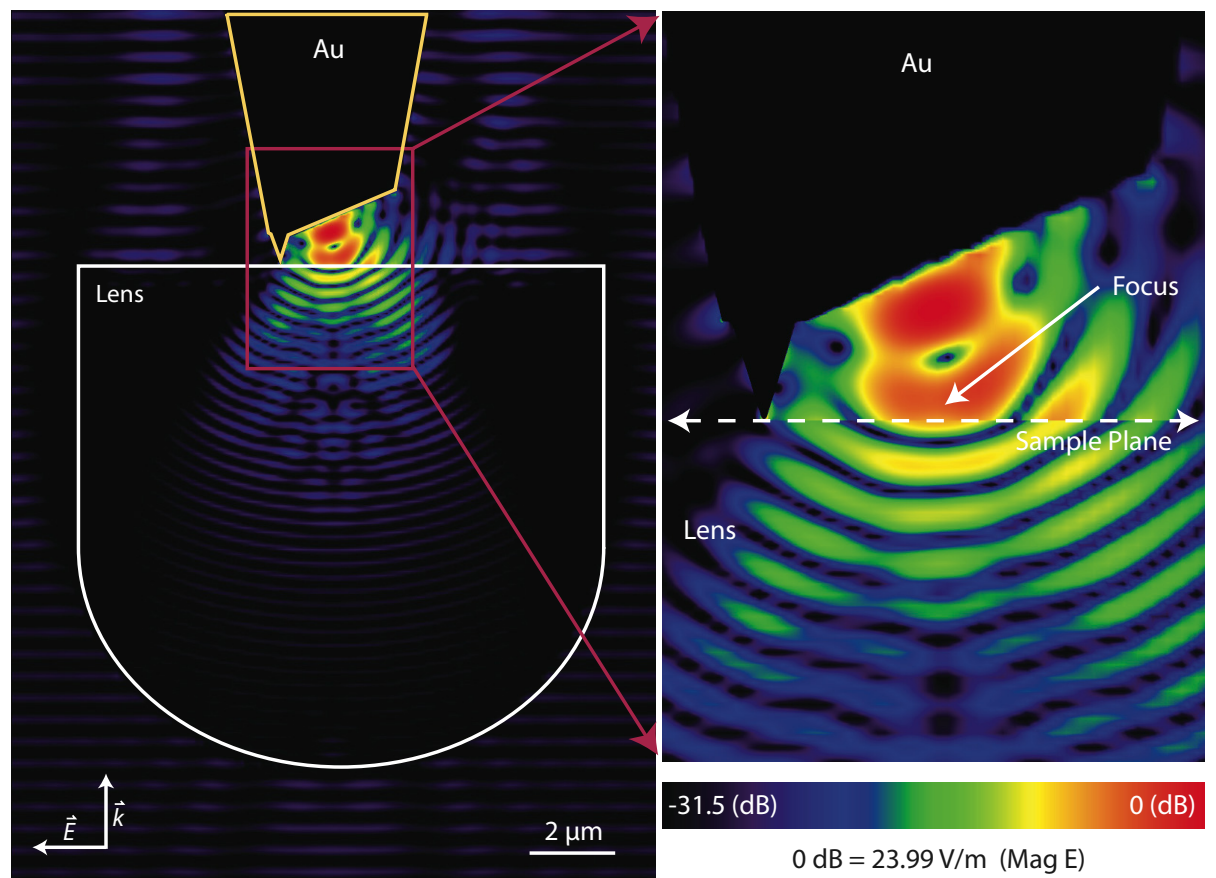

Figure 4.22 Asymmetric Au probe, illuminated from below with $800 \mathrm{~nm}$ light, focused at the sample surface. The highest electric field intensities are measured at the bulk of the tip several hundred nanometers above the imaging plane.

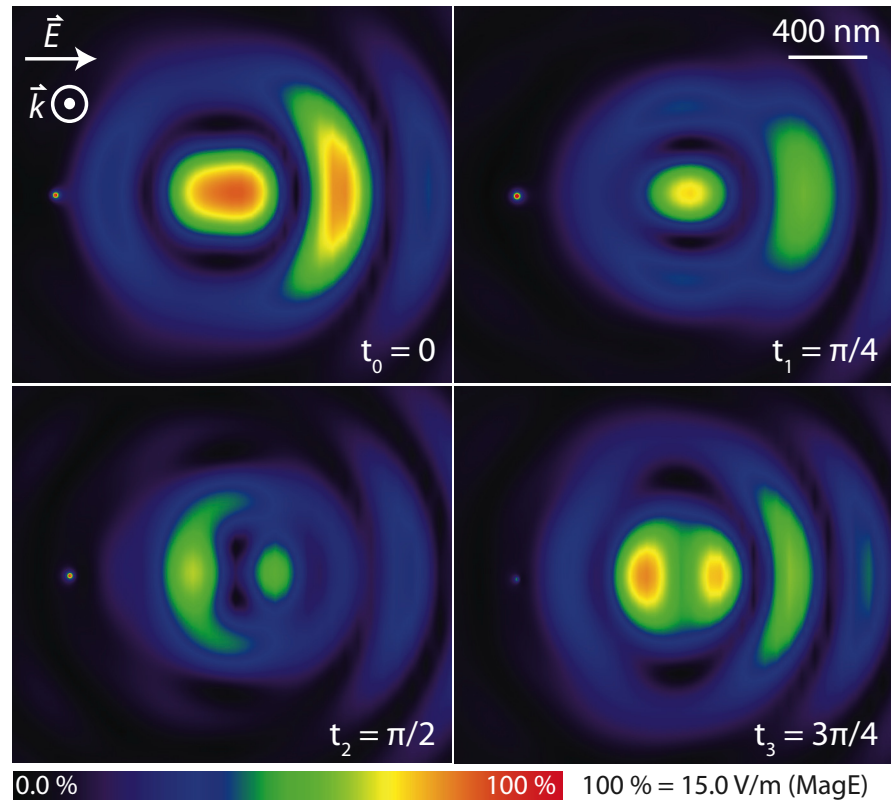

Figure 4.23 Imaging plane electric field magnitude distribution with the asymmetric $\mathrm{Au}$ probe and focus at sample surface (same model as Figure 4.22). One half of an optical cycle over four divisions. (Note: linear scale.) 
The TENOM probe is placed so that the middle of the bulk area is centered over the focal region. In this configuration, the electric field intensity increases from the $14.81 \mathrm{~V} / \mathrm{m}$ with no tip present to $23.99 \mathrm{~V} / \mathrm{m}$ with a tip present. Figure 4.23 shows that this increase is not in the sample-imaging plane. Furthermore, the overall area has a very large background contribution making it an unsuitable condition for near-field imaging. Moving the focus to the surface of the tip bulk gives a substantial increase in electric field at the near-field imaging point of the probe. Both Figures 4.24 and 4.25 demonstrate electric field intensities of $58.91 \mathrm{~V} / \mathrm{m}$ and $55.54 \mathrm{~V} / \mathrm{m}$, respectively. Moving the diffraction-limited focus from the imaging plane minimizes the far-field background contribution; the maximum-recorded background is roughly $6.0 \mathrm{~V} / \mathrm{m}$.
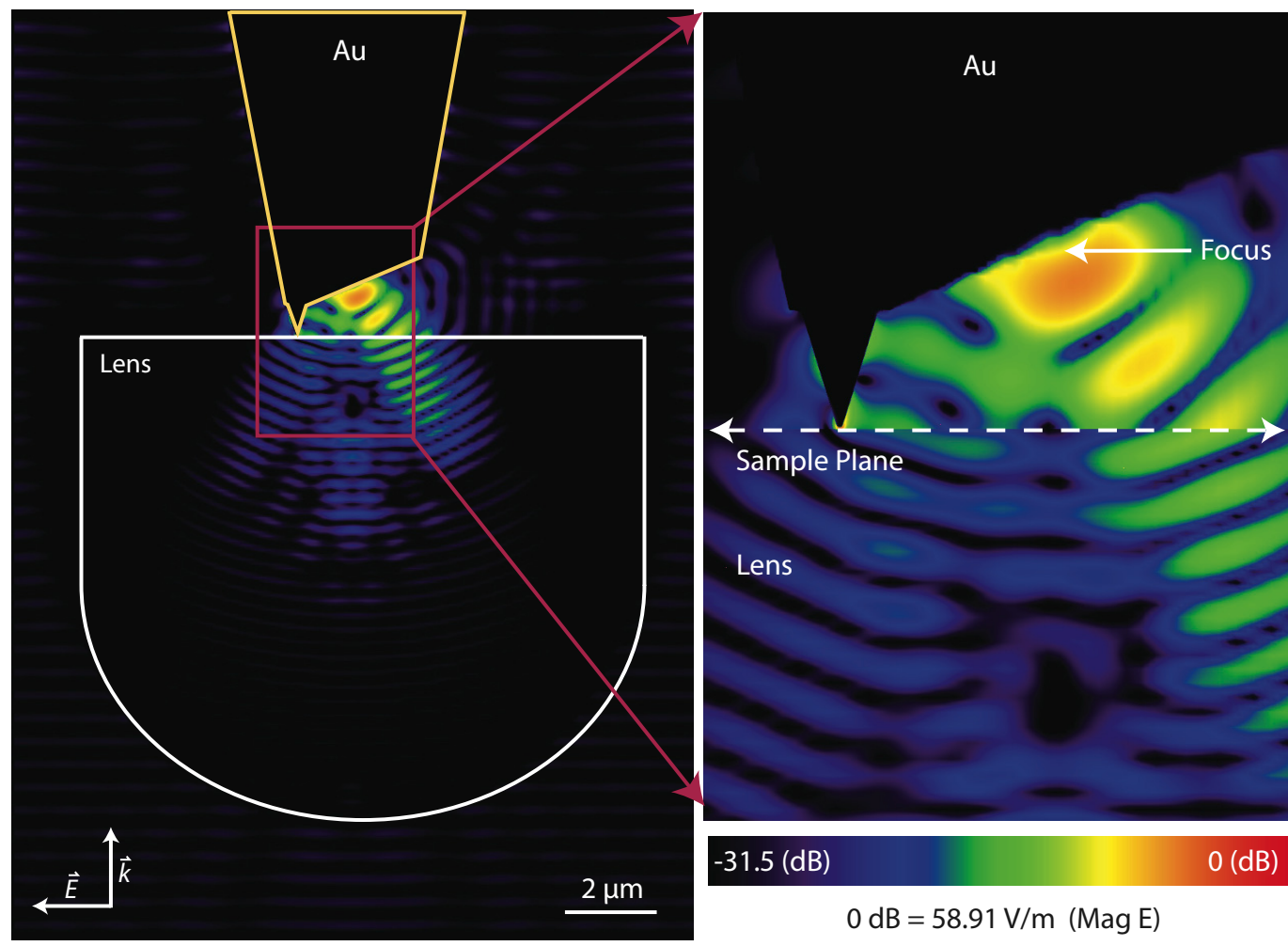

Figure 4.24 Asymmetric Au probe, illuminated from below with $800 \mathrm{~nm}$ light, focused at bulk surface on probe. The highest electric field intensities are measured at the end of the protrusion cone. 


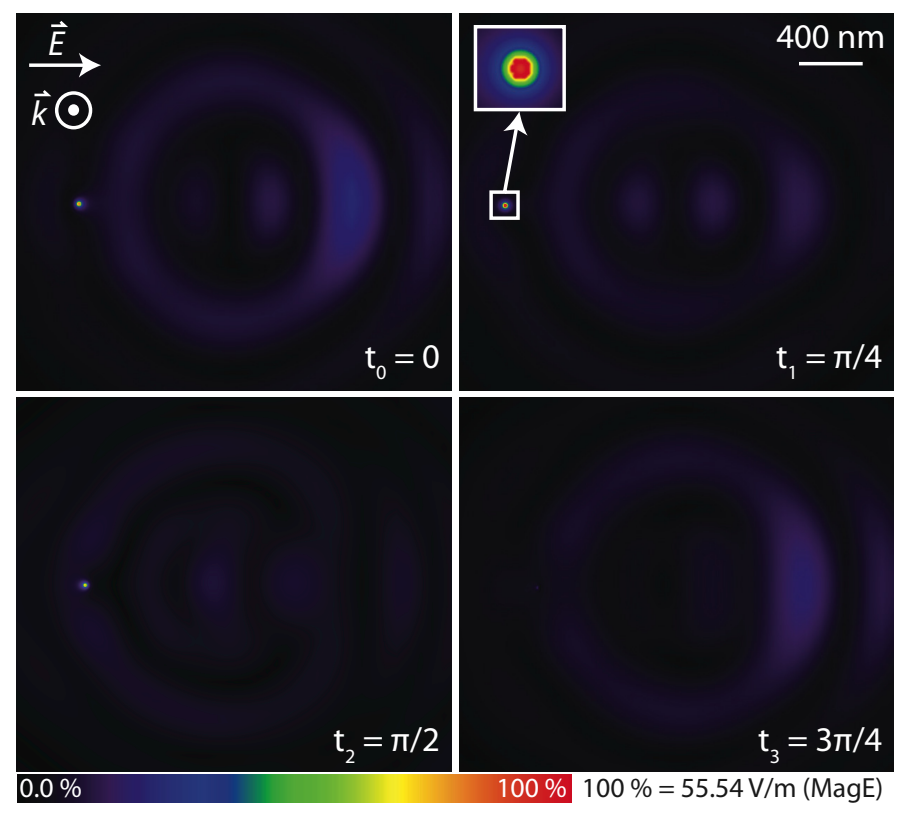

Figure 4.25 Imaging plane electric field magnitude distribution with the asymmetric $\mathrm{Au}$ probe and focus on probe bulk surface (same model as Figure 4.24). One half of an optical cycle over four divisions. (Note: linear scale.)

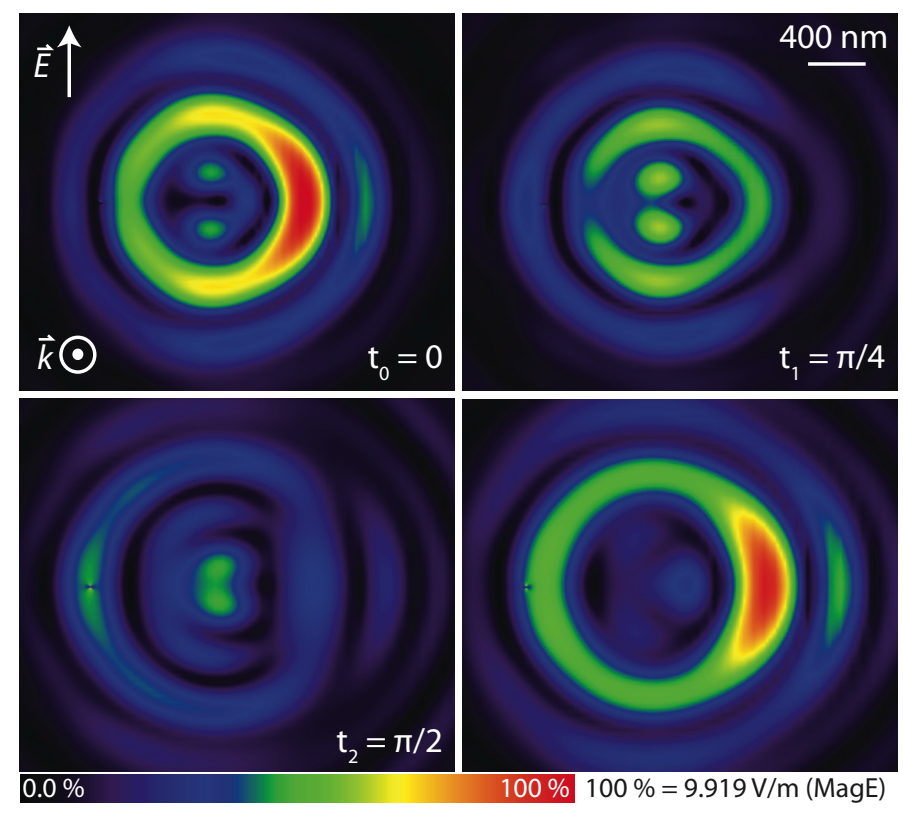

Figure 4.26 Imaging plane electric field magnitude distribution with the asymmetric $\mathrm{Au}$ probe and focus on probe bulk surface. Model differs from Figure 4.25, due to a rotation of incident electric field polarization by $90^{\circ}$. One half of an optical cycle over four divisions. (Note: linear scale.) 
Figure 4.26 shows the dependency of the asymmetric probe design on polarization. Rotating the polarization to be orthogonal to the direction of asymmetry in the probe does not drive surface charge from the bulk region into the sharp imaging region. Closer examination of the near-field electric field polarizations, present at the end of the imaging point, shows the strongest along the length of the probe with equal contributions in the orthogonal planes $y$ and $\mathrm{z}$, shown in Figure 4.27. This is the most favorable orientation of incident laser focus and delocalized near-field imaging point.

The final model examines the effects of placing the focus at the end of the protrusion cone. Both Figures 4.28 and 4.29 show the results of this model. The highest electric field of $57.22 \mathrm{~V} / \mathrm{m}$ is recorded along the protrusion cone, outside of the imaging plane. In the imaging plane, the highest electric field is $48.0 \mathrm{~V} / \mathrm{m}$. There is significantly more far-field contribution evident in Figure 4.29, in comparison to that shown in Figure 4.25 .

In all asymmetric conditions, having the large bulk area behind the protrusion cone aids in the driving of surface charge. The driving of this surface charge allows for the spatial confinement of a large electric field at the end of the protrusion cone. Ultimately, this allows for near-field imaging resolutions less than $30 \mathrm{~nm}$. Reduction of the protrusion cone will improve the spatial resolution, while diminishing the far-field contributions in the near-field imaging signal. The asymmetric design has demonstrated a quantifiable method to perform TENOM imaging with epi-illumination. 


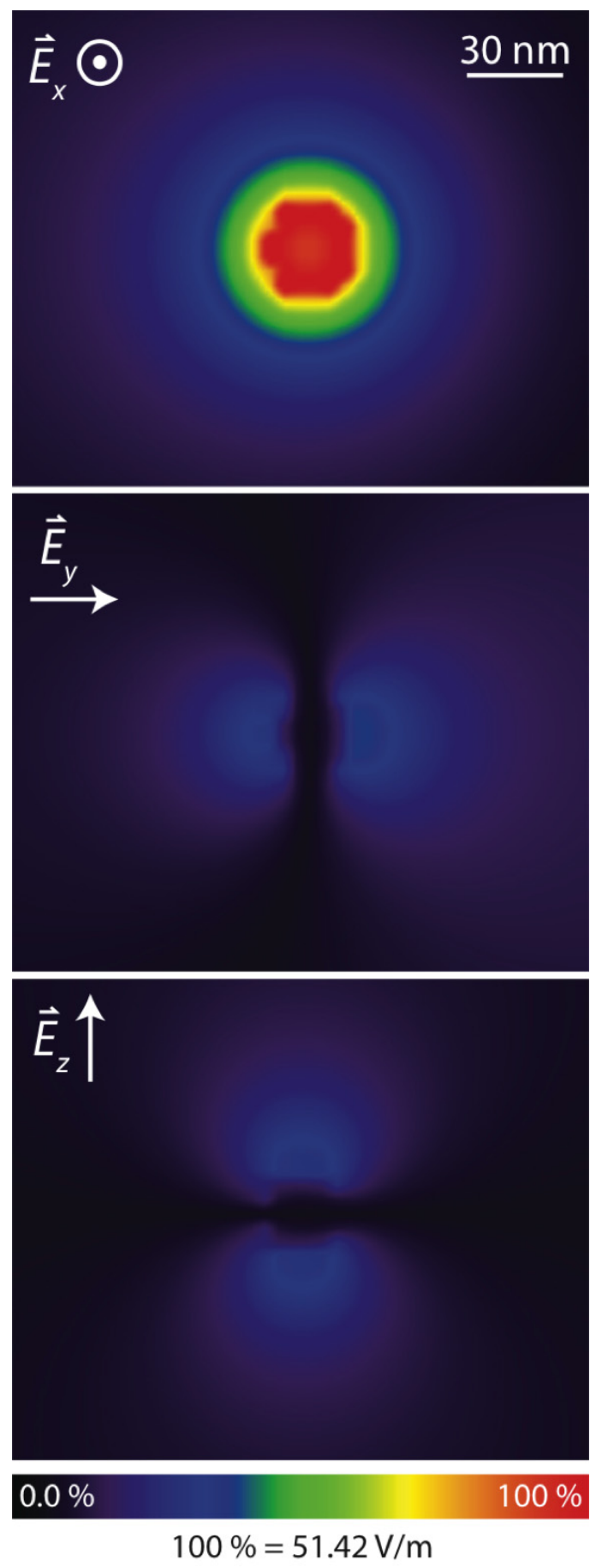

Figure 4.27 Imaging plane electric field components (X, Y, and Z) at the near-field imaging region. (Same area as zoomed feature in Figure 4.26) The same asymmetric Au probe and focus on probe bulk surface as model for Figure 4.25. (Note: linear scale.) 

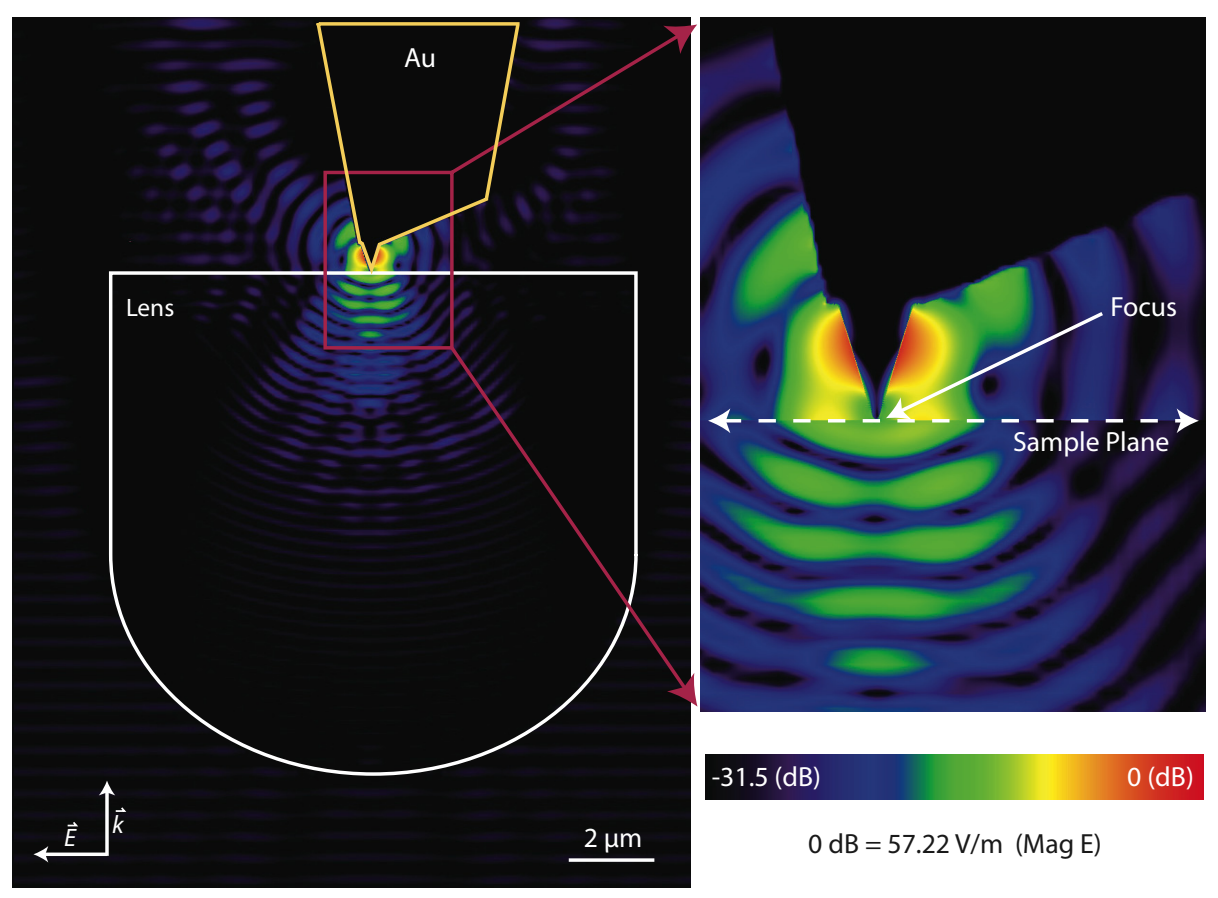

$-31.5(\mathrm{~dB})$

$0 \mathrm{~dB}=57.22 \mathrm{~V} / \mathrm{m}($ Mag $\mathrm{E})$

Figure 4.28 Asymmetric Au probe, illuminated from below with $800 \mathrm{~nm}$ light, focused at end of protrusion cone. The highest electric field intensities are measured a few hundred nanometers above the end of the protrusion cone.

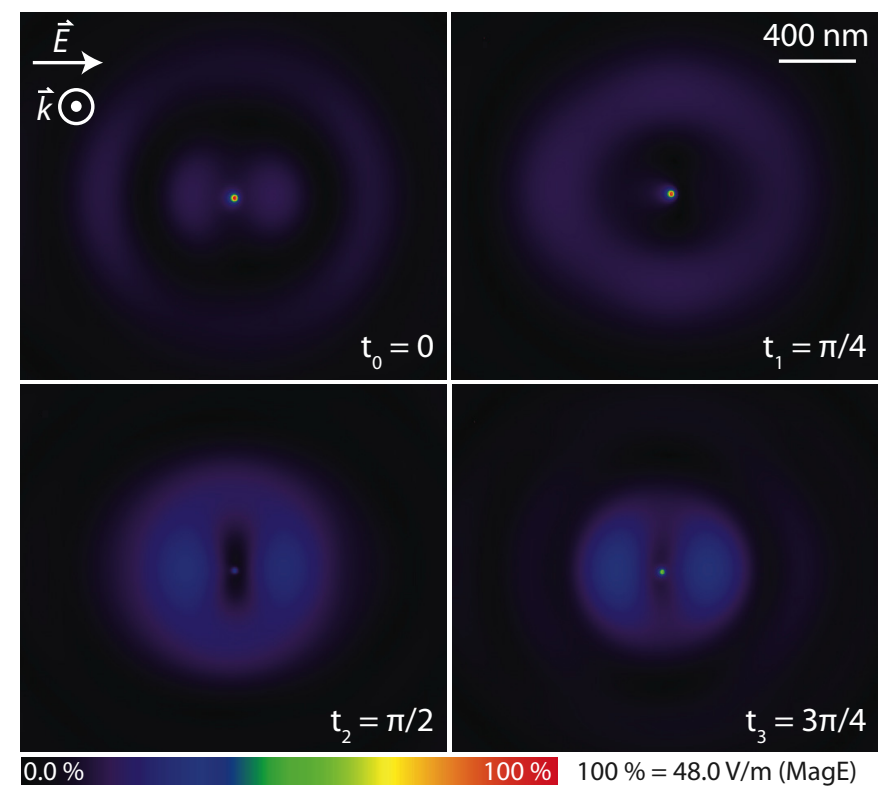

Figure 4.29 Imaging plane electric field magnitude distribution with the asymmetric Au probe and focus at protrusion cone (same model as Figure 4.28). One half of an optical cycle over four divisions. (Note: linear scale.) 


\section{CHAPTER 4 References}

Baer F. and Zhang B., "Optimizing computations in weather and climate prediction models”, Meterol. Atmos. Phys. 67, 153 (1998).

Barber D. J. and Freestone I. C., "An investigation of the origin of the colour of the Lycurgus Cup by analytical transmission electron microscopy," Archaeometry 32, 33-45 (1990).

Berger, M. J., Colella P., "Local Adaptive Mesh Refinement for Shock Hydrodynamics," J. Comp. Phys. 82, 64 (1989).

Debye P., “Zur Theorie der spezifischen Waerme,” Annalen der Physik 39, 789 (1912).

Drude P., “Zur Elektronentheorie der metalle,” Annalen der Physik 306, 566 (1900).

Drude P., "Zur Elektronentheorie der Metalle; II. Teil. Galvanomagnetische und thermomagnetische Effecte," Annalen der Physik 308 (11): 369 (1900).

Eustis S. and El-Sayed M. A., "Why Gold Nanoparticles are More Precious than Pretty Gold: Nobel Metal Surface Plasmon Resonance and Its Enhancements of Radiative and Nonradiatice Properties of Nanocystals of Different Shapes," Chem. Soc. Rev. 35, 209 (2006).

Faraday M., "The Bakerian Lecture: Experimental Relations of Gold (and Other Metals) to Light," Philos. Trans. R. Soc. London, 147, 145 (1857).

Forbes R. G., “A Generalised Theory of Standard Field Ion Appearance Energies," Surf. Sci. 61, 221 (1976).

Forbes R. G., "Charge Hopping and Charge Draining: Two Mechanisms of Field Desorption,” Surf. Sci. 102, 255 (1981).

Forbes, R. G., "Electrosprays and Liquid-Metal Ion Sources - Some Theoretical Similarites and Differences," J. Aero Sci. 30, 974 (1999).

Frens G., "Controlled nucleation for the regulation of the particle size in monodisperse gold suspensions," Nature Phys. Sci. 241, 20 (1973).

Gai H., Wang J. and Tian Q., "Modified Debye Model Parameters of Metals Applicable for Broadband Calculations," Appl. Opt. 46, 2229 (2007).

Goncharenko A. V., Chang H.-C. and Wang J.-K., "Electric Near-field Enhancing Properties of a Finite-size Metal Conical Nano-tip," Ultramicroscopy 107, 151 (2007). 
Kelly K. L., Coronado E., Zhao L. L., Schatz G. C., "The Optical Properties of Metal Nanoparticles: The Influence of Size, Shape, and Dielectric Environment," J. Phys. Chem. B 107, 668 (2003).

Krug, J. T., Sánchez, E. J., Xie, X. S., "Design of near-field optical probes with optimal field enhancement by finite difference time domain electromagnetic simulation," $J$. Chem. Phys. 11610895 (2002).

Krug J. T., Sánchez E. J. and Xie X. S., "Fluorescence Quenching in Tip-Enhanced Nonlinear Optical Microscopy,” Appl. Phys. Lett. 86, 233102 (2005).

Kunz K. S. and Luebbers R., The Finite Difference Time Domain Method for Electromagnetics, (CRC Press, New York, 1993) pp. 11-12 135-136.

Luebbers R., Hunsberger F., Kunz K., Standler R., and Schneider M., “A Frequencydependent Finite-difference Time-domain Formulation for Dispersive Materials," IEEE Trans. Electromag. Compat., 32, 222 (1990).

Luedi P. P., Dietrich F. S., Weidman J. R., Bosko J. M., Jirtle R. L., and Hartemink A. J., "Computational and experimental identification of novel human imprinted genes", Genone Res. 17, 1723 (2007).

McMahon J. M., computer code JFDTD (Northwestern University, Evanston, IL, 2010).

Mie G., "Beiträge zur Optik trüber Medien, speziell kolloidaler Metallösungen," Ann. Phys. 330, 377-445 (1908).

Nowak D. B., M.S. thesis, "Modeling and Extraction of Gilbert-Taylor cones for Apertureless Scanning Near-field Optical Microscopy Probes," Portland State University, 2004.

Novotny L., Sánchez E. J., Xie X. S., "Near-field Optical Imaging Using Metal Tips Illuminated by Higher-Order Hermite-Gaussian Beams," Ultramicroscopy 71, 21 (1998).

Rayleigh L., "On the Scattering of Light by Small Particles," Phil. Mag. 41, 275, 447 (1871).

Remcom Inc., “xFDTD Reference Manual, Version 6.3,” Product Manual, 2003.

Roundy D., computer program MEEP (Massachusetts Institute of Technology, Boston, MA, 2010).

Sau T. K., Pal A., Jana N. R., Wang Z. L. and Pal T., "Size controlled synthesis of gold nanoparticles using photochemically prepared seed particles," J. Nanoparticle Res. 3, 257 (2001). 
Taylor G. I., "Disintegration of Water Drops in an Electric Field," Proc. Royal Soc. London A 280, 383 (1964).

William G., Ewing L., Anthony S., Using MPI: Portable Parallel Programming with the Message Passing Interface, $2^{\text {nd }}$ ed. (MIT Press In Scientific And Engineering Computation Series, Cambridge, MA, USA, 1999).

Yee K., "Numerical solution of initial boundary value problems involving Maxwell's equations in isotropic media," Antennas and Propagation, IEEE Transactions 14, 302 (1966).

Zhang Z., Weber-Bargioni A., Wu S. W., Dhuey S., Cabrini S. and Schuck P. J., "Manipulating Nanoscale Light Fields with the Asymmetric Bowtie Nano-Colorsorter," Nano Lett. 9, 4505 (2009).

Zhu R.-J. Wang J. and Jin G.-F., "Mie Scattering Calcultation be FDTD Employing a Modified Debye Model for Gold Material." Optik 116, 419 (2005). 


\section{CHAPTER 5}

\section{Introduction to the Design of a TENOM-based Microscope}

In a nutshell, a tip enhanced near-field optical microscope (TENOM) is the combination of a shear force microscope (SFM) and an optical detection system that allows for the collection of photons generated in the near-field with a high collection efficiency (fluency) objective lens in the far-field. The design of a TENOM system requires careful consideration of the different required parameters. The foundation of the TENOM system requires low thermal expansion coefficients and carefully designed symmetries, as is the general case for scanning probe microscopy (SPM) [Park \& Barrett, 1993; Drake et. al., 1986; Gregory \& Rogers, 1988; Okayama et al., 1988]. Any large-scale thermal drift in the microscope will cause the microscope to lose optical alignment between the imaging probe and the laser excitation source. Ultimately, this will lead to imaging complications and artifacts from the act of manually compensating for drift during imaging. Vibrational sources have to be minimized, if not removed completely [Pohl, 1986]. Using floating optical tables and soft silicon mats helps to reduce environmental vibrations. Enclosing the microscope with acoustic dampening material removes acoustic noise and the opacity aids in the removal of background photon noise.

One of the major complications in TENOM imaging is the positioning of the probe in a focused laser spot that has a cross sectional diameter of $\sim 300 \mathrm{~nm}(1.4 \mathrm{NA}$ objective lens with $800 \mathrm{~nm}$ excitation light). This requires both manual coarse alignment capabilities combined with piezo positioners for fine alignment. Fine movement of the 
optical focus of the objective lens must also be included in the design, for this effectively moves the focus in the z-axis. The optical pathways can vary based on the type of sample. The focus of the TENOM design presented here will use transmissive samples. Transmissive samples allow for direct optical imaging from below the sample, making it an ideal candidate for an inverted optical microscope. Non-transmissive samples would require a more exotic optical system or the use of fiber-based aperture near-field techniques.

\subsection{Technical Motivation for Developing a TENOM System}

At the time of this publication, there are no commercial TENOM systems that can meet the needs and versatility in design of what is presented here. The main reason that there are no turnkey commercial systems available to reliably acquire TENOM images is the difficulty of probe design and manufacture. Previous systems have been custom, oneof-a-kind composites of many different pieces of hardware that were synchronized through multiple computer systems [Sánchez, 1999; Sánchez, Novotny, \& Xie, 1999; Hartschuh et al., 2003; Nowak et al., 2005]. The atomic force microscope (AFM/SFM) ${ }^{*}$ scan controller, monochromator, avalanche photodiodes (APDs), CCDs, laser control systems, and inverted microscope designs were all commercial units that would require modifications, in order to be integrated into a functional TENOM system. The difficulty incurred in such a design is the lack of cooperation from the commercial manufacturers to support such highly customized systems. This problem is compounded by continuous

\footnotetext{
*An atomic force controller is usually modified internally to support a shear force design suitable for TENOM.
} 
product upgrades and a lack of support for older systems. This becomes the primary motivation to develop a complete TENOM system from the ground up.

A secondary motivation is the use of the TENOM system subsections as functional stand-alone microscopes and tools. With the increasing popularity of SFMs in research environments as tools for the development of new imaging techniques where silicon cantilevers cannot be used, the TENOM system could function purely as an SPM. In a laboratory environment where far-field imaging is of interest, the TENOM system could be used purely as a far-field optical microscope. The motivation in this case is to develop the system to have a low cost burden, allowing an academic lab to build several TENOM systems for the typical cost of a single commercial unit. The TENOM system could also function as a learning platform for the understanding of scanning probe microscopy (SPM) and optical microscopy.

\subsection{Imaging Probe}

The spectroscopic response mechanism for TENOM imaging is completely dependent on the use of a conductive probe (typically metal). For biological imaging, Au probes are used to minimize chemical reactions with the biological specimen. Although $\mathrm{Ag}$ results in a much stronger enhancement, it is quite reactive. The metal probes are electro-chemically etched and then tailored to specific geometries (discussed in Chapter 4) with the use of a focused ion beam (FIB). A FIB system allows for the nanofabrication of materials through milling and chemical deposition at the nanometer scale $^{\dagger}$. Newer FIB systems include electron and ion columns for more precise work

\footnotetext{
$\dagger$ An outline of the use of the FIB to manufacture a Au probe is outlined in Chapter 11.
} 
[Sánchez, Krug, \& Xie, 2002]. The measure of the quality of tip design is based directly on the field enhancement obtainable. The greater the field enhancement of the tip, the more the ratio of near-field to far-field background contribution will increase. Ultimately, the elimination of the far-field signal by a decrease of laser illumination power will provide the best signal-to-noise ratio in the TENOM images. Further improvement in the signal-to-noise ratio can be made by moving the far-field focus out of the sample-imaging plane and onto locations on the tip suitable for surface plasmon generation. This plasmon is then directed by the geometry of the probe to a location outside of the far-field focal region. This method generates a smaller delocalized focal excitation region that overcomes the background signal. Sánchez originally proposed this method in 2002, which is one of the proposed mechanisms for the imaging contrasts seen in Chapter 12 and finite difference time domain (FDTD) modeled in Chapter 4 [Sánchez et al., 2002]. Recent near-field methods have looked at various methods of background suppression [Neacsu et al., 2010; Höppener, Beams, \& Novotny, 2009]. The geometry of the imaging probe will affect the recorded topographic features. The use of sharp (high aspect ratio) probes will provide realistic topography, while the use of blunt (or low aspect ratio) probes will produce topographic artifacts in the imaging. Figure 5.1 demonstrates this concept. Optical imaging can also exhibit artifacts based on the design of the tip. Figure 5.2 shows two effects that can affect optical imaging: movement of the probe in and out of the focus and optical quenching. The effect of the tip moving in and out the focus is exaggerated when the sample has high surface topography or low field enhancement. Optical quenching is an effect where the photon released from a fluorophore is reabsorbed by the imaging probe. Using FDTD modeling Krug and 
Sánchez determine the minimum distance the near-field imaging probe should be from a fluorophore to minimize quenching. They determined that distance to be $9 \mathrm{~nm}$.
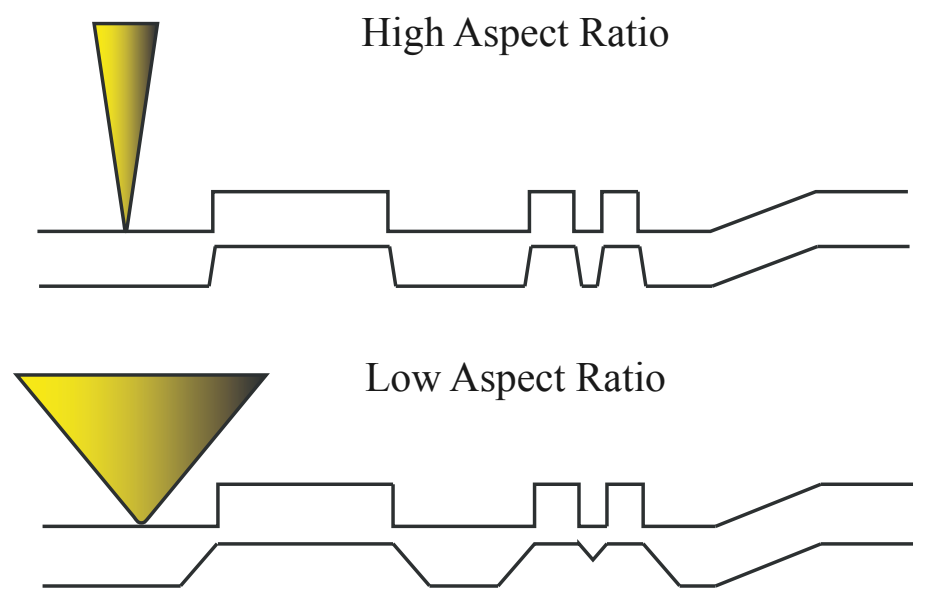

Figure 5.1 Effect of imaging probe aspect ratio on topographical imaging. (a) Represents the actual sample surface, (b) and (c) are the recorded sample surface for the high aspect ratio probe and low aspect ratio probe, respectively.

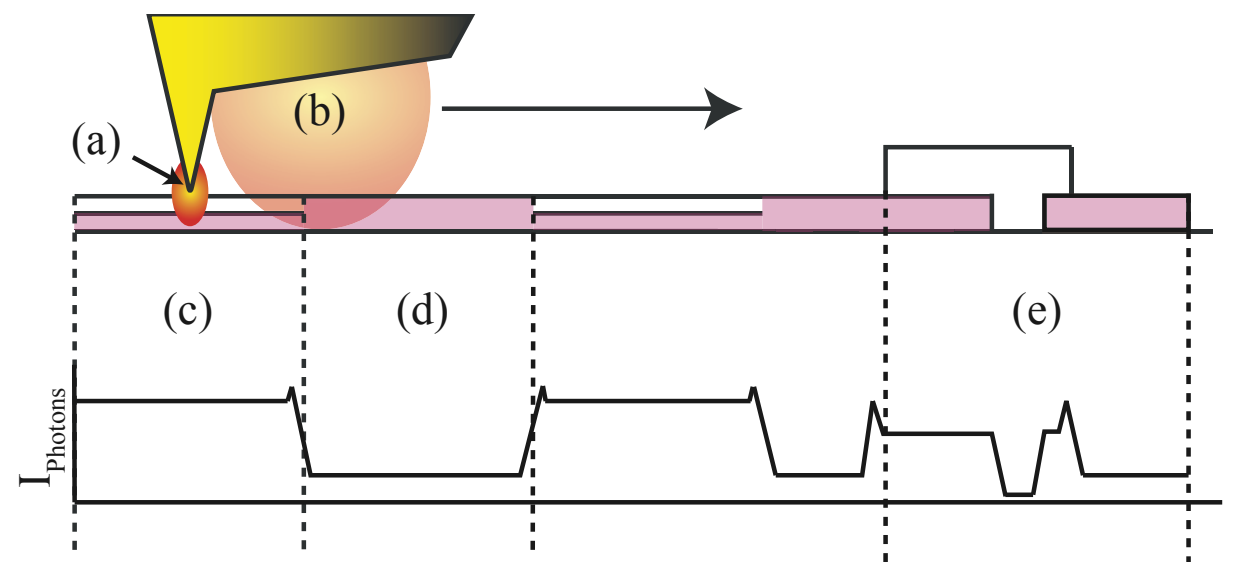

Figure 5.2 Effect of quenching and topographical features on image resolution. I $_{\text {Photons }}$ is the photon emission intensity from the sample surface (colored regions). (a) Area of localized field enhancement. (b) Far-field diffraction limited focal area. (c) Region with a spacing layer (polymer or lipid bi-layer) between the imaging probe and fluorophores (pink). (d) Region without spacing layer, which results in quenching. (e) Complex topography with fluorophores. It is important to note the peaks on, before and after the high topography surface. 
These optical effects can create very high-resolution features on the sample. The small peaks seen in section (e) of Figure 5.2 can give very high-resolution information but are a generated effect of quenching, topography, and tip geometry.

\subsection{Nano-Scale Movements: Piezo Electric Crystals}

Successful TENOM imaging requires the precise movement of the imaging probe with nanometer resolution. Crystals that exhibit piezoelectric properties can be used for this purpose, originally proposed by Synge [Synge, 1932]. Piezoelectricity is the ability to develop a net electrical potential throughout a material by the generation of an internal electric field. This electric field is developed by the deformation of a material that exhibits piezoelectric effects. In 1880, Pierre Curie and Jacques Curie observed the piezoelectric effect [Curie \& Curie, 1880]. In 1881, Gabrial Lippmann determined the mathematical framework for the piezoelectric effect, and would derive the foundation for the piezoelectric effect from thermodynamic principles [Lippmann, 1881]. The piezoelectric nature rises from an asymmetry in the crystal lattice structures within a material. Figure 5.3 shows a description of the asymmetric shape of the lattice structure and the electric field generated by this distortion. Each of the lattice structures will form an electric dipole with varying electric field strength based on the length of asymmetry and material composition. These dipoles are typically randomized throughout a material where the net electric field will be the superposition of all the dipole states [Birkholz, 1995]. In order to create a higher number of arranged dipole states, the material temperature can be raised above the Curie temperature in the presence of a high electric

field. Upon cooling of the material, a large number of the dipoles will maintain this alignment in a process called poling. 
(a)

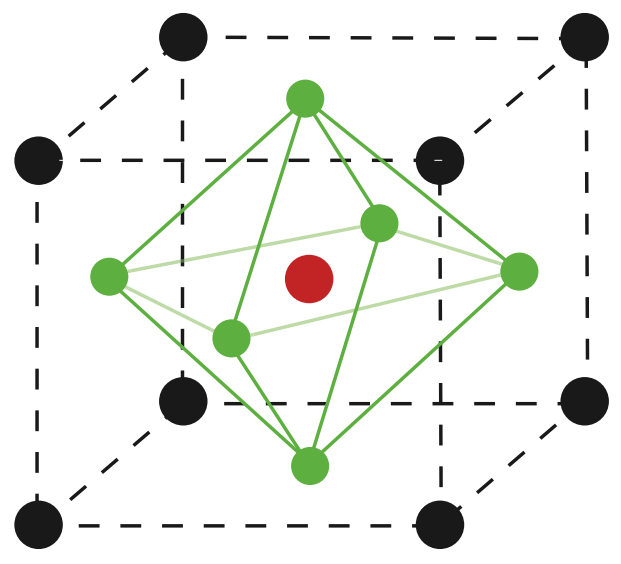

(b)

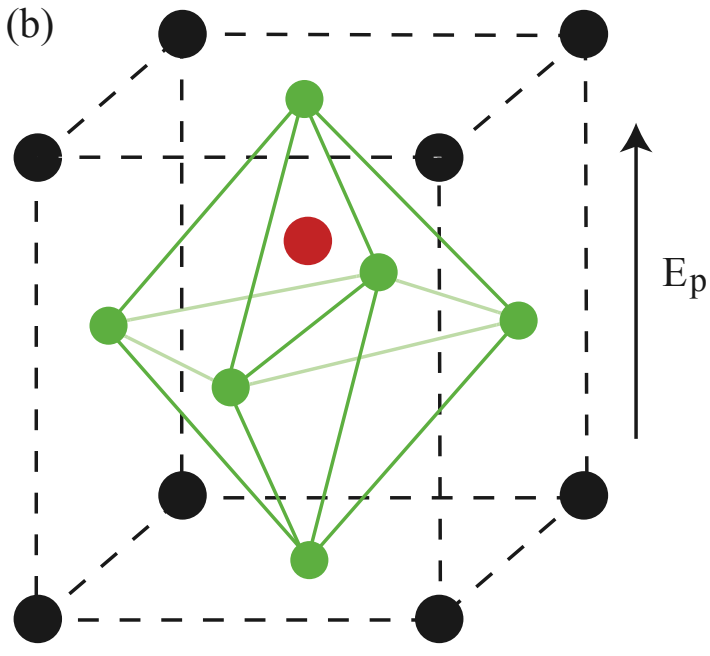

Figure 5.3 Lattice structure of a piezoelectric crystal, where black is $\mathrm{O}^{2-}$, green is $\mathrm{Pb}$ and red is $\mathrm{Ti}$ or $\mathrm{Zr}$. (a) is an unpoled lattice cube with no net electric field. (b) is the similar lattice cube with a poled dipole moment.

While piezo crystals have the ability to generate a net voltage when compressed, the converse is also true. If a voltage is applied to the piezo crystal, the crystal itself will experience an expansion or contraction based on the direction of the voltage. The crystal can be poled to expand in the radial or longitudinal directions. The net change in the piezo crystal as a function of voltage is defined by the following,

$$
\Delta x=d_{i k} V_{\text {applied }}
$$

where $d_{\mathrm{i}, \mathrm{k}}$ is a piezoelectric coefficient of the material $(\mathrm{m} / \mathrm{V})$ with $i$ and $k$ indexes denoting the poling directions, parallel or orthogonal. Popular piezo shapes for SPM are cylindrical tubes. Typically, one of the tubes has solid electrical coating on the inside and outside of the tube. The displacement of solid tube is modeled by the following equation;

$$
\Delta L=\frac{2 d_{31} V L}{(O . D .-I . D .)},
$$


where O.D. and I.D. are outer and inner diameters of the tube with voltage $\mathrm{V}$ and the total length of the tube $L, d_{31}$ represents movement in the longitudinal direction of the tube. The second type of tube is a segmented tube that has a solid inner tube (usually connected to a ground reference) and four symmetric outer layers that make up $+\mathrm{X},-\mathrm{X},+\mathrm{Y}$, and $-\mathrm{Y}$ directions. The displacement of the segmented tube is modeled by the following equation,

$$
\Delta L=\frac{\sqrt{2} d_{31} V L^{2}}{2 \pi r(\text { O.D. }- \text { I.D. })},
$$

where $r$ is the radius to the outside of the tube. Of course, these are theoretical equations of motion for the piezo displacement. The piezo will still have to be calibrated when used in an SPM, using a calibrated reference sample.

Piezoelectric crystals are non-linear materials that suffer from hysteresis and creep. Hysteresis in piezoelectric crystals is an artifact of molecular friction and polarization effects. The difficulty that is encountered due to hysteresis is the inability to return to the same displacement, based on voltage. For example, assume the piezo is positioned at some location $x_{o}$ at zero volts, the voltage is then increased to 100 volts and the piezo moves to $x_{f}$. If the voltage is returned to zero, the new piezo position becomes $x_{o}+x_{\text {error }}$. Figure 5.4 gives a hysteresis curve for a piezo demonstrating this effect. Creep is a similar issue that affects the ability to maintain a fixed location. Suppose a fixed voltage was applied to the piezo, causing it to move to a new displacement. The piezo would slowly creep away from this location with its rate decreasing logarithmically over time. There is a way to minimize, if not completely, remove hysteresis and creep by 
creating a closed loop feedback system that constantly monitors the location of the piezo and provides a correction to the voltage to remove the $x_{\text {error }}$ from the piezo.

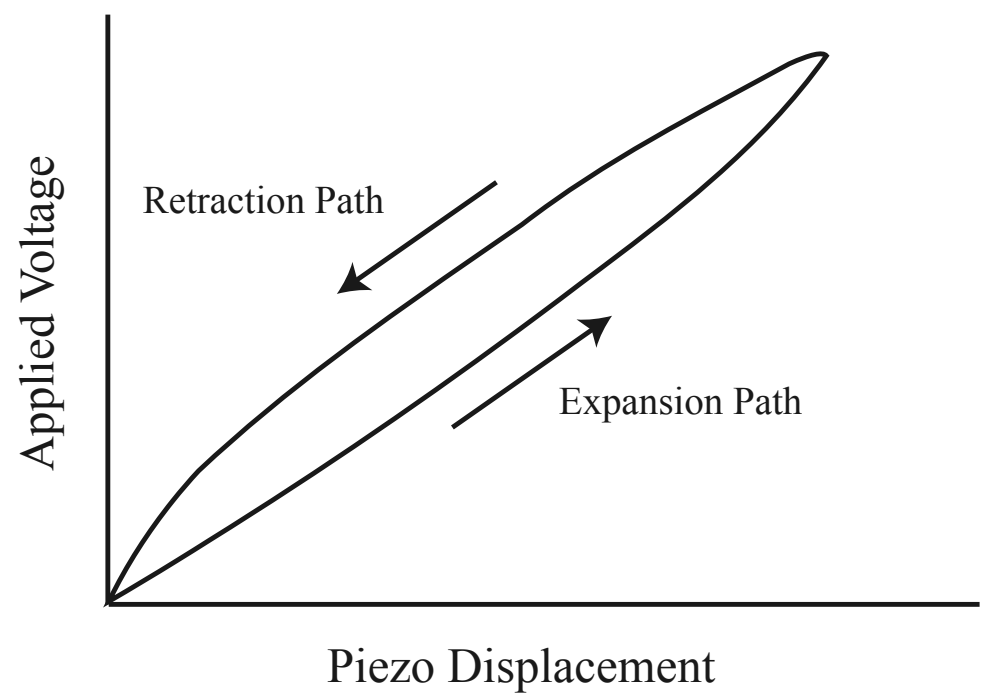

Figure 5.4 Hysteresis curve for open loop piezo positioners. The expansion and retraction of the piezo do not follow the same paths.

This can be accomplished with capacitive or resistance (strain gauge) feedback electronics. Chapter 10.3 shows the topographic differences between open loop and closed loop scanning.

\subsection{The Quartz Tuning Fork}

Quartz has natural piezoelectric characteristics. Walter Guyton Cady was the first to develop a quartz crystal oscillator, in 1921 [Marrison, 1948]. Dependent on the size and shape of the quartz crystal cuts, the resonant frequency of the crystal can be determined [Kahan, 1985]. The high resolution of the crystal oscillator has made it a useful device in the timing of watches and microcontrollers. Crystal oscillators are also used to maintain fixed frequencies for radio (RF) transmissions. 


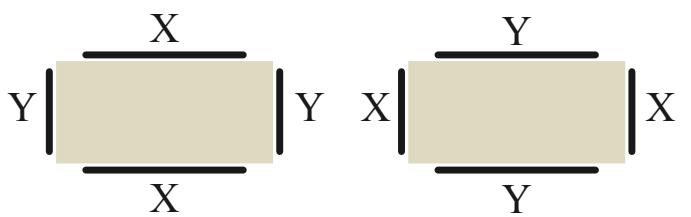

Figure 5.5 Electrode configuration for a tuning fork oscillator. In the case of an electrically driven oscillator, one of the electrodes (X) is oscillated and the piezo-induced electrical charge in (Y) is detected. In a mechanically driven oscillator, one electrode $(\mathrm{X})$ is grounded and piezoinduced electrical charge in (Y) is detected.

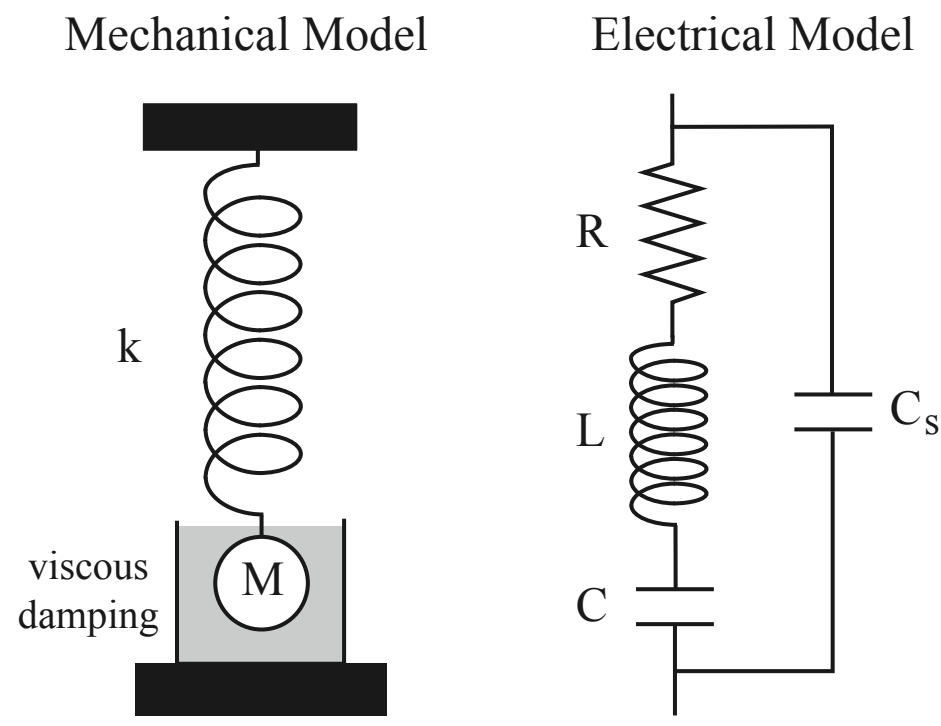

Figure 5.6 The mechanical and electrical models of a quartz tuning fork oscillator. $\mathrm{C}_{\mathrm{s}}$ is the parasitic capacitance of the tuning fork.

Karrai and Grober were the first to use a quartz tuning fork method for scanning probe microscope (SPM), in particular, for near-field scanning optical microscopy (as discussed in Chapter 3) [Karrai \& Grober, 1995]. The sensitivity of oscillation of the crystal oscillator would allow the crystal to be used to detect shear force (friction forces) near a sample with pico-Newton resolution [Karrai \& Grober, 1995]. Figure 5.5 shows the electrode arrangement for the tuning fork oscillator. 
The tuning fork can be modeled simply as a driven harmonic oscillator and electrically by the Butterworth-Van-Dyke equivalent circuit, shown in Figure 5.6. Starting with the equation of motion for a simple harmonic oscillator,

$$
m \ddot{x}(t)+k x(t)=0,
$$

where $m$ is the mass of the system and $k$ is the spring constant. Building upon this equation and factoring in the driving force for the oscillator and a damping force acting on the oscillator gives a more accurate equation for the oscillation of a tuning fork as a driven oscillator with damping,

$$
m \ddot{x}(t)+b \dot{x}+k x(t)=F_{0} \cos (\omega t),
$$

where $b$ is the viscous damping factor and $F_{o} \sin (\omega t)$ is the sinusoidal driving force for the tuning fork. The tuning fork can also be modeled by a similar $2^{\text {nd }}$ order differential equation. The electrical equivalent shown in Figure 5.6 is the Butterworth-Van-Dyke equation, given by,

$$
L \ddot{I}(t)+R \ddot{I}(t)+\frac{1}{C} I(t)=U \cos (\omega t)
$$

where $L$ is inductance, $R$ is resistance, $C$ is capacitance, and $U$ the potential added to the oscillator. Equation 5.6 can also be obtained using Kirchhoff's law of conservation of energy. The steady state amplitude solution for Equation 5.5 is,

$$
X(t)=\frac{F_{O}}{\left[b^{2} \omega^{2}+m^{2}\left(\omega_{O}^{2}-\omega^{2}\right)^{2}\right]^{\frac{1}{2}}} \cos (\omega t),
$$

where the phase is given as,

$$
\tan \varphi=\frac{b \omega}{m\left(\omega_{O}^{2}-\omega^{2}\right)} .
$$


The resonance condition for free oscillation frequency including damping is

$$
\omega_{O}^{2}=\frac{k}{m}
$$

The quality factor of the tuning fork oscillator can be defined by the constants in the system, both mechanical and electrical.

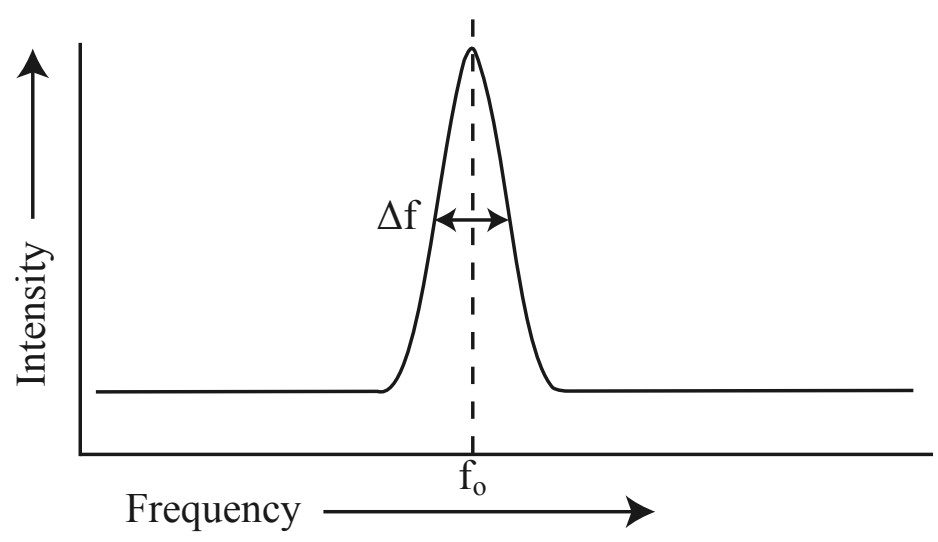

Figure 5.7 Measure of quality factor "Q" based on the amplitude and frequency response of the tuning fork. $\Delta \mathrm{f}$ is measured at the FWHM of the waveform.

A qualitative measure of the full-width at half-maximum (FWHM) of the oscillator as seen at resonance can also be used to calculate the quality factor. Figure 5.7 demonstrates the qualitative measure of the FWHM of a resonator. The relationships between these parameters are given by,

$$
Q=\frac{f_{o}}{\Delta f}=\frac{\sqrt{m k}}{b}=\frac{1}{R} \sqrt{\frac{L}{C}} .
$$

Combining the quality factor into the steady state solution for Equation 5.5 will allow the effect of quality factor on the response time of the oscillator to be examined. Equations 5.11 and 5.12 include $Q$ by substituting for $b$, including the resonance condition for the free oscillation damping. The amplitude is given by, 


$$
x_{O}=\frac{F_{O} / m}{\left[\left(\frac{\omega_{O} \omega}{Q}\right)^{2}+\left(\omega_{O}^{2}-\omega^{2}\right)^{2}\right]^{\frac{1}{2}}},
$$

with the phase defined as,

$$
\varphi_{O}=\tan ^{-1}\left(\frac{\omega_{O} \omega}{Q\left(\omega_{O}^{2}-\omega^{2}\right)}\right)
$$

If the amplitude $x_{\mathrm{o}}$ is changed by a perturbation, or in the case of an SPM, the distance between the tip and the changes due to topographical changes, a change in frequency will result [Albrecht, 1991]. The amplitude and phase become terms in the general solution,

$$
X(t)=x_{O} \cos \left(\omega t+\varphi_{O}\right) .
$$

The introduction of an instantaneous frequency change gives a solution with a steady state and transient term:

$$
X(t)=x_{O}^{\prime} \cos \left(\omega t+\varphi_{O}^{\prime}\right)+x_{t} e^{\left(-\omega_{o}^{\prime} t / 2 Q\right)} \cos \left(\omega_{t} t+\varphi_{t}\right) .
$$

The time constant for the transient term gives the settling time for the amplitude, and ultimately, the response time of the quartz oscillator. The time constant in terms of quality factor and frequency is given by,

$$
\tau=\frac{2 Q}{\omega_{O}}
$$

A typical tuning fork for the TENOM system presented here has a quality factor of $Q>4000$ with a system resonating at $2^{15} \mathrm{~Hz}$, and the response time for this tuning fork configuration would be $245 \mathrm{~ms}$. This makes the use of amplitude measurements for a feedback system undesirable. Instead, the phase measurement of the tuning fork should be used, which will be almost instantaneous in response. 

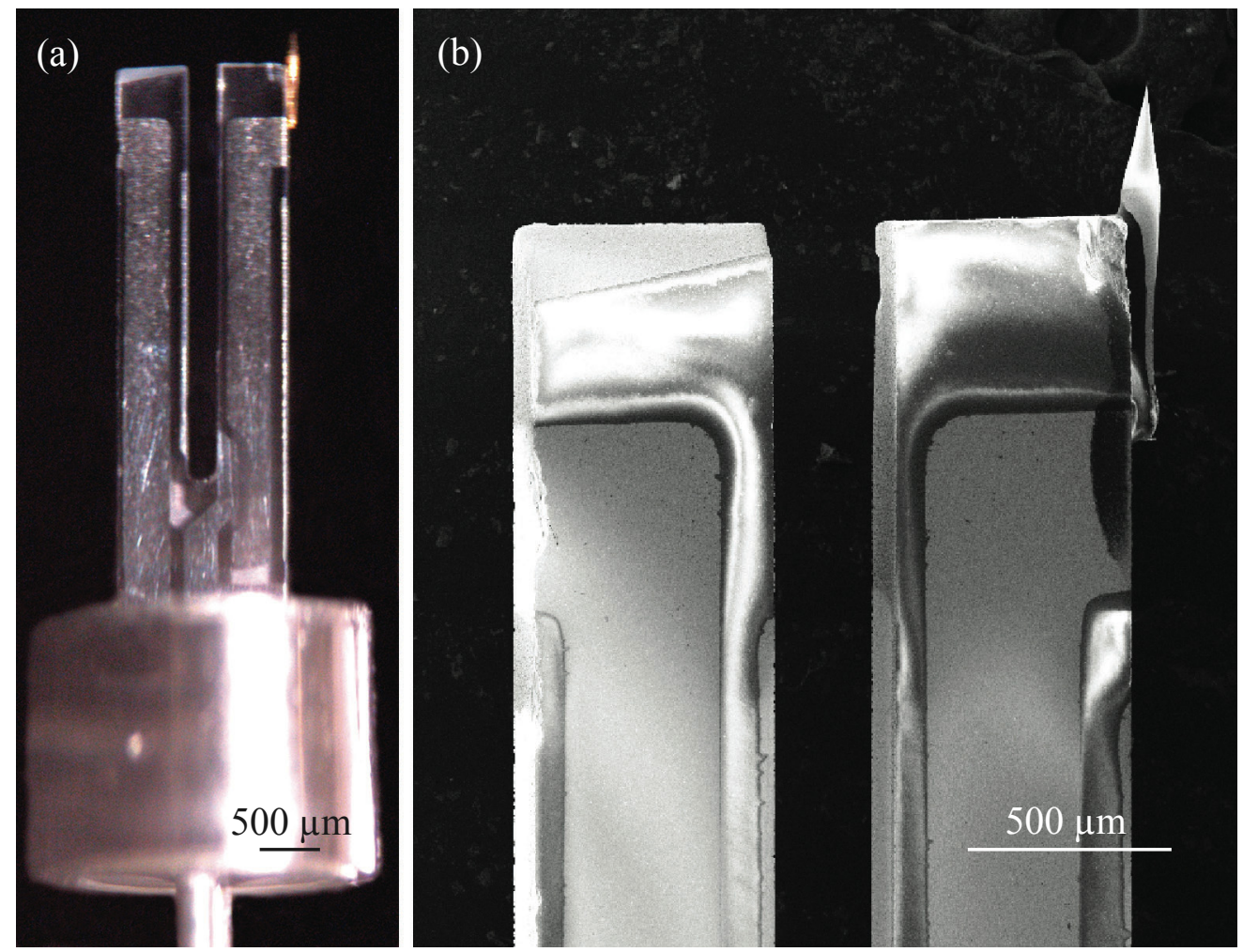

Figure 5.8 (a) Optical image of the quartz tuning fork exposed by the partial removal of the protective canister. (b) An electron micrograph of the same tuning fork. In both, an Au probe is mounted to the very end of the tuning fork. The size of the probe helps maintain high $Q$ factors ( 6000).

This, of course, is idealistic. A phase measurement would need a reference of at least two periodic cycles $\left(62 \mu \mathrm{s}\right.$ for $2^{15} \mathrm{~Hz}$ ), which is then multiplied and low pass filtered (see phase detection circuit design in Chapter 7). The low pass filter for the TENOM system is set at $1.7 \mathrm{kHz}$, which would place the response time at roughly a half of a millisecond.

\subsection{Shear Force Microscopy}

Binning and Rohrer started a revolution with their invention of the scanning tunneling microscope [Binning, 1982]. The piezo-based technology blossomed into the atomic force microscope that placed fewer constraints on the sample [Binning, Quate, \& 
Gerber, 1986]. AFMs detect the atomic interaction of the imaging probe and the sample surface. Two common imaging feedback modes for non-contact AFMs are lateral interaction, in which the tip deflection is parallel to sample surface, and tapping mode, in which the deflection is orthogonal. The lateral interaction feedback mechanism is commonly referred to as shear force microscopy (SFM). SFMs have become popular in the scientific community due to their high degree of customization. SFMs can be used as near-field microscopes [Pohl, Denk, \& Lanz, 1984; Lewis et al., 1984; Betzig et al., 1991], in situ liquid imaging systems [Rensen, Van Hulst, \& Kämmer, 2002], and ultrasonic imaging [La Rosa et al., 2005], just to name a few. Grober, Karrai and colleagues invented the ability to remove the laser feedback system, replacing it with a tuning fork shear force feedback, as previously mentioned [Karrai \& Grober, 1995].

Due to the interaction volume of the highly localized field enhancement, the probe must be within a few nanometers of the surface. To maintain this tip-sample interaction distance, an SFM is used, shown in Figure 5.9. SFMs have the ability to hold a probe just a few nanometers above the surface of the sample. High-speed electronics monitor the amplitude and phase of a quartz tuning fork, while a Proportional-IntegralDifferential (PID) feedback loop controls the Z-piezo. The role of the feedback loop is to maintain a constant height of the imaging probe above the sample topography while scanning. Using the imaging probes that are mounted to quartz tuning forks, angstrom level resolutions are possible [Grober et al., 2000]. Figure 5.8 demonstrates the mounting arrangement of the probe. The sample is moved under the probe so that laser illumination and probe alignment can be maintained during the raster scanning of the sample, shown in Figure 5.10. 


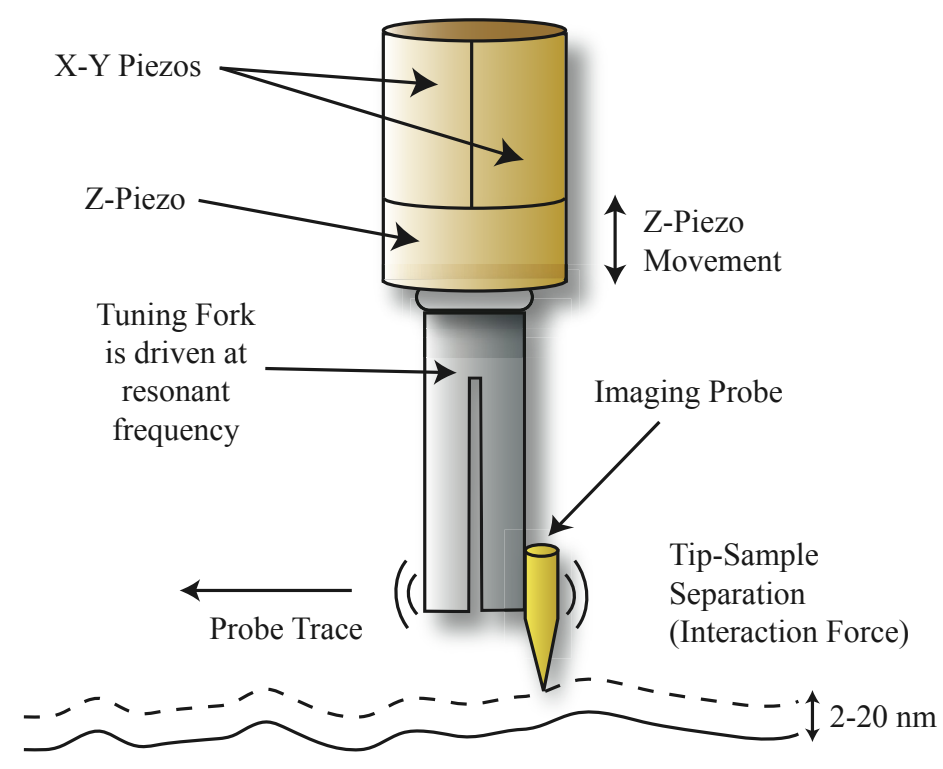

Figure 5.9 Conceptual drawing of the tuning fork SFM. The tuning fork is held by a tube-piezo stack comprised of a Z-axis piezo and a separate X$\mathrm{Y}$ axis segmented piezo.

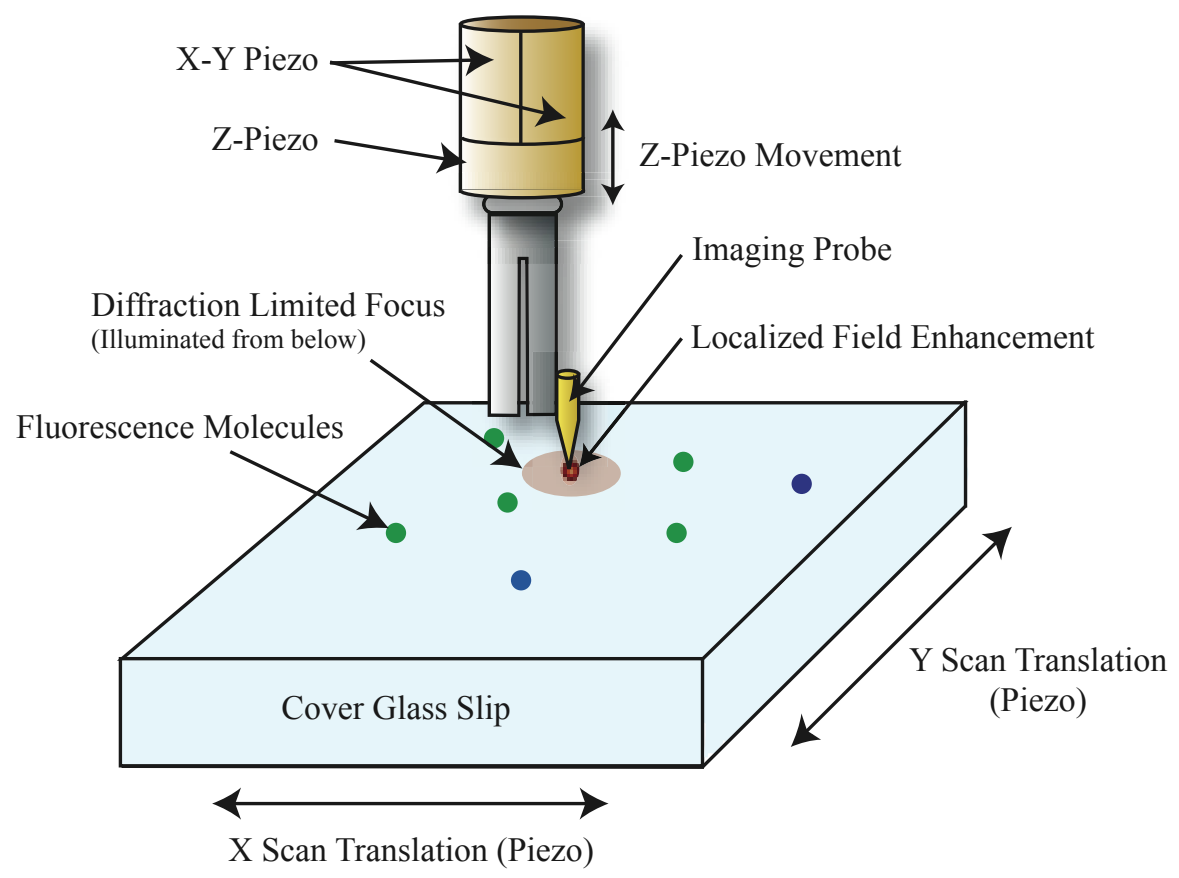

Figure 5.10 Conceptual drawing of a tuning fork SFM used in a TENOM system. The sample is raster scanned to compose a spatial near-field optical image. 


\subsection{Optical Requirements}

An inverted optical microscope is used as a base for the TENOM system. The inverted microscope provides the optical pathways needed for excitation and collection of nearfield and far-field optical responses.

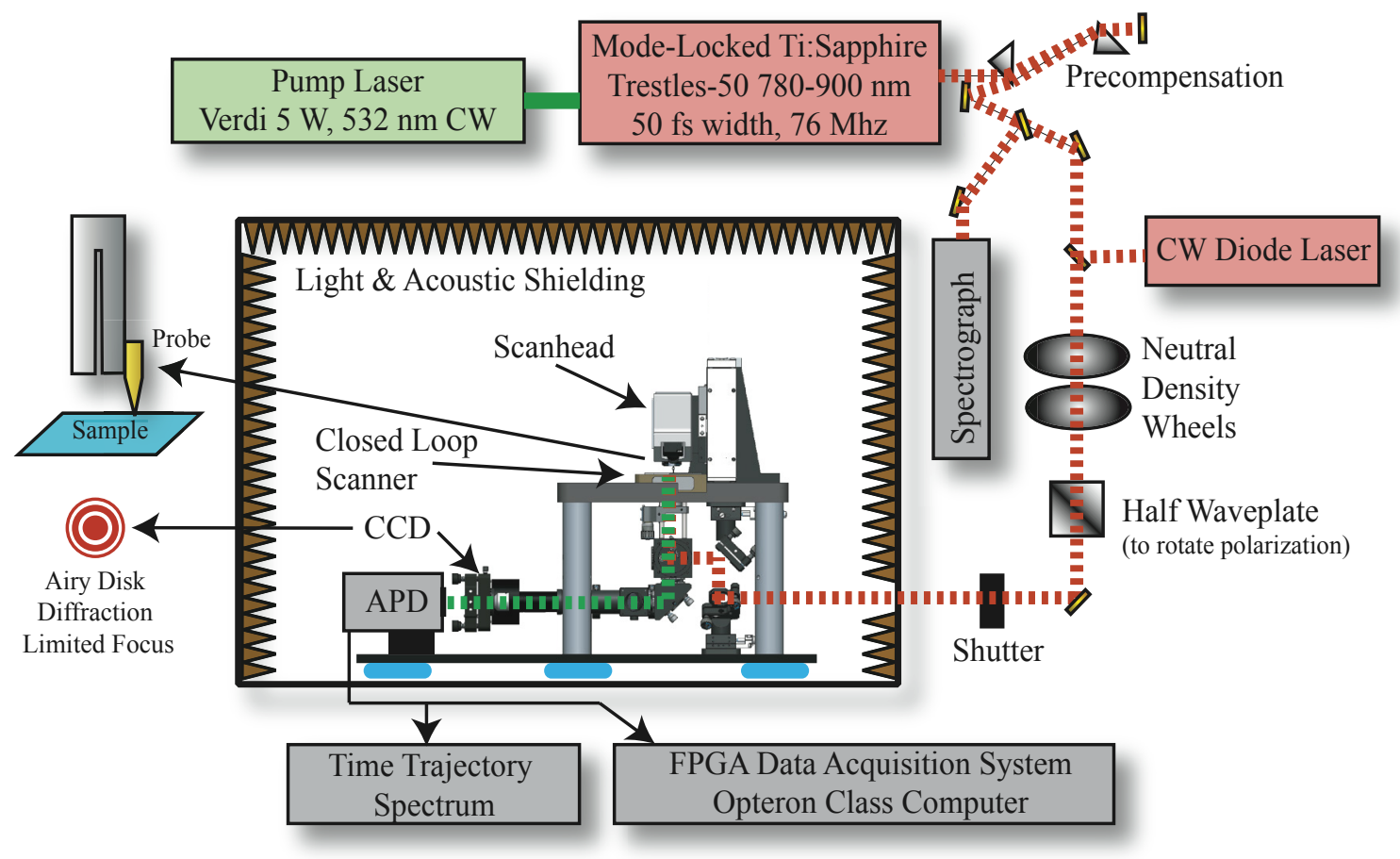

Figure 5.11 Optical arrangement for the TENOM experimental apparatus. (Note: the stepper motor is removed for clarity.)

The optical microscope is modified to support the piezo scan bed and SFM scanner [Sánchez, 1999; Sánchez, Novotny, \& Xie, 1999; Hartschuh et al., 2003; Nowak et al., 2005]. Two-photon excitation (TPE) is created with either a mode-locked Ti:sapphire laser or a CW light source in the near IR. Excitation and emission light are collected through a high numerical aperture objective lens under the scan bed, which leads to a single photon avalanche photodiode (SPAPD). Emission filters ( $>15$ OD blocking of excitation source) are used in combination with dichroic filters to remove the excitation 
light from the collection pathway. The sample is moved with a closed loop piezo scan bed, which allows for a scan area of $100 \times 100 \mu \mathrm{m}^{2}$ and a reproducible spatial resolution of $0.3 \mathrm{~nm}$. Figure 5.11 shows the overall layout required for TENOM imaging.

\subsection{Improvements Over Previous Designs}

Major motivations exist to centralize the electronic systems into one computer system and user interface, and to simplify the optical microscope pathways. Development of a TENOM system with this motivation in mind will make the operation of the system generally easy to use and intuitive. These features will set this system apart from previous system designs, starting with the use of an infinity-corrected objective lens. These lenses provide better efficiency of the emission optical filters and simplify the lens requirements for the focusing of light from the object for $\mathrm{CCD}$ images or collection by the fiber optic. Using a fiber optic coupler that collects the collimated light from the objective lens allows for direct coupling onto APD, PMT or monochromators. A major benefit of a fiber optic arrangement is the elimination of the need to realign optical pathways when changing from an APD, PMT or monochromator detector. Yet another advantage would be the implementation of a method to monitor the sample in real time from multiple views, while using an APD or other detector, by redirecting a very small percentage of the collected image from the objective lens to CCDs. Finally, the use of latest generation two-photon filters allows for the passing of nearly all of the collected fluorescent photons, while blocking the excitation wavelength at 8 O.D (optical densities), a further improvement over previous filter efficiencies. 


\subsection{Flexibility in Design: Modifying for Other Applications}

TENOM imaging is a specific technique that is based on basic function blocks. To make the most impact and allow the greatest flexibility within the design, each of the function blocks must have the ability to act independently from one another. This versatility would allow for the easy customization of the TENOM system. The TENOM system built, as is, can be used purely as an inverted microscope or purely as an SFM. Modifications could be made to extend the reach of the SFM into other SPM types, such as STM, magnetic field, or electric field imaging. Individual circuit boards could be used for various other experiments. The phase detection board (discussed in Chapter 7) has the flexibility to be used as a generic frequency / phase comparator. This is also true of the piezo driving amplifiers, which could be used to drive various piezos for other applications. 


\section{CHAPTER 5 References}

Albrecht T. R., Grutter P., Horne D., and Rugar D., "Frequency modulation detection using high-q cantilevers for enhanced force microscope sensitivity," J. Appl. Phys 69, 668 (1991).

Betzig E., Trautman J. K., Harris T. D., Weiner J. S., and Kostelar R. L., "Breaking the diffraction barrier: optical microscopy on a nanometric scale," Science 251, 1468-1470, (1991).

Birkholz M., "Crystal-field induced dipoles in heteropolar crystals - II. physical significance,” Z. Phys. B 96, 333 (1995).

Binnig G., Quate C. F., and Gerber Ch., “Atomic Force Microscope,” Phys. Rev. Lett. 56, 930 (1986).

Binnig G., Rohrer H., Gerber Ch., and Weibel E., "Surface Studies by Scanning Tunneling Microscopy,” Phys. Rev. Lett. 49, 57 (1982).

Curie J. and Curie P., "Contractions et dilatations produites par des tensions dans les cristaux hémièdres à faces inclines," C R Acad Sci Gen. 93, 1137 (1880).

Curie J. and Curie P., "Développement, par pression, de l'électricité polaire dans les cristaux hémièdres à faces inclines," Comptes Rendus de l'Académie des Sciences 91, 294 (1880).

Curie J. and Curie P., "Sur l'électricité polaire dans les cristaux hémièdres à faces inclines," C R Acad Sci Gen. 91, 383 (1880).

Drake B., Sonnenfeld R., Schneir J., Hansma P. K., Slough G., and Coleman R. V., "A tunneling microscope for operation in air or fluids," Rev. Sci. Inst. 57, 441 (1986).

Gregory S. and Rogers C. T., "High-Speed Scanning Tunneling Microscopes,” J. Vac. Sci. Technol. A 6, 390 (1988).

Grober R. D., Acimovic J., Schuck J., Hessman D., Kindlemann P. J., Hespanha J., Morse A. S., Karrai K., Tiemann I., and Manus S., "Fundamental Limits to Force Detection Using Quartz Tuning Forks,” Rev. Sci. Inst. 71, 2776 (2000).

Hartschuh A., Sánchez E. J., Xie X. S., and Novotny L., "Near-field Raman Spectroscopy of Single-Walled Carbon Nanotubes," Phys. Rev. Lett. 90, 9 (2003).

Höppener C., Beams R., and Novotny L., "Background Suppression in Near-Field Optical Imaging," Nano Lett. 9, 903 (2009). 
Kahan A., "Cut Angles for Quartz Crystal Resonators," U.S. Patent No. 4499395 (12 February, 1985).

Karrai, K., and Grober, R. D., "Piezoelectric Tip-Sample Distance Control for Near Field Optical Microscopes,” Appl. Phys. Lett. 66, 14 (1995).

Karrai, K., and Grober, R. D., "Piezo-electric Tuning Fork Tip-Sample Distance Control for Near Field Optical Microscopes," Ultramicroscopy 61, 197 (1995).

Krug J. T., Sánchez E. J. and Xie X. S., "Fluorescence Quenching in Tip-Enhanced Nonlinear Optical Microscopy,” Appl. Phys. Lett. 86, 233102 (2005).

La Rosa A., Cui X., McCollum J., Li N., and Nordstrom R., "The ultrasonic/shear-force microscope: Integrating ultrasonic sensing into a near-field scanning optical microscope," Rev. Sci. Inst. 76, 093707 (2005).

Lewis A., Isaacson M., Harootunian A. and Murray A., "Development of a $500 \AA$ spatial resolution light microscope. I. Light is efficiently transmitted through $\lambda / 16$ diameter apertures," Ultramicroscopy 13, 227 (1984).

Lippman, G., "Principe de la conservation de l'électricité" (in French). Annales de chimie et de physique 24, 145 (1881).

Marrison W. A., "The Evolution of the Quartz Crystal Clock," Bell System Technical Journal (AT\&T) 27, 510 (1948).

Neacsu C. C., Berweger S., Olmon R. L., Saraf L. V., Ropers C., and Raschke M. B., "Near-Field Localization in Plasmonic Superfocusing: A Nanoemitter on a Tip," Nano Lett, 10, 592 (2010).

Nowak D. B., Krug J. T., Xie X. S., and Sánchez E. J., "Pushing the Limits of Near-field Microscopy," World Multi-conference on Systemics, Cybernetics and Informatics, WMSCII Proceedings, Orlando, Florida, 2005.

Okayama S., Komuro M., Mizutani W., Tokumoto H., Okano M., Shimizu K., Kobayashi Y., Matsumoto F., Wakiyama S., Shigeno M., Sakai F., Fujiwara S., Kitamura O., Ono M., and Kajimura K., "Observation of microfabricated patterns by scanning tunneling microscopy,” J. Vac. Sci. Technol. A 6, 440 (1988).

Park S. and Barrett R. C., "Design Considerations for an STM System," in Methods of Experimental Physics, edited by Stroscio J. A. and Kaiser W. J. (Academic Press, New York, 1993) Vol. 27, pp. 30-76.

Pohl D. W., "Some Design Criteria in Scanning Tunneling Microscopy", IBM J. Res. Develop. 30, 417-427 (1986). 
Pohl D. W., Denk W., and Lanz M., "Optical stethoscopy: Image recording with resolution $\lambda / 20$," Appl. Phys. Lett. 44, 651 (1984).

Rensen W. H. J., Van Hulst N. F., and Kämmer S. B., "Imaging Soft Samples in Liquid with Tuning Fork Based Shear Force Microscopy," Appl. Phys. Lett. 77, 1557 (2002).

Sánchez, E. J., Ph.D. dissertation, "A Novel Scheme for High Resolution Near-Field Fluorescence Microscopy”, Portland State University, 1999 (UMI No. 3018652).

Sánchez E. J., Novotny L., and Xie X. S., "Near-field Fluorescence Microscopy Based on Two-Photon Excitation With Metal Tips," Phys. Rev. Lett. , 82, 20 (1999).

Sánchez E. J., Krug J. T., and Xie X. S., "Ion and electron beam assisted growth of nanometric $\mathrm{Si}_{\mathrm{m}} \mathrm{O}_{\mathrm{n}}$ structures for near-field microscopy”, Rev. Sci. Inst. 73, 11 (2002).

Synge E. H., “An application of piezoelectricity to microscopy,” Phil. Mag. 13, 297 (1932). 


\section{CHAPTER 6}

\section{The Physical TENOM Microscope}

Building a microscope with a reproducible design and the ability to image below the diffraction limit requires precision machining and well-documented parts. Based on the level of complexity involved, it is essential to check for form and function by initially designing the microscope in a virtual environment. Virtual design has other commonly overlooked benefits: the ability to express your design ideas to others, collaborate on the design process, and instantly document the experimental apparatus. Using 3D CAD modeling software for the design of the microscope saved months of work by eliminating a majority of the complications that can be encountered through traditional trial-and-error techniques. In particular, building the small-scale parts for the scan head and the placement of the scan head above the objective lens was greatly simplified. Determining the material requirements and outlining the major features were of first priority. The focus of this chapter is the design fundamentals and optical pathways for both the inverted microscope and the shear force microscope (SFM) mechanical components.

\subsection{Virtual Design Tool}

The entire TENOM microscope was designed in the 3D CAD modeling program SolidWorks (SW hereafter). Its acceptance by industry, user-friendly environment, and compatibility with $\mathrm{CNC}$ milling machines made SW an ideal candidate for designing the TENOM system. Choosing industry standard software ensures long-term support of the software and acts as a teaching vehicle for students to gain usable skills. It is worth 
mentioning that SW supports plug-ins that perform optical ray tracing, aiding the designer in the accurate alignment of optical components [OPTIS, 2010].

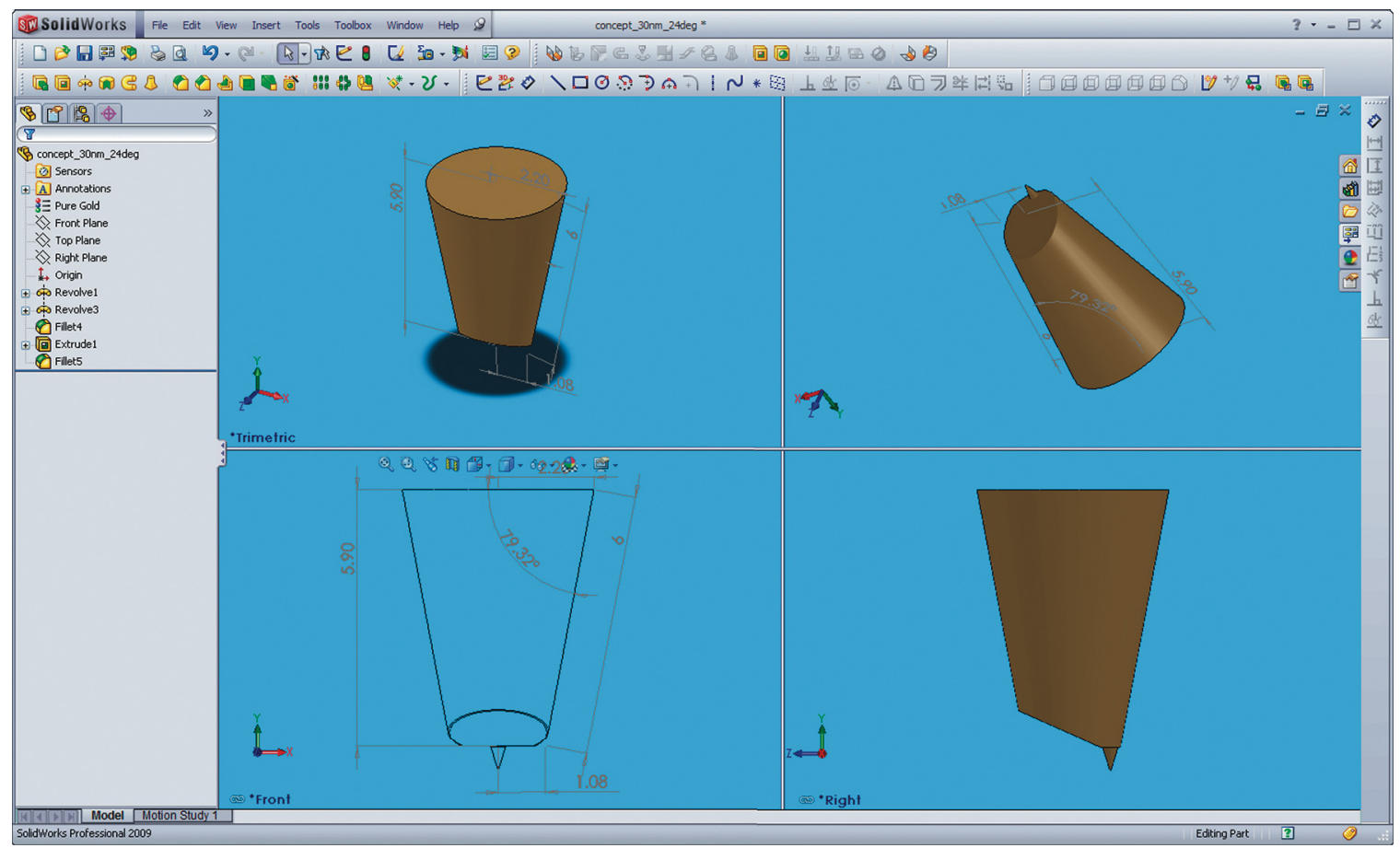

Figure 6.1 SolidWorks 2009 3D CAD software main GUI. The design concept for an asymmetric tip design for TENOM imaging, shown in four different perspectives.

The SW user environment allows the designer to approach 3D modeling from a top-down approach. Similar to working in a machine shop, a basic primitive is created and then embossed or extruded. Edges of parts can be filleted and lathing tools are available for rotational cuts. This is all done in a 3D environment, shown in Figure 6.1, allowing the user to rotate objects and change views in real time. SW uses three main file types: part, assembly, and sheets. Parts are essentially the smallest whole unit of a design, i.e., metal rods, gears, and screws. Assemblies are collections of parts that are mated together along tangent lines, concentric lines or at fixed distances. Assemblies can 
also be checked for collisions and mating incompatibilities between parts. Parts and assemblies can be exported to mechanical drawings referred to as "Sheets", which give scale dimensions and engineering perspectives in order to facilitate the actual creation of the physical part by a machinist. Appendix B.1 and B.2 show all of the mechanical drawings and part list for the TENOM system, as created in SW.

Most of the elements for the inverted microscope are purchased from optic mechanical supply stores. Oftentimes, these suppliers have 3D drawings of their products, which port right into SW. Having access to these virtual parts allows for quick virtual prototyping of a design.

\subsection{Material Requirements}

The design of the optical pathways for the TENOM system is based on a custom inverted microscope. The custom design allows for the incorporation of an SFM scan head, while maintaining a simplified optical system. Due to the sensitivity of SFM imaging, the height of the microscope is kept to a minimum. The TENOM system parts were manufactured from cast iron, stainless steel, and aluminum. The main reason for this choice in material was ease of manufacture, allowing students to build the parts themselves in a student machine shop. This material selection is not the most optimum for the SPM requirements, but with careful machining and design, it's possible to place the sensitive locations of the microscope in the center of most parts. This allows for the radial expansion and contraction to be minimized at those locations. Many groups have proposed radial symmetric designs for scanning tunneling microscopes (STM) that can be applied to SFM [Drake et. al., 1986; Gregory \& Rogers, 1988; Okayama et al., 1988]. 
Table 6.1 is a list of metal expansion coefficients. Ideally, exotic materials such as Invar, Super Invar, and Zerodur would be best, but these tend to be difficult to machine and are generally more expensive.

\begin{tabular}{|l|c|}
\hline \multicolumn{1}{|c|}{ Material } & $\begin{array}{c}\text { Coefficient of linear thermal } \\
\text { Expansion }(\mu \mathrm{m} /(\mathrm{m} \cdot \mathrm{K})) \text { at } 20^{\mathrm{O}} \mathrm{C}\end{array}$ \\
\hline Aluminum & 23 \\
Stainless Steel & 17.3 \\
Cast Iron (grey iron) & 11.1 \\
Titanium & 8.6 \\
Invar & 1.2 \\
Super Invar & .63 \\
Zerodur* & -.052 \\
\hline
\end{tabular}

Table 6.1 Comparison of coefficients of linear thermal expansion for commonly used materials. Note: the negative value for Zerodur. *[Lindig and Pannhorst]

\subsection{Inverted Optical Microscope}

The inverted optical microscope was built using primarily Thorlabs parts, but some custom design parts were implemented, where required. Using SW, the virtual microscope components were assembled to ensure fit and function. Figures 6.3 through 6.6 and Figure 6.9 show examples of these virtual assemblies. The placement of optics was determined virtually, using ray tracing, and also experimentally. The microscope, as presented, is designed primarily for infinity corrected objective lenses. The optical pathways are enclosed as much as possible to minimize stray light from adding to the background noise of the photon detection devices. The microscope itself is also enclosed in a light and acoustically isolated enclosure, similar to Figure 5.11. The use of tube 
lenses (Thorlabs SM1L30) allows for the modification of lens placement to adapt to other focal length objectives and direct mounting of CCD cameras.

The light collected by the objective lens is directed with a steering mirror (Thorlabs KCB1) and then split with a 92:8 beam splitter (Thorlabs CM1-BP108). The first path allows $92 \%$ of the light to be passed through 1.0 inch emission filters held in a tube lens, then directly coupled into a fiber optic (Thorlabs F810SMA-543). This allows for direct fiber coupling to an SPAPD (Perkin Elmer SPCM-AQR-14-FC) / PMT (Hamamatsu 2P8) or Monochromator (Acton SP-150) with attached ICCD (Princeton Instruments Pentamax-512-EFT/1EIA). The remaining $8 \%$ is focused with a $200 \mathrm{~mm}$ achromatic tube lens (Thorlabs AC254-200-A). This signal is further divided with a 50/50 beam splitter, between two CCDs (KT\&C KPC-S500B) - one for imaging the sample surface and one which is equipped with a Bertrand lens system for a fish-eyed view of the imaging probe above the surface for tip focus alignment. To improve the sample view, a magnifying lens can be used (Thorlabs AC254-040-A). Images (B) and (C) in Figure 6.7 shows these views. A Bertrand lens is commonly used for conoscopic imaging, to aid in the alignment of apertures placed in front of the condenser lens [Murphy, 2001]. In the TENOM system, the Bertrand lens is composed of two lenses (Thorlabs AC254-050-A; A375TM-A mounted to S1TM09). Figure 6.2 shows the overall optical excitation and emission pathways of the inverted microscope.

Excitation sources, such as laser or arc-lamp illumination enter through back of the inverted microscope assembly through two steering mirrors configured as a periscope. The mirrors both have $\theta$ and $\phi$ tilt adjustment capabilities using standard kinematic mirror mounts (Thorlabs K100), held at $45^{\circ}$. Linear translation of the beam is 
accomplished by mounting each of the mirrors assembles to a translation stage (Melles Griot 07 TXS 223). The two mirrors are set orthogonal from each other, providing two linear directions of beam translation $(\mathrm{x}, \mathrm{y})$. The pathway of the excitation source then leads to a tube lens (Thorlabs SM1L10), which hold excitation filters that are mounted to the back of a dichroic filter holder (Thorlabs C4W, B4C, B5C). The dichroic filter holder allows for the precise alignment of the incident light so that 45 -degree incidence can be achieved with the dichroic filter. Appendix B gives more details on part placement for the microscope. Figure 6.3 shows two computer rendered views of the inverted microscope, highlighting parts of interest.

Using current generation two photon and Raman filters from Chroma Technology, we are able to filter nearly $100 \%$ of laser excitation wavelengths from the sample, while allowing $>95 \%$ of the light signal to pass through [Chroma Technology Corp, 2009]. Combining these filters with direct optical pathways from the collection objective lens to a fiber-coupled avalanche photodiode (APD), the microscope obtains photo detection efficiency of $\sim 10.2 \%$, assuming a 1.4 NA objective lens $4 \pi$ collection efficiency of $38 \%$. This efficiency is a result of the following experimentally determined results, using $532 \mathrm{~nm}$ light: objective lens throughput (Olympus 1.4 NA 60x) (78\%), collection efficiency $(4 \pi)(38 \%)$, dichroic (Chroma 725dcspxr) (94\%), steering mirror (ThorLabs PF10-03-P01) (98\%), emission filters (Chroma ET750sp-2p8) (3 x 96\%), Beamsplitter (ThorLabs CM1-BP108) (90\%), fiber optic coupling and fiber (85\%), and SPAPD (Perkin Elmer SPCM-AQR-14-FC) (55\% @ 633 nm). 


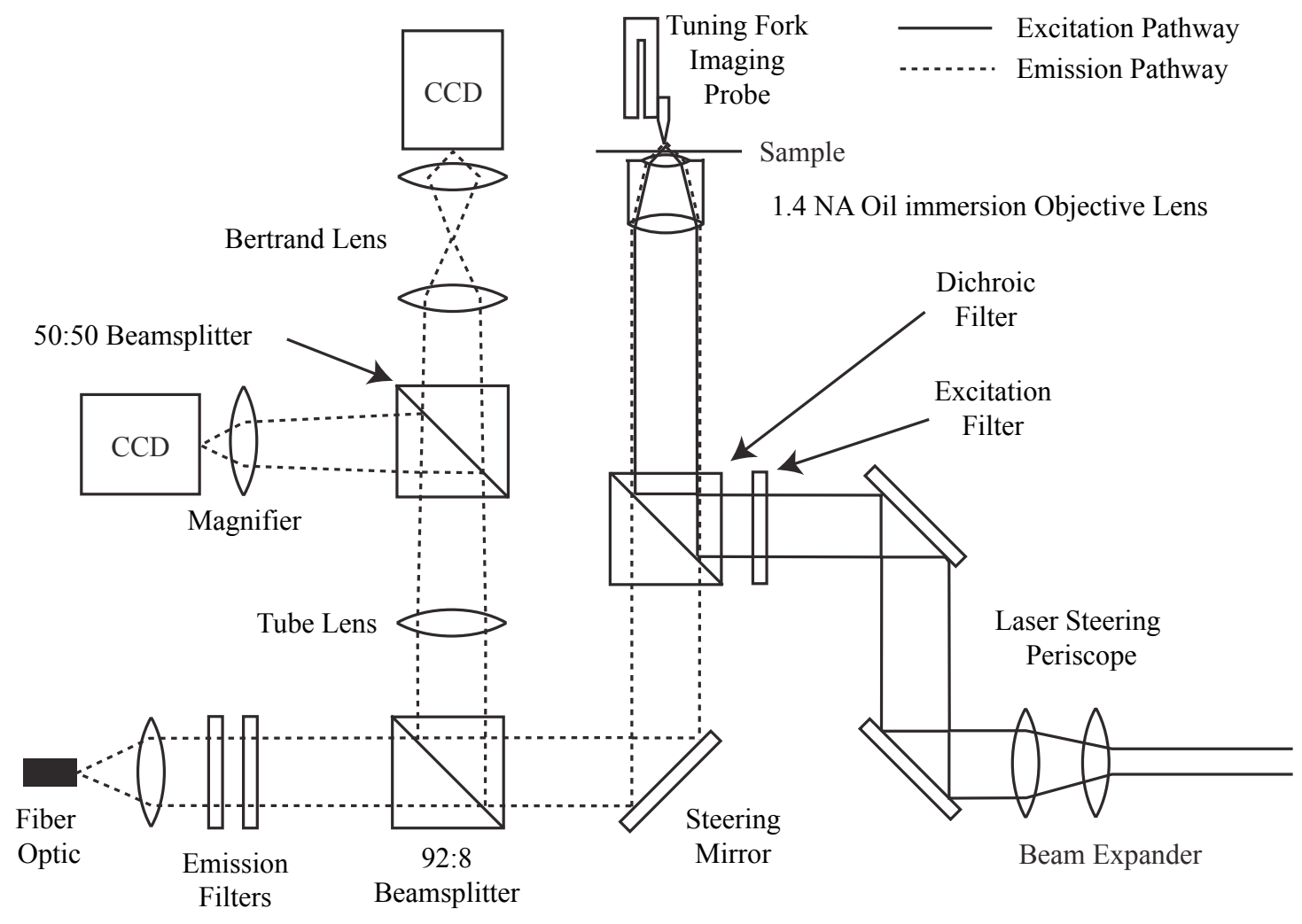

Figure 6.2 Optical pathways of the TENOM system. The Bertrand lens system allows for imaging of the back aperture of the objective lens. This provides a fish fish-eye view of the tip above the imaging surface, allowing for the tip to be positioned in the center of the objective lens. Side illumination of the sample with LEDs produces a shadow of the imaging probe across the sample surface. The CCD is focused on the sample surface to provide this image and aid in the focus of the excitation laser. 


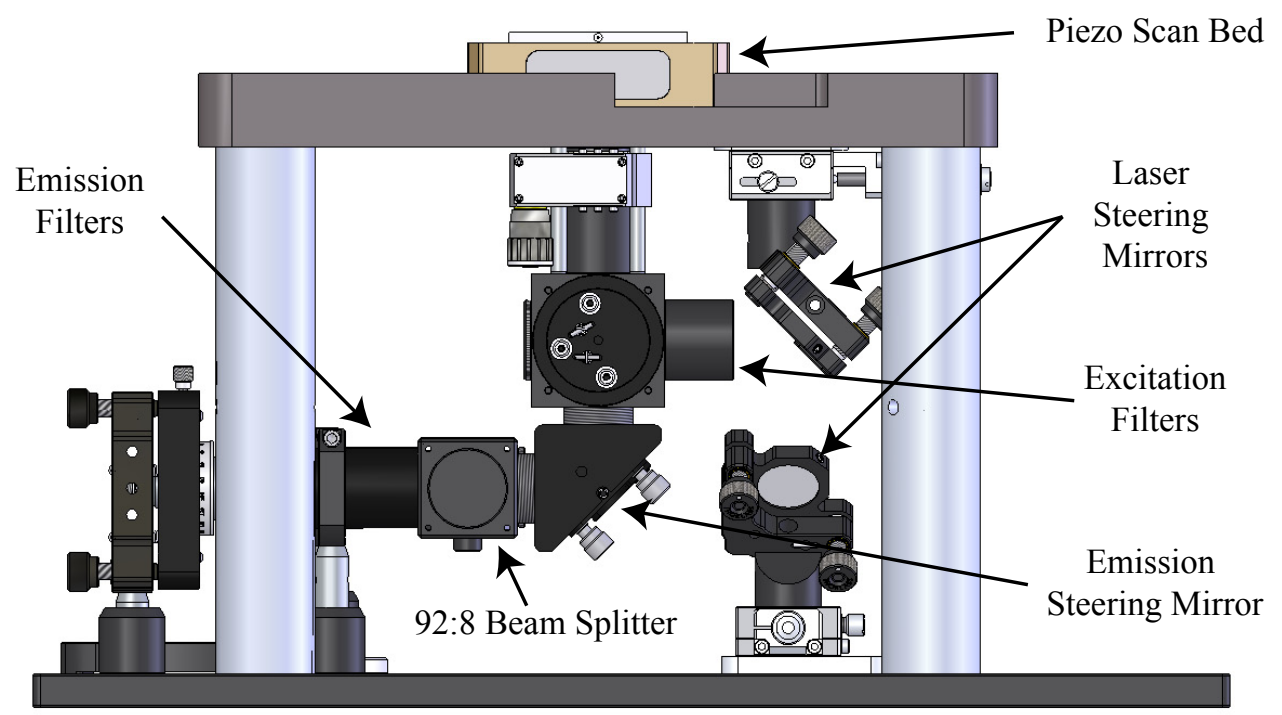

Side View

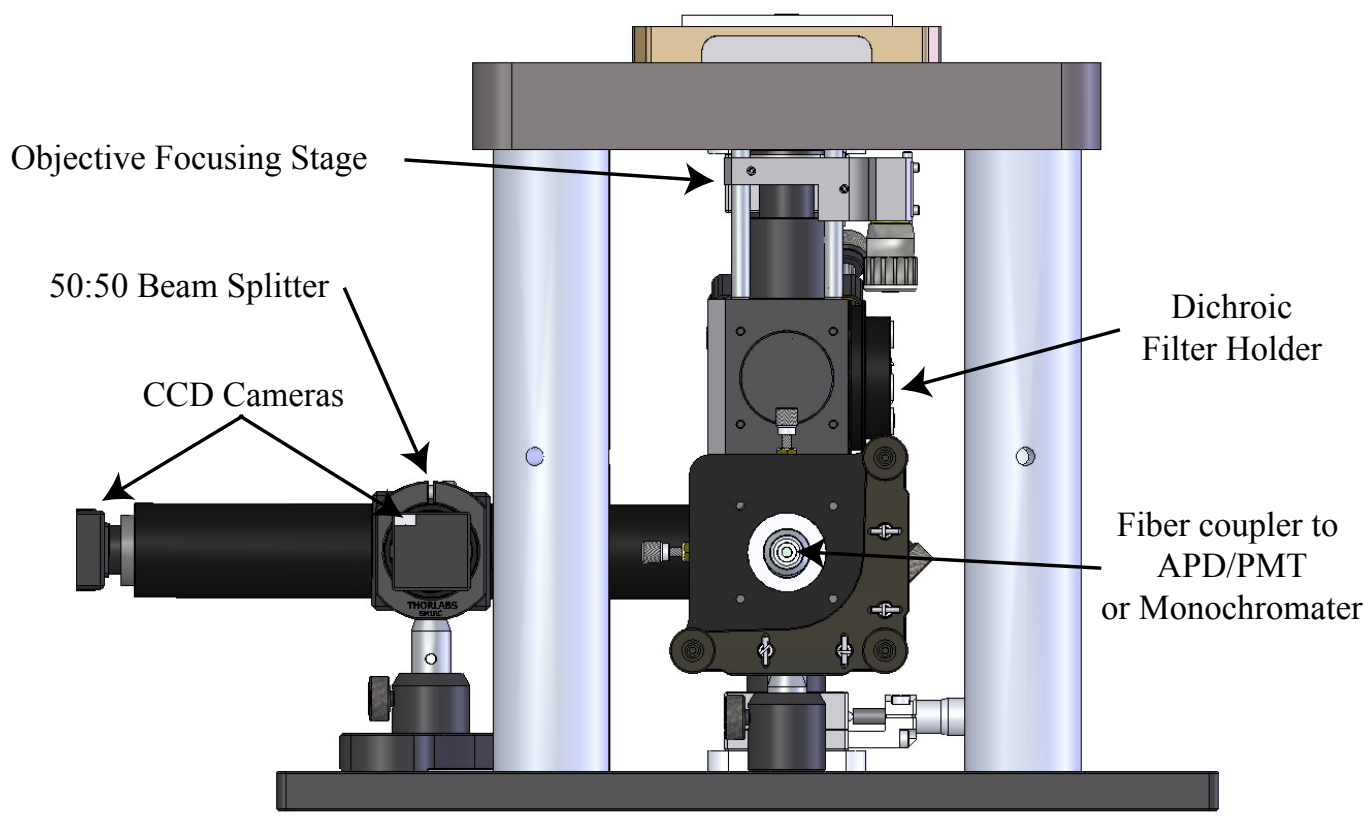

Front View

Figure 6.3 3D representation of the inverted microscope. The laser light enters through the back of the dichroic filter holder. The fluorescence will return down the same path where $92 \%$ will go to the photon detection devices and the remaining $8 \%$ will be directed to the CCDs for user alignment. 


\subsection{Shear Force Microscope Scan Head}

A major complication in the alignment of a TENOM imaging probe is its positioning in the focal spot of the excitation laser. To overcome this complication, the scan head houses a piezo stack which has been designed for alignment flexibility. The stack is comprised of a z-piezo tube (Boston Piezo-Optics, PZT-5A) and x-y outersegmented tube with a solid inner ground layer (Boston Piezo-Optics, PZT-5A Segmented). These tubes are epoxied together and held in a flexure mount, with MACOR used as an insulator. The flexure mount (Newport MFM-075) allows for the coarse alignment (several mm's) of the tip into the laser spot when viewed through the Bertrand lens system (ThorLabs AC245-050-A and A375TM-A Lenses). The x-y outersegmented piezo tube then allows for the precise nanometer positioning of the tip over tens of microns for maximum field enhancement. Figure 6.4 shows the piezo stack assembly.

(a)
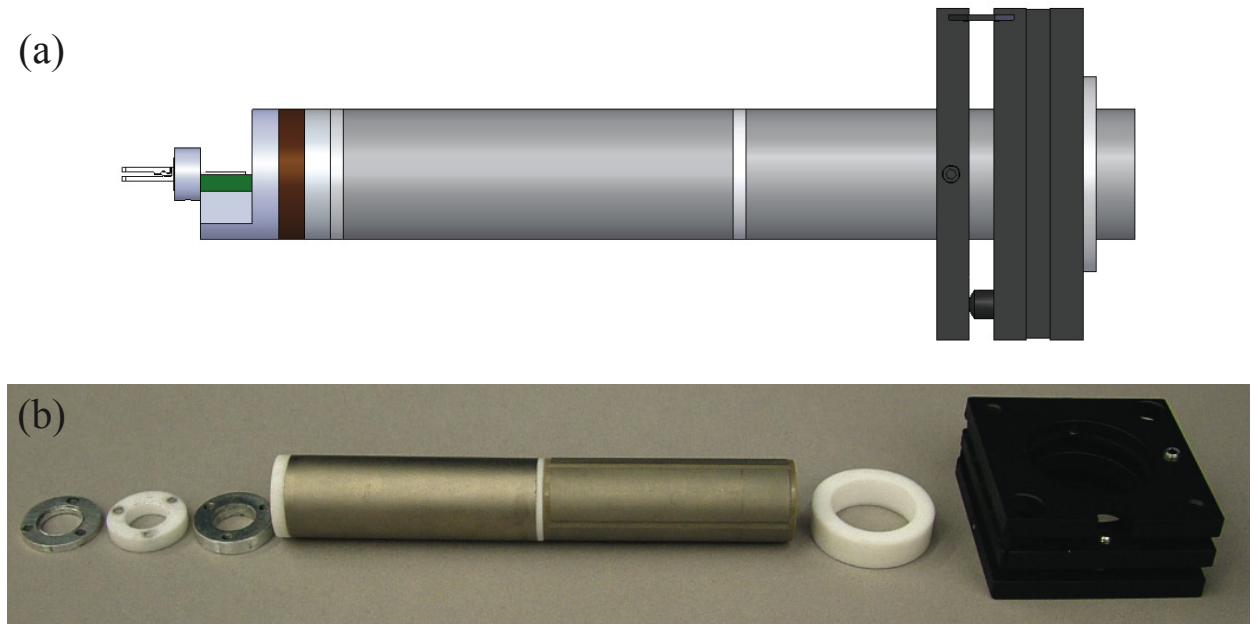

Figure 6.4 Piezo stack: (a) is a 3D assembly of all the parts for the piezo stack generated in SolidWorks, (b) is a photograph of the parts before physical assembly. (See Appendix B for complete details.) 


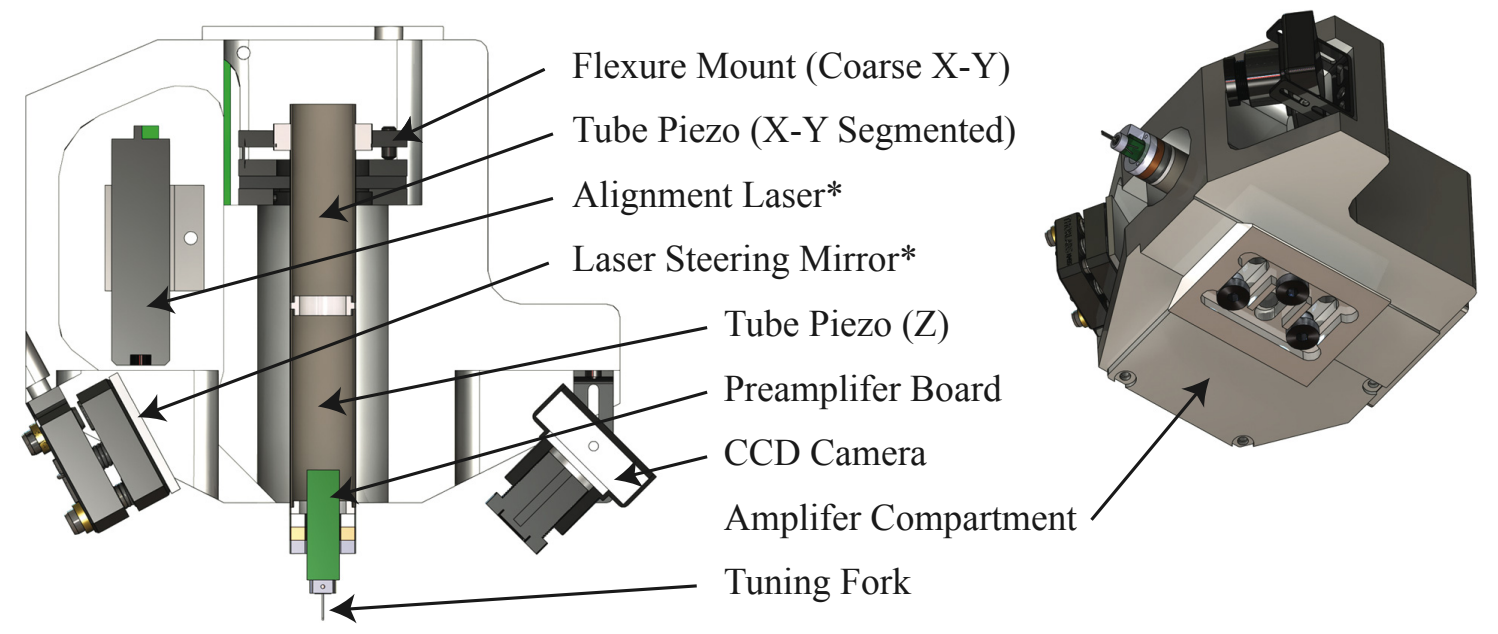

Figure 6.5 3D representations of the SFM scan head. Including the amplifier section within the scan head helps remove noise from the feedback signal.

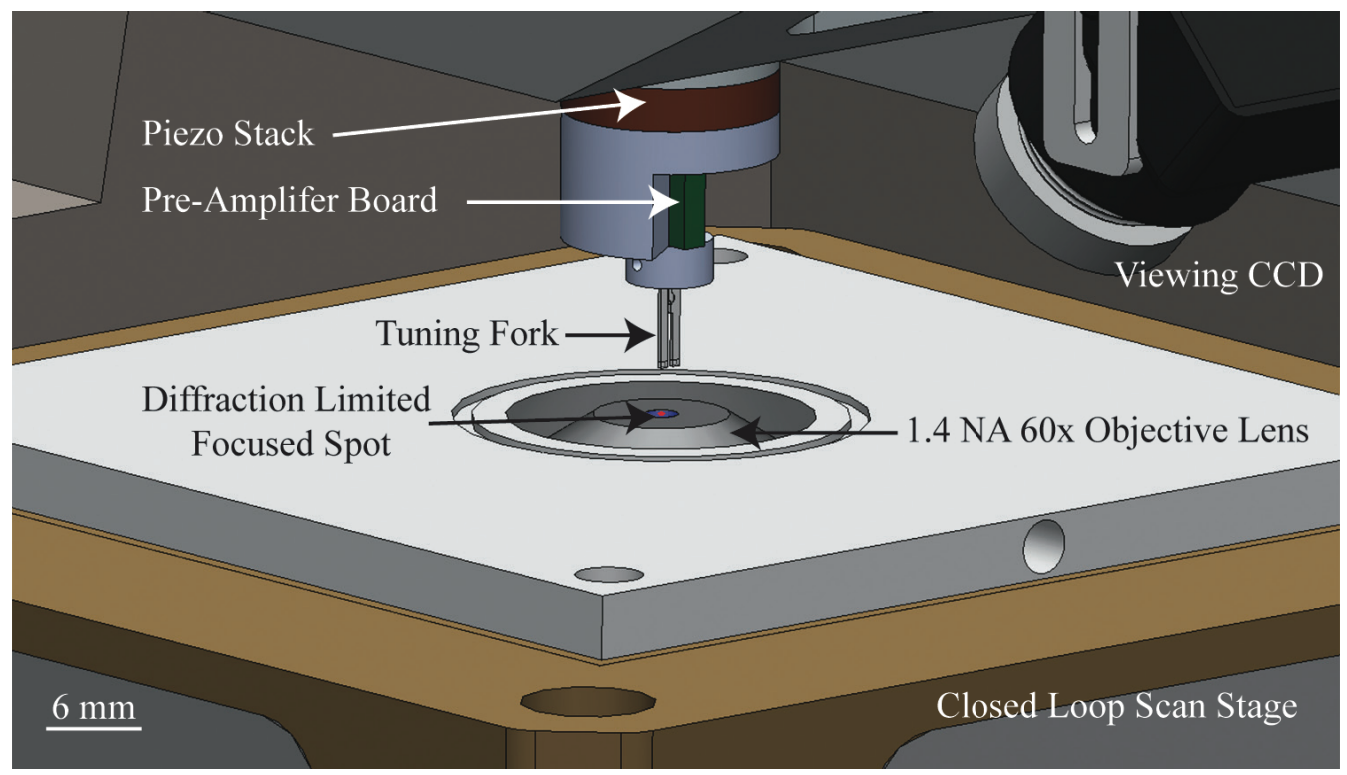

Figure 6.6 3D representation of the alignment flexibility needed to place the probe within the focused laser spot. 
The scan head design includes a low power adjustable laser pointer, steering mirror, tuning fork preamplifier circuit board, and miniature CCD camera, shown in Figure 6.5. The scan head is constructed out of aluminum, where the piezo stack is placed in the center of the unit to minimize the effects of scan drift, due to thermal expansion of the aluminum. The laser pointer and CCD are used to align the probe into the focused laser spot from the objective lens in the inverted microscope, shown in Figure 6.6. It should be noted that the laser pointer was originally intended to illuminate the end of the probe; when the probe was near the diffraction limited spot, the diffraction pattern for the tip would give an approximation of the location of the end tip of the probe. It was discovered that side illumination of the tip with multiple LEDs would provide shadows of the tip when viewed from below, providing the same function. The current design has a modification that allows for two LEDs to be mounted on the CCD camera. The next design update for the scan head will remove the laser and mirror components and simplify the number of cuts necessary to manufacture the scan head. A future design will also include a four point illumination with LEDs to provide a cross pattern that would intersect when the tip reaches the surface. Movement of the CCD from the scan head to a holder on the base of the microscope is also being investigated. The current design still has merit if one would like to integrate an AFM cantilever optical feedback system on the microscope, which would require the laser and mirror components and the introduction of a quadrant photodiode.

Figure 6.7 demonstrates all of the alignment views for the TENOM system. Using the three different views during the approach of the probe on to the sample surface guaranties reproducible placement of the tip in the far-field focal spot. 


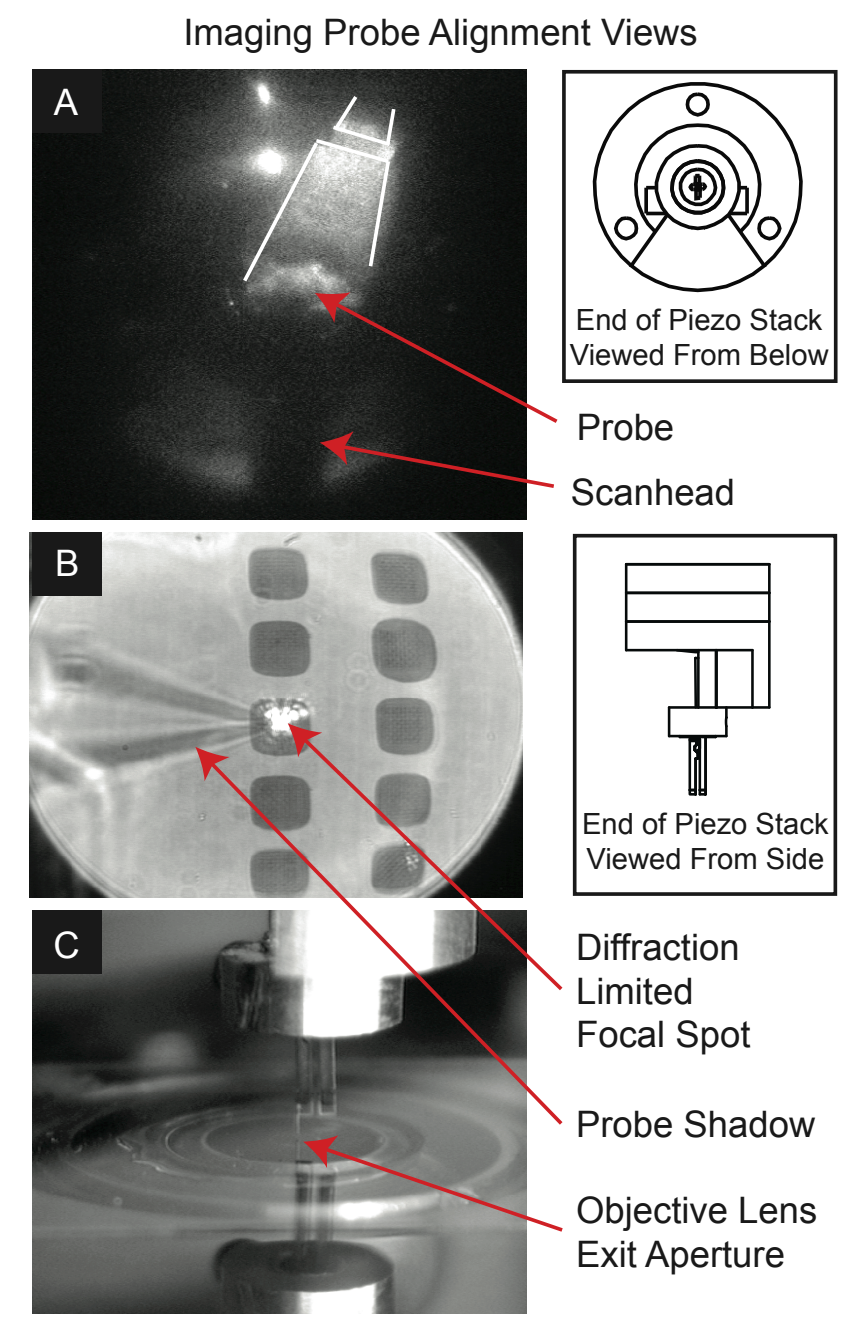

Figure 6.7 Views from the three alignment CCDs: (A) is from the CCD mounted after the Bertrand Lens, (B) is from the CCD focused on the sample surface with probe side illuminated with LEDs, $(C)$ is from the CCD mounted to the scan head or microscope base plate, focusing on the tuning fork and imaging probe.

\subsection{Assembly of the TENOM Design}

The scan head is mounted to a vertical translation stage (Thorlabs LNR50). The position of scan head is controlled with a stepper motor (Oriental Motor PK243B1ASG36), with 0.01 degree-per-step precision and 36:1 gear reduction. Coupled to a 40 turns-per-inch lead screw (Universal Thread), the motor gives a theoretical, per step 
resolution of $18 \mathrm{~nm}$. This, of course, is only theoretical; in application, the stage assembly demonstrates resolutions just under a micron for a full step. Partial steps can obtain smaller resolutions, if needed. The role of the stepper motor arrangement is to provide the safe approach of the imaging probe onto the surface without damaging the probe through tip-sample contact. The rugged design is used to minimize backlash and create a smooth approach that is predictable for the feedback electronics. Park and Barrett stress this requirement in the design for STMs, and the requirement is extremely valid in this application, as well. Any tip damage will prevent near-field imaging [Park $\&$ Barrett, 1993]. The locations of the stepper motor and lead screw are shown in Figure 6.9 .

A majority of the parts were purchased from Thorlabs mainly due to their support of SW. Other vendors include Melles Girot, Olympus, Physik Instrumente, Oriental Motor, Hammond Mfg., and Universal. The custom part requirements include the base plate, back plane, scan head, and adapter plates, all of which can be machined on a CNC or traditional milling machine. The machining time is roughly one to two weeks for all custom parts needed. Assembly can be accomplished in a few hours with standard tools. The only specialty tool required is a spanner wretch, used to mount the tube lens for the CCD cameras, lenses, and filters. Alignment of the optical pathway requires a little more time and some experience with aligning laser systems. The dichroic filter can be aligned before assembly into the microscope using a laser pointer or compact $\mathrm{HeNe}$ laser. Figure 6.8 shows the layout of the TENOM system with monochromator, camera, APD, and laser systems on an optical table (Newport RS4000). 


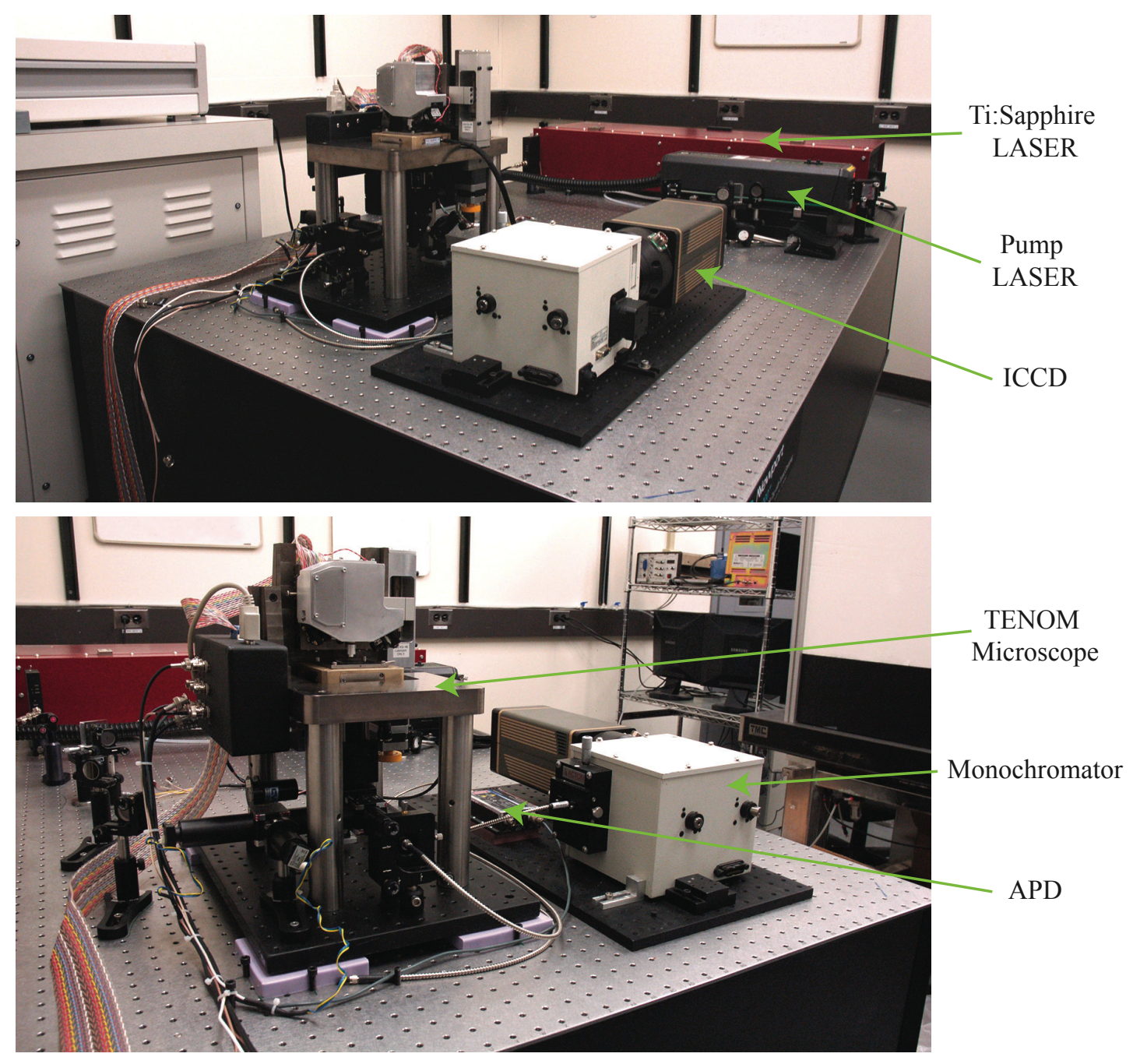

Figure 6.8 TENOM system layout: The system is placed on silicon vibration dampening material to reduce high frequency vibrations. The optical table dampens the lower frequency floor vibrations. Lasers, camera, and monochromator are mounted to the table for ease of alignment. 


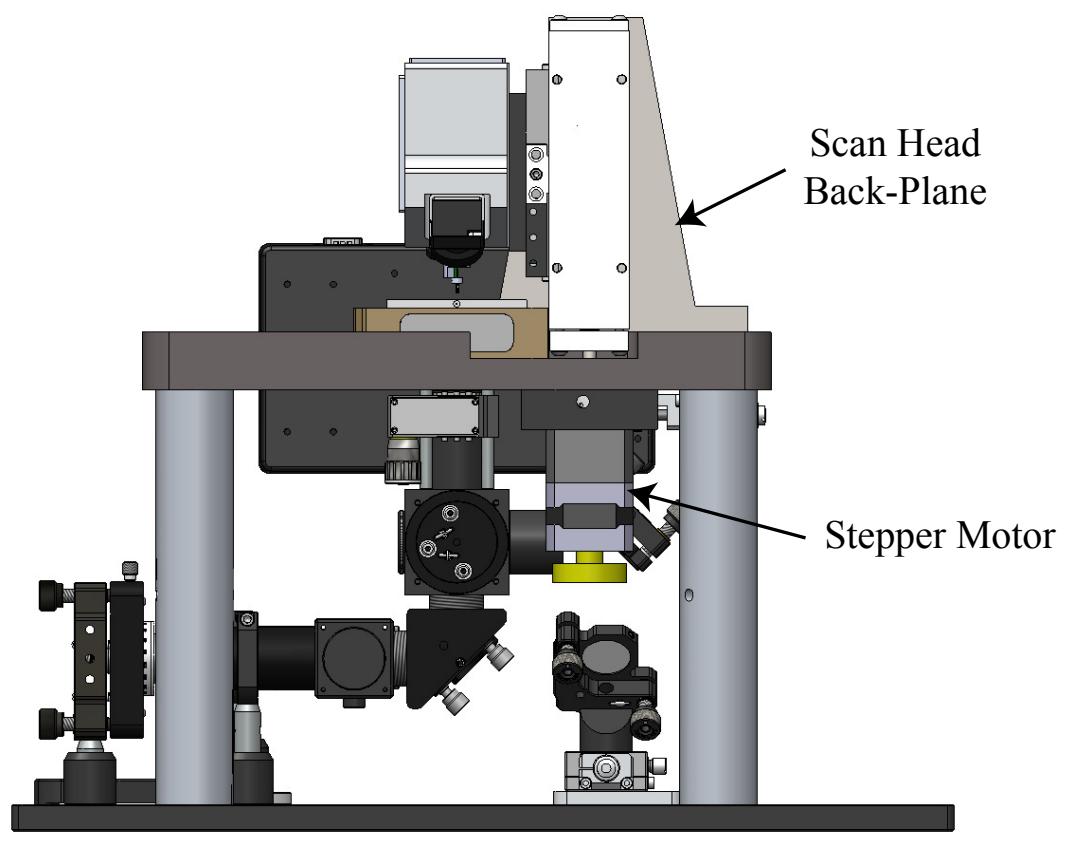

Side View

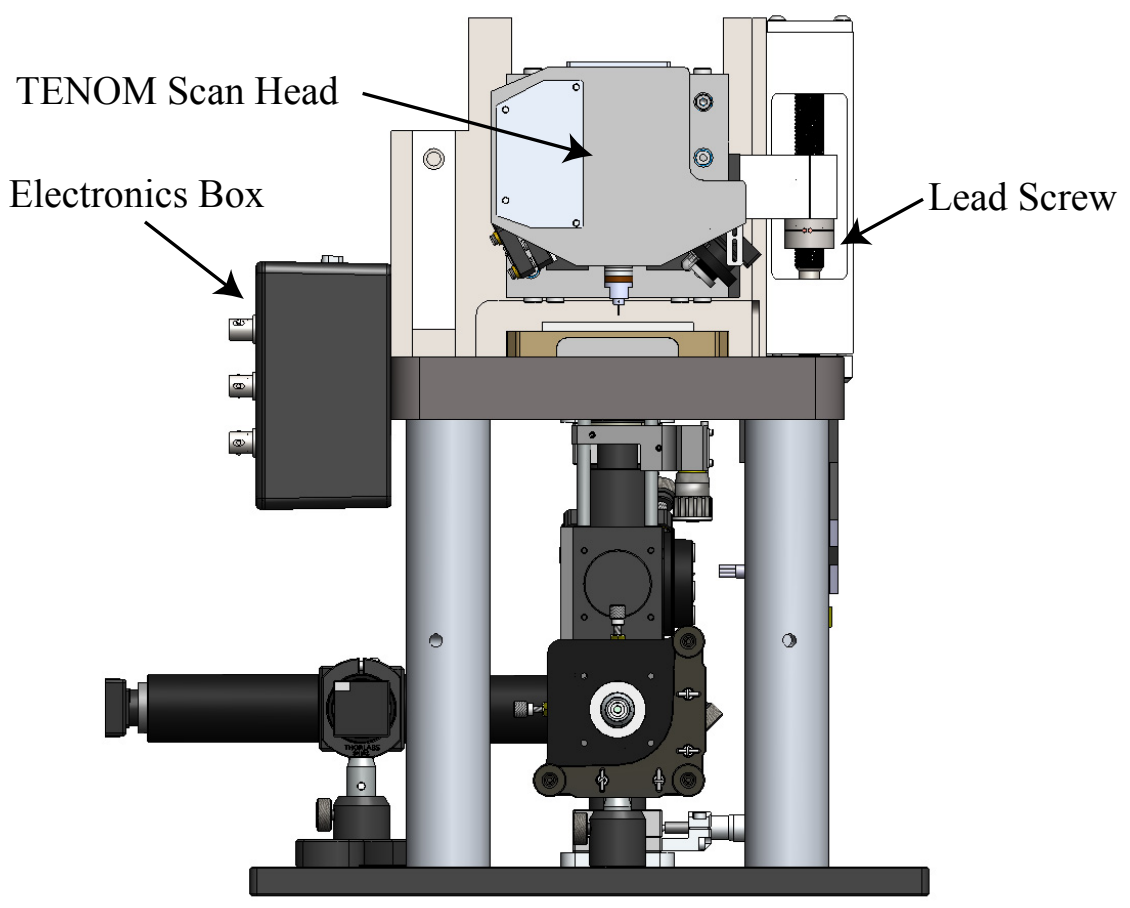

Front View

Figure 6.9 3D representation of the TENOM system. The scan head is supported by the back-plane. The lead screw and stepper motor ensure a soft landing of the imaging probe onto the surface. The electronic box directs the electrical signals to the scan head, cameras, and stepper motor. 


\section{CHAPTER 6 References}

Chroma Technology Corp. "Catalog of Filters and Mirrors for Laser Applications," Product Catalog, 2009 pp. 25-30.

Drake B., Sonnenfeld R., Schneir J., Hansma P. K., Slough G., and Coleman R. V., "A tunneling microscope for operation in air or fluids," Rev. Sci. Instrum. 57, 441 (1986).

Gregory S. and Rogers C. T., "High-Speed Scanning Tunneling Microscopes," J. Vac. Sci. Technol. A 6, 390 (1988).

Lindig O. and Pannhorst W., "Thermal expansion and length stability of Zerodur in dependence of temperature and time," Applied Optics 24, 20 (1985).

Okayama S., Komuro M., Mizutani W., Tokumoto H., Okano M., Shimizu K., Kobayashi Y., Matsumoto F., Wakiyama S., Shigeno M., Sakai F., Fujiwara S., Kitamura O., Ono M., and Kajimura K., "Observation of microfabricated patterns by scanning tunneling microscopy,” J. Vac. Sci. Technol. A 6, 440 (1988).

OPTIS, computer program OptisWorks--for Solidworks (OPTIS North America, Troy, MI, 2010).

Park S. and Barrett R. C., "Design Considerations for an STM System," in Methods of Experimental Physics, edited by Stroscio J. A. and Kaiser W. J. (Academic Press, New York, 1993) Vol. 27, pp. 30-76.

Murphy, D. Fundamentals of Light Microscopy and Digital Imaging, (Wiley-Liss, New York, 2001) pp. 17. 


\section{CHAPTER 7}

\section{Design of the TENOM Electronics}

Early generations of tip enhanced near-field optical microscopes (TENOM) relied heavily on the modification of commercial hardware and the integration of multiple computers to control the third party hardware [Sánchez, 1999; Sánchez, Novotny \& Xie, 1999]. It is this integration of hardware that allows the user to simultaneously obtain topographic, spectral, and optical information from the sample. The complexity of a TENOM system requires a computer system to monitor and make real-time decisions without the computational overhead of operating systems, which interfere with high-speed data acquisition. The design motivation of the TENOM electronics is to simplify this complexity with a single computer in which the OS runs the user interfaces, and a single embedded processor deals with all of the high-speed timing requirements. The hardware requirements to make a functional TENOM system are discussed in this chapter.

The problem stems from common day operating systems. Graphical user interfaces (GUIs) used on modern day operating systems work using interrupt request (IRQ) logic. An IRQ is the method in which a keyboard, mouse, or internal hardware requests resources from the central processing unit (CPU) to process the input of user information. The timing response of the operating system is designed to make the user's input, such as mouse movement and keyboard typing, seem fluid. This overhead burden on the operating system makes it inefficient when high-speed timed or real-time decision processes are needed. 


\subsection{Field Programmable Gate Array (FPGA) Microcontroller}

A solution to bulky operating system overhead is to use embedded processors that are customized to calculate simple processes without interaction from the computer operating system. The embedded processors fall into a few different categories: digital signal processors (DSPs), field programmable gate arrays (FPGAs), and microcontrollers. A detailed understanding of embedded design is available in Steve Heath's book "Embedded System Design" [Heath, 2003]. Peter Bishop gives a comparison of the three technologies of embedded processing, ultimately relying on the designer to choose the best for their application, based on the benefits or limitations of the different processors [Bishop, 2009]. DSPs are high-speed devices that are used primarily in audio processing hardware, such as sound cards, audio synthesizers, and audio mixing platforms. The DSP is very efficient at floating-point mathematic algorithms and other hard-wired algorithms. A microcontroller is a simplified computer, designed to fit on a single integrated circuit (IC). Many microcontrollers contain onboard read-only and random access memory, serial and parallel output ports for interfacing with other computer systems, and a CPU. Microcontrollers are very good at processing basic algorithms in a serial processing fashion. FPGAs represent a good combination of the high-speed signal processing of a DSP with the logic processing of a microcontroller. FPGAs use an array of logic blocks that are configured and linked to generate a custom-processing unit that can be programmed to meet the end-user's needs. One advantage the FPGA has over the microcontroller is the ability to process multiple instructions in parallel. Over the last decade, FPGAs have grown in popularity [McGrath, 2006] and have been replacing DSPs due to the customizable benefit of the FPGA. A majority of embedded processing 
solutions are attracted to the FPGA market due to the simplicity of design, easy firmware upgrades, and future expandability offered in an FPGA package, as opposed to a hardwired custom microcontroller IC die. Based on these benefits, the design on the TENOM system will be implemented on a FPGA.

Ultimately, the FPGA is only a function block when it comes to data acquisition. The FPGA chip will have to be mated to digital-to-analog converters (DAC) to generate voltage outputs to control devices, and to analog-to-digital converters (ADC) to record and process real world signals from various sensors. Several DACs and ADCs are required to effectively operate a TENOM microscope due to the number of signals needed to drive and monitor the system. Converters must be carefully chosen to maximize the sensitivity (bit-resolution) and speed (bandwidth) of the TENOM system. To build a TENOM system or any scanning probe microscope (SPM) system from this level would require a depth of knowledge in mixed analog and digital circuit design, in order to correctly implement the DAC and ADC circuit requirements. To ease the complexity of development, a compromise was made that allows for the use of commercially available data acquisition cards which provide a good balance of bit resolution and bandwidths of the on-board DACs and ADCs.

While not the focus in the design presented in this dissertation, it should be mentioned that the continued development of a data acquisition system for the TENOM system that has a custom set of ADCs and DACs would be advantageous. This selection of ADCs and DACs should have bandwidths and bit depths optimized for each of the microscope's input and output signals. In fact, many commercial SPM instruments use a variety of ADCs and DACs for just this purpose. 


\subsection{National Instruments Reconfigurable I/O}

To simplify the arrangement and development of the embedded processing elements of the TENOM system, a data acquisition card with an FPGA processor and integrated DAC and ADC with direct memory access (DMA) transfer capabilities was chosen. The TENOM electronics were developed around a National Instruments (NI) (Austin, TX, USA) reconfigurable data acquisition (DAQ) card (PCI-7833R). This card utilizes a user-programmable FPGA microprocessor. The NI FPGA allows for custom programming in LabVIEW of the logic needed for TENOM imaging and runs that logic in real-time. The 7833-R DAQ card has 3 million logic gates, all of which are programmable. The DAQ card has 8 analog inputs (ADC) with bandwidths of $200 \mathrm{kHz}$, 8 analog outputs (DAC) with bandwidths of $1 \mathrm{MHz}$, and 96 digital lines (DIO) at 40 MHz. The FPGA is clocked at $40 \mathrm{MHz}$, giving digital timing resolutions and instruction clock cycles of $25 \mathrm{~ns}$. The parallel nature of the FPGA allows all of the analog I/O and digital I/O ports to be accessed simultaneously. At the time of this writing, a newer version of FPGA based DAQs has been released from NI and preliminary investigation shows that there is direct compatibility with the previous generations. Benefits include faster bandwidth converters, increased number of logic gates, and a transition from PCI to PCI-express computer bus interfacing [National Instruments, 2009]. This level of compatibility was one of the reasons that NI was chosen as a platform, as they have a history of supporting hardware for extended periods of time.

Initially, the intent was to use the PCI-7833R DAQ to control all aspects of the TENOM system with minimal signal conditioning, but it was determined early on that the DACs and ADCs did not support the bandwidth or the bit-resolution needed for seamless 
integration into the physical microscope. The development of analog circuitry for a highresolution oscillation signal for driving the shear force microscope (SFM) tuning fork and summing multiple analog outputs from the DAQ to create higher bit resolutions for the scan algorithm was the solution to the limited bandwidth of the FPGA.

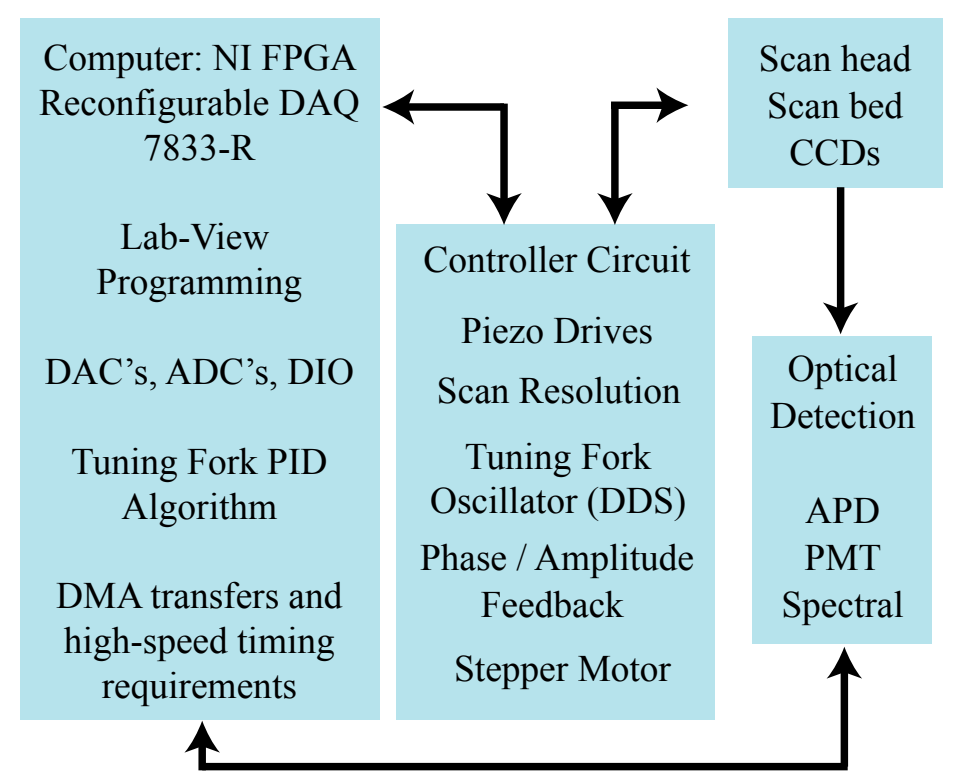

Figure 7.1 A block diagram of the TENOM control system. The arrows indicate direction of data signal flow.

The bandwidth limitations of the ADCs made it difficult to digitally monitor amplitude and phase measurements of the tuning fork. The workaround for this limitation was the development of two analog circuits to detect amplitude and phase, independently. Figure 7.1 shows a block diagram of the TENOM microscope with computer integration, the DAQ card, and analog circuitry.

\subsection{Calculating a Noise Baseline}

Before discussing the details of the analog circuit design, it is important to determine a baseline for the noise level requirements for the SFM elements of the 
TENOM electronics and to develop a method to quantify the electrical noise in the system. Fundamentally, the noise level of the FPGA - DAQ card from National Instruments will be the limiting factor in lowering the noise floor of the microscope. Most of the output voltages from the DAQ card will be used to directly drive piezo crystals. This will affect the measurements, i.e., metrology capabilities of the microscope. Thus, it will be useful to begin thinking about the voltage noise in terms of a unit of measure. For this application, nanometer measurements that are converted from millivolt to microvolt of measure will be appropriate.

The DAQ card has different noise levels for digital to analog conversion and analog to digital conversion, outlined in the product specification data sheet [National Instruments, 2009]. The manufacturer specifies the input noise to be $\pm 7.78 \mathrm{mV}$ and the output noise as $\pm 5.88 \mathrm{mV}$ for the $7833 \mathrm{R}$ card. In the next generation $7852 \mathrm{R}$, the noise levels are about half that of the 7833R. The noise levels of the different analog circuits will be determined by measuring the propagation of signals from the DAQ to the circuit of interest. The calculation and noise of the analog circuits will be presented with the discussion of the individual components in their respective sections of this chapter.

\subsection{Analog Circuitry}

The analog requirements for the detection of phase and amplitude from the AFM tuning fork, along with the high voltage requirements for the piezo crystals, are designed onto custom printed circuit boards (PCBs). The controller circuit boards are designed to be modular, which allow for easy future revisions and upgrades. Ideally, as the line of National Instruments FPGA - DAQs improves, analog elements of the system can be removed. Figure 7.2 shows a data flow layout for the modular circuits. 


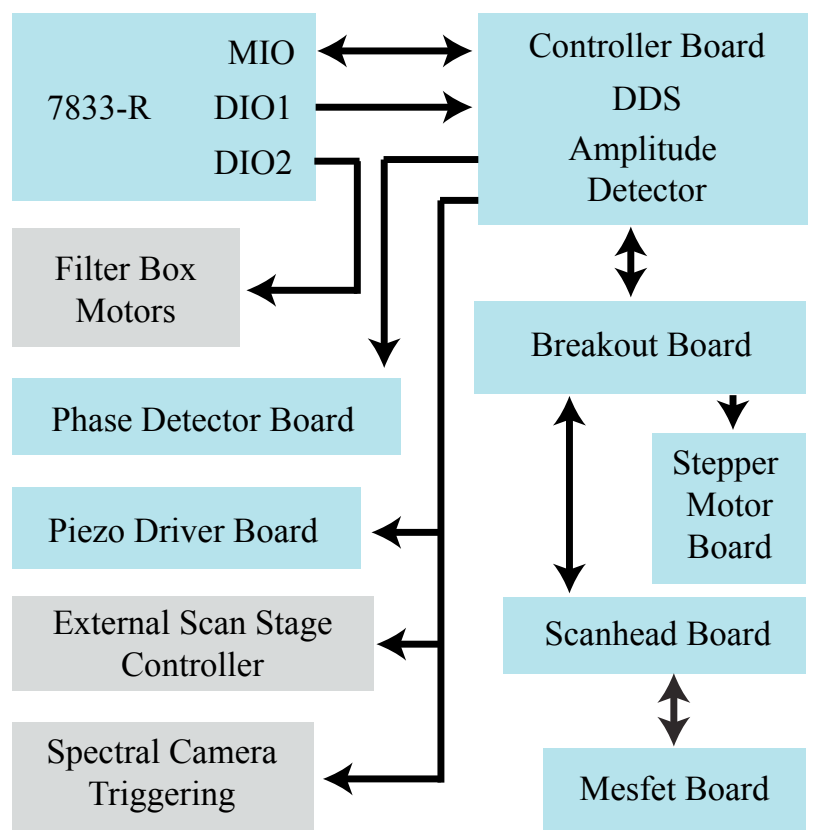

Figure 7.2 A block diagram of the analog circuitry layout on multiple PCBs to allow for modularity in design. The arrows indicate the direction of data flow throughout the controller.

All of the PCBs were developed in the freely available software package PCB123 (v2), developed by Sunstone Circuits (Mulino, OR, USA). PCB123 allows the user to layout the electrical schematics and then link these schematics to a layout program. The schematic and layout programs share file information using a net list, allowing the user to make changes to the schematic and update the changes to the layout without complication. The finished designs are emailed to the manufacturing facility from the PCB123 program. At the time of publication, a newer PCB123 (v3) has been released which follows the same basic functionality as version 2 with the schematic and layout program combined into one application. Figure 7.2 shows the layout file image of the main controller board and a picture of the finished PCB. All of the circuit schematics and PCB layouts for the microscope are presented in Appendix C. 

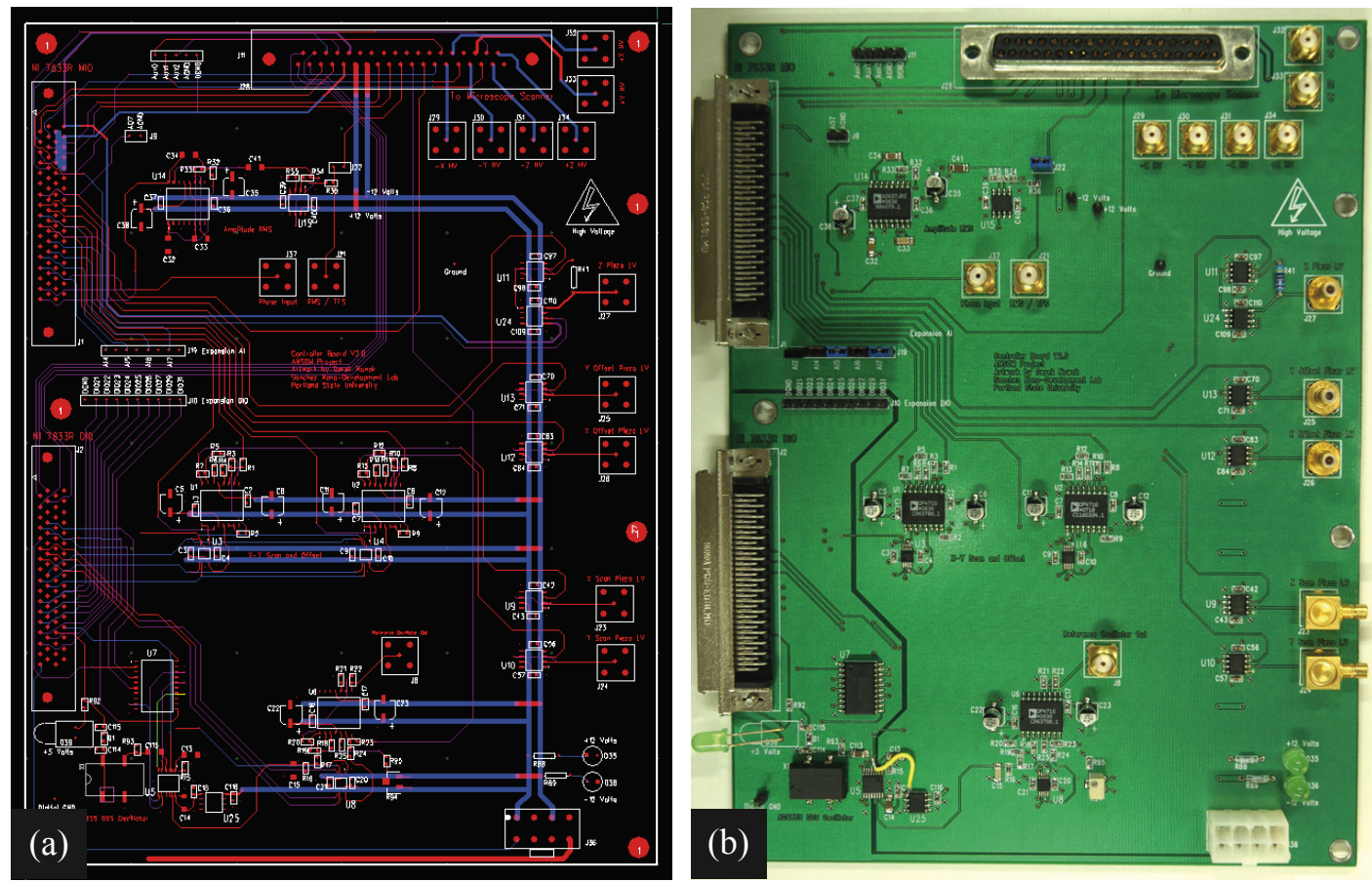

Figure 7.3 (a) Gerber file image from the PCB123 layout program, showing all four layers of the PCB stacked together. Each color represents one layer. (Ground plane layers removed for clarity.) (b) Main controller board after production with all components placed.

\subsection{Considerations in PCB Design}

The TENOM electronics involve the mixing of analog and digital circuitry. The use of high speed digital communication with sensitive analog voltage requires careful choices in power supply filtering, ground planes and paths, and routing of signals on the circuit board [Johnson, 1993]. To address these concerns, all PCBs for the TENOM electronics were designed with 4 layers. This gives enough flexibility in routing that one plane can be a solid ground plane, which minimizes stray ground paths that can couple unwanted signal return paths into sensitive analog paths [Brockman \& Williams]. The digital and analog components are isolated from one another, whenever possible, by isolating ground layers. All ICs have power supply filtering capacitors and high-speed 
devices with ferrite beads to isolate noise from coupling back onto the power supplies. All low voltage PCBs are powered by a $\pm 15 \mathrm{~V}$ linear power supply (International Power IHAA14-0.8). Finally, all boards have a common star ground on the main power supply to minimize ground loops.

\subsection{X-Y Scanning}

Due to the 16 bit resolution of the DAC on the NI FPGA - DAQ card, it was determined that the best scanning resolution obtainable with a $100 \times 100 \mu \mathrm{m}^{2}$ scan stage would result in $1.5 \mathrm{~nm}$ per DAC voltage division, theoretically. With a DAC output noise floor of $\pm 5.88 \mathrm{mV}$ for the $7833 \mathrm{R}$ card, an error of $\pm 29.4 \mathrm{~nm}$ would result. This is assuming that the bipolar output $\pm 10 \mathrm{~V}$ of the DAQ was applied across the whole scan area. Scan resolutions that are below one nanometer are much more desirable for highresolution imaging. In order to achieve sub $20 \mathrm{~nm}$ optical resolutions, an improvement in the scanning resolution is required. To circumvent this issue, the output of two DACs, one that applies a static voltage offset and one that applies the raster scanning voltage, is summed together. The scanning output is sent to a programmable potentiometer (Analog Devices AD5290), configured as a voltage divider to adjust the scan size. This serial peripheral interface (SPI) programmable voltage divider circuit has 256 possible divisions of resistance, lowering the theoretical scanning resolution to $0.01 \mathrm{~nm}$. When running the microscope, a static offset is first applied to move the scan stage to the desired location. This offset location becomes the origin for the raster scanning voltage. This voltage is then sent to either an external piezo scan bed controller or to piezo driving amplifiers available on the circuit board. Figure 7.4 shows the logic for the scan circuit, with Figure 7.5 showing the implemented PCB for the scan circuit. 


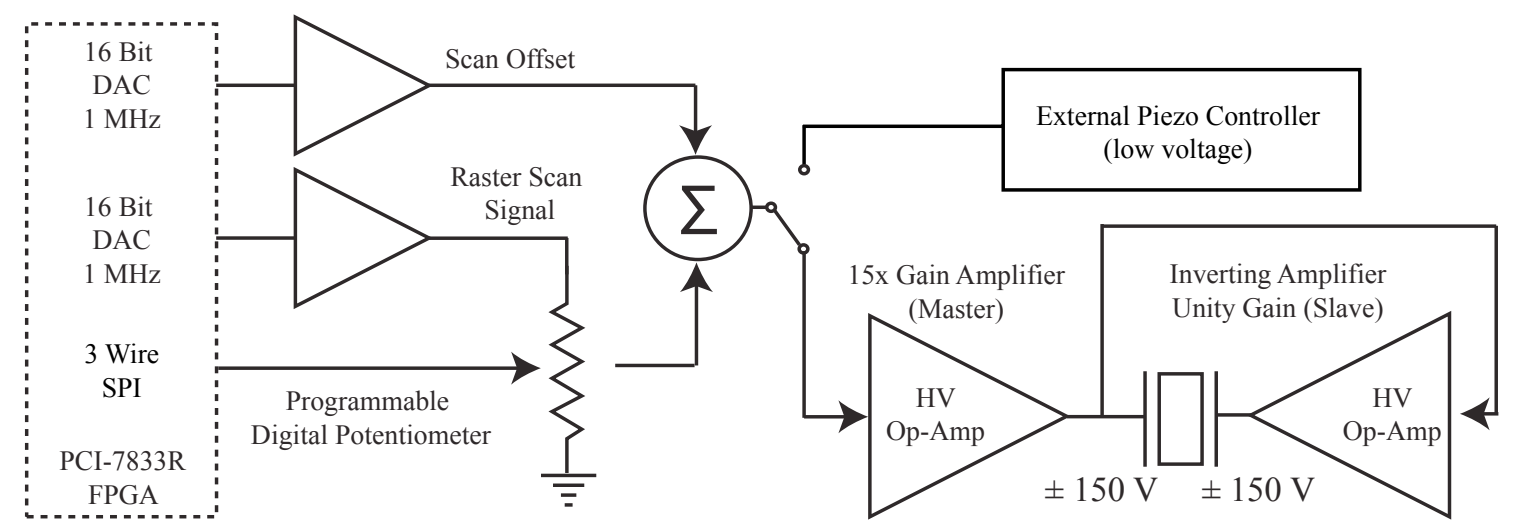

Figure 7.4 Circuit logic for the summing of the two analog outputs for the scanning offset and raster scan signals. The microscope implements two summing circuits for independent control of the $\mathrm{X}$ and $\mathrm{Y}$ axis. The user has the flexibility to send the low voltage output to the piezo driving PCB or to a third party piezo controller.

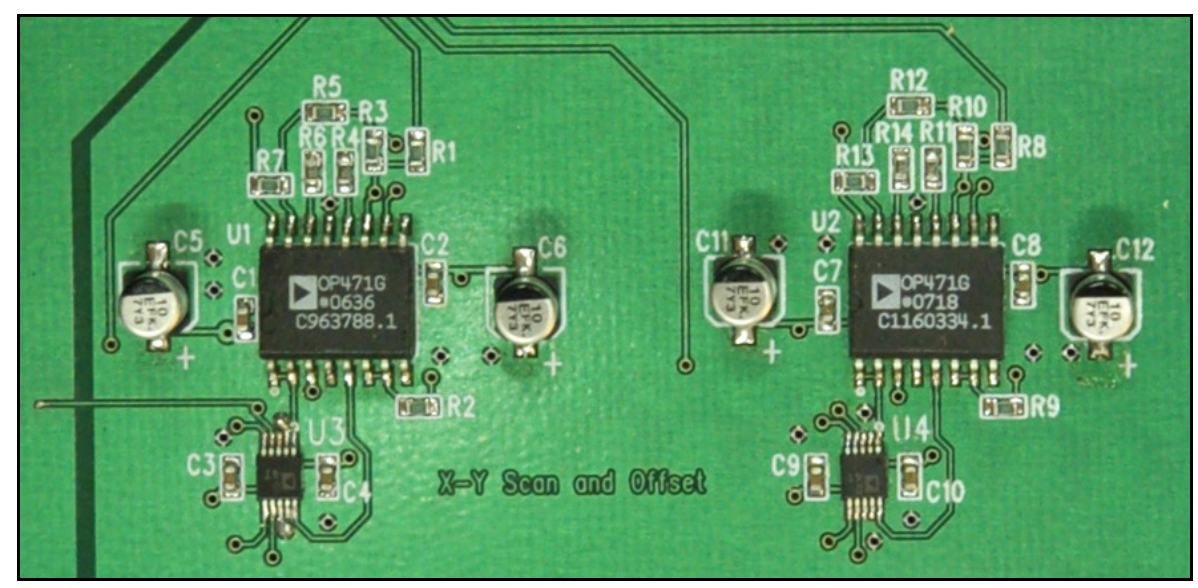

Figure 7.5 Scanning circuit on PCB, OP471 (U1, U2) operational amplifiers sum and amplify the signals to the users specifications. U3 and $\mathrm{U} 4$ are the digital potentiometers.

This flexibility of design allows researchers to scan with a simple open loop piezo scan tube or a more precise closed loop piezo scan stage. The FPGA then controls the scan and data acquisition, based on the number of pixels, scan area, and scan speed, all of which are specified by the user (detailed in Chapter 8). 


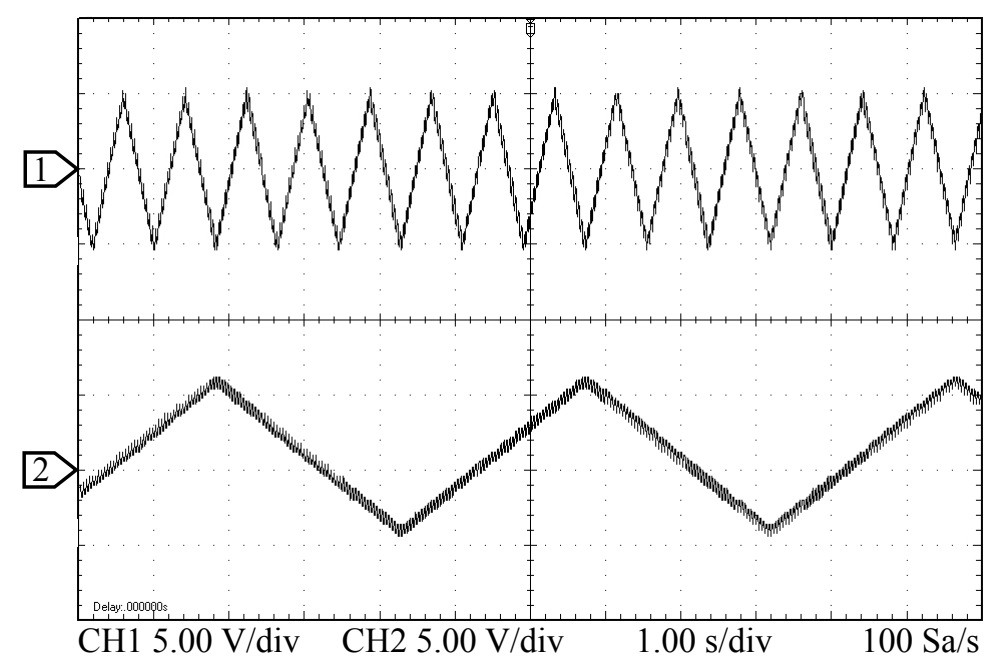

Figure 7.6 X (upper), Y (lower) scan waveforms for the TENOM system. Both channels displayed at $5.0 \mathrm{~V} / \mathrm{div}$ with a $1.0 \mathrm{sec} / \mathrm{div}$ time base. (See Chapter 8.2.e for complete algorithm details.)

The scan circuitry must have low electrical noise. The operational amplifiers (opamps) chosen for signal conditioning and summing are a pair of OP471s. The OP471 is a quad op-amp packaged IC based on the OP27, which allows for a very low noise floor with a small signal bandwidth of $8.0 \mathrm{MHz}$ [Analog Devices, 2006]. The low speed of the OP27 is ideal for the relatively slow scanning system; it will reject much of the high frequency noise, acting like a low-pass filter around 8.0 Mhz. The electronics should then be naturally immune to environmental high frequency RF noise (microwave, cellular and wifi frequencies). Figure 7.6 shows the output of the scanning waveforms.

\subsection{Piezo Driving Circuits}

The voltage output of the DAC on the FPGA card is rated for $\pm 10 \mathrm{~V}$. Typical piezos will require hundreds of volts to generate microns of displacement. In order to generate this driving voltage and retain the ability to change this voltage in the low tens of kilohertz bandwidth, high voltage (HV) operational amplifiers from APEX 
Microtechnology (Cirrus Logic) are used. These operational amplifiers are configured in a bridge circuit, which consists of two amplifiers connected in a master and slave configuration, shown in Figure 7.7 [Cirrus Logic, 2009]. The benefit of this arrangement is that the output voltage swing is twice that of a single amplifier, and the slew rate is also doubled.

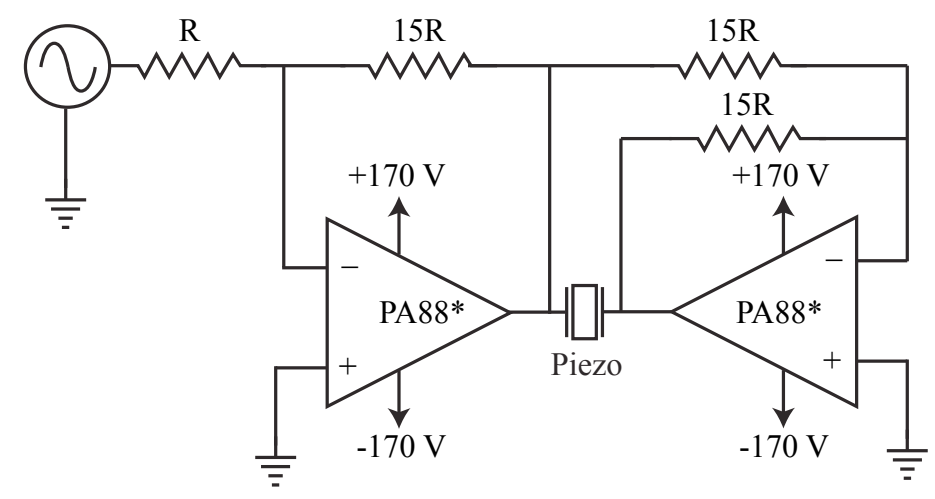

Figure 7.7 A resistor diagram of a bridge circuit configuration of two PA88 HV operational amplifiers. *Support components, capacitors, protection diodes, compensation linear components are removed for clarity. (Appendix $\mathrm{C}$ has complete details.)

For example, the PA88 is one such high-voltage amplifier, with a voltage output rating of $\pm 225 \mathrm{~V}$ and a slew rate of $2.0 \mathrm{~V} / \mu$ s. In the bridge configuration, the maximum differential voltage available to drive the piezo is $900 \mathrm{~V}$ with a slew rate of $4.0 \mathrm{~V} / \mu \mathrm{s}$. PA88s are popular in SPM for their low noise floor of $2.0 \mu \mathrm{V}_{\mathrm{rms}}$ with a load on the power supply of $1.7 \mathrm{~mA}$ [Behler, 1997; Cirrus Logic 2009]. The increase output swing is a result of allowing the master amplifier to reach a maximum voltage output of $225 \mathrm{~V}$ while the slave is outputting $-225 \mathrm{~V}$, thus having a forward voltage of $450 \mathrm{~V}$. If the logic is reversed, allowing the master to reach $-225 \mathrm{~V}$ while the slave is outputting $225 \mathrm{~V}$, then a backward voltage of $-450 \mathrm{~V}$ is created. The differential of these two conditions gives a 
full swing of $900 \mathrm{~V}$. The increase of slew rate is just the effect of a change of $1.0 \mathrm{~V}$ in the master and consequent change of negative $1.0 \mathrm{~V}$ in the slave, with a differential of 2.0 V.

The TENOM design has the ability to drive as many as five independent bridge circuits for the ability to control five different piezos. For this particular application, the linear power supplies that provide the high voltage for the PA88s are two $155 \mathrm{~V}$ units (International Power IHB155-0.12). The gain used in the bridge circuit of the TENOM system is $x 15$, so the maximum output will be $\pm 150 \mathrm{~V}$. To provide the headroom needed to prevent clipping of the PA88s, the power supplies output voltage is adjusted to \pm 170 V. The first power supply is configured normally using the +output set at $170 \mathrm{~V}$. For the second one, the +output is connected to the -output (floating ground) of the first. Now the -output on the second supply will be $-170 \mathrm{~V}$. The +output on the first supply and output on second will be the ground reference and then can be wired to the star ground location in the chassis. Appendix $\mathrm{C}$ shows a detailed diagram, outlining the power and ground wiring for the electronics chassis. Please note that some adjustments to the voltage output on the power supply may be necessary.

The design of the PCB is shown in Figure 7.8. Due to the higher voltages that exist on the circuit, spacing minimums were increased to prevent arcing conditions [IPC, 1998]. Component spacing was also considered to prevent arcing between components such as heat sinks and ground pins on capacitors. To calculate the minimum noise floor for the bridge circuit, the input voltage is shorted to ground and the outputs of the amplifiers are recorded. Figure 7.9 shows the outputs recorded by an oscilloscope. 


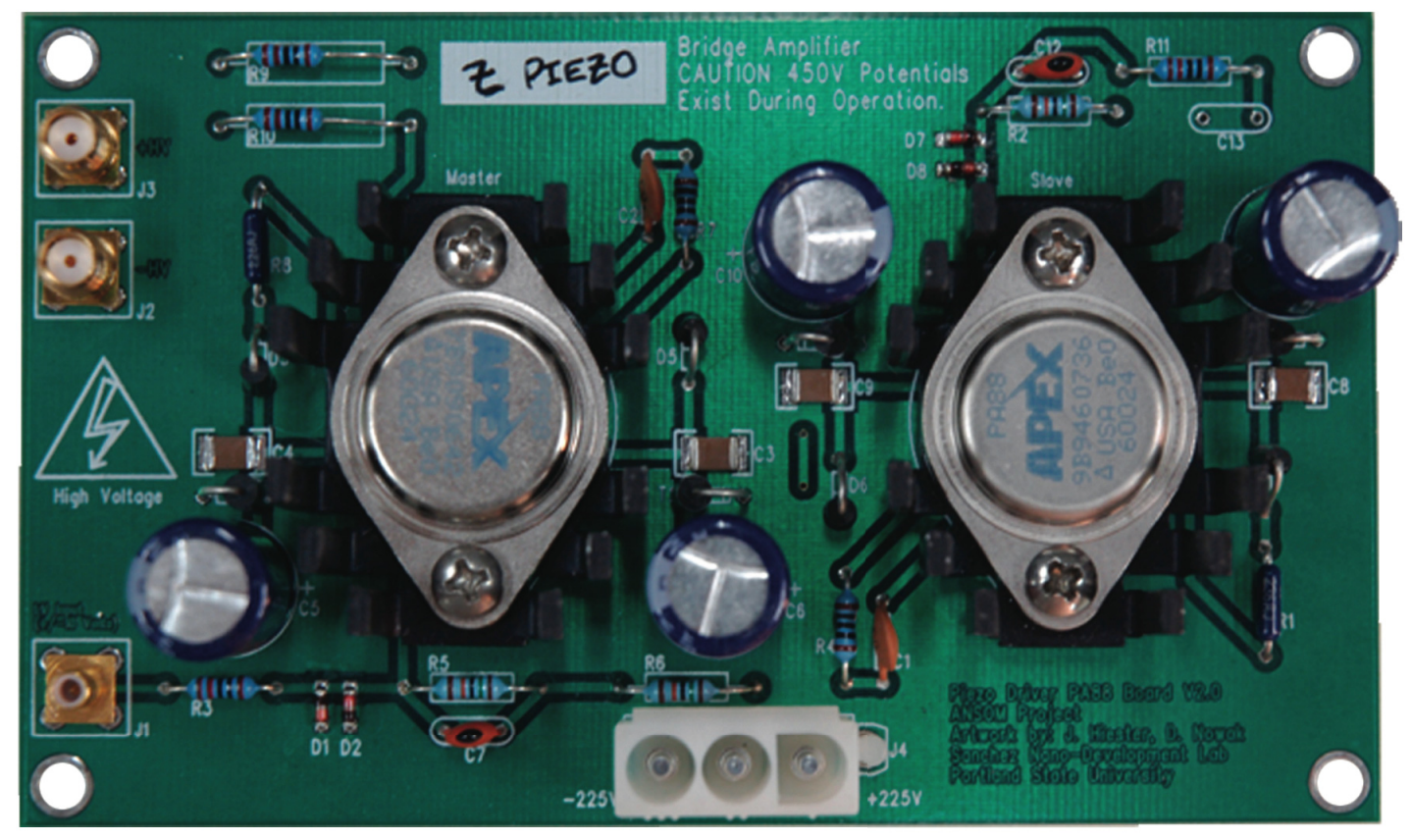

Figure 7.8 PCB of bridge circuit piezo driver with components. The two amplifiers are Apex PA88 chips with heat sinks. The remaining components configure the gain and slew rates with phase compensation.

The $\mathrm{Z}$ tube piezo used to obtain the data presented later in this dissertation has a displacement of $10.8 \mathrm{~nm} / \mathrm{V}$. Based on the waveform in Figure 7.9, the noise floor is 18.4 $\mathrm{mV}_{\text {rms }}$ or $0.2 \mathrm{~nm}_{\mathrm{rms}}$. The choice of a high displacement per volt piezo was made, based on the high topography usually found on biological samples. The final test for the bridge circuit is an input step function to determine the response time for the amplifiers. Figure 7.10 shows the input step test to verify that the each amplifier is responding at a rate of 3.0 V/ $\mu$ s for a combined effect of $6.0 \mathrm{~V} / \mu \mathrm{s}$ [Cirrus Logic, 2009]. This is not the limiting bottleneck for the piezo response of the microscope. The tube piezo chosen for the microscope design has a capacitance of $50 \mathrm{nF}$. When combined with two current-limiting resistors of $1.0 \mathrm{kohms}$ each, the bridge circuit will have a resultant bandwidth of 1.89 $\mathrm{V} / \mu \mathrm{s}$. 


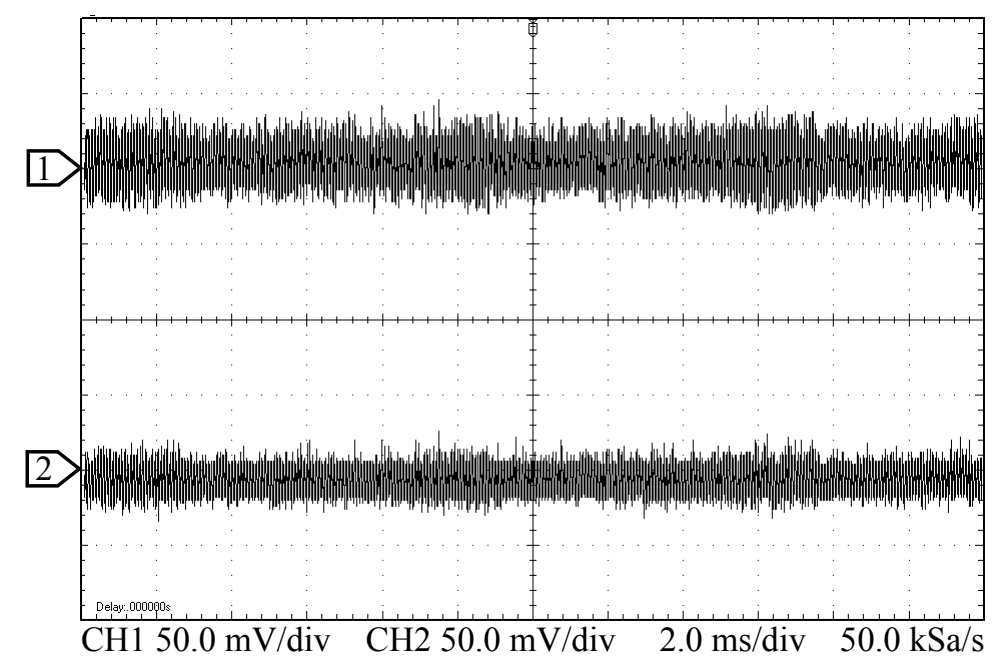

Figure 7.9 Noise output of both $+\mathrm{HV}$ (upper) and $-\mathrm{HV}$ (lower) outputs with a shorted input. Both channels displayed at $50 \mathrm{mV} / \mathrm{div}$ with a 2.0 $\mathrm{ms} /$ div time base.

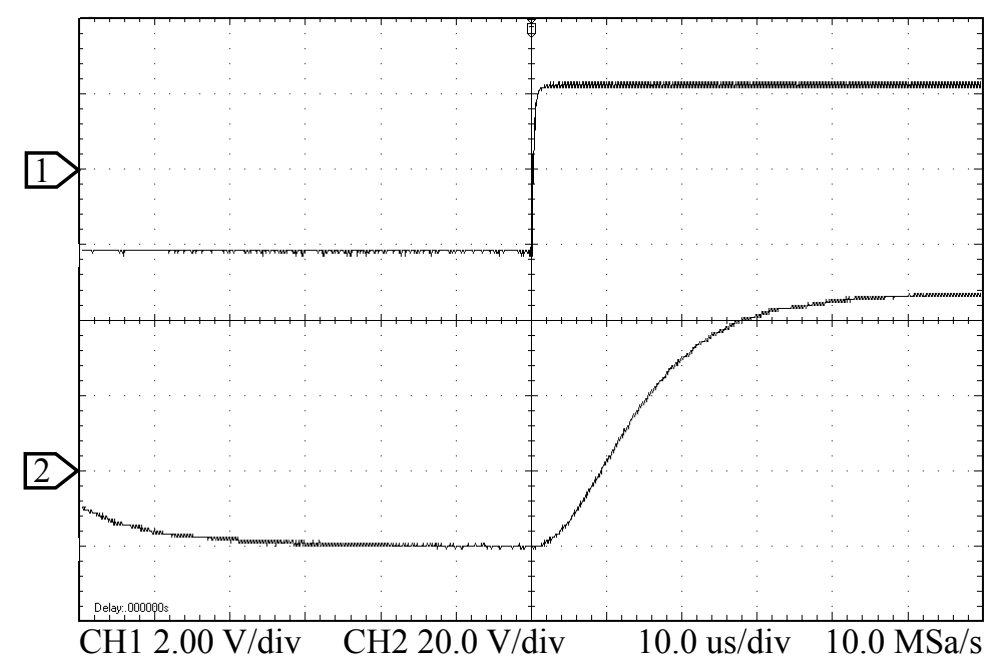

Figure 7.10 Positive output of bridge circuit and its response to an input step function. Channel one (upper) $2.0 \mathrm{~V} / \mathrm{div}$, channel two (lower) 20 $\mathrm{V} / \mathrm{div}, 10 \mu \mathrm{s} / \mathrm{div}$ time base. 


\subsection{Direct Digital Synthesis}

In order to oscillate the tuning fork at its resonance frequency, a high-resolution sine wave is required with frequency adjustability on the order of hundredths of Hz. For example, a tuning fork with a quality factor $\left(\mathrm{f}_{0} / \Delta \mathrm{f}_{\mathrm{FWHM}}\right)$ of 1,000 has a spectral bandwidth of $3.2 \mathrm{~Hz}$. The driving oscillation must have the resolution to drive the tuning fork between the range of $32,769.7$ and $32,766.3 \mathrm{~Hz}$. One solution is to use a direct digital synthesis (DDS) IC (Analog Devices AD9835) to generate the frequencies needed. A DDS uses a 48-bit look up table, which is preprogrammed with sine and/or cosine functions. Output frequency is controlled by the ratio of a clock divider referenced against a master clock, typically an external oscillator. A specified frequency request is sent to the DDS IC via a 3-wire SPI communication as 32-bit hexadecimal frequency division value, as outlined in Figure 7.11 [Colm, 2003]. The output frequency is then sent to the phase detection circuit as a reference oscillator, and to a voltage divider where output will electrically drive the tuning fork (See Figure 7.13) [Grober et al., 2000; Jahncke et al., 2004].

Implementing the DDS on a PCB requires careful attention to detail between the analog and digital ground planes for the device [Analog Devices, 1998]. The IC requires the analog and digital voltages to be powered independently at $5.0 \mathrm{~V}$ each; this is possible with the use of voltage regulators. The DDS IC also requires CMOS logic level voltages for digital communication. The low level TTL logic from the FPGA DAQ card is insufficient to make the needed digital level transitions. This is remedied with the use of a line driver 74HCT244 to convert the TTL to CMOS voltage levels. 


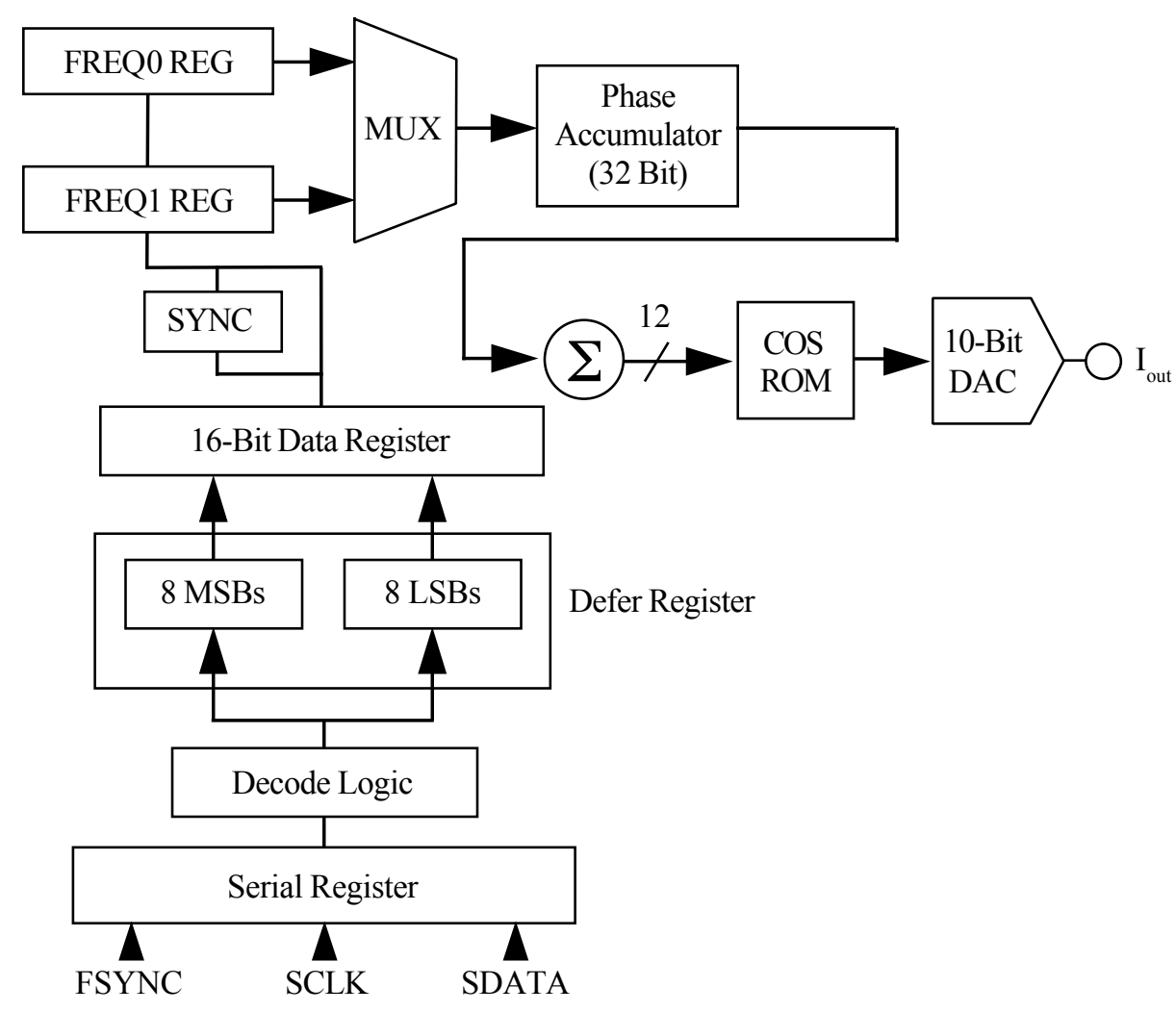

Figure 7.11 Block Diagram of the internal function of an AD9835 DDS, adapted from Analog Devices data sheet [Analog Devices, 1998]. The frequency is set using four 32 bit hexadecimal values. 8 bits of each of the four contain the frequency, high MSBs, low MSBs, high LSBs, and low MSBs. The remaining bits are instructions for the DDS to decode the incoming logic. 


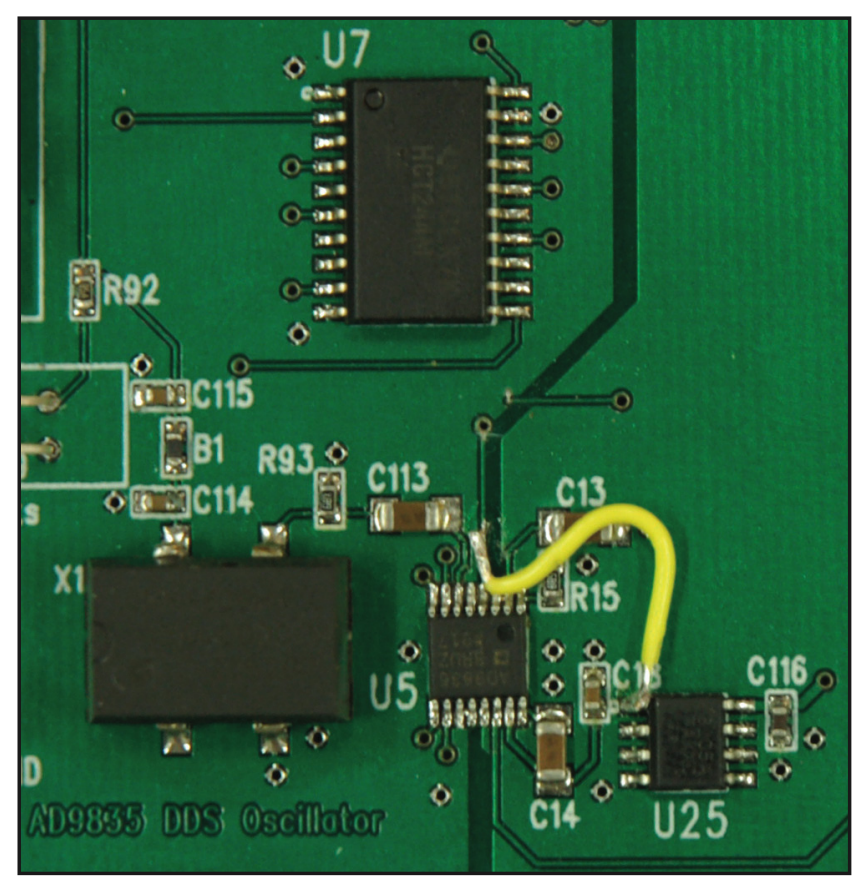

Figure 7.12 DDS Oscillator circuit on PCB, U5 is the AD9835, X1 is a 50 $\mathrm{MHz}$ clock oscillator, $\mathrm{B} 1$ is the ferrite bead, and $\mathrm{U} 7$ is the line driver (74HCT244) to convert from low voltage TTL to CMOS logic voltages. The yellow wire is a testing modification on an earlier version of the PCB.

\subsection{Tuning Fork Signal Amplifier}

The oscillation of the quartz tuning fork will generate an oscillating current on the order of several nanoamps [Friet \& Carry, 2007]. This current is converted to a voltage and amplified with a gain of $10^{6}$ that brings the voltage level up into the microvolt range. It is important that this initial pre-amplification be done as close as possible to the tuning fork, in order to minimize the amplification of noise. The signal is later amplified by a two-stage amplifier with a dual channel operational amplifier with a gain of $10^{3}$. Figure 7.13 shows the logic of the amplification, while Figure 7.14 shows the result of the amplification as a tuning fork is passed through resonance. 


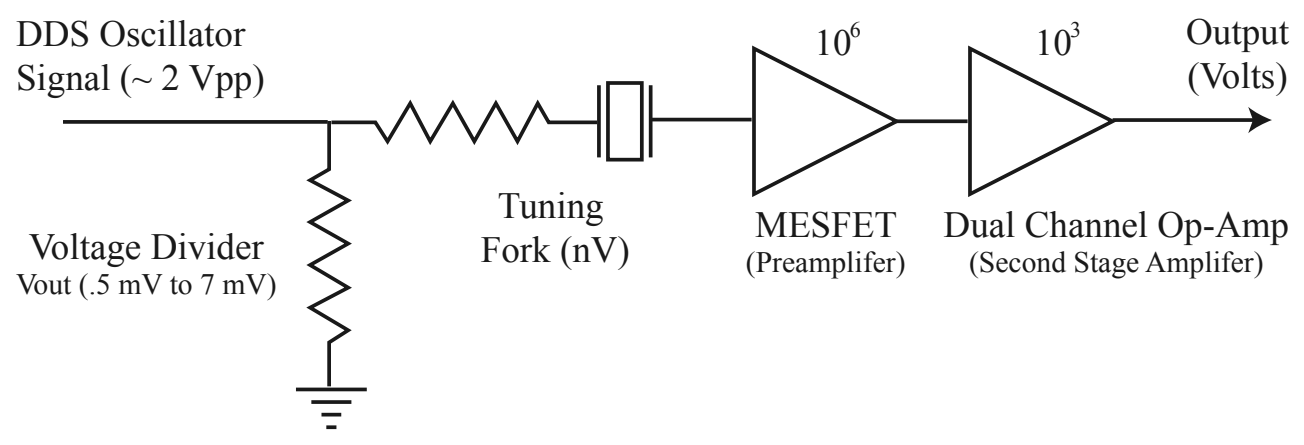

Figure 7.13 The DDS signal is used to directly drive the tuning fork, the signal is voltage divided in close proximity to the tuning fork to minimize signal degradation from the DDS chip, which is generated in the controller over a meter away from the tuning fork.

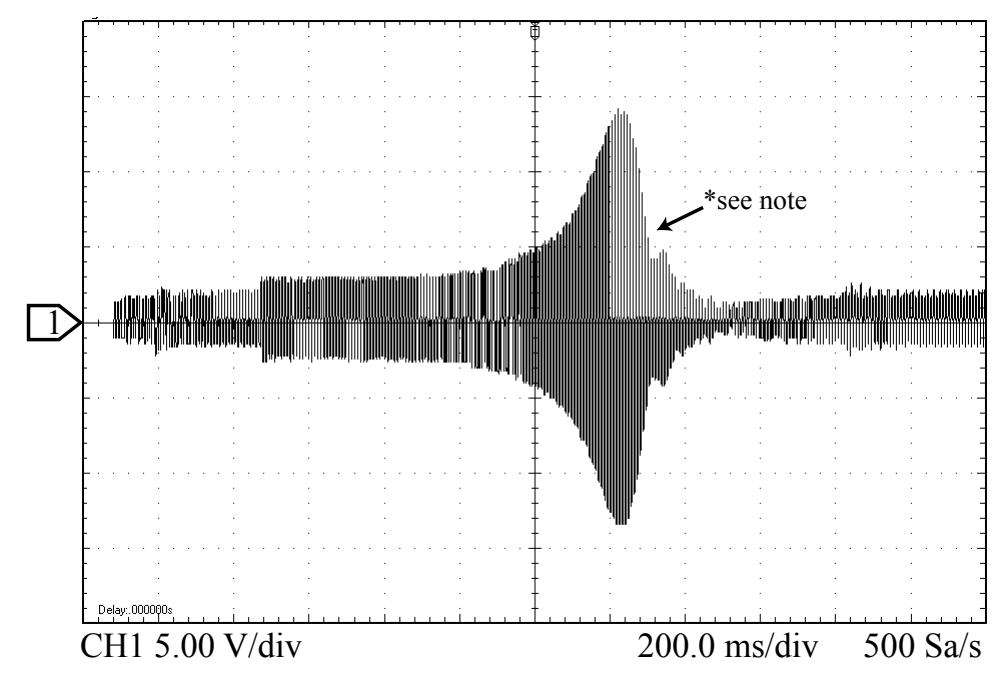

Figure 7.14 Response of the tuning fork with a sweep of frequencies, passing the tuning fork through resonance. $5.0 \mathrm{~V} / \mathrm{div}, 200 \mathrm{~ms} / \mathrm{div}$ time base. *Note: Irregularity in traces is a result of oversampling with respect to the output resolution of oscilloscope to PC dataset.

A small circuit board is placed within the piezo tube, shown in Figure 7.15 (b). The close proximity of the tuning fork to the preamp is crucial to reducing capacitive effects that slow the system response, as well as to lower the noise that can occur in long wire runs. The amplifier's proximity to the preamp exists for the same reason. 


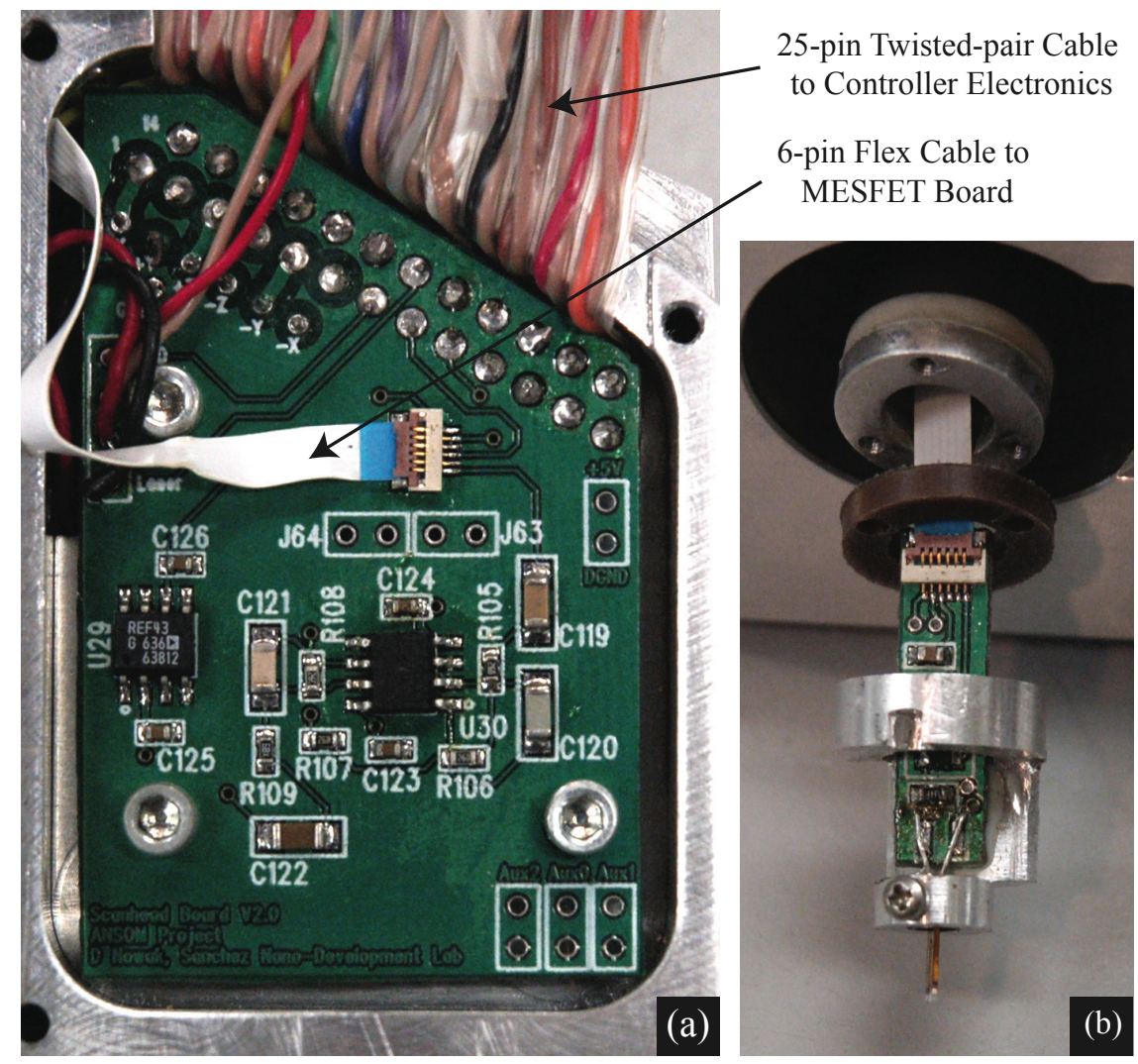

Figure 7.15 (a) The dual stage AD8599 (U30) amplifier circuit board, which is also placed in the scan head. U29 is the precision voltage source for the MESFET. (b) Small MESFET board and holder used to obtain the high gain of $10^{6}$ required to amplify the signal from the tuning fork.

The MESFET (NXP Semiconductors BF992) is configured as a common drain (source follower) amplifier circuit. The MESFET preamplifier is powered by a $2.5 \mathrm{~V}$ precision power source (Analog Devices AD REF43). The precision voltage source limits the oscillation of the MESFET due to power supply noise.

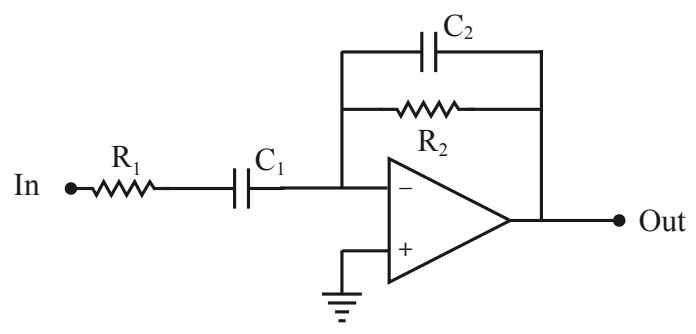

Figure 7.16 Inverting active band-pass filter. 
The dual stage amplifier AD8599 is configured as an inverting active band pass filter. Figure 7.16 shows an active band-pass filter design. The frequency of the low pass is given by,

$$
f_{L P}=\frac{1}{2 \pi R_{2} C_{2}},
$$

and the high pass is given by,

$$
f_{H P}=\frac{1}{2 \pi R_{1} C_{1}},
$$

with the gain of the inverting amplifier by the following,

$$
V_{\text {out }}=-\frac{R_{2}}{R_{1}} V_{\text {in }} .
$$

The low end is configured at $11 \mathrm{kHz}$ with the upper end configured at $73 \mathrm{kHz}$. Each stage is configured as its own filter.

\subsection{Amplitude and Phase Detection}

Monitoring small changes in the amplitude and phase of the signal returning from the tuning fork is accomplished with analog circuitry. Traditionally, lock-in amplifiers have been used for detecting the changes in the return signal but tend to be too slow to update the feedback circuitry. As discussed in Chapter 5, the quartz tuning fork oscillator suffers from a slow amplitude response to changes in interaction force, typically on the order of hundreds of milliseconds. For this reason, the measurement of the amplitude strength of the tuning fork is used to aid the user in correctly tuning the oscillator for the feedback system. From that point, the phase lock loop will be used as the feedback 
system to monitor probe height. Figure 7.17 shows the overall signal pathways for the intergration of FPGA, DDS, phase, amplitude, and tuning fork elements.

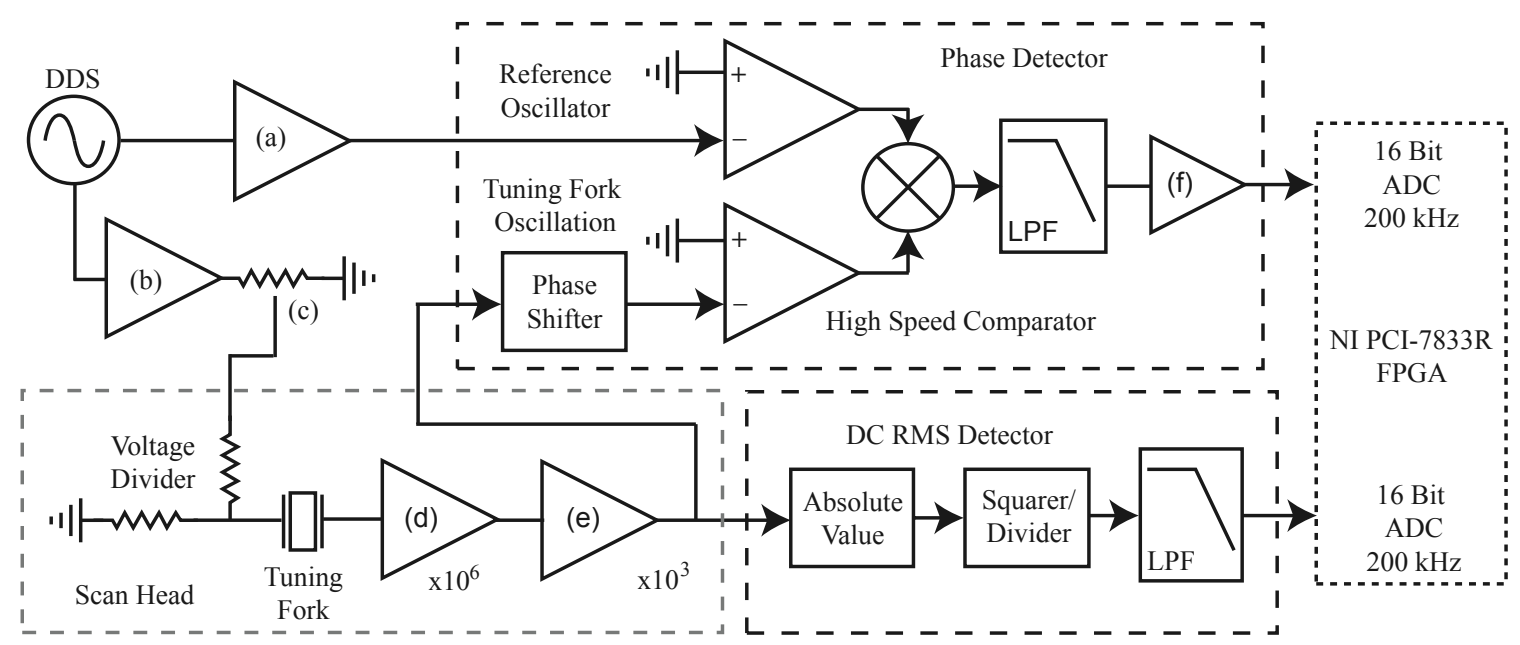

Figure 7.17 Tuning fork oscillation and detection scheme: The reference oscillator is compared to the return oscillation from the tuning fork. The tuning fork oscillation can be phase-shifted to correct for any stray system capacitance. The tuning fork oscillation amplitude is monitored to aid in tuning of oscillator, determining the quality factor of the system. (a) Summing setpoint amplifier to condition the reference signal $\left( \pm 5 \mathrm{~V}_{\max }\right)$. (b) Amplifier conditions the drive signal for the long signal pathway to the scan head, where the signal is then voltage-divided for a range of 0.3-7.0 $\mathrm{mV}$ near the tuning fork. (c) Digital potentiometer (3-wire SPI) controls the drive voltage. (d) MESFET preamplifier. (e) Two-stage amplifier, band-pass filtered between $11-73 \mathrm{kHz}$. (f) Conditions phase output to dynamic range of ADC. Details for phase detector outlined in text.

The amplitude detection for SPM involves the processing of the returning sine wave through an RMS to DC converter [Hsu, McDaniel, \& Hallen, 1997]. The RMS converter IC (Analog Devices AD637) simply takes the oscillating input voltage and computes the RMS value of the voltage it outputs as a static positive DC voltage [Analog Devices, 2007]. The general calculation performed by the AD637 RMS IC is defined by, 


$$
V_{r m s}=A v g\left(\frac{V_{i n}^{2}}{V_{r m s}}\right) \text {. }
$$

The average is calculated with a 2-pole Sallen-Key Filter [Sallen \& Key, 1955] that filters $-12 \mathrm{~dB}$ at the cutoff frequency given by,

$$
f_{c}=\frac{\sqrt{2}}{2 \pi C_{1} R} .
$$

Figure 7.18 shows the outline of a 2-pole Sallen-Key Filter. For the TENOM system, the cutoff frequency of the filter is set to $640 \mathrm{~Hz}$. The ouput voltage from the RMS circuit is then sampled by one of the analog inputs of the FPGA card. By sending the tuning fork a sweep of frequencies that center around the resonance frequency, an RMS amplitude curve can be mapped for the oscillator. The quality factor for the tuning fork and determination of the resonance frequency after a sweep of frequencies is shown in Figure 7.23. Figure 7.19 shows the PCB implementation of the AD637 RMS to DC converter. Figure 7.20 shows the effect of the RMS calculations on an oscillating signal passing through the RMS detector.

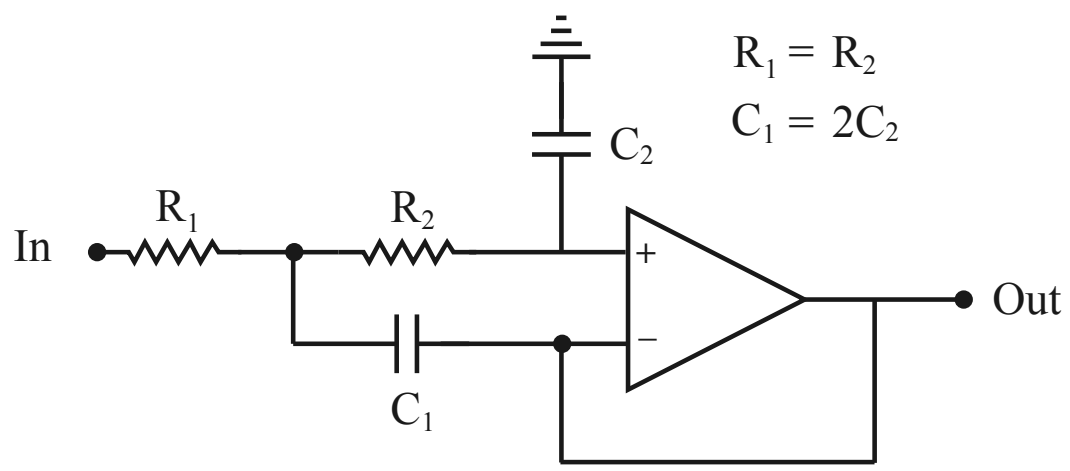

Figure 7.18 2-pole Sallen-Key filter design. 


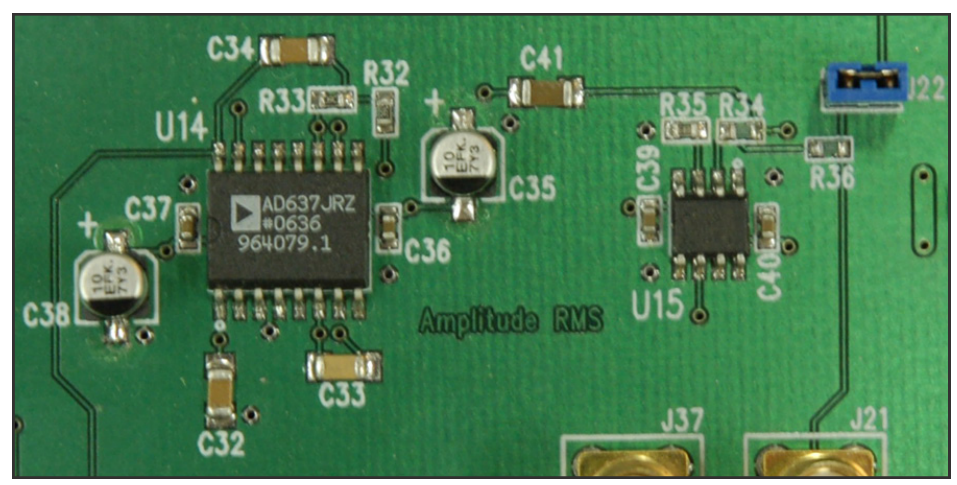

Figure 7.19 PCB implementation of AD637 RMS to DC converter. U14 is the AD637 and U15 is an OP27 to aid in conditioning the return signal from the amplifier stages in the scan head. The Sallen-Key filter is designed around a unity gain buffer amplifier built into the AD637.

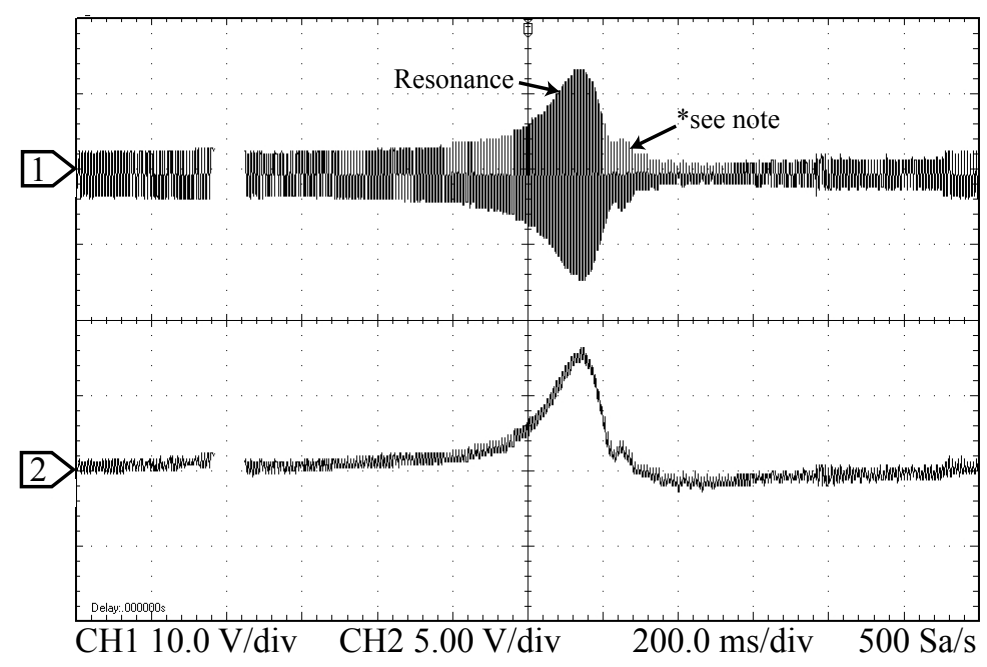

Figure 7.20 Response of tuning fork with a sweep of frequencies, passing the tuning fork through resonance (upper). RMS calculated response of the upper signal with the AD637 (lower). Channel one (upper) $10 \mathrm{~V} / \mathrm{div}$, channel two (lower) $5.0 \mathrm{~V} / \mathrm{div}$, with a $200 \mathrm{~ms} / \mathrm{div}$ time base. *Note: Irregularity in traces is a result of oversampling with respect to the output resolution of oscilloscope to $\mathrm{PC}$ dataset.

A solution to the low bandwidth response of the tuning fork is to monitor the frequency change near resonance. The frequency change is detected by monitoring a phase difference between the reference signal from the DDS and the return signal from 
the tuning fork, shown in Figure 7.17. Each signal is independently processed through a high speed comparator IC (Linear Technologies LT1712), which monitors the signals for their zero crossovers with a 4.5 ns response time. An important benefit of the LT1712 over other comparator ICs is the fast response time while maintaining TTL/CMOS logic levels, in an easily implemented circuit design. The high speed comparator compensates for hysteresis to minimize ringing when the signal crosses through zero volts. The role of the comparator is to convert the incoming sinusoidal waveforms into square waveforms. Both the noninverting output and the inverting output of the comparator are sent to a four quadrant multiplier IC (AD633). The general transfer function $(W)$ for the multipler is given by,

$$
W=\frac{\left(X_{1}-X_{2}\right)\left(Y_{1}-Y_{2}\right)}{10 V}+Z,
$$

where $X_{1,2}$ and $Y_{1,2}$ are the inputs and $Z$ is a added offset. The output signals for the comparator are configured for $4.0 \mathrm{~V}$ in the phase detector $\mathrm{PCB}$ when true, and zero volts when false. Equation 7.6 would govern that the output of the multipler has a dynamic range of $\pm 1.6 \mathrm{~V}$ when $\mathrm{Z}$ is held at zero. To increase the dynamic range of the output, the multipler is configured to include a variable scale factor, this modification to the transfer function is,

$$
W=\frac{\left(X_{1}-X_{2}\right)\left(Y_{1}-Y_{2}\right)}{10 \text { volts }}\left(\frac{R_{1}+R_{2}}{R_{1}}\right)+S
$$

By setting $R_{1}$ and $R_{2}$ to 10 kohms, an increase of 2 can be applied to the dynamic range of the output of the multipler, making a new range of $\pm 3.2 \mathrm{~V}$ while $\mathrm{S}$ set to zero. Figure 7.21 shows the configuration of the multiplier chip with a variable scale factor [Analog 
Devices, 2010]. Effectively, the multipler will only give two possible values by multiplying the four square waves, $3.2 \mathrm{~V}$ or $-3.2 \mathrm{~V}$. The result of the multiplication is processed through a $4^{\text {th }}$ order Butterworth low-pass filter IC (Linear Technologies LTC1563). The effect of the low-pass filter is to integrate with respect to time when the multipler's output. If the multiplier is outputting a continuous maximum value, then the intergration value will also be a maxima, concluding that the two input signals must be in phase with each other.

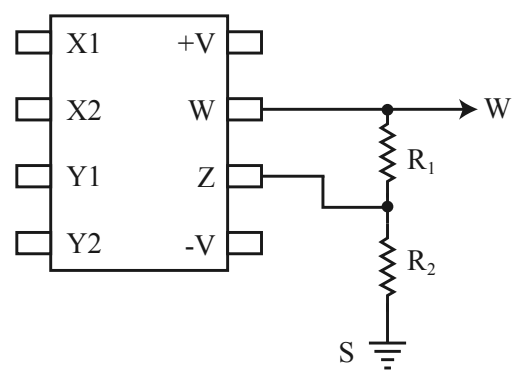

Figure 7.21 Four quadrant multiplier configured with a scaling factor. All other components removed for clarity. This allows the device to use a voltage that is scaled by the ratio of resistors multiplied by the intrinsic gain of the multiplier. S applies an offset to the output W. Setting S to ground will null any offset.

If the output square wave from the multiplier produces a contiuous minimum value then the two input signals are 180 degrees out of phase with each other. Of course, any combination within these extremes would give a intergration term somewhere between a minimum and a maximum. DC output value is digitized by the FPGA as a relative phase difference between the reference and tuning fork signal and is directly used by the PID algorithm. Figure 7.22 outlines this logic and Figure 7.24 show the phase change of the tuning fork when passed through resonance.

The finished PCB design for the phase detection circuitry is shown in Figure 7.23. 
The board is powered by $\pm 15 \mathrm{~V}$, and the voltage regulators supply the $\pm 5 \mathrm{~V}$ needed by the linear technology ICs. The analog mathematics are outlined for each stage of phase calculations in Figures 7.25 through 7.28. The figure captions specify where the measurements were taken on the phase detector PCB.

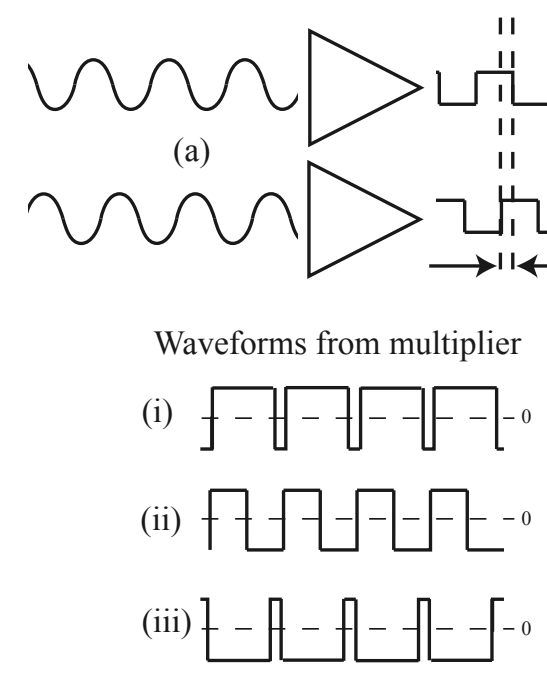

(b)
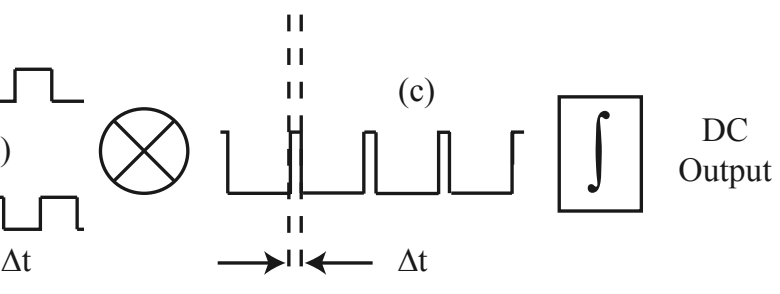

Figure 7.22 Phase detector logic: (a) Input signals pre-conditioned for comparators, (b) signals after comparison, (c) signals after multiplication. Waveforms (i), (ii), (iii) are example conditions and the effect of integration on those waveforms.

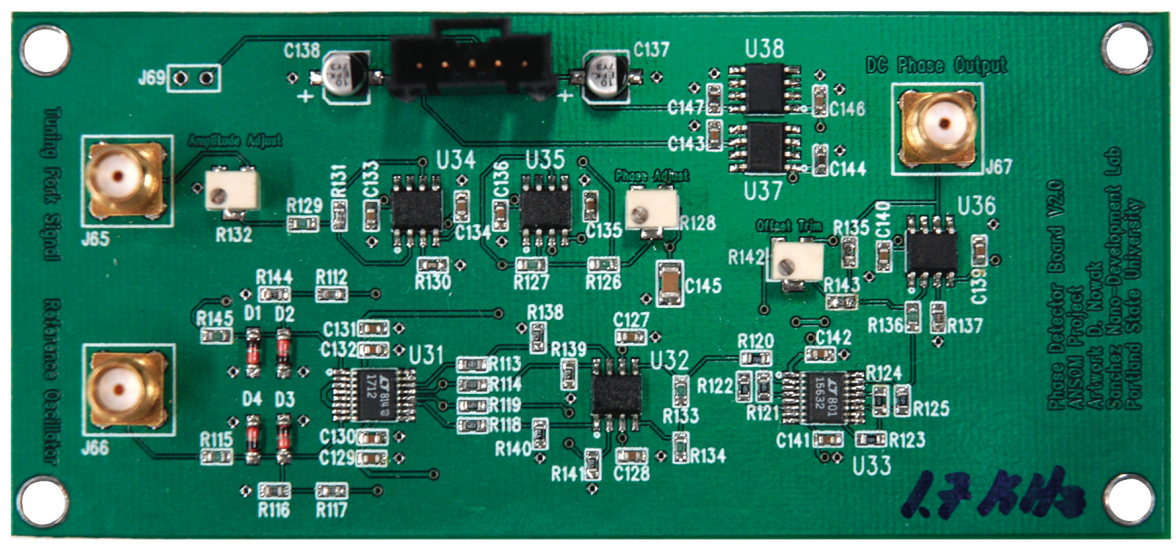

Figure 7.23 PCB implementation of phase detection circuitry. U31 is the high-speed comparator LT1712, U32 is the AD633 multiplier, U33 is the $4^{\text {th }}$ order low-pass filter, U35-36 are OP27 operational amplifiers for signal conditioning, and U37-U38 are voltage regulators. 


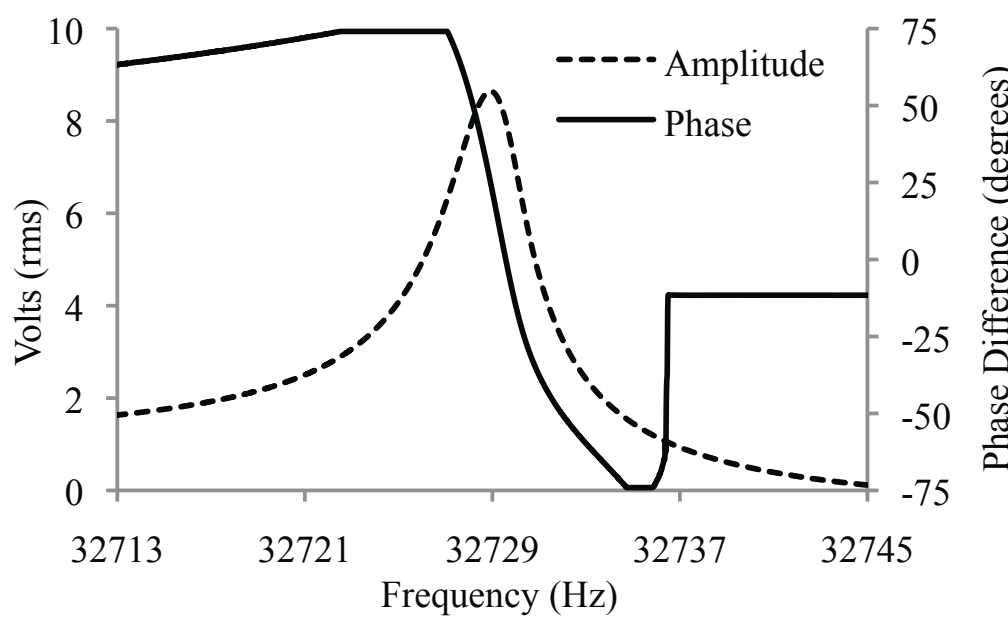

Figure 7.24 Amplitude and phase relationship for the quartz-tuning fork recorded by the feedback electronics. The saturation of the phase at $\pm 75^{\circ}$ is an artifact of the phase detector using the full dynamic range of the ADC. The phase region after $32,737 \mathrm{~Hz}$ is an artifact of the return signal (outside of resonance region) being too low for comparison. Amplitude demonstrates a quality factor of $\sim 7000$ for a bare tuning fork in ambient conditions, with the asymmetry due to the parasitic capacitance of the tuning fork.

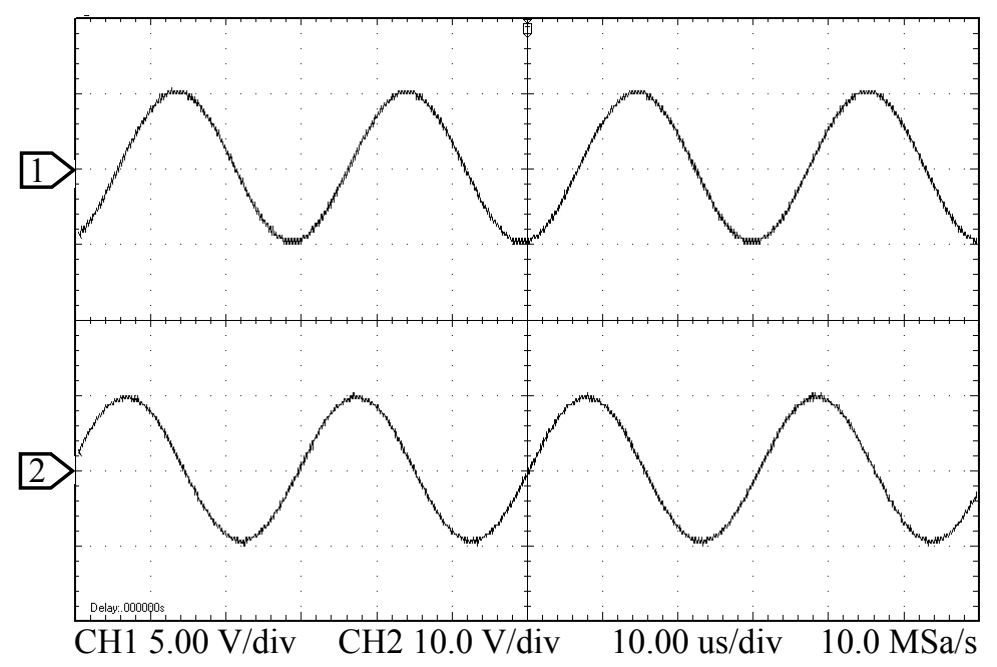

Figure 7.25 DDS reference oscillator signal, conditioned for the comparator IC (upper). Tuning fork signal after amplification, before reaching the phase detection board (lower). The tuning fork will be further conditioned through a voltage divider and phase shifting circuit, before reaching the comparator IC. Channel one (upper) $5.0 \mathrm{~V} / \mathrm{div}$, channel two (lower) $10 \mathrm{~V} / \mathrm{div}$, with a $10 \mu \mathrm{s} / \mathrm{div}$ time base. 


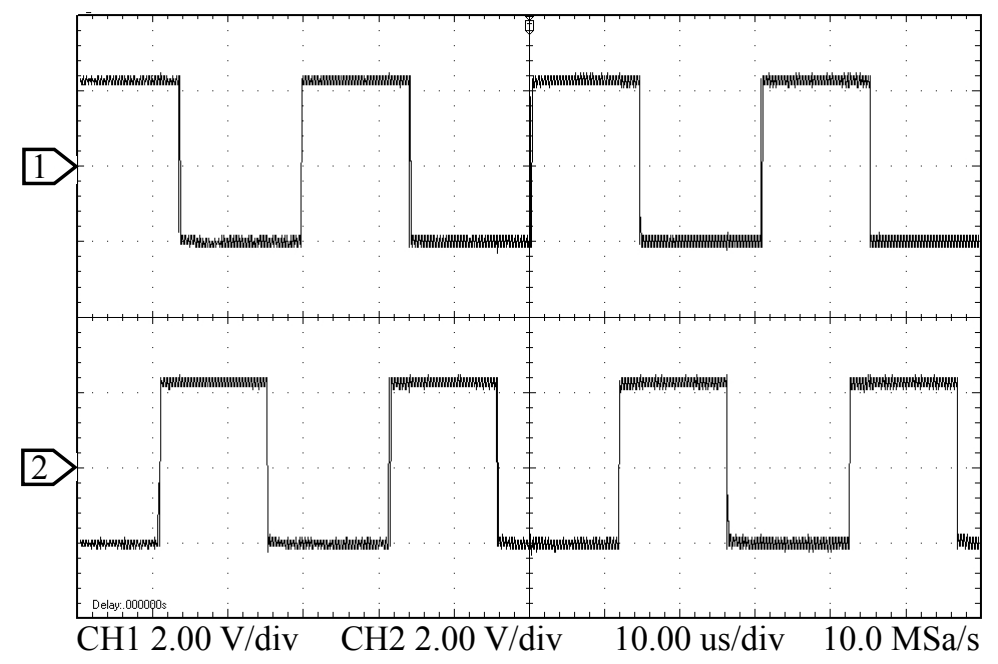

Figure 7.26 DDS reference oscillator signal after comparator IC measured at R140 (upper). Tuning fork signal after comparator IC measured at R138 (lower). Channel one (upper) and two (lower) are $2.0 \mathrm{~V} / \mathrm{div}$, with a 10 $\mu \mathrm{s} / \mathrm{div}$ time base.

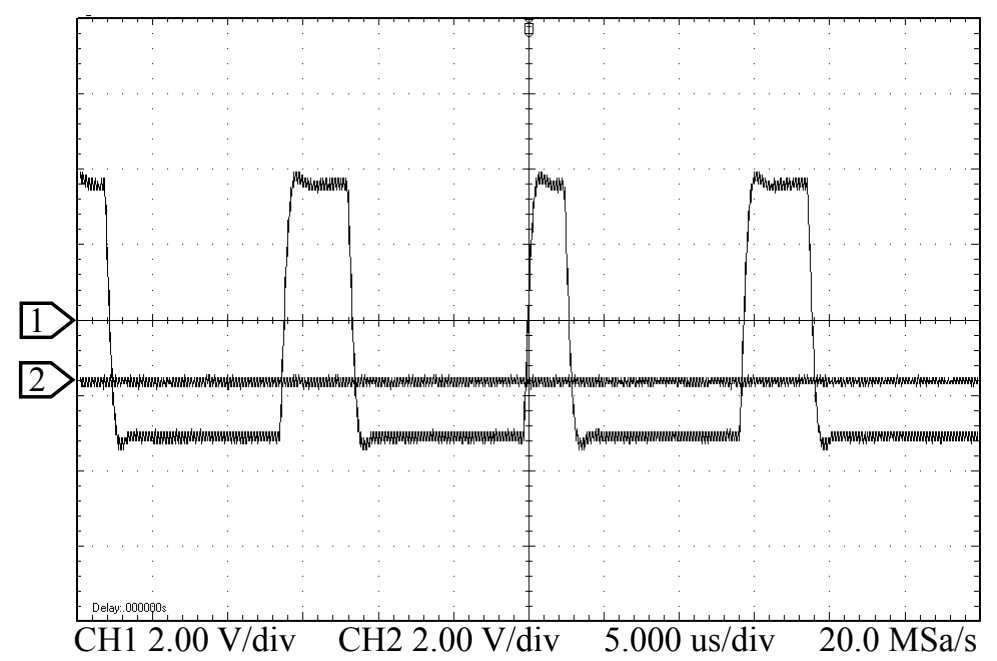

Figure 7.27 Result of the $-55^{\circ}$ relative phase difference and the variable transfer function of the four-quadrant multiplier (square wave), measured at R122. The constant voltage line represents the DC output of the lowpass filter, measured at R136. Both channel one and channel two are set at $2.0 \mathrm{~V} / \mathrm{div}$, with a $5.0 \mu \mathrm{s} / \mathrm{div}$ time base. 


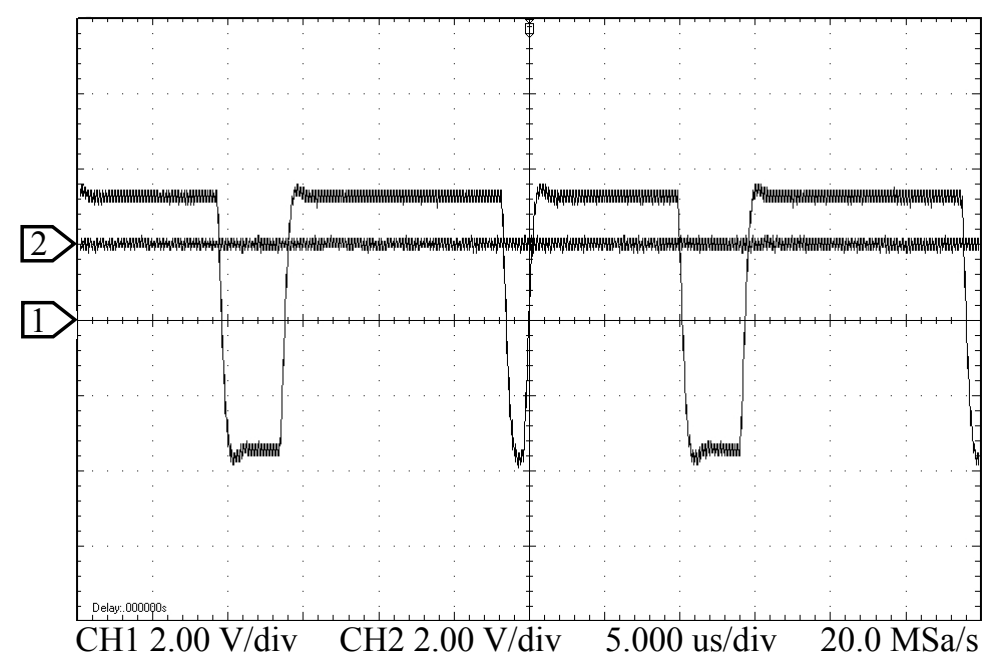

Figure 7.28 Result of the $55^{\circ}$ relative phase difference and the variable transfer function of the four-quadrant multiplier (square wave), measured at R122. The constant voltage line represents the DC output of the low pass filter, measured at R136. Both channel one and channel two are set at $2.0 \mathrm{~V} / \mathrm{div}$, with a $5.0 \mu \mathrm{s} / \mathrm{div}$ time base.

It is worth mentioning that Analog Devices has a demodulating phase detection IC (AD8302) that works in the $\mathrm{GHz}$ range and can be implemented at lower frequencies (10s of Hz) [Analog Devices, 2002; Pilotte, 2005]. Prototyping with this device showed noise complications from interference with cellular frequencies. This interference directly affected the feedback signal.

\subsection{Stepper Motor Current Control}

The Z-piezos used in this TENOM design have typical travel ranges of tens of microns. It is impractical to try to manually engage the probe with the sample, so a stepper motor combined with a high ratio gear reduction drive is. The stepper motor used in the TENOM system is an Oriental Motor (PK243B1A-SG36), with $0.01^{\circ}$ per step precision with 36:1 gear reduction. To further increase the resolution, the motor is attached to a 40 turns per inch lead screw from Universal Thread. The motor is run with 
a quarter step routine [Lo, 1978; Markus, 1980], shown in Figure 7.29, (and discussed in detail in Chapter 8) using the FPGA DIO, optocoupled (Vishay TCMT4100) to TIP31 high current transistors. The TIP31 transistors will handle half an ampere each. The FPGA controls the approach by extending the Z-piezo until the surface is found. If the surface is not found, the piezo retracts and the stepper motor steps the number of quarter steps specified by the user. The process is repeated until the surface is located. Once the surface is located, the FPGA switches from the approach algorithm to the PID loop. The PCB design for the stepper motor focuses on isolating the grounds of the stepper motor and the $7833 \mathrm{R}$ card to prevent current spikes from damaging the card or computer system. The board is powered by a $5 \mathrm{~V}$ linear power supply that does not share the star ground location of the controller electronics. Figure 7.30 shows the layout of the stepper motor PCB.

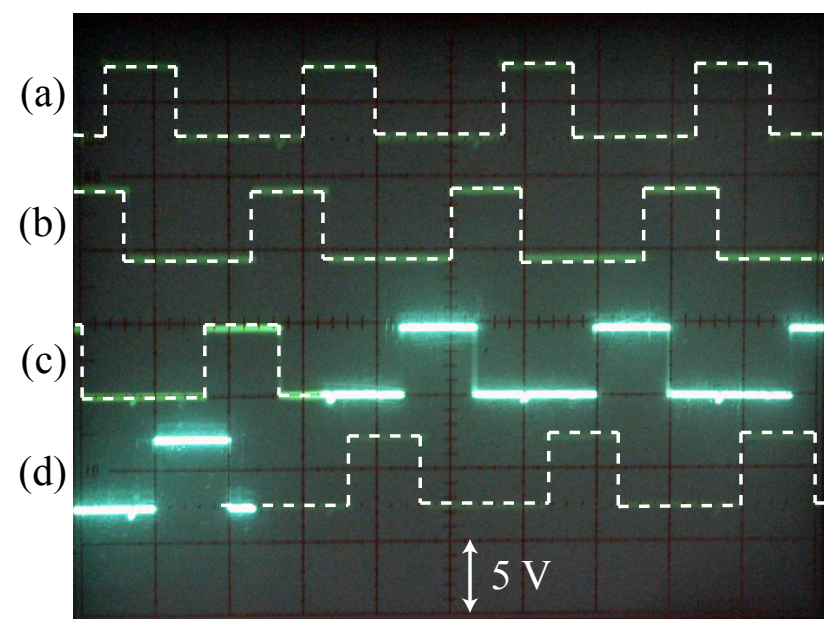

Figure 7.29 Oscilloscope trace of the four phases (a-d) of the stepper motor. Each phase is a quarter step lagging or advancing the previous based on motor rotation direction. 


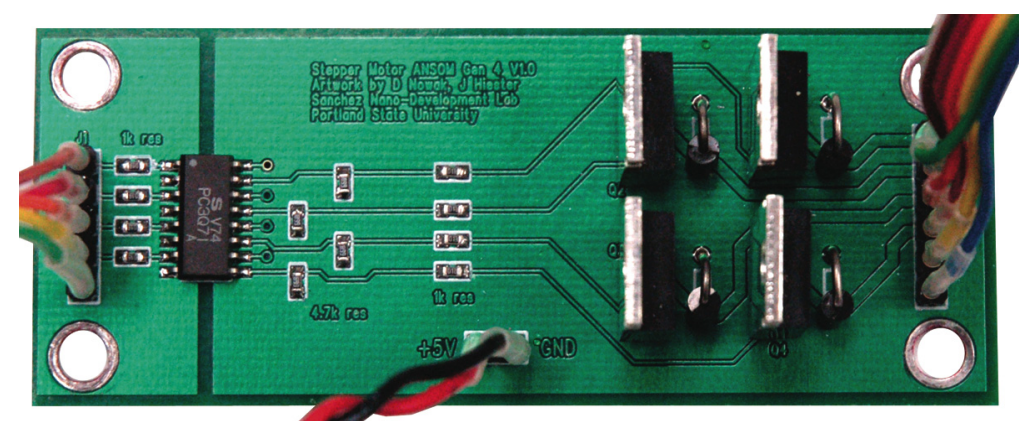

Figure 7.30 PCB implementation of stepper motor circuitry. The IC is a four-channel optocoupler and the ground planes have been separated for ground isolation. The TIP31 are protected with diodes, preventing back emf (which is generated from manually turning the motor) from damaging the transistors.

\subsection{Parasitic Noise}

In the early prototyping of the TENOM electronics, a parasitic ground noise was discovered on the analog ground locations. The ground noise affects the output of most of the analog cirucitry, including the piezo driver amplifers. Figure 7.31 shows the parasitic ground noise on the outputs of the piezo bridge circuit. There is an increase from $18.4 \mathrm{mV}_{\mathrm{rms}}$ to roughly $50 \mathrm{mV}_{\mathrm{rms}}$. This increase will change the $0.2 \mathrm{~nm}_{\mathrm{rms}}$ to 0.6 $\mathrm{nm}_{\mathrm{rms}}$. Careful analysis of the noise revealed the source to be the result of the $50 \mathrm{MHz}$ clock oscillator used as a master reference clock for the DDS IC. Figure 7.32 shows the parasitic ground noise, oscillating at the same frequencies as the clock. Various isolation troubleshooting methods proved effective at removing the oscillation noise. It was determined that the oscillation coupled into the FPGA - DAQ board through the digital ground plane, then over to the analog grounds of the DAQ, itself. The oscillation ultimatly found its way onto the analog power supplies for the PCBs. 


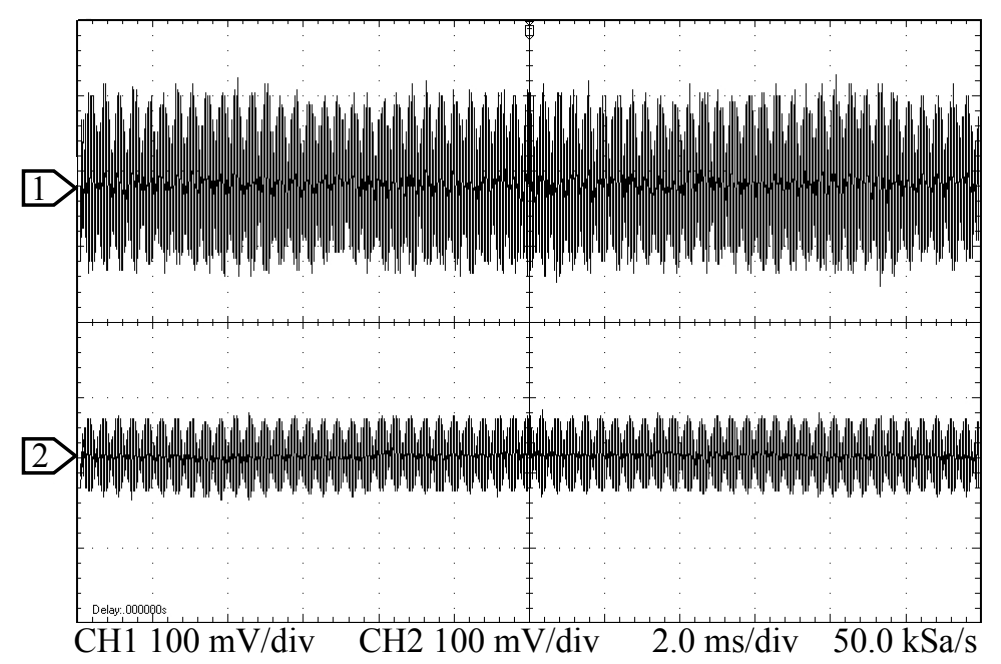

Figure 7.31 Noise output of both $+\mathrm{HV}$ (upper) and $-\mathrm{HV}$ (lower) outputs when connected to 7833R DAQ card. Both channels $100 \mathrm{mV} / \mathrm{div}$ with a $2.0 \mathrm{~ms} /$ div time base.

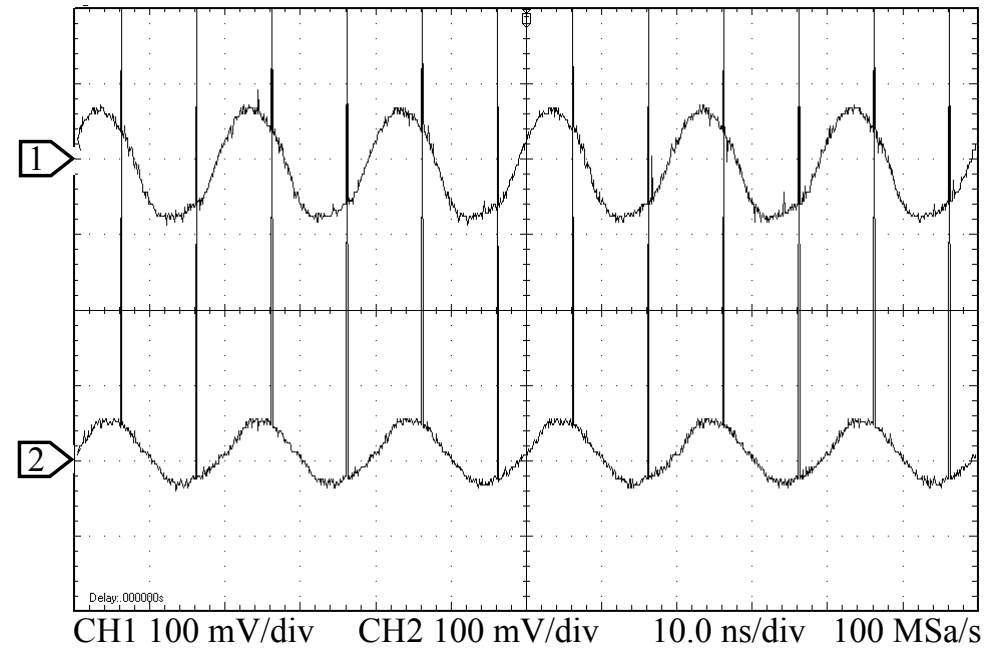

Figure 7.32 Noise output of both $+\mathrm{HV}$ (upper) and $-\mathrm{HV}$ (lower) outputs when connected to 7833R DAQ card, showing the $50 \mathrm{MHz}$ oscillations from the clock oscillator. Both channels $100 \mathrm{mV} / \mathrm{div}$ with a $10 \mathrm{~ns} / \mathrm{div}$ time base. 
A proposed method to remove the oscillations is to completely isolate the DDS circuitry. The required SPI signals would need to be isolated with an optocoulper IC and ground routing would be filtered and returned to the analog power supplies. Placing the DDS on its own PCB board would be advantangous for this isolation. 


\section{CHAPTER 7 References}

Analog Devices, “AD633 Low Cost Analog Multiplier,” Product Data Sheet, Rev. G, (2010).

Analog Devices, “AD637 High Precision, Wideband RMS-to-DC Converter”, Product Data Sheet, Rev. J, (2007).

Analog Devices, “AD8302 2.7GHz RF / IF Gain Phase Detector,” Product Data Sheet, Rev. A, July 2002.

Analog Devices, “AD9835 50 MHz CMOS Complete DDS”, Product Data Sheet, Rev. 0, July 1998.

Analog Devices, “OP27 Low Noise, Precision Operational Amplifer," Product Data Sheet, Rev. F (2006).

Behler S., Rose M. K., Dunphy J. C., Ogletree D. F., Salmeron M., Chapelier C., Rev. Sci. Inst. 68, 2479 (1997).

Bishop P., "A tradeoff between microcontroller, DSP, FPGA and ASIC technologies," EE times Europe, February 25, 2009.

Brockman D. and Williams A., Analog Devices, "Ground Rules for High-Speed Circuits," Appl. Note AN-214.

Cirrus Logic - Apex, “Driving Piezoelectric Actuators,” Appl. Note 44, May 2009.

Cirrus Logic - Apex, "PA88 High Voltage Power Operational Amplifers," Product Data Sheet, Rev. N, May 2009.

Colm Slattery, Analog Devices, "Programming the AD9832 / AD9835", Appl. Note AN621, Rev. 0 (2003).

Friet J.-M. and Carry É., "Introduction to the quartz tuning fork," Am. J. Phys. 72, 5 (2007).

Grober R. D., Acimovic J., Schuck J., Hessman D., Kindlemann P. J., Hespanha J., Morse A. S., Karrai K., Tiemann I., and Manus S., "Fundamental Limits to Force Detection Using Quartz Tuning Forks,” Rev. Sci. Inst. 71, 2776 (2000).

Heath S., Embedded Systems Design $2^{\text {nd }}$ ed. (Newsnes, New York, 2003) pp. 2. 
Hsu J. W. P., McDaniel A. A., and Hallen H. D., "A shear force feedback control system fro near-field scanning optical microscope without lock-in detection”, Rev. Sci. Inst. 68, 3093 (1997).

IPC - The Institute for Interconnection and Packaging Electronic Circuits, "Generic Standard on Printed Board Design," IPC-2221, February 1998.

Jahncke C. L., Brandt O., Fellows K. E., and Hallen H. D., "Choosing a Preamplifier for Tuning Fork Signal Detection is Scanning Force Microscopy,” Rev. Sci. Inst. 75, 2759 (2004).

Johnson H., High-Speed Digital Design (Prentice Hall, 1993).

Lo H., "Digital Display of Stepper Motor Rotation," Computer Design 147-148, 150-151, April 1978.

Markus J., Modern Electronic Circuits Reference Manual (McGraw-Hill Book Company, 1980) pp. 580.

McGrath D., "FPGA Market to Pass \$2.7 Billion by '10, In-Stat Says," EE Times, May $24,2006$.

National Instrument Corporation, "NI R Series Multifunction RIO Integrated Analog and Digital I/O with FPGA Technology", Product Brochure, July 2009.

National Instrument Corporation, "NI R Series Multifunction RIO User Manual", Product Manual, June 2009.

Pilotte M., Analog Devices, "Operation of RF Detector Products at Low Frequency," Appl. Note AN-691, Rev. 0, 2005.

Sallen R. P. and Key E. L., “A Practical Method of Designing RC Active Filters,” IRE Transactions on Circuit Theory 2, 74-85 (1955).

Sánchez, E. J., Ph.D. dissertation, “A Novel Scheme for High Resolution Near-Field Fluorescence Microscopy”, Portland State University, 1999 (UMI No. 3018652).

Sánchez E. J., Novotny L., and Xie X. S., "Near-field Fluorescence Microscopy Based on Two-Photon Excitation With Metal Tips”, Phys. Rev. Lett. 82, 20 (1999). 


\section{CHAPTER 8}

\section{Computer Control of the TENOM Microscope}

Controlling the tip enhanced near-field optical microscope (TENOM) is completely dependent on the use of computers to make real time decisions at speeds faster than typical human response times. The computer automation of the TENOM system has two independent layers of computer processing. The field programmable gate array (FPGA) will make up the first layer. The FPGA runs all the core logic and automated routines: oscillator tuning, data acquisition scan, approach, and proportional integral differential (PID) algorithm. The FPGA program is really the personality behind the microscope. The second layer is the user interface that runs the graphical user interface (GUI) that interacts with the FPGA program to control the microscope. The second layer requests information from the FPGA to display and save incoming data, allowing the user to change parameters in real-time in a Windows operating system environment. An overview of the FPGA algorithms are presented in this chapter with a general overview of important tools developed to make the microscope user friendly.

\subsection{LabVIEW Programming Environment}

The programming environment used for the development of the microscope is LabVIEW (Laboratory Virtual Instrumentation Engineering Workbench), versions 8.2, 8.5, 8.6, and 2009. Programming in LabVIEW allows for the rapid integration of a Windows-based GUI. Figure 8.1 shows a basic algorithm implemented in the LabVIEW environment. LabVIEW also allows for the seamless integration of (FPGA) programming 
in a Windows user environment. Traditionally, this would require a vast understanding of C, C++, Visual Basic, Assembly Language, VHDL, Direct Memory Access (DMA) transfers, and Windows device drivers. LabVIEW removes a lot of the complex overhead, and allows for quick prototyping and modification of code. This greatly accelerates data acquisition programming time.

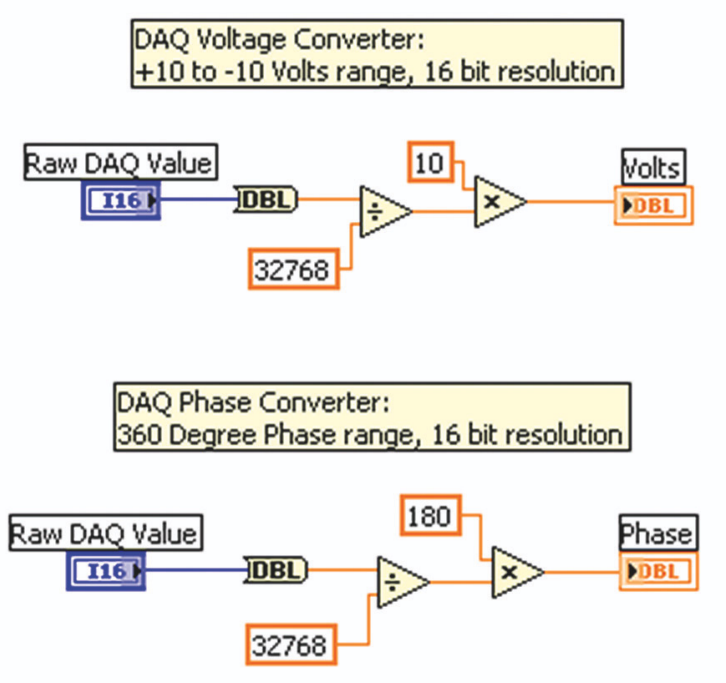

Figure 8.1 Demonstration of LabVIEW programming environment. DAQ voltage converter takes the raw 16-bit integer and converts it to a double precision floating point value, representing the recorded voltage. DAQ phase converter does the same but scales the value to represent a predefined phase value.

LabVIEW uses files referred to as VIs (Virtual Instruments) that are composed of a GUI window (front panel) and a block diagram. The block diagram is built with icons and virtual wires to specify data flow direction (function blocks, as shown in Figure 8.1), integrated with other VIs or subVIs, used as GUIs, or forced to run in the background. These VIs can then be compiled into executable files for Windows, Mac OS X, and Linux operating systems. Here, the focus will be on the Windows operating system for full compatibility with the PC based FPGA hardware. 


\subsection{FPGA Real Time Programming}

Programming for FPGA hardware uses the same programming environment in LabVIEW that is used for Windows GUI program. The VIs that are created for the FPGA will be compiled into VHDL before runtime, then saved as a bit image to be flashed* onto the FPGA. Once the required logic has been programmed into the FPGA hardware, recompiling will only be necessary if there is a logic change in the development environment. From this point, all references that the Windows (Host) files make will be in reference to the bit image. In other words, the host programs will pass variables to or request variables from the FPGA (compiled) reference VI, through an interrupt request. For high-speed data transfer, the FPGA has DMA first-in first-out (FIFO) buffers, to bypass the slower interrupt request method. The only drawback in VI programmability is a reduced programming function set that can be used with the FPGA.

\section{2.a Responsibilities of the FPGA DAQ}

For the TENOM system, the FPGA will run subroutines that require autonomy from the Windows OS. The execution of these subroutines will be done in real-time in order to successfully run the microscope, relying on Windows to determine the start and stop of these routines as the user requires. These routines include a scanning algorithm, which must synchronize timing of data, SPI communication, timing for a four-phase stepper motor, sample surface approach, and high speed digital counters. The FPGA will also control dynamic variable timing control and interlock controls. Windows could run these controls but implementation on the FPGA will allow the developers and builders of

\footnotetext{
* Process of writing the image onto the FPGA IC.
} 
the microscope to debug code and electronics within the FPGA VI development environment.

The dynamic variable timing control is an implemented feature that limits the speed of a variable change on the FPGA. For example, if a user would like to change the setpoint for the PID loop of the microscope, the variable would only be allowed to change at a specified rate. In other words, the variable would not change directly to the newly requested value. It would, instead, inclement or decrement at the user specified rate. The implemented logic is to minimize the reaction rate of certain parameters of the shear force microscope. A detailed discussion of the major autonomous algorithms will be discussed in the following subsections, along with the presentation of important code in Appendix D.

\section{2.b FPGA Toolkit}

The FPGA Toolkit has a reduced number of functions when compared to the more general Windows LabVIEW functions. As a discrete device, the FPGA toolkit provides loop structures, array building and manipulation, data clustering, integer mathematics, Boolean logic, FIFO buffers, local memory allocation, and commands to extract data from the DAQ ports. The LabVIEW FPGA architecture does not allow for native support of floating point datatypes. This limits the number of complex mathematical computations that can be processed on the FPGA. This forces the end user to be very conscious of the finite FPGA resources available to them. It is still possible to implement fractional integer values using a fixed-point datatype that is similar to floating-point with limited precision, using scaled integers. The IEEE Standard for Binary Floating-Point Arithmetic (IEEE 754) allows a programmer to encode floating- 
point values (of definable resolution) into unsigned integers [IEEE 754, 1985]. IEEE 754 defines the datatype by,

$$
(-1)^{\text {sign }} \times 2^{\text {exponent }-127} \times 1 . \text { mantissa }
$$

where the sign bit simply determines the sign of the number. The exponent is 8-bits in width and biased by 127 for efficient floating-point conversions. Finally, the mantissa determines the precision of the number. The LabVIEW FPGA allows for the use of fixed-point datatypes, which allows for correct implementation of integration and differentiation algorithms used in the PID feedback loop.

The values used to interface with the $\mathrm{ADC}$ and the $\mathrm{DAC}$ are numerical representations of binary. For example, the 7833R card used in the TENOM system has 16-bit ADCs and DACs that operate between $+10 \mathrm{~V}$ to $-10 \mathrm{~V}$. The 16-bit numerical representations are values ranging from $-32,768$ to 32,767 ; only values in this range will be sent to the host program. It will then be the job of the host program to convert that binary representation to a floating-point voltage value, demonstrated in Figure 8.1. One immediate benefit of this conversion is the ability to add constants to the converting functions, allowing for calibration values to be entered into the host without the need to recompile the FPGA VIs.

\section{2.c SPI Communication}

Serial peripheral interface (SPI) bus is a data communication protocol, in which one device transmits data to another via synchronous serial communication. The SPI protocol requires at least three wires for communication. These include SDATA (serial data), SCLK (serial clock), and CS (chip select). SDATA is the wire that carries the 
digital programming word to the device. SCLK provides a reference timer for the loading of the programming word. When CS is set too low, the device is ready to receive a data transmission. At this point, when SCLK rises from high to low, the bit on the SDATA line will be loaded. This process repeats until the entire word is read, at which time the CS returns to high, ending the transmission process. Figure 8.2 demonstrates the basic SPI three-wire process.

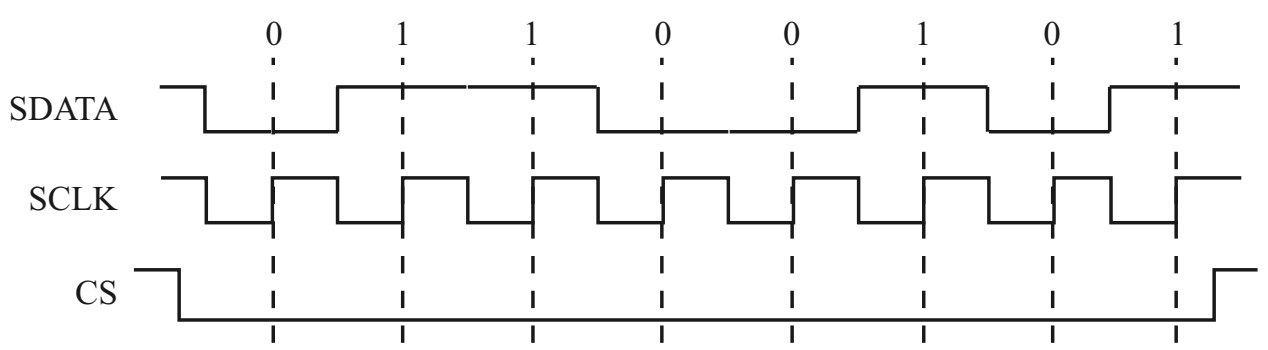

Figure 8.2 Three-signal communication of SPI. The CS signal opens the SDATA for communication, while the SDATA is read with the rising of the clock signal (SCLK).

Implementing SPI on a FPGA requires three digital output lines for the three communication ports of the SPI device. Timing characteristics vary between different ICs. For example, the AD9835 DDS oscillator and the AD5290 digital potentiometer (both used in the TENOM system) have a maximum SCLK period of $20 \mathrm{MHz}$ and 4 MHz, respectively [Analog Devices, 1998; \& 2010], since the SPI communication within the TENOM system will be setting quasi-static values in the ICs. The timing needs for SPI communication is simplified by making a robust algorithm with the SCLK period set to $2 \mu \mathrm{s}$ in the FPGA. The algorithm functions as follows: The CS line is set to false, followed by a $1 \mu$ s wait time, at which point the first bit is set on the SDATA line, the SCLK is cycled from false to true, then back to false, symmetrically across $2 \mu \mathrm{s}$. This 
continues until the final bit is sent, after which CS and SDATA are set to false. A flow chart of the algorithm is presented in Figure 8.3, along with a piece of example SPI communication code presented in Appendix D.

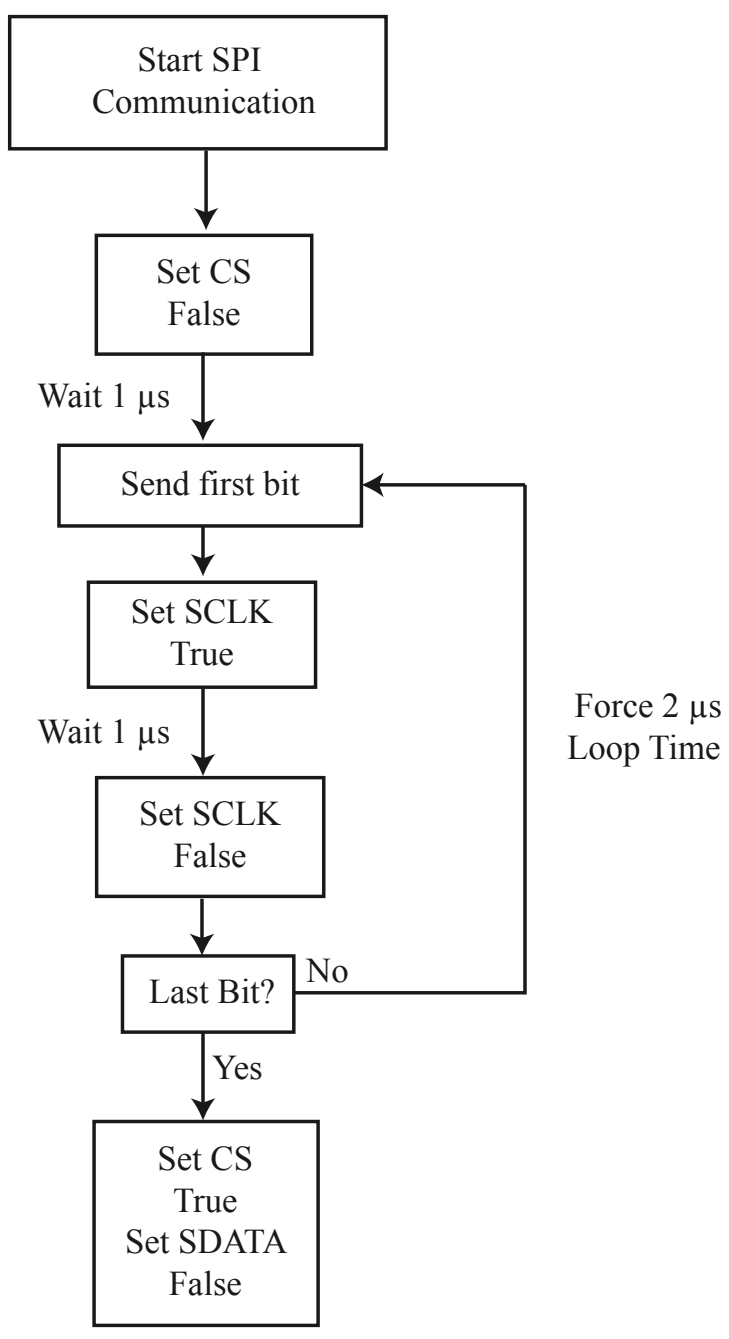

Figure 8.3 FPGA communication and timing algorithm for the SPI protocol in the TENOM system. The algorithm will be used to send 8 or 16-bit words to various ICs in the system. 


\section{2.d PID Algorithm}

The Proportional Integral Differential (PID) algorithm is arguably the most important aspect of the TENOM system. The generic algorithm for a PID loop is shown in Figure 8.4. The goal of the PID loop is to maintain a setpoint [D'Azzo \& Houpis, 1981; Rowland, 1986]. The setpoint for a shear force microscope is usually set to maintain a constant interaction force between the imaging tip and the surface of the sample. This is typically accomplished by monitoring amplitude or a phase difference between a reference oscillator and the return signal from the tuning fork [Binning, Quate, \& Gerber, 1986; Albrecht, 1991]. The PID takes the current difference (error) between the setpoint and measured feedback signal. This difference is then processed through a proportional gain equation, which directly multiplies a proportional constant to the error. The difference is also processed by an integral gain equation, which retains a prior history of the error by including the previous error values in the integration. This equation is also multiplied by an integral constant to add weight to its effect in the PID algorithm. Finally, a differential equation is used to process the error value.

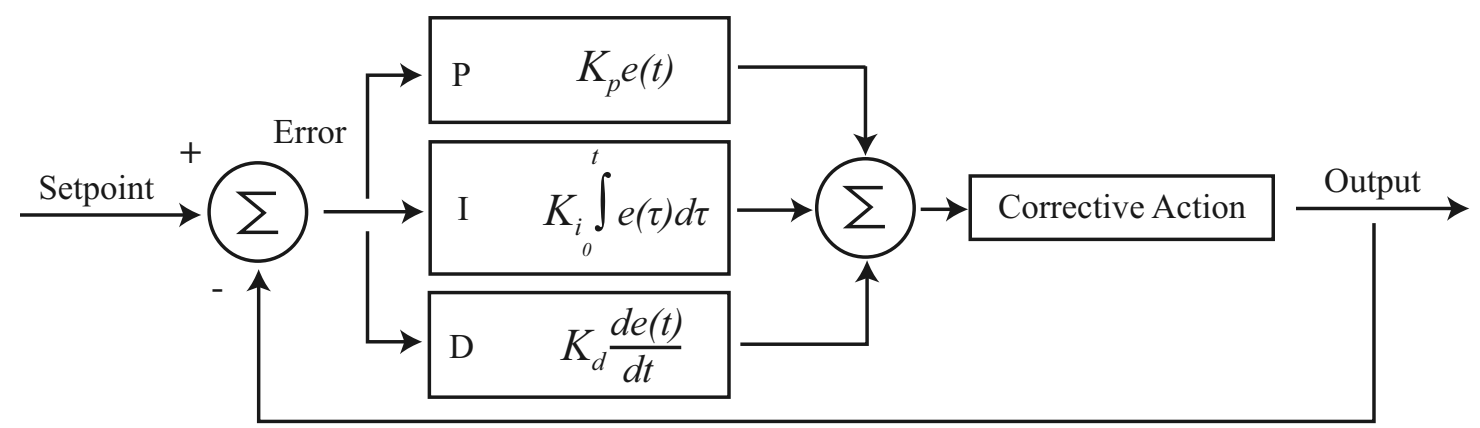

Figure 8.4 PID algorithm flow chart: The difference between the desired setpoint and the feedback signal is parallel processed by a proportional, integral, and differential gain equations, then summed together as the correction needed to maintain the setpoint. 
In SFM, differential gain equations are rarely used since they tend to magnify electronic noise from the microscope itself [Grober et al., 2000]. The user of a PID loop will have to select values for P, I, and D. Tuning of the PID is done manually to obtain the best tracking of the surface.

Complications in the PID algorithm will affect the microscope's ability to successfully scan a surface by maintaining a constant interaction force. Ultimately, this will lead to irreproducible scans and damaged tips. During the development of the TENOM system, difficulties with the PID algorithm plagued surface scans for several months, until a complete analysis and understanding of the National Instruments provided discrete PID for LabVIEW FPGA implementation could be made. During scanning, the proportional and integral gain equations serve two different but similar tasks. While they are both designed to take the error in the feedback signal and create a correction action to restore the setpoint, they both do this in their own way, with different effects. The proportional gain reacts immediately to any abrupt change in surface topography. The proportional gain has no history, so it will always reference the error by applying a negative scale value of the error. To temper this effect, the integral gain will allow for the tracking of long-term sample changes such as sample tilt, by factoring in the previous error values into its integration. The difficulty in the LabVIEW discrete PID toolkit is that the integral gain equation is lacking the long-term history needed for SFM bandwidths, causing the integral gain to act as if it was a proportional gain. The lack of dynamic range in the integral scaling term created this effect. To remedy this complication, the LabVIEW provided algorithm for the integral gain equation was 
modified at the source to increase the dynamic range of the integral term. A detailed discussion showing the code change is presented in Appendix D.

\section{2.e Scan Algorithm}

The most complicated algorithm in the FPGA logic is the scan algorithm. The scan algorithm requires the synchronization of multiple sub-routines that are interacting with each other. The highest level of the algorithm is the raster scan generator. The raster scan creates a trace and re-trace line in the $\mathrm{x}$-axis for each pixel depth in the $\mathrm{y}$-axis. Figure 8.5 shows a dashed line that represents the raster scan path of the probe. The raster scan is composed of two triangle waveforms that are generated in real-time as the

probe scans. A linear interpolation function is used in conjunction with Boolean flags to determine if the scan is in a trace, re-trace, up, or down parts of the waveforms. The xaxis and y-axis are generated from the same algorithm, with the y-axis function slowed to create a longer period than that of the x-axis. The two scanning waveforms are synchronized to start simultaneously by the FPGA. The relationship between the $\mathrm{x}$ and $\mathrm{y}$-axis periods is given by,

$$
t_{y}=2\left(\# \text { pixels }_{x}\right) t_{x} .
$$

If $t_{x}$ is normalized to one for 512 pixels, then the scan rate for $y$ will be $1 / 1024^{\text {th }}$ that of the scan rate for $\mathrm{x}$. Square scan areas where the number of pixels in $\mathrm{x}$ and $\mathrm{y}$ are the same is the current limitation of this algorithm.

The data collection during the scan is handled by three routines that are continuously checking for the start of pixel, midpoint of pixel, and end of pixel conditions, while calculating the new values for the next pixel. Figure 8.5 shows an 
overview of the data collection per pixel. The start pixel routine and the midpoint pixel routine check every clock cycle of the FPGA in a single cycle timed loop for the value that defines the start pixel or midpoint pixel from the linear interpolation waveform function in real time for the x-axis. Since the scan waveforms can only update once a clock cycle, this guaranties that values in the waveform will not be missed. The start pixel routine will also handle the DMA transfer for the pixel information, counts, topographic, feedback error, current y line, and x pixel.
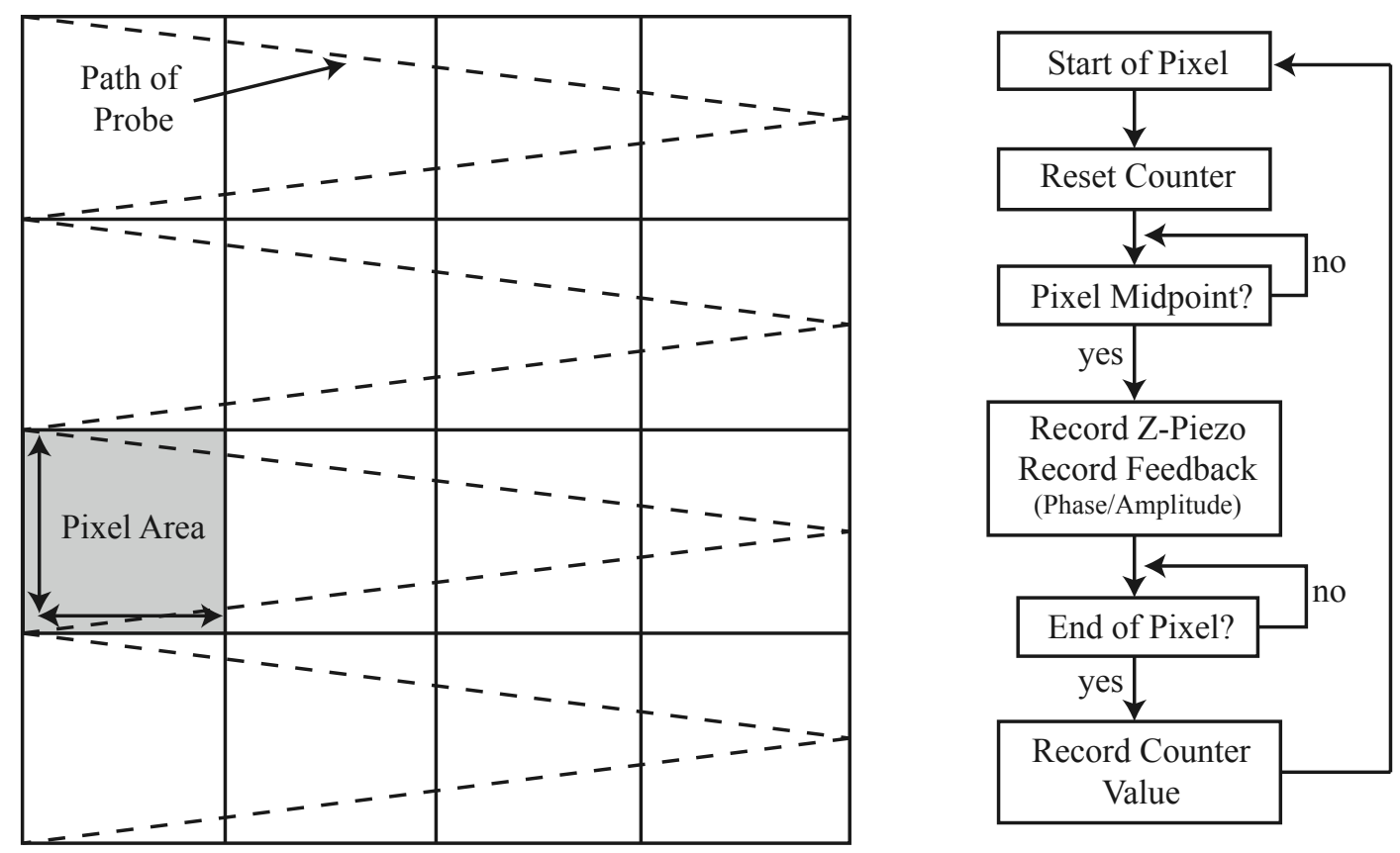

Figure 8.5 Scan algorithm for a four by four pixel scan area. The dashed line represents the path of the probe as it creates a trace and retrace line for each y-axis pixel. The algorithm for data synchronization runs in its entirety for each pixel.

Based on the current direction of the microscope (trace, retrace) and the number of imaging pixels, the FPGA will calculate the next pixel parameters (start, stop, and midpoint) in real-time, based on the current location the imaging probe in the scan 
waveform algorithm. Any Windows request to move the tip to a new location (i.e. top of page, bottom of page, start and stop scanning) will be interpolated to the new location in single DAQ step increments, at the imaging scan rate defined by the user.

\section{2.f Stepper Motor $1 / 4$ Step Algorithm}

The stepper motor was developed to generically drive any type of $1 / 4$ step fourphase stepper motor, independent of manufacture, current, or timing requirements [Lo, 1978; Markus, 1980]. The algorithm uses an array of motor states (8 in total), where an index pointer flags the appropriate logic state of the motor. Based on the direction of motor drive, the pointer is either incremented or decremented through the array to determine the next motor state. Figure 8.6 demonstrates this logic. The algorithm incorporates the ability to run in a single pulse mode, where the motor takes a discrete number of $1 / 4$ steps predetermined by the user, or in a continuous mode, where the motor is driven directly by a push button on the Windows GUI at run-time.
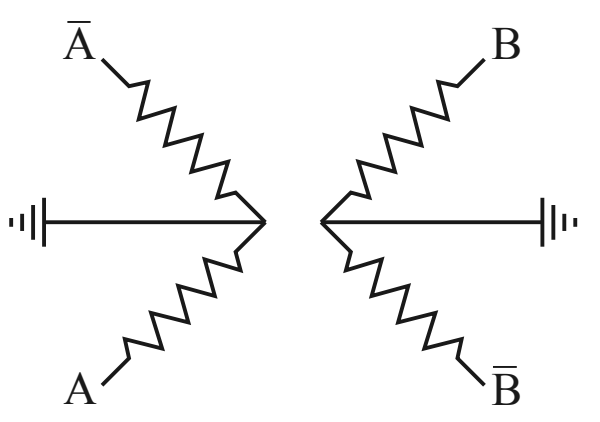

\begin{tabular}{ccccc}
$\mathrm{A}$ & $\overline{\mathrm{A}}$ & $\mathrm{B}$ & $\overline{\mathrm{B}}$ & Decimal Eq. \\
\hline 1 & 0 & 0 & 0 & 8 \\
1 & 1 & 0 & 0 & 12 \\
0 & 1 & 0 & 0 & 4 \\
0 & 1 & 1 & 0 & 6 \\
0 & 0 & 1 & 0 & 2 \\
0 & 0 & 1 & 1 & 3 \\
0 & 0 & 0 & 1 & 1 \\
1 & 0 & 0 & 1 & 9
\end{tabular}

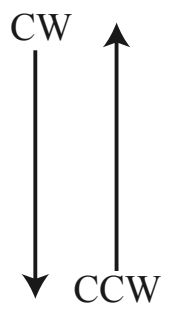

Figure 8.6 Stepper motor four-phase logic for $1 / 4$ steps. The FPGA represents the phase conditions (on, off) with the decimal equivalent of binary representation of the phase.

Continuous mode allows for course positioning of the probe, millimeters above the sample surface, while pulse mode is used almost exclusively for the surface approach 
algorithm discussed in the following section. The phase logic is sent to a current driver (which would be motor specific) and the timing of the steps can be altered to accommodate different motor timing requirements.

\section{2.g Surface Approach Algorithm}

Automated computer control is required to engage the imaging probe on the surface without impacting the surface and damaging the probe. The average distance between the sample and the tip during imaging is only $2-20 \mathrm{~nm}$. An analogy of the difficulty of moving the tip from $2.0 \mathrm{~mm}$ above the surface to only a few $\mathrm{nm}$ above the surface would be similar to moving the moon from its orbit to 1 meter above the earth's surface in roughly 60 seconds, without hitting the surface of the earth [West, 2007].

The surface approach algorithm uses a combination of the z-piezo and the stepper motor to "stitch" the imaging probe onto the surface, shown in Figure 8.7. First, the zpiezo is extended forward at a predetermined rate.

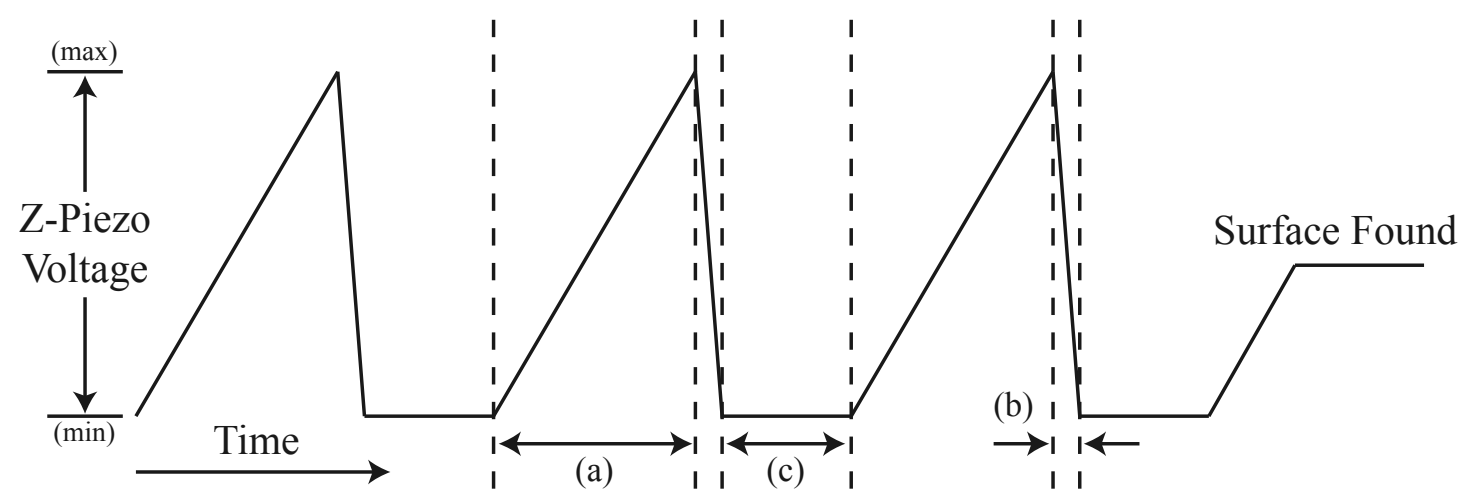

Figure 8.7 Sample approach algorithm: (a) is the approach swing of the zpiezo, followed by (b) which is a quick retract swing, after (b), the stepper motor is pulsed a predetermined distance, (c) is the delay time to allow for the stepper motor movement before the next approach swing. The process is repeated until the surface is located. 
During the extension of the piezo, the feedback signal is closely monitored for a change (signaling the location of the surface). If a change is found, then the approach algorithm is stopped and the control of the z-piezo is passed to the PID algorithm. If the surface is not located, then the piezo is retracted and the stepper motor is instructed to take a step toward the surface. A delay is used after the step instruction to allow for motor settling time. This process is repeated until the surface is located. During run-time, the rates for piezo extension and retraction and stepper motor delay time are changeable, at the user's request.

\subsection{Programming the GUI}

The Windows GUI will be the program that the user interacts with during microscope run-time. Keeping with the modular theme of the electronics of the TENOM system, the Windows GUI is also modular. Having a modular GUI allows the microscope user to customize the functions they choose to have running while imaging. For example, if running the microscope as a pure SFM, there would be no need for photon detection information to be displayed. This reduces clutter and allows one to focus on their specific experiment by making the system easier to understand and use. The following sections give a detailed account of the microscope's major GUI interfaces. Specific LabVIEW code that deserves closer investigation is presented in Appendix D.

\section{3.a Program Structure}

The microscope GUI is designed around a central VI (ANSOMEntry.vi) that was programmed to allow several subVIs to run in parallel. Figure 8.8 shows the front page of the VI. The central VI gives the user access to all the functions, programmed for 
running the TENOM microscope. These functions are broken up into five main categories: file options, diagnostic programs, main system controls, third party image processing, and help menus. The entry VI also allows the user to toggle the power of the SPM hardware controller on and off, in order to facilitate imaging probe exchanges and tuning fork tuning with the Z-piezo disabled.

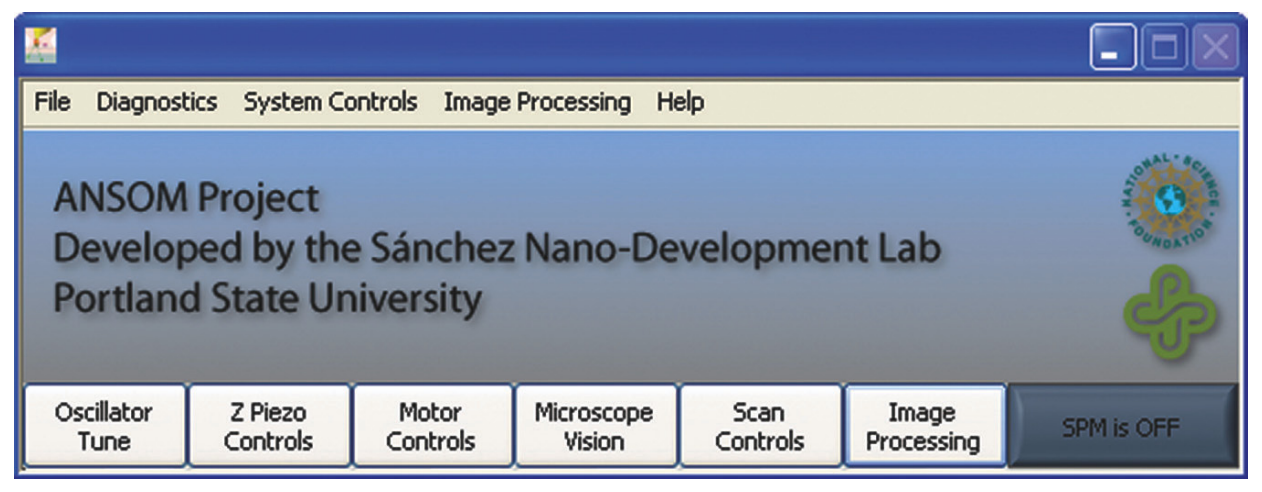

Figure 8.8 Main Entry VI for the TENOM microscope uses a combination of action buttons and drop down menus. ANSOM project is the dissemination name for the microscope design and documentation as an open system design. See Chapter 15 for more information.

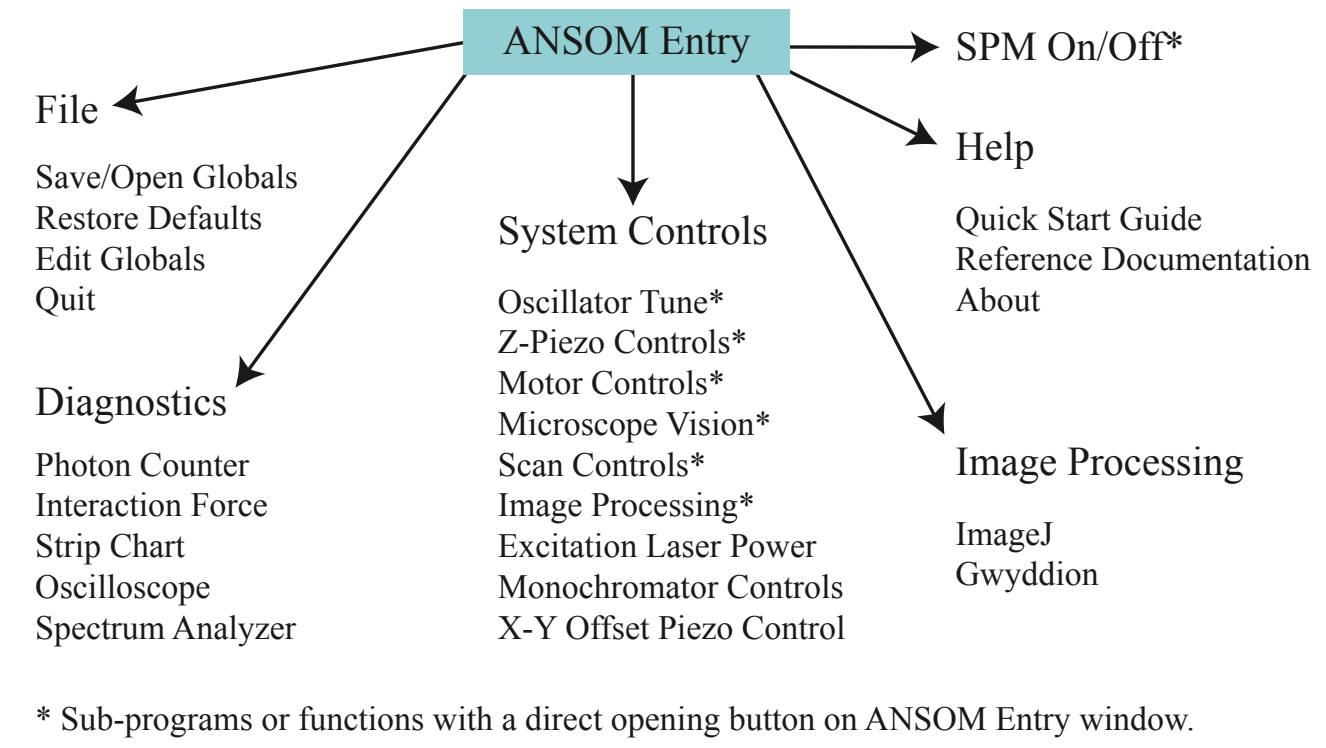

Figure 8.9 Windows program tree overview. 
Figure 8.9 gives the details of the programs that are available under each of the five categories. The entry VI also handles the loading of the global constants for the microscope programs. LabVIEW programming-specific details for the some the subVIs presented in this and the following subsections are located in Appendix D, including a list of the global constants for the software.

\section{3.b Z-Piezo Control}

The Z-Piezo Control GUI, shown in Figure 8.10, allows the microscope user direct access and control of the z-piezo element of the SFM. The GUI provides real-time information in regards to the voltage and position of the z-piezo, along with real-time feedback values.

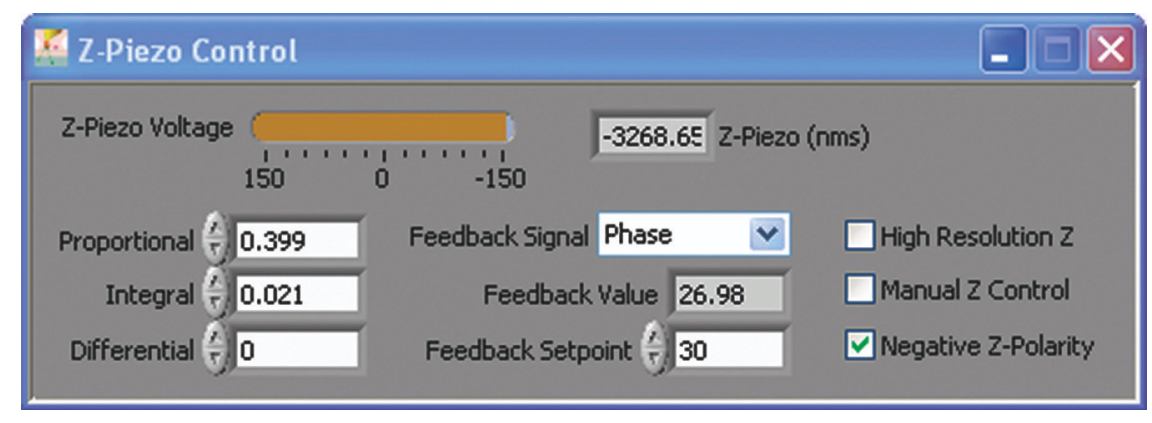

Figure 8.10 Z-Piezo control GUI. Shown with typical operation values for proportional, integral and differential gain.

Real-time z-piezo information is invaluable in determining false engagement conditions and whether or not the piezo has been retracted from the surface for sample repositioning. The GUI allows the user to input values for the PID algorithm and the feedback setpoint. These values are updated immediately after entry, by flagging a value change interrupt condition in the program. The user also has the ability to select a high-resolution $\mathrm{z}$ mode of imaging, control the z piezo manually, and set the polarity of the z-piezo. 


\section{3.c Oscillator Tune}

The Oscillator Tune GUI allows the user to tune the tuning fork oscillator before imaging. The user defines a center frequency in which to sweep along with the resolution of frequency steps in the sweep. The amplitude of the drive voltage is variable over 256 different values ranging for $0.3 \mathrm{mV}$ to $7.0 \mathrm{mV}$. The GUI includes advanced controls that allow the user to change the number of frequency samples per sweep period. Another advanced control allows for command signals to be sent directly to the DDS IC. This allows for rapid debugging of the oscillator circuitry.

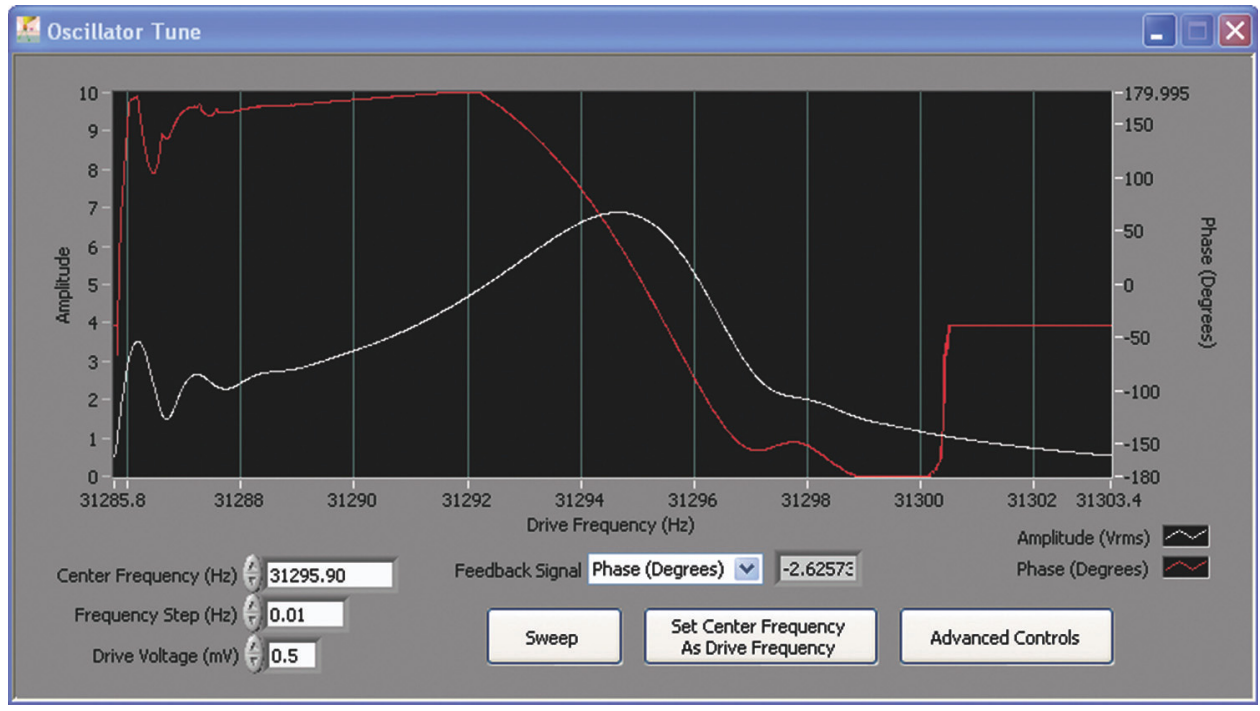

Figure 8.11 Oscillator Tune GUI. The oscillator tune shows the simultaneous real-time measurement of phase (red) and amplitude (white). The amplitude is showing a quality factor of $>6000$ for a normal tip mounted tuning fork in air.

The sweep algorithm uses DMA transfers to and from the FPGA to generate a smooth waveform. The command structure for the DDS requires 32-bit frequency words, where 16-bits of the 32-bit word are instructions for the DDS intertwined with 16-bits of frequency information. Two of these instructions will send a complete 32-bit frequency 
value [Colm, 2003]. Based on the users selection of center frequency and frequency step, an array of DDS commands is created and DMA transferred to the FPGA. Amplitude and phase values are recorded as the FPGA increments through the array, sending DDS commands through the SPI algorithm. These recoded values are then DMA transferred back to the host via the oscillator tune program.

\section{3.d Stepper Motor Control}

The Motor Control GUI gives the user direct access to driving the approach stepper motor for the TENOM system. The GUI allows for course approach and withdrawal of the motor, as well as the microscope scan head. The buttons are mouse activated to give a fluid feel to the motor. The motor moves with a mouse down action and stops with a mouse up action. These types of actions are easily programmed in LabVIEW, using event structures.

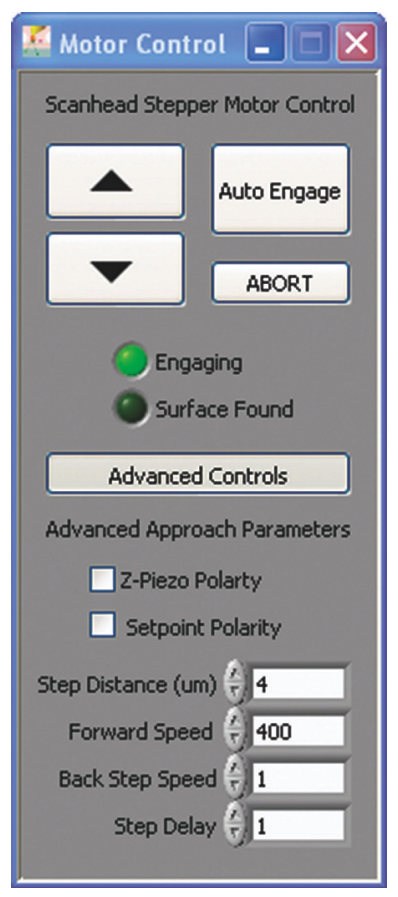

Figure 8.12 Motor Control GUI. 
The GUI has an auto-engage start button and an abort button to stop the engage process at anytime. The advanced controls allow the user to change the 'stitching' profile (presented in Chapter 8.2.g) by setting parameters for piezo extension speed, retraction speed, number of $1 / 4$ steps of the motor to execute, and stepper motor delay time.

\section{3.e Scan Data Controls}

The Scan Data Controls GUI is where all data from the microscope is displayed and recorded, shown in Figure 8.13.

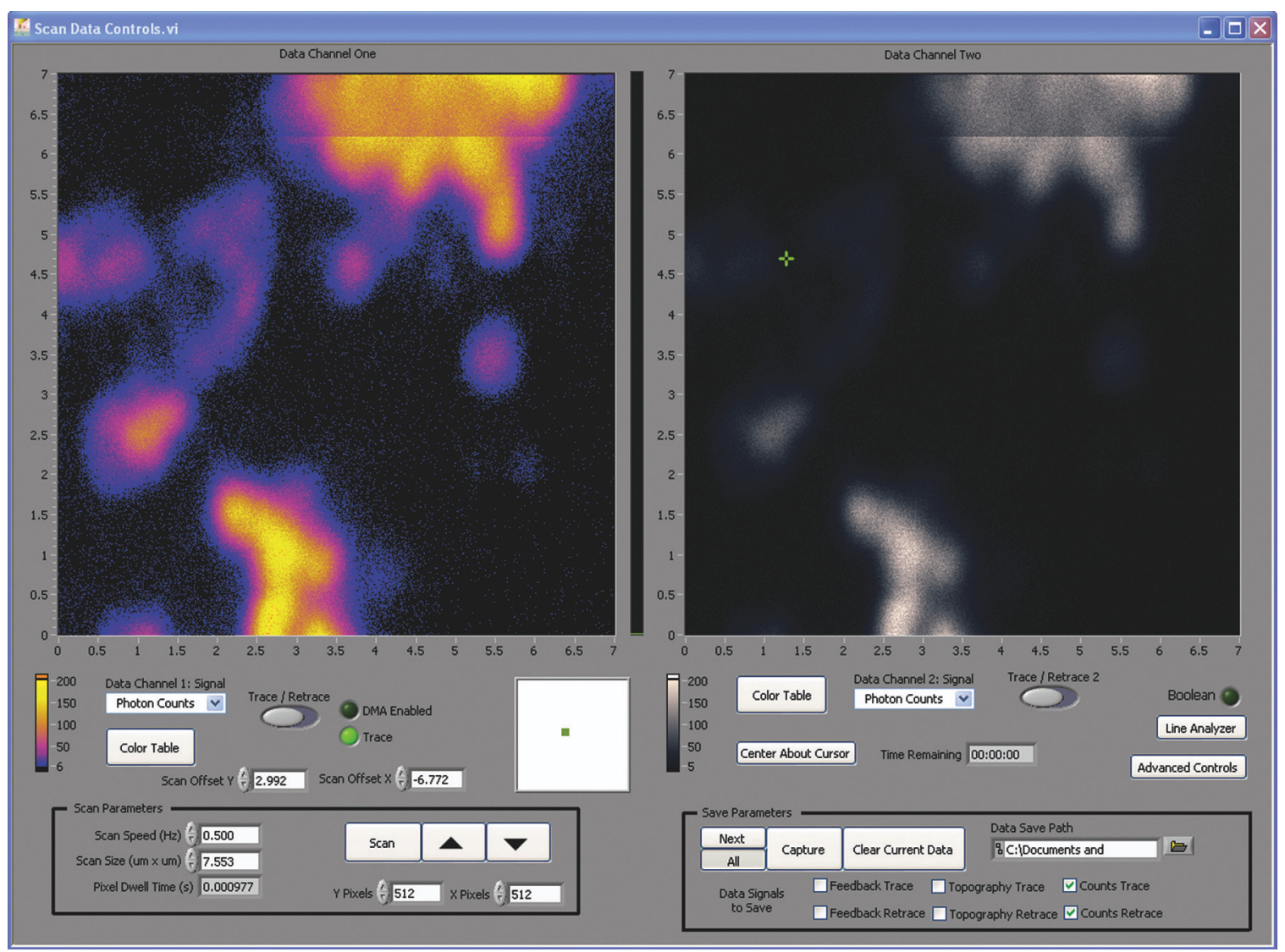

Figure 8.13 Scan Data Controls GUI. The images are multi-photon induced fluorescence of chlorophyll; both images are false color look-uptables. 
The GUI is laid out to allow for the simultaneous viewing of two data signals in a $2 \mathrm{D}$ plot, representing the area that is being imaged; the $3^{\text {rd }}$ dimension of the graphs is represented by color look-up-tables. The look-up-tables can be customized to help with the visualization of fine features in the images.

The user can change the data type displayed in the graphs to any combination of data by selecting from the trace or retrace images of photon counts, topography, or the feedback signal. Scan parameters such as scan speed, scan size (in microns), number of pixels, and starting and stopping of the scan are available. The user can also specify which of the six data channels should be saved. The GUI can save data when the top or bottom of the image is detected from the incoming data stream, along with a force save option that can be executed anytime during the scan.

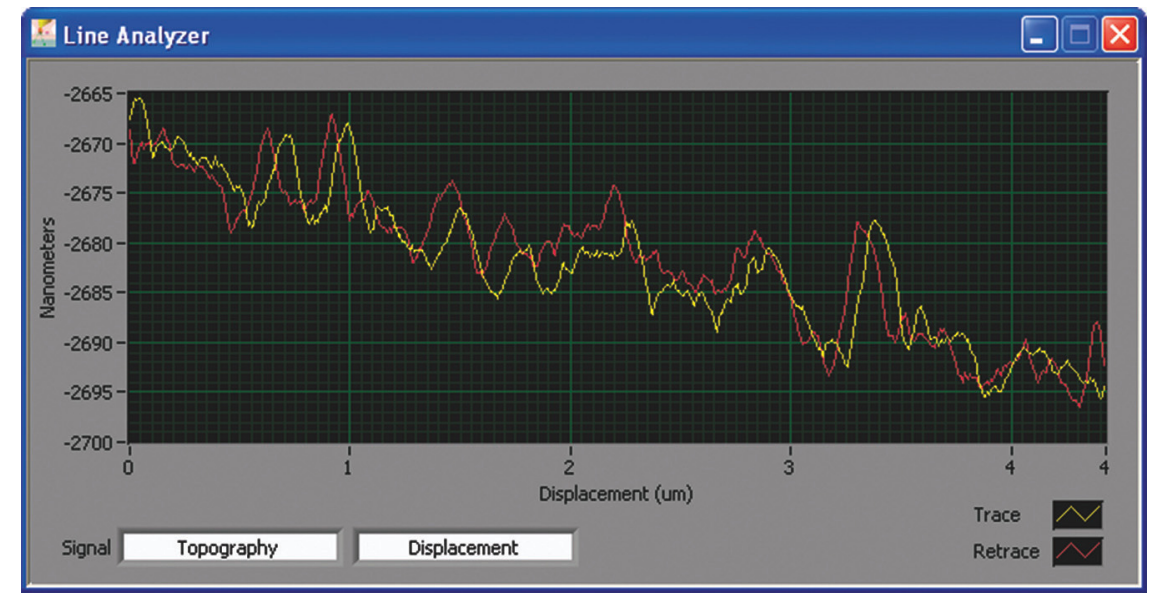

Figure 8.14 Line Analyzer GUI invoked from the Scan Data Controls GUI. Displays the previous scan line in real-time.

\section{3.f Microscope Vision}

The Microscope Vision GUI interfaces with IMAQ extensions for USB frame capture devices and allows those images to be displayed in LabVIEW. The program 
allows for direct control over LED or Laser illumination from the scan head or secondary illumination from a fiber lamp source. A stutter can be controlled by the program, using one of the FPGA digital outputs connected to a current relay for a mechanical shutter. Multiple camera views are toggled with an external mechanical switch designed for audio/visual signals. This simple solution reduces the cost and software complexity of multiple video frame grabbers.

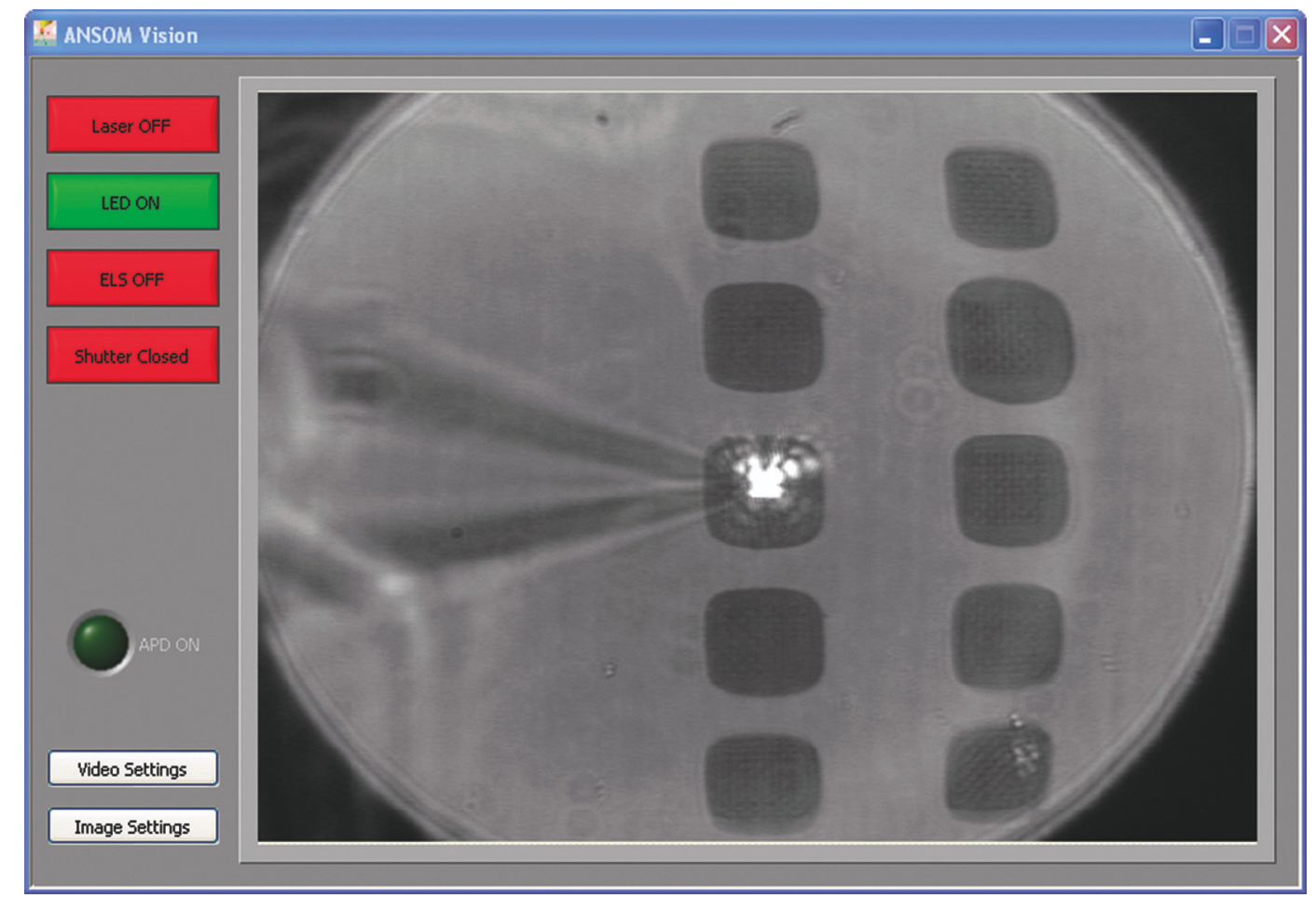

Figure 8.15 ANSOM Vision GUI. Image shows a sample surface imaged from below with a high numerical aperture objective lens. Both the tip and diffraction limited focused laser spot is shown.

\section{3.g Excitation Laser Controls}

The excitation laser control GUI interfaces with the FPGA code, in order to control stepper motors that are mounted to optical, neutral density filter wheels. This allows the user to dynamically change the illumination power at the sample without 
disturbing the laser source or the microscope while scanning. The excitation power can be monitored by the addition of a photodiode and a cover glass slip mounted at 45 degrees, somewhere in the laser beam pathway before its arrival at the microscope. The photodiode should be mounted to record the intensity of the reflection off of the coverglass slip, somewhere after the optical filters. Assuming the photodiode is configured as a linear detector, a calibration factor can be entered for both continuous wave (CW) and pulsed laser sources. Typically, the pulsed source acts differently from the CW laser when incident on the photodiode. To improve measurements, the photodiode should be shielded from ambient light sources. Figure 8.16 shows the GUI available for the user to monitor the laser power. The calibration scale factors have to be determined for different laser sources and have to be recalibrated if there are any changes to the beam pathway to maintain accuracy.

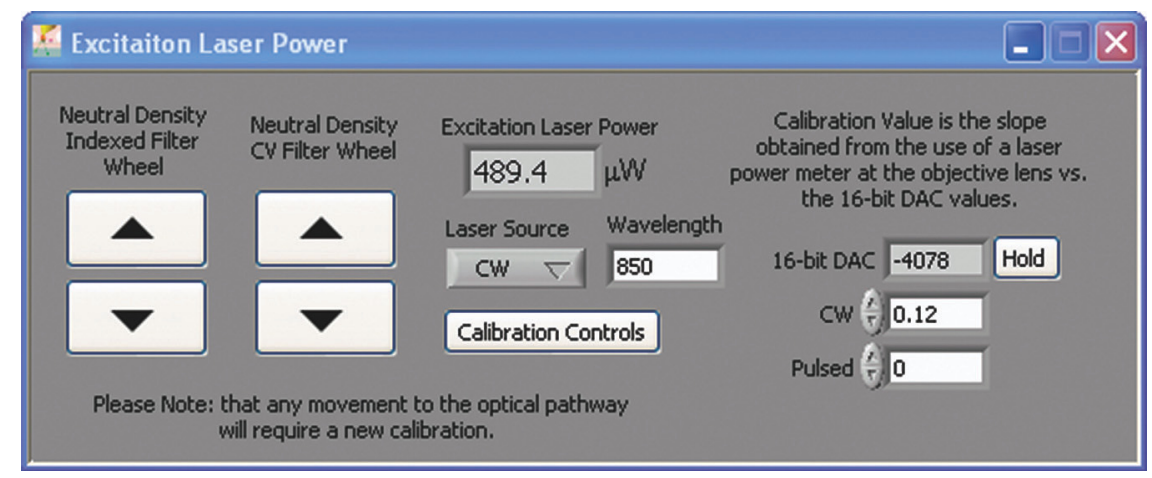

Figure 8.16 Excitation Laser Power GUI. The user has control over filter wheels that are mounted to stepper motors that use the same routine as the stepper motor control for Z-approach. A photodiode that monitors the laser output can be interfaced with the FPGA through an extra analog input. The user can then calibrate a linear relationship against the laser power measured at the sample surface. 


\section{3.h Image Processing}

The Imaging Processing GUI, shown in Figure 8.17 and 8.18, aids in the removal of imaging artifacts commonly associated with topographic imaging with SPMs.

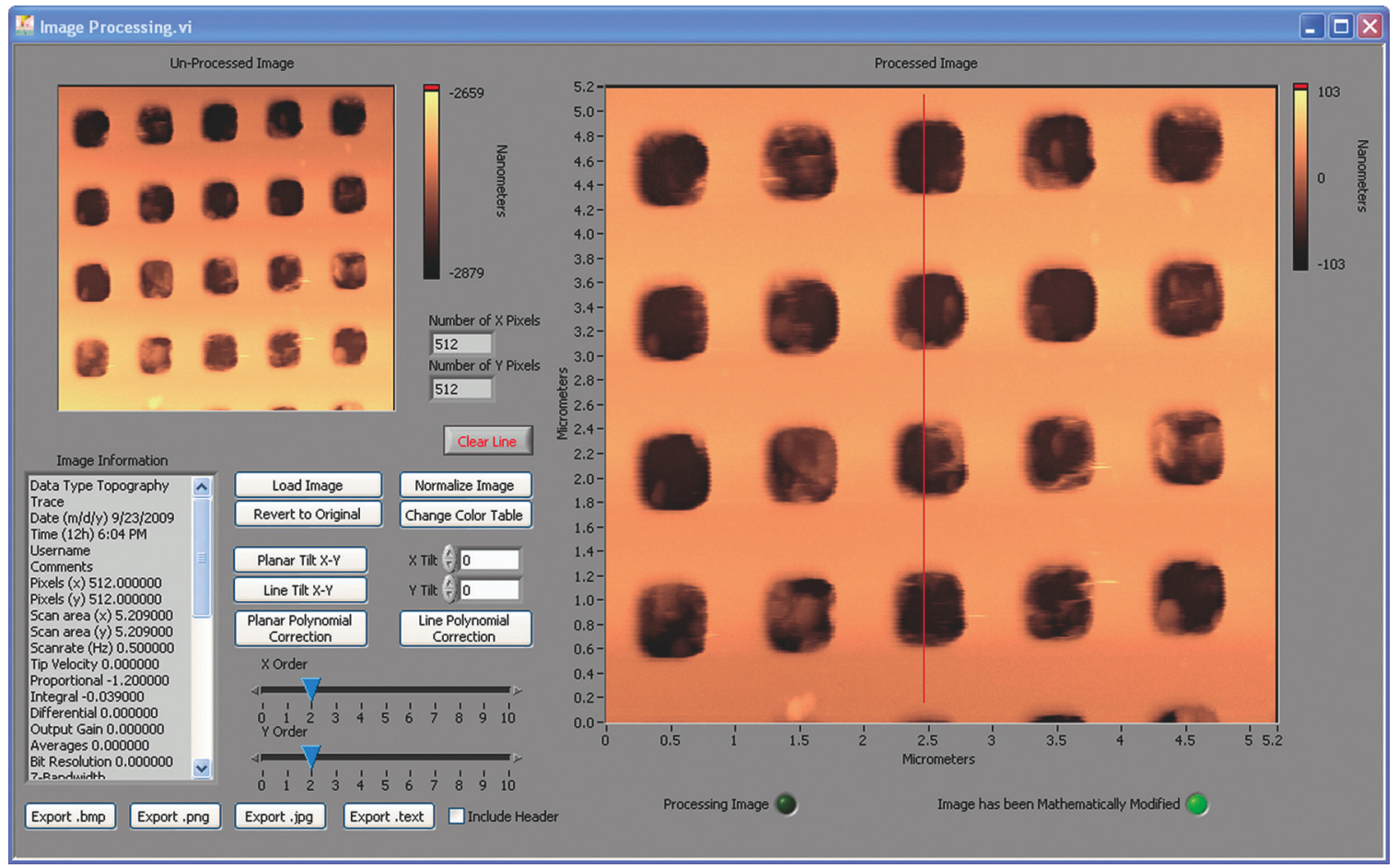

Figure 8.17 Image Processing GUI, displays the raw unprocessed image, image header information, and the processed image. The program flags the user if the image has been mathematically modified.

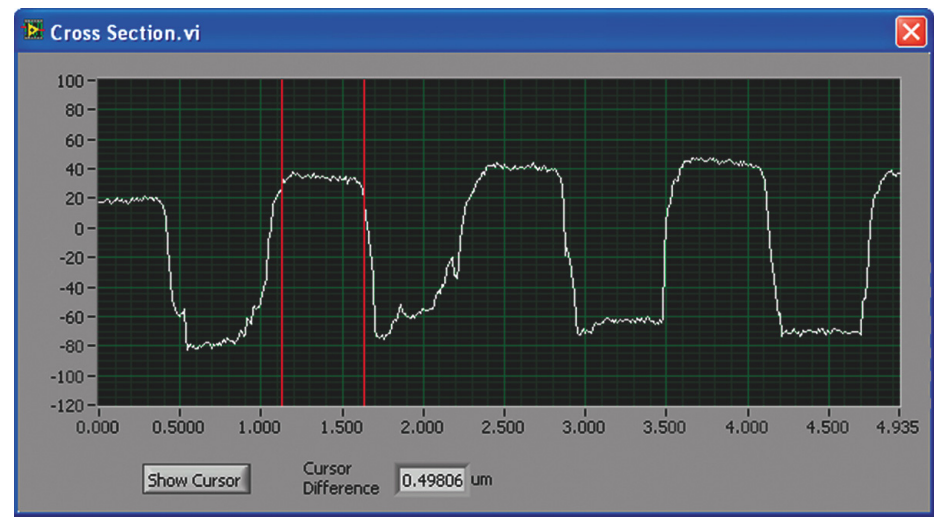

Figure 8.18 Cross section GUI, invoked from the Image Processing VI. Shows a line profile from the vertical red line on the processed image in Figure 8.17. 
The first artifact is caused by sample tilt. Sample tilt can easily wash out nanometer features in an imaging area of several microns. The second artifact is caused by piezo hysteresis (discussed in Chapter 5). While piezo hysteresis affects movement in all three dimensions, the image-processing program will focus only on the z-axis. To process an image, the program reads in a previously saved file from the scan data controls program. The header of the file is read to inform the program of the image parameters (\# pixels, scan size, date, etc.). The user then has the flexibility of changing look-up-tables and data scaling to aid in image correction. The two modes of tilt correction are accomplished by manual planar correction or computer-interpolated tilt correction, either as a planar correction or a line-by-line correction. Hysteresis artifacts are compensated by polynomial fitting of the data in either planar or line-by-line, and then subtracting the fitted polynomial from the data set.

The planar tilt correction is the simplest of the algorithms to analyze. Since the image is stored as a two-dimensional array, $(A)$ of size $n$ and $m$, a planar tilt will be processed about the midpoints of the $n$ dimension and $m$ dimension. Of course, the $n$ and $m$ dimension correlated to the spatial $X$ and $Y$ directions, respectively, will be used interchangeably for the following discussion. The midpoints are determined by the element size (\# of pixels) of the array given by,

$$
i=\frac{n+1}{2},
$$

with,

$$
n=2^{m}-1 \text {, where } m=0,1,2,3 \ldots .
$$


Extracting the $i$ values from the $X$ dimension and the $i$ values from the $Y$ dimension, two one-dimensional arrays containing the scan data can be graphed as y-values where the $\mathrm{x}$ values are the array index integers from 0 to $n$. Using a least squares fit, a linear slope can be calculated for the two one-dimensional arrays. The formulism for the slope calculation from a least squares fit to the arrays, as they pertain to the spatial scan directions $\mathrm{x}$ and $\mathrm{y}$ are given by,

$$
m_{X(\text { direction })}=\frac{n\left(\sum_{k=0}^{n-1} x_{k} y_{k}\right)-\left(\sum_{k=0}^{n-1} y_{k}\right)\left(\sum_{k=0}^{n-1} x_{k}\right)}{n\left(\sum_{k=0}^{n-1} x_{k}^{2}\right)-\left(\sum_{k=0}^{n-1} x_{k}\right)^{2}}
$$

and,

$$
m_{Y(\text { direction })}=\frac{n\left(\sum_{k=0}^{n-1} x_{k} y_{k}\right)-\left(\sum_{k=0}^{n-1} y_{k}\right)\left(\sum_{k=0}^{n-1} x_{k}\right)}{n\left(\sum_{k=0}^{n-1} x_{k}^{2}\right)-\left(\sum_{k=0}^{n-1} x_{k}\right)^{2}}
$$

respectively. Using the slopes $m_{x}$ and $m_{y}$ two new two-dimensional arrays will be created $F_{x}$ and $F_{y}$. These new arrays will be the correction constants for the original scan data array $A . F_{x}$ will be composed of $n$ values that map the linear function with slope $m_{x}$ and an intercept of zero, and all values in $m$ constant at each $n$ value. The same process will be done for and be stored in the array $F_{y}$. The sample tilt in the $X$ direction and $Y$ direction in image $A$ is now represented by two arrays, $F_{x}$ and $F_{y}$ (one for each direction $\mathrm{X}$ and $\mathrm{Y}$ ). To remove the sample tilt from the original scan image, the arrays $F_{x}$ and $F_{y}$ are be negated and then added to the array $A$. The addition of the arrays for the $X$ direction and the $Y$ direction is given by; 


$$
\begin{aligned}
& A_{0}=-\left(F_{x}\right)+A, \\
& A_{1}=-\left(F_{y}\right)+A_{0} .
\end{aligned}
$$

First array $A$ is added to $-F_{x}$ giving the resulting array $A_{0}$ now corrected in the $X$ directional for tilt. This new array $A_{0}$ is added to $-F_{y}$ resulting in array $A_{1}$ that now has both $X$ and $Y$ directional tilts removed.

Z-axis piezo hysteresis removal uses a similar mathematical process to that of the tilt removal, but a polynomial fit will be applied instead of a linear fit. The midpoints of the array to be corrected are found using Equation 8.1, as was the case for tilt correction. The image processing is done for both $X$ and $Y$ because the Z-hysteresis is compounded with off-axis movement in $X$ and $Y$. The user will input the desired order, denoted by the constant $k$, of the correction polynomial. The $k^{\text {th }}$ degree polynomial is defined by the following;

$$
\begin{aligned}
& y_{x}=a_{x 0}+a_{x 1} x+\ldots+a_{x k} x^{k}, \\
& y_{y}=a_{y 0}+a_{y 1} x+\ldots+a_{y k} x^{k} .
\end{aligned}
$$

A least squares polynomial-fitting algorithm will be applied to the one-dimensional data, in order to find the equation of the curve, as follows;

$$
\begin{aligned}
& a_{0} n+a_{1} \sum_{j=0}^{n-1} x_{j}+\ldots+a_{k} \sum_{j=0}^{n-1} x_{j}=\sum_{j=0}^{n-1} y_{j}, \\
& a_{0} \sum_{j=0}^{n-1} x_{j}+a_{1} \sum_{j=0}^{n-1} x_{j}^{2}+\ldots+a_{k} \sum_{j=0}^{n-1} x_{j}^{k+1}=\sum_{j=0}^{n-1} x_{j} y_{j} \\
& \text { - } \\
& \text { - } \\
& a_{0} \sum_{j=0}^{n-1} x_{j}^{k}+a_{1} \sum_{j=0}^{n-1} x_{j}^{k+1}+\ldots+a_{k} \sum_{j=0}^{n-1} x_{j}^{2 k}=\sum_{j=0}^{n-1} x_{j}^{k} y_{j} .
\end{aligned}
$$


For a given $k$ th degree polynomial, $k$ number of equations are required in order to solve for $k$ number of coefficients (up to $a_{k}$ ). Each value in $x$ will be plugged into the equation 8.5 and solved for $y_{x}$. The same procedure is done for $y$. These solutions will be used to create two new two-dimensional arrays will be created $F_{x}$ and $F_{y} . F_{x}$ will be composed of $n$ values that map the polynomial function, and all values in $m$ constant at each $n$ value. The same process will be done for and be stored in the array $F_{y}$. These two arrays are now negated and processed with the following functions,

$$
\begin{aligned}
& A_{0}=-\left(F_{x}\right)+A, \\
& A_{1}=-\left(F_{y}\right)+A_{0} .
\end{aligned}
$$

First, array $A$ is added to $-F_{x}$ giving the resulting array $A_{0}$, now corrected in the $X$ directional hysteresis. This new array $A_{0}$ is added to $-F_{y}$ resulting in array $A_{l}$ that now has both $X$ and $Y$ directional hysteresis removed.

\section{3.k Other Helper Programs}

Various other programs were created to add functionality and usability of the TENOM system. These are real time control and diagnostic tools that help give the user feedback about the microscope alignment and status. Two of the GUIs for these programs, a photon counter and X-Y piezo offset programs are presented in Figures 8.19 and 8.20. The Photon Counter GUI give the operator real time count rates from either a photomultiplier tube (PMT) configured in photon counting mode or an single photon avalanche photodiode (APD) detector. Real-time access to photon rates, aids the user in optical alignment of detectors and focusing of the microscope. The X-Y Piezo Offset GUI provides real-time user control over the $\mathrm{X}$ and $\mathrm{Y}$ alignment piezo in the scan head. 
Used in conjunction, the photon counter provides feedback to proper alignment (high count rates) when setting the $\mathrm{X}-\mathrm{Y}$ piezo offsets.

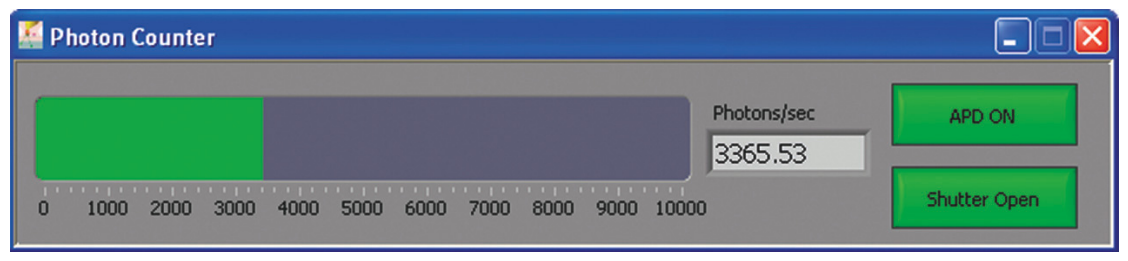

Figure 8.19 Photon Counter GUI, displays live photon counts rates (\# of photons per second) in both a graphic indicator and a numeric indicator. "APD ON" flags a digital I/O to trigger a solid-state relay to toggle on and off the detector. "Shutter Open" flags a digital I/O to trigger a mechanical shutter.

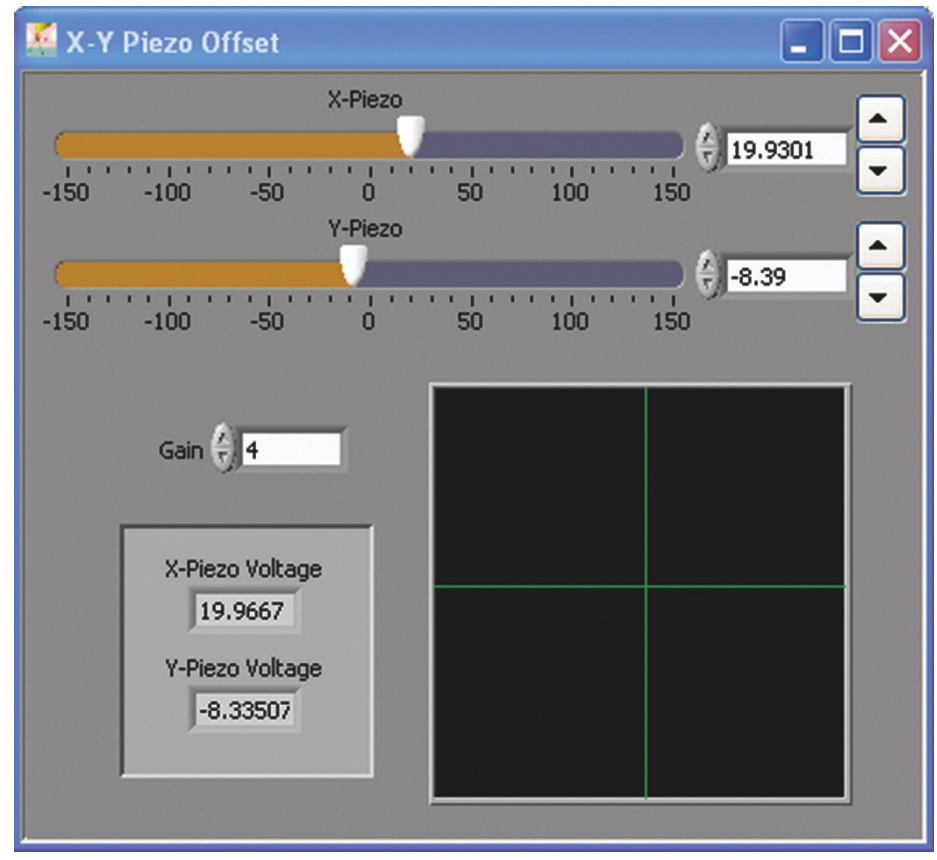

Figure 8.20 X-Y Piezo Offset GUI, allows the user to directly interface with the segmented alignment tube piezo in the scan head.

Not shown here are three other programs that are under development and will further aid the user in SPM diagnostics: an oscilloscope, spectrum analyzer, and a chart graph. These three programs have the same basic goal: to analyze in real-time 
microscope variables for diagnostics. The oscilloscope will provide voltage verses time graphs with triggering capabilities. The spectrum analyzer will provide live fast Fourier transform (FFT) of the oscilloscope data, transforming data from time domain to frequency domain. And finally the strip chart maintains a running log of signals over time. 


\section{CHAPTER 8 References}

Albrecht T. R., Grutter P., Horne D., and Rugar D., "Frequency modulation detection using high-q cantilevers for enhanced force microscope sensitivity," J. Appl. Phys 69, 668 (1991).

Analog Devices, “AD9835 50 MHz CMOS Complete DDS”, Product Data Sheet, Rev. 0, July 1998.

Analog Devices, "Compact +30 V / \pm 15 V 256-Position Digital Potentiometer", Product Data Sheet, Rev. B (2010).

Binnig G., Quate C. F., and Gerber Ch., “Atomic Force Microscope,” Phys. Rev. Lett. 56, 930 (1986).

Colm Slattery, Analog Devices, "Programming the AD9832 / AD9835", Appl. Note AN621, Rev. 0. (2003).

D’Azzo J. J. and Houpis C. H., Linear Control System Analysis and Design (McGrawHill, New York, 1981).

Grober R. D., Acimovic J., Schuck J., Hessman D., Kindlemann P. J., Hespanha J., Morse A. S., Karrai K., Tiemann I., and Manus S., "Fundamental Limits to Force Detection Using Quartz Tuning Forks,” Rev. Sci. Inst. 71, 2776 (2000).

IEEE Computer Society, "IEEE Standard for Binary Floating-Point Arithmetic," IEEE 754-1985.

Lo H., "Digital Display of Stepper Motor Rotation," Computer Design 147-148, 150-151, April 1978.

Markus J., Modern Electronic Circuits Reference Manual (McGraw-Hill Book Company, 1980) pp. 580.

Rowland J. R., Linear Control Systems: Modeling, Analysis and Design (Wiley, New York, 1986).

West P. E., Introduction to Atomic Force Microscopy Theory, Practice, Applications (AFMUniversity.org, 2007) pp. 36. 


\section{CHAPTER 9}

\section{Nonlinear Far-field Diffraction Limited Imaging}

Using nonlinear two-photon excitation (TPE) for fluorescence imaging improves the spatial imaging resolution to $\lambda / 3$. This is accomplished by suppressing out-of-focus fluorescence that hinders linear excitation. A further advantage of the TPE method includes a larger optical bandwidth for excitation of fluorescent molecules at optical wavelengths several hundred nanometers lower than the emission wavelength of the molecule. As a result, optical filter selections that encompass many different fluorescent molecules can be used. For near-field imaging, TPE is beneficial due to the background suppression and penetration depth of the near-infrared radiation commonly used in TPE. This chapter demonstrates the far-field nonlinear TPE imaging capabilities of the TENOM system. Imaging examples showing TPE on polymers, single molecules, quantum dots, pulmonary heart cells, and Caenorhabditis Elegans are presented. The use of pulsed laser sources and continuous wave (CW) laser sources are specified where appropriate. In addition, the use of photomultiplier tubes (PMT) and single photon avalanche photodiodes (SPAPD) is demonstrated.

\subsection{Diffraction Limited Imaging of Polymers}

To show the far-field imaging versatility of the microscope, a far-field multiphoton fluorescence image of J-Aggregates (PVS Poly vinyl Sulfate and PseudoIsocyanine dye) was obtained [Higgins \& Barbara, 1995; Higgins et al. 1996]. Preparation of J-aggregates requires $3.0 \mathrm{~mL}$ of $0.75 \%$ by weight of PVS solution (Aldrich 
$\# 278424$ ) heated to $90^{\circ} \mathrm{C}$, while stirring. Once the temperature of $90^{\circ} \mathrm{C}$ is obtained, 0.2 $\mathrm{mL}$ of $10 \mathrm{mM}$ PIC dye is added. The solution should be left to cool with gentle agitation. As the solution returns to room temperature, the color should change from a semitranslucent red to an opaque red with a thin film of metallic green. The J-Aggregates were spin coated on a cover glass slip (VWR Scientific \# 48366-045) at $300 \mathrm{rpm}$.

Two-photon excitation has a quadratic relationship with the excitation power [Denk, Stickler \& Webb, 1990; Sánchez et al., 1997]. In order to justify that the microscope is capable of multi-photon imaging, a series of images of the region of interest was acquired at different power levels. For imaging a PMT (Hamamatsu 1P28) configured for photon counting was used with a 60x 1.4 NA Olympus infinity corrected objective lens.
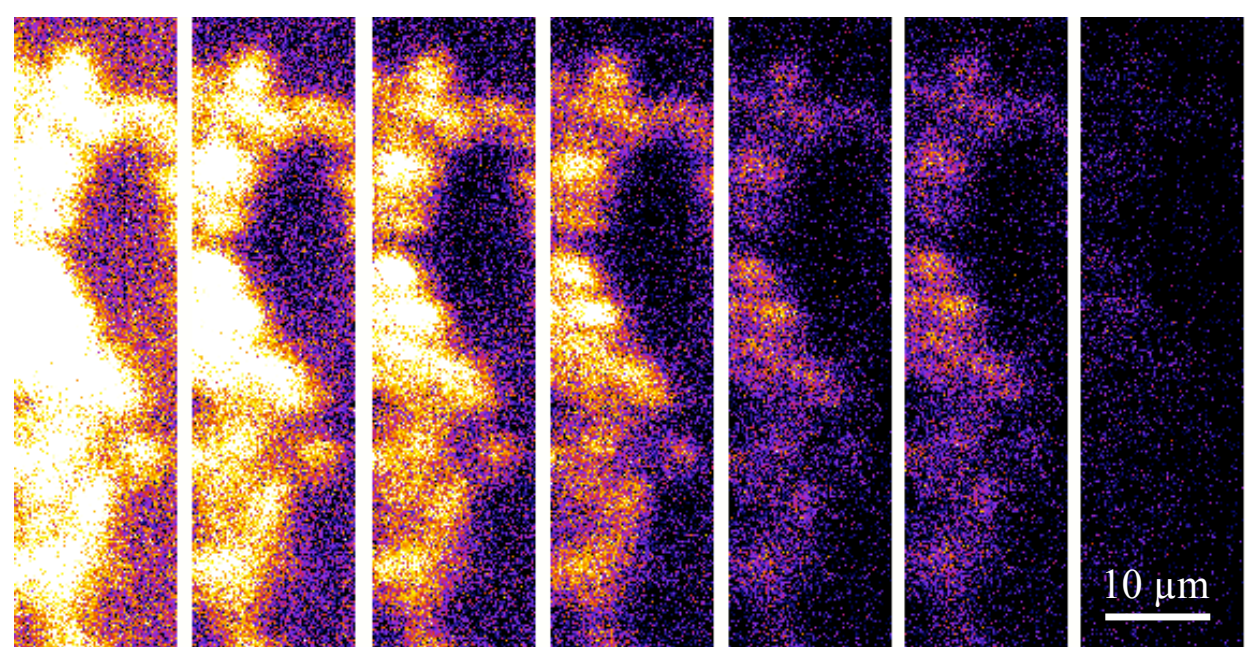

Figure 9.1 J-Aggregates, where the same region of interest was imaged at increasing (from right to left) excitation power levels.

The excitation source is a New Focus Stablewave CW diode laser with a wavelength output of $850 \mathrm{~nm}$ with a line width of less than one nanometer. The beam is modified with an anamorphic prism pair (Thorlabs PS875-B) and beam expander (5XBX CVI 
Laser Corporation). The excitation source is filtered with a dichroic filter (Chroma 725dcspxr) and two excitation filters (Chroma ET700sp-2p8) placed in front of the PMT.

Figure 9.1 shows the series of images of J-Aggregates, at different power levels of the $850 \mathrm{~nm}$ New Focus StableWave CW diode laser. Figure 9.2 shows the quadratic relationship determined experimentally.

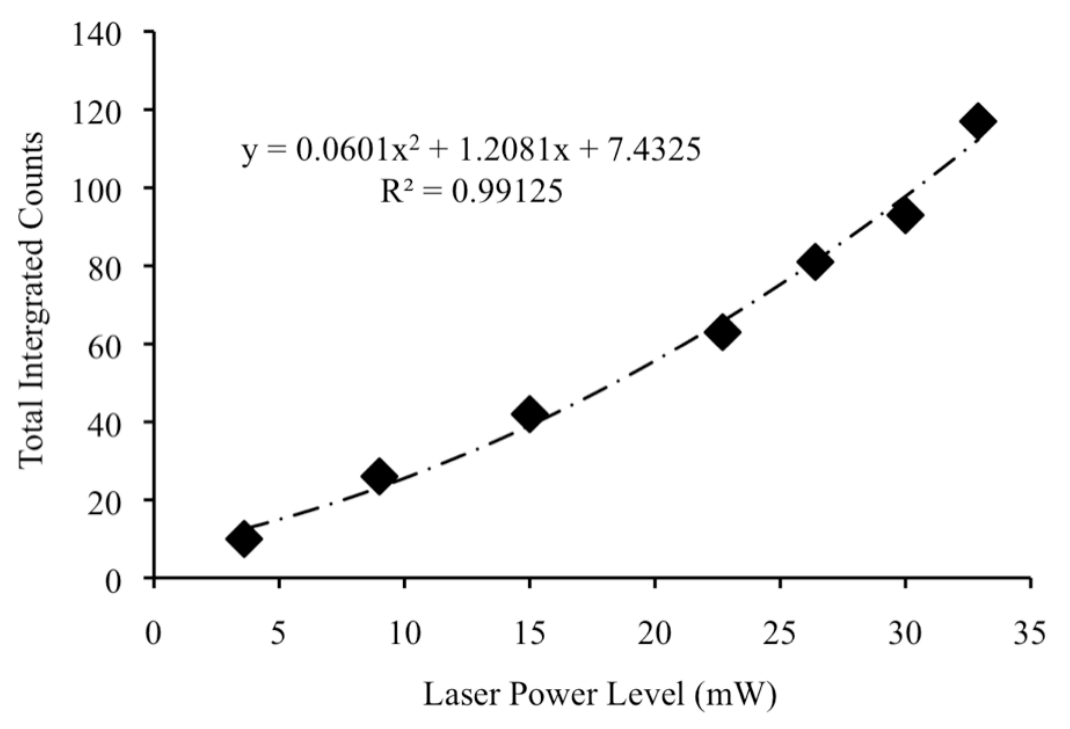

Figure 9.2 The quadratic nature of multi-photon fluorescence.

Figure 9.3 (a) shows a CCD image of the area of interest and (b) shows a multi-photon fluorescence image of the same region. The multi-photon image is 2048 x 2048 pixels square, scanned at $0.3 \mathrm{~Hz}$ per line. The color scale used in Figures 9.3 (b) and Figure 9.4 is arbitrary; in this case, yellow represents the regions of highest photon count and black is the background level. All images were processed in ImageJ analysis suite (version 1.4) [ImageJ, 2007]. Figure 9.4 (a) shows the same area of interest scanned at $512 \times 512$ pixels and a scan speed of $0.5 \mathrm{~Hz}$ per line. Figure 9.4 (b) demonstrates the highest obtainable resolution with the quality of laser alignment used for imaging. 

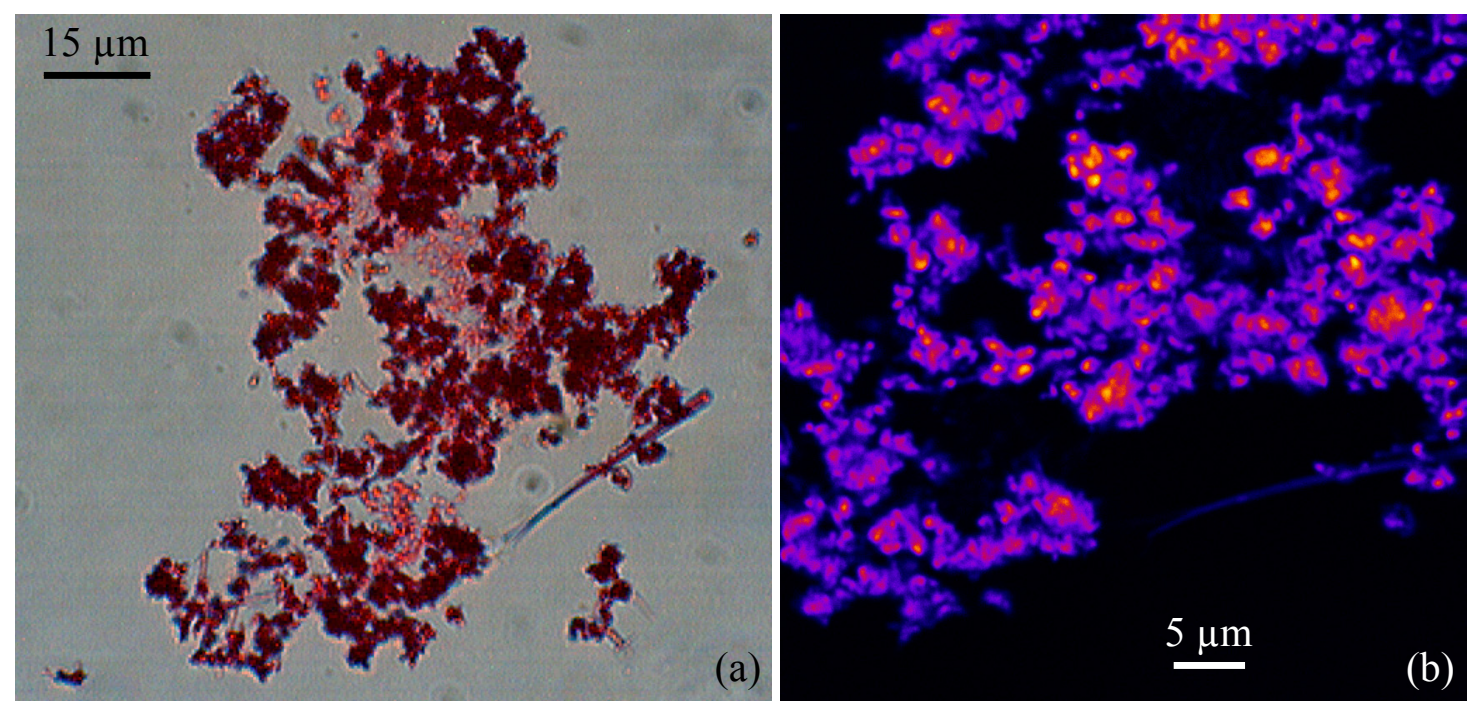

Figure 9.3 (a) CCD optical image of J-Aggregates. (b) CW Far-field Multi-photon fluorescence image of the same region acquired by point-bypoint raster scan with a PMT.
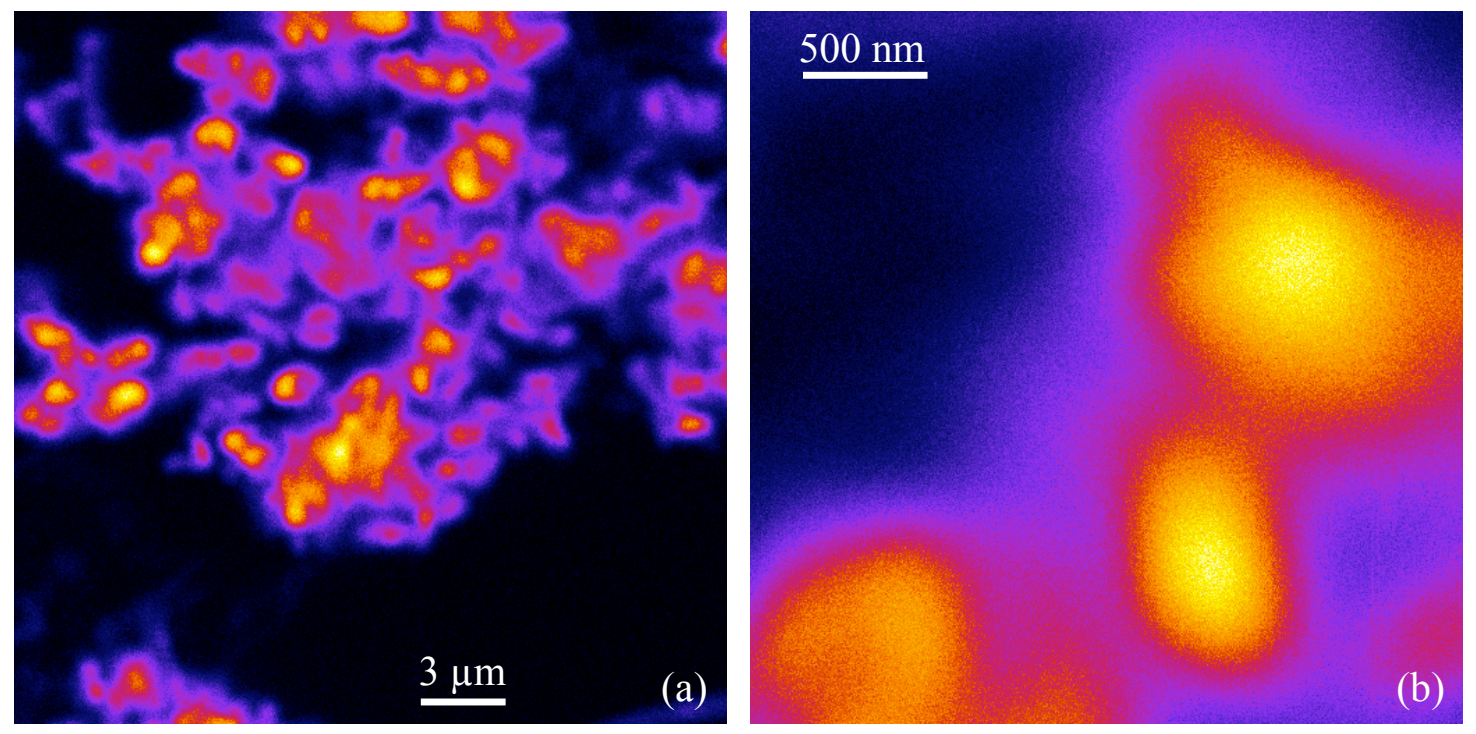

Figure 9.4 (a) CW Far-field Multi-photon fluorescence point-by-point raster scanned image of J-Aggregates (b) is a magnified image of the same area showing near diffraction limited imaging.

Using the same stable wave laser, Figure 9.5 shows a contrast between power levels and background noise while using a SPAPD (Perkin Elmer SPCM-AQR-14-FC). The higher efficenciy of the SPAPD allowed for lower excitation powers to be used. CW laser 
excitation power as low as $350 \mu \mathrm{W}$ was able to obtain a nice quality image of the Jaggregates (Figure 9.5.b).
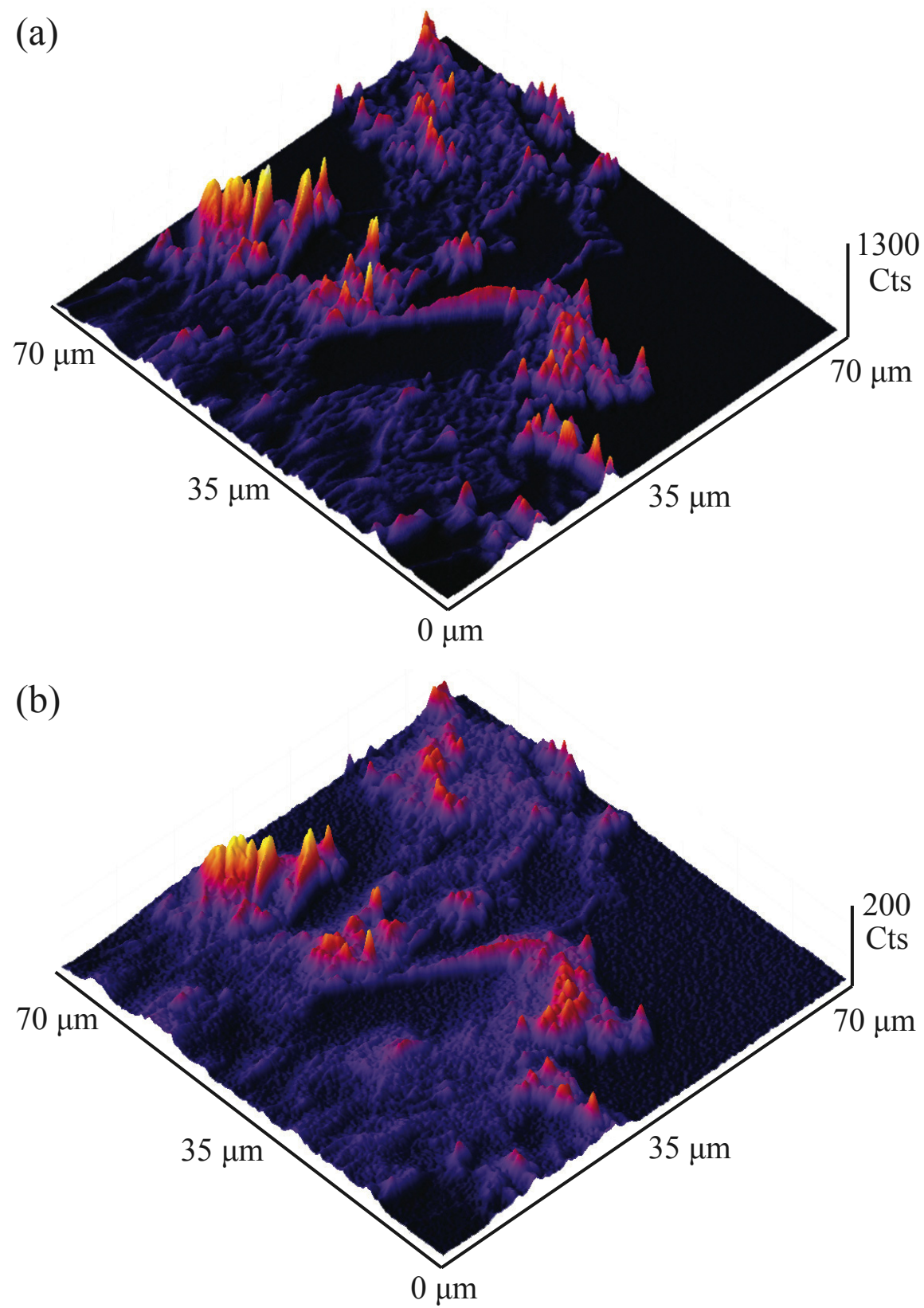

Figure 9.5 J-aggregates on glass, imaged with CW laser light at $850 \mathrm{~nm}$. (a) Using an excitation power at the sample of $1.3 \mathrm{~mW}$. (b) Using an excitation power at the sample of $350 \mu \mathrm{W}$. 


\subsection{Single Molecule Sensitivity}

The sensitivity of the microscope allows for the direct imaging of single molecules at room temperature and atmospheric pressure, Figure 9.6.

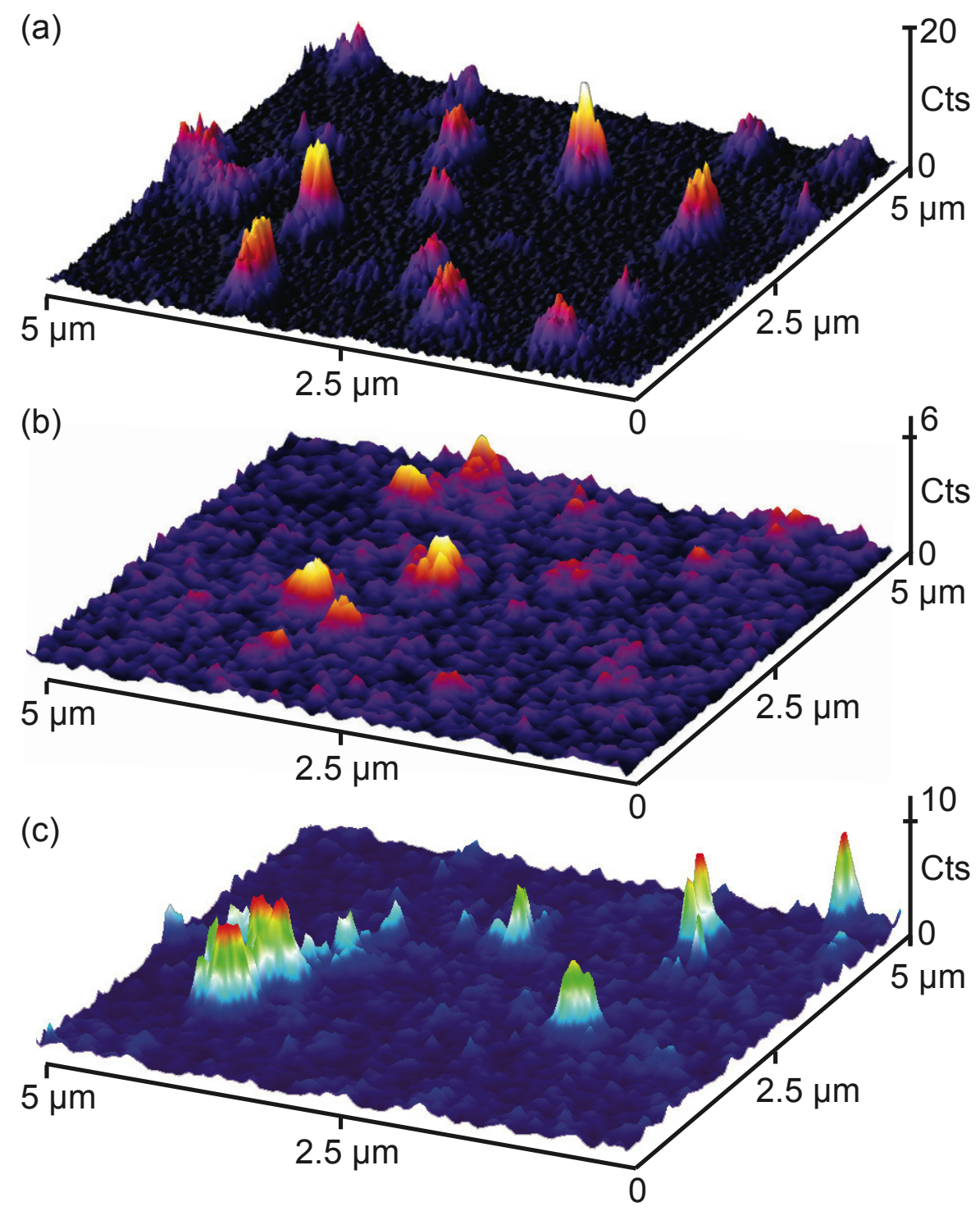

Figure 9.6 Single molecule sensitivity - Images $(a, b)$ are far-field optical responses of two photon excitation of single Rhodamine $6 \mathrm{G}$ molecules. (a) Acquired with $50 \mathrm{fs}$ laser pulses at $824 \mathrm{~nm}$ and an excitation power of 563 $\mu \mathrm{W}$. (b) Acquired with the same laser configured for $\mathrm{CW}$ at $817 \mathrm{~nm}$ at 32 $\mathrm{mW}$. (c) Shows two photon excited Si quantum dots. 
Imaging at this level has been demonstrated with a mode-locked Ti:sapphire lasers [Sánchez, 1997]). Figure 9.6 demonstrates both mode-locked pulsed mode and CW mode imaging of single molecules using Del Mar Photonics Trestles 50 Ti:sapphire laser. The single molecules of Rhodamine 6G were prepared in methanol to a concentration of $0.1 \mathrm{nM}$, then dropped onto a glass cover slip (gold seal $25 \mathrm{~mm}$ x $25 \mathrm{~mm}$ ), rotating at 4000 rpm in a spin coater. The full width half maximum (FWHM) of the single molecules is $275 \mathrm{~nm}$, roughly $\lambda / 3$, which is expected for two-photon excitation.

\subsection{Biological Application: Imaging of Bovine Pulmonary Artery Endothelial Cells}

Figure 9.7, shows a multi-photon image of bovine pulmonary artery endothelial (BPAE) cells, excited with an mode-locked Ti:Sapphire laser with 52 fs pulses (Del Mar Photonics Trestles 50 Ti:sapphire) while using a Monochromator (Acton SP-150) with an attached ICCD (Princeton Instruments Pentamax-512-EFT/1EIA). All fluorescence from both MitoTracker red CMXRos and Alexa Fluor 488 (Phalloidin) were collected by the single photon avalanche photodiode detector. In this figure, MitoTracker Red stains the F-actin (green fluorescence emission) and Alexa Fluor 488 stains the nuclear DNA.

\subsection{Biological Application: Imaging of Caenorhabditis Elegans}

Caenorhabditis Elegans are microscopic worms that are commonly used for mitochondria studies. Figure 9.8 shows Caenorhabditis Elegans that have been dyed with MitoTracker red. The images in Figure 9.8 show two different Z-axis imaging positions, confirming the confocal nature of two-photon fluorescence. Moreover, the increased penetration depth of near-IR light is also demonstrated. 

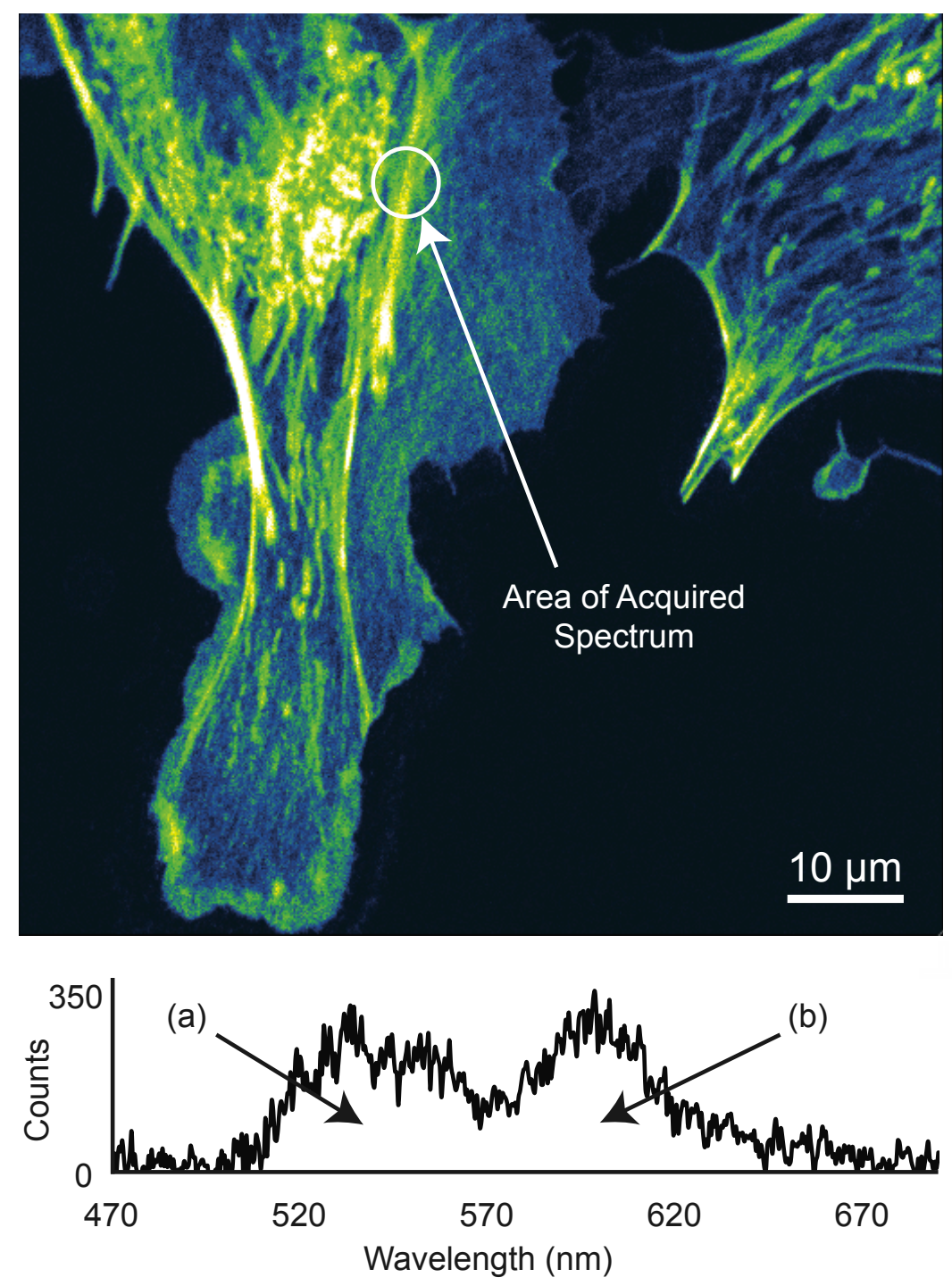

Figure 9.7 Two-photon far-field fluorescence mapping of BPAE cells mounted on a cover glass slip. Sample was excited at $800 \mathrm{~nm}$ using $52 \mathrm{fs}$ laser pulses with an average intensity of $112 \mu \mathrm{W}$. Image shows combined fluorescence of MitoTracker red CMXRos, Alexa Fluor 488 (Phalloidin) and DEHP labels. The spectrum of MitoTracker red CMXRos (a) and Alexa Fluor 488 (Phalloidin). (b) Acquired with a 1.0 second integration time at the area marked in the image. 

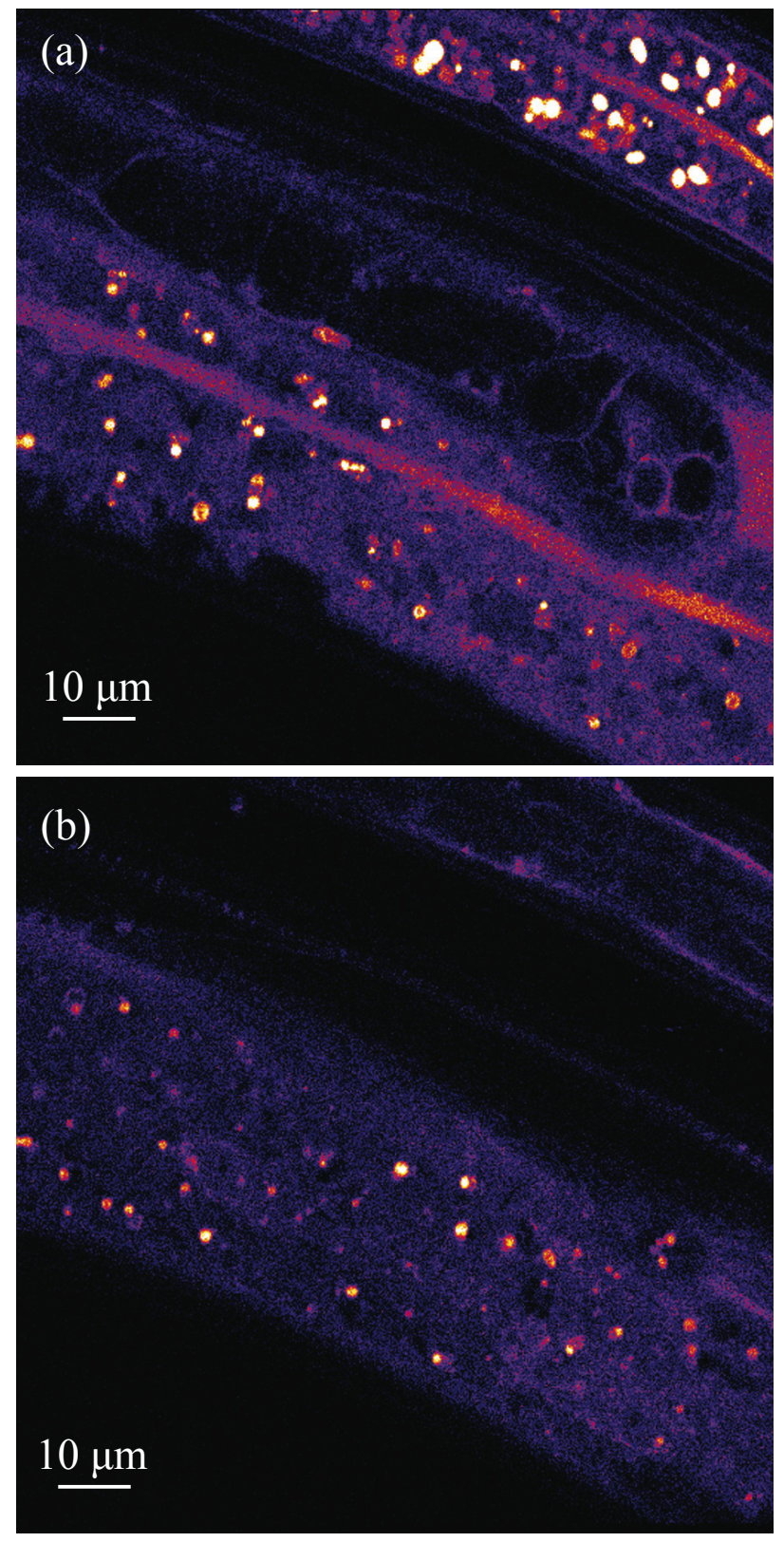

Figure 9.8 Two-photon far-field fluorescence mapping of Caenorhabditis Elegans mounted on a cover glass slip. (a) The lower part of the nematode on a glass slide in agar. (b) The focus was increased in $\mathrm{z}$ and another image taken. Due to the highly confined Z-focal range, little out-of-focus fluorescence is seen. 


\section{CHAPTER 9 References}

Denk W., Strickler J., and Webb W., "Two-Photon Laser Scanning Fluorescence Microscopy," Science 248, 73-6 (1990).

Higgins D. A. and Barbara P. F., "Excitonic Transitions in J-aggregates Probes by Nearfield Scanning Optical Microscopy,” J. Phys. Chem. 99, 3 (1995).

Higgins D. A., Kerimo J., Vanden Bout D. A., and Barbara P. F., “A Molecular Yarn: The Self Assembly of Flexible, Fluorescent Fibers Studied by Near Field Scanning Optical Microscopy," J. Am. Chem. Soc. 118, 4049 (1996).

Rasband, W. S., ImageJ, U. S. National Institutes of Health, Bethesda, Maryland, USA, http://rsb.info.nih.gov/ij/, 1997-2007.

Sánchez E. J., Novotny L., Holtom G. R., and Xie X. S., "Room-Temperature Fluorescence Imaging and Spectroscopy of Single Molecules by Two-Photon Excitation,” J. Phys. Chem. A 101, 7020 (1997). 


\section{CHAPTER 10}

\section{Shear Force Imaging}

Scanning near-field imaging requires the placement of an imaging probe within one wavelength of the incident excitation radiation. This requirement is further complicated by the need to protect the imaging probe from damage, typically inflicted by interaction with the sample surface. For the imaging probes used in the tip enhanced near-field optical microscope (TENOM), any geometry changes caused by surface interaction will render the probes useless. This requires the scanning probe microscope (SPM) to operate in a non-contact, constant height mode. Finding the correct parameters for the shear force feedback loop and the approach algorithms to ensure a "soft" landing is paramount for near-field imaging. These parameters were determined with tungsten tips on standard calibration grids. Similar to the evaluation of optical imaging and sensitivity demonstrated in Chapter 9, this chapter shows the shear force microscopy (SFM) capabilities of the TENOM system. Included are topographic images and an example of closed loop piezo scanning versus open loop piezo scanning.

\subsection{Preparing the Probes}

To adequately test the SFM capabilities of the TENOM system, $100 \mu \mathrm{m}$ tungsten wire (Alfa Aesar \#10404) is used. Tungsten was chosen for its ease of electrochemical etching, yielding reproducibly high-aspect ratio, sharp tips with tens of nanometer end diameters, while using simple etching circuits and basic electrolyte chemistry [Melmed, 1991]. Many groups discuss methods for the electrochemical etching of $\mathrm{W}$ wire, with a 
range of applications from scanning tunneling microscopy (STM) to electron sources of scanning electron microscopes and liquid metal ion sources (LMIS) for focused ion beam microscopes (FIBs) [Bryant et al., 1987; Wagner \& Hall, 1977].

The $\mathrm{W}$ etching circuit uses a variable direct current (DC) etching voltage from 0.0 to $12 \mathrm{~V}$. The voltage is converted to a current using an OP27 operational amplifier that is wired to the $\mathrm{W}$ wire, which becomes the anode for the electrochemical cell. The cathode is a Pt ring that is wired to another OP27 as a current-to-voltage converter. This voltage is compared to a reference voltage by a LM311 comparator IC. The comparator determines when the shut off voltage for the etching has been reached. The user sets the reference shut off voltage before the etching begins. The output of the comparator controls the gate of a transistor to cut off the etching voltage. The electrochemicaletching cell uses $7.5 \mathrm{M} \mathrm{KOH}$ and a platinum ring as the cathode; the $1.0 \mathrm{~cm}$ long tungsten wire is the anode. Both the $\mathrm{W}$ and $\mathrm{Pt}$ wires are held in place with $\mathrm{Cu}$ clips, in an etching apparatus. The $\mathrm{W}$ is placed in solution in the middle of the Pt ring, which is submerged roughly a millimeter below the surface of the solution. W etches take several seconds and result in sub-50 nm end diameters that are constantly obtained with an etch voltage of $3.0 \mathrm{~V}$ and a reference voltage of $0.8 \mathrm{~V}$.

The results of the $\mathrm{W}$ etch are analyzed, first optically, with a low power stereo zoom microscope, and then further in a SEM for quality. The $\mathrm{W}$ probes are then shortened with a pair of sharp scissors to 1-2 $\mathrm{mm}$ of length. The probes are glued with CA (Cyanoacrylate) glue to one of the exposed tuning fork sides. Figure 5.8 in Chapter 5 shows the Au probe mounted to a tuning fork; the same basic idea applies for the $\mathrm{W}$ probe. The tuning fork is exposed by cutting the protective can open. Using a laser- 
diode can opener (Thorlabs WR1), the base of the can will be left intact, allowing the tuning fork to maintain a high mechanical rigidity. The W-tuning-fork assembly can be used immediately, but it is best to let it cure in a vacuum chamber. Otherwise, as the glue cures, the resonance frequency shifts, which can affect shear force scanning. Furthermore, it is a good habit to store the probes in a vacuum or argon environment to prevent dust collection.

\subsection{Interaction Force}

The TENOM system will require minimal interaction with the sample surface. To determine optimal setpoints for the feedback system, a measure of the interaction force as a function of distance away from the sample is required. Figure 10.1 shows this relationship for the TENOM system, using a $\mathrm{W}$ probe.

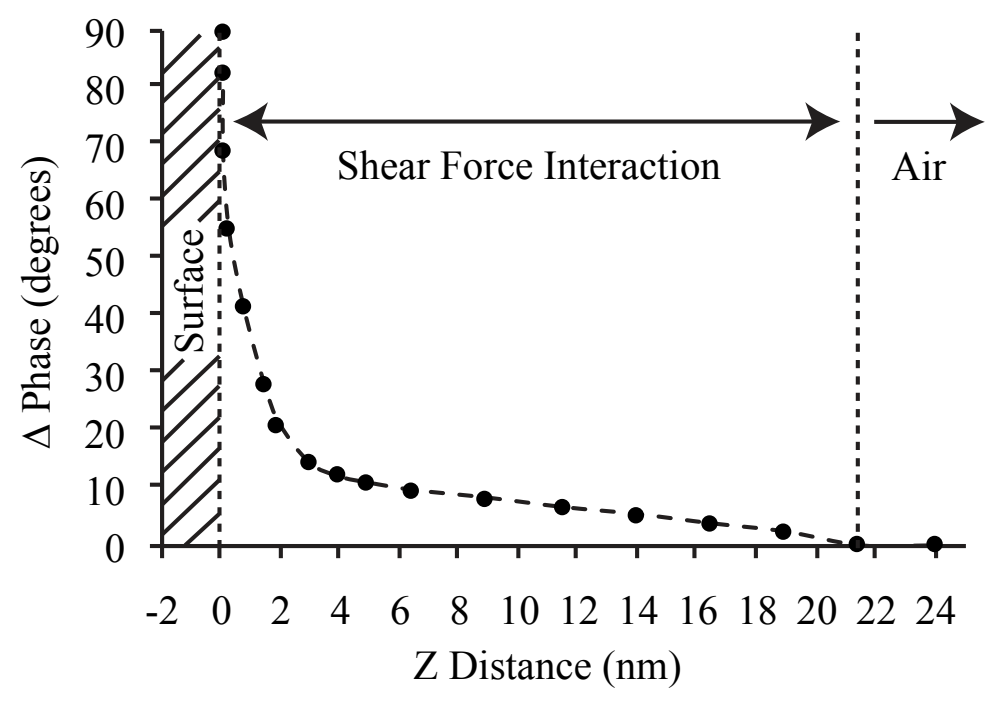

Figure 10.1 Relative phase as a function of distance from the surface of the sample. The shear force interaction begins about $20 \mathrm{~nm}$ away from the sample surface. 
Shear force interaction becomes detectable at roughly $21 \mathrm{~nm}$, which benefits the nearfield imaging. Maintaining distances greater that $9.0 \mathrm{~nm}$ greatly reduces optical quenching. From distances of $21 \mathrm{~nm}$ to $3.0 \mathrm{~nm}$, a very gradual change to the phase in the feedback signal occurs. Distances less then $3.0 \mathrm{~nm}$ experience a much larger rate of change in phase, as related to a change in distance. For typical scanning experiments, the phase is set $3^{\circ}$ to $5^{\circ}$ below the phase measurement, when the tip is far away from the sample surface. In this condition, the scanning should maintain a tip-sample separation distance of 10 to $20 \mathrm{~nm}$.

\subsection{Calibration Grids}

Calibrations of SPMs require a calibration grid as a three dimensional spatial standard. The critical dimensions of the grid provide a method to derive accurate conversion factors from voltages to nanometers.
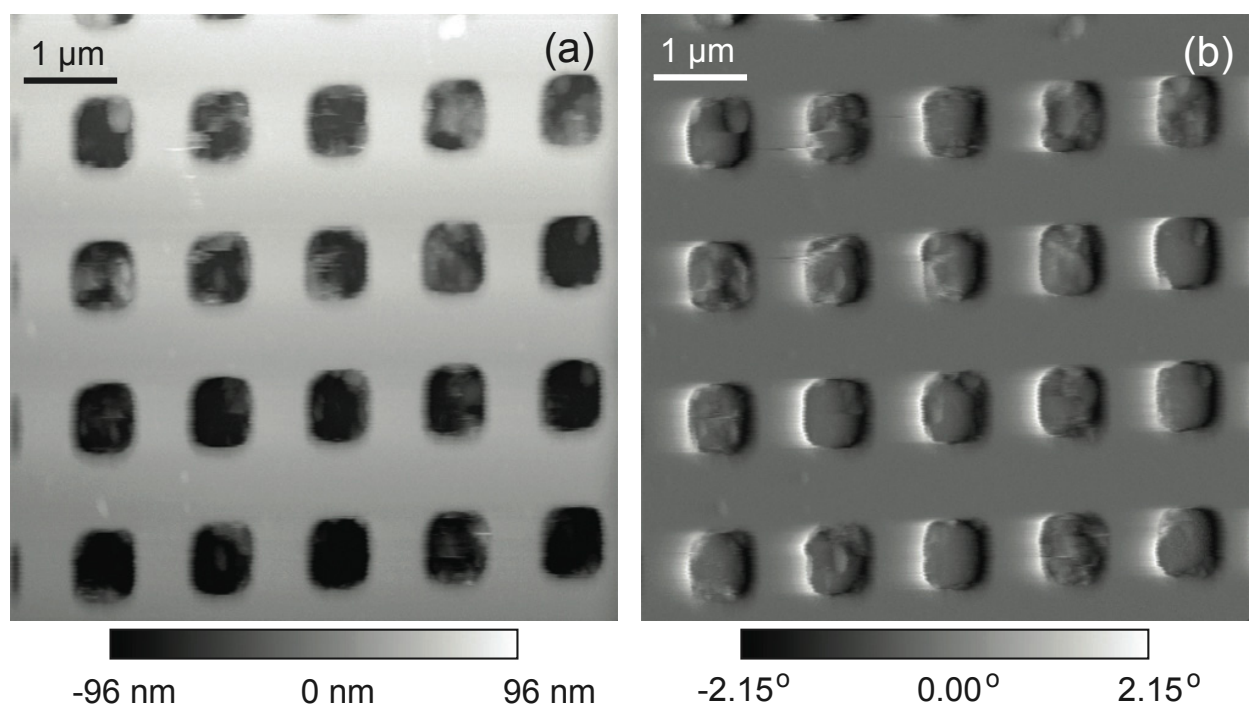

Figure 10.2 Shear force closed-loop images of a calibration grid (Digital Instruments, Veeco, PG platinum coated calibration grid $1.0 \mu \mathrm{m}$ x $1.0 \mu \mathrm{m}$ period). (a) The topographic image in the trace direction (left to right). (b) The error in the phase feedback signal in the retrace direction (right to left). Both images were acquired at a scan rate of $0.3 \mathrm{~Hz}$. 
Figure 10.2 is a closed-loop scanned topographic and phase feedback image of a platinum coated calibration grid with a $1.0 \mu \mathrm{m} \times 1.0 \mu \mathrm{m}$ periodicity. The calibration grid consists of pits etched into $\mathrm{Si}$. The scan rate in Figure 10.2 was $0.3 \mathrm{~Hz}$ with a proportional gain of 0.4 and integral gain of 0.021 ; the differential gain was set to zero. Figure 10.3 shows images with the same calibration grid in closed-loop (Physik Instrumente P-733.2CL) but over a larger region of interest. During the scan, the phase setpoint was varied from $11^{\circ}$ to $14^{\circ}$ to $17^{\circ}$. The topographic image does not show the change in Z-displacement since this only corresponds to about a nanometer of change. Although with the increased interaction force, the imaging probe has a higher probability of wear.
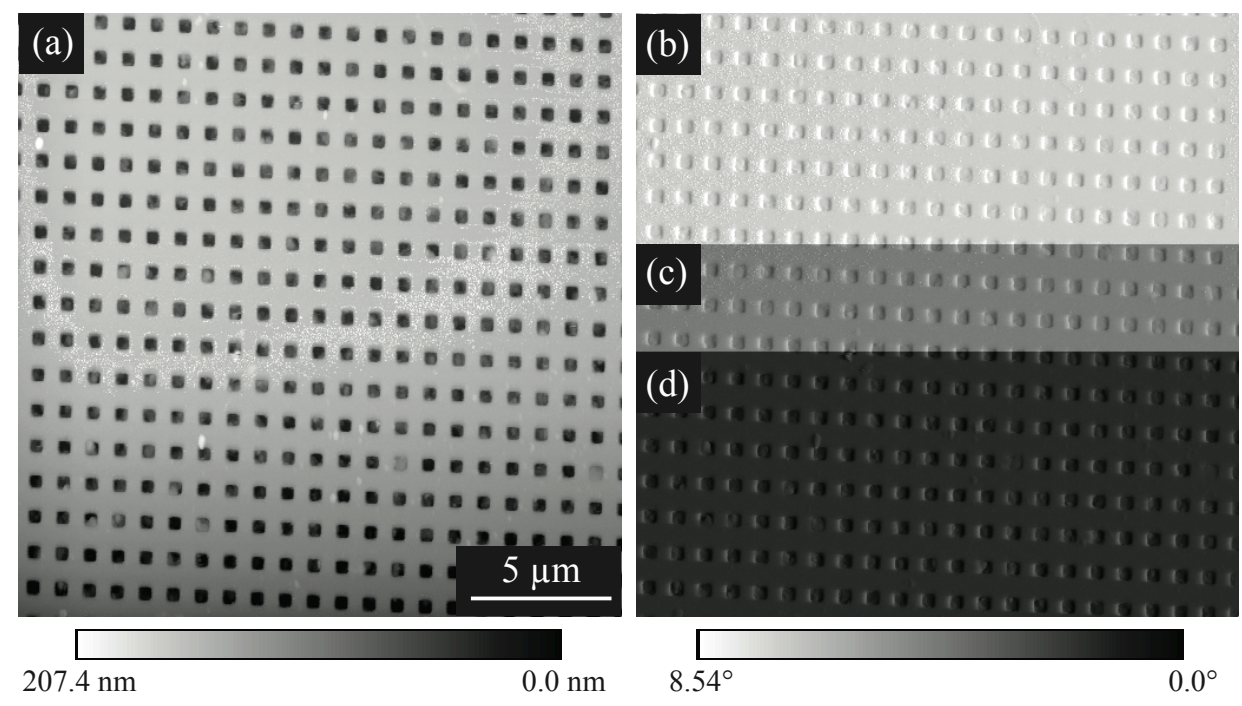

Figure 10.3 Shear force closed-loop imaging of a calibration grid with a larger field of view (Digital Instruments, Veeco, PG platinum coated calibration grid $1.0 \mu \mathrm{m} \times 1.0 \mu \mathrm{m}$ period). (a) The topographic image in the trace direction (left to right). (b) The error in the phase feedback signal engaged at $11^{\circ}, 14^{\circ}$, and $17^{\circ}$.

Figure 10.4 shows the same grid area as Figure 10.3 but with the closed-loop position feedback disabled. This results in significant distortion of the scan image, especially 
along the edges of the images, where the quick directional change shows a lot of piezo hysteresis. If imaging had been done purely in open-loop piezo configurations, complex nonlinear correction equations would be required to remove the distortion in the image. These would have to be determined for every piezo element used for metrology.

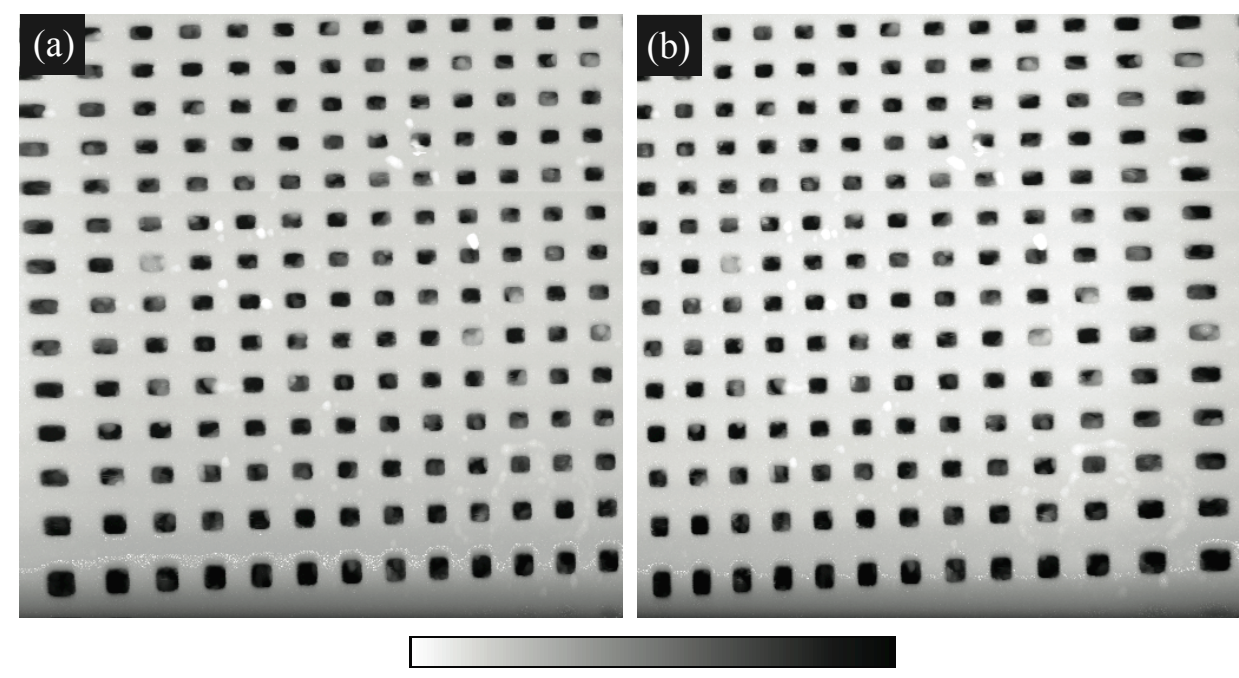

$228.4 \mathrm{~nm} \quad 0.0 \mathrm{~nm}$

Figure 10.4 Shear force open-loop images of calibration grid (Digital Instruments, Veeco, PG platinum coated calibration grid $1.0 \mu \mathrm{m} \times 1.0 \mu \mathrm{m}$ period). (a) The topographic image in the trace direction (left to right). (b) The topographic image in the retrace direction (right to left). Both images were acquired at a scan rate of $0.2 \mathrm{~Hz}$.

The $1.0 \mu \mathrm{m} \times 1.0 \mu \mathrm{m}$ periodicity calibration grid images showed a lot of damage from age and misuse. Most of the damage was from contaminates becoming embedded in the etched pits over time. To obtain an accurate measure of the z-piezo, a second calibration grid was used; Figure 10.5 shows an image obtained from the new standard. The grid is $5.0 \mu \mathrm{m} \times 5.0 \mu \mathrm{m}$ periodicity with square pillars that are $119 \mathrm{~nm}$ in height, on average (nanoSurf BT00250). The scan shown in Figure 10.5 was one of the better images acquired with the default PID algorithm, provided with LabVIEW's FPGA 
package. For a successful image to be obtained, the integral term was set to the smallest possible value; any increase in value resulted in instability in scanning. The scan line artifacts and specks of noise in the Figure 10.5 were all caused by PID complications. This became the real motivation for modifying the PID algorithm to allow for an increase in the dynamic range of the integral term. The previous Figures 10.2 through 10.4 were all obtained with the improved PID algorithm.

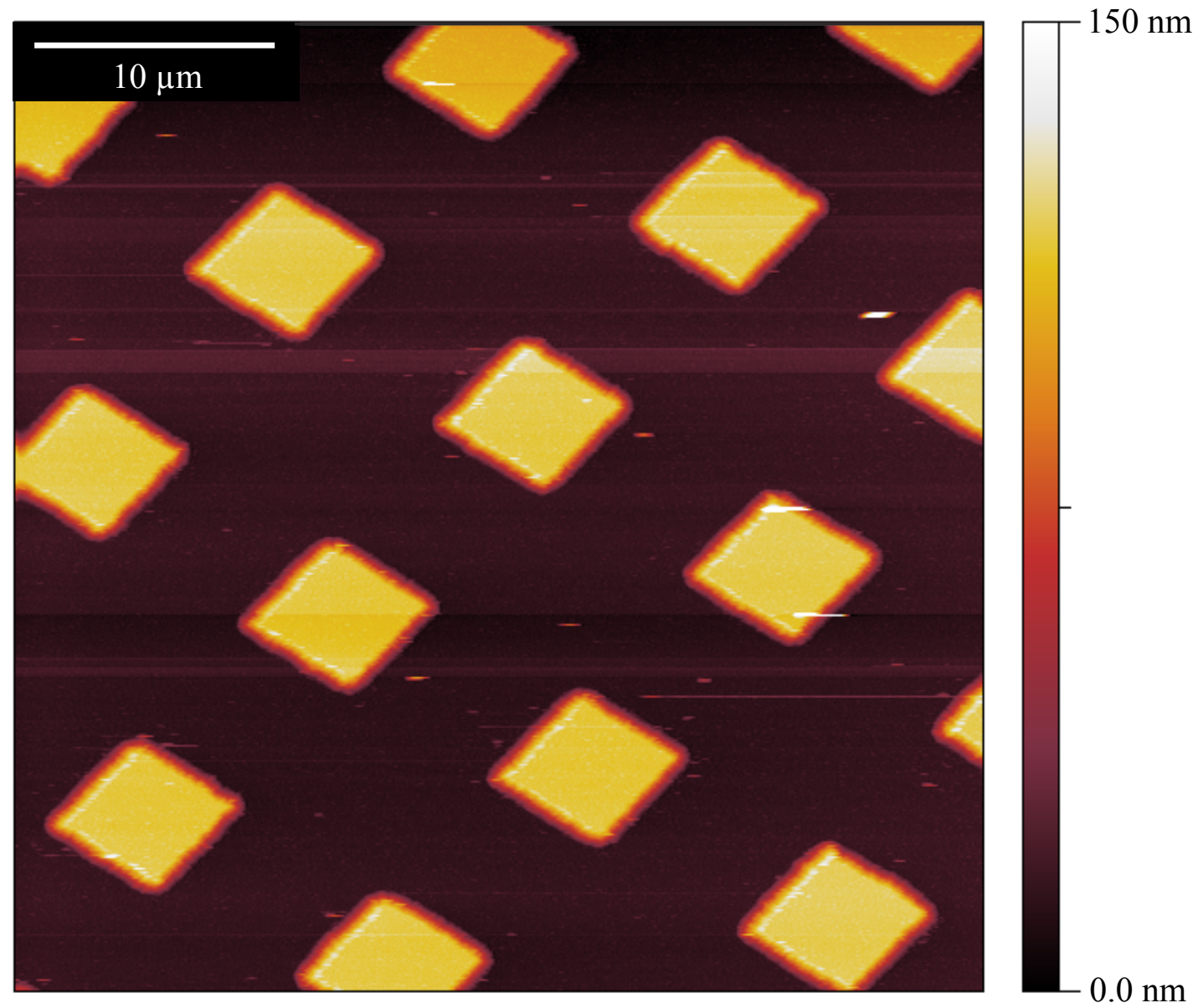

Figure 10.5 Topographic shear force image of $5.0 \mu \mathrm{m} \times 5.0 \mu \mathrm{m}$ periodicity, $119 \mathrm{~nm}$ high pillars. Scan noise is a result of an overly sensitive integral gain term in the PID feedback loop. 


\subsection{Topographic Examples}

The TENOM system can be used specifically to image topography of opaque samples for surface studies. In this case, the quality of the imaging probe will determine the obtainable spatial resolution in the $\mathrm{x}$ and $\mathrm{x}$ dimensions. The $\mathrm{z}$ resolution is less dependent on probe geometries, since it measures the distance from the end of the final atoms on the probe to sample surface. Some care should be used to maintain sharp tips, even if the primary concern is in the $\mathrm{z}$ dimension. As the end radius of the imaging probe changes from a small to a larger, blunter shape due to surface interaction, the parameters for the PID loop change. This change is a consequence of the larger interaction area of the probe.

To highlight the topographic capabilities of the TENOM system, a few topographic images are presented. Figure 10.6 shows the surface mica, covered with an unknown contamination layer. Figure 10.7 shows the surface of a DVD imaged at 1024 x 1024 pixel resolution. DVDs can be used as low cost rough calibration grids. Figure 10.8 shows the surface of a rewritable DVD as a comparison to the traditional, read-only DVD in Figure 10.7. 

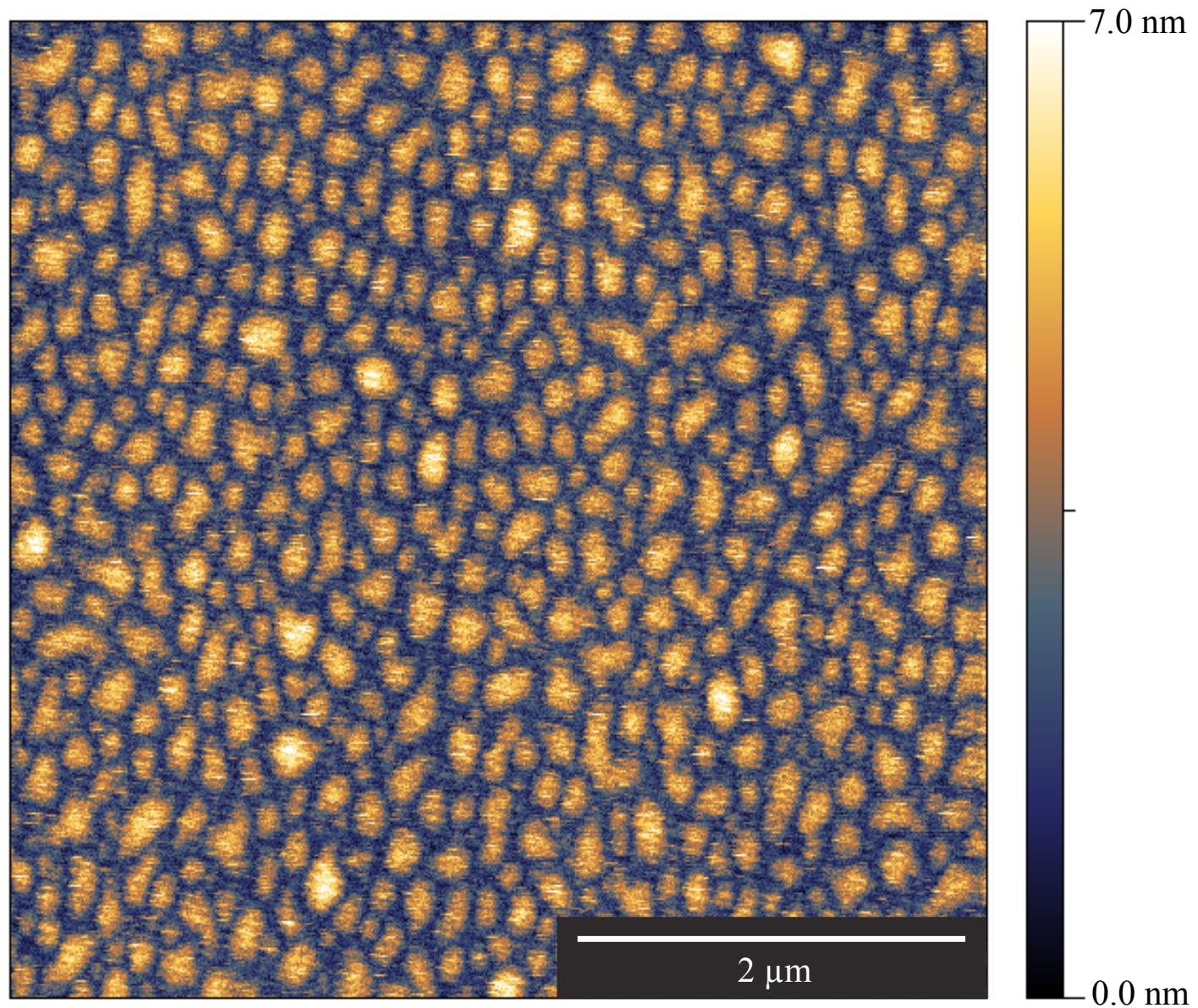

Figure 10.6 Topographic shear force image of an unknown contamination layer on mica. The image was flattened in post-processing to remove the sample tilt and a $2^{\text {nd }}$ order distortion present in $\mathrm{z}$ along the $\mathrm{y}$ dimension, caused by the closed-loop sample stage's off-axis movement. 


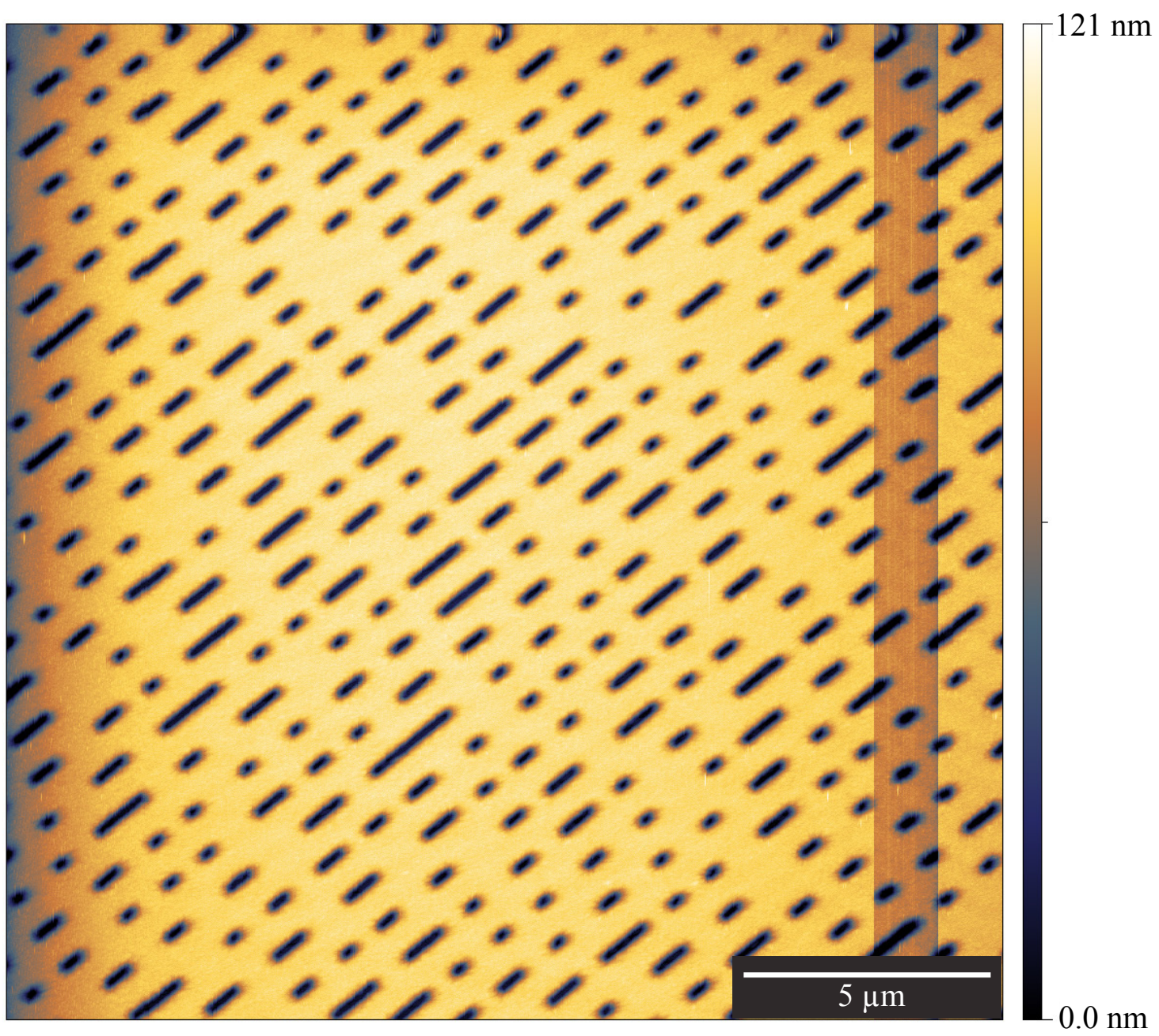

Figure 10.7 Topographic shear force image of DVD. The band on the right side of the image results from changing the PID settings, then returning them to the original scan values. The image was flattened in post-processing to remove the sample tilt and a $2^{\text {nd }}$ order distortion present in $\mathrm{z}$ along the $\mathrm{x}$ dimension and the $\mathrm{y}$ dimension, caused by the closed-loop sample stage's off-axis movement. 


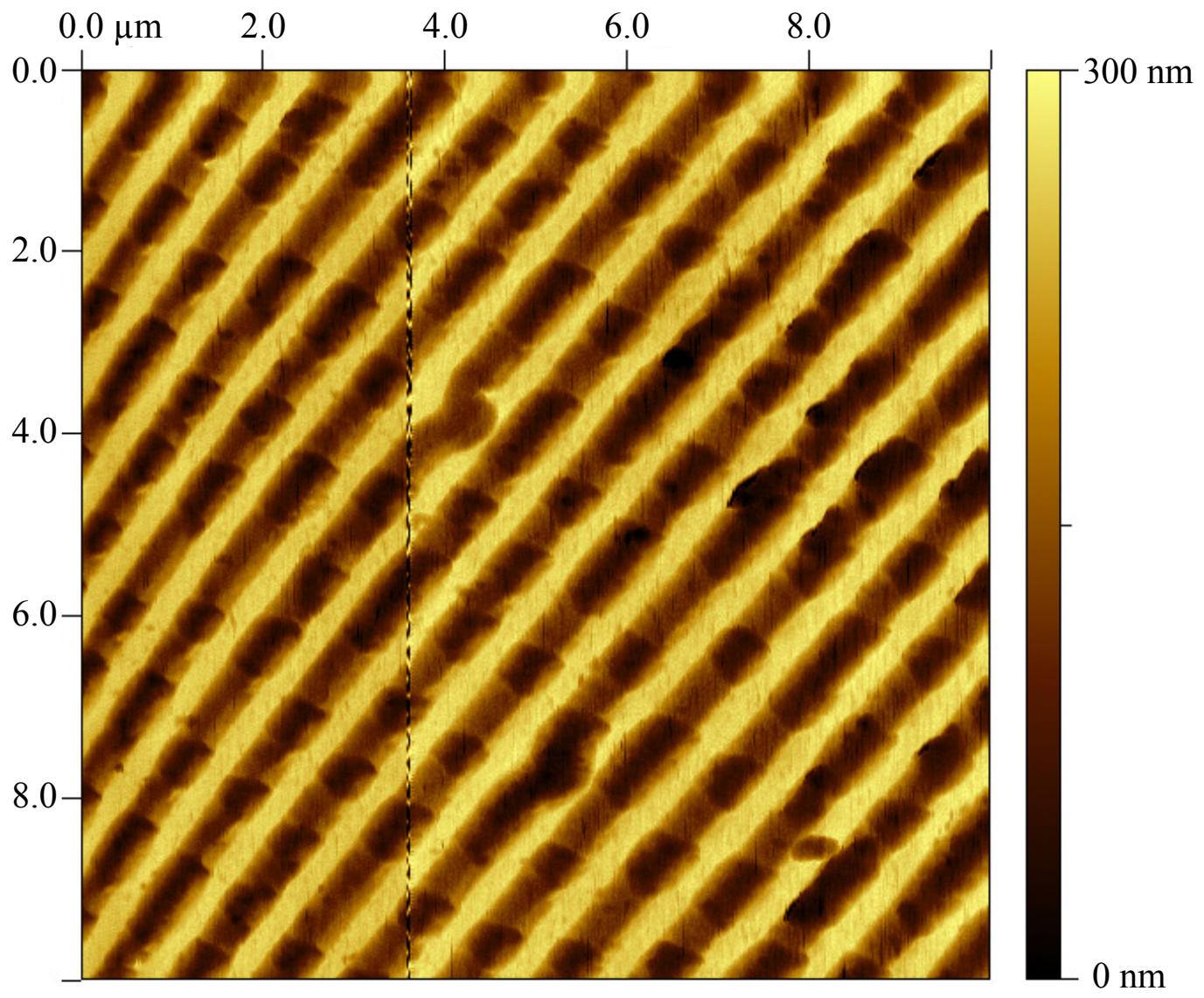

Figure 10.8 Topographic shear force image of a rewritable DVD. The band on the left of $4.0 \mu \mathrm{m}$ location on the image results from changing the PID settings, then returning them to the original scan values. The image was flattened in post-processing to remove the sample tilt and a $2^{\text {nd }}$ order distortion present in $\mathrm{z}$ along the $\mathrm{y}$ dimension, caused by the closed-loop sample stage's off-axis movement. 


\section{CHAPTER 10 References}

Bryant P. J., Kim H. S., Zheng Y. C. and Yang R., "Technique for Shaping Scanning Tunneling Microscope Tips,” Rev. Sci. Instrum. 58, 1115 (1987).

Melmed A. J., "The Art and Science and Other Aspects of Making Sharp Tips.” J. Vac. Sci. Technol. B 9, 601 (1991).

Wagner A. and Hall, T. M., "Liquid Gold Ion Source,” J. Vac. Sci. Tech. 16, 1871 (1979). 


\section{CHAPTER 11}

\section{Fabrication of Probes for TENOM Imaging}

As determined by the finite difference time domain (FDTD) modeling in Chapter 4, the asymmetric probe design should give successful near-field images. The feasibility of the asymmetric design was tested with its production in a focused ion beam (FIB) microscope. The fabrication of the asymmetric probe requires a top down approach. As with many scanning probe microscope probe designs, a bulk metallic wire is required. The wire is electrochemically etched and then milled in the FIB, to create the custom geometry required. This chapter outlines the process to custom manufacture TENOM probes, specifically the asymmetric design, including details of the complications encountered during fabrication.

\subsection{Chemical Etching of Au Wire}

$\mathrm{Au}$ wire, $100 \mu \mathrm{m}$ thickness, was chosen as the metallic probe material for TENOM imaging (Sigma-Aldrich \#349283). In general, Au does not give the highest field enhancements when compared to other materials such as Ag. But Au has an advantage in that it does not react with biological compounds. Moreover, Au does not oxidize as quickly as most metals, and is fairly easy to handle and chemically etch with chemicals of low toxicity.

In Chapter 10, the goal was to chemically etch $\mathrm{W}$ to the smallest possible tip apex diameters. It is easy to become fixated on the same goal with the Au etching. Luckily, probe sharpness is not of utmost importance with the Au probe fabrication. In many 
ways, very sharp Au etchings $(<100 \mathrm{~nm})$ complicate the fabrication process by increasing the milling times in the FIB. Precision cuts in the FIB are done at lower beam currents, resulting in slower milling times. The more material that has to be removed to fabricate the tips results in a compromise between precision and milling speed. The ideal Au etch would have a reproducible tip apex on the order of $500 \mathrm{~nm}$, with an overall parabolic shape. From this point, milling in the FIB will be predicable and fast.

\section{1.a DC Etching Difficulties}

Initial etching tests applied the direct current (DC) design for $\mathrm{W}$ etching presented in Chapter 10, using an electrolytic solution of $37 \% \mathrm{HCl}$ with an etch voltage of $7.0 \mathrm{~V}$ and a reference voltage of $0.05 \mathrm{~V}$ to etch the Au wire. Following the data presented by Sánchez's dissertation, a good reproducible etch was unattainable with the new DC circuit design [Sánchez, 1999]. Novonty proposed an etch circuit design for $\mathrm{Au}$, combining an AC component to a DC etch [Novotny \& Hecht, 2006]. Attempts to recreate his design resulted in poor etching results.
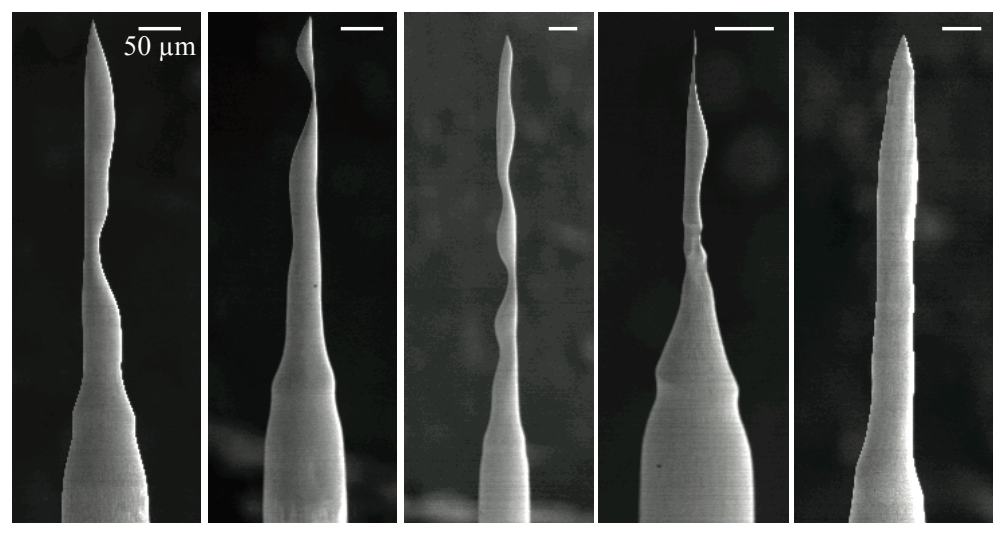

Figure 11.1 Five different Au probes etched with DC etch circuit. Details of etch parameters are outlined in text. Scale bar is $50 \mu \mathrm{m}$ for all images. 
Most of the DC etches resulted in long taper lengths with wavy thicknesses. Figure 11.1 shows the results of several DC Au etches. Many upon many trials were run, varying both etching and reference voltage settings, but good settings were not found. Other groups have discussed methods of Au etching, but ran into similar complications [Ren, Picardi \& Pettinger, 2004]. For their needs, the etched probes are acceptable, but will require more work in the FIB to make a functional TENOM probe.

\section{1.b Reproducible AC Etching}

Alternating current for etching is usually used to electrochemically polish surfaces and etched tips. Experiments with $\mathrm{W}$ and $\mathrm{AC}$ etching demonstrated parabolic shapes with tip apexes on the order of a micron. The AC W etches used the electrochemical solution that was used with the DC W etches; it was then assumed that the same relation would exist for the Au wire. Figure 11.2 shows the electrical signal connections for the AC etching circuit.
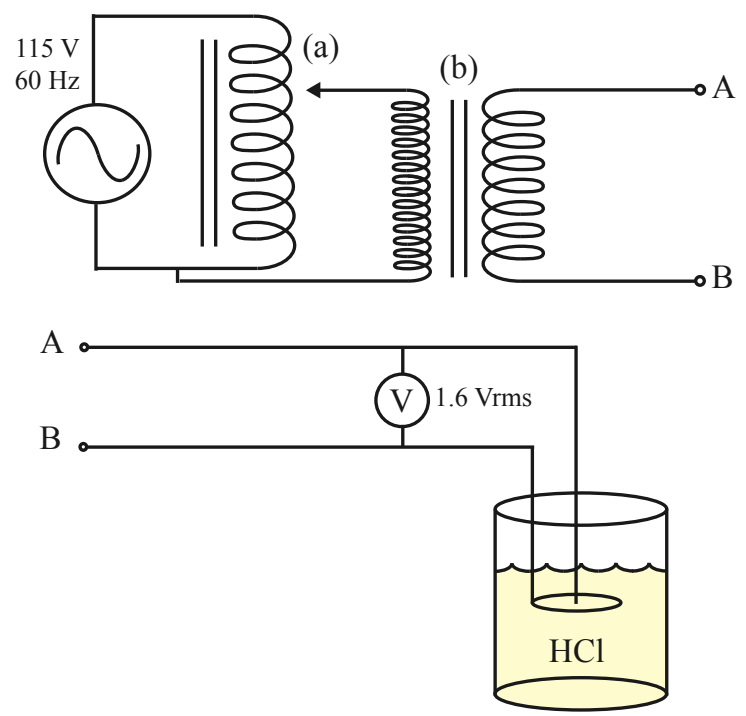

Figure 11.2 Electrical schematic of AC circuit for electrochemically etching Au wire. The circuit consists of a laboratory Variac (a) and a step down transformer (b). 
The Variac controls the input voltage into a step down transformer; this configuration maintains a clean sinusoidal waveform. A Variac or autotransformer tends to show a distorted waveform at very low voltages. For the AC, the same electrolytic solution of $37 \% \mathrm{HCl}$ was used, with an etching voltage of $1.6 \mathrm{~V}_{\text {rms. }}$. After etching, the wire was dipped into a beaker of deionized water to rinse away any residual $\mathrm{HCl}$ solution. A successful tip etching is usually observable under a stereo-zoom microscope, but a SEM can be used for better inspection. Figure 11.3 shows an SEM inspection of a typical $\mathrm{Au}$ probe shape with the AC etching circuit.
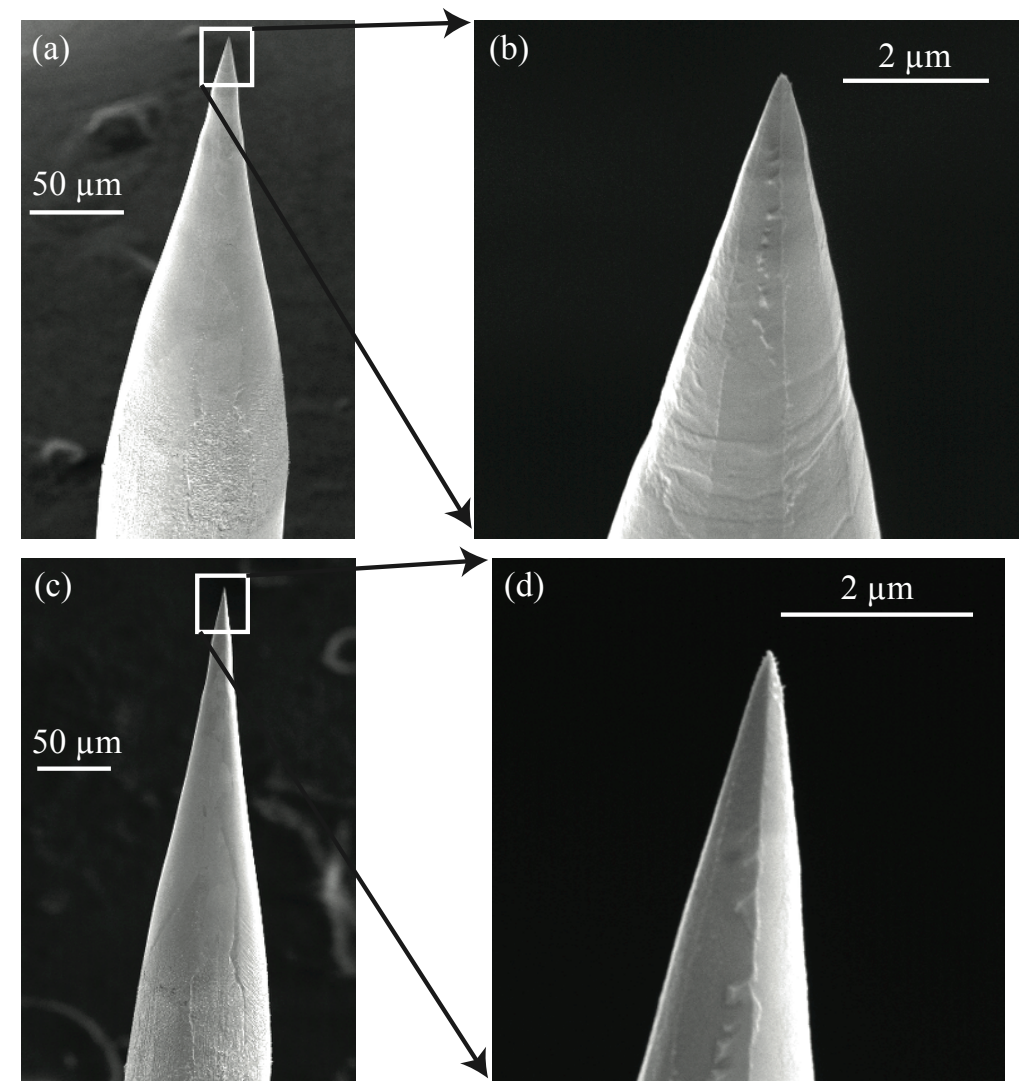

Figure 11.3 Scanning electron micrographs of Au wire after AC etching procedure. These two examples are very typical results. (b), (d) Zoomed areas from (a) and (c), respectively. 


\subsection{Mounting Probes to Tuning Forks}

Before milling the probes in the FIB, it is best to mount them to the tuning forks. There are two main benefits to this. First, the mounting of the probes is the step that has the highest probability of incurring damage to the probes themselves. Second, mounting followed by milling allows control over the orientation of the cuts, with respect to the tuning fork features. This helps determine the optimum polarization before bringing the probe onto the sample surface.

To mount the probes onto the tuning fork, the metal can containing the tuning fork needs to be opened to expose the forks. This can be accomplished with a tool typically used to open the containment cans for laser diodes. The tool is not much more than a miniaturized can opener, and is named accordingly (Thorlabs WR1). Figure 11.4 shows the tool with a tuning fork still in its can being prepared for opening.

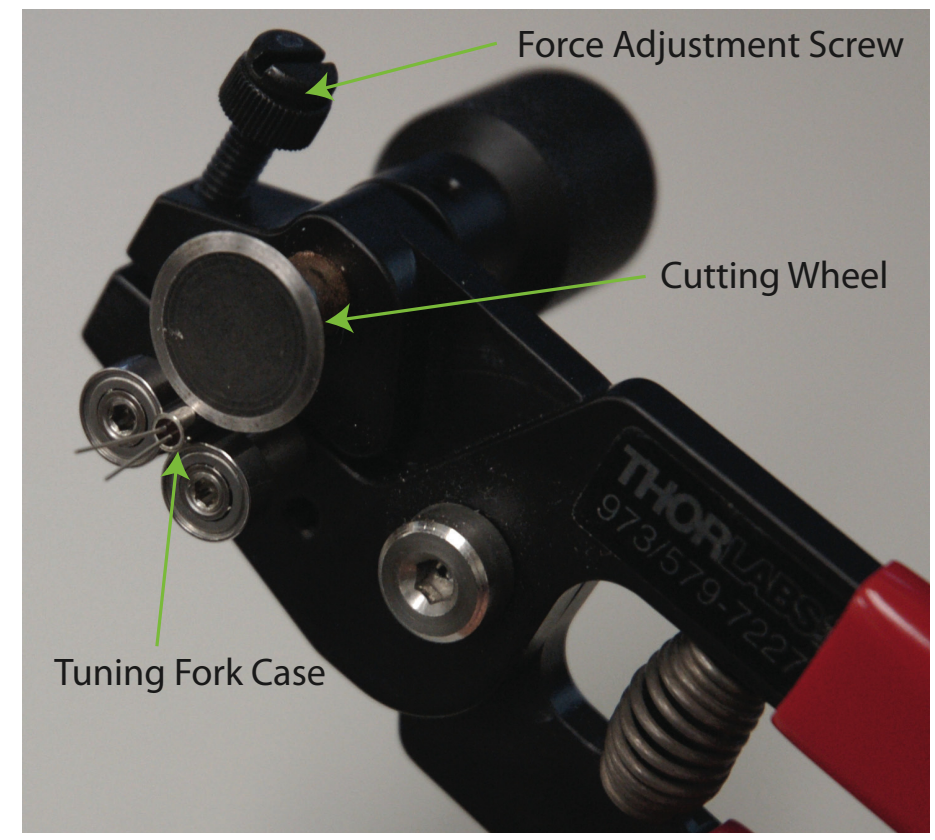

Figure 11.4 Tuning fork in can, in preparation of opening with Thorlabs laser diode opener. 
The tuning fork is very fragile and can be broken if care is not taken during the removal process. It is very important to start with a small amount of force and build up to a greater force that will eventually open the can. During the first few rotations of the cutting wheel, the tuning fork can start to drift perpendicular to the cutting axis. To minimize this, add just a little extra squeezing force, while moving the cutting wheel back and forth. Once the can remains in place while rotating the cutting wheel, it is important to pick a rotation direction with the cutting wheel and maintain it until the can has been opened. During this process, slowly increase force, and then rotate the cutting wheel rapidly for many rotations. Continue this process until the can falls out of the tool. While this last step may seem violent, the tuning fork typically survives.

Mounting the Au probe to the tuning fork requires a stereo-zoom microscope. The tuning fork should be positioned in the center of view. Holding the tuning fork in place can be accomplished by taping it around the remaining portion of the can. A small drop of CA (cyanoacrylate, "superglue") is placed on a flat metal surface. There is very little mounting time with glue, so it is important to have the Au probe ready in tweezers ahead of time. Using the sharp corner of a razor blade or Exact-O knife, pick up a small amount of the glue. Dab the glue onto one of the tuning fork prongs so that the liquid spot is about two millimeters long. Be careful not to short the electrical connections of the tuning fork, by using probes that are too long. Furthermore, be careful not to let the probe hang too far off the tuning fork, since this will give lower quality factors. 


\subsection{Milling Probes with a Focused Ion Beam}

The Au probes are milled in a dual beam microscope Strata DB-237, manufactured by the FEI Company. A dual beam is a system that has both FIB and SEM columns. The benefit of this combination is to use the SEM as an inspection tool and the FIB as a dissection tool. Dual-beam systems are heavily used to create thin sections for transmission electron microscopy (TEM) inspection. A complication with dual-beam systems can arise from limited stage movements due to a large number of analytical tools placed in the same imaging chamber. This requires some foresight about the desired cuts for the TENOM probes. One solution is to make custom sample holders to introduce tilt to the sample; Figure 11.5 (b) shows a custom stub design for this purpose. In DB-237, the electron column is directly above the sample stage, and the ion column is positioned at $52^{\circ}$ to the sample stage, or $38^{\circ}$ from the electron column.
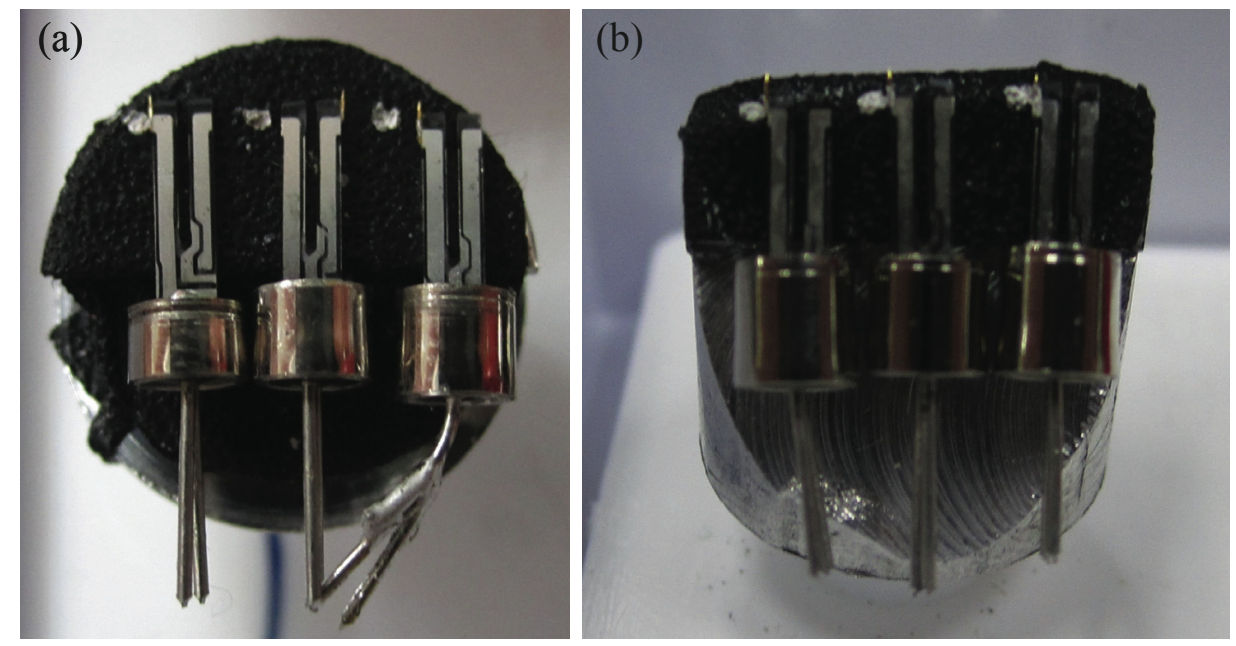

Figure 11.5 Sample stubs with $\mathrm{Au}$ probes mounted on tuning forks for milling. (a) Flat aluminum sample stub that has a notch milled to allow space for the remaining portion of the tuning fork can. (b) Aluminum stub milled at $52^{\circ}$ (not used for the asymmetric tip design). The small drops of silver connect the Au probes to the conductive carbon tape are to prevent charging. 
For the asymmetric probe design, the flat sample stub shown in Figure 11.5 (a) is used. The sample is placed in the DB-237, so the probes are pointing away from the chamber door (front of microscope). The initial milling cut is a solid polygon pattern that is made at $100 \mathrm{pA}$ of ion beam current, with no tilt of the microscope's sample stage. The result of this cut is shown in Figure 11.6, image (b). This current could be higher since the first few cuts do not require high precision. The quality of the chemical etch will determine the time needed for this step.

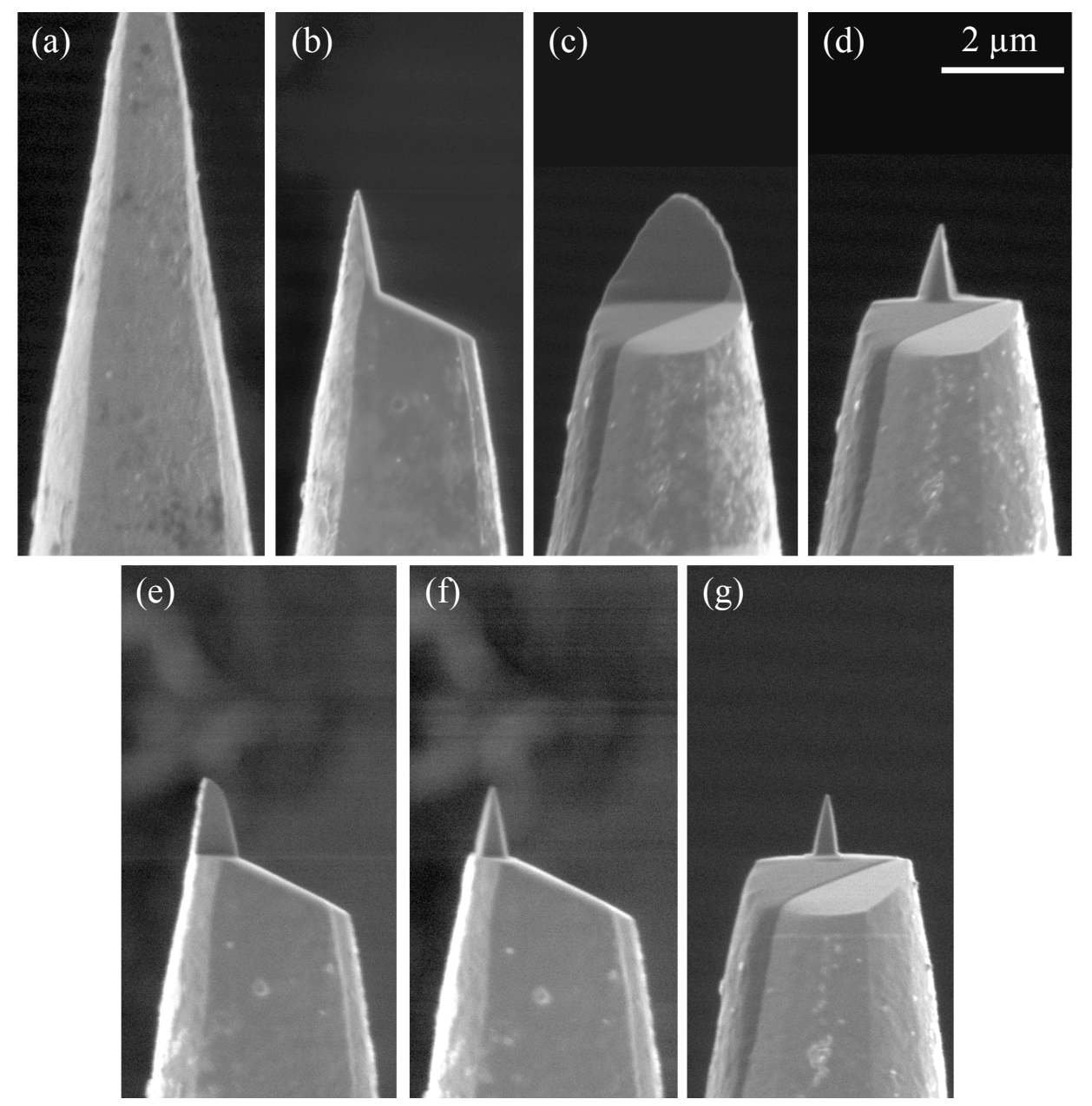

Figure 11.6 Milling cuts for the asymmetric TENOM probe design as outlined in the text. Shown in order from first cut to last (a) to (g). 
With the completion of the first step, the ion beam current should be decreased to $10 \mathrm{pA}$. The sample is rotated $180^{\circ}$ clockwise, as shown in Figure 11.6, image (c). Milling two solid polygons, simultaneously, at the $10 \mathrm{pA}$ ion beam current, will create the shape shown in Figure 11.6 image (d). Rotating the sample $180^{\circ}$ counterclockwise is shown as (e) in Figure 11.6 and allows for further cleanup of the protrusion cone that is being created, shown by (f). Transitioning between these two different sample positions should be repeated until an adequate protrusion cone is created. Lowering the milling current to $1.0 \mathrm{pA}$ can be helpful with fine cuts, but the sample may drift during the longer milling times. From this point, the TENOM probes are ready to be used as near-field imaging probes. 


\section{CHAPTER 11 References}

Novotny L. and Hecht B., Principles of Nano-Optics (Cambridge University Press, New York, 2006) pp. 213.

Ren B., Picardi G., Pettinger B., "Preparation of Gold Tips Suitable for Tip-Enhanced

Raman Spectroscopy and Light Emission by Electrochemical Etching," Rev. Sci. Inst. 75, 837 (2004).

Sánchez, E. J., Ph.D. dissertation, "A Novel Scheme for High Resolution Near-Field Fluorescence Microscopy”, Portland State University, 1999 (UMI No. 3018652). 


\section{CHAPTER 12}

\section{TENOM Imaging}

Tip enhanced near-field optical microscopy (TENOM) imaging is dependent on driving surface charge in a metallic probe. As demonstrated thus far, geometries that are optimal for epi-illumination are polarization dependent. The superposition of the incident electric field will determine the direction of surface charge oscillation. There are many variables that can affect the quality of TENOM images; these include polarization, location of the focus of the incident field, height of the sample topography, and quality of the imaging probe. Using fluorescent J-aggregates, Rhodamine 6G (R6G) dye crystals, and thylakoid membranes of Spinacea Oleracea, the functionality of the TENOM system with an asymmetric probe design is presented. Included are examples of probe scattering effects and near-field quenching effects that can be false indicators of high-resolution imaging. Comparisons are also made with mode-locked pulsed laser and continuous wave (CW) laser sources, as a motivation for developing lower cost imaging capabilities while mitigating damage due to the higher power requirements of the $\mathrm{CW}$ sources. Finally, all images were acquired with a single photon avalanche photodiode SPAPD (Perkin Elmer SPCM-AQR-14-FC), dichroic filter (Chroma 725dcspxr), three emission filters (Chroma ET750sp-2p8), and one excitation filter (Chroma r785lp), as outlined in Chapter 6.

\subsection{Near-Field Imaging of J-aggregates}

A sample that lends itself well to successful excitation by the multi-photon process is comprised of aggregates of a polymer (PVS) with Pseudo-Isocyanine (PIC) 
dye. J-aggregates are long and thin tube-like structures that are typically only a few nanometers across and several microns in length, making them ideal for near-field studies [Higgins \& Barbara, 1995; Higgins et al. 1996; Sánchez, Novotny \& Xie, 1999]. An added benefit when imaging J-aggregates is the lower susceptibility of photobleaching because of energy transfer between dye molecules [Horng \& Quitevis, 1993]. As mentioned in Chapter 9, preparation of J-aggregates requires $4.0 \mathrm{~mL}$ of $0.75 \%$ by weight of PVS solution (Aldrich \#278424) heated to $90^{\circ} \mathrm{C}$, while stirring. Once the temperature of $90^{\circ} \mathrm{C}$ is obtained, $0.4 \mathrm{~mL}$ of $10 \mathrm{mM}$ PIC dye is added. The solution should be left to cool with gentle agitation. As the solution returns to room temperature, the color should change from a semi-translucent red to an opaque red with a thin film of metallic green. The J-aggregates should then be spin coated onto cover glass slips at 4,000 rpm, while the solution is slightly above room temperature. As the J-aggregates continue to cool, the samples become thicker, which can be less desirable for near-field imaging.

\section{1.a Probe Interaction and Scattering Effects}

The TENOM imaging probe should be designed with geometries that promote high field enhancements. Typically, these enhancements should be at least an order of magnitude greater than the far-field background contributions. If the highest magnitude of enhancements during alignment demonstrates a simple doubling in the photon signal over the background signal, this can be a sign of the probe scattering background fluorescence into the objective lens. In this case, the probe is increasing the collection efficiency of the objective lens by reflecting off-axis photons back into the collection pathway. Figure 12.1 demonstrates the effect of scattering by the probe. Caution must be exercised, since it is possible that the lack of high electric field enhancement could 
have resulted from poor alignment of the imaging probe. In this case, the scattering effect was amplified due to a damaged probe. After exhausting all of the alignment degrees of freedom, the probe was analyzed in a scanning electron microscope (SEM). The SEM verified that the probe was damaged by sample probe interactions;
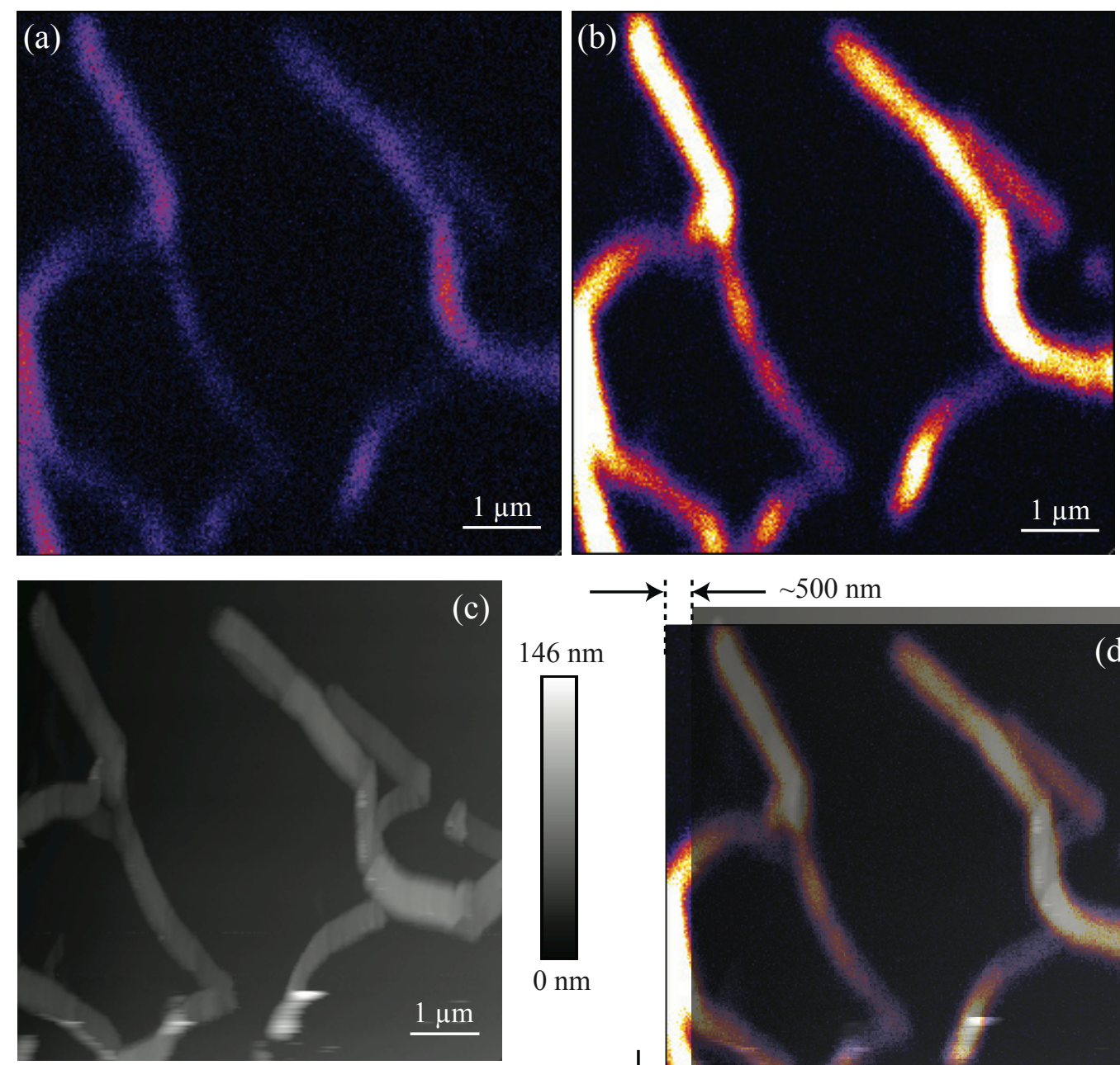

(c)
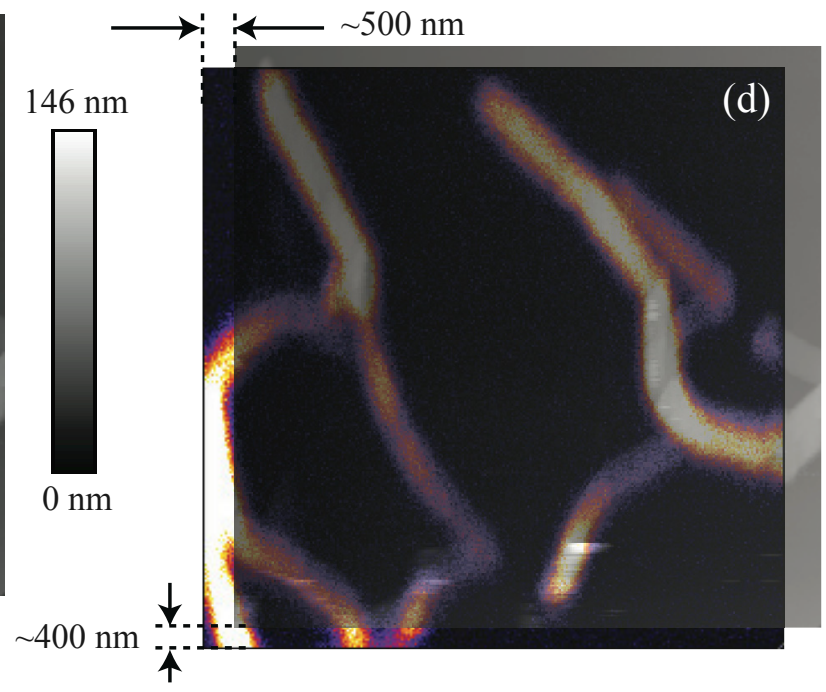

Figure 12.1. Scattering effects from a near-field probe on two-photon imaging of J-aggregates with PIC dye. (a) Far-field diffraction limited optical image without tip present. (b) Diffraction limited resolution optical image with tip present. (c) Surface topography of imaging ROI. (d) Shows a total offset of $640 \mathrm{~nm}$ between the optical image and the topographic image. 
The small protrusion cone had been smashed all the way to the bulk region. This damage is evident in the topographic image of the J-aggregates in Figure 12.1, where the sample appears to be flat ribbon-like structures as a result of the imaging probe's blunt profile.

\section{1.b Effects of Quenching}

Optical quenching results from the reabsorption of a fluorescent photon into the metallic imaging probe. The effect of quenching increases as the imaging probe moves closer to the sample surface.
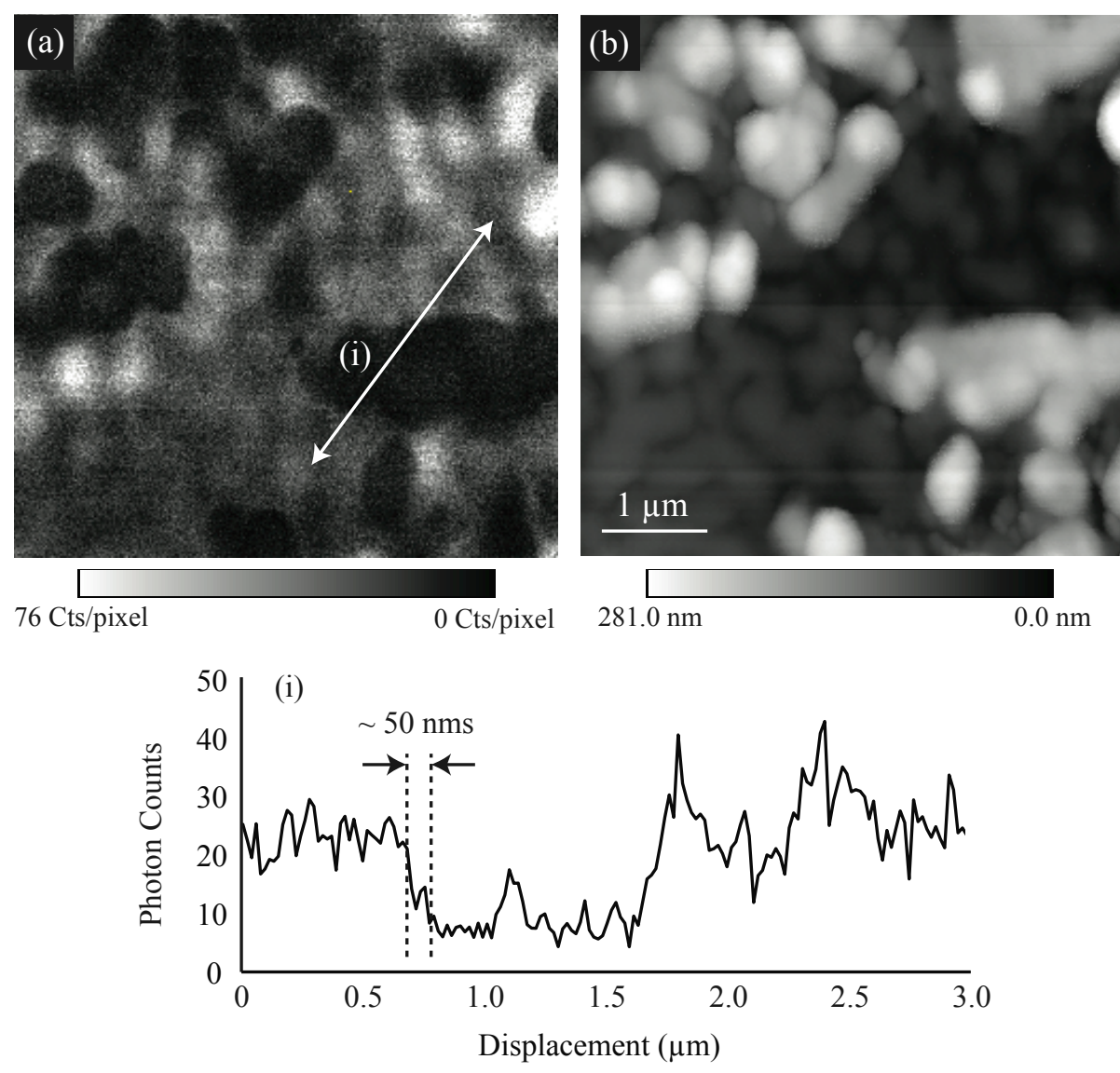

Figure 12.2 Quenching effects from the metallic near-field probe on twophoton imaging of R6G dye crystals. (a) CW excited near-field optical image, demonstrating below $50 \mathrm{~nm}$ resolution, measured from edgecontrast. The dark regions are areas of optical quenching. (b) Topography of the sample surface, acquired simultaneously. The regions of high topography correspond with the quenching regions. 
The break-even point for quenching appears to occur at $9.0 \mathrm{~nm}$, well within the typical size of the localized electric field [Krug, Sánchez \& Xie 2005]. In near-field optical images, quenching is observed as well-defined dark regions that correspond to areas of raised topography. Figure 12.2 shows the effects of quenching on R6G dye crystals. The crystals were made from a solution with $1.0 \mu \mathrm{M}$ concentration of the R6G molecules in methanol. A small droplet of solution was placed on a cover glass slip and allowed to air dry. For imaging, a location that had a thin coating of dye was found and examined. The sample was excited with a Del Mar Photonics Trestles-50 Ti:sapphire laser configured for $\mathrm{CW}$ output at $817 \mathrm{~nm}$ with an excitation power of $550 \mu \mathrm{W}$ at the sample surface.

\section{1.c Kerr-Mode Locked Pulsed Laser Source}

The use of mode-locked pulsed laser sources have been used in TENOM fluorescence imaging to maximize photon density to increase the probability of a twophoton event. The peak power of the pulsed laser is on the order of a kilowatt, but the duration is only 52 fs long. Due to the short duration of the pulses, the overall average power of the pulsed laser is in the milliwatts. It is assumed that these short pulses will not contribute to any heat-induced modification of the imaging probes. Moreover, it will be seen that successful TENOM images will use excitation powers of 10's of microwatts; the resulting peak power per pulse is approximately $100 \mathrm{~mW}$. Figure 12.3 shows the first successful high-resolution near-field image with the TENOM system. The 512 by 512 pixel images were acquired with the Trestles-50 Ti:sapphire laser operating in femtosecond pulsed mode, operating at $833 \mathrm{~nm}$ with $52 \mathrm{fs}$ pulses and an average power at 
the sample of $10.6 \mu \mathrm{W}$. The laser power at the surface was verified with a power meter (Coherent FieldMaster \#33-0506-000 with LM-2 VIS power head).
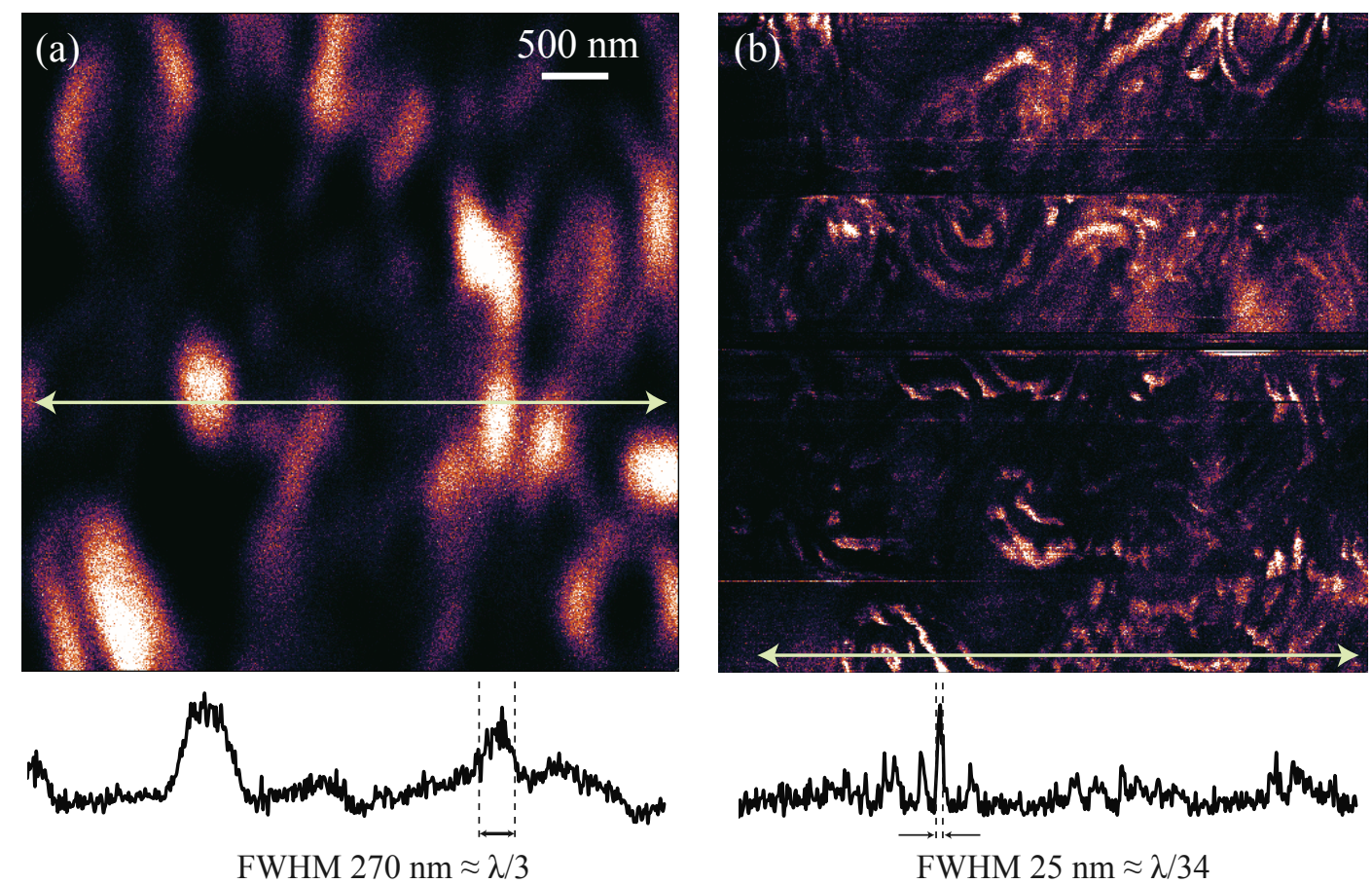

FWHM $270 \mathrm{~nm} \approx \lambda / 3$

FWHM $25 \mathrm{~nm} \approx \lambda / 34$
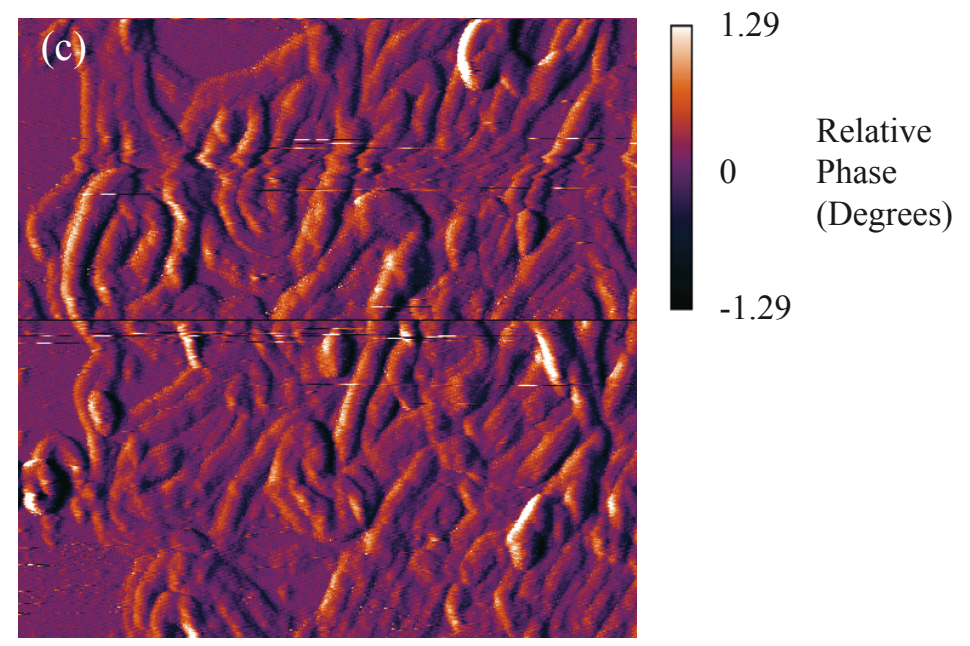

$-1.29$

Figure 12.3 Two-photon imaging of J-aggregates with PICI dye excited at $833 \mathrm{~nm}$ with a mode-locked laser operating at 52 fs pulses. (a) Far-field diffraction limited at $10.1 \mu \mathrm{W}$. (b) Near-field with average excitation power of $9.8 \mu \mathrm{W}$, same ROI as (a). (c) Phase feedback image generated by the error signal for the topography scan, simultaneously acquired with (b). 

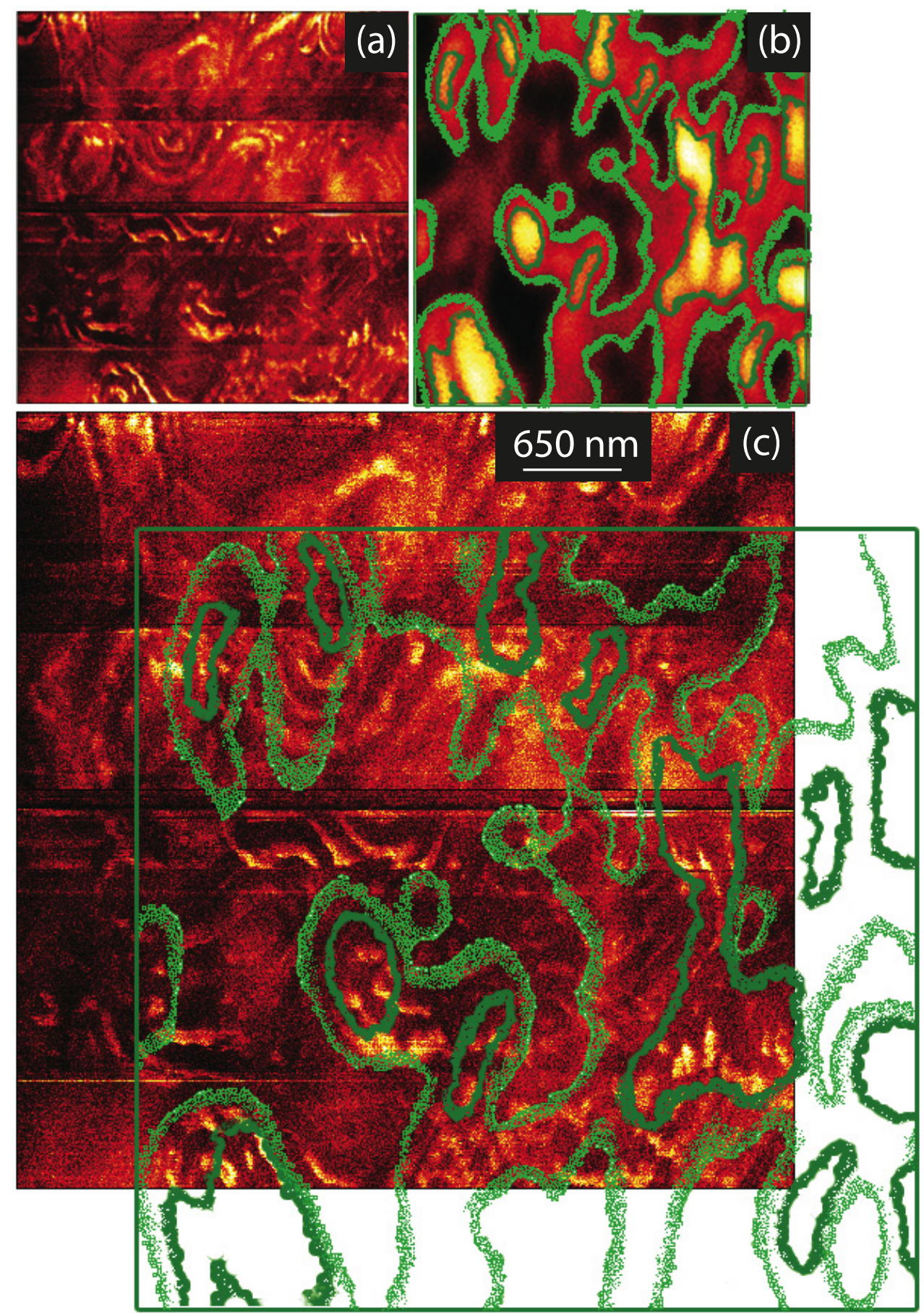

Figure 12.4 Proposed displacement between the near-field and far-field images presented in Figure 12.2, due to focal delocalization. Both images (a) and (b) are of the same ROI, one with the TENOM imaging probe (a) and one with the probe removed (b). The green contour maps were generated with ImageJ and Photoshop from image (b). (c) Shows the amount of displacement is roughly $1.0 \mu \mathrm{m}$. 
The diffraction limited image in Figure 12.3(a) demonstrates the $\lambda / 3$ expected from the nonlinear two-photon process. The near-field image shows full width half maximum (FWHM) optical resolutions of $25 \mathrm{~nm}$ or roughly $\lambda / 34$. The relative phase feedback image shows very little interaction with the sample. During imaging, it appeared that the imaging location of the highest near-field signal was shifted from the background diffraction limited far-field fluorescent image. Figure 12.4 examines this lateral shift between the near-field and far-field images. The modeling in Chapter 4 suggests this is a possible focus delocalization imaging condition. The lateral shift is on the order of one micron, placing the far-field focus over the bulk area in the asymmetric imaging probe, away from the imaging protrusion cone. Both Figure 4.22 and Figure 5.2 demonstrated these ideas.

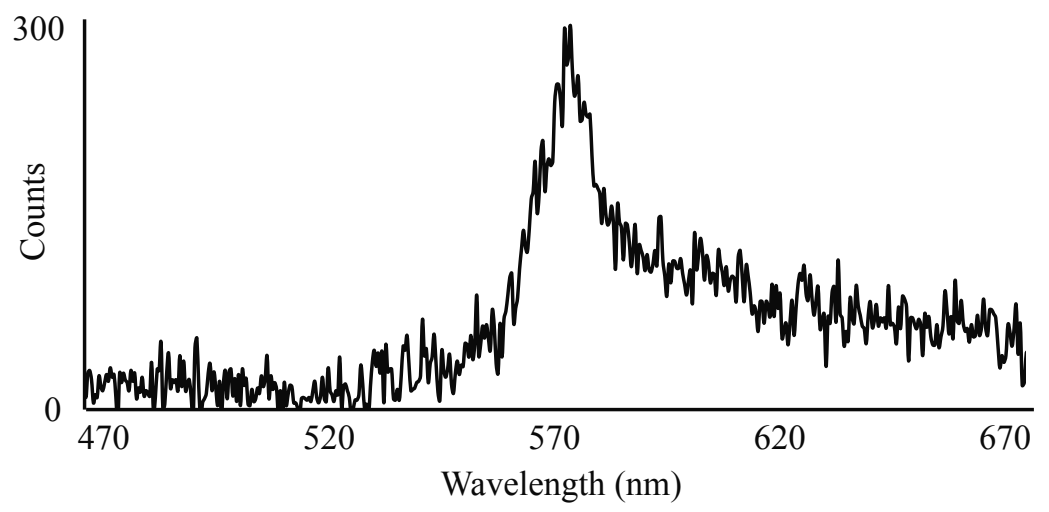

Figure 12.5 Fluorescence spectrum from the emission of the PIC dye. Sampled at the origin of the ROI shown in Figure 12.3, two-photon excitation with $833 \mathrm{~nm}$ mode locked laser source at $52 \mathrm{fs}$ at $110 \mu \mathrm{W}, 1.0$ second acquisition time.

To justify the two-photon fluorescence process, the spectrum shown in Figure 12.5 was acquired with the J-aggregate sample from the previous Figure 12.3. This is the same spectrum acquired with one-photon excitation [Sánchez et al., 1997]. The spectrum 
was acquired with a monochromator (Acton SP-150) with an attached ICCD (Princeton Instruments Pentamax-512-EFT/1EIA).

The final images of J-aggregates using the mode locked laser source are presented in Figure 12.6. The motivation was to use these images as a comparison ROI for the pulsed laser excitation verses the CW laser excitation, discussed in the following section.

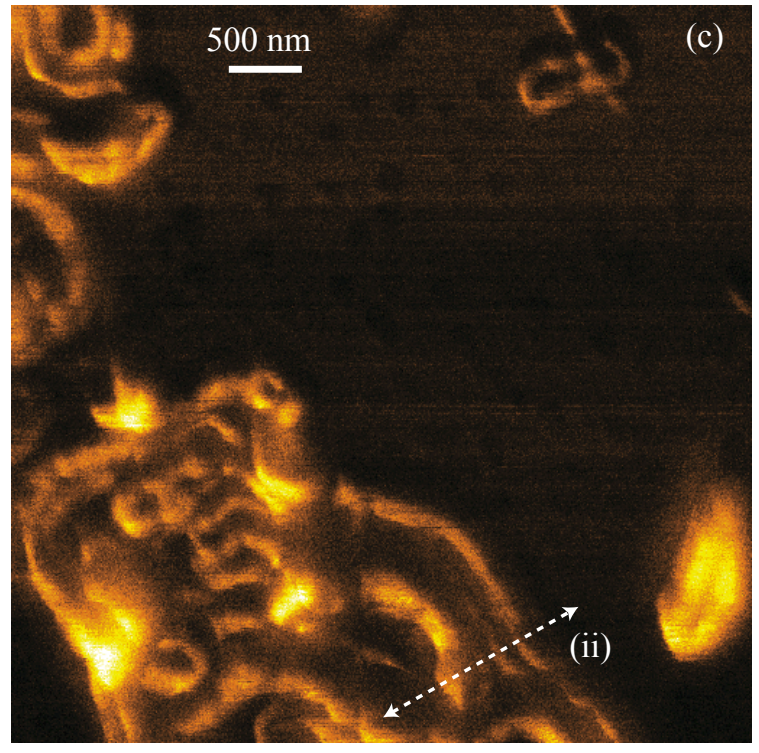

Cts/pixel 0 194

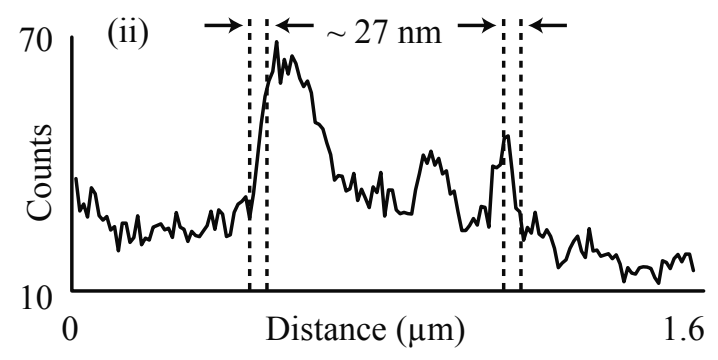

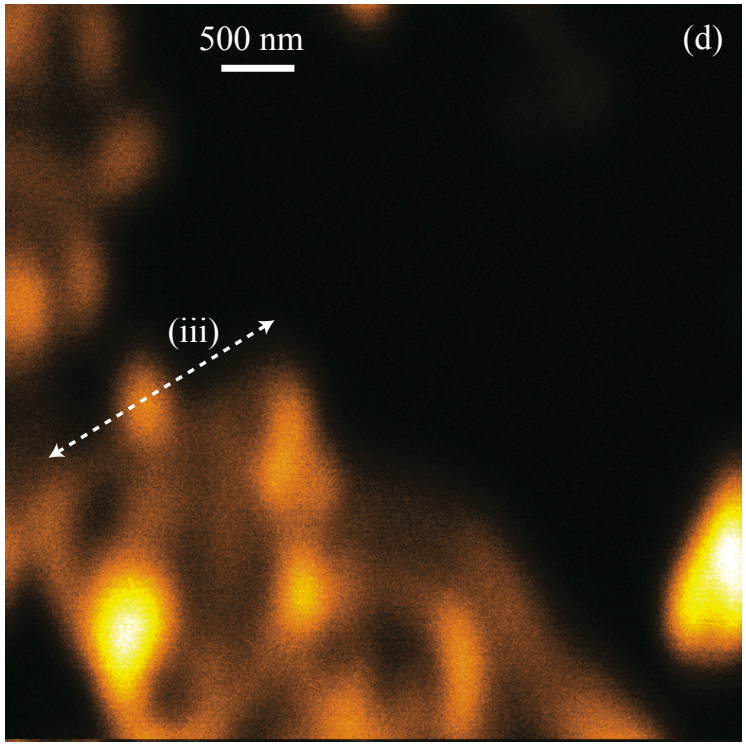

Cts/pixel 0 390

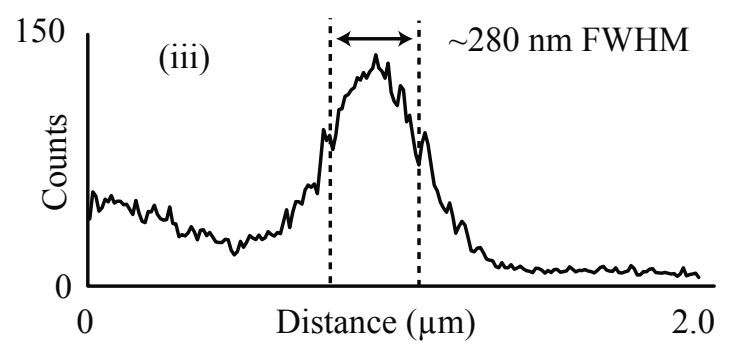

Figure 12.6 Near-field two-photon excitation fluorescence image of Jaggregates of PIC dye in a PVS film on a glass substrate (c), $\lambda_{e}=833 \mathrm{~nm}$ at $10.2 \mu \mathrm{W}$ mode locked at 52 fs. (d) Diffraction limited far-field twophoton excitation fluorescence of same ROI and excitation source as (c) at $31.5 \mu \mathrm{W}$. Both (ii) and (iii) are line analysis of image features. 
A near-field image, 512 by 512 pixels, is obtained using the mode locked laser source at $833 \mathrm{~nm}$ and $10.2 \mu \mathrm{W}$, demonstrating an optical resolution of $27 \mathrm{~nm}$. This resolution is represented by three pixels. It could be argued that the resolution is lower, but a higher pixel resolution image would be required. A diffraction limited image is also included as a baseline, which demonstrates the expected $\lambda / 3$ resolution. Both images were acquired at 20 minutes each.

\section{1.d Continuous Wave Coherent Laser Source}

Using CW illumination requires, on average, about 100x the power that is used in mode-locked illumination [Hell et al., 1998]. To show that the increase in power needed would not cause damage to either the sample or the imaging probe, a region of interest of J-aggregates was imaged with a mode-locked laser source at $10.6 \mu \mathrm{W}$ (Figure 12.6) and again with a collimated CW diode laser source (SDL-5411-G1, Thorlabs LT220P-B) at $1.3 \mathrm{~mW}$, as shown in Figure 12.7, while using the same imaging probe. The wavelength was held constant at $833 \mathrm{~nm}$. The acquisition time for the $\mathrm{CW}$ image and simultaneous topography image was about 20 minutes. The main difference between the $\mathrm{CW}$ and mode-locked was the increased far-field contribution from the CW illumination. This was a result of the poor beam quality of most inexpensive diode lasers. This could be improved by adding optics to correct the beam profile of the $\mathrm{CW}$ diode laser. Improvements in tip design to yield higher field enhancements would also minimize the far-field contribution during imaging. The $\mathrm{CW}$ image demonstrates the same optical resolution as its mode-locked counterpart, of $27 \mathrm{~nm}$. The topography of the scan area suggests that some damage to the imaging probe had resulted from surface interaction 
(ribbon-like J-aggregate appearance). This could have been a result of focusing the objective lens or have occurred during the approach routine. The topography for the mode-locked laser source image (not shown) demonstrated the same ribbon-like appearance. SEM imaging revealed that, in fact, the tip had been slightly damaged. The resultant damage actually improved imaging by flattening the protrusion cone and creating a sharp corner, which resulted in a strong localized field.
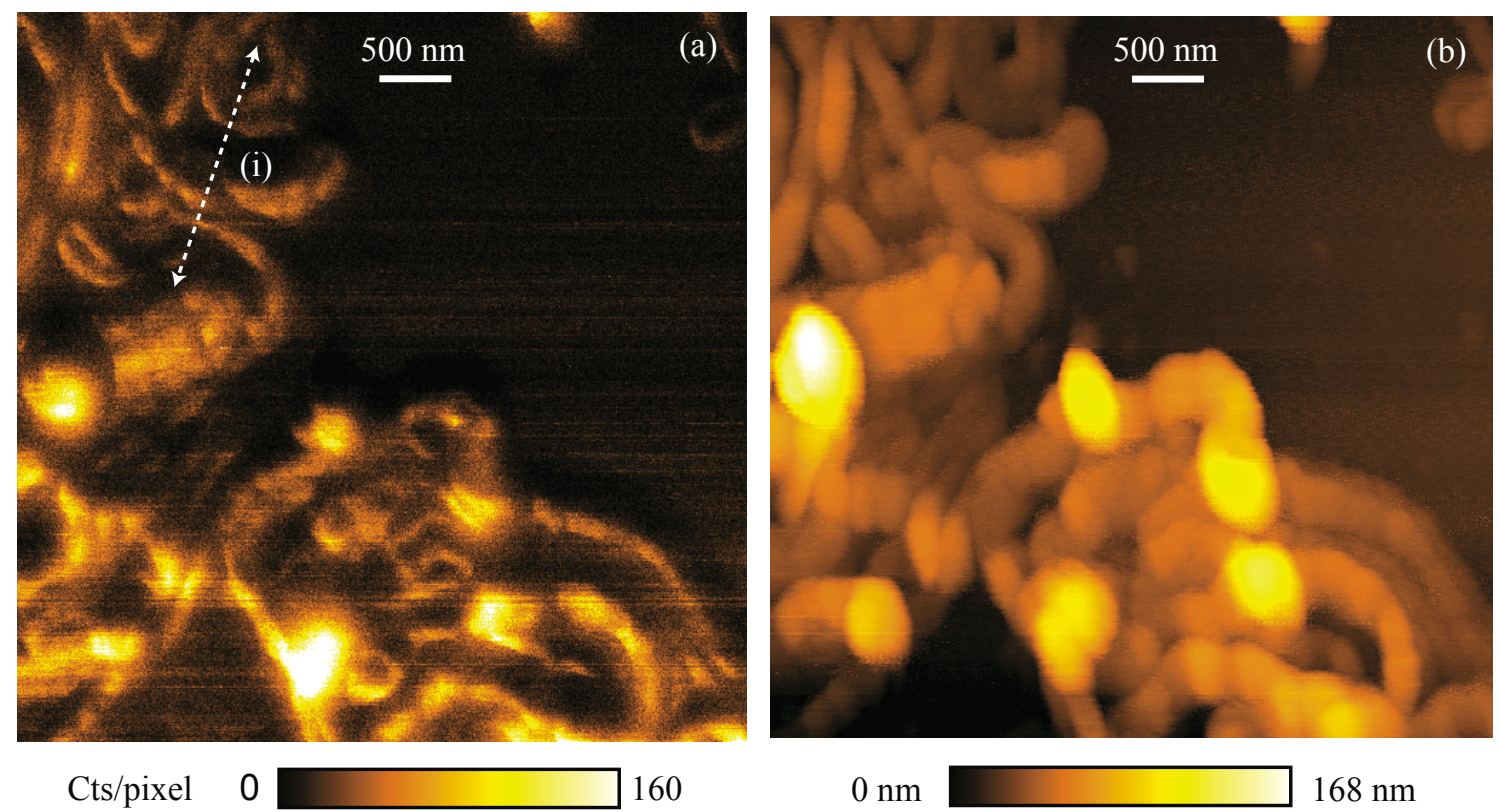

Cts/pixel 0 160 $0 \mathrm{~nm}$ $68 \mathrm{~nm}$

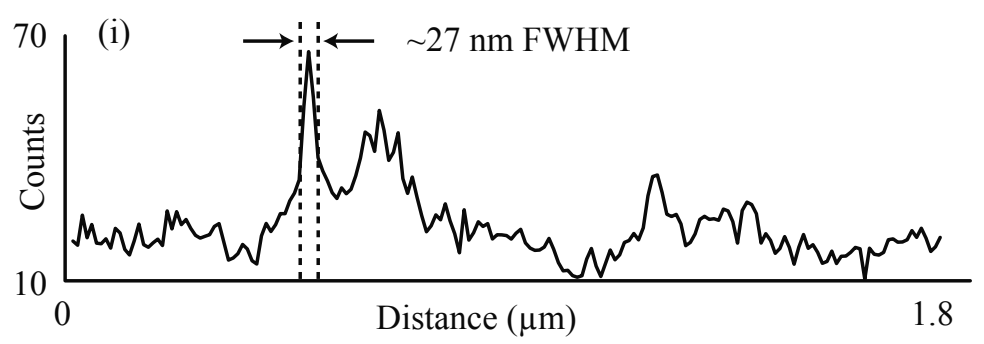

Figure 12.7 Near-field two-photon excitation fluorescence image of Jaggregates of PIC dye in a PVS film on a glass substrate. (a) $\lambda_{e}=834 \mathrm{~nm}$ at $1.3 \mathrm{~mW} \mathrm{CW}$. (b) Topography of J-aggregates taken simultaneously with an optical image. (i) Line scan profile demonstrating a FWHM resolution of $27 \mathrm{~nm}$. The ROI is similar to Figure 12.5. 
The offset is caused by a lateral shift between the pulsed laser and CW laser alignment. Unfortunately, the topography suffered from this damage. Careful inspection shows that the topographic image and the optical image are laterally shifted by about 200 $\mathrm{nm}$, suggesting that the middle of the protrusion cone was not generating the near-field optical image.

The higher power requirement for the $\mathrm{CW}$ diode laser adds an excitation efficiency complication. While all efforts were made to make the collection of emitted photons as high as possible, the TENOM system's optics were not efficient in the nearinfrared wavelengths. About ten percent of the power measured at the laser output aperture reaches the sample, assuming optimum alignment and good beam quality. A large percentage of light is lost through the objective lens itself. The maximum output power of the diode laser used is $143 \mathrm{~mW}$, with the maximum power attainable at the surface of $2.5 \mathrm{~mW}$. This low power leaves little room for improvement. Reconfiguring the Ti:sapphire laser to operate in $\mathrm{CW}$ mode allows for excitation powers of up to $50 \mathrm{~mW}$ to be available at the sample surface, with tunable output wavelength. Figure 12.8 uses power levels comparable to the $\mathrm{CW}$ diode laser used in the previous imaging, demonstrating near-field imaging in this configuration. Only a thin section of the image is shown, since the probe was destroyed after violent oscillations in the feedback loop caused continuous tip sample interactions. The oscillations corresponded with the alignment of the probe and when peaked, photon emission would occur from the sample surface. It is speculated that the tip exhibited a very high field enhancement; due to the high field, it may have been pulled into the sample in a process that is commonly used in 
atomic laser trapping. The oscillations resulted from the inability for the PID loop to adjust for this new force.
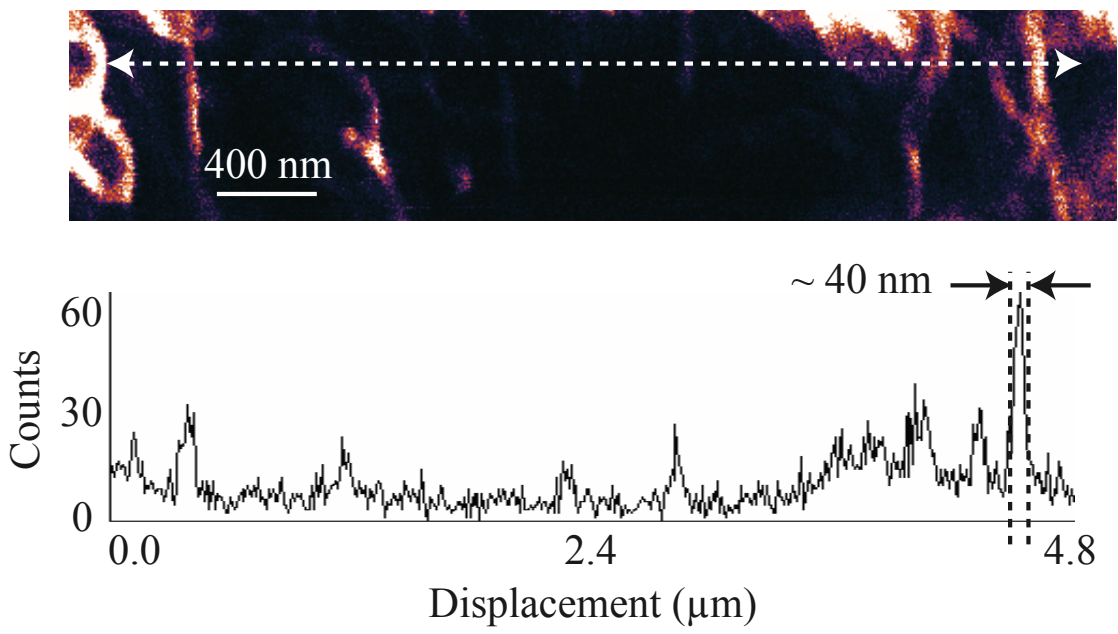

Figure 12.8. Two-photon imaging of J-aggregates with PIC dye excited at $817 \mathrm{~nm}$ with a Ti:sapphire operating in $\mathrm{CW}$ mode with $1.18 \mathrm{~mW}$ of excitation power at the sample surface. Resolutions of $40 \mathrm{~nm}$ and less are demonstrated.

\subsection{Biological Application: Imaging of Thylakoid Membranes}

Demonstration of near-field imaging on a biological sample was accomplished by imaging thylakoid membranes from Spinacea Oleracea. Common variety spinach is puréed, and then centrifuged at $8000 \mathrm{rpm}$ for twenty minutes. Centrifuging separates the chlorophyll (dark green) from the sugars (clear). The chlorophyll is siphoned from the bottom of the separated solution and spin-coated at 2000 rpm onto a cover glass slip. The near-field and far-field images were acquired $\lambda_{e}=833 \mathrm{~nm}$ at $16.5 \mu \mathrm{W}$ mode locked at 52 fs, shown in Figure 12.9. The near-field imaging probe proved to have poor field enhancement, in combination with a sample ROI that exhibited a large number of compact fluorescent chlorophyll locations that were difficult to resolve. 


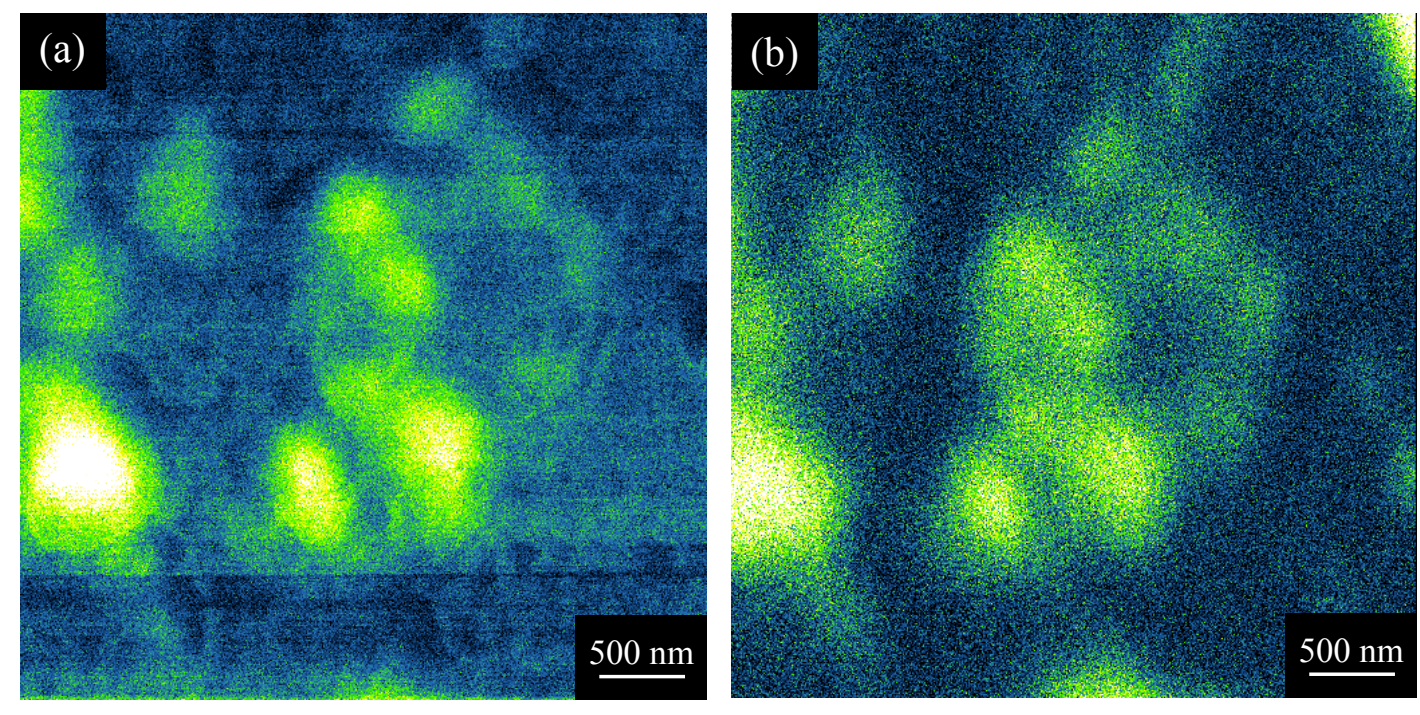

(c)

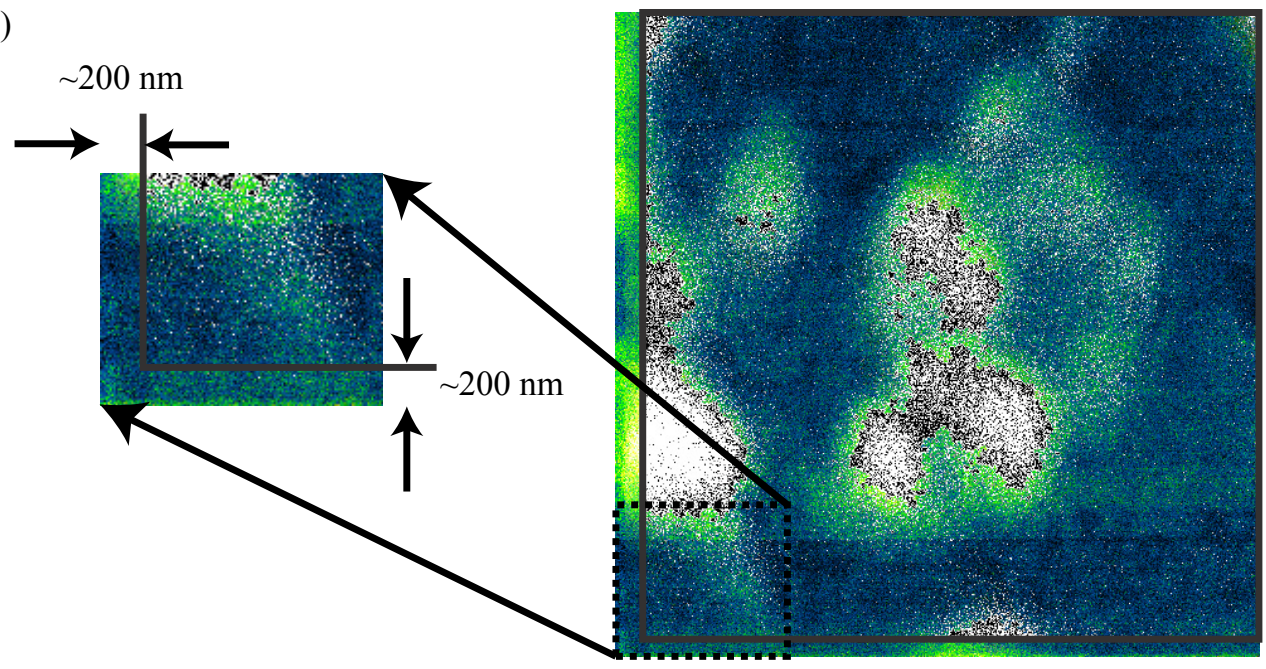

Figure 12.9 Near-field two-photon excitation fluorescence image of thylakoid membranes from Spinacea Oleracea on a glass substrate. (a) Near-field image with $\lambda_{e}=833 \mathrm{~nm}$ at $16.5 \mu \mathrm{W}$ mode locked at $52 \mathrm{fs}$. (b) Diffraction limited far-field image of same ROI. (c) Demonstrating the offset between the near-field and far-field images. The white regions are generated by saturating the bright regions of $(b)$.

Further investigation should be pursued with the Spinacea Oleracea. Since there appears to be little to no sample damage from imaging, $\mathrm{CW}$ excitation should provide similar results as seen with the J-aggregates. A demonstration of a biological sample with $\mathrm{CW}$ excitation will validate the motivation for developing a low cost, high- 
resolution biological tool. To alleviate the cost of a Ti:sapphire laser, a high power $(\sim 500$ $\mathrm{mW}$ ) $\mathrm{CW}$ diode laser will be required for imaging with flexible power ranges. Since the two-photon wavelength cross sections can spread over tens to hundreds of nanometers, a flexible $\mathrm{CW}$ laser wavelength can be determined. This will allow for the imaging of different samples and dyes using the same $\mathrm{CW}$ wavelength. 


\section{CHAPTER 12 References}

Hell S. W., Booth M., Wilms S., Schnetter C. M., Kirsch A. K., Arndt-Jovin D. J., and Jovin T. M., "Two-photon Near- and Far-field Fluorescence Microscopy with Continuous-Wave Excitation," Opt. Lett. 23, 1238 (1998).

Higgins D. A. and Barbara P. F., "Excitonic Transitions in J-aggregates Probes by Nearfield Scanning Optical Microscopy,” J. Phys. Chem. 99, 3 (1995).

Higgins D. A., Kerimo J., Vanden Bout D. A., and Barbara P. F., “A Molecular Yarn: The Self Assembly of Flexible, Fluorescent Fibers Studied by Near Field Scanning Optical Microscopy," J. Am. Chem. Soc. 118, 4049 (1996).

Horng M.-L. and Quitevis E. L., "Excited-State Dynamics of Polymer-Bound JAggregates" J. Phys. Chem. 97, 12408 (1993).

Krug J. T., Sánchez E. J. and Xie X. S., "Fluorescence Quenching in Tip-Enhanced Nonlinear Optical Microscopy,” Appl. Phys. Lett. 86, 233102 (2005).

Sánchez E. J., Novotny L., Holtom G. R., and Xie X. S., "Room-Temperature Fluorescence Imaging and Spectroscopy of Single Molecules by Two-Photon Excitation,” J. Phys. Chem. A 101, 7020 (1997).

Sánchez E. J., Novotny L., and Xie X. S., "Near-field Fluorescence Microscopy Based on Two-Photon Excitation With Metal Tips," Phys. Rev. Lett. 82, 20 (1999). 


\section{CHAPTER 13}

\section{Discussion}

The fundamental goal of near-field optical microscopy is to spatially resolve unknown features at resolutions below the diffraction limit. The motivation for the development of a high-resolution optical microscope is to make subdiffraction limit imaging an everyday tool for the researcher of any discipline. To make this a reality, continuous refinement of current microscope designs and the creation of new imaging techniques will be required. The tip enhanced near-field optical microscope (TENOM) based system presented throughout this dissertation outlines the elements of design needed and the importance of imaging probe geometries that successfully image below the diffraction limit. Breaking the diffraction limit is not a trivial task for any of the mentioned far-field or near-field techniques. How does the current capability of TENOM system aid in improving nearfield imaging? This discussion chapter will focus on the important features of the microscope that either benefit or need to be improved for near-field imaging. These design limitations are mentioned in context throughout this discussion, starting with a recap of the introduction to near-field optical imaging techniques.

The diffraction limit classically sets the spatial resolution of a microscope to half of the wavelength of incident imaging radiation. Near-field microscopes are able to obtain high resolutions by confining the excitation light to regions smaller than the diffraction limit. Of course, this is not without limitation. The near-field probes must excite a spectroscopic change in the sample within a distance less than that of the desired 
level of resolution. In other words, if the obtainable near-field resolution is $20 \mathrm{~nm}$, then the imaging probe will need to be within a maximum of $20 \mathrm{~nm}$ of the response location on the sample. This fundamentally restricts near-field imaging to planar samples with small topographic features. Fortunately, a lot of near-field imaging is applied to nanomaterials and nanoscale biological processes that can be prepared on planar surfaces. Far-field "super-resolution" techniques, which have shown $20 \mathrm{~nm}$ resolutions, have less planar requirements for the samples, but require functionalize dye labels to be implanted into the sample. It is difficult to determine how these dyes change the physiology of the sample. The far-field methods currently demonstrate long image acquisition times.

It is important to realize that all techniques give some type of information that others cannot. A few compromises have to be made in order to break the diffraction limit, and one must choose which ones are most applicable for their sample. The TENOM system design addresses some near-field imaging complications, but is limited by the constraints of epi-illumination. The main advantage of epi-illumination is the high collection efficiency of the microscope. The disadvantages are complex alignment and the requirement of planar transmissive samples. The TENOM system was designed to be flexible enough to allow for modification of other near-field or even far-field imaging techniques to be implemented on the basic platform.

Development of TENOM imaging probes requires an understanding of the technical limitations of the controlled manufacturing process at the nanoscale level. Luckily, finite difference time domain (FDTD) modeling provides insight to potential TENOM probe designs, without having to spend months trying to fabricate and test each design. Initially modeling designs to determine what provides high field enhancement 
and potential focal delocalization, also gives a good conceptual starting point. However, when applying these designs to real world imaging environments, it becomes obvious that variables that seem trivial in modeling have much more impact in experimentation. For instance, the modeling of the focusing lens presented in Chapter 4 generated a numerical aperture (NA) of 1.1. For the near-field imaging presented in Chapter 12, the objective lens used had a NA of 1.4. The modeling predicted that focusing the light on the bulk area of the probe would provide a focus delocalization several nanometers away. This effect was observed experimentally, but only when the protrusion cone was manufactured to be smaller, or had been damaged to the point that it was smaller than the size of the protrusion cone used for modeling. This discrepancy could be attributed to the complicated polarizations of the electric field in the focus of the 1.4 NA objective lenses and how those wave fronts act as they leave the cover glass slip. This difference between modeling and experimentation will require further investigation.

The nanoscale movement of the TENOM imaging probe to obtain high-resolution images requires piezo electric crystals. Piezo technology is not without its limitations. Bandwidth of piezo movement is typically low, in the $1-20 \mathrm{kHz}$ range. The slow rates of movement limit typical image acquisition times, on the order of several minutes, as is the case for most of the images presented throughout Chapters 9, 10, and 12. The far-field optical images also suffer from this limitation since a closed-loop piezo scan bed is used for sample movement. Groups have implemented methods to increase scan rates for scanning probe microscopes (SPMs) [Rost et al., 2005; Picco et al., 2007]. In these cases, higher bandwidth piezos are required. These piezo have to be operated in an openloop configuration, since closed-loop feedback systems lose tracking accuracy due to the 
rapidly changing piezo position. One could still monitor the error signal from the closedloop feedback system to provide a correction factor for the scanned image, as a postprocessing step. The piezo artifacts will be greatest during changes in direction at the end of a scan line. To minimize this effect, the triangular waveforms that are generated for the raster scans will have to be changed to sine waveforms [Rost et al., 2005]. Changing the scanning waveforms will require more post-processing of the acquired images. Due to the intrinsic capacitance of piezos, another complication results from faster piezo movement. The faster rate of applied voltage to the piezo requires faster charging rates. Different designs of the piezos from tube, disk, or flexure movement will determine the overall capacitance, but the increase in current availability requires significant changes in the driving amplifiers and electronics. The current carrying ability of the amplifier circuit and the power supplies for the amplifiers will have to be carefully redesigned. Originally, the PA98 was chosen for its $1000 \mathrm{~V} / \mu$ s slew rates, which is about two orders of magnitude higher than the PA88s used in the final TENOM system design. Initial design tests using the PA98 proved more difficult than initially assumed. Complications with the PA98s resulted from the distance the amplifiers were from the piezo, the power supply current needs, and heat dissipation of the amplifier.

Further limitations of scan speed are caused by the tuning fork feedback system. The low resonance frequency of the tuning fork $\left(2^{15}=32,768 \mathrm{~Hz}\right)$ limits the response time of the $\mathrm{Z}$ motion of the microscope. Higher resonance oscillators could be used, but these devices have smaller dimensions, making them more difficult to work with. The TENOM system is flexible enough to adapt to different resonance frequencies. A modification of capacitor values for the active low and high pass filters would need to be 
adjusted for the new frequency. The direct digital synthesizer (DDS) will support frequencies up to $40 \mathrm{Mhz}$, but higher frequencies would require a new solution. Most of the operational amplifiers (OP27) throughout the microscope have a small signal bandwidth of 8.0 Mhz. This would have to be considered if oscillators in the Mhz range are desired.

As a basic inverted microscope platform, the design of the TENOM system gives the microscope a lot of versatility. A few design modifications could add more to this versatility. The TENOM system, as presented, uses a two-axis, closed-loop scan stage. Exchanging this for a three-axis, closed-loop scan stage would allow three dimensional confocal two-photon imaging, by providing precise control of Z-focal depth. Moreover, having a closed-loop Z-axis in the scan stage will limit artifacts generated in Z, during Xaxis and $\mathrm{Y}$-axis scanning, in a two-axis scan stage. These artifacts are usually a few nanometers of off-axis movement, so far-field imaging would see little to no benefit, but topography measurements in near-field imaging would benefit a great deal.

The addition of filter holders that allow rapid filter exchanges without the need for optical realignment would also be an added benefit. The current microscope design does not allow for the quick exchange of filters, mainly to minimize the number of complicated custom part designs.

The shear force microscope (SFM) design elements of the TENOM system have shown successful subdiffraction limited, high-resolution images as shown in Chapter 12. The usability of the near-field imaging probe on the microscope benefits by the electrical driving of the tuning forks. Several trials were performed with mechanical oscillation, since it provides better signal-to-noise ratios over the electrical driven methods [Jahncke 
et al., 2004]. It was determined that the reproducibility of obtaining a good resonance feedback signal was much easier with the electrical oscillation than mechanical oscillation. The larger Q-factors obtainable with electrical oscillation and rigid mechanical coupling to the tuning fork holder allow the microscope to sense the surface of the sample from $20 \mathrm{~nm}$ away. However, a side effect of rigid mechanical coupling is an increase in false engagement conditions during the approach process. As the Z-piezo extends to find the surface, a stiffening of the piezo itself occurs. Any coupling to the tuning fork will result in a frequency change. If the frequency change is great enough, then the feedback system will assume the surface has been found, resulting in false engagement. False engagements can be minimized by having a spacer material between the piezo stack and the tuning fork holder, usually a disk of nylon or acrylic. Being able to maintain distances greater that $9.0 \mathrm{~nm}$ is a further benefit, since it greatly reduces the amount of optical quenching in the near-field images [Krug, Sánchez \& Xie, 2005]. Finally, including a piezo to control fine focusing distances of the collection objective placed under the sample would greatly improve the usability of the TENOM system, by maintaining optimal focus through computer control while scanning.

The electronics for the TENOM system presented have been redesigned several times. The initial designs were prototyped on breadboards for functionality. Once the design details were worked out, the needed analog electronics were implemented on single printed circuit boards (PCBs). Difficulties with design errors and the original attempts with the PA98 amplifier that required modification of commercial power supplies [Steele, 1994] spurred the first redesign. Further difficulties with power spikes during the initial powering of the power supplies resulted in a second redesign, using the 
PA88s. With the design of the second version of the PCB came the realization that making the system modular would facilitate the exchange and upgrades to basic circuits, resulting in the third and current design. Even with all of the design changes, one issue remains: the parasitic noise that is discussed at the end of Chapter 7. A possible solution would be to remove the oscillation circuit from the main circuit board and isolate its ground return path, so that it is not allowed to migrate onto the data acquisition card. This could be accomplished with opticouplers and voltage regulators. Moving the DDS oscillation circuit into the scan head could also minimize noise that is picked up on the signal pathway from the main circuit board to the scan head.

Computer control of the TENOM system has demonstrated very good control of the imaging probe. Once good operating parameters for the proportional, integral, and differential gain equations and the approach timing constants were determined, the computer control of the microscope was very predictable. There are still a few complications with the computer program that need to be addressed to improve usability of the TENOM system. These include the data flow for the imaging system, improvement in the handling of large arrays, and the introduction of lithography control of the imaging probe.

The imaging system is not consistent in what direction, left-to-right or right-toleft, when starting the scan routine. This causes the trace and re-trace data sets to occasionally flip between the end of one image and start of the next. The very first execution of a scan results in a shift in the imaging arrays. This is overcome by stopping and restarting the scan. Both of these issues are directly related to the direct memory access (DMA) transfer functions and first in first out (FIFO) buffers. Implementing error 
checks in the field programmable gate array (FPGA) code will remedy this complication. Flexibility of displaying data is limited, due to the use of the intensity graphs packaged with LabVIEW. The intensity graphs are very memory intensive, limiting the TENOM system imaging to $512 \times 512$ pixels. This limitation could be overcome by the development of better array handling and compression for live image viewing. The addition of complex probe controls, such as lithography capability and Z-axis control for magnetic and electric field shear force imaging, would greatly add to the versatility of the TENOM system. 


\section{CHAPTER 13 References}

Jahncke C. L., Brandt O., Fellows K. E., and Hallen H. D., "Choosing a Preamplifier for Tuning Fork Signal Detection is Scanning Force Microscopy,” Rev. Sci. Inst. 75, 2759 (2004).

Krug J. T., Sánchez E. J. and Xie X. S., "Fluorescence Quenching in Tip-Enhanced Nonlinear Optical Microscopy," Appl. Phys. Lett. 86, 233102 (2005).

Picco L. M., Bozec L., Ulcinas A., Engledew D. J., Antogonozzi M., Horton M. A. and Miles M. J., "Breaking the Speed Limit with Atomic Force Microscopy,"

Nanotechnology 18, 044030 (2007).

Rost M. J., Crama L., Schakel P., Van Tol E., Van Velzen-Williams G. B. E. M., Overgauw C. F., Ter Horst H., Dekker H. Okhuijsen B., Seynen M., Vijftigschild A., Han P., Katan A. J., Schoots K., Schumm R., Van Loo W., Oosterkamp T. H. and Frenken J. W. M., "Scanning Probe Microscopes Go Video Rate and Beyond," Rev. Sci. Inst. 76, 053710 (2005).

Steele J., "Modify Power Supply to Enhance Performance," Analog Applications Issue Electronic Design, pp. 57, June 27, 1994. 


\section{CHAPTER 14}

\section{Conclusion}

The tip enhanced near-field optical microscope (TENOM) design developed for this dissertation has successfully imaged at resolutions below $30 \mathrm{~nm}$ on various samples. The use of nonlinear two-photon excitation has further simplified the optical filter requirements for the microscope. This allows for the imaging of different dyes without changing filter sets. Moreover, the use of two-photon excitation reduces the background fluorescence normally associated with one-photon excitation. The design demonstrates versatility from high-resolution, topographic imaging to single molecule, sensitive, farfield imaging to high-resolution, fluorescence imaging, using both a femtosecond modelocked pulsed laser source and a low cost continuous wave (CW) diode laser source. The integration of an intensified CCD camera, paired with a monochromator, demonstrates the spectroscopic abilities of the microscope. The design further demonstrates highresolution topography capabilities as a stand-alone shear force microscope.

The cost and complexity of pulsed laser systems can place two-photon imaging fluorescence imaging out of reach at smaller research labs. Showing that a diode laser can accomplish similar imaging resolutions and responses greatly simplifies the laser pathways and places less of a burden on the researcher to maintain the alignment and stability of a pulsed source. The cost of the diode source is roughly one-thousandth of that of the pulsed sources. Of course, using $\mathrm{CW}$ excitation will require higher power levels (as demonstrated) and won't allow the user to distinguish time dynamics in 
molecular fluorescence events. For time dynamics, a pulsed system is still required, since the dead time in between pulses is long enough for pump-probe experiments.

By developing the system in a standard programming environment with commercially supported, off-the-shelf components and simplified usability, the system can be easily recreated. In fact, two similar systems have been developed in-house and, with slight modifications, have been developed into electrochemical scanning and farfield magnetic imaging tools. This versatility was the original motivation for the system design, and should spark interest in the scientific community.

Continued development of the TENOM system with added functionality is currently under way. As a shear force microscopy tool, the addition of electric field and magnetic field imaging would add to the system's versatility. A fiber-based design and simplification of the current scan head to allow for non-transmissive samples will also be investigated for more versatile near-field imaging. Raman spectroscopy capabilities that include the integration of an intensified CCD camera with a high-resolution monochromator will allow for spectral imaging of multiple Raman lines, simultaneously. This will eliminate the need for multiple Raman notch filters, making the system more dynamic and universal. The TENOM technique will be an added benefit by amplifying the weak Raman spectral response. Ultimately, developing the microscope into a user friendly Raman imaging system will benefit the longevity of the design. With increasing advancements in far-field "super-resolution" techniques, near-field fluorescence imaging will most likely fall out of favor to its far-field counterpart. Raman spectroscopy signals are instantaneous and impossible to analyze with "super-resolution" methods. 


\section{CHAPTER 15}

\section{Dissemination Proposal}

Traditionally, scientific findings are disseminated through a peer reviewed publication process that is managed by independent publishing groups. The publishing groups require monetary compensation and typically retain the rights to the published works for their services. Furthermore, there has been an increase in incentives for academic environments to seek intellectual property rights by acquiring patents, in order to monetarily protect inventions [Benkler, 2004]. However, the Internet is changing traditional dissemination avenues. Electronic media and data sharing are creating new forms of worldwide collaborations and many question if new methods of dissemination should be encouraged [Harnad, 1999]. Based on the current trends in the sciences and changing trends of the grant funding agencies, a proposed method of dissemination for the TENOM system presented in this dissertation shall be discussed.

Many wonder who should own scientific papers [Bachrach et al. 1998]. The general consensus among the open source community is that a majority of these findings be made freely available to the public, with or without peer review. The counterargument contends that the peer review process could fall apart if there is little incentive to maintain scientific publishing integrity in an open review environment [Nature, 2006]. Ultimately, the question becomes whether or not the current method of publication hinders or benefits the scientific method. How does it affect the impact of the scientific community and what effect would open source publishing have in the sciences? 
Moreover, should open source publications still be peer reviewed?

Currently, peer-reviewed publications are the most accepted measure of scientific validity. A submitted publication to a journal is sent to reviewers that are considered to be experts in the scope of the material presented. The depth of knowledge of the reviewers is used to determine how novel the article is, as well as the potential impact of the information contained therein. One school of thought suggests that open access to journals would generate more citations and exposure in the scientific community, but this has yet to materialize [Evans, 2009]. Current open access, non-peer reviewed publications have not received the scientific support of the traditional, peer-reviewed process. The increasing number of submissions to journals is greater than the peer review process can support, resulting in rejections before proper review [Lawrence, 2003]. How this will affect whether or not researchers pursue open access, non-reviewed publication avenues has yet to be seen.

Grant funding agencies have the greatest power over how information is to be disseminated. In the last few years, the United States government has taken several steps, in order to make scientific publications freely available. Through the Consolidated Appropriations Act of 2008, the US Congress requires scientists funded by the National Institutes of Health (NIH) to make their publications available through PubMed, a free database. This trend is expected to spread to other government funding agencies as well [Butler, 2010]. While the subject of dissemination will continue to be debated for some time, the grant funding agencies will make the final call. Of course, this will most likely be influenced by public opinion. 
Where does the dissemination of this dissertation fit into the grand scheme of this debate? While the elements of novelty and scientific impact will be submitted through the peer review process, the technical details of the TENOM system will be presented in an open access environment where it will be referred to as the ANSOM Project. An open source, content management system (CMS) called Drupal was chosen to create a centralized website environment in which to disseminate the project. Drupal provides a CMS that allows for dynamic website and information control [drupal.org, 2010]. Using the Drupal format, it is possible to create user access controls and forums to allow fellow researchers to exchange ideas about the project, and consequently, improve upon the original project framework. The project site is structured to give general information about the science and technique of TENOM imaging to the unauthorized user, while the authorized user gains full access to the complete details of the project. These details include, but are not limited to, mechanical drawings, electric circuit diagrams, including design files, microscope software, and user manuals. Users can easily become authorized by simple registration. The purpose of this registration requirement is to compile a list of the users and their academic or industry affiliations for grant progress reports and to measure the overall success of the project.

The ultimate goal of the ANSOM Project is to share the technical details of design that cannot be published in a traditional scientific journal. Due to size limitations in publications, it is difficult to discuss every nut and bolt that encompasses the design of the scientific apparatus. More often than not, it is these design details that make the apparatus and the experiment in general functional. This also helps researchers whose primary focus may interest them in some small detail that will help in their particular 
discipline, rather than the entire scope of the project. Connections to the project can also help to forge interdisciplinary, collaborative relationships. The stress of finding, creating or maintaining the modular components of the TENOM system may also contribute to these collaborative relationships. For more information or to access and contribute to the ANSOM Project, please visit the project website at www.ansom.research.pdx.edu. 


\section{CHAPTER 15 References}

Bachrach S., Berry R. S., Blume M., Von Foerster T., Fowler A., Ginsparg P., Heller S., Kestner N., Odlyzko A., Okerson A., Wigington R., and Moffat A., "Intellectual

Property: Who Should Own Scientific Papers?” Science 281, 1459 (1998).

Benkler Y., "Commons-Based Strategies and the Problems of Patents," Science 305, 1110 (2004).

Butler D., "US Seeks to Make Science Free for All,” Nature 464, 822 (2010).

Buytaert D., computer program Drupal version 6.14 (Drupal.org, 2010).

Evans J. "Electronic Publication and the Narrowing of Science and Scholarship," Science 321, 395 (2008).

Harnad, S., "Free at Last: The Future of Peer-Reviewed Journals," D-Lib Magazine 5, 12 (1999).

H.R. 2764--110th Congress: Consolidated Appropriations Act, 2008. (2007).

Lawrence P. A., "The Politics of Publication: Authors, Reviewers and Editors Must Act to Protect the Quality of Research," Nature 422, 259 (2003).

Nature Editorial, "Peer Review and Fraud: Two Assessments of the Refereeing Process Highlight Challenges for Journals," Nature 444, 971 (2006). 


\section{BIBLIOGRAPHY}

Abbe E., "Beiträge zur Theorie des Mikroskops und der mikroskopischen Wahrnehmung," Archiv f. Miroskop. Anat. 9, 413 (1873).

Airy G. B., "On the Diffraction of an Object-glass with Circular Aperture," Transactions of the Cambridge Philosophical Society 5, 283-291 (1835).

Albrecht G. M. and Creighton A. J., "Anomalously Intense Raman Spectra of Pyridine at a Silver Electrode," Journal of the American Chemical Society 99, 5215-5219 (1977).

Albrecht T. R., Grutter P., Horne D., and Rugar D., "Frequency modulation detection using high-q cantilevers for enhanced force microscope sensitivity," J. Appl. Phys 69, 668 (1991).

Ambrose, W. P., Goodwin, P. M., Martin, J. C., and Keller, R. A., "Alterations of Single Molecule Fluorescence Lifetimes in Near-Field Optical Microscopy," Science 265, 364 (1994).

Analog Devices, “AD633 Low Cost Analog Multiplier," Product Data Sheet, Rev. G, (2010).

Analog Devices, “AD637 High Precision, Wideband RMS-to-DC Converter”, Product Data Sheet, Rev. J, (2007).

Analog Devices, "AD8302 2.7GHz RF / IF Gain Phase Detector," Product Data Sheet, Rev. A, July 2002.

Analog Devices, “AD9835 50 MHz CMOS Complete DDS”, Product Data Sheet, Rev. 0, July 1998.

Analog Devices, “Compact +30 V / \pm 15 V 256-Position Digital Potentiometer", Product Data Sheet, Rev. B (2010).

Analog Devices, “OP27 Low Noise, Precision Operational Amplifer," Product Data Sheet, Rev. F (2006).

Ash E. A. and Nichols G., "Super-resolution aperture scanning microscope," Nature 237, 510 (1972).

Bachrach S., Berry R. S., Blume M., Von Foerster T., Fowler A., Ginsparg P., Heller S., Kestner N., Odlyzko A., Okerson A., Wigington R., and Moffat A., "Intellectual Property: Who Should Own Scientific Papers?” Science 281, 1459 (1998). 
Baer F. and Zhang B., "Optimizing computations in weather and climate prediction models", Meterol. Atmos. Phys. 67, 153 (1998).

Barber D. J. and Freestone I. C., "An investigation of the origin of the colour of the Lycurgus Cup by analytical transmission electron microscopy," Archaeometry 32, 33-45 (1990).

Behler S., Rose M. K., Dunphy J. C., Ogletree D. F., Salmeron M., and Chapelier C., "Scanning tunneling microscope with continuous flow cryostat sample cooling," Rev. Sci. Inst. 68, 2479 (1997).

Benkler Y., "Commons-Based Strategies and the Problems of Patents," Science 305, 1110 (2004).

Berger, M. J., and Colella P., "Local Adaptive Mesh Refinement for Shock Hydrodynamics," J. Comp. Phys. 82, 64 (1989).

Bethe, H. A., “Theory of Diffraction by Small Holes,” Phys. Rev. 66, 163, (1944).

Betzig E., Finn P. L., and Weiner S. J., "Combined shear force and near-field scanning optical microscopy,” Appl. Phys. Lett. 60, 2484-2486 (1992).

Betzig E., Grubb S. G., Chichester R. J., DiGiovanni D. J., and Weiner J. S., "Fiber Laser Probe for Near-Field Scanning Optical Microscopy," Appl. Phys. Lett. 63, 3550 (1993).

Betzig E., Patterson G. H., Sougrat R., Lindwasser O. W., Olenych S., Bonifacino J. S., Davidson M.W., Lippincott-Schwartz J., and Hess H. F., "Imaging Intracellular Fluorescent Proteins at Nanometer Resolution," Science 313, 1642-1645 (2006).

Betzig E., Trautman J. K., Harris T. D., Weiner J. S., and Kostelar R. L., "Breaking the diffraction barrier: optical microscopy on a nanometric scale," Science 251, 1468- 1470 (1991).

Bielefeldt, H., Hörsch I., Krausch G., Lux-Steiner M., Mlynek J. and Marti O., "Reflection-scanning near-field optical microscopy and spectroscopy of opaque samples," Appl. Phys. Lett. 59, 103 (1994).

Binnig G., Rohrer H., Gerber Ch., and Weibel E., "Surface Studies by Scanning Tunneling Microscopy,” Phys. Rev. Lett. 49, 57 (1982).

Binnig G., Quate C. F., and Gerber Ch., “Atomic Force Microscope,” Phys. Rev. Lett. 56, 930 (1986).

Birkholz M., "Crystal-field induced dipoles in heteropolar crystals - II. physical significance,” Z. Phys. B 96, 333 (1995). 
Bishop P., "A tradeoff between microcontroller, DSP, FPGA and ASIC technologies," EE times Europe, February 25, 2009.

Bouwkamp, C. J., “On Bethe's Theory of diffraction by Small Holes,” Phillips. Res. Rep. 5, 401 (1950).

Brockman D. and Williams A., Analog Devices, "Ground Rules for High-Speed Circuits," Appl. Note AN-214.

Bryant P. J., Kim H. S., Zheng Y. C. and Yang R., "Technique for Shaping Scanning Tunneling Microscope Tips,” Rev. Sci. Instrum. 58, 1115 (1987).

Butler D., "US Seeks to Make Science Free for All,” Nature 464, 822 (2010).

Buytaert D., computer program Drupal version 6.14 (Drupal.org, 2010).

Cirrus Logic - Apex, “Driving Piezoelectric Actuators,” Appl. Note 44, May 2009.

Cirrus Logic - Apex, "PA88 High Voltage Power Operational Amplifers," Product Data Sheet, Rev. N, May 2009.

Colm Slattery, Analog Devices, "Programming the AD9832 / AD9835", Appl. Note AN621, Rev. 0. 2003.

Courjon, D., Banier, C., Spajer, M., "Imaging of submicrion index variations by scanning optical tunneling,” J. Vac. Sci. Tech. B 10, 2436 (1992).

Chroma Technology Corp. "Catalog of Filters and Mirrors for Laser Applications," Product Catalog, 2009 pp. 25-30.

Curie J. and Curie P., "Développement, par pression, de l'électricité polaire dans les cristaux hémièdres à faces inclines," Comptes Rendus de l'Académie des Sciences 91, 294 (1880).

Curie J. and Curie P., "Sur l'électricité polaire dans les cristaux hémièdres à faces inclines," C R Acad Sci Gen. 91, 383 (1880).

Curie J. and Curie P., "Contractions et dilatations produites par des tensions dans les cristaux hémièdres à faces inclines," C R Acad Sci Gen. 93, 1137 (1880).

D’Azzo J. J. and Houpis C. H., Linear Control System Analysis and Design (McGrawHill, New York, 1981). 
De Broglie L., Ph.D. dissertation. "Recherches sur la théorie des quanta (Researches on the quantum theory)," (Paris, 1924).

Debye P., “Zur Theorie der spezifischen Waerme,” Annalen der Physik 39, 789 (1912).

Denk, W., Piston, D. W., and Webb, W. W., Handbook of Biological Confocal Microscopy, ed. J. Pawley (Plenum Press: New York, 1995), pp 445-458.

Denk W., Strickler J., and Webb W., "Two-Photon Laser Scanning Fluorescence Microscopy," Science 248, 73-6 (1990).

Dobell C., Antony van Leeuwenhoek and His 'Little Animals'. (Dover Publications, New York, 1932).

Drake B., Sonnenfeld R., Schneir J., Hansma P. K., Slough G., and Coleman R. V., “A tunneling microscope for operation in air or fluids," Rev. Sci. Instrum. 57, 441 (1986).

Drude P., “Zur Elektronentheorie der metalle,” Annalen der Physik 306, 566 (1900).

Drude P., "Zur Elektronentheorie der Metalle; II. Teil. Galvanomagnetische und thermomagnetische Effecte," Annalen der Physik 308 (11): 369 (1900).

Duncan M., Reintjes J., and Manuccia T. J., "Scanning Coherent Anti-Stokes Raman Microscope," Opt. Lett. 7, 350-352 (1982).

Dunn, R. R., "Near-field scanning optical microscopy,” Chem. Rev. 99, 2891-2927 (1999).

Erni Rolf, Rossell M. D., Kisielowski C., and Dahmen U., "Atomic-Resolution Imaging with a Sub-50-pm Electron Probe,” Phys. Rev. Lett. 102, 096101 (2009).

Eustis S. and El-Sayed M. A., "Why Gold Nanoparticles are More Precious than Pretty Gold: Nobel Metal Surface Plasmon Resonance and Its Enhancements of Radiative and Nonradiatice Properties of Nanocystals of Different Shapes," Chem. Soc. Rev. 35, 209 (2006).

Evans J. "Electronic Publication and the Narrowing of Science and Scholarship," Science 321, 395 (2008).

Faraday M., "The Bakerian Lecture: Experimental Relations of Gold (and Other Metals) to Light," Philos. Trans. R. Soc. London 147, 145 (1857).

Farahani, J. N., Pohl D. W., Eisler, H. J., and Hecht B., "Single quantum dot coupled to a scanning optical antenna: A tunable super emitter," Phys. Rev. Lett. 95, 017402 (2005). 
Fine S., and Hansen W. P., "Optical Second Harmonic Generation in Biological Systems,” Appl. Opt. 10, 2350-2353 (1971).

Fleischmann M., Hendra P. J. and McQuillan A. J., "Raman Spectra of Pyridine Adsorbed at a Silver Electrode," Chemical Physics Letters 26, 163-166 (1974).

Forbes R. G., "A Generalised Theory of Standard Field Ion Appearance Energies," Surf. Sci. 61, 221 (1976).

Forbes R. G., "Charge Hopping and Charge Draining: Two Mechanisms of Field Desorption," Surf. Sci. 102, 255 (1981).

Forbes, R. G., "Electrosprays and Liquid-Metal Ion Sources - Some Theoretical Similarites and Differences," J. Aero Sci. 30, 974 (1999).

Förster T., "Zwischenmolekulare Energiewanderung und Fluoreszenz,” Ann. Physik 437, 55 (1948).

Franken P. A., Hill A. E., Peters C. W., and G. Weinreich, "Generation of Optical Harmonics," Phys. Rev. Lett. 7, 118-121 (1961).

Freund I., Deutsch M., and Sprecher A., "Connective Tissue Polarity,” Biophys. J. 50, 693-712 (1986).

Friet J.-M. and Carry É., "Introduction to the quartz tuning fork," Am. J. Phys. 72, 5 (2007).

Gai H., Wang J. and Tian Q., "Modified Debye Model Parameters of Metals Applicable for Broadband Calculations," Appl. Opt. 46, 2229 (2007).

Garcia-Parajo, M. F., Cambril, E., and Chen Y., "Simultaneous scanning tunneling microscope and collection mode scanning near-field optical microscope using gold coated optical fiber probes,” Appl. Phys. Lett. 65, 1498 (1994).

Goeppert-Mayer M., "Über Elementarakte mit zwei Quantensprüngen,” Ann Phys 9, 273-95 (1931).

Goldstein J. I. et al., Scanning Electron Microscopy and X-Ray Microanalysis, $2^{\text {nd }}$ ed. (Plenum Press, New York, 1992) pp. 51.

Goncharenko A. V., Chang H.-C. and Wang J.-K., "Electric Near-field Enhancing Properties of a Finite-size Metal Conical Nano-tip," Ultramicroscopy 107, 151 (2007). 
Gregory S. and Rogers C. T., "High-Speed Scanning Tunneling Microscopes," J. Vac. Sci. Technol. A 6, 390 (1988).

Grober R. D., Acimovic J., Schuck J., Hessman D., Kindlemann P. J., Hespanha J., Morse A. S., Karrai K., Tiemann I., and Manus S., "Fundamental Limits to Force Detection Using Quartz Tuning Forks,” Rev. Sci. Inst. 71, 2776 (2000).

Grober R. D., Schoelkopf R. J., and Prober D. E., "Optical Antenna: Towards a Unity Efficiency Near-Field Optical Probe,” Appl. Phys. Lett. 70, 1354 (1997).

Harnad, S., "Free at Last: The Future of Peer-Reviewed Journals," D-Lib Magazine 5, 12 (1999).

Hartschuh A., Sánchez, E. J., Xie, X. S., and Novotny L., "Near-field Raman Spectroscopy of Single-Walled Carbon Nanotubes,” Phys. Rev. Lett. 90, 9 (2003).

Heath S., Embedded Systems Design $2^{\text {nd }}$ ed. (Newsnes, New York, 2003) pp. 2.

Hecht E., Optics, $3^{\text {rd }}$ ed. (Addison Wesley, New York, 1998) pp. 1-2.

Hell S. W., Booth M., Wilms S., Schnetter C. M., Kirsch A. K., Arndt-Jovin D. J., and Jovin T. M., "Two-photon Near- and Far-field Fluorescence Microscopy with Continuous-Wave Excitation,” Opt. Lett. 23, 1238 (1998).

Hell S. W. and Wichmann J. "Breaking the Diffraction Resolution Limit by Stimulated Emission: Stimulated-Emission-Depletion Fluorescence Microscopy,” Optics Letters 19, 780-782 (1994).

Hellwarth R. and Christensen P., "Nonlinear Optical Microscopic Examination of Structure in Polycrystalline ZnSe," Optics Comm. 12, 318-322 (1974).

Hess S. T., Girirajan T. P. K., and Mason M. D., "Ultra-High Resolution Imaging by Fluorescence Photoactivation Localization Microscopy," Biophysical Journal 91, 42584272 (2006).

Higgins D. A. and Barbara P. F., "Excitonic Transitions in J-aggregates Probes by Nearfield Scanning Optical Microscopy,” J. Phys. Chem. 99, 3 (1995).

Higgins D. A., Kerimo J., Vanden Bout D. A., and Barbara P. F., "A Molecular Yarn: The Self Assembly of Flexible, Fluorescent Fibers Studied by Near Field Scanning Optical Microscopy," J. Am. Chem. Soc. 118, 4049 (1996).

Horng M.-L. and Quitevis E. L., "Excited-State Dynamics of Polymer-Bound JAggregates" J. Phys. Chem. 97, 12408 (1993). 
Hooke R., Micrographia: or, Some physiological descriptions of minute bodies made by magnifying glasses (J. Martyn and J. Allestry, London, 1665).

Höppener C., Beams R., and Novotny L., "Background Suppression in Near-Field Optical Imaging,” Nano Lett. 9, 903 (2009).

H.R. 2764--110th Congress: Consolidated Appropriations Act, 2008. (2007).

Hsu J. W. P., McDaniel A. A., and Hallen H. D., "A shear force feedback control system fro near-field scanning optical microscope without lock-in detection", Rev. Sci. Inst. 68, 3093 (1997).

Huygens C., Traité de la Lumière (Pieter van der Aa, Leiden, 1690).

Ichimura T., Hayazawa N., Hashimoto M., Inouye Y. and Kawata S. et. al. "Tip Enhanced Coherent Anti-Stokes Raman Scattering for Vibrational Nanoimaging", Phys. Rev. Lett. 92, 220801 (2004).

Ilardi V., "Eyeglasses and Concave Lenses in Fifteenth-Century Florence and Milan: New Documents," Renaissance Quarterly 29, 341-360 (1976).

Inouye Y. and Kawata S., "Near-field Scanning Optical Microscope with a Metallic Probe Tip," Opt. Lett. 19, 159 (1994).

IPC - The Institute for Interconnection and Packaging Electronic Circuits, "Generic Standard on Printed Board Design,” IPC-2221, February 1998.

Jahncke C. L., Brandt O., Fellows K. E., and Hallen H. D., "Choosing a Preamplifier for Tuning Fork Signal Detection is Scanning Force Microscopy,” Rev. Sci. Inst. 75, 2759 (2004).

Jensen T. R., Malinsky M. D., Haynes C. L., and Van Duyne R. P., "Nanosphere Lithography: Tunable Localized Surface Plasmon Resonance Spectra of Silver Nanoparticles," J. Phys. Chem. B 104, 10549 (2000).

Jeanmaire, D. L. and Van Duyne R. P., "Surface Raman Electrochemistry Part I. Heterocyclic, Aromatic and Aliphatic Amines Adsorbed on the Anodized Silver Electrode," Journal of Electroanalytical Chemistry 84, 1-20 (1977).

Johnson H., High-Speed Digital Design (Prentice Hall, 1993).

Kahan A., "Cut Angles for Quartz Crystal Resonators," U.S. Patent No. 4499395 (12 February, 1985). 
Kalkbrenner T., Ramstein M., Mlynek J., and Sandoghdar V., "A Single Gold Particle as a Probe for Apertureless Scanning Near-Field Optical Microscopy," J. Microscopy 202, $72-76$ (2001).

Karrai, K. and Grober, R. D., "Piezoelectric Tip-Sample Distance Control for Near Field Optical Microscopes,” Appl. Phys. Lett. 66, 14 (1995).

Karrai, K. and Grober, R. D., "Piezo-electric Tuning Fork Tip-Sample Distance Control for Near Field Optical Microscopes,” Ultramicroscopy 61, 197 (1995).

Kelly K. L., Coronado E., Zhao L. L., and Schatz G. C., "The Optical Properties of Metal Nanoparticles: The Influence of Size, Shape, and Dielectric Environment," J. Phys.

Chem. B 107, 668 (2003).

Klar T. A. and Hell S. W., "Subdiffraction Resolution in Far-Field Fluorescence Microscopy," Optics Letters 24, 954-956 (1999).

Krug, J. T., Sánchez, E. J., and Xie, X. S., "Design of near-field optical probes with optimal field enhancement by finite difference time domain electromagnetic simulation," J. Chem. Phys. 116, 10895 (2002).

Krug J. T., Sánchez E. J. and Xie X. S., "Fluorescence Quenching in Tip-Enhanced Nonlinear Optical Microscopy,” Appl. Phys. Lett. 86, 233102 (2005).

Kunz K. S. and Luebbers R., The Finite Difference Time Domain Method for Electromagnetics, (CRC Press, New York, 1993) pp. 11-12 135-136.

La Rosa A., Cui X., McCollum J., Li N., and Nordstrom R., "The ultrasonic/shear-force microscope: Integrating ultrasonic sensing into a near-field scanning optical microscope," Rev. Sci. Inst. 76, 093707 (2005).

Lawrence P. A., "The Politics of Publication: Authors, Reviewers and Editors Must Act to Protect the Quality of Research," Nature 422, 259 (2003).

Leeuwenhoek A., "De natis è semine genitali animalculis". R Soc (London) Philos Trans. 12, $1040-1043$ (1678).

Lewis A., Isaacson M., Harootunian A. and Murray A., "Development of a $500 \AA$ spatial resolution light microscope. I. Light is efficiently transmitted through $\lambda / 16$ diameter apertures," Ultramicroscopy 13, 227 (1984).

Lippman, G., "Principe de la conservation de l'électricité" (in French). Annales de chimie et de physique 24, 145 (1881). 
Lindig O. and Pannhorst W., "Thermal expansion and length stability of Zerodur in dependence of temperature and time," Applied Optics 24, 20 (1985).

Lo H., "Digital Display of Stepper Motor Rotation," Computer Design 147-148, 150-151, April 1978.

Luebbers R., Hunsberger F., Kunz K., Standler R., and Schneider M., “A Frequencydependent Finite-difference Time-domain Formulation for Dispersive Materials," IEEE Trans. Electromag. Compat., 32, 222 (1990).

Luedi P. P., Dietrich F. S., Weidman J. R., Bosko J. M., Jirtle R. L., and Hartemink A. J., "Computational and experimental identification of novel human imprinted genes", Genone Res. 17, 1723 (2007).

Maker P. D. and Terhune R. W., "Study of Optical Effects Due to an Induced Polarization Third Order in the Electric Field Strength," Phys. Rev. 148, 990 (1966).

Marchman, H.M., Griffith, J.E., and Filas, R.W., "Fabrication of optical fiber probes for nanometer-scale dimensional metrology,” Rev. Sci. Inst. 65, 2538-2541 (1994).

Markus J., Modern Electronic Circuits Reference Manual (McGraw-Hill Book Company, 1980) pp. 580.

Martin O. J. F., and Girard C., "Controlling and Tuning Strong Optical Field Gradients at a Local Probe Microscope Tip Apex.”, Appl. Phys. Lett. 70, 705 (1997).

Marrison W. A., “The Evolution of the Quartz Crystal Clock,” Bell System Technical Journal (AT\&T) 27, 510 (1948).

McGrath D., "FPGA Market to Pass \$2.7 Billion by '10, In-Stat Says,” EE Times, May 24, 2006.

McMahon J. M., computer code JFDTD (Northwestern University, Evanston, IL, 2010).

Melmed A. J., "The Art and Science and Other Aspects of Making Sharp Tips.” J. Vac. Sci. Technol. B 9, 601 (1991).

Mie G., "Beiträge zur Optik trüber Medien, speziell kolloidaler Metallösungen,” Ann. Phys. 330, 377-445 (1908).

Minsky, M. U. S. Patent No. 3,013,467 (19 December, 1961).

Murphy, D. Fundamentals of Light Microscopy and Digital Imaging, (Wiley-Liss, New York, 2001) pp. 17. 
National Instrument Corporation, "NI R Series Multifunction RIO User Manual”, Product Manual, June 2009.

National Instrument Corporation, "NI R Series Multifunction RIO Integrated Analog and Digital I/O with FPGA Technology”, Product Brochure, July 2009.

Nature Editorial, "Peer Review and Fraud: Two Assessments of the Refereeing Process Highlight Challenges for Journals," Nature 444, 971 (2006).

Neacsu C. C., Berweger S., Olmon R. L., Saraf L. V., Ropers C., and Raschke M. B., "Near-Field Localization in Plasmonic Superfocusing: A Nanoemitter on a Tip," Nano Lett. 10, 592 (2010).

Novotny L. and Hecht B., Principles of Nano-Optics (Cambridge University Press, New York, 2006) pp. 213.

Novotny L., Sánchez E. J., and Xie X. S., "Near-field Optical Imaging Using Metal Tips Illuminated by Higher-Order Hermite-Gaussian Beams," Ultramicroscopy 71, 21 (1998).

Nowak D. B., Krug J. T., Xie X. S., and Sánchez E. J., "Pushing the Limits of Near-field Microscopy," World Multi-conference on Systemics, Cybernetics and Informatics, WMSCII Proceedings, Orlando, Florida, 2005.

Nowak D. B., M.S. thesis, "Modeling and Extraction of Gilbert-Taylor cones for Apertureless Scanning Near-field Optical Microscopy Probes," Portland State University, 2004.

Okayama S., Komuro M., Mizutani W., Tokumoto H., Okano M., Shimizu K., Kobayashi Y., Matsumoto F., Wakiyama S., Shigeno M., Sakai F., Fujiwara S., Kitamura O., Ono M., and Kajimura K., "Observation of microfabricated patterns by scanning tunneling microscopy,” J. Vac. Sci. Technol. A 6, 440 (1988).

O'Keefe J. A., "Resolving power of visible light," J. of the Opt. Soc. of Am. 46, 359 (1956).

OPTIS, computer program OptisWorks--for Solidworks (OPTIS North America, Troy, MI, 2010).

Palomba S., Danckwerts M., and Novotny L., "Nonlinear Plasmonics with Gold Nanoparticle Antennas," J. Opt. A.: Pure Appl. Opt. 11, 114030 (2009).

Pettinger B., Ren B., Picardi G., Schuster R., and Ertl G., "Nanoscale Probing of Absorbed Species by Tip-Enhanced Raman Spectroscopy," Phy. Rev. Lett. 92, 096101 (2004). 
Park S. and Barrett R. C., "Design Considerations for an STM System," in Methods of Experimental Physics, ed. Stroscio J. A. and Kaiser W. J. (Academic Press, New York, 1993) Vol. 27, pp. 30-76.

Pilotte M., Analog Devices, "Operation of RF Detector Products at Low Frequency," Appl. Note AN-691, Rev. 0, 2005.

Pohl D. W., Denk W., and Lanz M., "Optical stethoscopy: Image recording with resolution $\lambda / 20$," Appl. Phys. Lett. 44, 651 (1984).

Pohl D. W., "Some Design Criteria in Scanning Tunneling Microscopy", IBM J. Res. Develop. 30, 417-427 (1986).

Raman C. V. and Krishnan K. S., "A New Type of Secondary Radiation,” Nature (London) 121, 501 (1928).

Rasband, W.S., ImageJ, U. S. National Institutes of Health, Bethesda, Maryland, USA, http://rsb.info.nih.gov/ij/, 1997-2007.

Rayleigh L., "Investigations in optics, with special reference to the spectroscope," Phil. Mag. 8, 261-274/403-411/477-486 (1879).

Rayleigh L., "On the Scattering of Light by Small Particles,” Phil. Mag. 41, 275, 447 (1871).

Remcom Inc., “xFDTD Reference Manual, Version 6.3,” Product Manual, 2003.

Ren B., Picardi G., and Pettinger B., "Preparation of Gold Tips Suitable for TipEnhanced Raman Spectroscopy and Light Emission by Electrochemical Etching," Rev. Sci. Inst. 75, 837 (2004).

Rensen W. H. J., Van Hulst N. F., and Kämmer S. B., "Imaging Soft Samples in Liquid with Tuning Fork Based Shear Force Microscopy,” Appl. Phys. Lett. 77, 1557 (2002).

Roundy D., computer program MEEP (Massachusetts Institute of Technology, Boston, MA, 2010).

Rowland J. R., Linear Control Systems: Modeling, Analysis and Design (Wiley, New York, 1986).

Rust M. J., Bates M. and Zhuang X., "Sub-Diffraction-Limit Imaging by Stochastic Optical Reconstruction Microscopy (STORM)" Nature Meth. 3, 793-796 (2006).

Sallen R. P. and Key E. L., "A Practical Method of Designing RC Active Filters," IRE Transactions on Circuit Theory 2, 74-85 (1955). 
Sánchez, E. J., Ph.D. dissertation, “A Novel Scheme for High Resolution Near-Field Fluorescence Microscopy”, Portland State University, 1999 (UMI No. 3018652).

Sánchez E. J., Novotny L., Holtom G. R., and Xie X. S., "Room-Temperature Fluorescence Imaging and Spectroscopy of Single Molecules by Two-Photon Excitation,” J. Phys. Chem. A 101, 7020 (1997).

Sánchez E. J., Novotny L., and Xie X. S., "Near-field Fluorescence Microscopy Based on Two-Photon Excitation With Metal Tips," Phys. Rev. Lett. 82, 20 (1999).

Sánchez E. J., Krug J. T., and Xie X. S., "Ion and electron beam assisted growth of nanometric $\mathrm{Si}_{\mathrm{m}} \mathrm{O}_{\mathrm{n}}$ structures for near-field microscopy”, Rev. Sci. Inst. 73, 11 (2002).

Sau T. K., Pal A., Jana N. R., Wang Z. L. and Pal T., "Size controlled synthesis of gold nanoparticles using photochemically prepared seed particles," J. Nanoparticle Res. 3, 257 (2001).

Schoch, B., Jones, B.E., and Franks, A., "A simple technique for the manufacture of optical probes for scanning near-field optical microscopes," Meas. Sci. Tech. 5, 663 (1994).

Sheppard C. J. R., Kompfner R., Gannaway, and Walsh D., "Scanning Harmonic Optical Microscope,” IEEE J. Quantum Electronics 13, 100 (1977).

Synge E. H., "A suggested method for extending the microscopic resolution into the ultramicroscopic region." Phil. Mag. 6, 356 (1928).

Synge E. H., "An application of piezoelectricity to microscopy,” Phil. Mag. 13, 297 (1932).

Taylor G. I., "Disintegration of Water Drops in an Electric Field," Proc. Royal Soc London A 280, 383 (1964).

Valaskovic, G.A., Holton, M., and Morrison, G. H., "Parameter control, characterization, and optimization in the fabrication of optical fiber near-field probes," Applied Optics 34, 1215 (1995).

Veerman, J. A., Otter, A. M., Kuipers, L., and Van Hulst, N. F., "High definition aperture probes for near-field optical microscopy fabricated by focused ion beam milling," Appl. Phys. Lett. 72, 3115-3117 (1998).

Wessel J., "Surface-Enhanced Optical Microscopy,” J. Opt. Soc. Am. B 2, 1538 (1985).

West P. E., Introduction to Atomic Force Microscopy Theory, Practice, Applications (AFMUniversity.org, 2007) pp. 36. 
Westphal V., Kastrup L. and Hell S.W., "Lateral Resolution of $28 \mathrm{~nm}(\lambda / 25)$ in Far-Field Fluorescence Microscopy,” Appl. Phys. B 77, 377-380 (2003).

Wagner, A. and Hall, T. M., "Liquid Gold Ion Source," J. Vac. Sci. Tech. 16, 1871 (1979).

William G., Ewing L., and Anthony S., Using MPI: Portable Parallel Programming with the Message Passing Interface, $2^{\text {nd }}$ ed. (MIT Press In Scientific And Engineering Computation Series, Cambridge, MA, USA, 1999).

Xu C. and Webb W. W., "Measurement of Two-Photon Excitation Cross Sections of Molecular Fluorophores with Data from 690 to 1050 nm," J. Opt. Soc. Am. B 13, 481491 (1996).

Yakobson, B. I., Moyer, P. J., and Paesler, M. A., "Kinetic limits for sensing tip morphology in near-field scanning optical microscopes," J Appl. Phys. 73, 7984 (1994).

Yee K., "Numerical solution of initial boundary value problems involving Maxwell's equations in isotropic media," Antennas and Propagation, IEEE Transactions 14, 302 (1966).

Young T. "The Bakerian Lecture: Experiments and Calculations Relative to Physical Optics," Philosophical Transactions of the Royal Society of London 94, 1-16 (1804).

Zenhausern F., Martin Y., and Wickramsinghe H. K., "Scanning Interferometric Apertureless Microscopy: Optical Imaging at 10 Angstrom Resolution," Science 269, 1083 (1995).

Zhang Z., Weber-Bargioni A., Wu S. W., Dhuey S., Cabrini S. and Schuck P. J., "Manipulating Nanoscale Light Fields with the Asymmetric Bowtie Nano-Colorsorter," Nano Lett. 9, 4505 (2009).

Zhu R.-J. Wang J. and Jin G.-F., "Mie Scattering Calculation be FDTD Employing a Modified Debye Model for Gold Material." Optik 116, 419 (2005). 


\section{APPENDIX A}

\section{MPI Computer Clustering}

This appendix is a complement to the discussion in Chapter 4 about the benefits of MPI computer clustering to distribute large numerical calculation sets to multiple computers for parallel processing. The first half of this appendix gives specific instructions for the building of a MPI based computer cluster. The second half of the appendix is specific to the installation of a commercial MPI based FDTD package, purchased from Remcom. A detail account of the installation of the Remcom MPI version of XFDTD 6.3.1 will be presented.

The installation presented was developed from the ground up, using the Fedora Core 4 Linux distribution. There are utilities that aid in the clustering of computers. One in particular is 'Rocks', developed to make the deployment of cluster environments more seamless when installing onto many computer nodes. The use of Rocks for the development of a MPI cluster for the FDTD modeling was not investigated.

\section{A.1 General Considerations}

Networking: The networking of the server and nodes will be the main bottleneck of the cluster. Personally, I would recommend at minimum gigabit Ethernet connections. Exotic networking methods such as Myrinet and Infiniband have shown possible network bandwidths of $2 \mathrm{Gbit} / \mathrm{s}$ and $2.5 \mathrm{Gbits} / \mathrm{s}$, respectively. Infiniband is also scalable, allowing the speed to be multiplied by $1 \mathrm{x}, 4 \mathrm{x}, 12 \mathrm{x}$, and supports single, double and quad data rates, giving a theoretical maxim of $96 \mathrm{Gbit} / \mathrm{s}$.

Server: An installation with a GUI is helpful in managing the nodes. Because of this, make the server a little more beefy than the nodes. More RAM and a slightly faster processor can be helpful. Using two Ethernet cards allows the server to talk to the cluster and act as a firewall to the actual nodes, and allows access to the outside world as well. Also, it is important to have the software development tool compilers included.

Nodes: Basic install (here you have some choices).

1. If you install one node correctly, an install image can be made to help install the remaining nodes.

2. If you intend to install all nodes by hand, they will all need gcc support for the installation of mpich (which is the mpi software need to run mpi processes).

3. Either way the node must have compilers installed to install mpich, which can be done on the first node if an install image is to be made (homogeneous clusters). 


\section{A.2 Installation notes for an MPI based cluster}

Installation: During the graphical installation of Fedora Core 4, you will be asked what type of install you would like to perform (server, workstation, personal, custom). Here you want custom. Then for the nodes, deselect all but the software development. For the server you may want other services, such as ftp, graphical environments (gnome), and server tools.

Partitions: Linux will automatically partition for you.

But if you would like to do it yourself for flexibility, you'll need a "swap" partition. This is the virtual memory for Linux around $2+\mathrm{Gb}$, depending on how big your hard drive is and the size of the programs you would like to run. Next is the "/" partition which will incorporate all of the remaining space on the drive. It can also take two hard drives and treat it as one large drive. The swap is formatted as such and the "/" is formatted normal as ext3 file system.

Networking: Disable DHCP on network device (normally - Eth0). Define the IP address for the node (for example 100.0.0.2 for node2). Set the default gateway and the Netmask as 255.255 .255 .0

Firewall: None, nothing, disable secure SElinux settings. (Proceed on firewall warning.)

Package: For the nodes, select "development tools" only. Deselect everything else. (Note: Do not select min when installing but unselect.)

Setting up the network: Give the nodes static IP addresses for communication, and use an unmanaged dedicated gigabit Ethernet hub for communications. (The nodes will already have static IP addresses if the installation instructions are followed. If not, these settings can be edited as follows.)

The computer name can be changed by editing: /etc/sysconfig/network.

There are several ways to define the IP address for the server and nodes. For the server, the networking cards, either eth0 or eth1, should be set to DHCP so that the server can talk to the outside world. The other network card should be given a static IP address. This can be accomplished during install when the installer asks for the IP information about the computer. Or one can define it by hand. If you're unfamiliar with networking in Linux, here are some helpful tools.

"ifconfig" gives the current status on all networking devices. There are many tools in ifconfig that will allow you to control the network devices, from turning on and off the devices to setting the IP address. One issue is that many of the changes to the ifconfig will only remain in effect during run time; if you reboot the system, the settings will return to their default settings. To change the default settings, the following file can be changed: 
/etc/sysconfig/network-scripts/ifcfg-eth*

There should be a file for each hardware device.

Using a text editor the file should look like the following (node1)

DEVICE $=$ eth0

BOOTPROTO $=$ static

HWADDR $=00: 00: 00: 00: 00: 00$ (this is the unique MAC address for the hardware)

IPADDR $=100.0 .0 .2$

NETMASK $=255.255 .255 .0$

Here, I have chosen the following naming convention, with IP addresses.

nanocluster.pdx.edu (server) 100.0.0.1

node( $\mathrm{x}$ ) 100.0.0.n where $\mathrm{n}=\mathrm{x}+1$

The text should be placed in the /etc/hosts file.

(The third line will be unique on each computer of the cluster.)

\# Do not remove the following line, or various programs

\# that require network functionality will fail.

127.0.0.1 node1 localhost.localdomain localhost

100.0.0.1 nanocluster.pdx.edu

100.0.0.2 node 1

100.0.0.3 node 2

100.0.0.4 node 3

100.0.0.5 node 4

100.0.0.6 node 5

100.0.0.7 node6

100.0.0.8 node 7

100.0.0.9 node 8

100.0.0.10 node9

100.0.0.11 node 10

100.0.0.12 node 11

100.0.0.13 node 12

100.0.0.14 node13

100.0.0.15 node 14

100.0.0.16 node 15

100.0.0.17 node 16

This file gives names to all the used IP addresses for ease of following communication and finding the nodes by name instead of by number. All the computers in the cluster should have a similar file, except for the first line 127.0.0.1 that should be the hostname of the system in which the file resides. If you like, test ssh node5. Does it work? (The next section shows how to remove the password request when using ssh.) 
Streamlining ssh communication: This section is critical in removing the ssh password prompting that happens when you attempt to ssh to one of the nodes of the cluster. For MPI communication, ssh calls can happen quite often, requiring the user to enter a password for every request. To remove this request, encrypted keys need to be shared between the machines.

In the home account generate a pair of public/private keys:

ssh-keygen - $\mathrm{t}$ dsa

(Do not enter a passphase.)

In your $\sim /$ ssh directory you will find something like this:

id_dsa

id_dsa.pub

Now you have to copy the public key to every single machine you want to be able to log onto. Copy the file directly to the remote host using scp:

scp.ssh/id_dsa.pub host:.ssh/authorized_keys2

Or you can go step by step:

scp .ssh/id_dsa.pub host:.ssh/id_rsa.pub

Then on the target host:

cp .ssh/id_dsa.pub.ssh/authorized_keys2

chmod go-rwx.ssh/authorized_keys2

chmod $755 . \mathrm{ssh} /$

Finally on the server host you want to run:

ssh-agent \$SHELL or ssh-agent

and finally:

ssh-add

After this, you should be able to log into your nodes remotely from the server without a password prompt.

Installing MPICH: (This is the mpi engine needed to execute mpi based programs.) Download the mpi version that you want to use (for Remcom's software, I used mpich1.2.6). This is an open source program. 
Download and untar the program using the following command:

tar -xvf mpich-1.2.6.tar.gz

This will extract the files into a directory mpich-1.2.6:

cd mpich-1.2.6

Run the following configuration script. This sets up the installation directory. It is critical that this directory be identical in all nodes:

$$
\text { ./configure --prefix=/usr/local/mpich --with-common-prefix=/usr/local/mpich }
$$

Finish with the following commands:

make

make install

After mpich is installed, go to the directory $/ \mathrm{usr} / \mathrm{local} / \mathrm{mpich} / \mathrm{share}$ and edit the file machines.LINUX. The file should list all the names of the computers on the cluster.

For Example:

\# if you have dual processor machines the machine can be listed twice to access \# the other processors

nanocluster.pdx.edu

node1

node2

node3

node4

node5

node6

node7

node 8

node9

node 10

node 11

node 12

node 13

node 14

node 15

node 16 
Testing MPICH: If you go to the /usr/local/mpich/example directory you can type: make cpi. This will compile a cpi program that can be used to test and debug the mpi installation. This cpi program will need to be compiled on all machines in the cluster.

To run the test (after compiling the program cpi), $\log$ in to the server and the directory /usr/local/mpich/example and type the following:

$$
\text { .mpirun -np } 1 \text { cpi }
$$

This will execute the program on one machine. To do the run on multiple machines, change the number 1 to any number not greater than the number on machines in your machine file list.

Setting up nfs mounting on the server: This is required for any mpi calculation that will be writing data to a file to be analyzed later. The idea is that the server will host any project files needed by the mpi executable. The nodes will look for this project file on the server and write their output to the same directory. One can get confused by this step, but the logic is that the mpich programs will need to be on all the local nodes. The mpi executable (third party) needs to be on all the local nodes as well. This is typically installed in the /usr/local directory. The project file and any execution scripts need to be in the nfs accessible mount location on the server. The server, by convention, should be configured to have its /opt directory and nfs mount location for the node computers.

On the server the /etc/exports file indicates the directory and machines allowed access.

$$
\text { lopt node1(rw, no_root_squash, sync) node2 (rw, no_root_squash, sync) }
$$

To force the nfs daemon to reread the exports file after a change, run the command:

$$
\text { exportsfs -ra }
$$

Note: Only editing exports can create potential security hazards, since the cluster is primary used for modeling. There should be no need for internet connectivity. Including no-root-squash was done to correct a complication with the Remcom mpi-xFDTD software.

Setting up nfs mounting on the nodes: On the node computers, the nfs file location can be mounted using the command:

$$
\text { mount nanocluster.pdx.edu:/opt /opt }
$$

To insure mounting of the nfs directory at boot up, the following line should be added to the etc/ fstab file:

nanocluster.pdx.edu:/opt $\quad$ opt $\quad$ nfs $\quad$ bg, intr, noac $\quad \begin{array}{lll}0 & 0\end{array}$ 
Installation of Remcom MPI-calcfdtd: Using Fedora core 4 and xfdtd mpi-calcfdtd engine version 6.3 , the following procedure should be followed while using the install script provided by the vendor.

1. Use Linux-i686EL as the platform type.

2. Install the compat-libstdc++-33-3.2.3-47.3.i386.rpm (the basic FC4 install will be missing a required binary file for xfdtd.

Remcom License file: Is required. Information on implementing the flexlm license file should be available from the vendor. Below is an example license file:

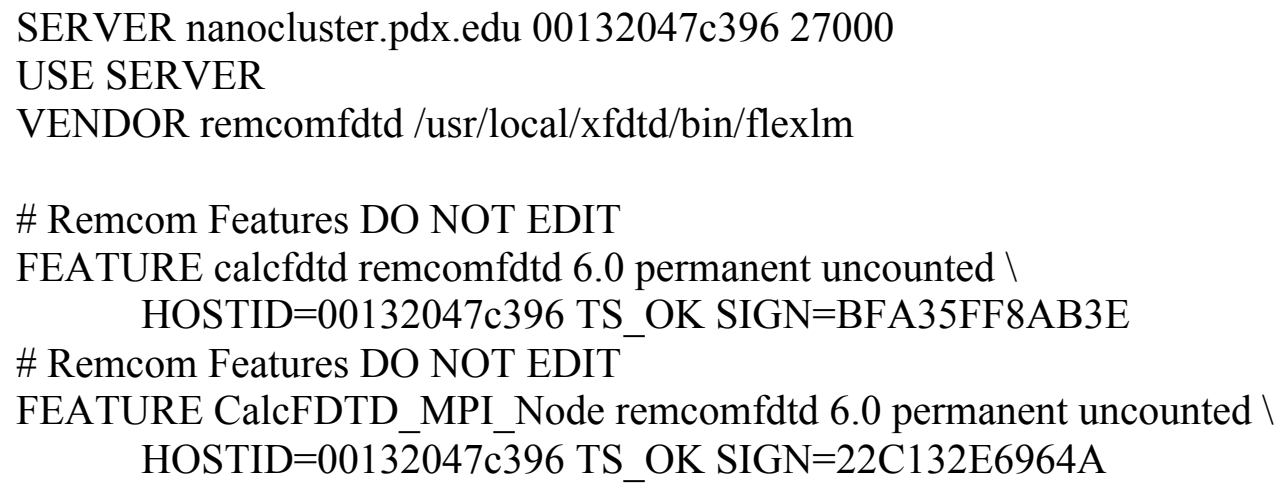

\section{A.3 Execution Routine for an MPI xFDTD Calculation}

\section{Execution Script:}

\#!/bin/csh

\# Determine where the script is located

set currentdir='echo $\$ 0 \mid$ awk ' $\{$ sz=split $(\$ 0$, foo,"/") $\}$ \{print $\operatorname{substr}(\$ 0,1$, length $(\$ 0)$ length(foo[sz])-1) \}'

\# Set up a variable for the binary directory

setenv XFDTD_BIN_DIR/usr/local/xfdtd/bin

\# Set up the dynamic linker path

\# This should be set to rh8 or rh72 depending on your distribution

\# setenv LD_LIBRARY_PATH /usr/local/xfdtd/lib/rh8

\# Specify the license path

setenv REMCOMFDTD_LICENSE_FILE 27000@nanocluster.pdx.edu

\# Formulate the execution command

\# The default setting is for rh8 mpich 1.2.5 which is the most common

\# If you are running RedHat 7.2 you must change this!

setenv XFDTD_CMD "/usr/local/xfdtd/bin/mpich-1.2.6-ssh/dynamic/calcfdtd63 \$*" 
echo Executing command: \$ $\{$ XFDTD_CMD $\}$

\# Run calcfdtd61

\$ $\{$ XFDTD_CMD $\}$

\section{Execution Steps:}

1. Create project and geometry files in xFDTD 6.3 GUI, usually a Windows computer.

2. If the cluster is on a network, the files can be transferred via ftp to the /opt directory on the server computer. Otherwise the files can be transferred via flash drive to the same directory.

3. Open a terminal on the server. (From the GUI, right click with the mouse and select open new terminal.)

4. In terminal type: $\mathrm{cd} / \mathrm{opt}$

5. Type: mkdir "name of your data directory"

6. Use the cp command to place the .id and .fdtd file in this new directory.

7. Place a copy of the run script calcfdtd63.csh in the same directory

a. Example: cp /opt/calcfdtd63.csh /opt/"name of your data directory"

8. Execute the MPI calculation with the following command

a. /usr/local/mpich/bin/mpirun -np 16 calcfdtd.csh $-v$ yourfile.fdtd

9. If an error is generated saying that a license found but CalcFDTD not present, restart the license server by typing: /usr/local/xfdtd/bin/flexlm/lmgrd 


\section{APPENDIX B}

\section{Microscope Mechanical}

\section{B.1 Microscope Drawings}

Table B.1 List of Mechanical Drawings*

Drawing Number

ANSOM_Assembly_001

ANSOM_Assembly_002

ANSOM_Assembly_003

ANSOM_Assembly_004

ANSOM_Assembly_005

ANSOM_Assembly_006

ANSOM_Assembly_007

ANSOM_Assembly_008

ANSOM_Part_001

ANSOM_Part_002

ANSOM_Part_003

ANSOM_Part_004

ANSOM_Part_005

ANSOM_Part_006

ANSOM Part 007

ANSOM_Part_008

ANSOM_Part_009

ANSOM_Part_010

ANSOM_Part_011

ANSOM_Part_012

ANSOM_Part_013

ANSOM_Part_014

ANSOM_Part_015

ANSOM_Part_016

ANSOM_Part_017

ANSOM_Part_018

ANSOM_Part_019

ANSOM_Part_020

ANSOM Part 021

ANSOM_Part_022

ANSOM_Part_023
Description

Far-field Microscope

Far-field Optical Pathways

Backplane Assembly

Piezo Stack

Scan Head V1

Microscope Camera Coupler

Breakout Box

Complete ANSOM Microscope

Baseplate

Backplane

Brace 1

Brace 2

Front Brace

Motor Bracket

Baseplate Mirror Adapter

Breadboard Mirror Adapter

Z Translation Adapter

Dovetail Rail

Scan Head V1 Shell

Scan Head Top Cover

Scan Head Front Cover

Scan Head Back Cover

Piezo Stack: Macor Top Piece

Piezo Stack: Macor Coupler Piece

Piezo Stack: Macor End Piece

Piezo Stack: Al Mounting Piece

Breakout Box Lid Cuts

Breakout Box Cuts

Piezo Stack: Tuning Fork Holder

Scan Head V1 Laser Bracket

P.I. Piezo Stage: Sample Holder
Page

269-271

272,273

274

275

276,277

278

279

280,281

282-284

285,286

287

288

289

290

291

292

293

294

295-301

302

303

304

305

306

307

308

309

310,311

312

313

314

*Drawings are reduced $75 \%$ from the scale references indicated on the sheets.

To enlarge them to scale they need to be reproduced at $133.3 \%$. 


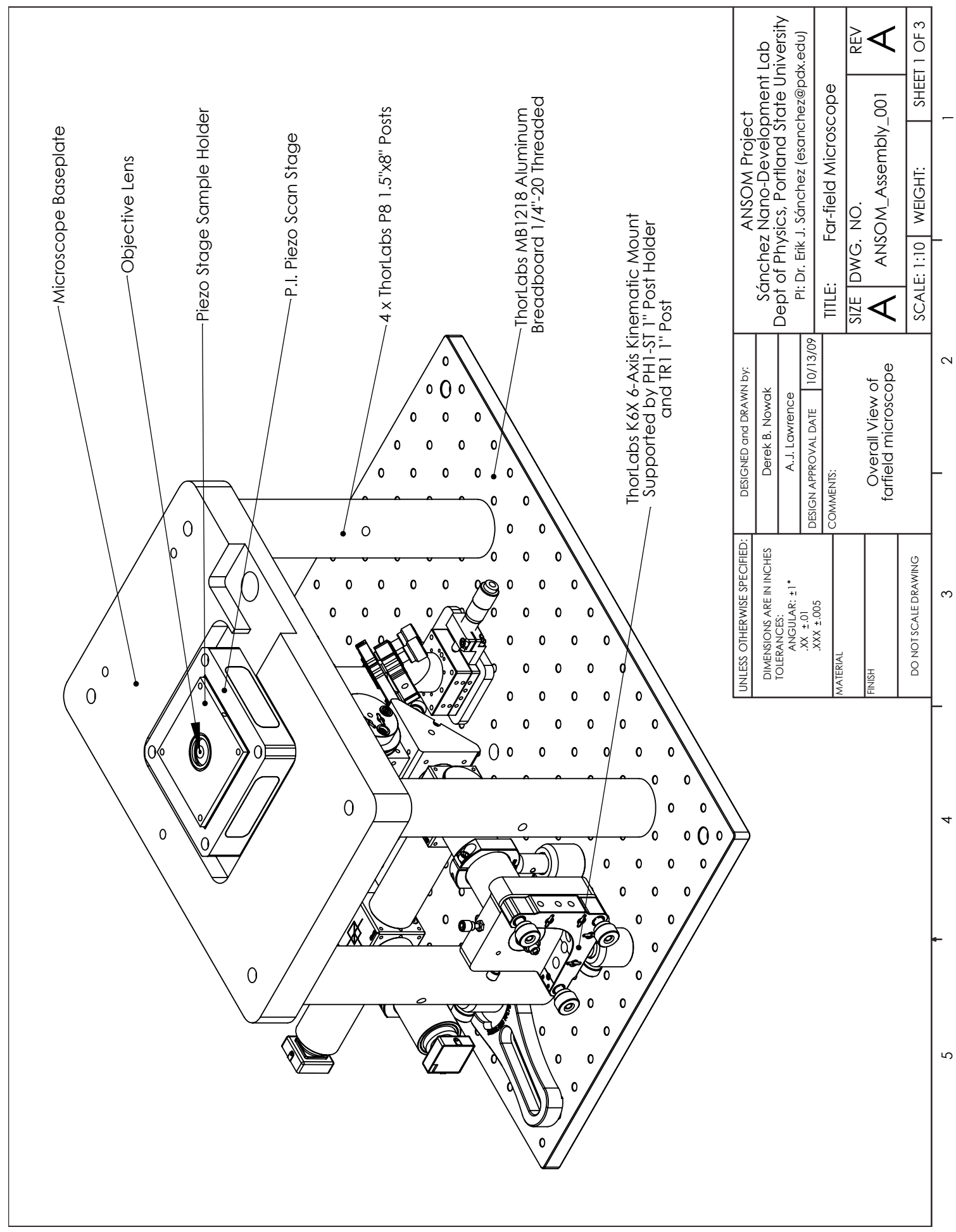




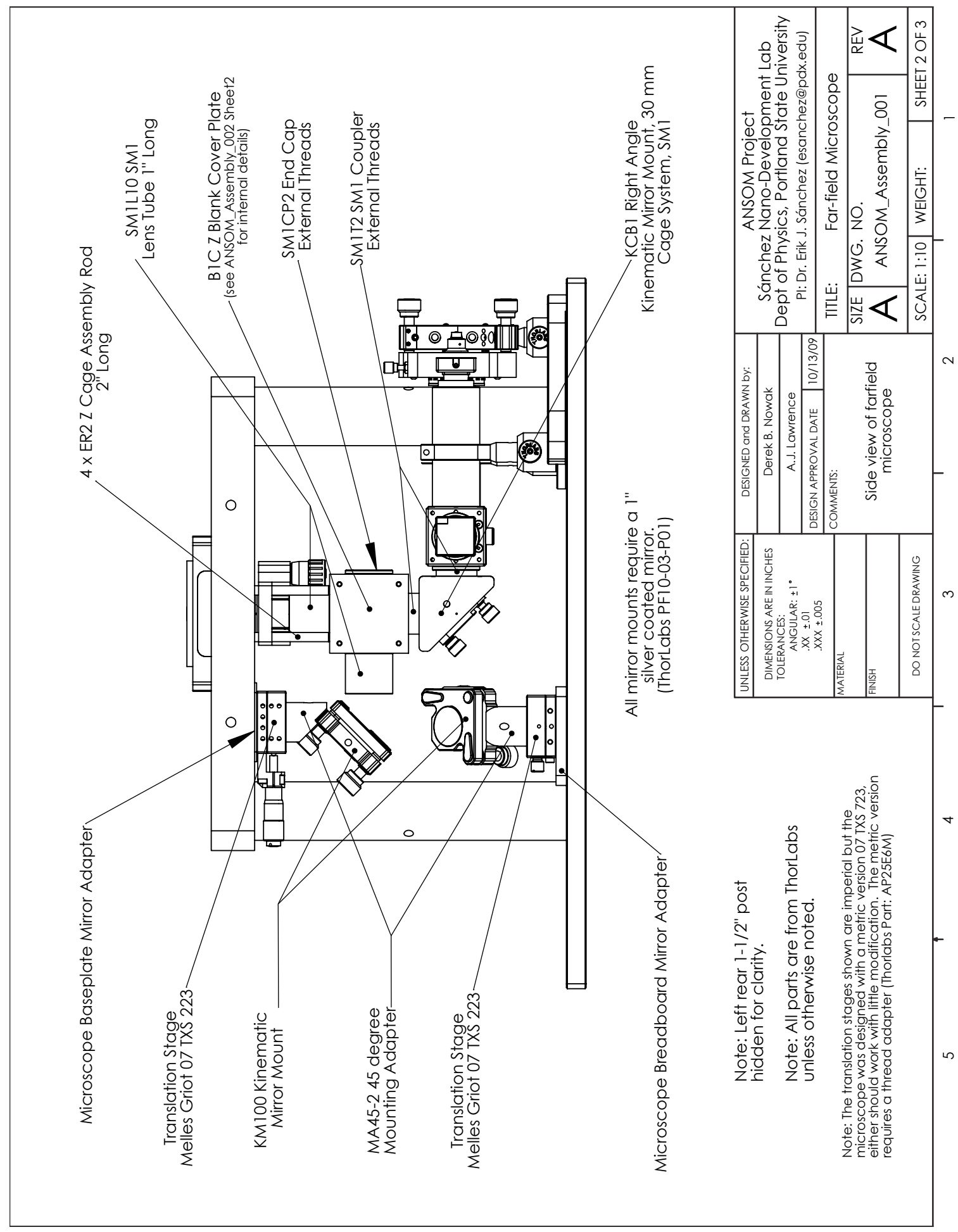




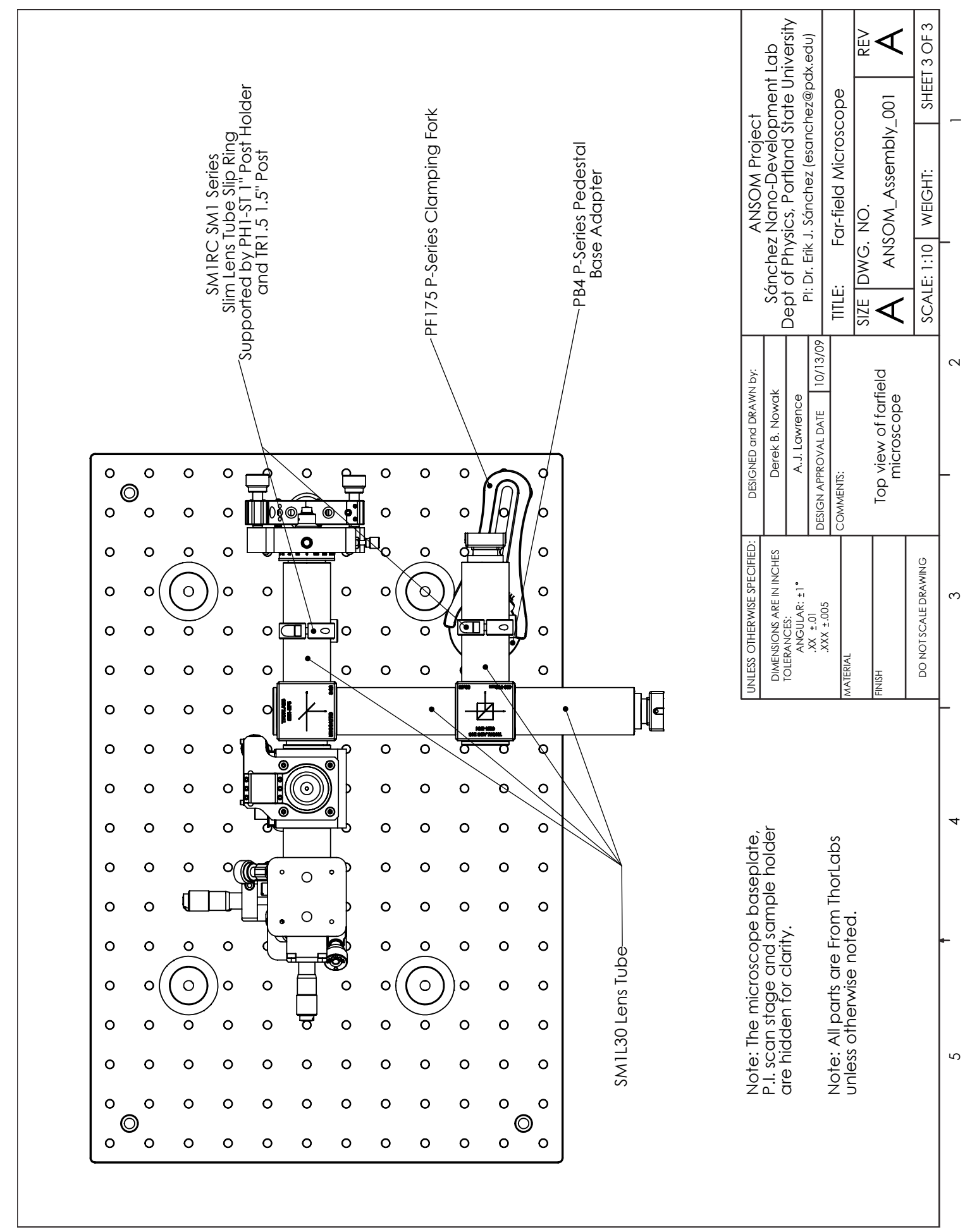




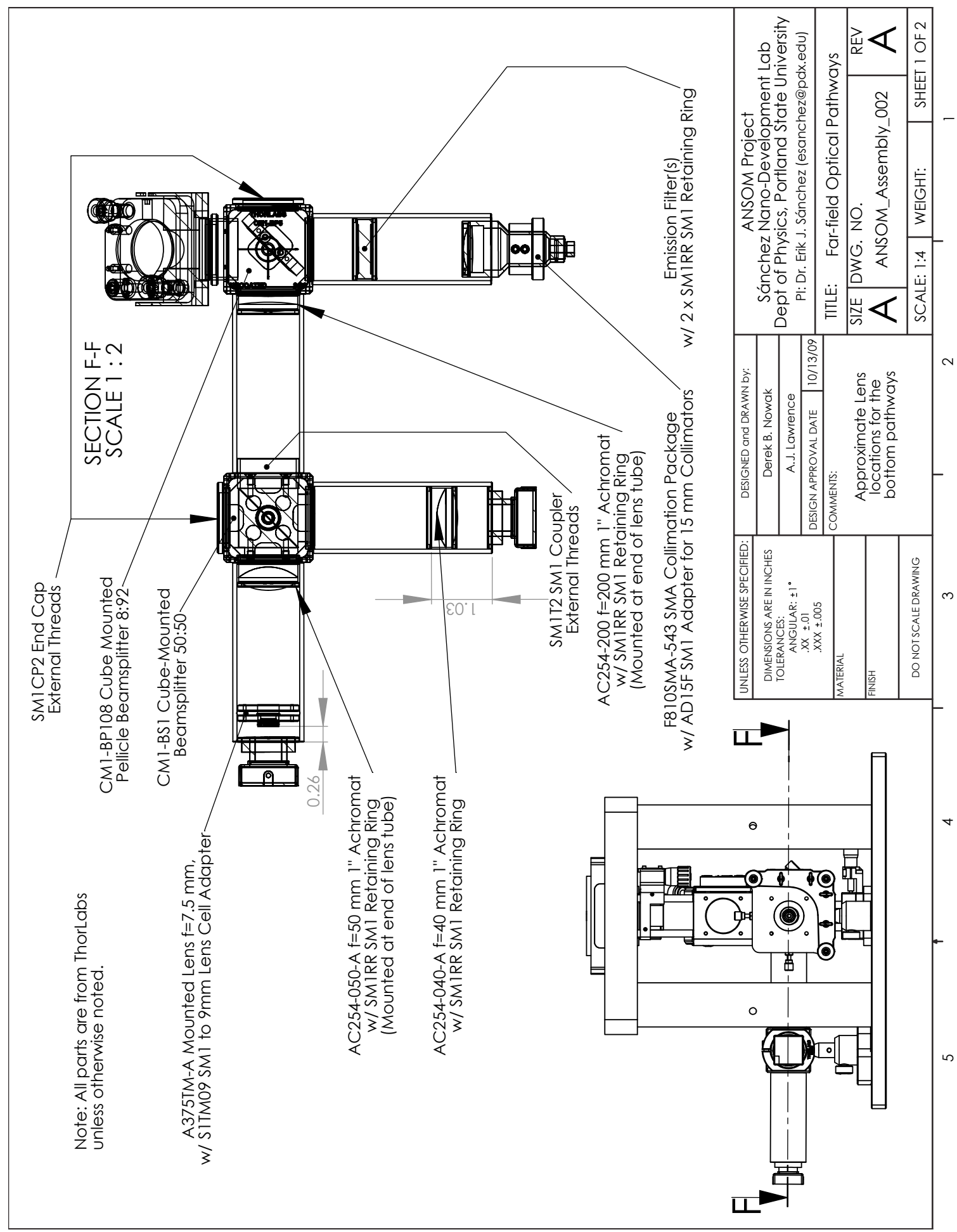




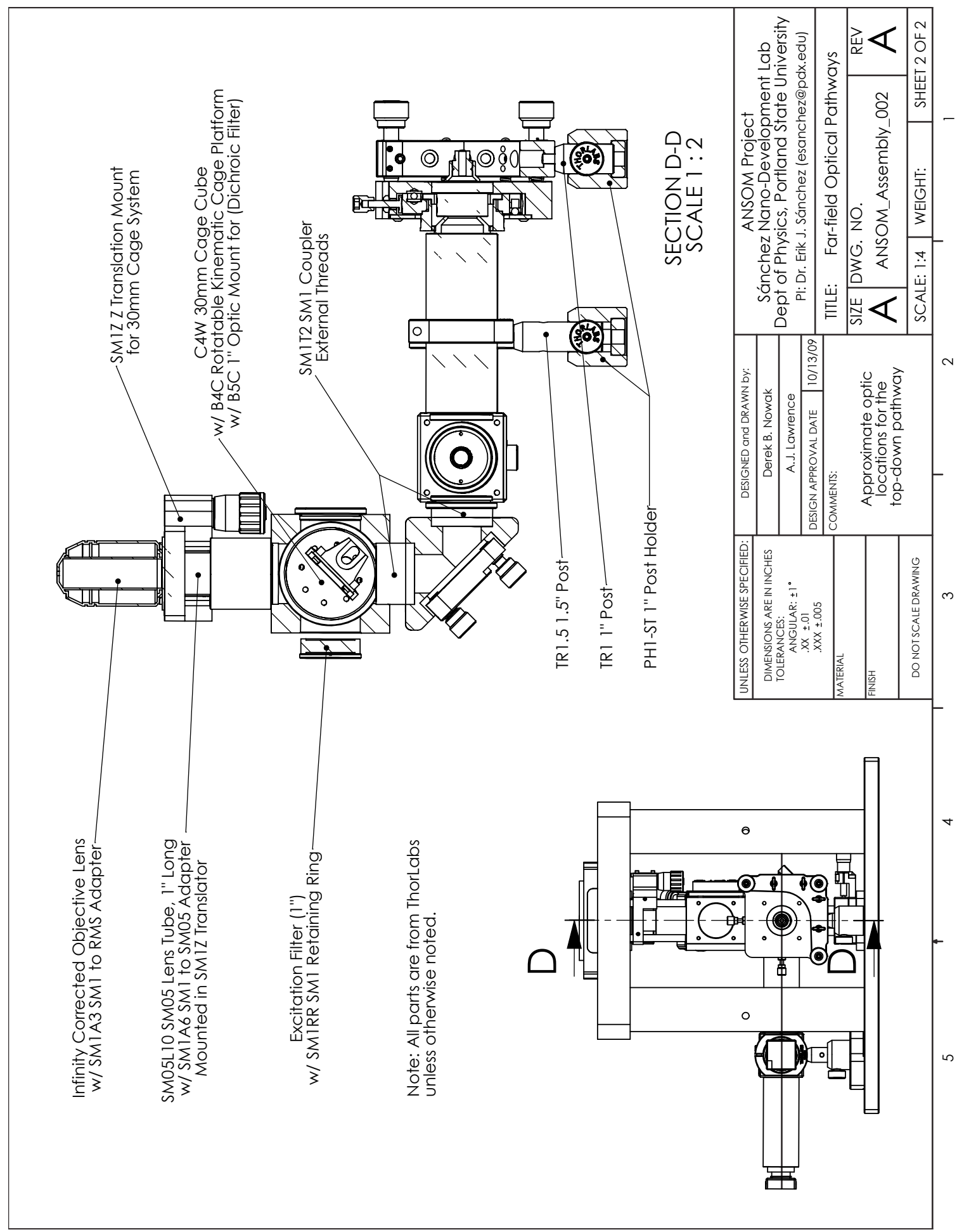




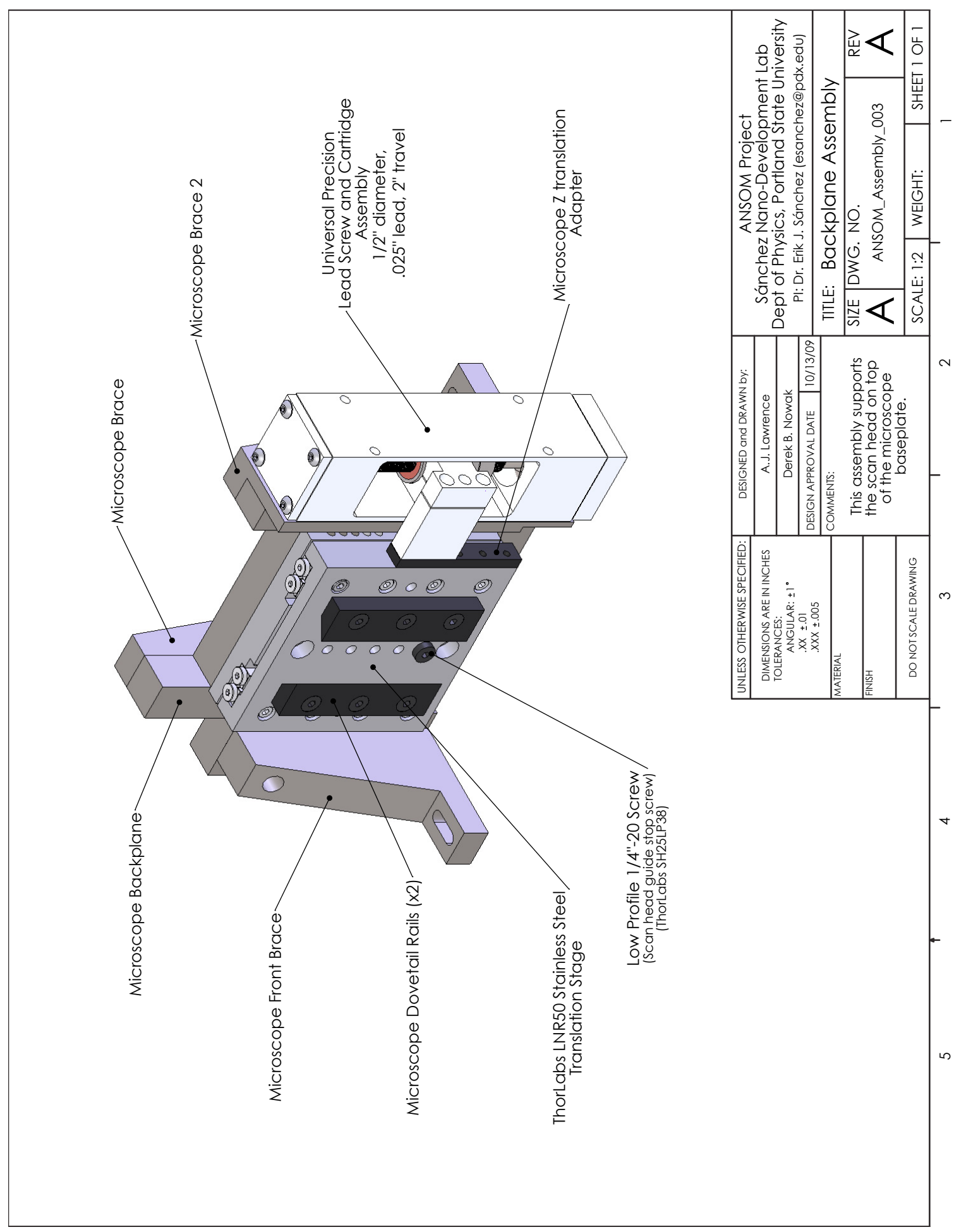




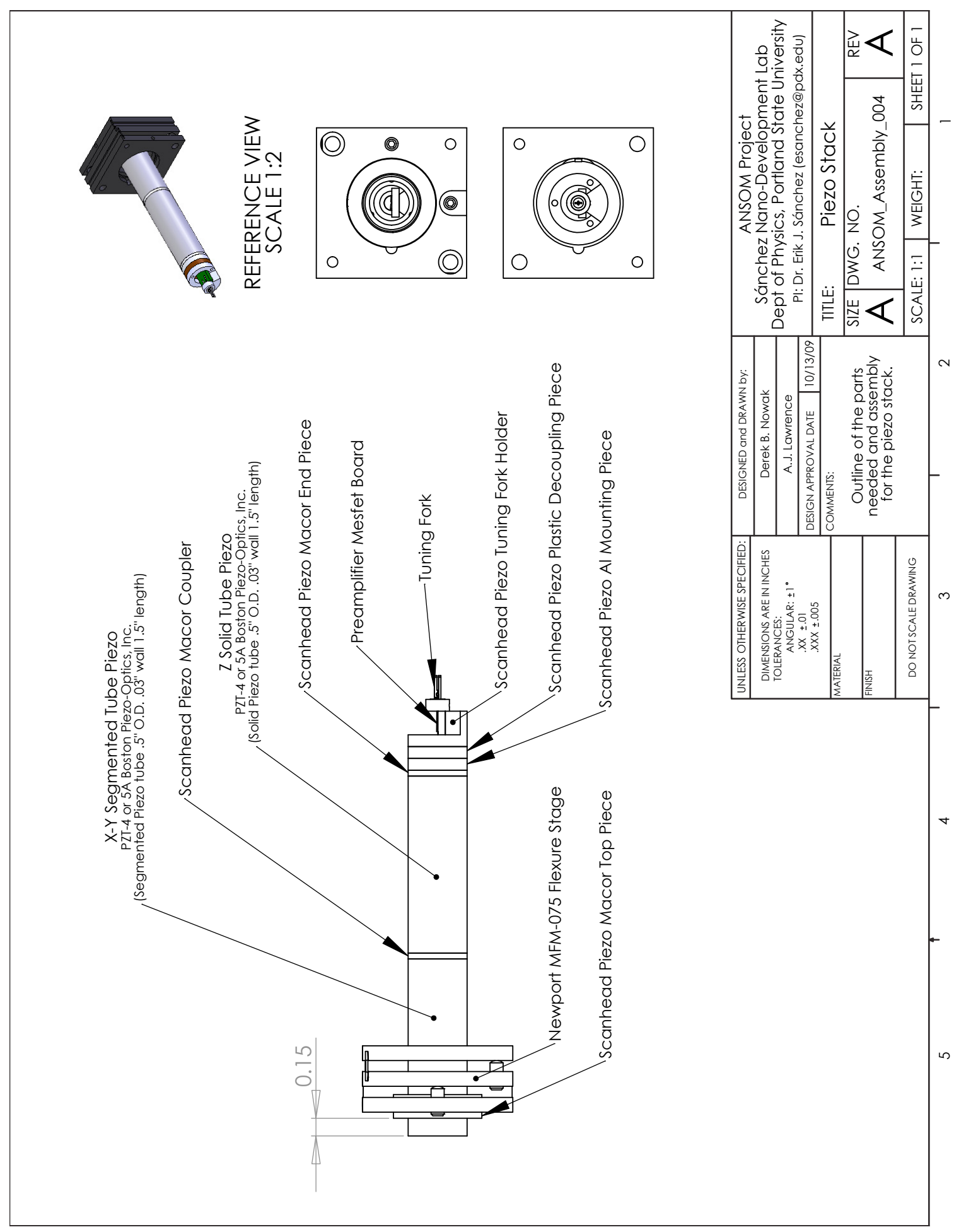




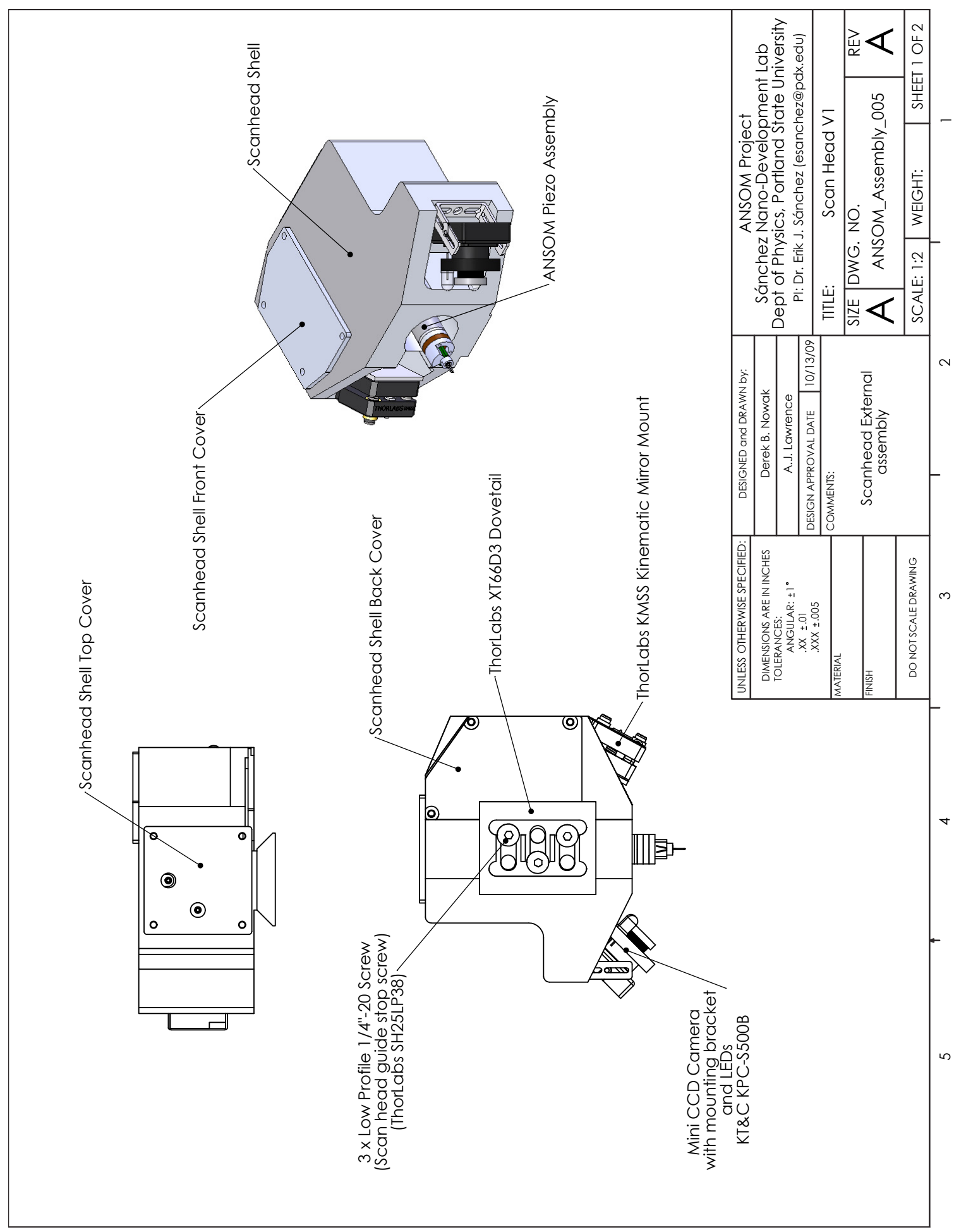




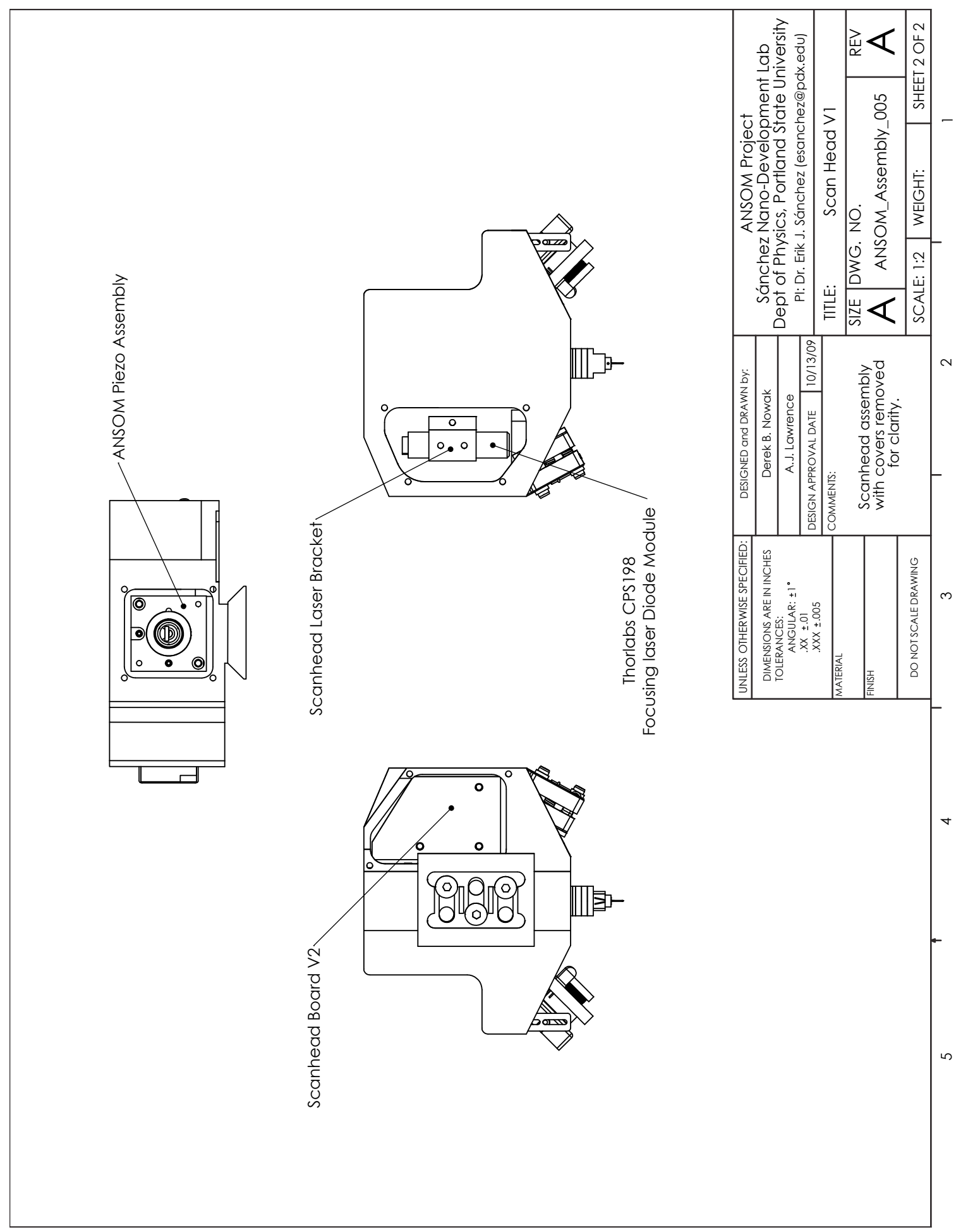




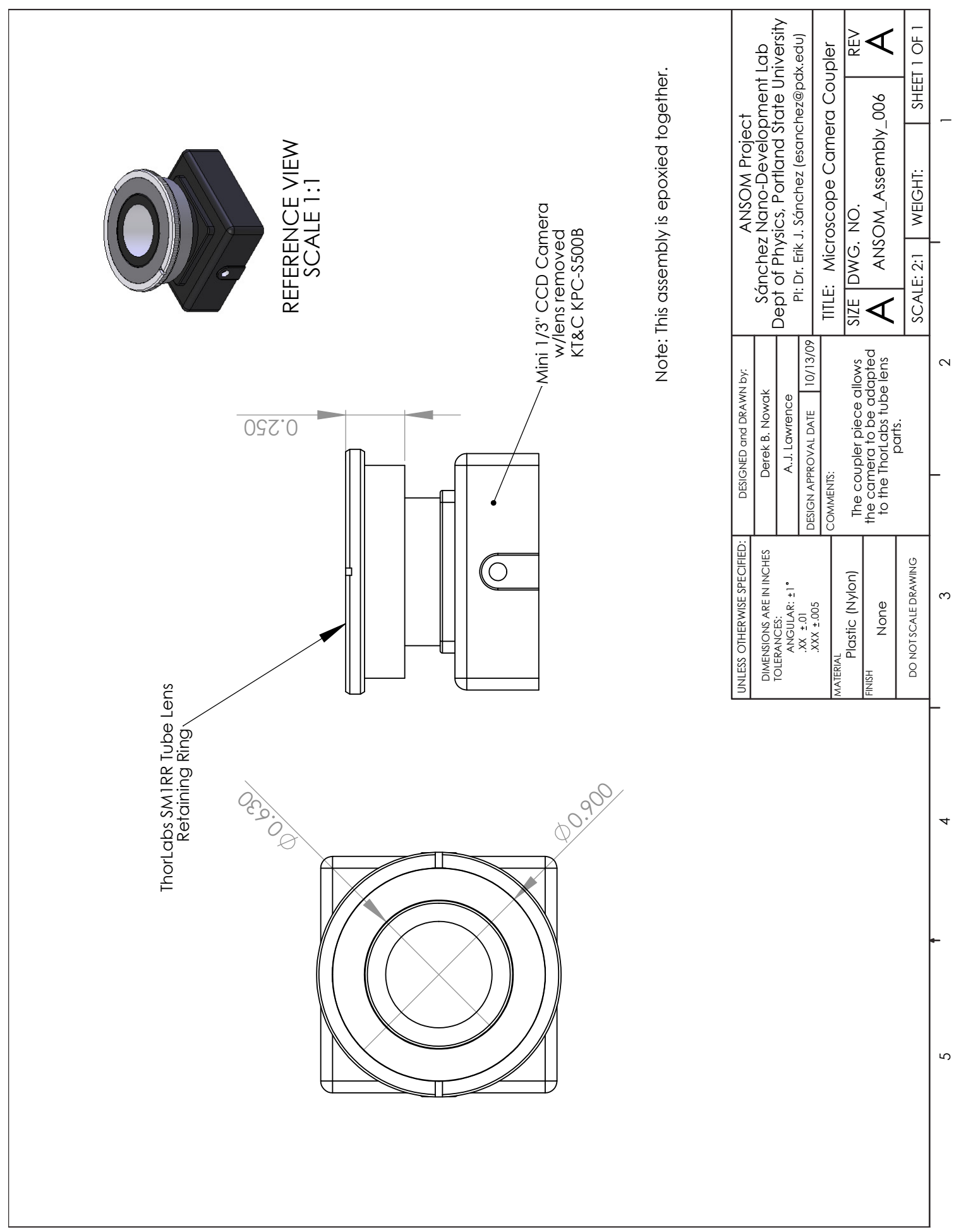




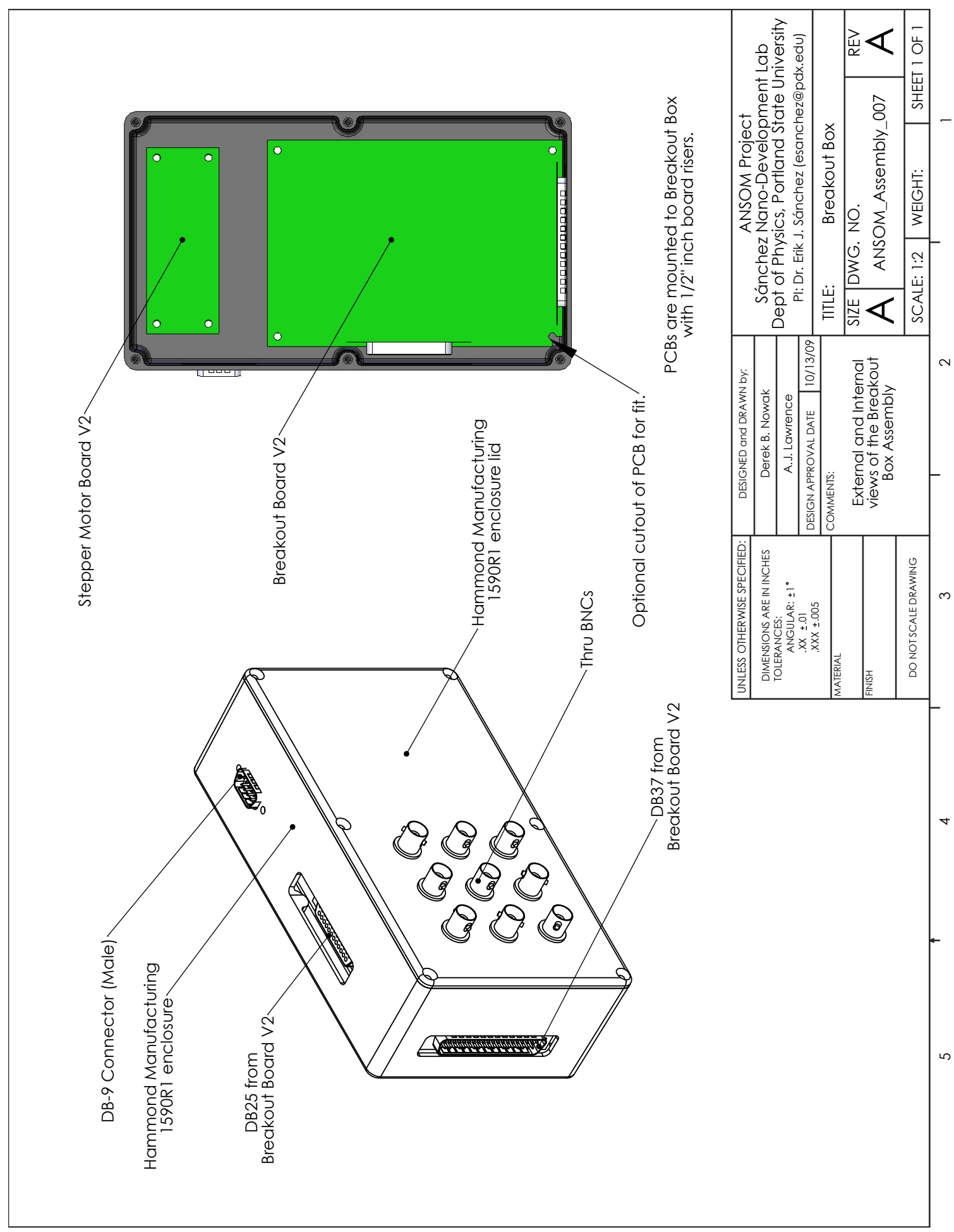




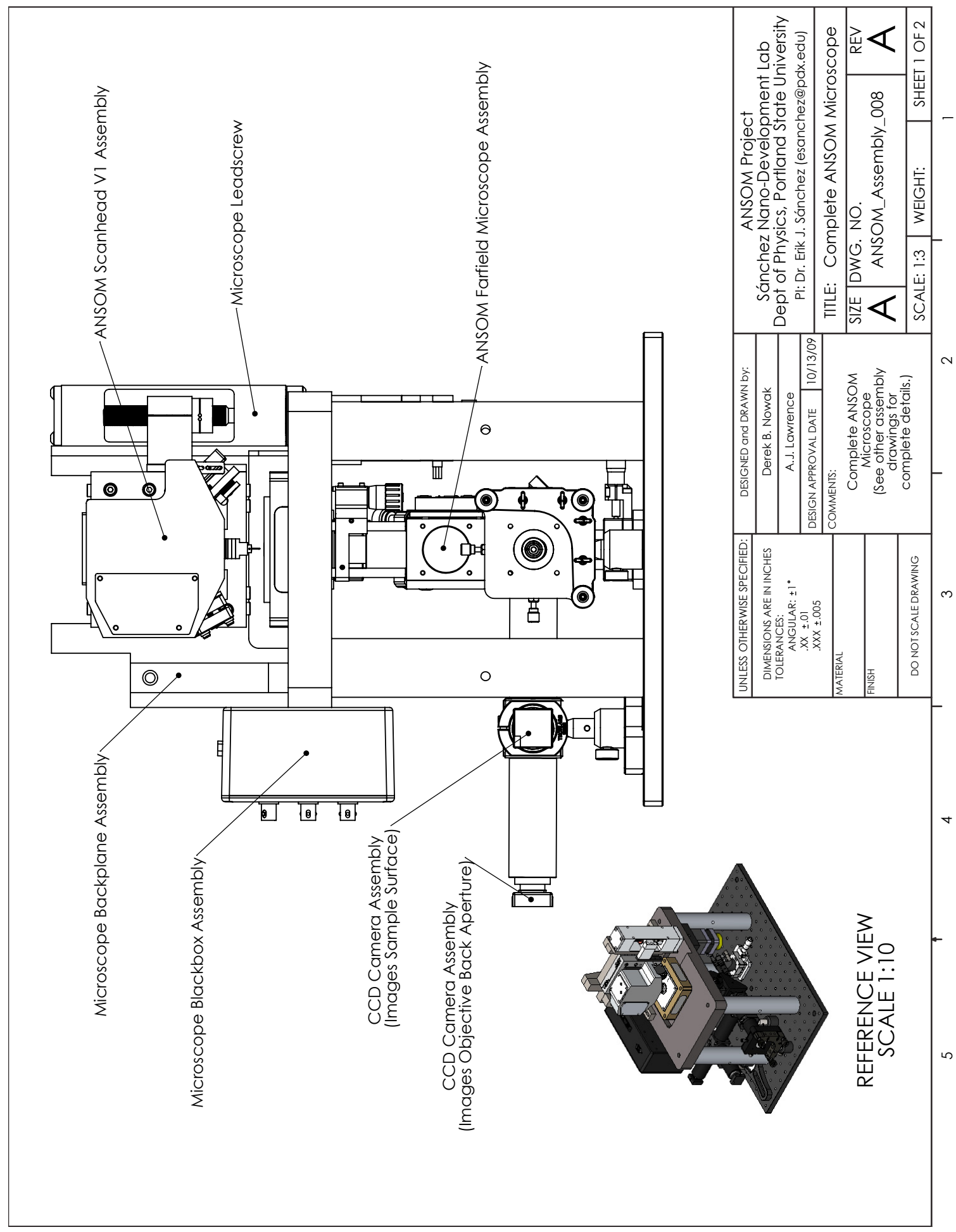




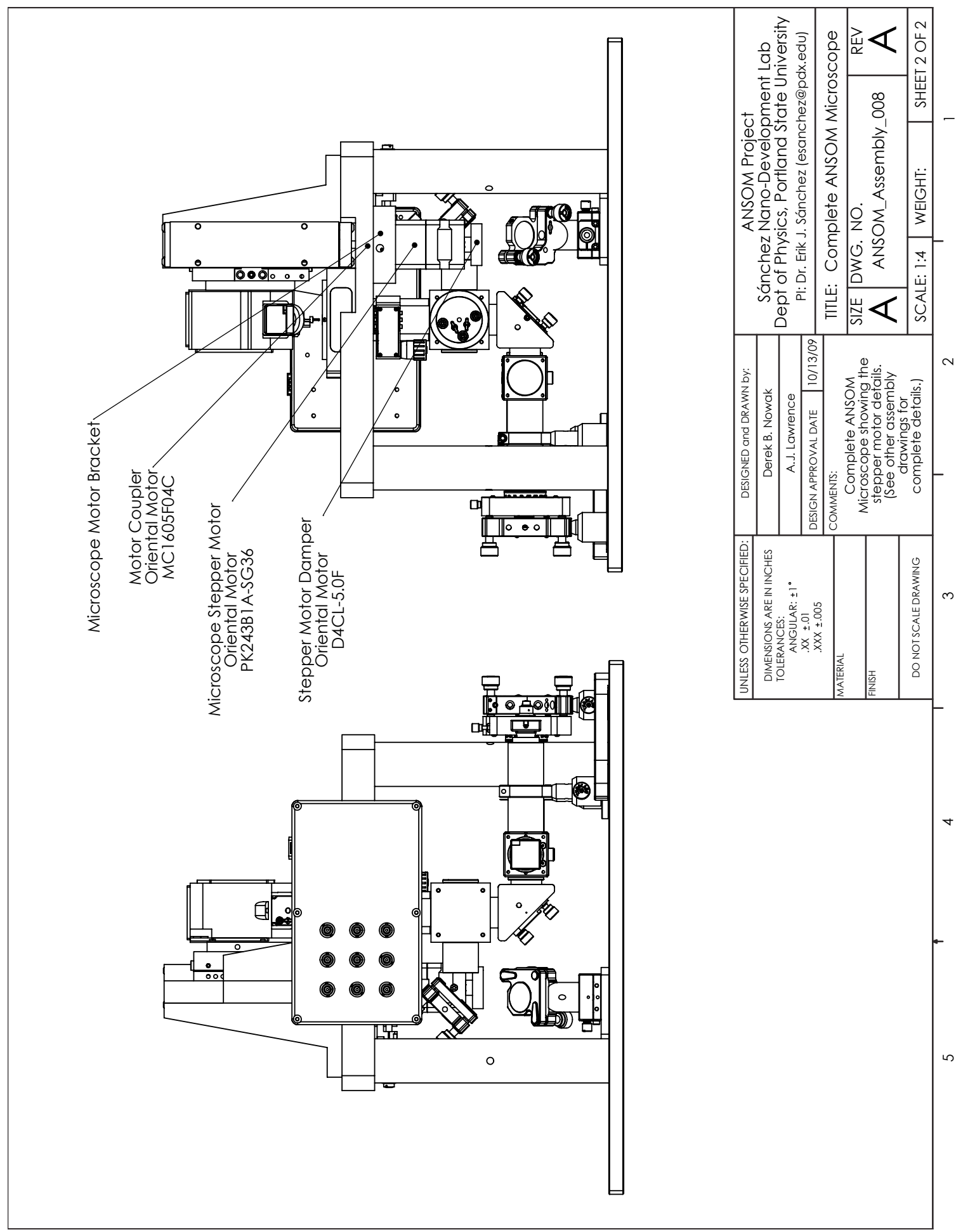




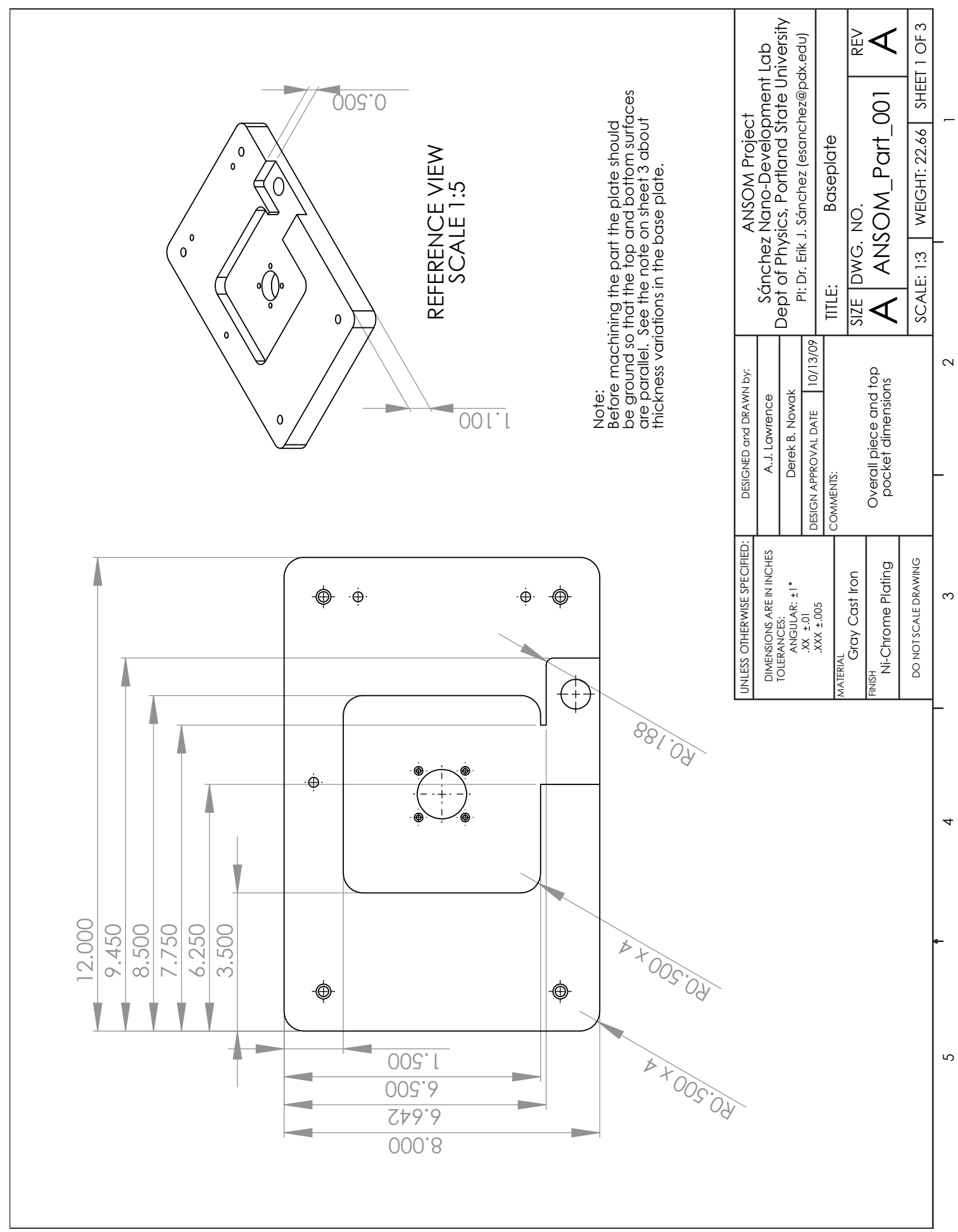




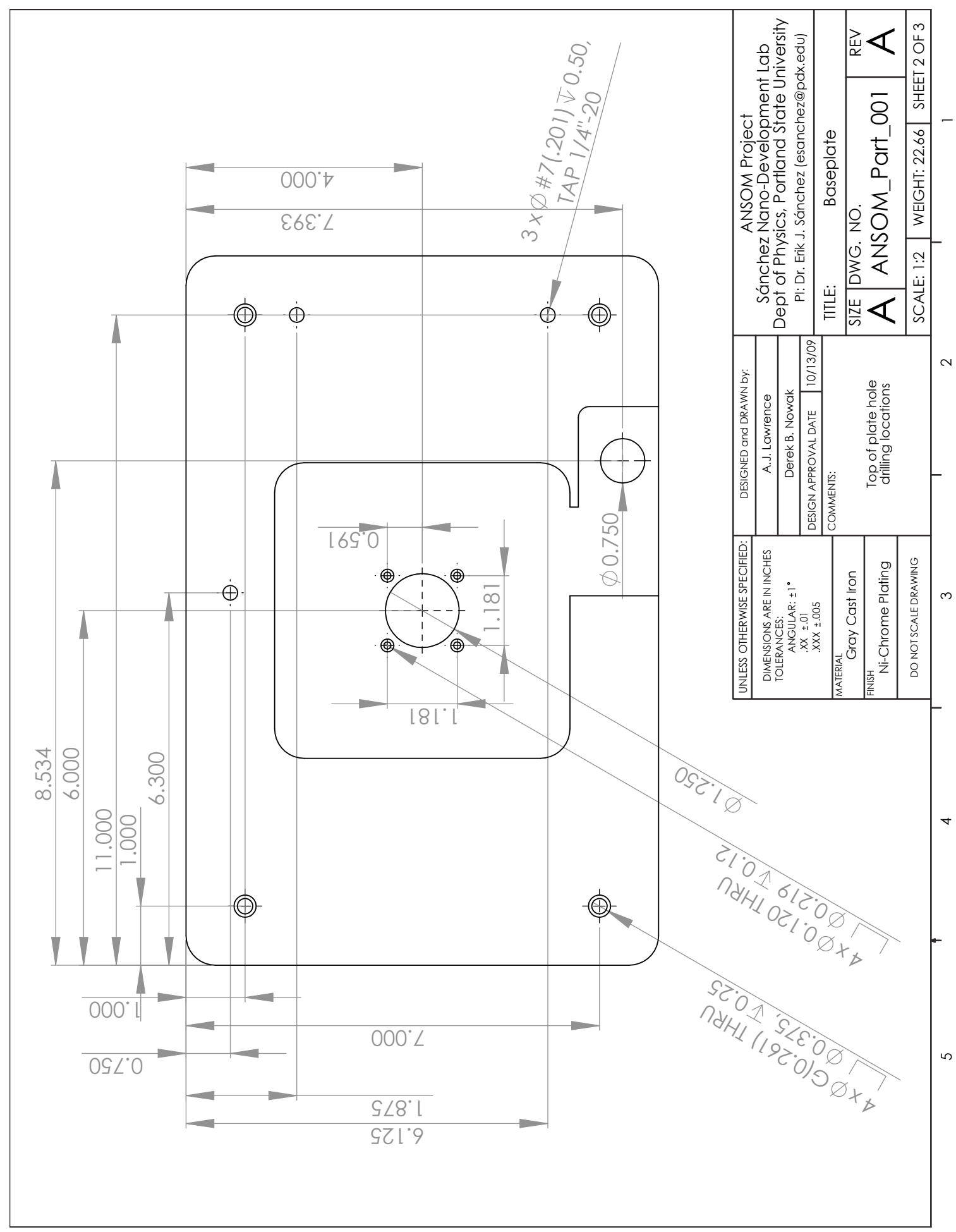




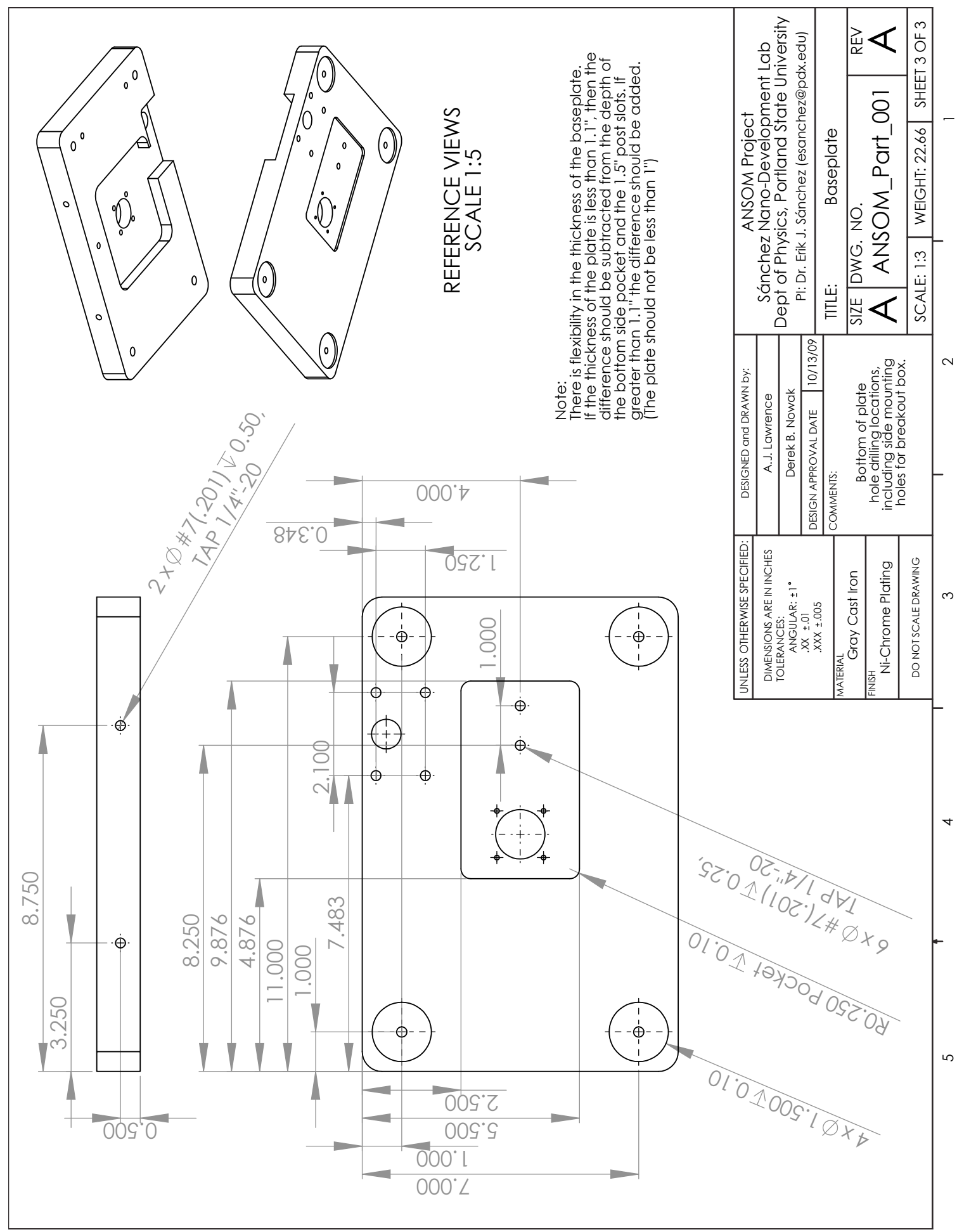




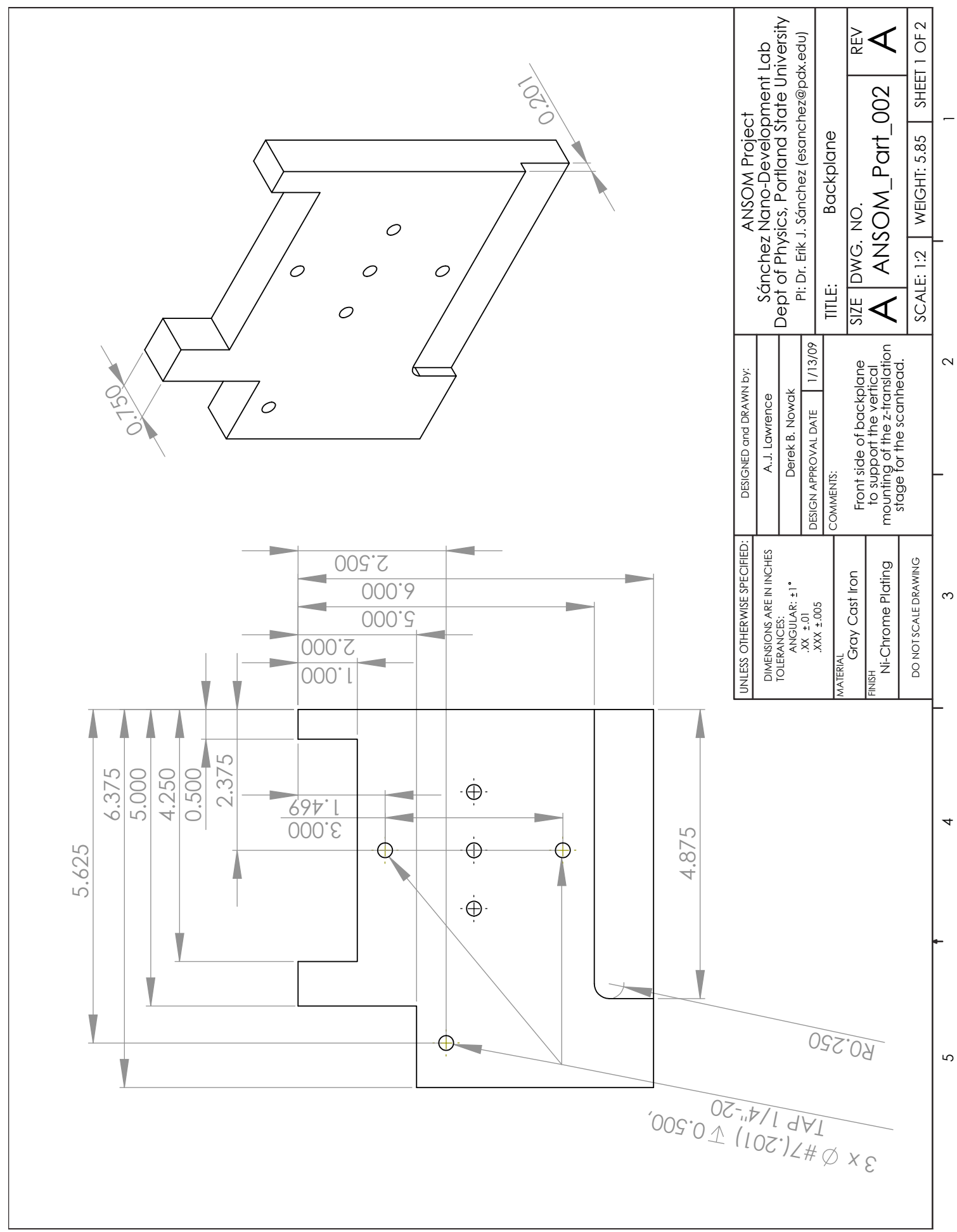




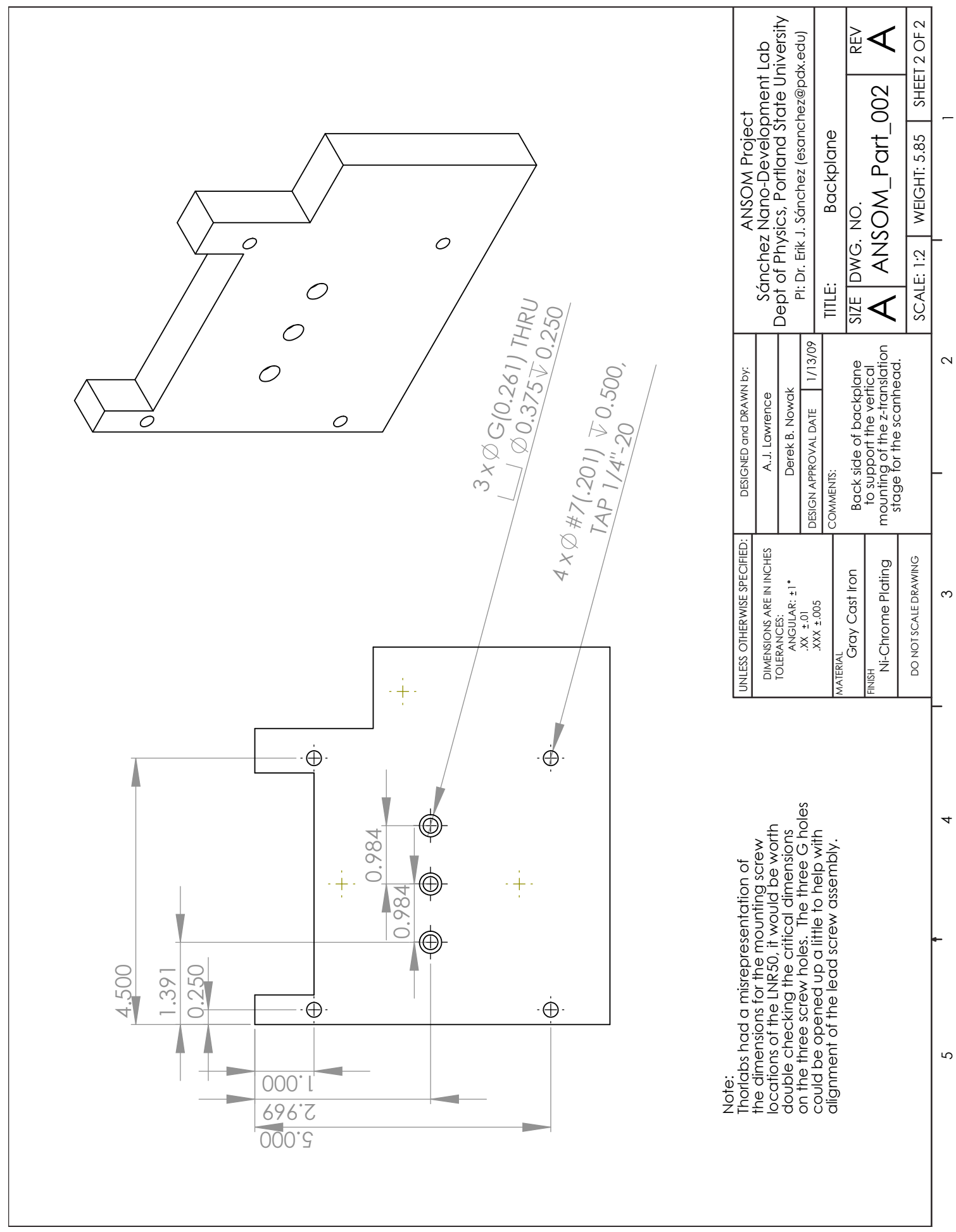




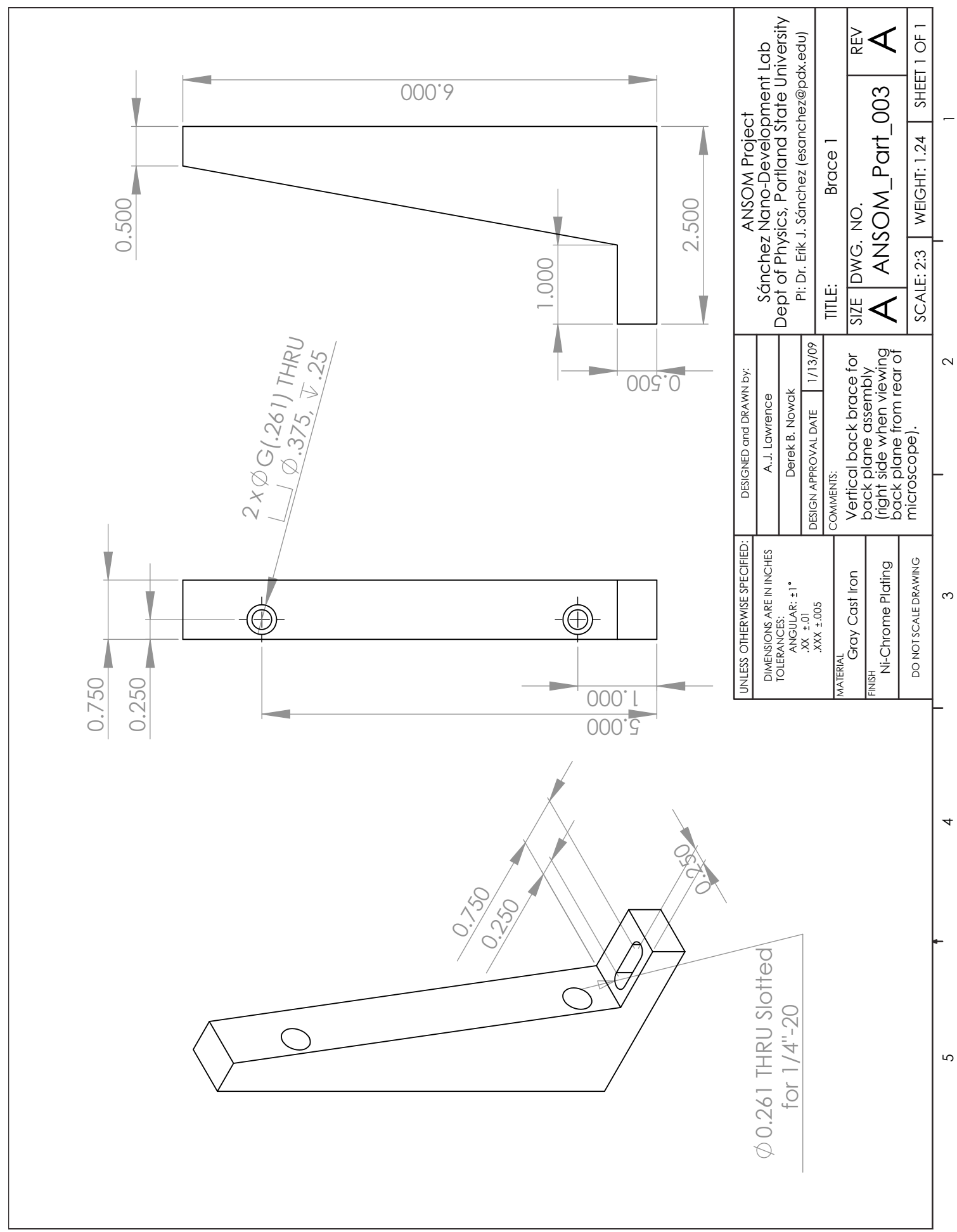




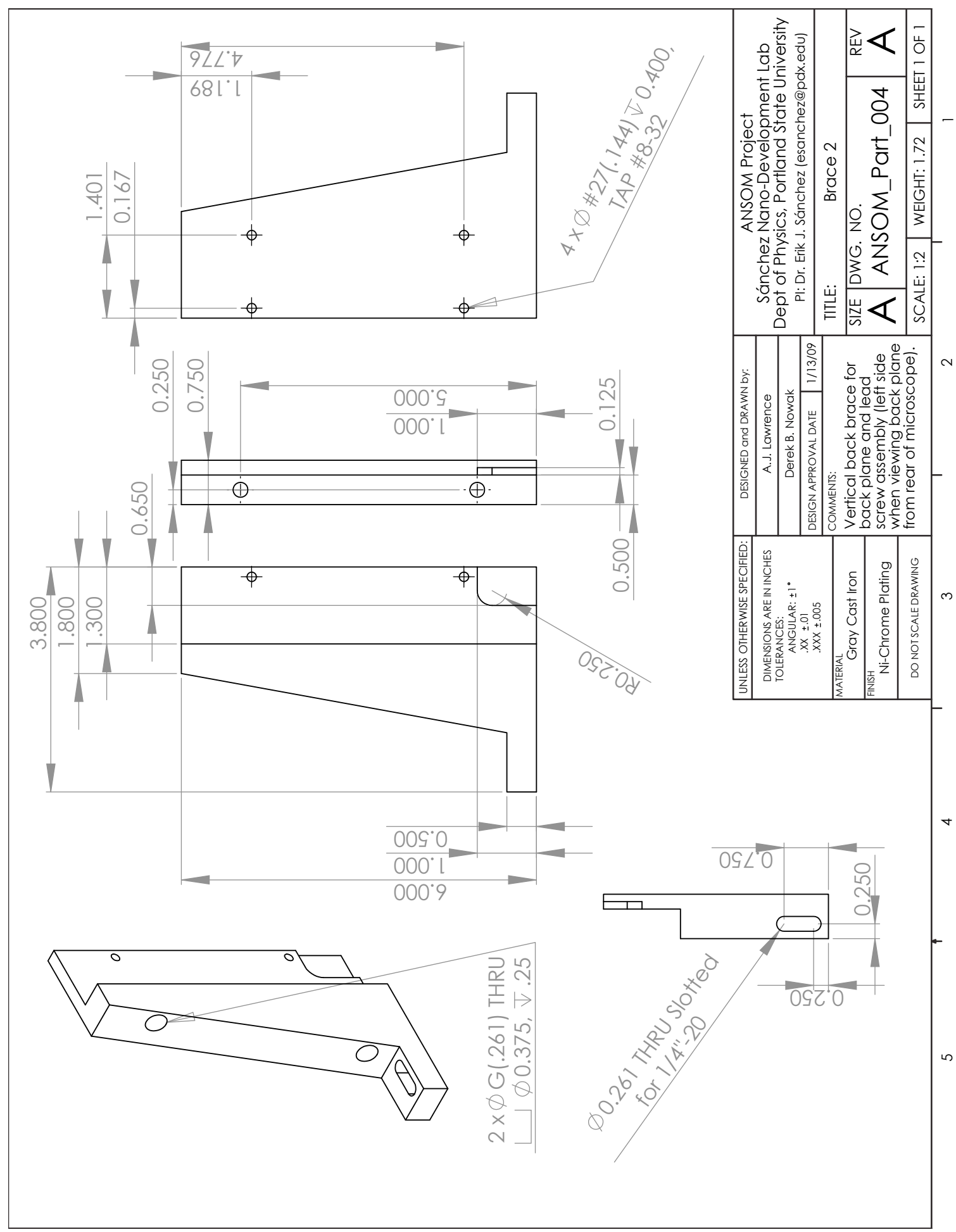




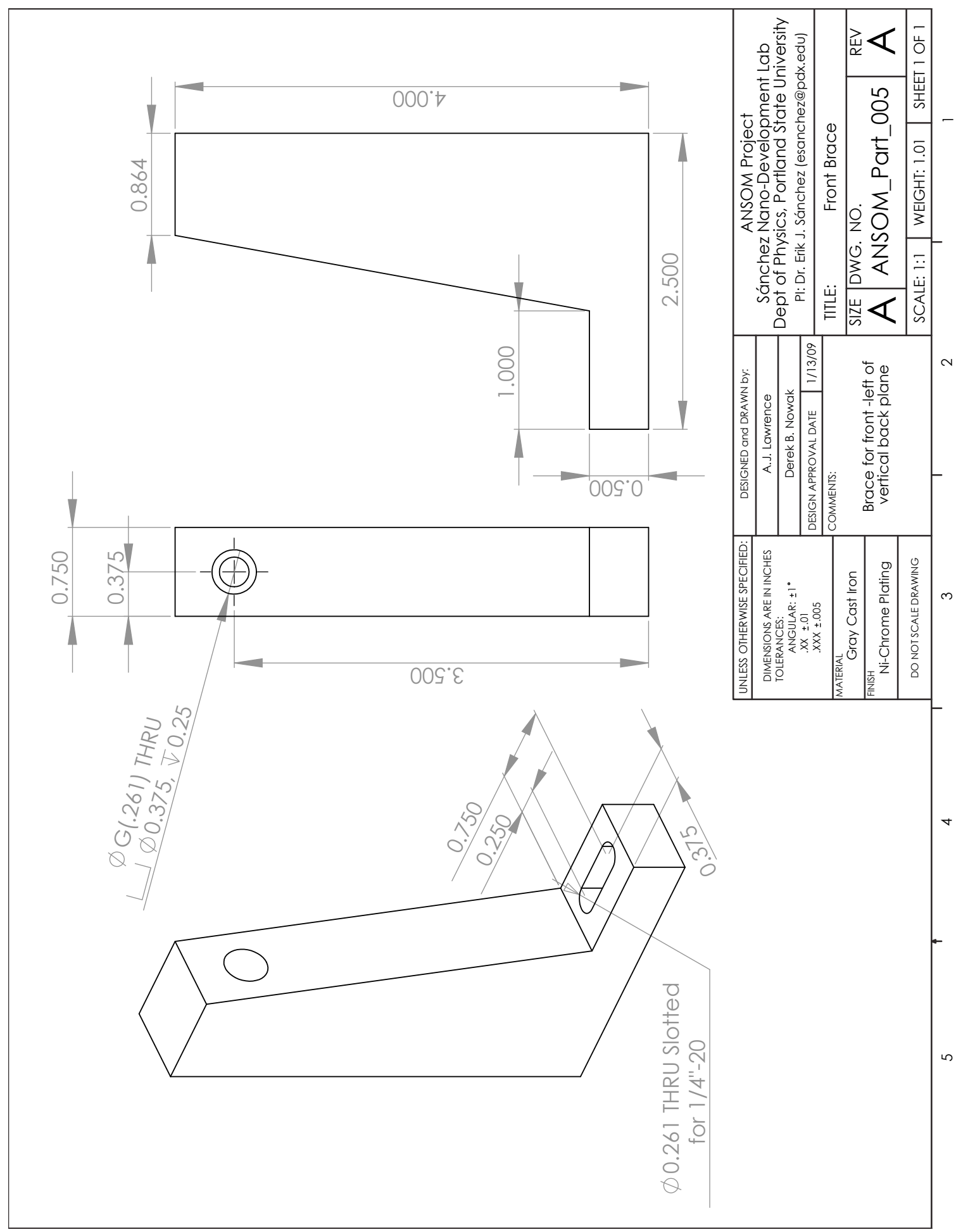




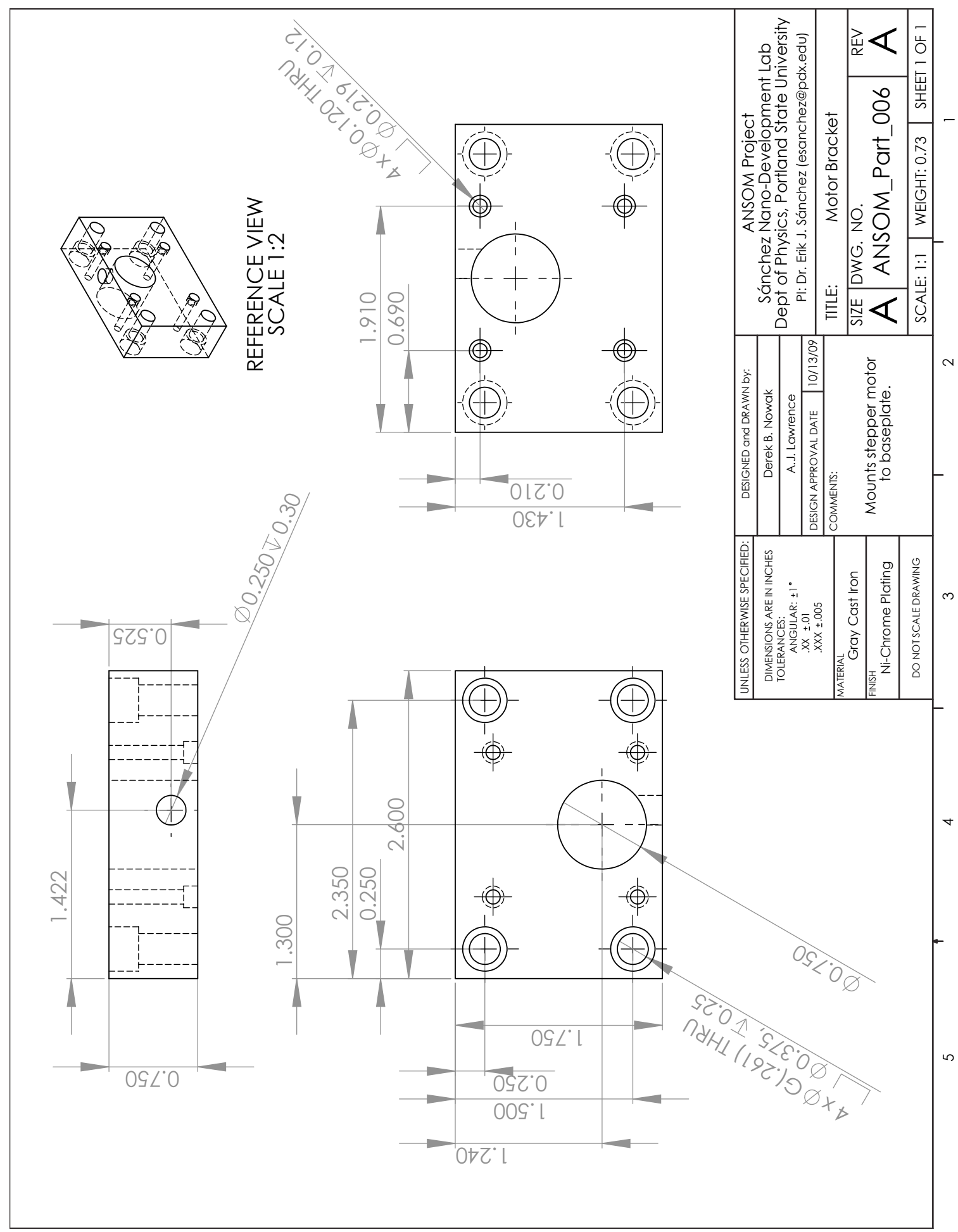




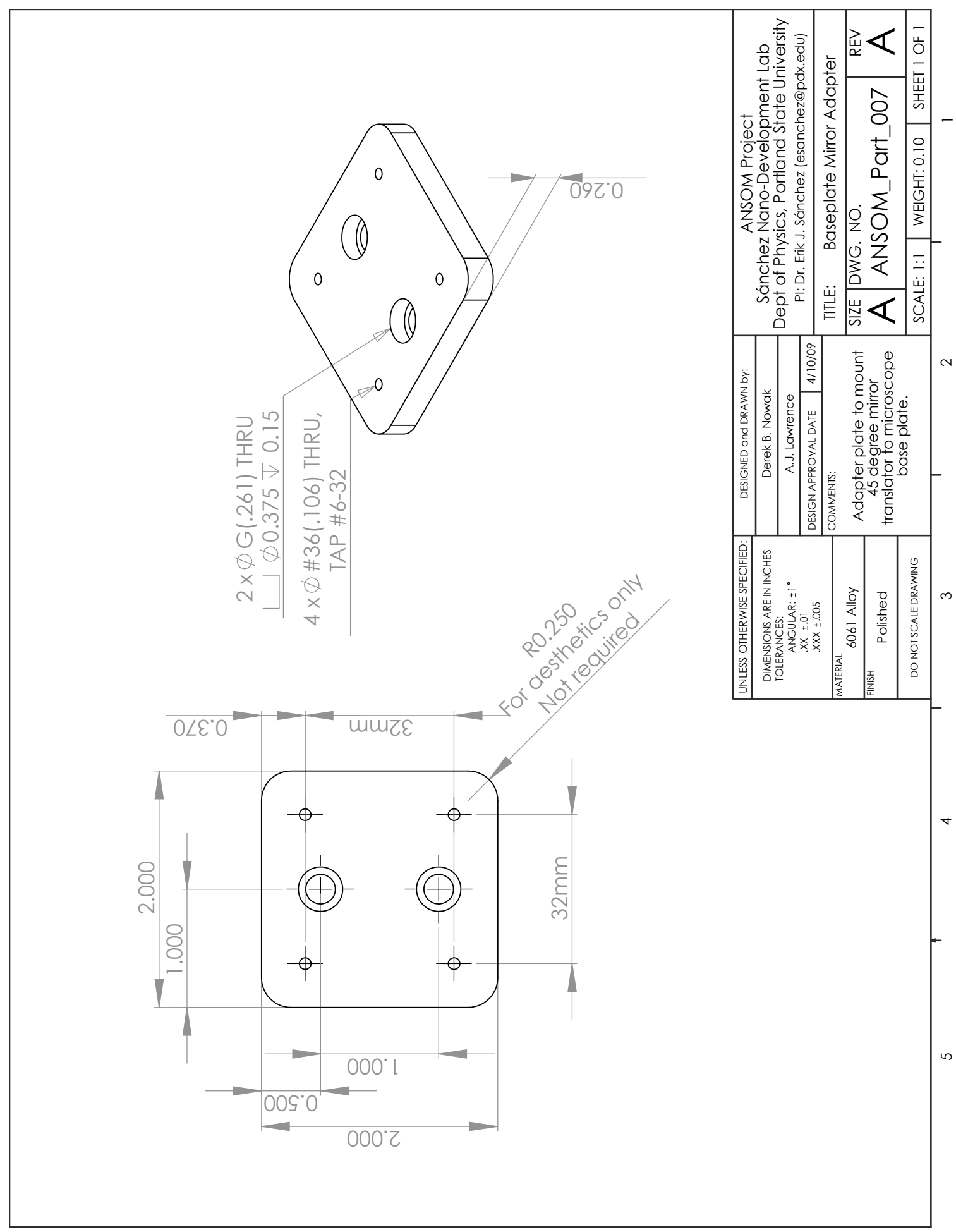




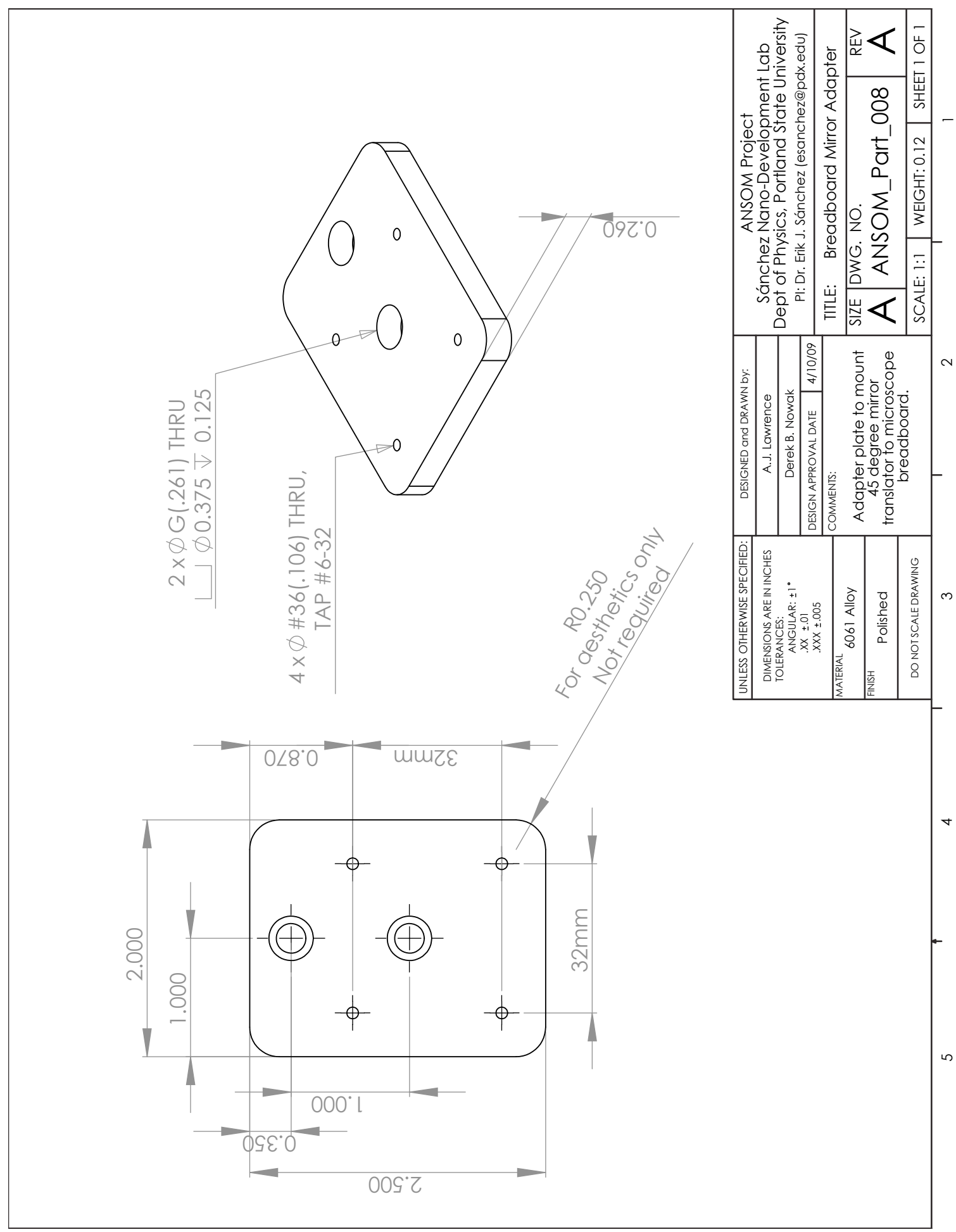




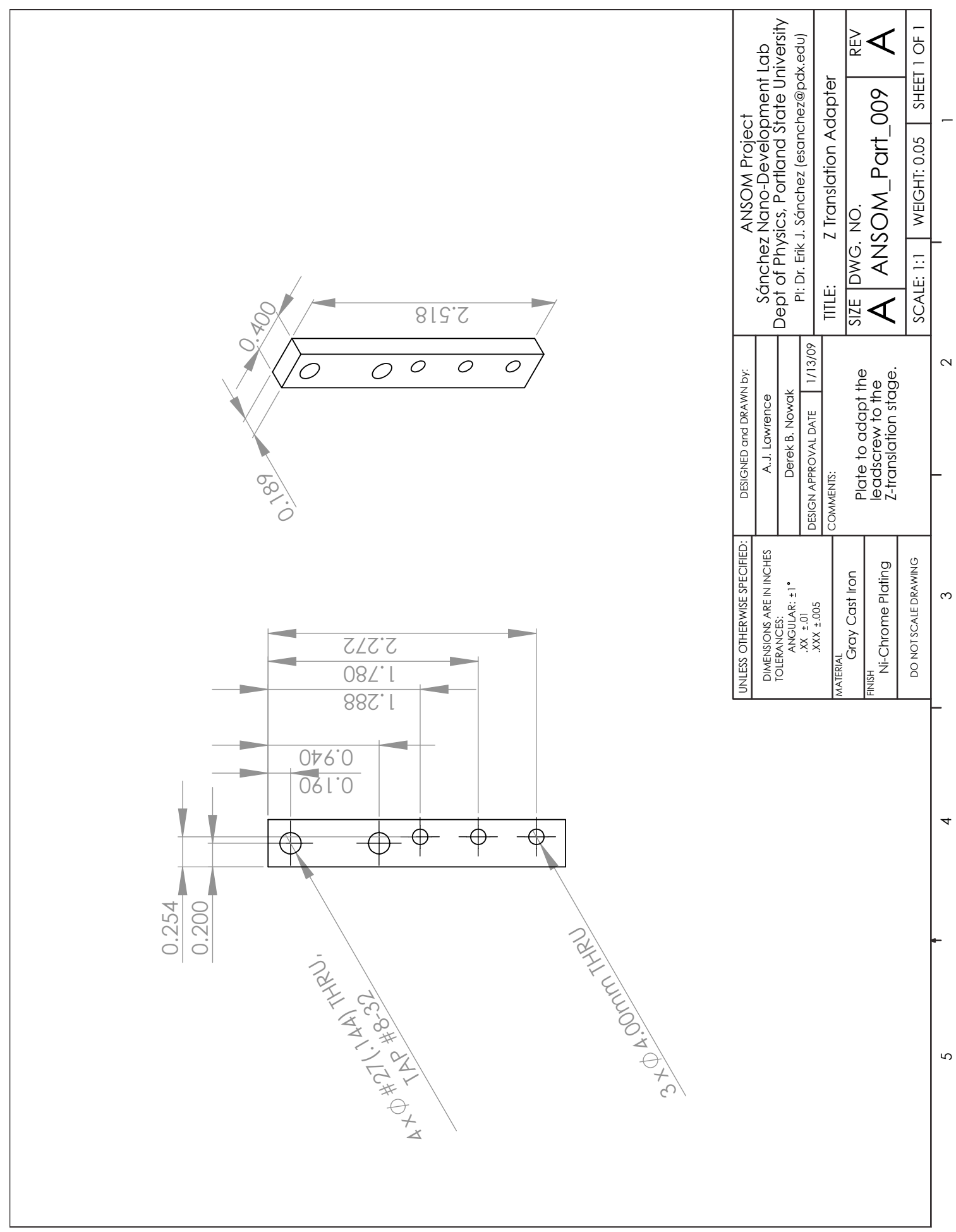




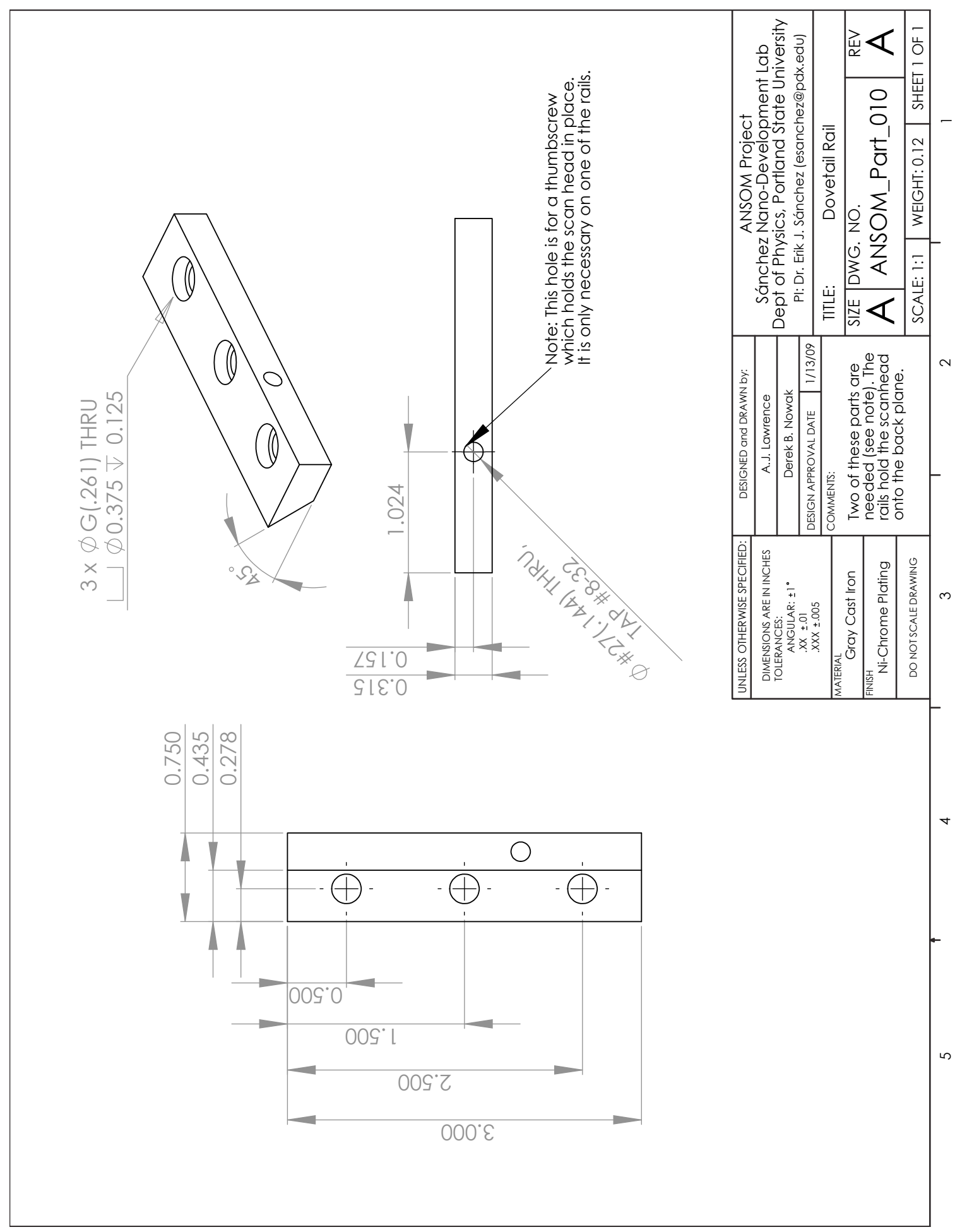




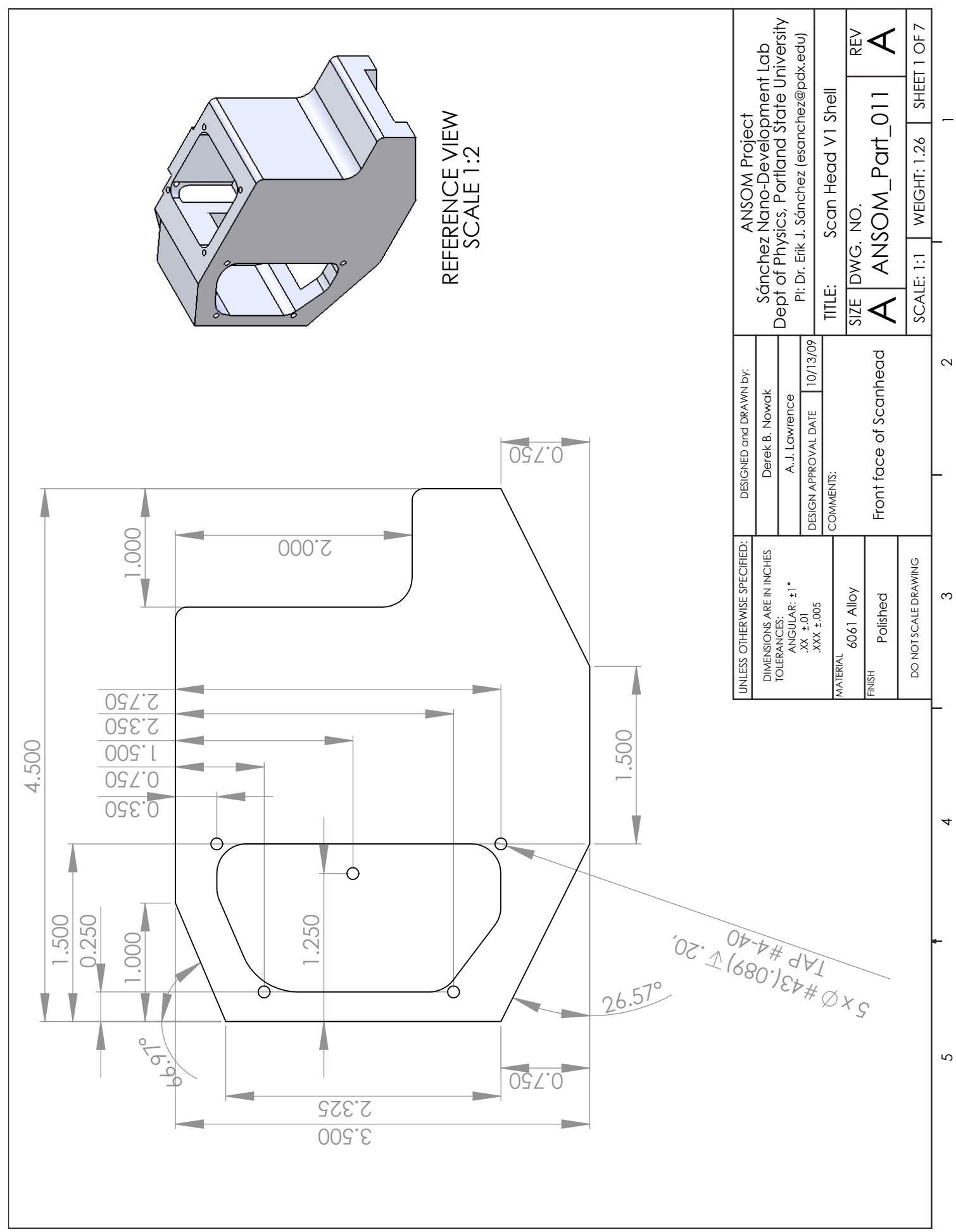




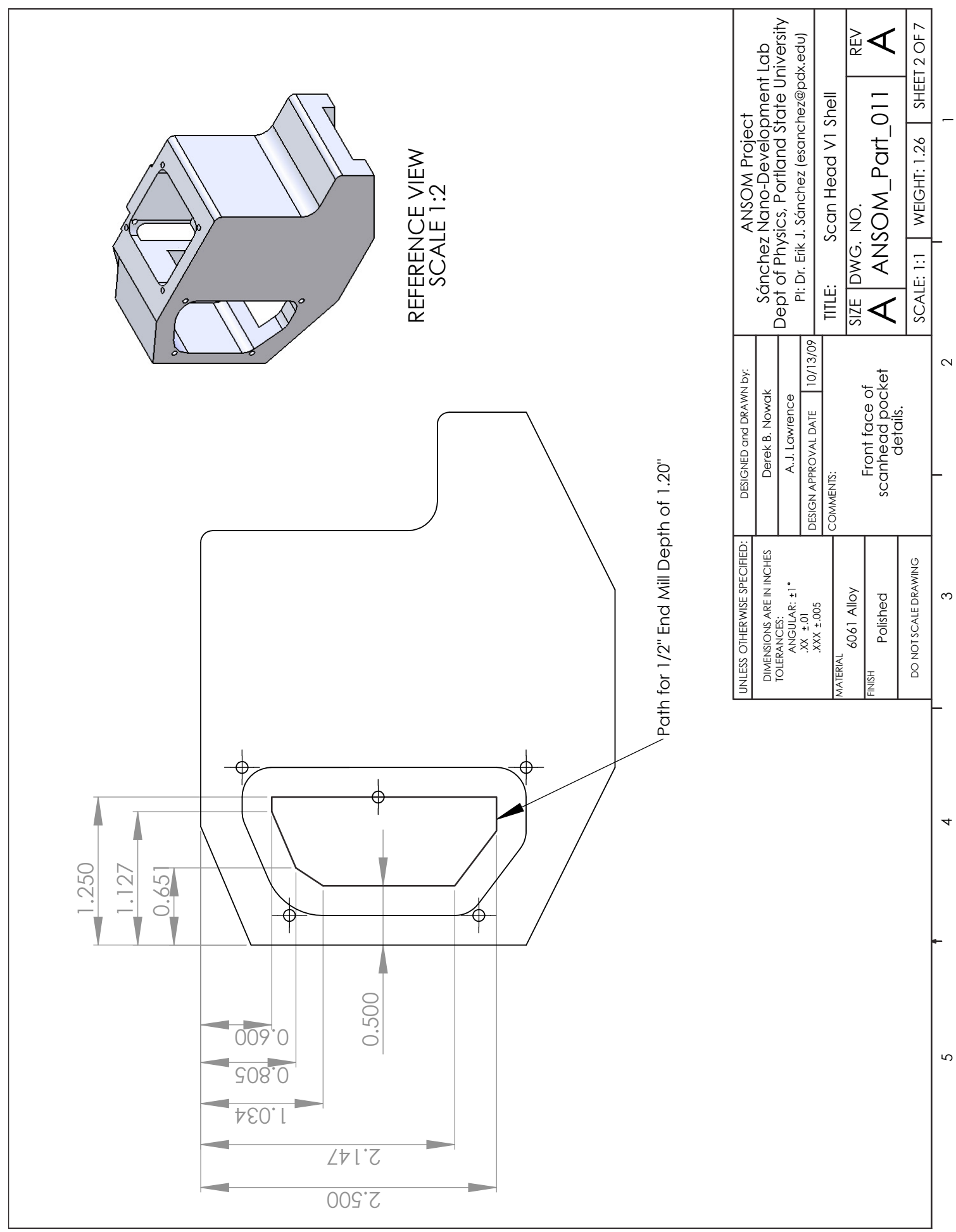




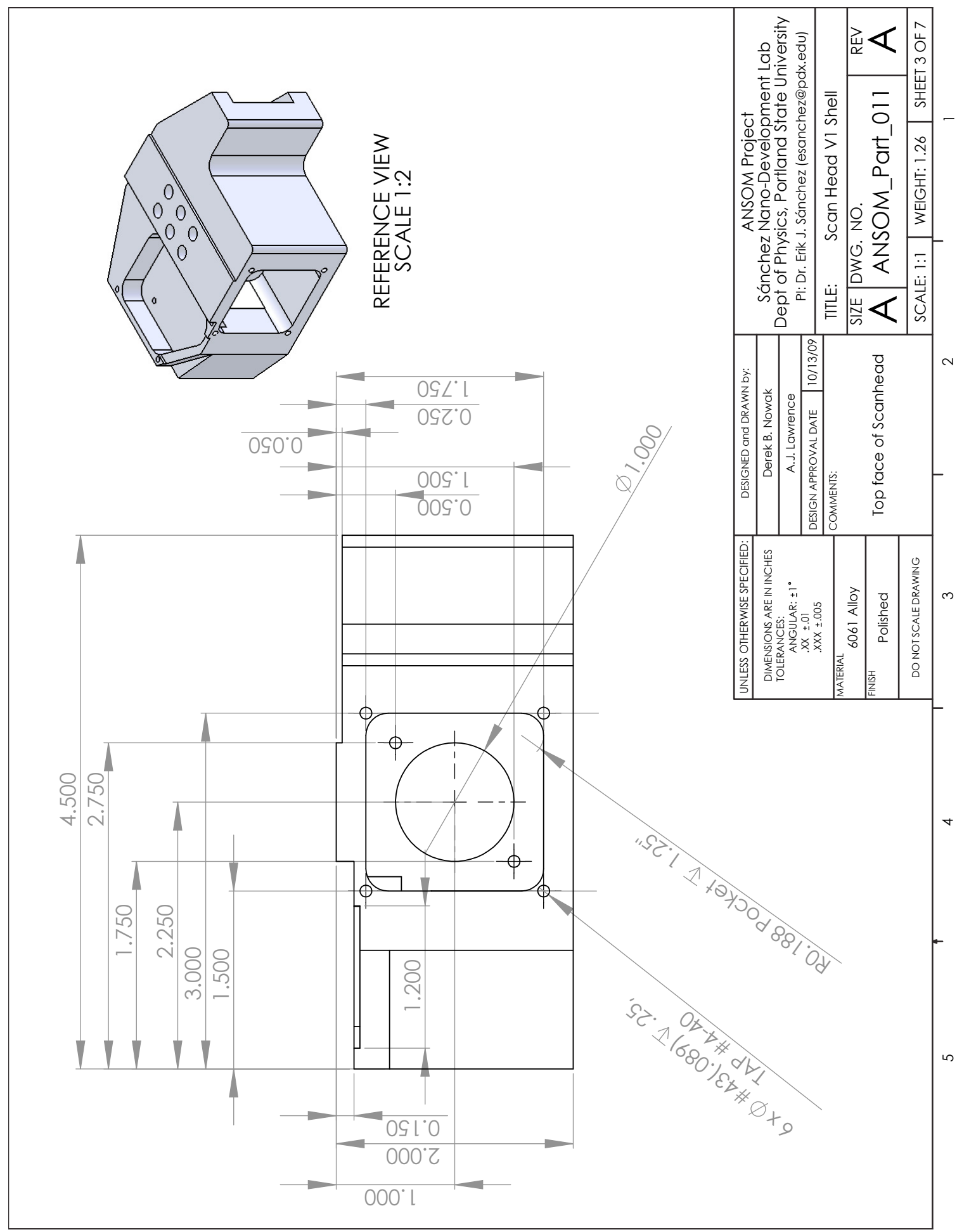




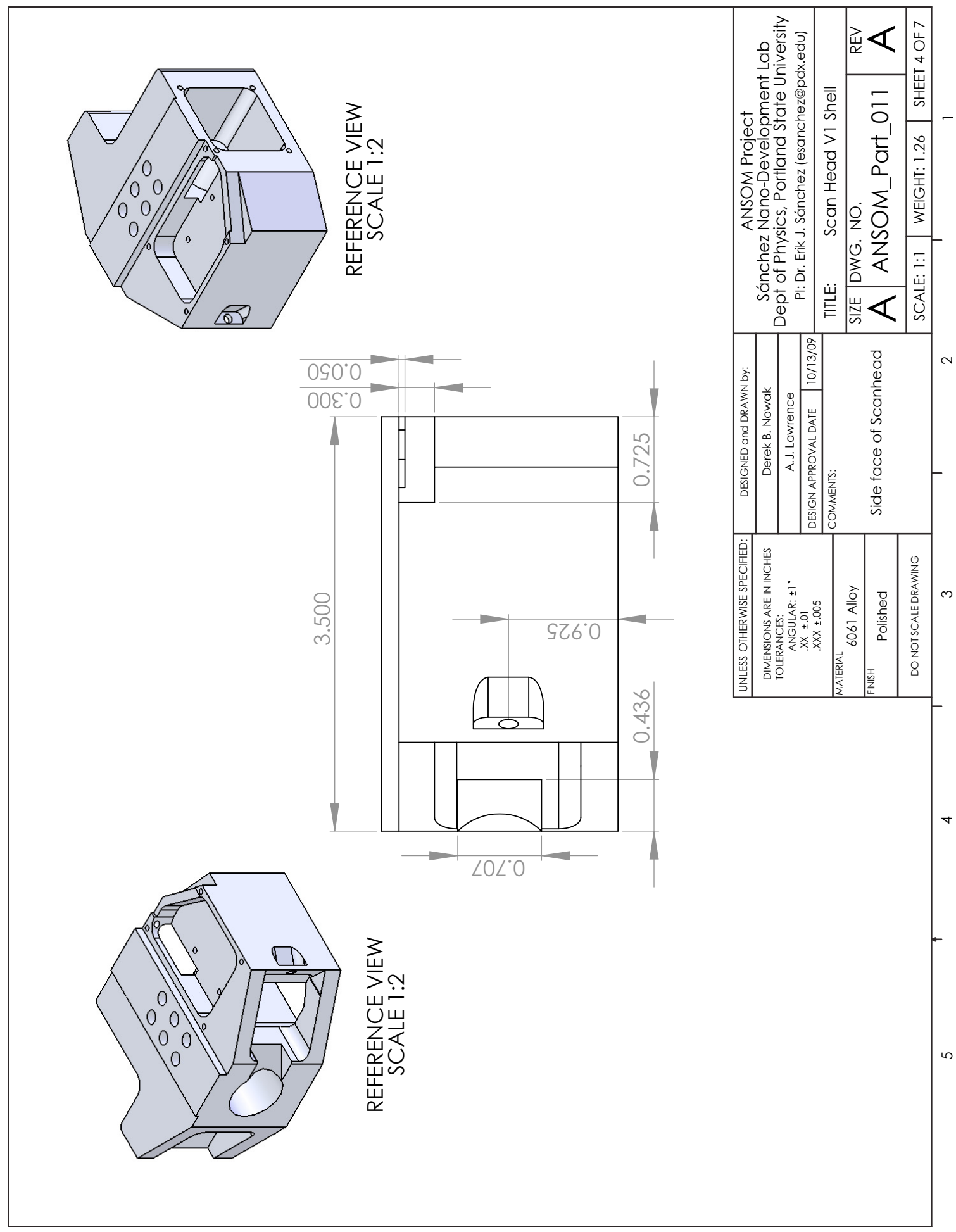




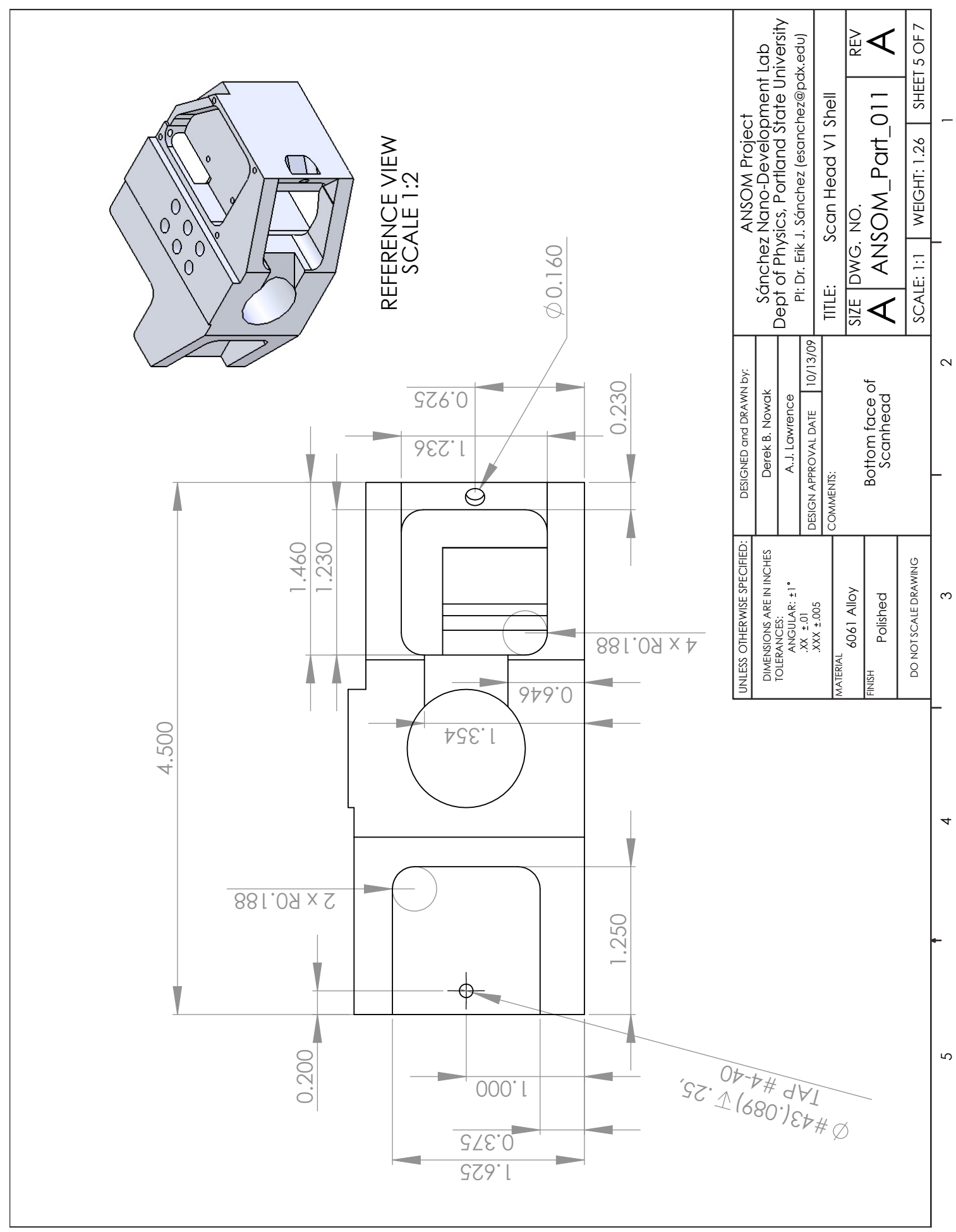




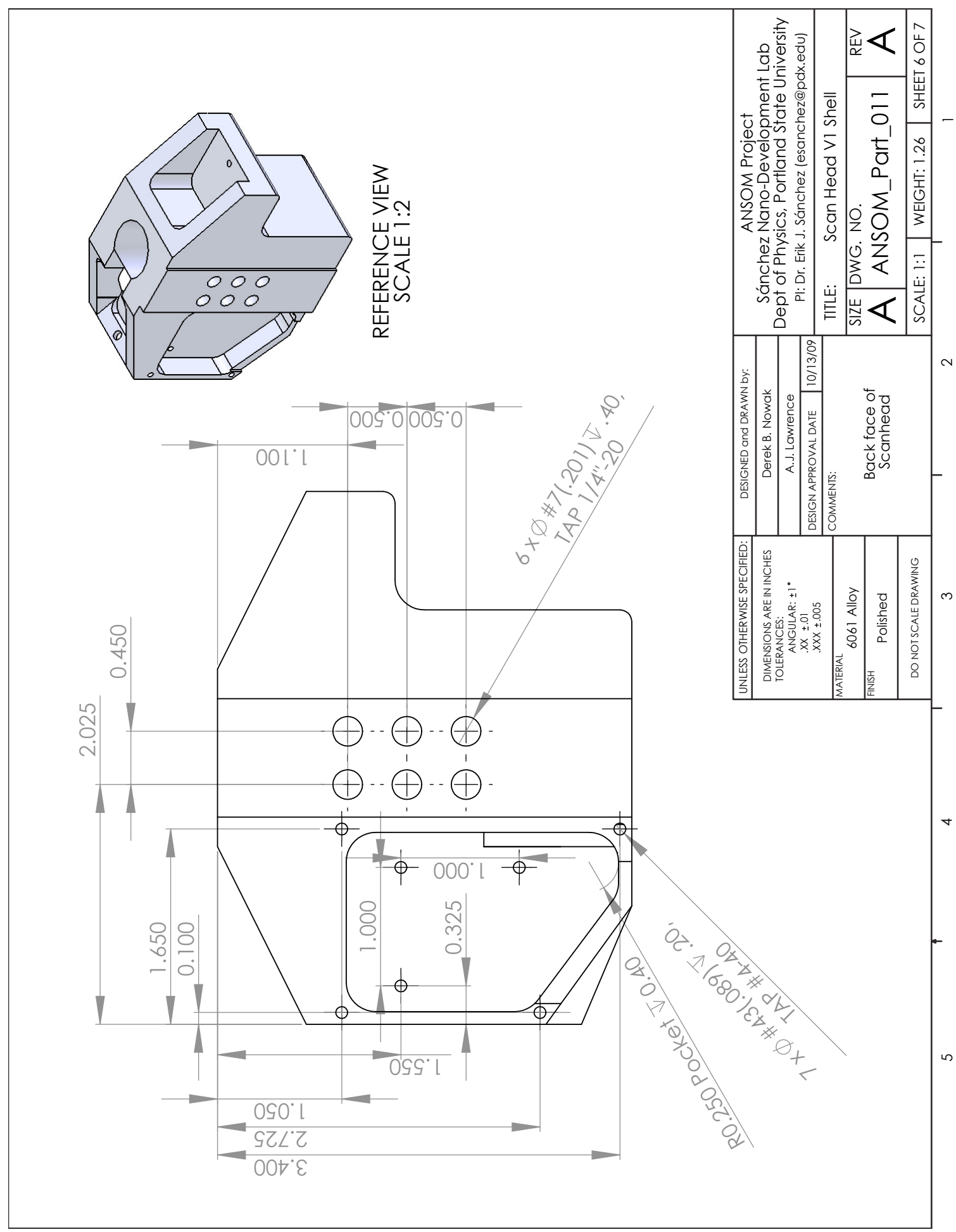




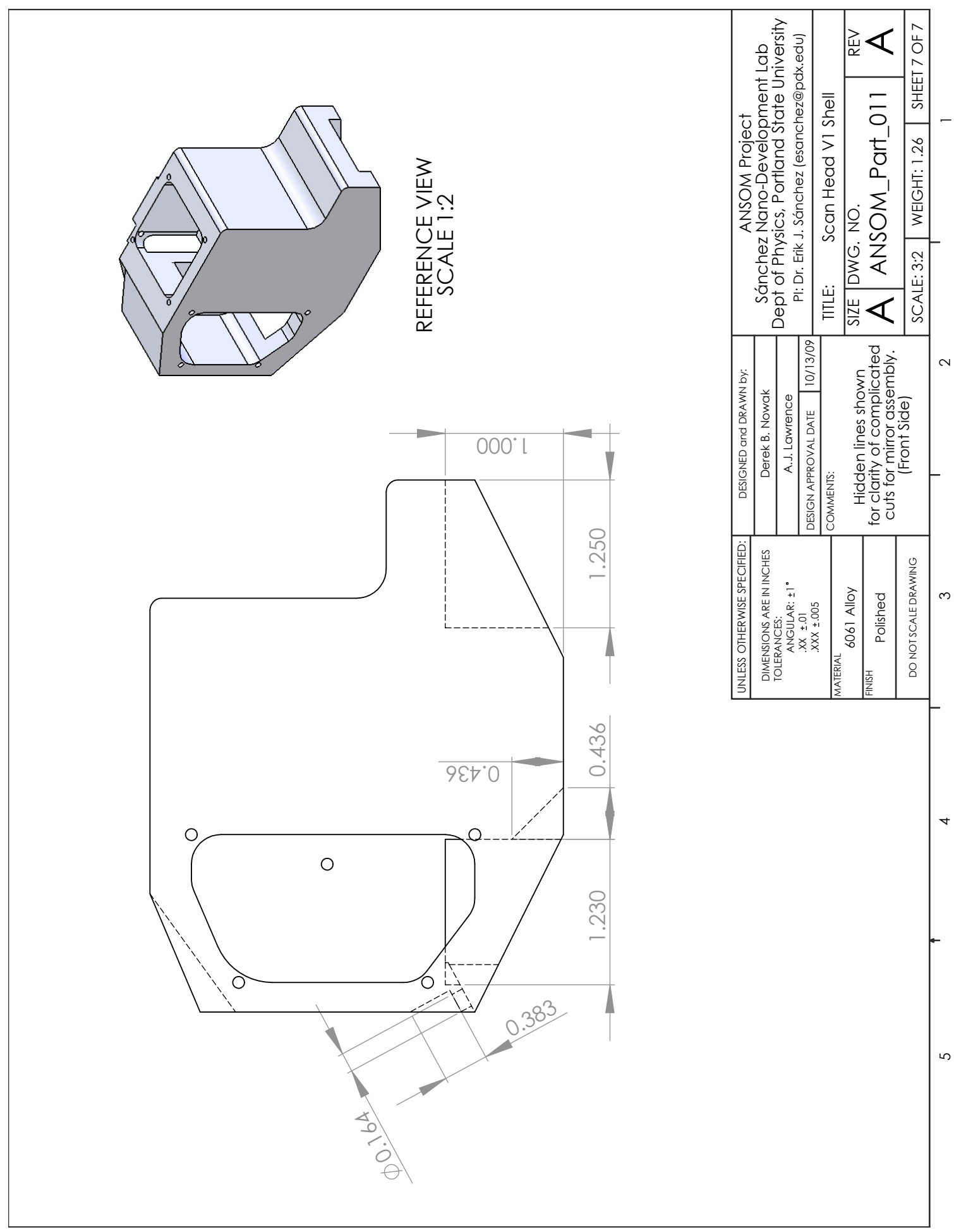




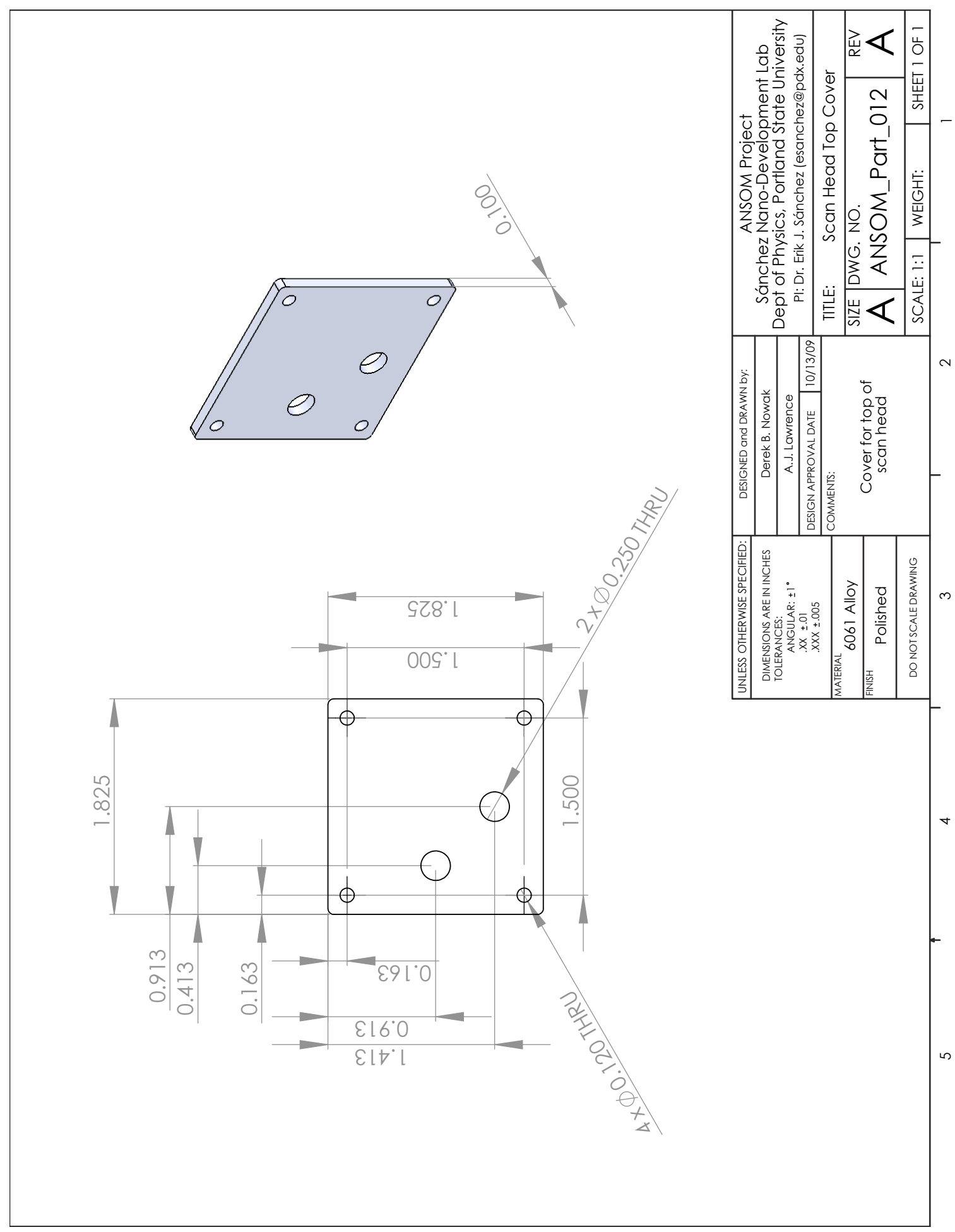




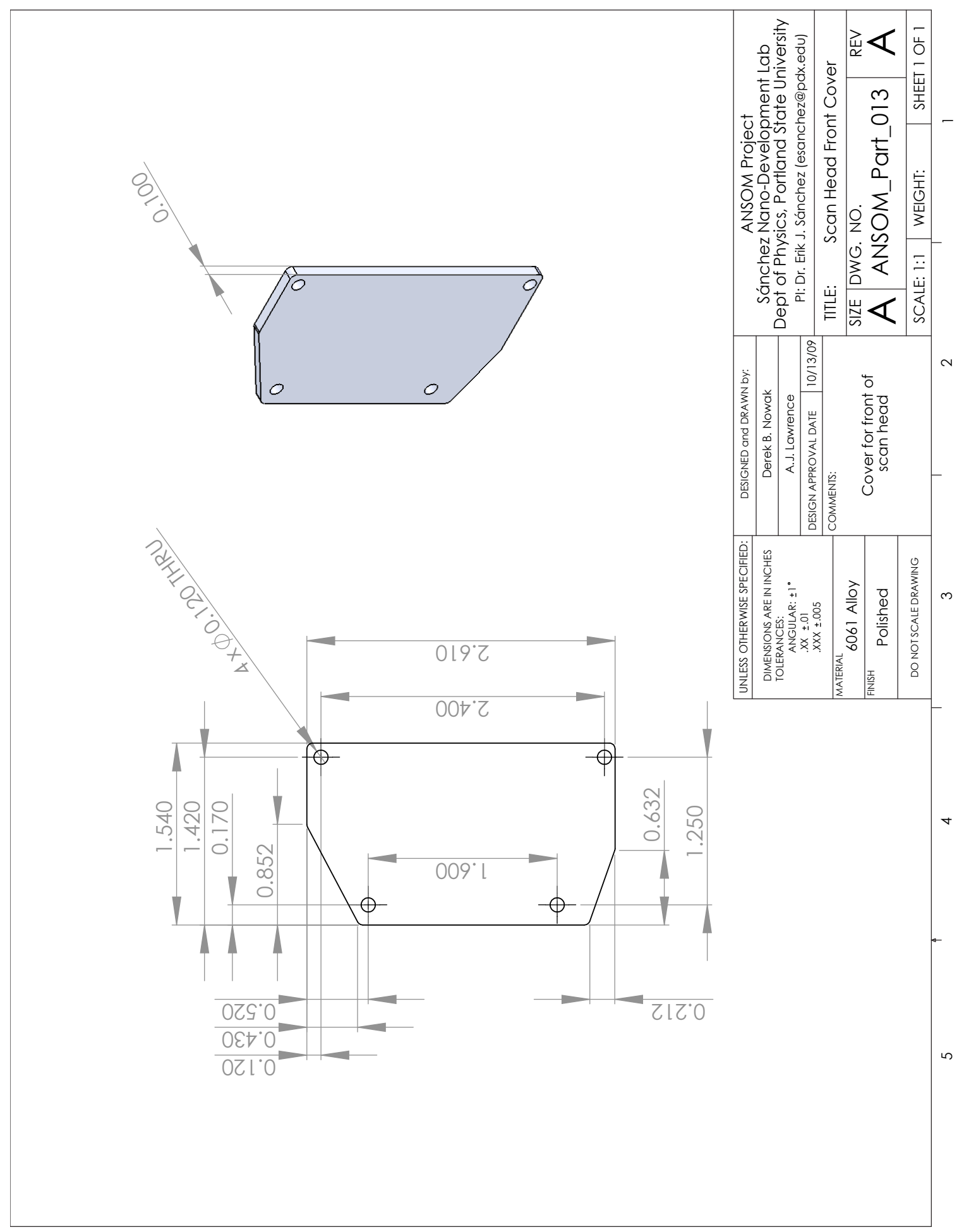




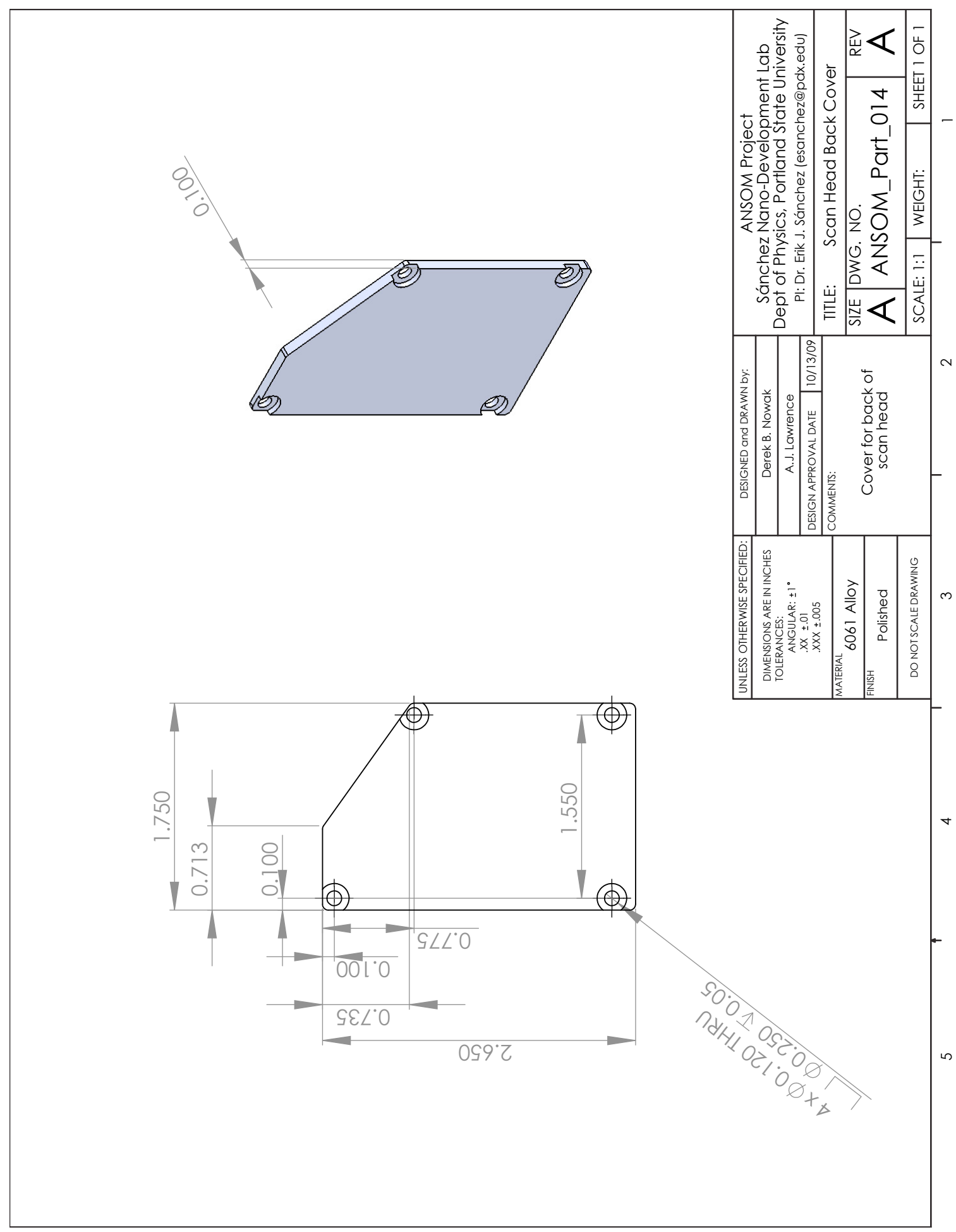




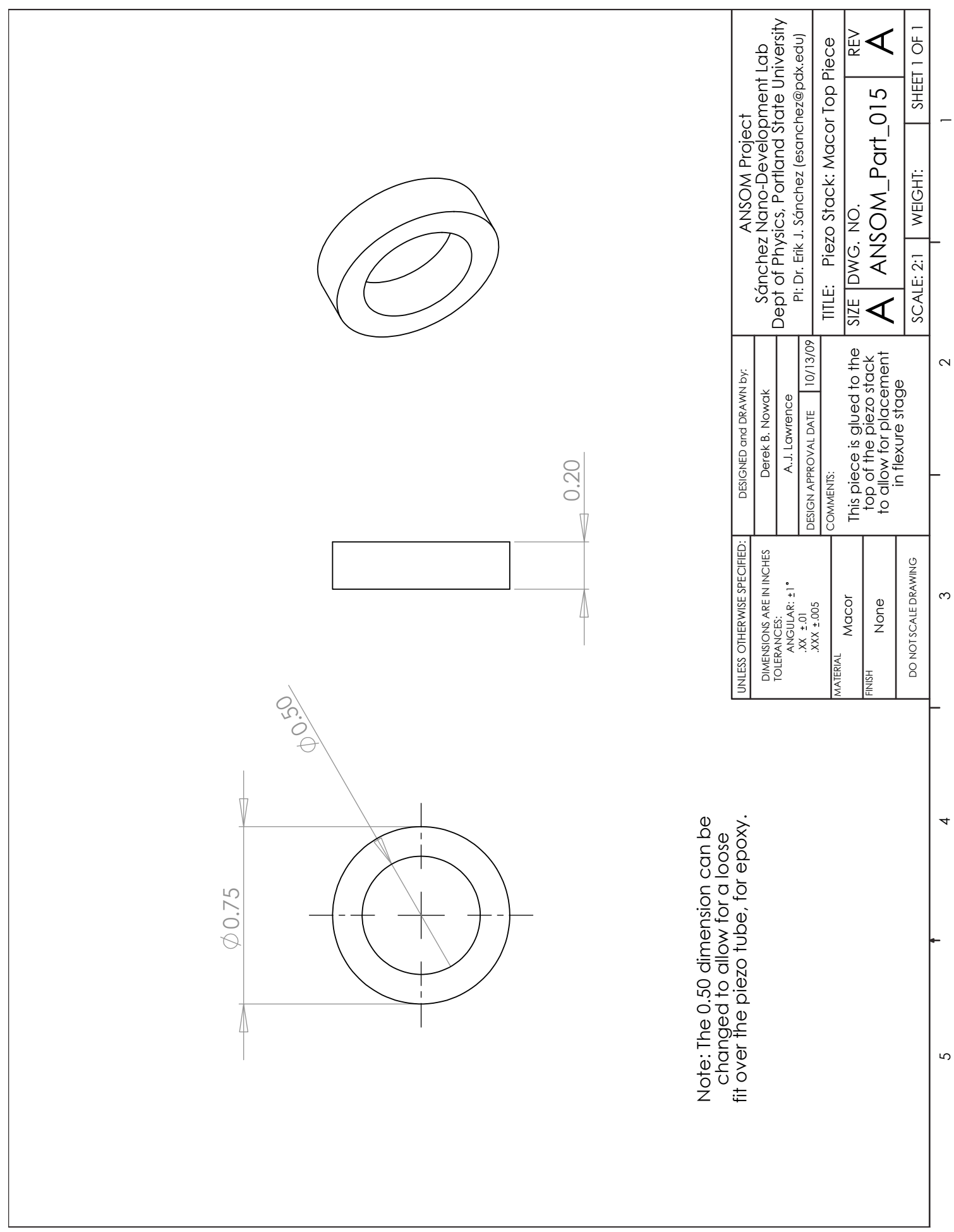




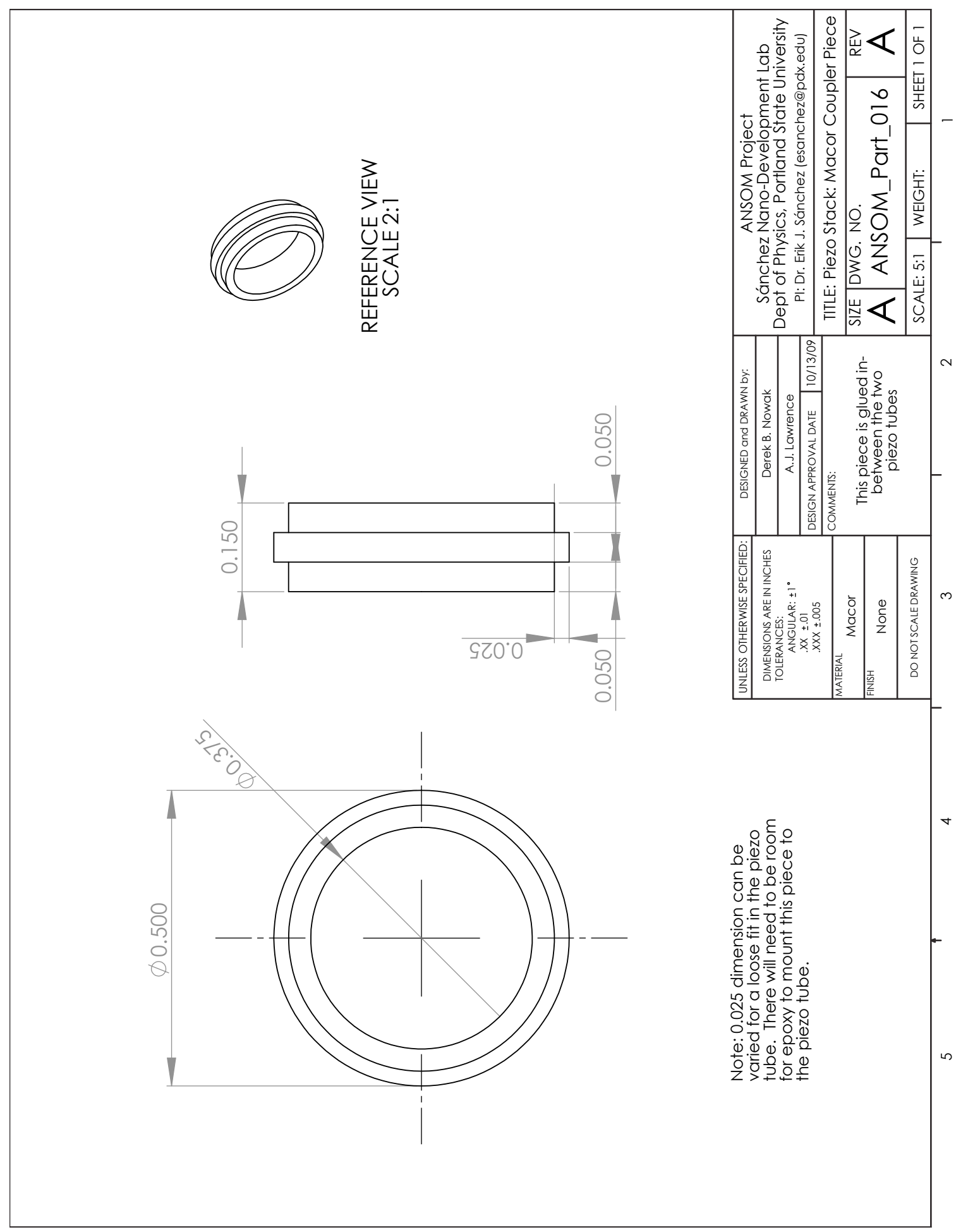




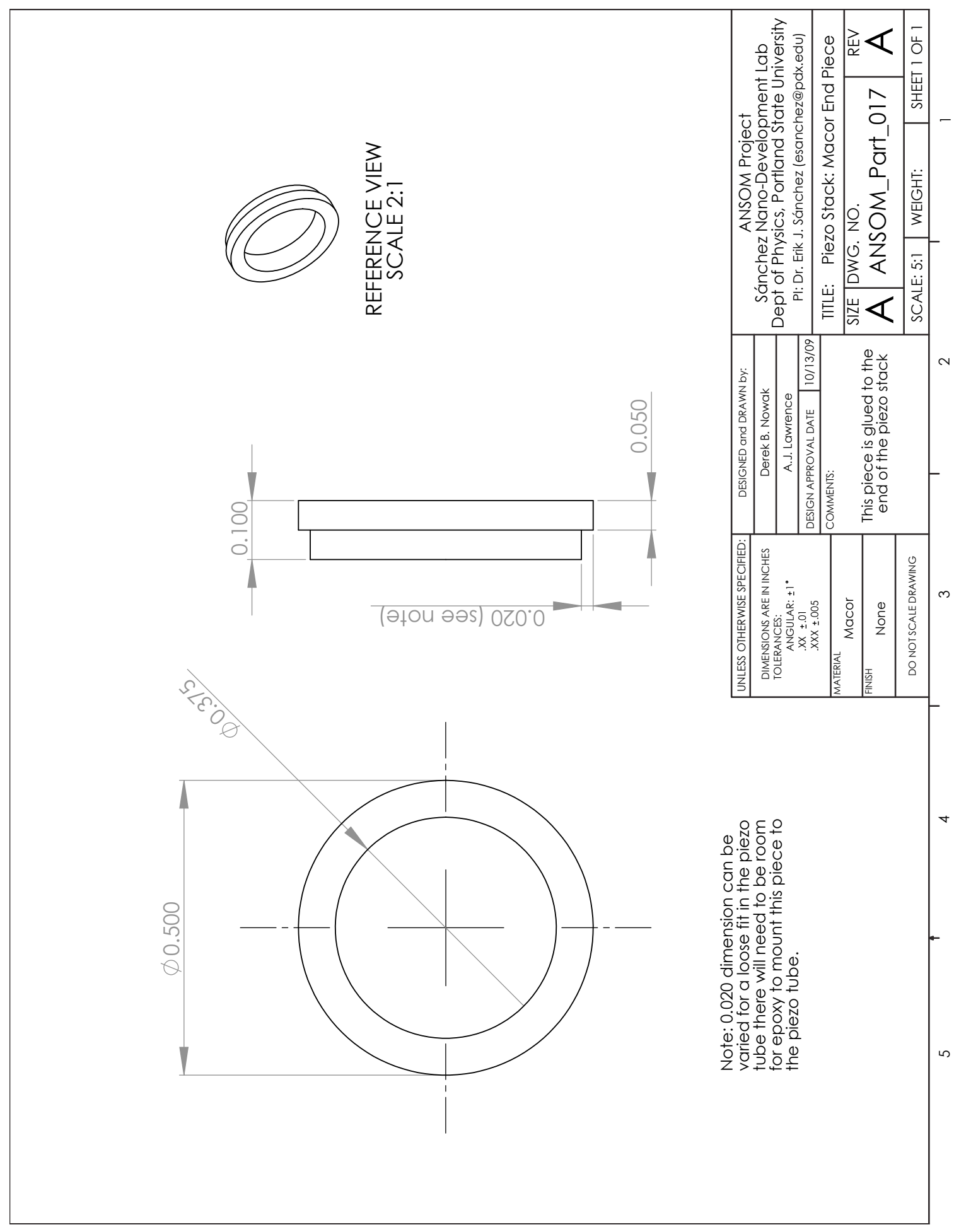




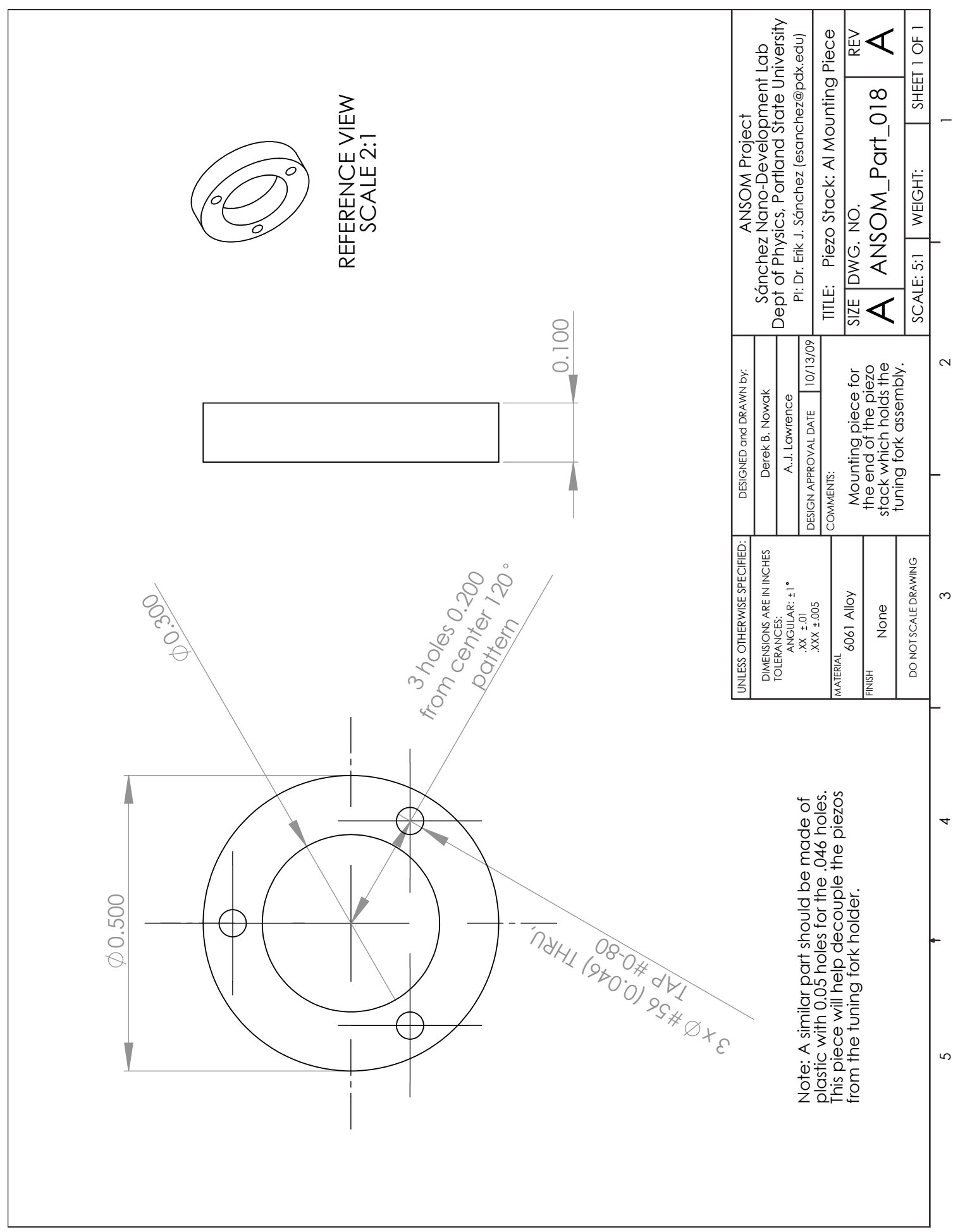




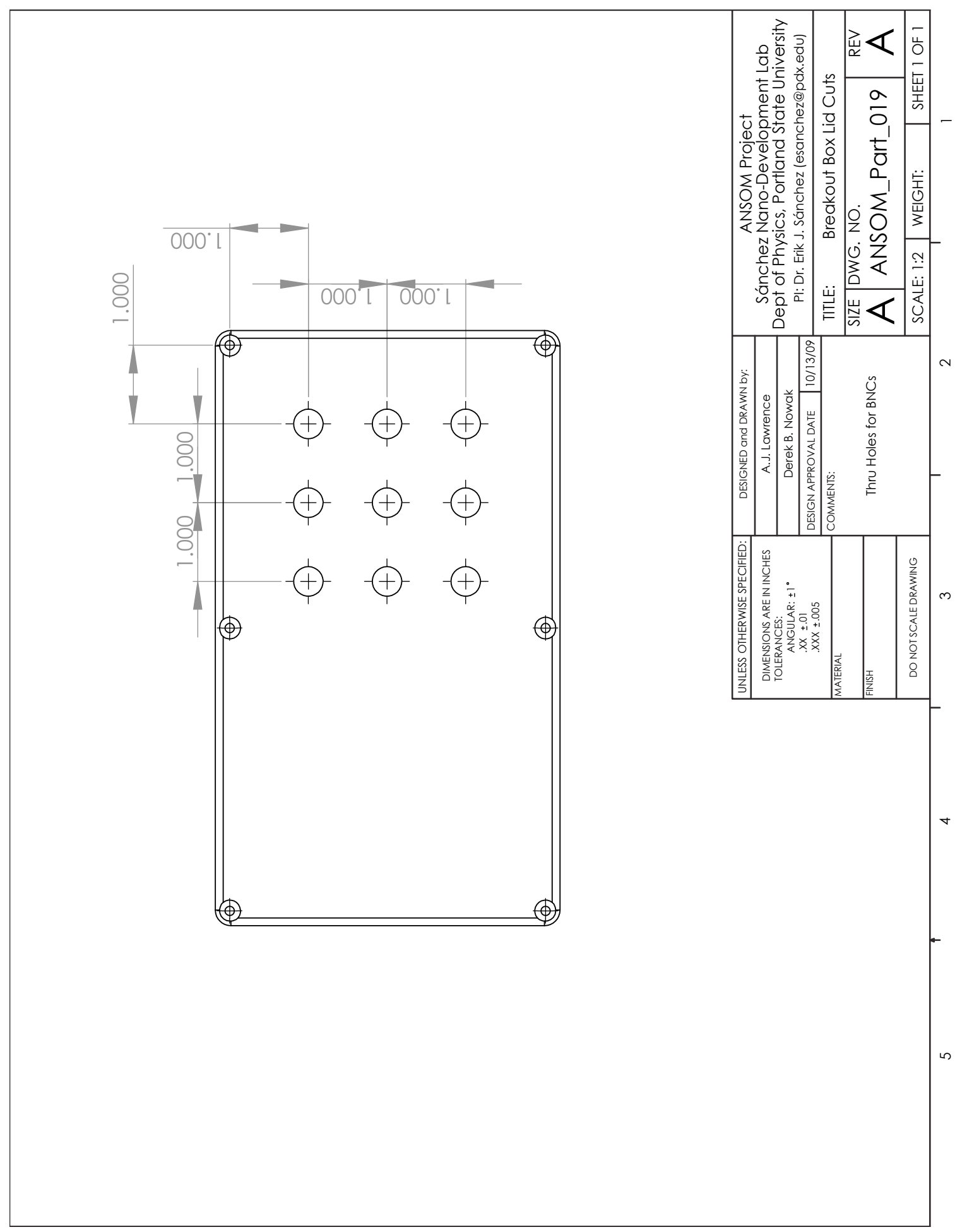




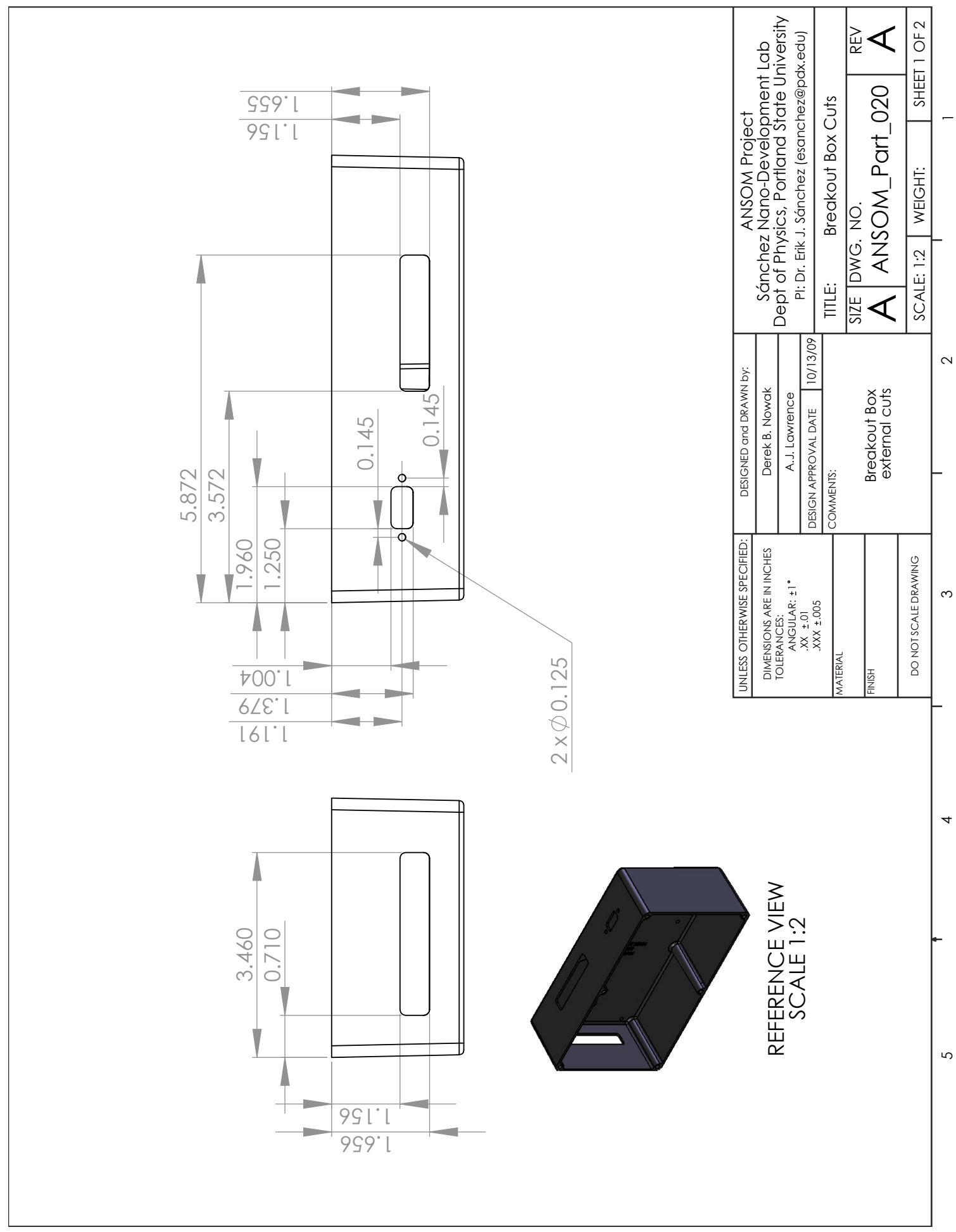




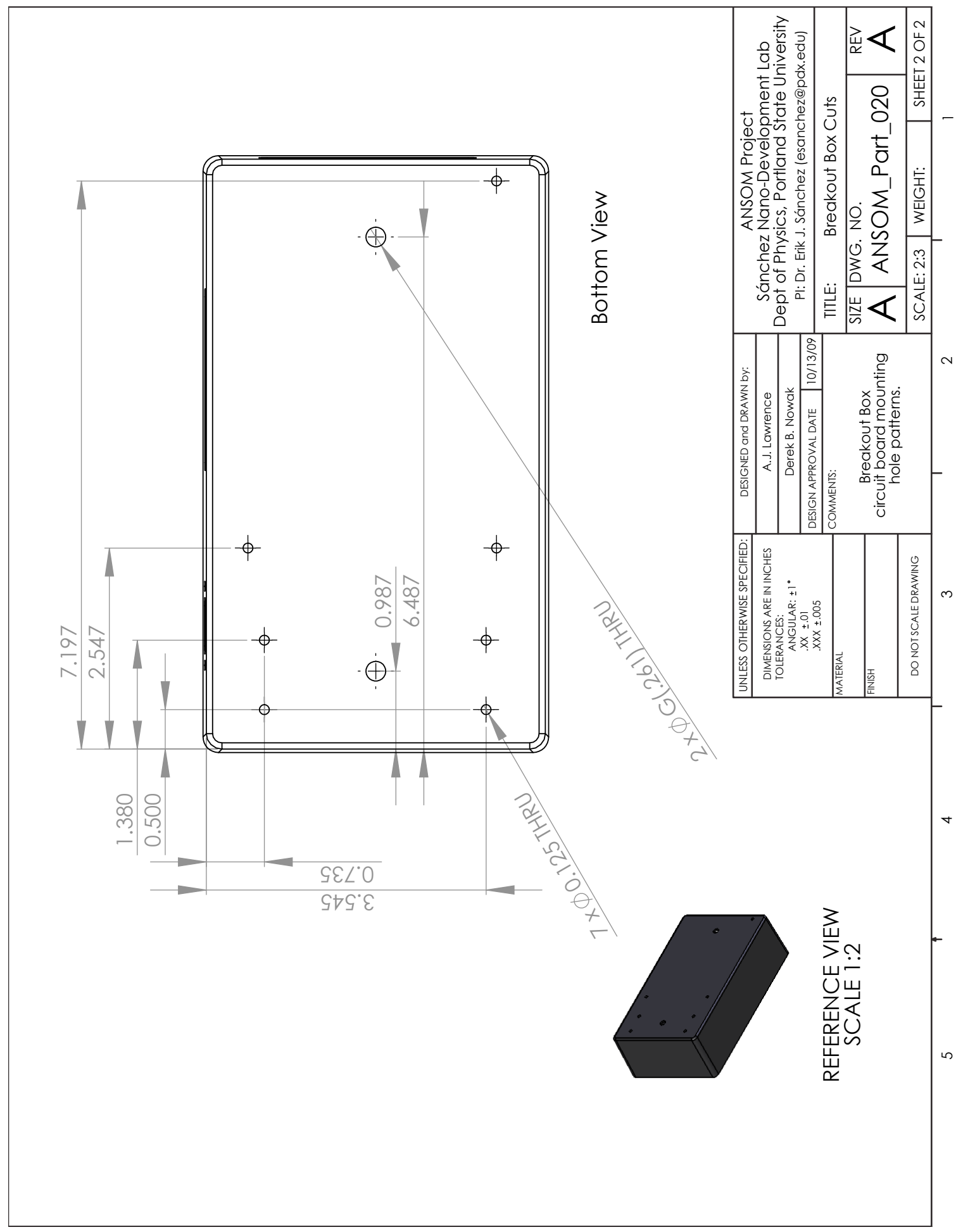




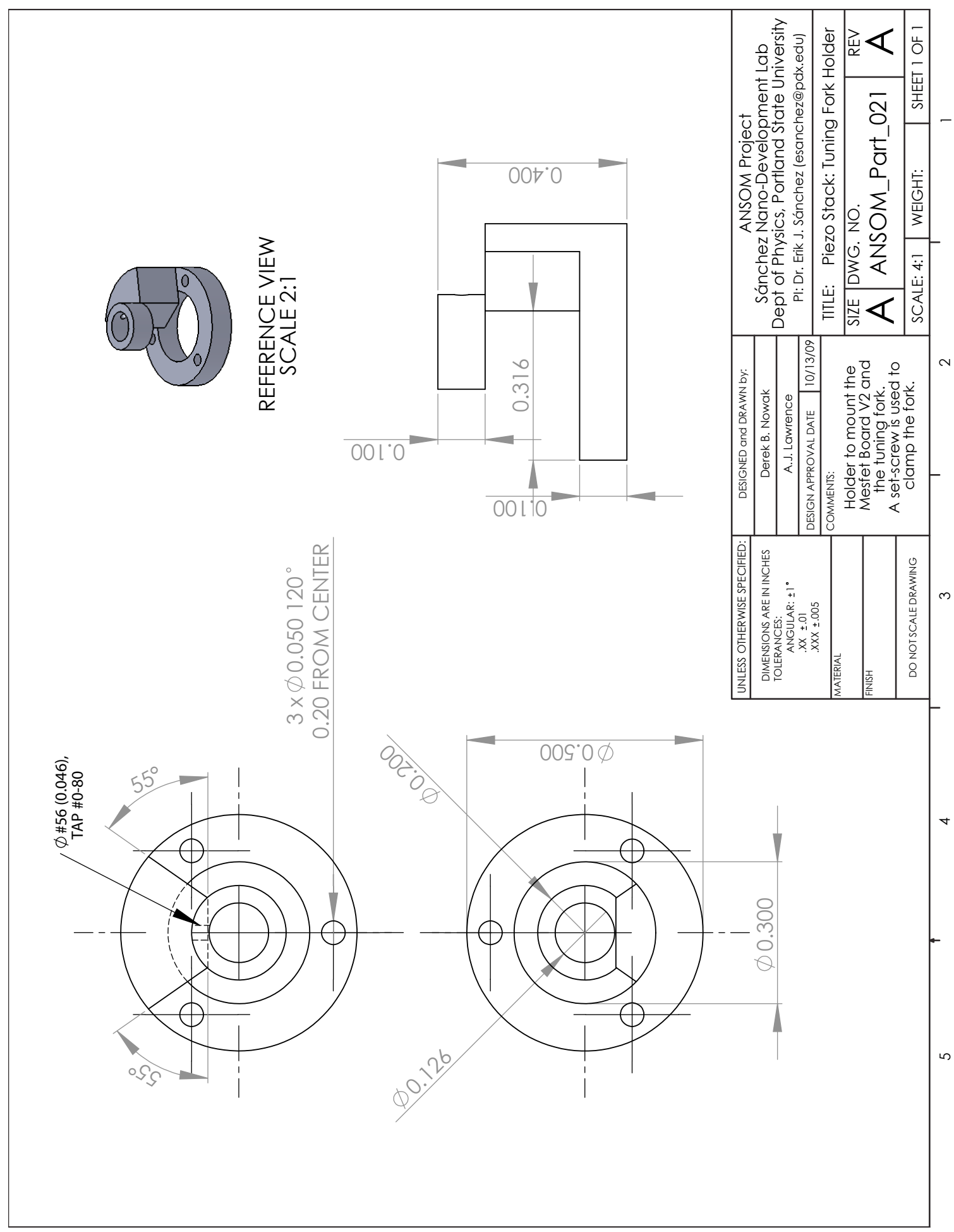




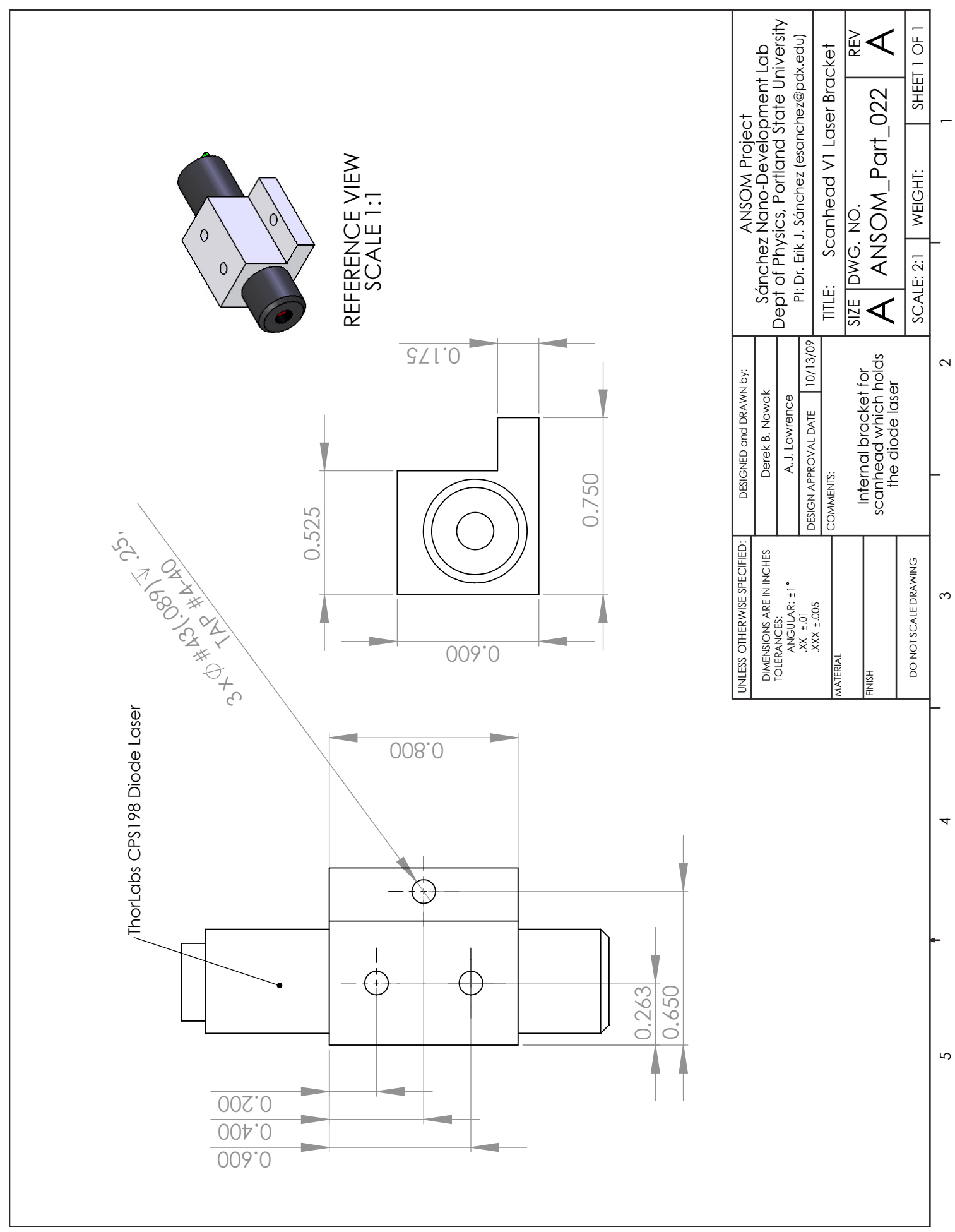




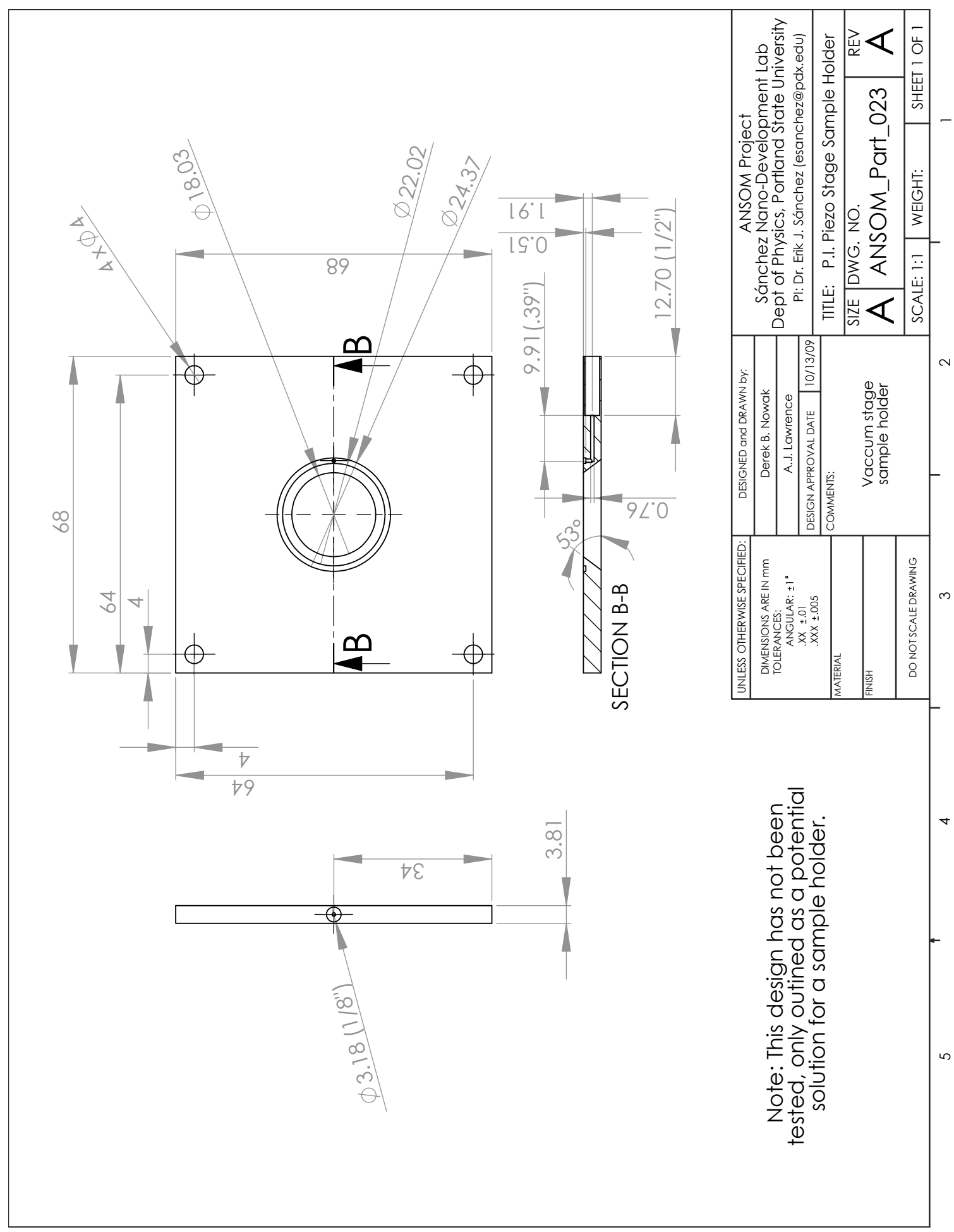




\section{B.2 Bill of Materials}

Table B.2 List of Microscope Mechanical Parts (BOM)

$\begin{array}{lc}\text { BOM List } & \text { Page } \\ \text { Thorlabs Components } & 315 \\ \text { Non Thorlabs Parts } & 316 \\ \text { Fasteners } & 316 \\ \text { Material Costs } & 316\end{array}$

\begin{tabular}{|c|c|c|c|c|}
\hline \multicolumn{5}{|l|}{ Thorlabs Components } \\
\hline Part Number & Description & Quantity & Price (2009) & Total \\
\hline B1C & Blank Cover Plate with Rubber O-Ring for C4W/C6W & 1 & 16 & 16 \\
\hline B5C & Ø1" Cage Cube Optic Mount For B3C & 1 & 31 & 31 \\
\hline C4W & $30 \mathrm{~mm}$ Cage System Cube, 4-Way & 1 & 58 & 58 \\
\hline CM1-BP108 & Cube-Mounted Pellicle Beamsplitter, 8:92, Uncoated & 1 & 215 & 215 \\
\hline KCB1 & Right Angle Kinematic Mirror Mount, 30 mm Cage System, SM1 & 1 & 104 & 104 \\
\hline PF10-03-P01 & Ø1" (Ø25.4mm) Protected Silver Mirror, 0.24" (6.0 mm) Thick & 3 & 50 & 150 \\
\hline ER2 & Cage Assembly Rod - 2", Ø6 mm & 4 & 6 & 24 \\
\hline KM100 & Kinematic Mirror Mount For 1 inch Optics & 2 & 42 & 84 \\
\hline MA45-2 & $45^{\circ}$ Mounting Adapter & 2 & 23 & 46 \\
\hline SM1Z & SM1 Series Z Translator & $\overline{1}$ & 176 & 176 \\
\hline SM1A6 & Adapter with External SM1 Threads and Internal SM05 Threads & 1 & 19 & 19 \\
\hline SM1A3 & Internal RMS to External SM1 Thread Adapter & 1 & 17 & 17 \\
\hline SM1CP2 & End Cap External Threads, SM1 Series & 3 & 19 & 57 \\
\hline MB1218 & Aluminum Breadboard, 12" x 18" x 1/2", 1/4-20 Threaded & 1 & 208 & 208 \\
\hline PB4 & P-Series Pedestal Base Adapter - Imperial & 1 & 13 & 13 \\
\hline PF175 & P-Series Clamping Fork & 1 & 17 & 17 \\
\hline PH1-ST & Post Holder with Spring-Loaded Thumbscrew, L= 1" & 3 & 8 & 24 \\
\hline SM1RC & SM1 Series Slim Lens Tube Slip Ring & 2 & 22 & 44 \\
\hline SM1RR & SM1 Retaining Ring for $\varnothing 1 "$ Lens Tubes and Mounts & 10 & 6 & 60 \\
\hline TR1.5 & $\varnothing 1 / 2 "$ x 1.5" Post & 2 & 5 & 10 \\
\hline TR1 & $\varnothing 1 / 2 " \times 1 "$ Post & 1 & 5 & 5 \\
\hline K6X & 6 Axis Kinematic Optic Mount & 1 & 294 & 294 \\
\hline AFS105/125Y-CUSTOM* & $\begin{array}{l}\text { AFS105/125Y Custom Patch Cord Length: } 1 \mathrm{~m} \text { End A: FC/PC End B: } \\
\text { SMA Jacket: FT030BK inside FT051SS }\end{array}$ & 1 & 80 & 80 \\
\hline & AFS105/125Y Custom Patch Cord & & & \\
\hline AFS105/125Y-CUSTOM* & Length: 1m End A: SMA End B: SMA Jacket: FT030BK inside & 1 & 80 & 80 \\
\hline XT66D3 & X-Axis Dovetail, $50 \mathrm{~mm}$ Length & 1 & 14 & 14 \\
\hline KMSS & Kinematic Mirror Mount, Hex Adjuster & 1 & 32 & 32 \\
\hline LNR50 & $50 \mathrm{~mm}$ TravelMax Stage, Basic, Imperial & 1 & 810 & 810 \\
\hline CPS198 & Focusing laser Diode Module & 1 & 120 & 120 \\
\hline${ }^{*}$ The fiber optic cables $v$ & re custom made to interface & & Total: & 4132 \\
\hline
\end{tabular}




\begin{tabular}{|c|c|c|c|c|}
\hline Part Number & Description & Quantity & Price & Total \\
\hline N/A & Olympus or any Objective lens (Varies greatly) & 1 & Varies & \\
\hline KPC-S500B & CCD Camera KT\&C KPC-S500B & 3 & 47.5 & 142.5 \\
\hline 07 TXS 223 & Translation Stage Melles Griot 07 TXS 223 & 2 & 300 & 600 \\
\hline N/A & Universal Lead Screw and Coupling (see part drawing) & 1 & 850 & 850 \\
\hline PK243B1A-SG36 & Oriental Stepper Motor & 1 & 250 & 250 \\
\hline D4CL-5. OF & Oriental Stepper Motor Damper & 1 & 50 & 50 \\
\hline MC1605F04C & Motor Coupling Unit & 1 & 50 & 50 \\
\hline Boston Piezo & Z Piezo Segmented (see part drawing for more information) & 1 & 200 & 200 \\
\hline Boston Piezo & X-Y Piezo Segmented (see part drawing for more information) & 1 & 200 & 200 \\
\hline \multirow[t]{2}{*}{ MFM-075 } & Newport XY Flexure Stage & 1 & 100 & 100 \\
\hline & & & Total: & 2442.5 \\
\hline
\end{tabular}

\begin{tabular}{|c|c|c|c|c|c|c|c|}
\hline \multicolumn{8}{|l|}{ Fasteners } \\
\hline Part Number & Description & Application: & Thread & Length & Quantity & Price & Total \\
\hline 91746 A226 & Knurled Head Thumb & For Dovetail Rails & $8-32$ & $.75^{\prime \prime}$ & 1 & 2.99 & 2.99 \\
\hline 91251A183 & Standard Head Socket Cap & Leadscrew to Adapter Plate & $8-32$ & $1.125 "$ & 2 & 0.36 & 0.72 \\
\hline 91251A188 & Standard Head Socket Cap & Leadscrew to Bracket & $8-32$ & 1.75" & 4 & 0.64 & 2.56 \\
\hline 91251A112 & Standard Head Socket Cap & Cage Assembly Rods to Baseplate & $4-40$ & $.625 "$ & 4 & 0.13 & 0.52 \\
\hline $91251 \mathrm{~A} 113$ & Standard Head Socket Cap & Motor to Motor Bracket & $4-40$ & $.75^{\prime \prime}$ & 4 & 0.13 & 0.52 \\
\hline \multirow[t]{2}{*}{ 91251A551 } & Standard Head Socket Cap & Motor Bracket to Backplane & $1 / 4 "-20$ & 2.25 & 2 & 0.29 & 0.58 \\
\hline & & $\begin{array}{l}\text { Forks to Breadboard (4), Dovetail } \\
\text { Brackets to LNR50 (6), Dovetail }\end{array}$ & & & & & \\
\hline 92220A182 & Low Head Socket Cap & Stopper (1) & $1 / 4 "-20$ & .375" & 11 & 0.18 & 1.98 \\
\hline 91251A537 & Standard Head Socket Cap & Rear Bracket to Backplane & $1 / 4 "-20$ & $.5 "$ & 2 & 0.12 & 0.24 \\
\hline \multirow[t]{2}{*}{ 91251A540 } & Standard Head Socket Cap & $\begin{array}{l}\text { Bracket to Baseplate (3), LNR50 to } \\
\text { Backplane (2) }\end{array}$ & $1 / 4 "-20$ & .75" & 5 & 0.13 & 0.65 \\
\hline & & $\begin{array}{l}\text { Baseplate to Posts (4), Front } \\
\text { Bracket to Backplane (1), }\end{array}$ & & & & & \\
\hline 91251A542 & Standard Head Socket Cap & Backplane to LNR50 (3) & $1 / 4 "-20$ & 1" & 8 & 0.15 & 1.2 \\
\hline 91251A544 & Standard Head Socket Cap & Rear Bracket to Backplane & $1 / 4 "-20$ & 1.25" & 2 & 0.18 & 0.36 \\
\hline 91290A144 & Standard Head Socket Cap & Adapter Plate to LNR50 & M4 & $10 \mathrm{~mm}$ & 3 & 0.05 & 0.15 \\
\hline SH25LP38 & Channel Screws Low Profile & Dovetail Rails to translation stage & $1 / 4 "-20$ & $.25 "$ & 1 & 22 & 22 \\
\hline${ }^{*}$ All parts from & ИcMaster Carr except SH25LF & $h$ is from Thorlabs & & & & Total: & 31.48 \\
\hline
\end{tabular}

\begin{tabular}{|c|c|c|c|c|}
\hline \multicolumn{5}{|c|}{ Material Costs } \\
\hline Part Number (McMaster) & Description & Quantity & Price & Total \\
\hline 8928K961 & Cast Iron 1" x 12" x 8" (Baseplate) & 1 & 106 & 106 \\
\hline $8928 K 63$ & Cast Iron 3/4" x 6" x 24" (Backplane, Dovetail rails, Motor bracket) & 1 & 149 & 149 \\
\hline $8975 K 566$ & Aluminum 3' x 4" x 6" (Scanhead, adapter plates) & 1 & 50 & 50 \\
\hline 8489K419 & Macor Rod 3/4" diameter 4" length (piezo stack components) & 1 & 50 & 50 \\
\hline $9062 K 311$ & $1 / 2 "$ x 5" Aluminum Rod & 1 & 10 & 10 \\
\hline $9785 \mathrm{~K} 18$ & Stainless Steel sheet polished & 1 & 87 & 87 \\
\hline 8975 K322 & Aluminum 1' x 4" x 6" (possible scanstage sub*) & 1 & 31 & 31 \\
\hline \multicolumn{2}{|c|}{${ }^{*}$ Machined to mimic the height of the PI scanbed, if the scanbed is not included in the design. } & \multicolumn{2}{|c|}{ Total: } & 377 \\
\hline
\end{tabular}




\section{APPENDIX C}

\section{Microscope Electrical}

\section{C.1 Circuit Schematics}

Table C.1 List of Electrical Drawings

Drawing Number

ANSOM Electrical-001

ANSOM Electrical-002

ANSOM Electrical-003

ANSOM Electrical-004

ANSOM Electrical-005

ANSOM Electrical-006

ANSOM Electrical-007

ANSOM Electrical-008

ANSOM Electrical-009

ANSOM Electrical-010

ANSOM Electrical-011

ANSOM Electrical-012

ANSOM Electrical-013

ANSOM Electrical-014

ANSOM Electrical-015

\section{Description}

Controller Board V2 (1 of 4)

Controller Board V2 (2 of 4)

Controller Board V2 (3 of 4)

Controller Board V2 (4 of 4)

Piezo Driver PA88 Board V2

Breakout Board V2

Scan Head Board V2

MESFET Board V2

Phase Detection Board V2

Stepper Motor Board V2

Controller Chassis Wiring Diagram

DB37 Cable Assembly

DB25 Cable Assembly

Piezo Wiring Diagram

DB9 Cable for Stepper Motor
Page

318

319

320

321

322

323

324

325

326

327

328

329

330

331

332

\section{C.2 Bill of Materials}

Table C.2 List of Electrical Circuit Parts (BOM)

\section{BOM List}

Controller Board V2

Piezo Driver PA88 V2

Breakout Board V2

Scan Head Board V2

MESFET Board V2

Phase Detection Board V2 336

Stepper Motor V2 336

Computer / Cables and Electronics Enclosure / Power supplies 337

(Project Controller) 


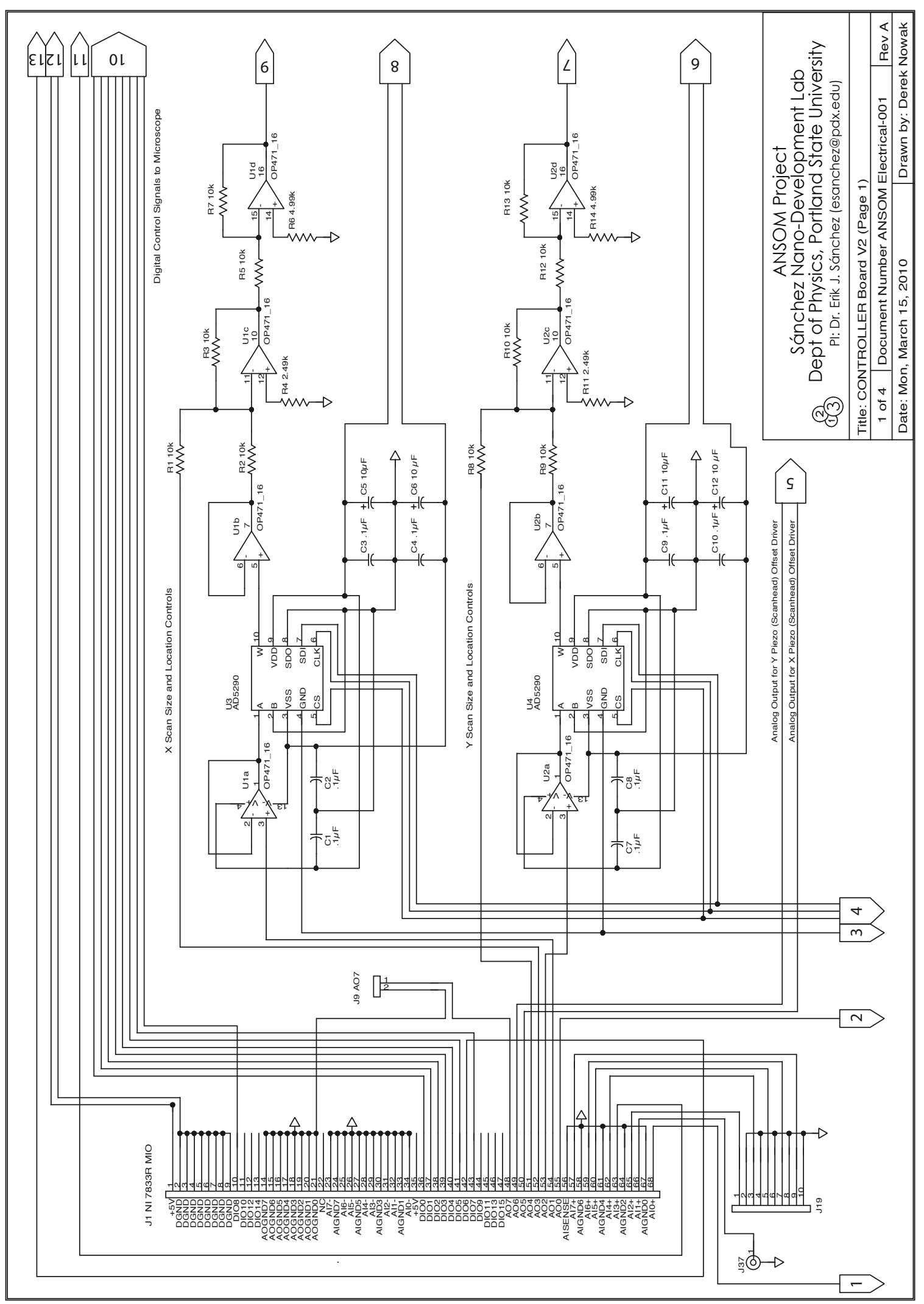




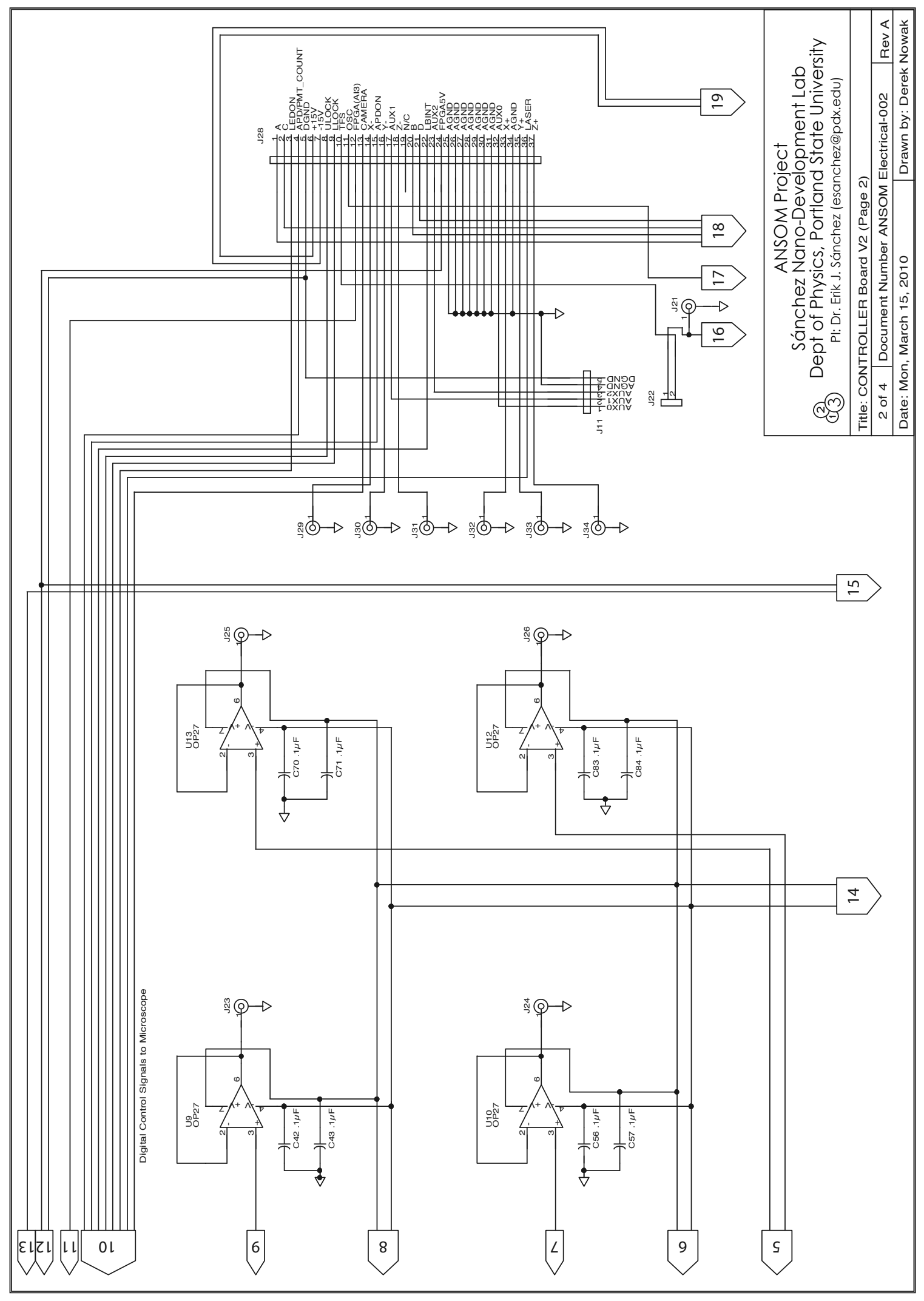




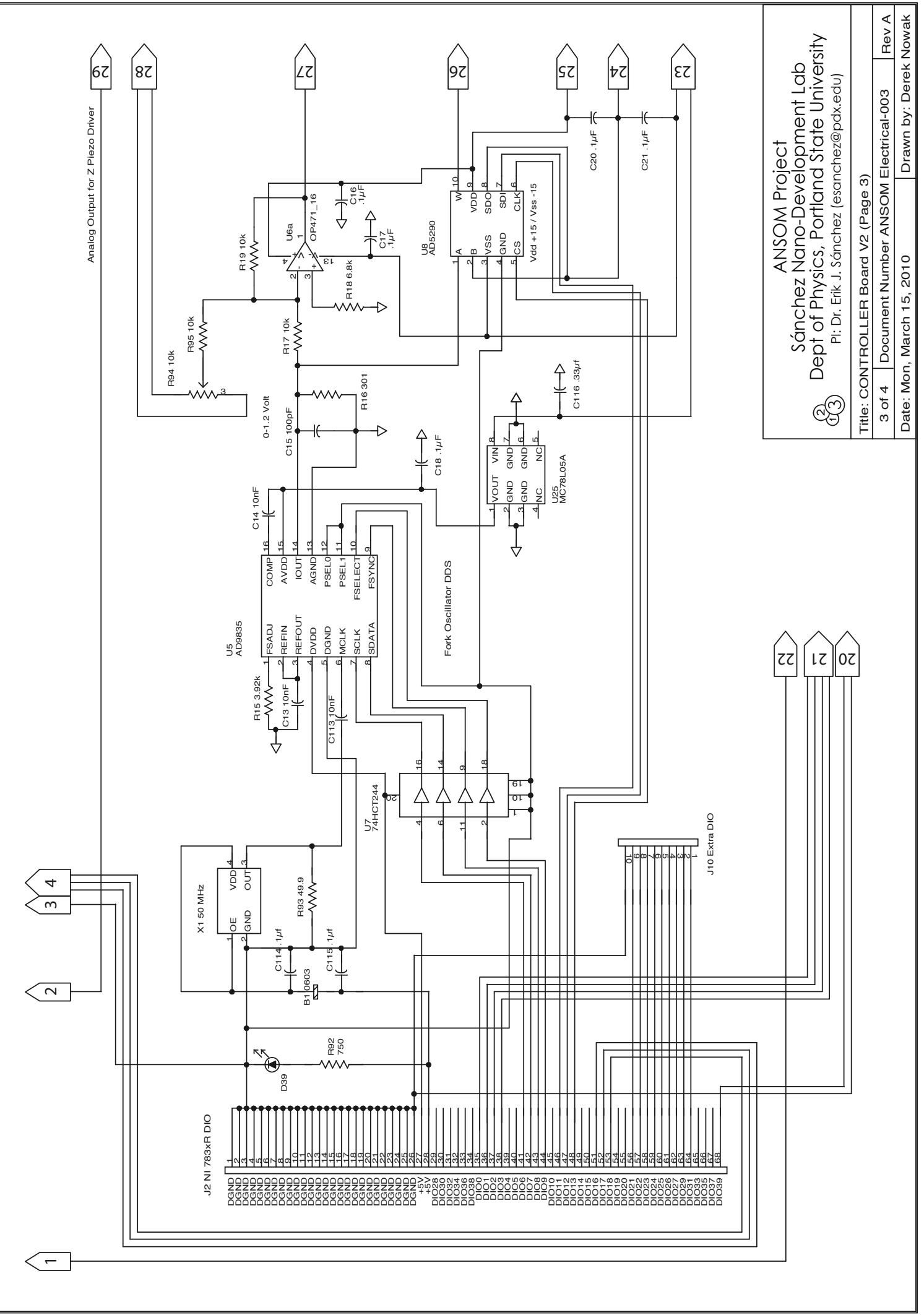




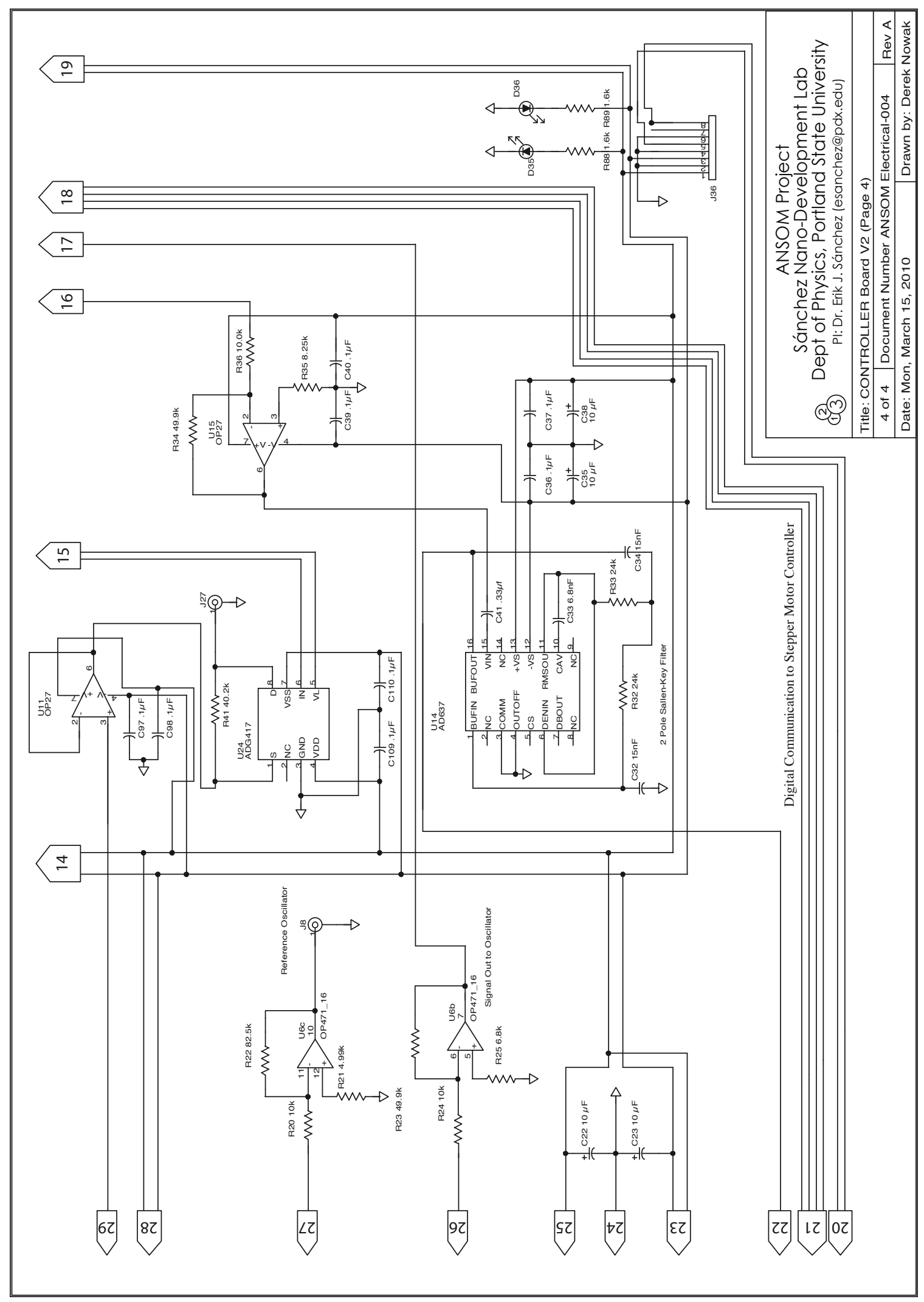




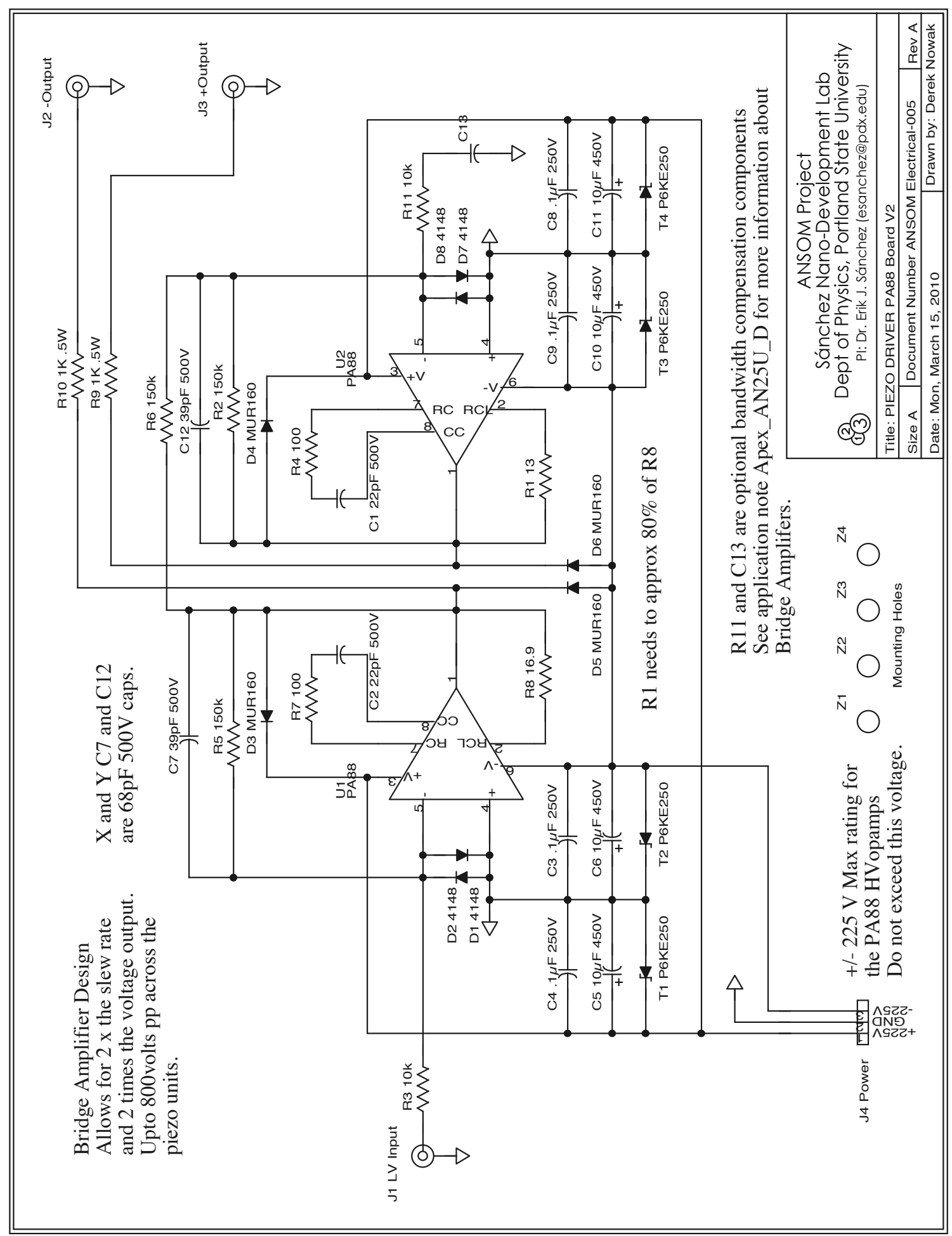




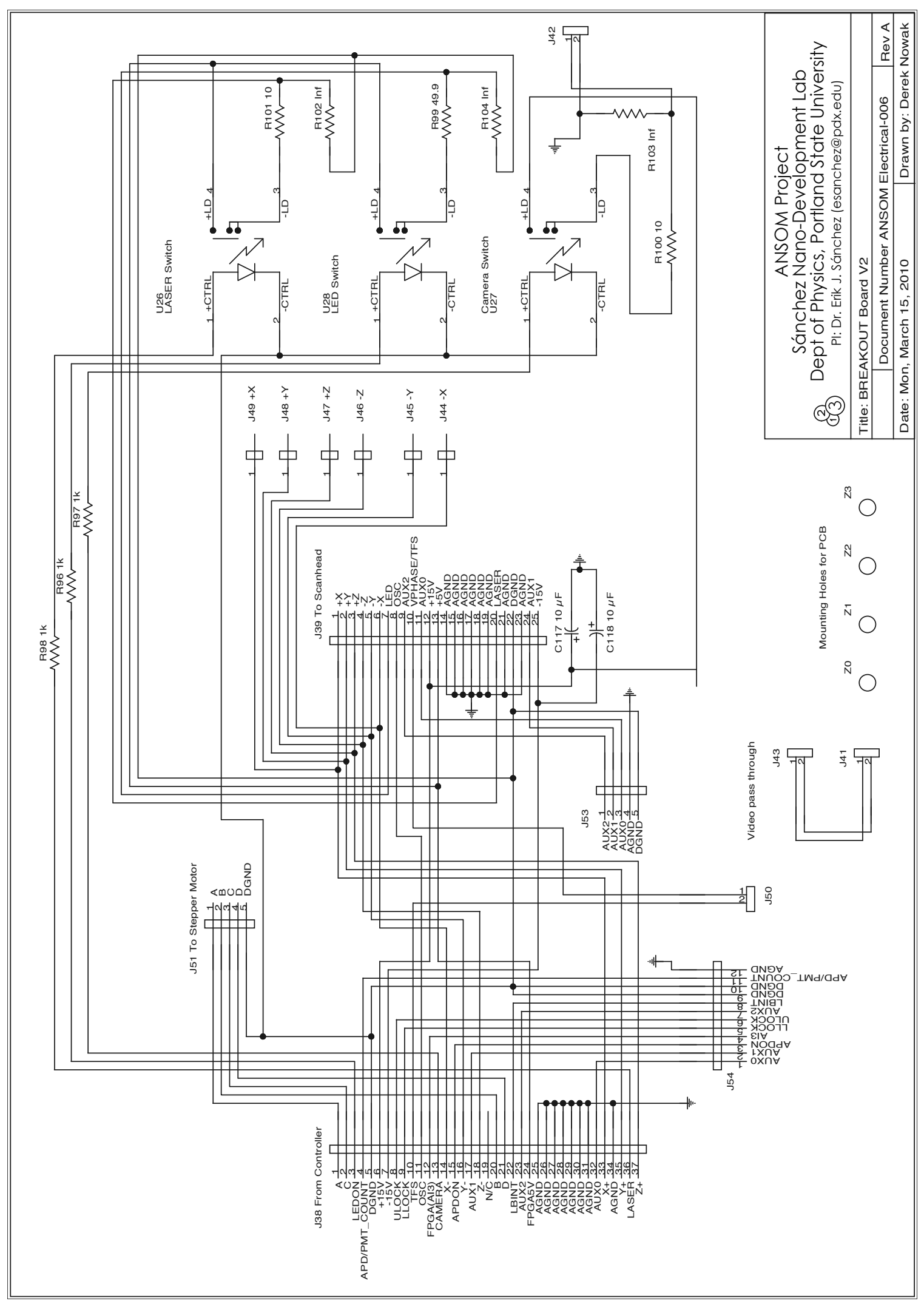




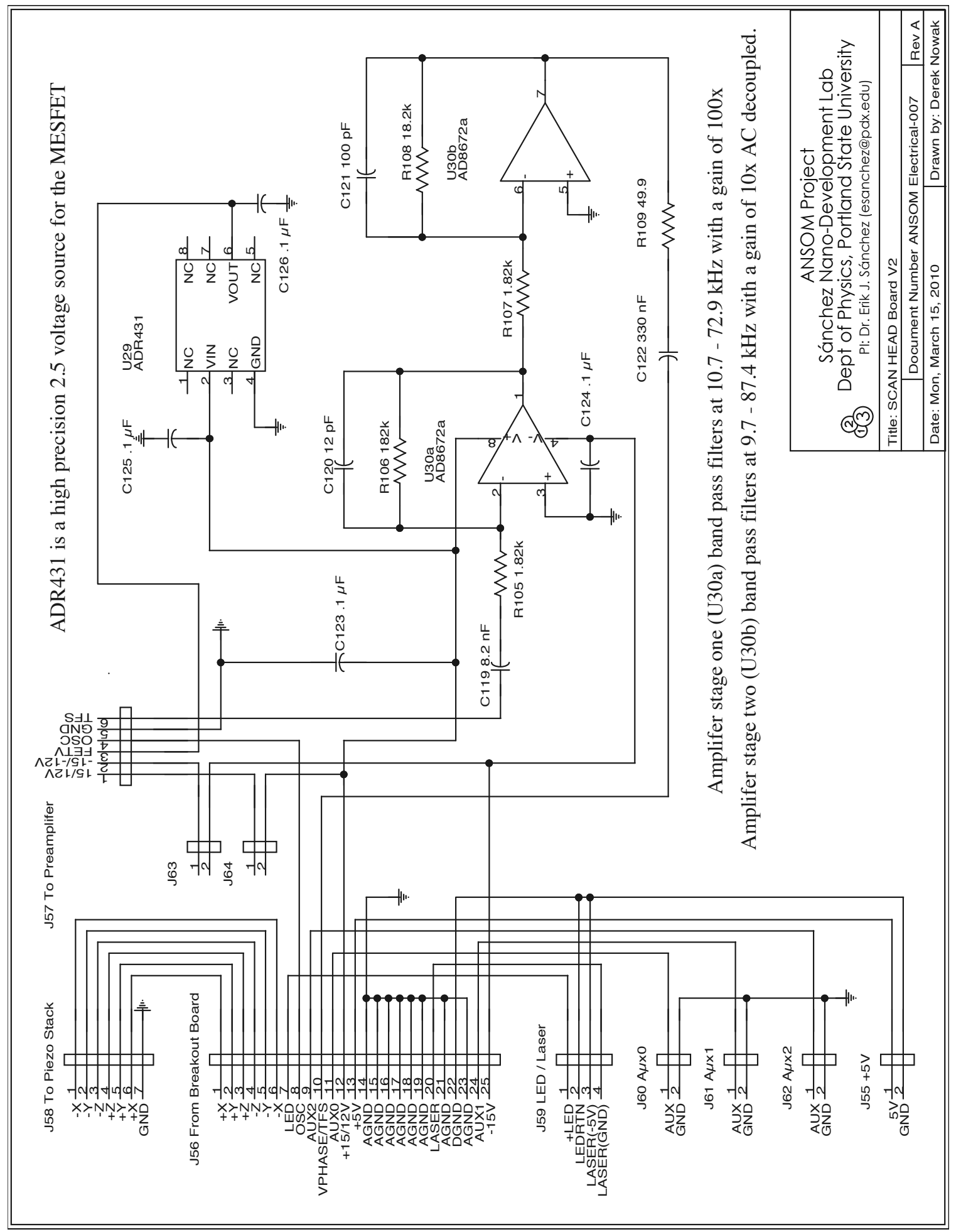




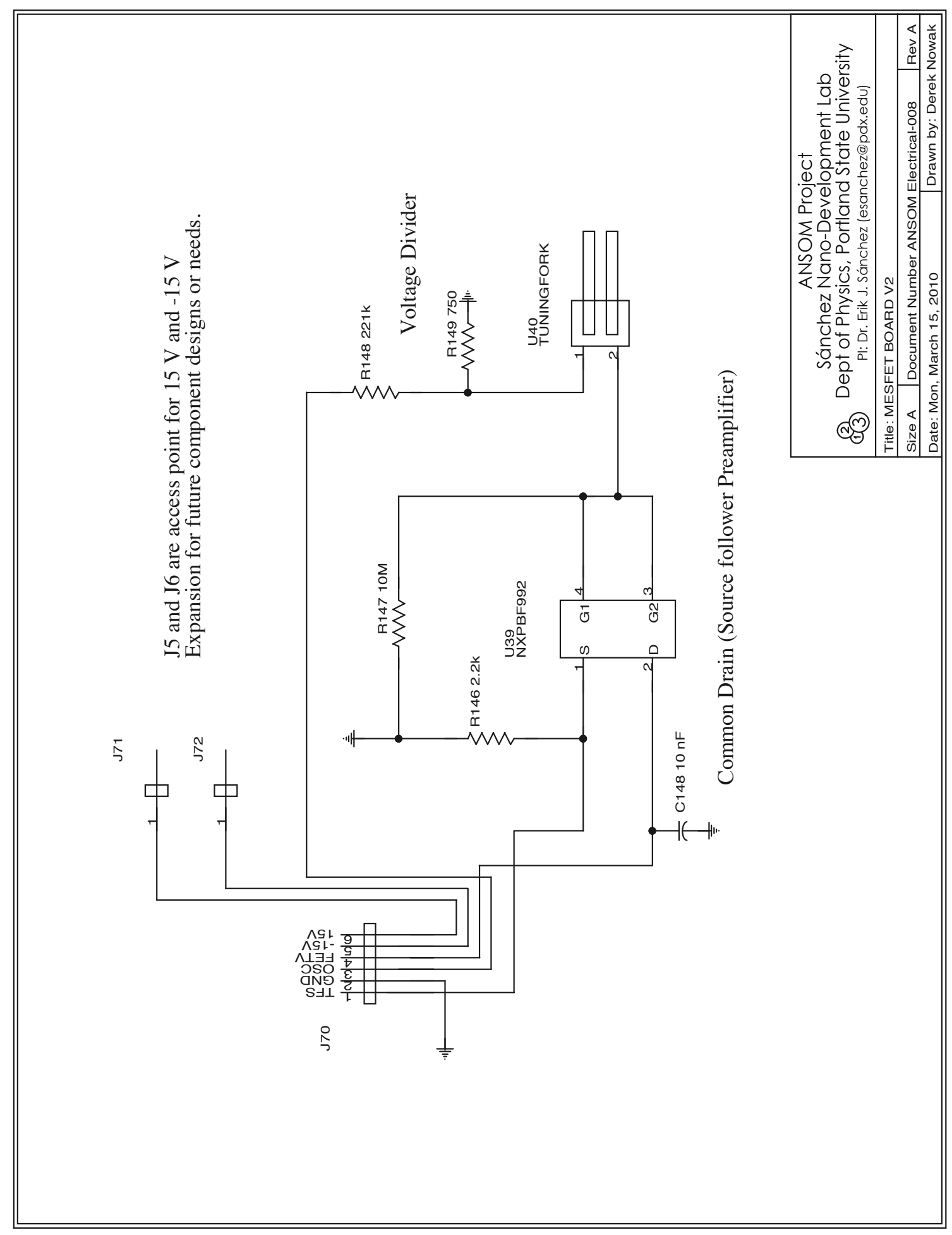




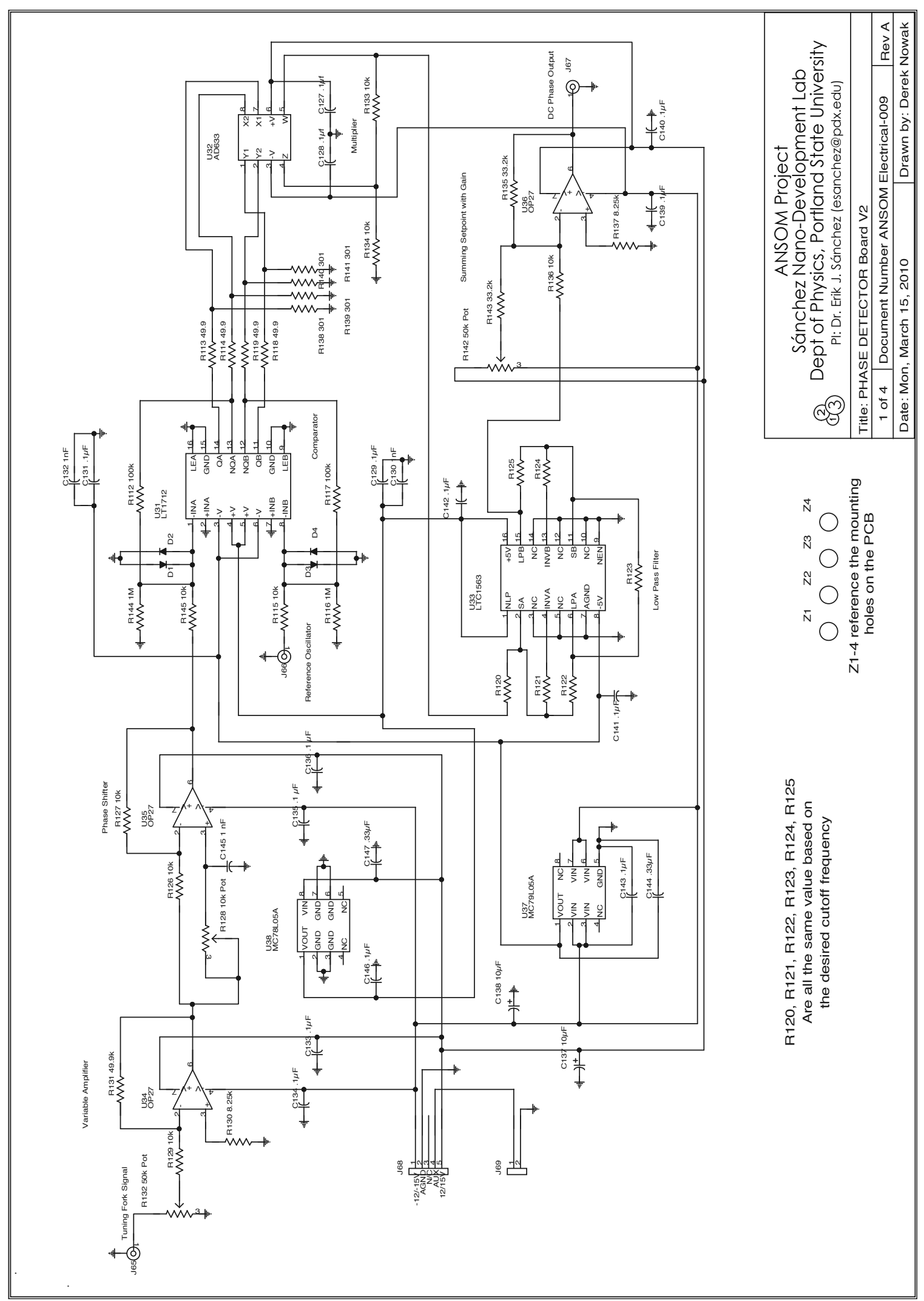




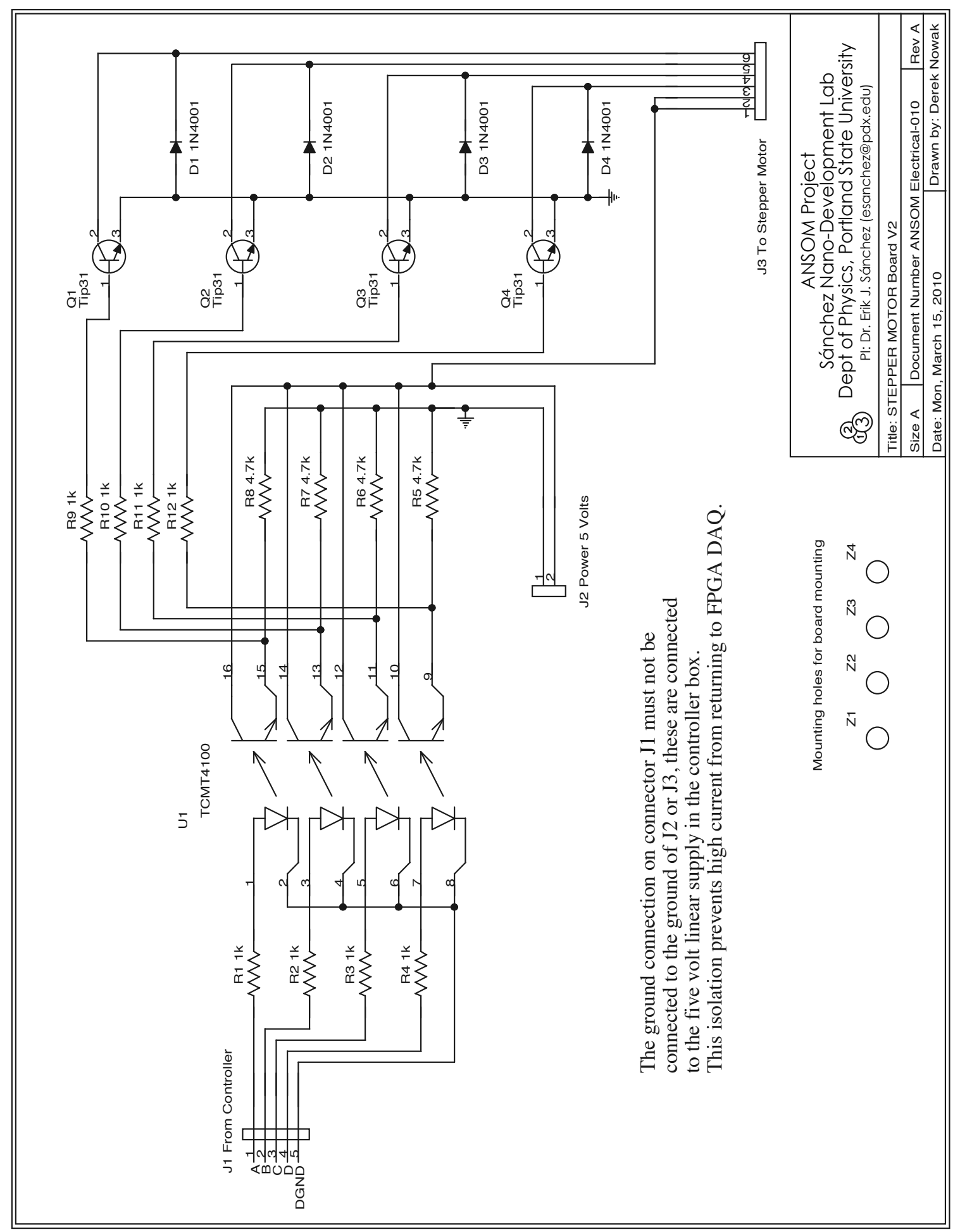




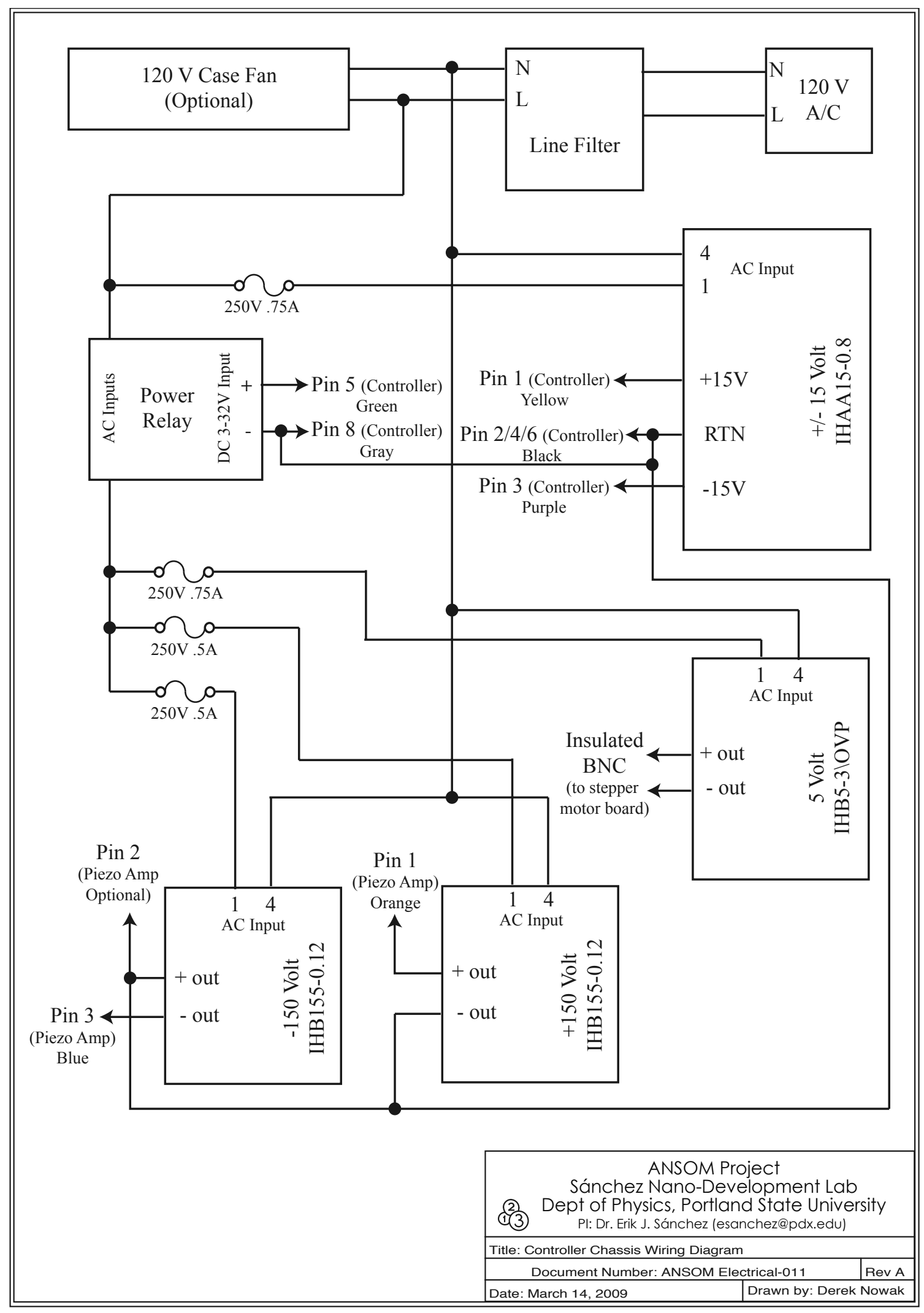




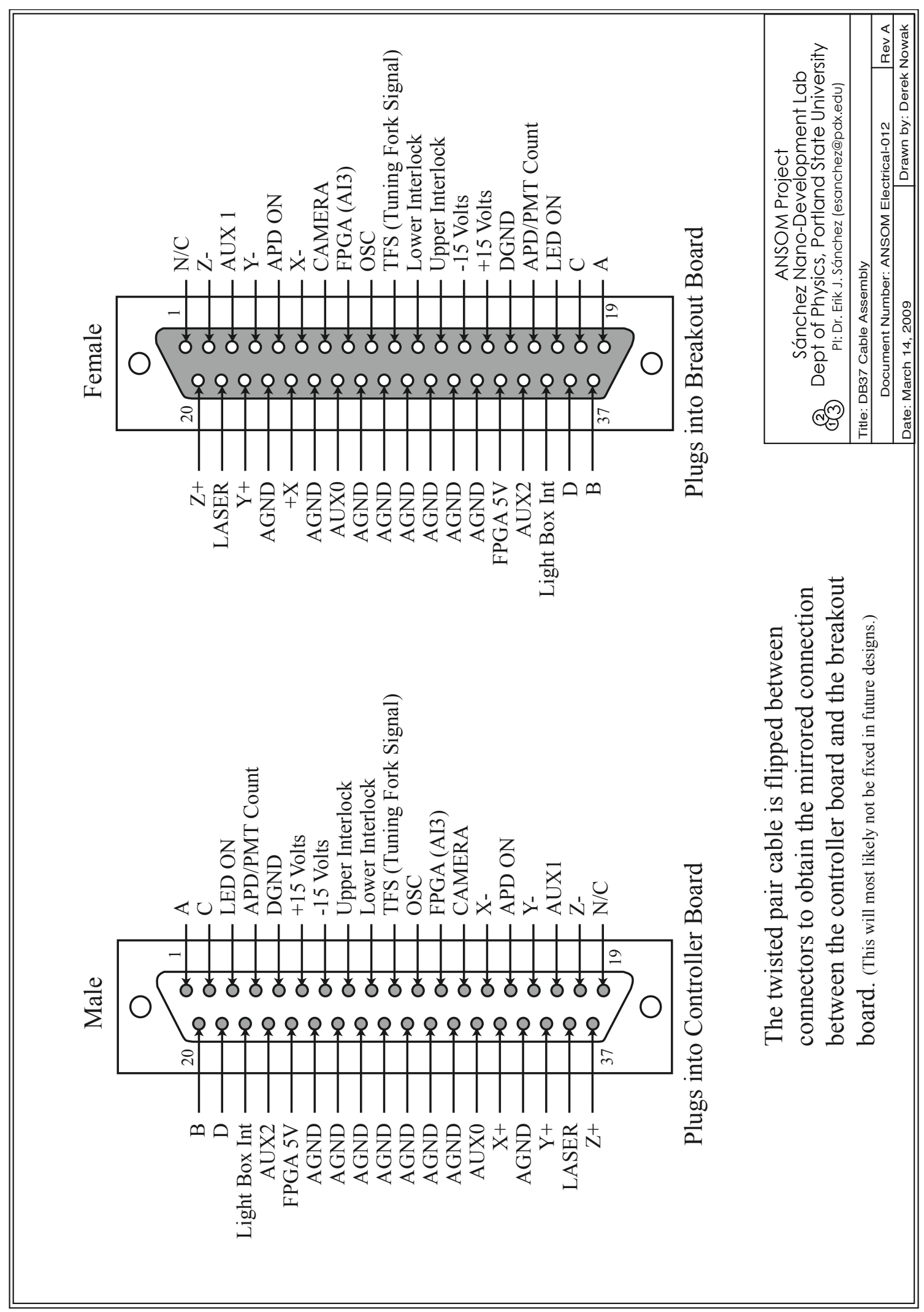




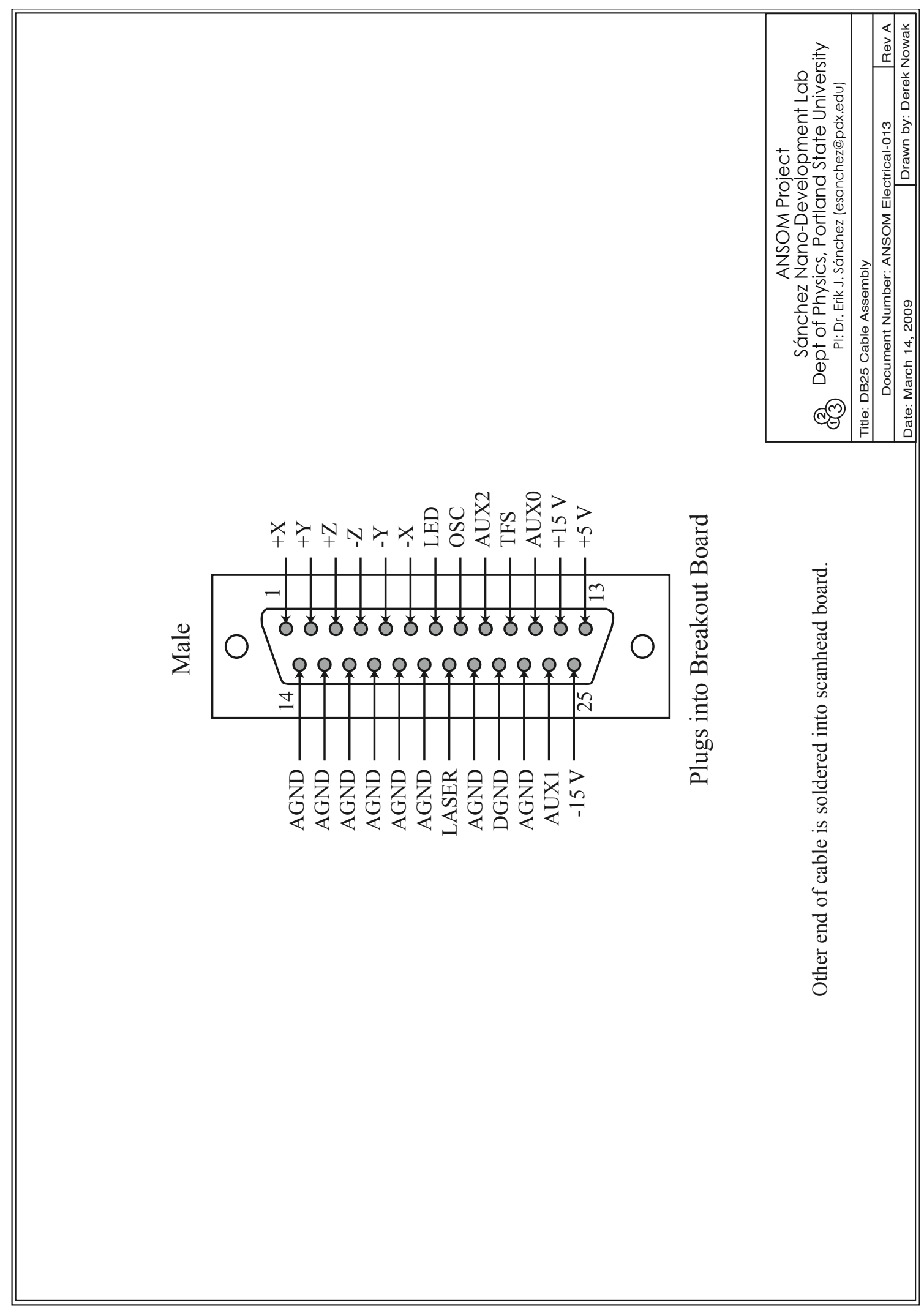




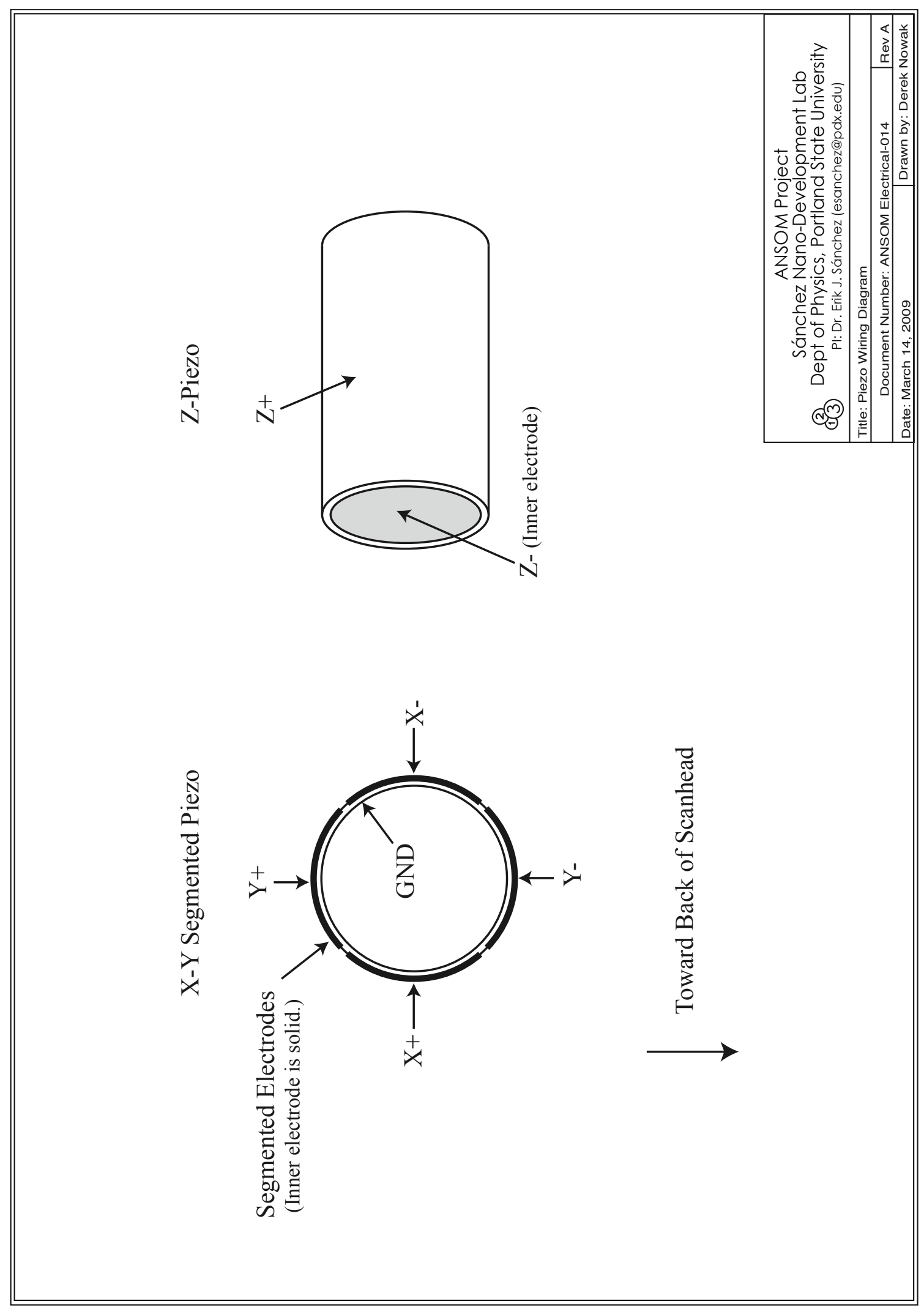




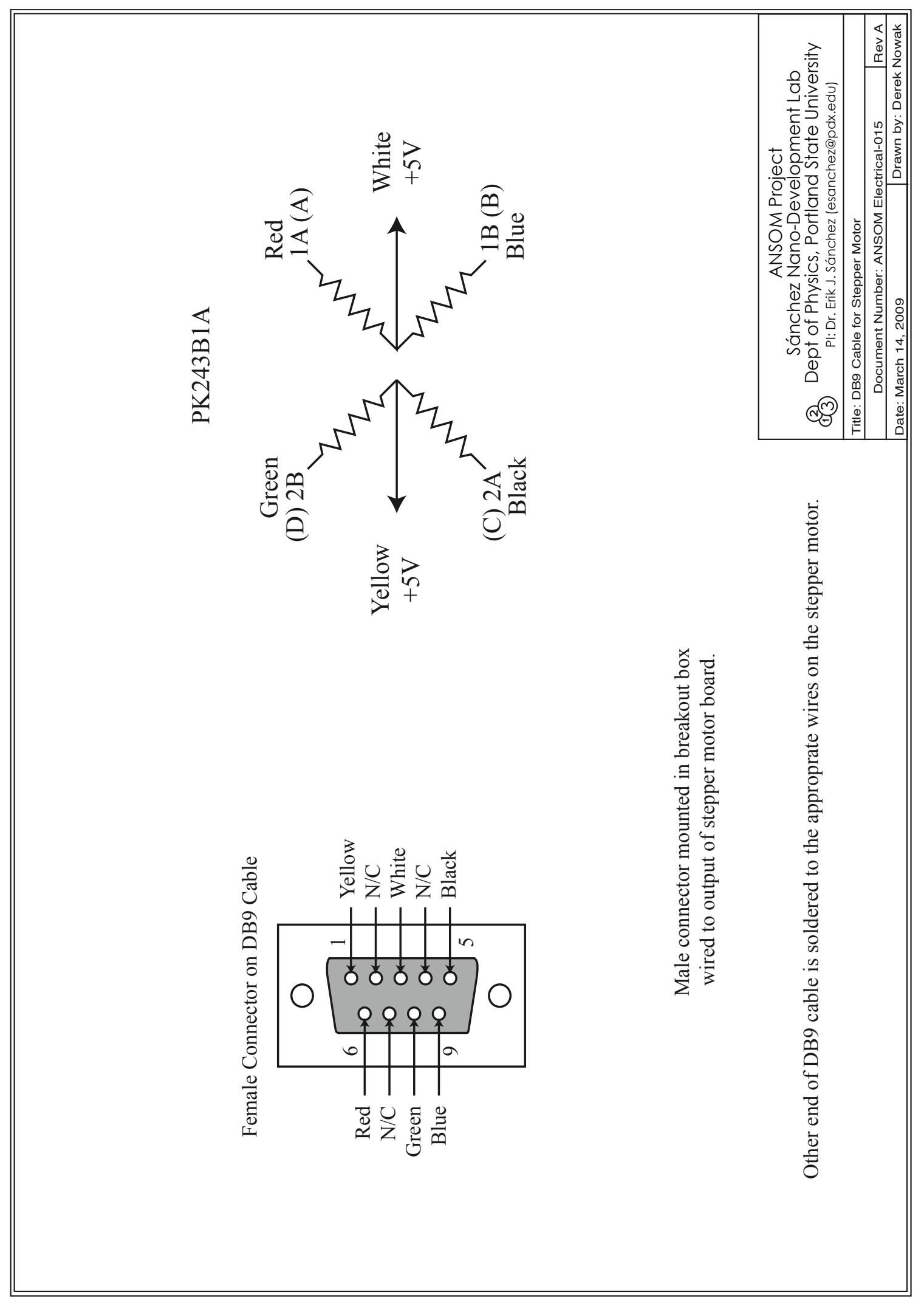




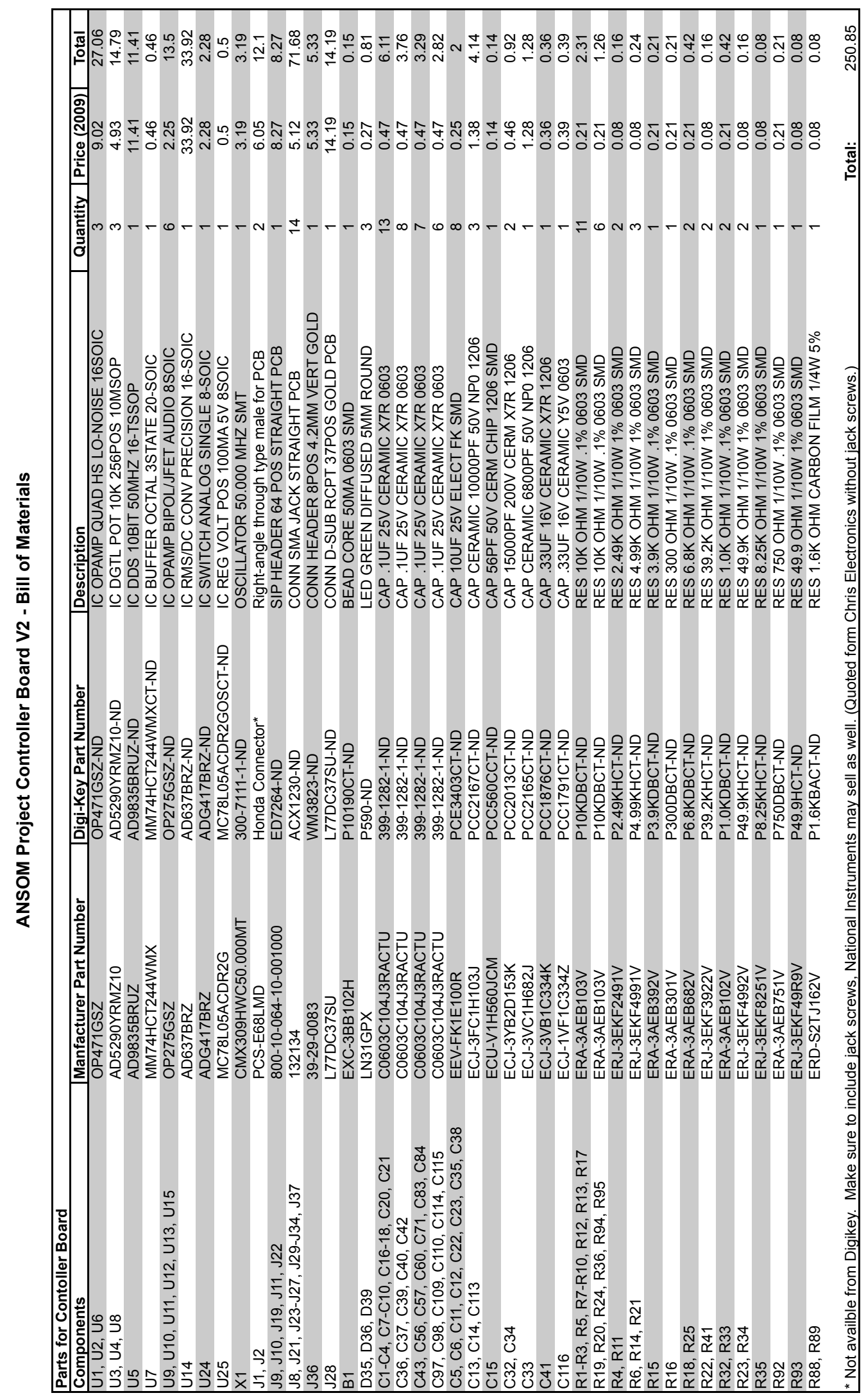



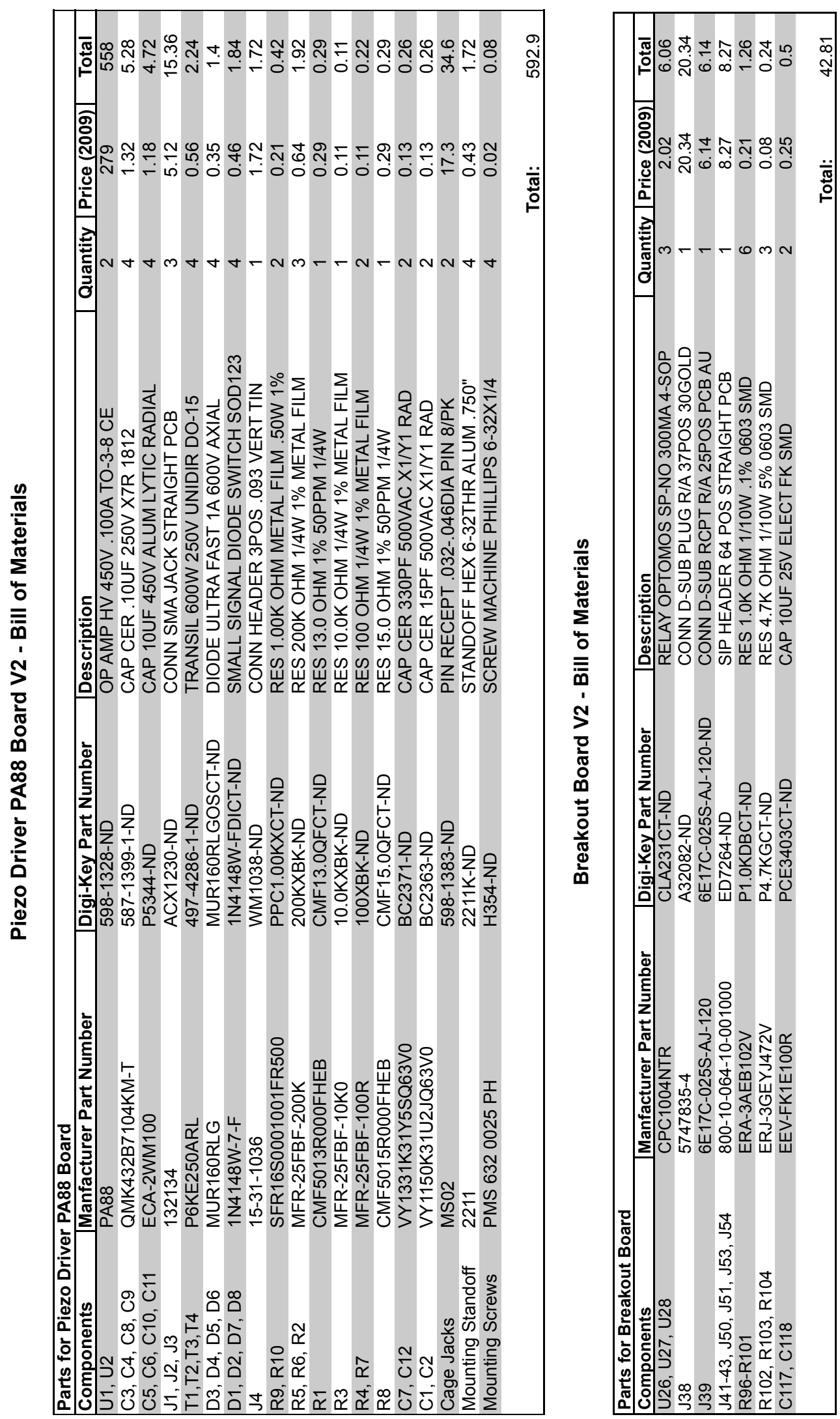

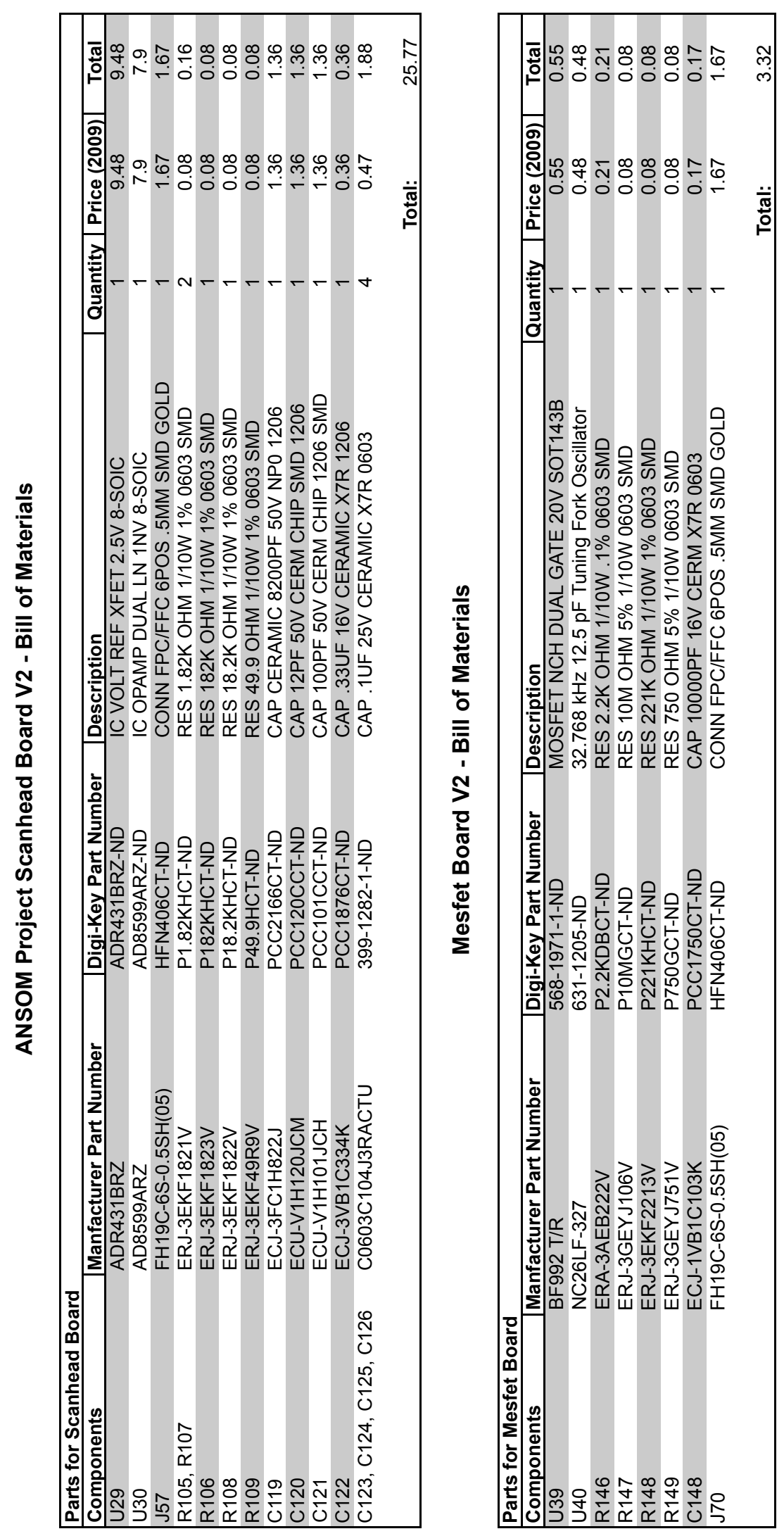

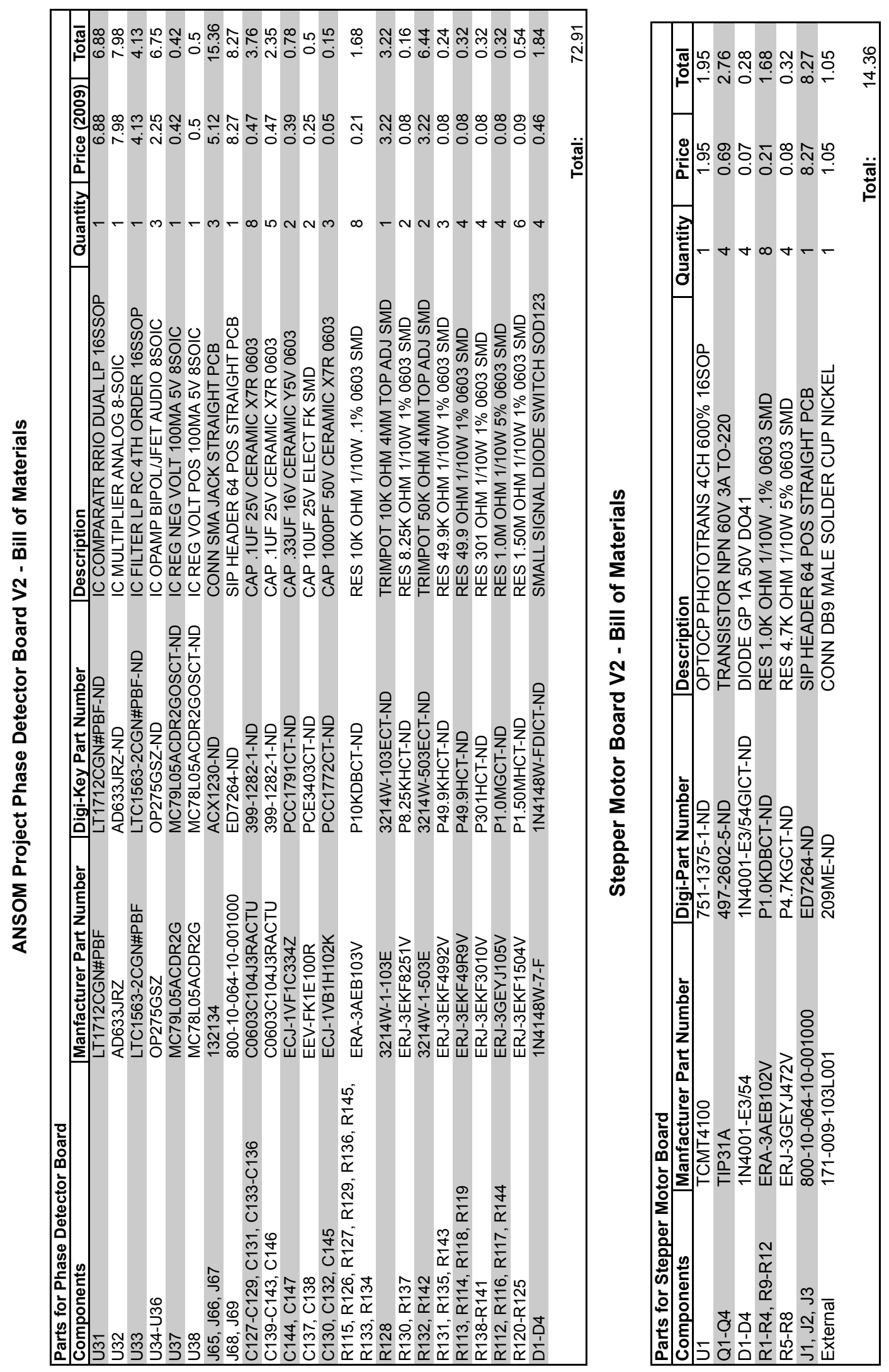

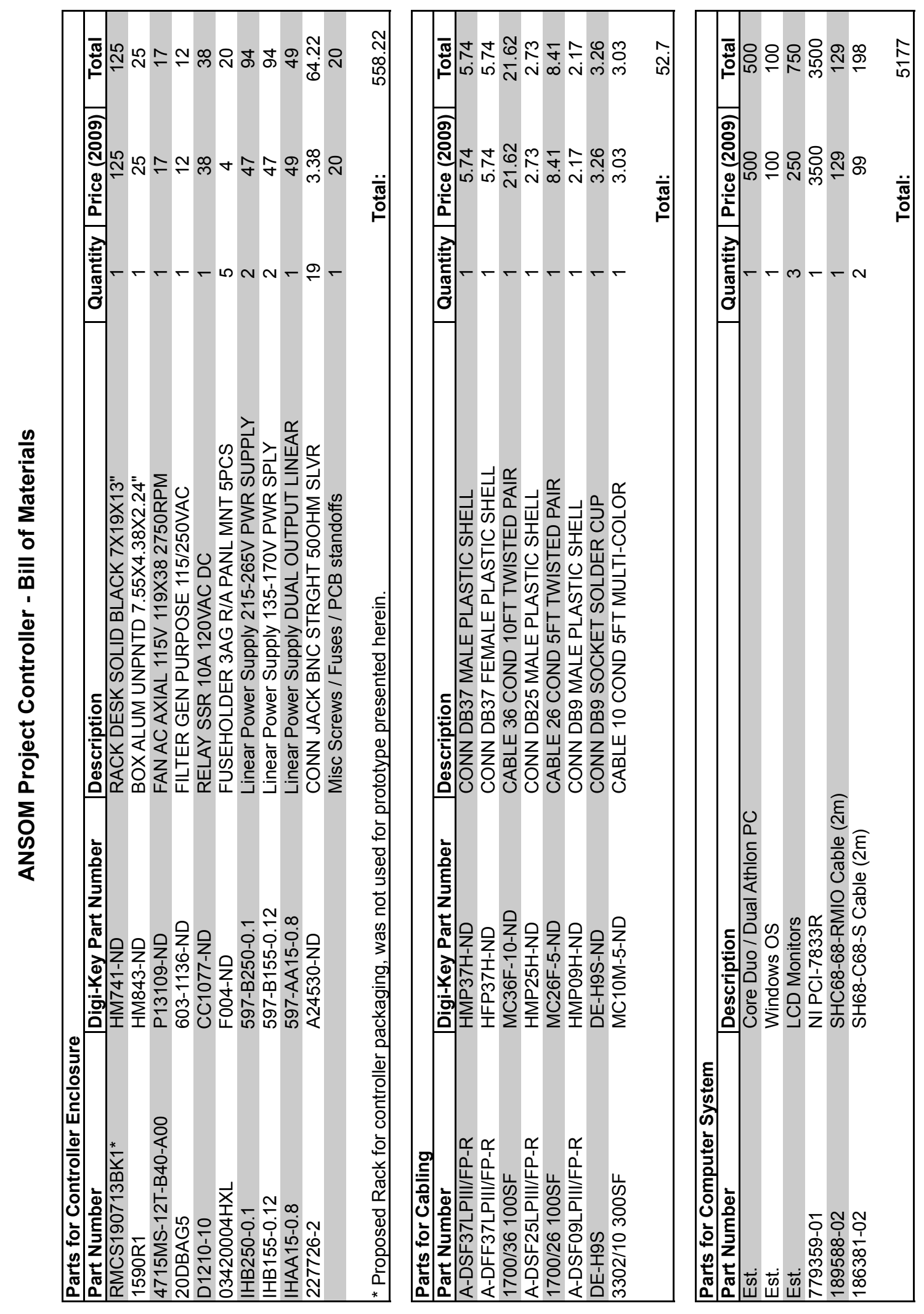


\section{C.3 PCB Layout Artwork}

Table C.3 List of PCB Drawings

\section{Drawing}

Controller Board V2 (Silk Screen Layer) $\quad 339$

Controller Board V2 (Top Layer) 340

Controller Board V2 (First Inner Layer) 341

Controller Board V2 (Second Inner Layer) 342

Controller Board V2 (Bottom Layer) 343

Piezo Driver PA88 V2 (Silk Screen Layer) $\quad 344$

Piezo Driver PA88 V2 (Top Layer) 344

Piezo Driver PA88 V2 (First Inner Layer) 345

Piezo Driver PA88 V2 (Second Inner Layer) 345

Piezo Driver PA88 V2 (Bottom Layer) 346

Breakout Board V2 (Silk Screen Layer) 346

Breakout Board V2 (Top Layer) 347

Breakout Board V2 (First Inner Layer) 347

$\begin{array}{ll}\text { Breakout Board V2 (Second Inner Layer) } & 348\end{array}$

$\begin{array}{ll}\text { Breakout Board V2 (Bottom Layer) } & 348\end{array}$

Scan Head Board V2 (Silk Screen Layer) 349

Scan Head Board V2 (Top Layer) 349

Scan Head Board V2 (First Inner Layer) 349

Scan Head Board V2 (Second Inner Layer) 349

Scan Head Board V2 (Bottom Layer) $\quad 349$

MESFET Board V2 (Silk Screen Layer) 349

MESFET Board V2 (Top Layer) 349

MESFET Board V2 (First Inner Layer) $\quad 349$

MESFET Board V2 (Second Inner Layer) $\quad 349$

MESFET Board V2 (Bottom Layer) $\quad 350$

Phase Detection Board V2 (Silk Screen Layer) 350

Phase Detection Board V2 (Top Layer) 350

Phase Detection Board V2 (First Inner Layer) 351

Phase Detection Board V2 (Second Inner Layer) 351

Phase Detection Board V2 (Bottom Layer) 351

Stepper Motor V2 (Silk Screen Layer) 352

Stepper Motor V2 (Top Layer) 352

Stepper Motor V2 (Bottom Layer) 352 


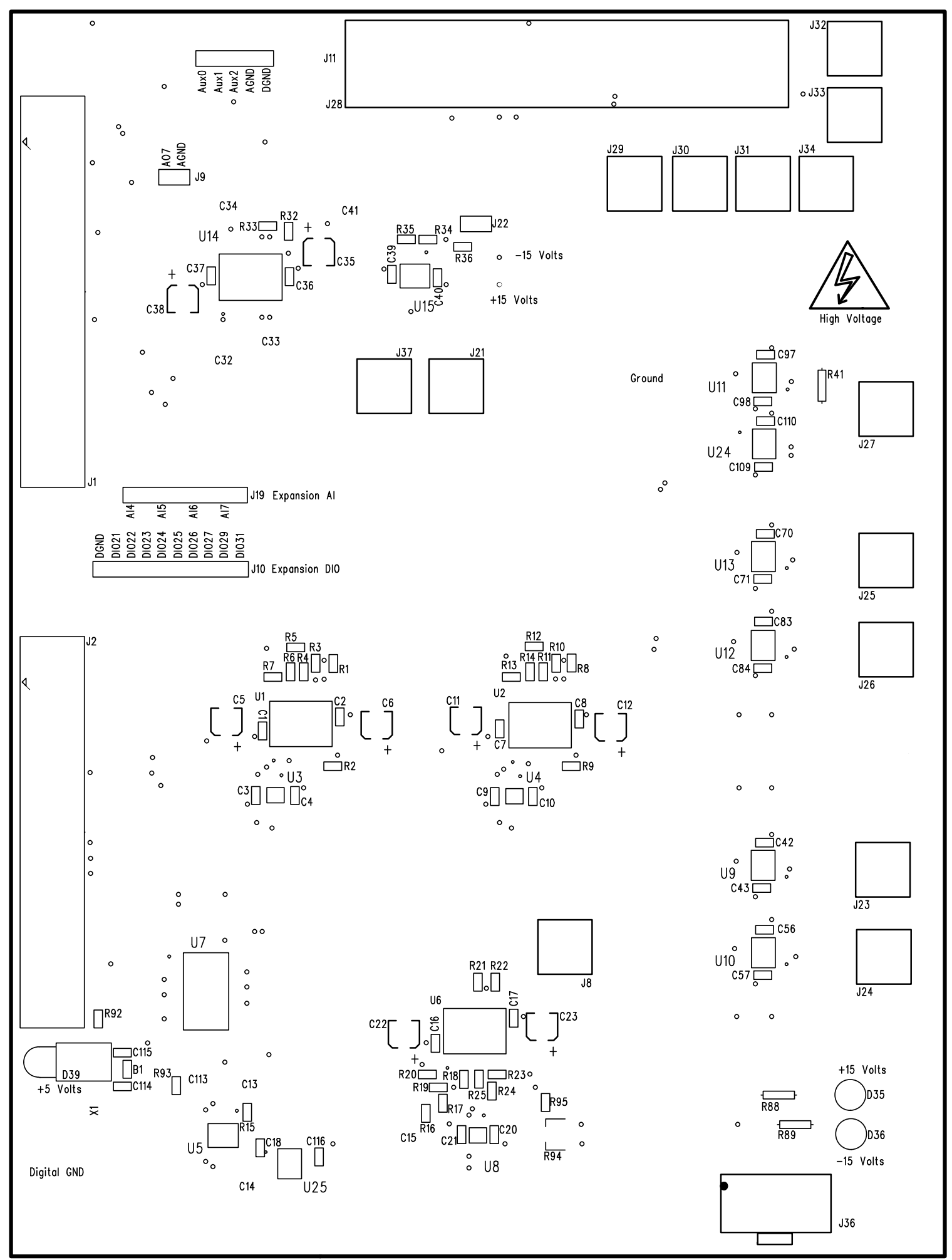

Controller Board V2 (Silk Screen Layer) Scale 1:1 


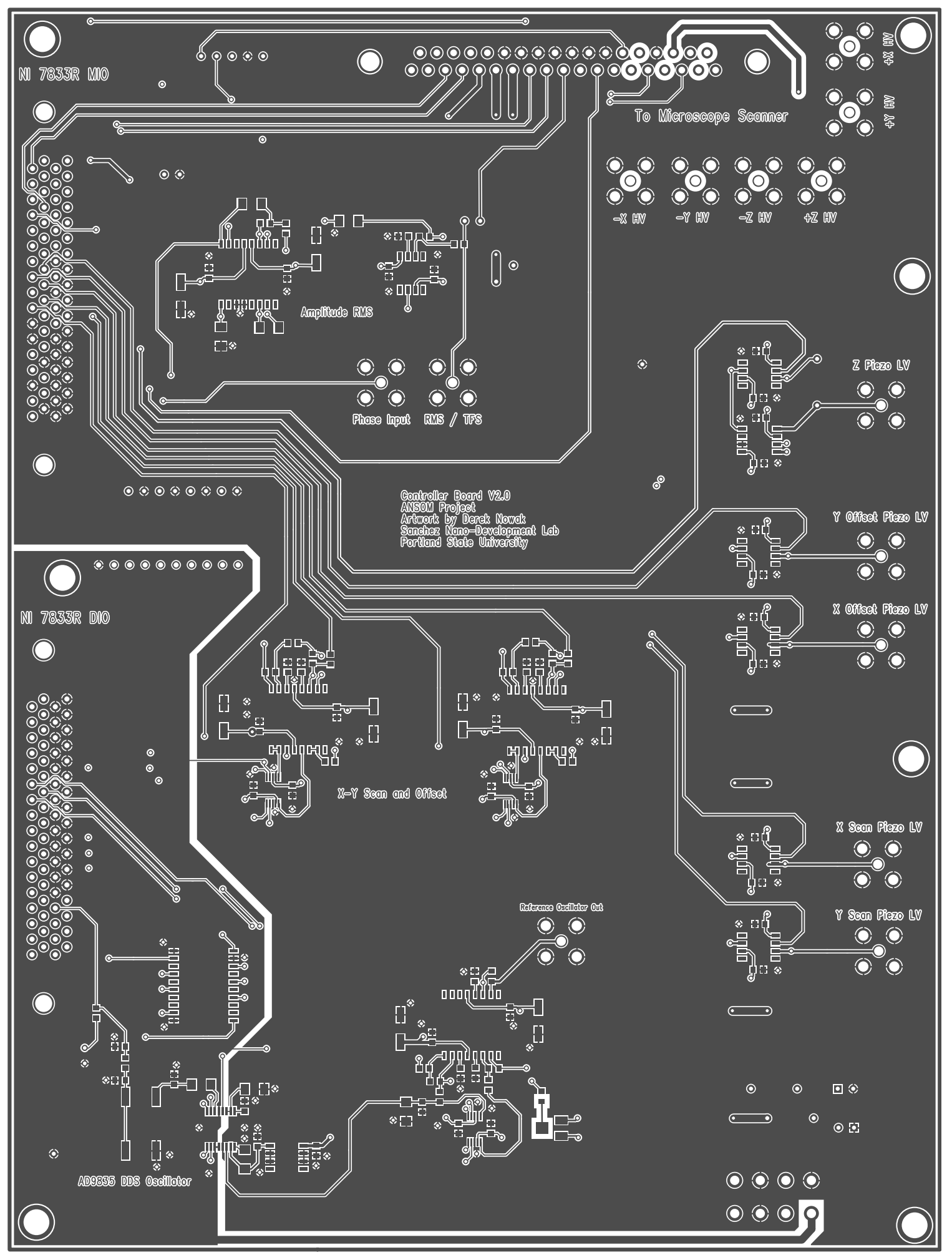

Controller Board V2 (Top Layer) Scale 1:1 


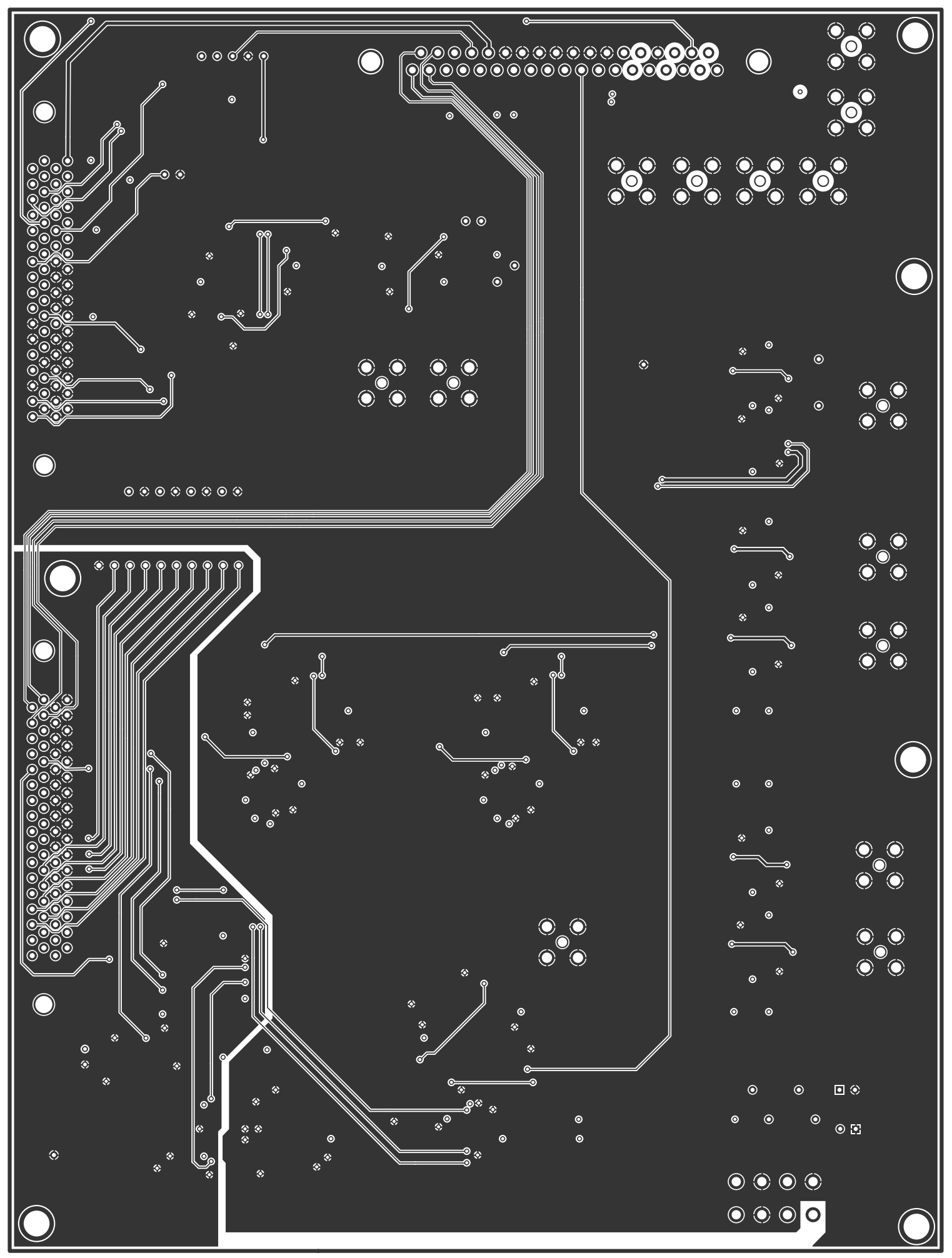

Controller Board V2 (First Inner Layer) Scale 1:1 


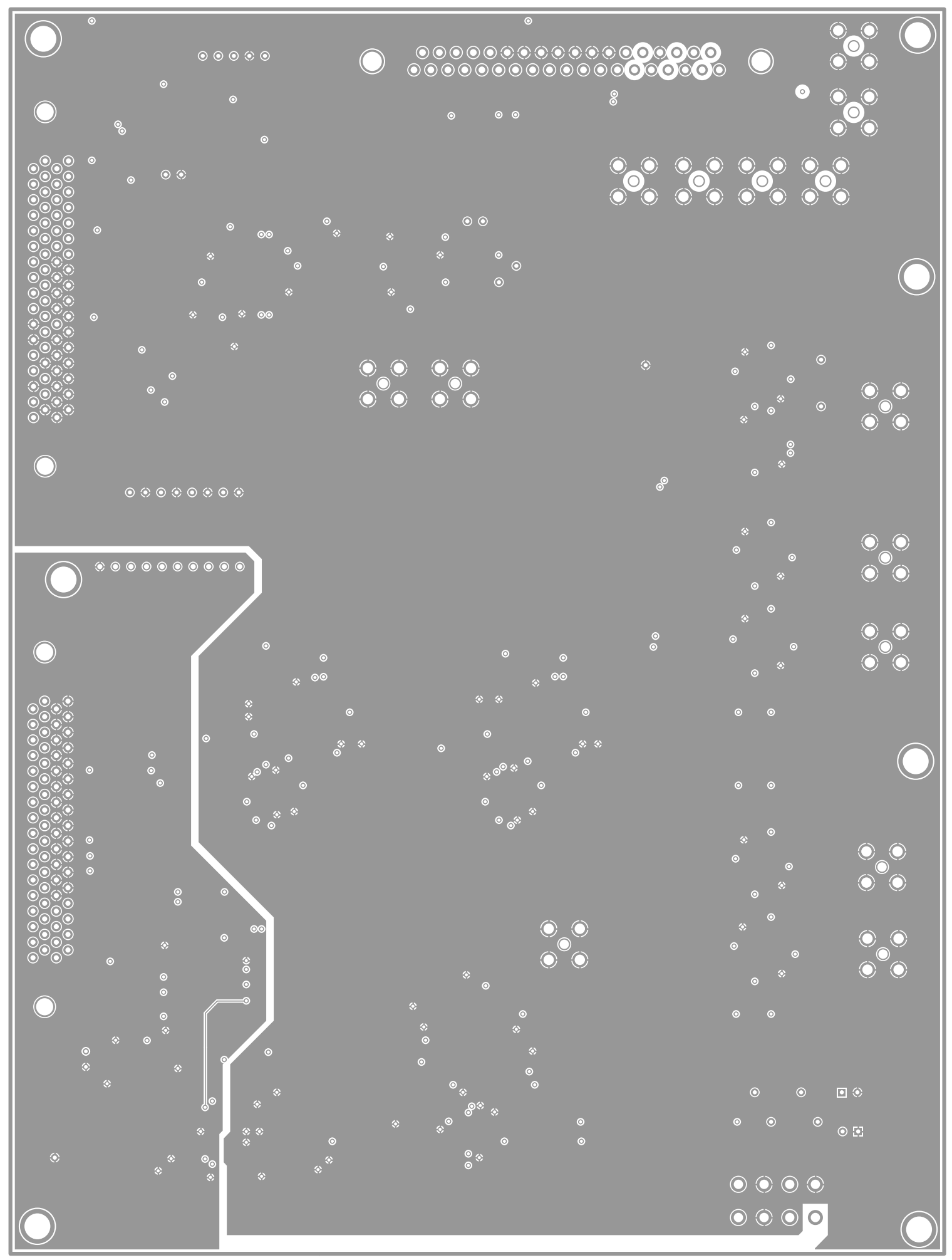

Controller Board V2 (Second Inner Layer) Scale 1:1 


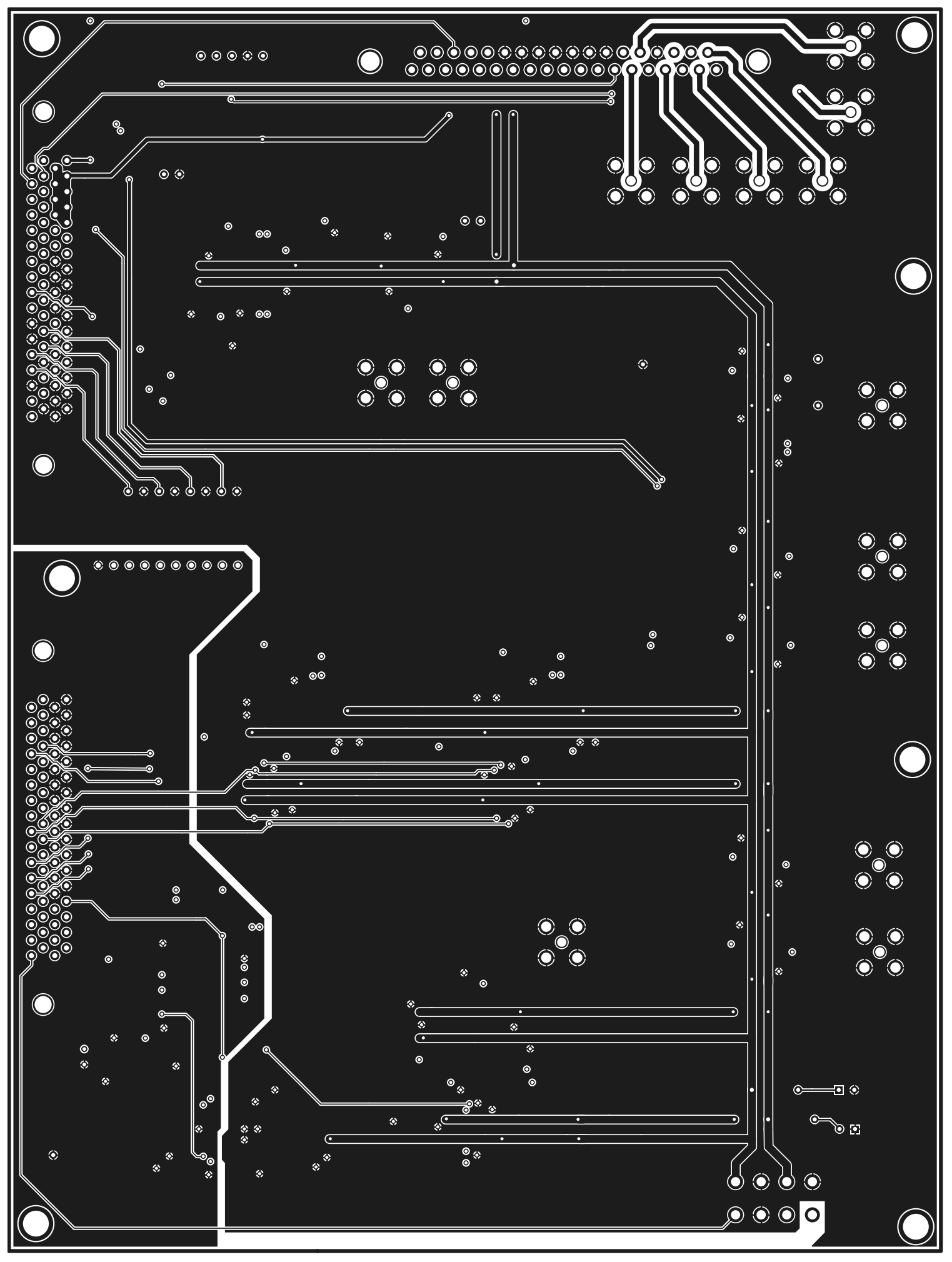

Controller Board V2 (Bottom Layer) Scale 1:1 


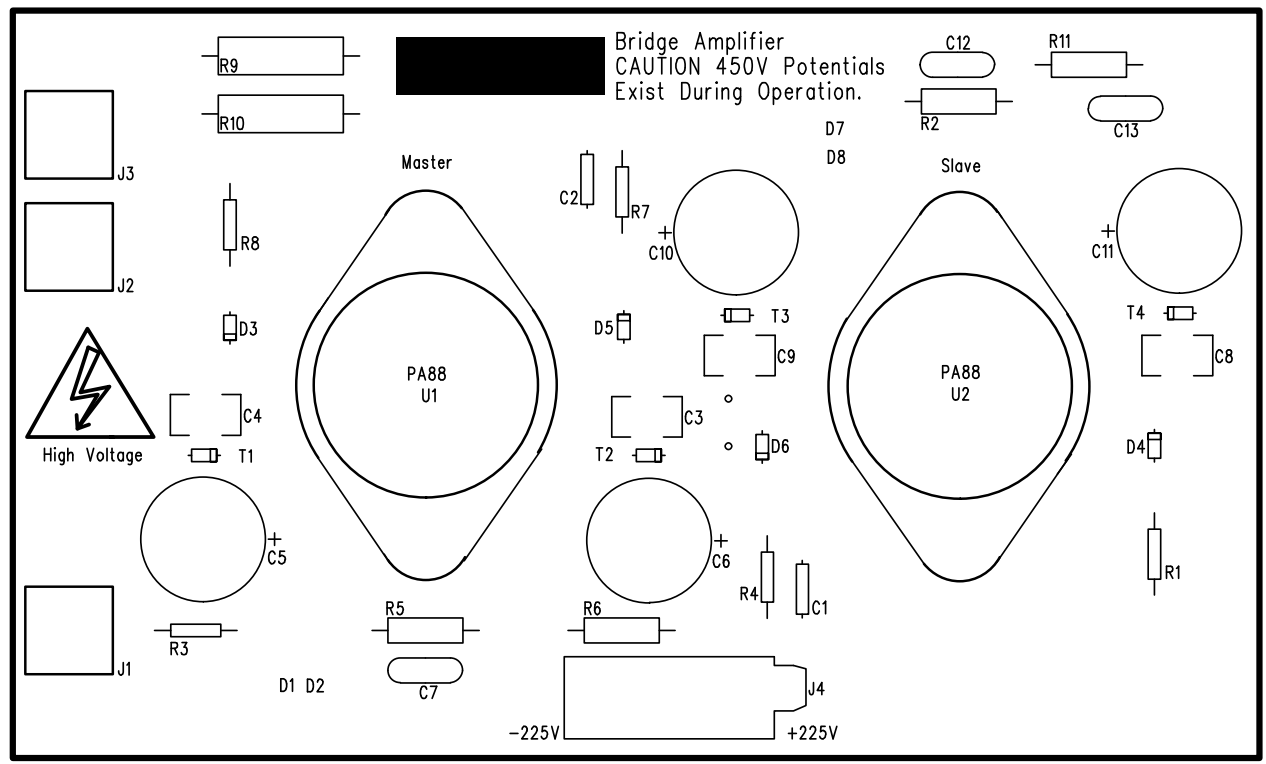

Piezo Driver PA88 Board V2 (Silk Screen Layer) Scale 1:1

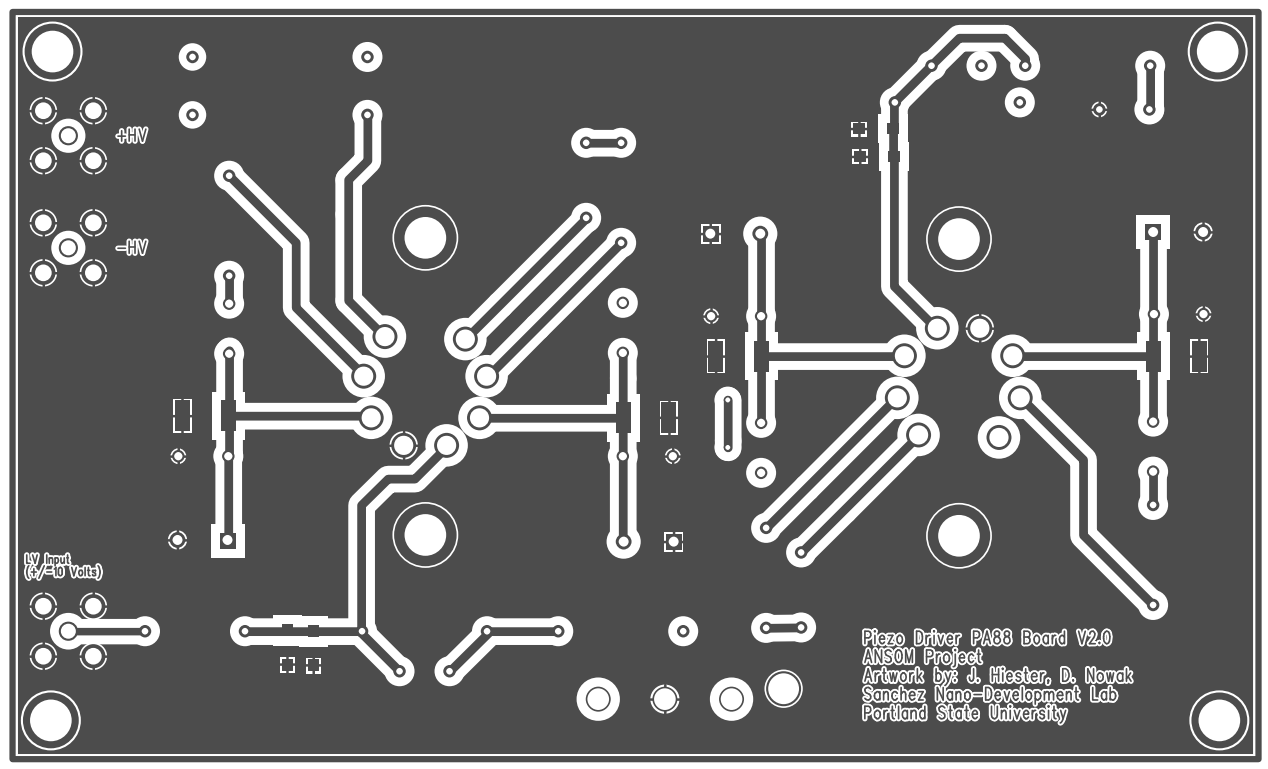

Piezo Driver PA88 Board V2 (Top Layer) Scale 1:1 


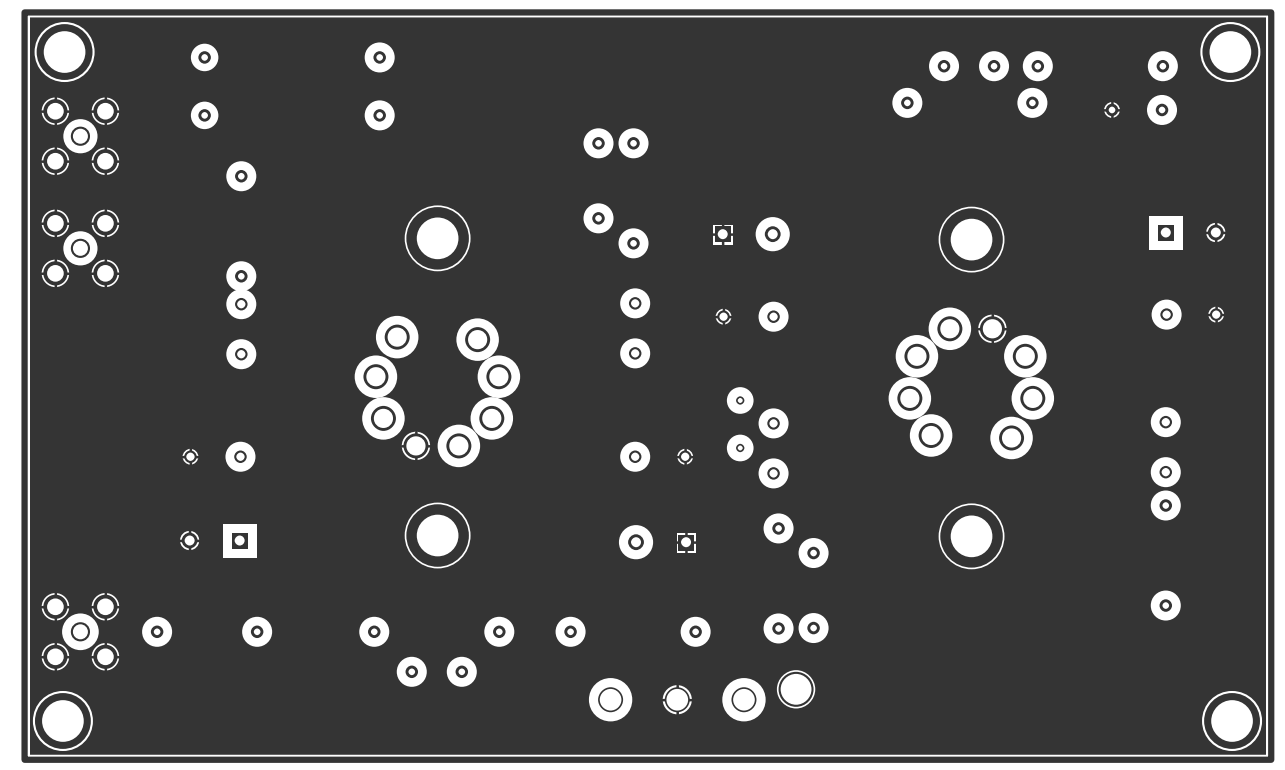

Piezo Driver PA88 Board V2 (First Inner Layer) Scale 1:1

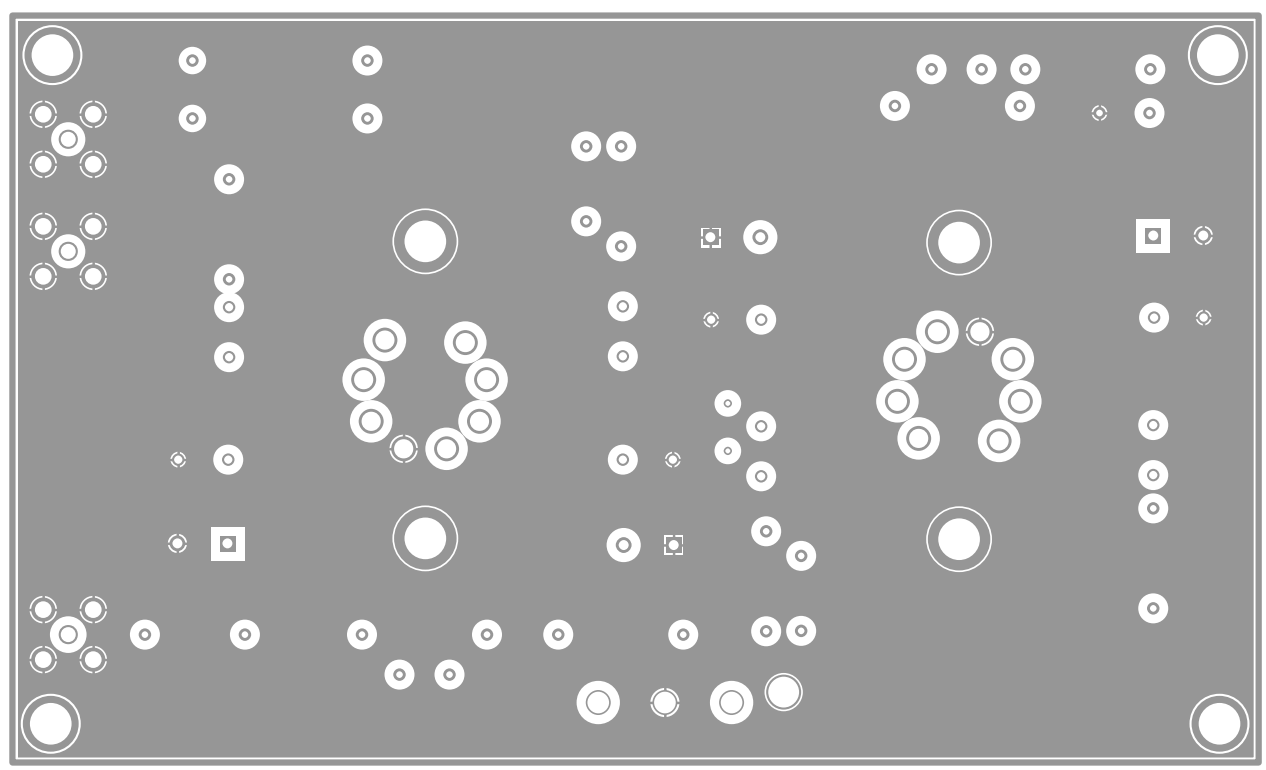

Piezo Driver PA88 Board V2 (Second Inner Layer) Scale 1:1 


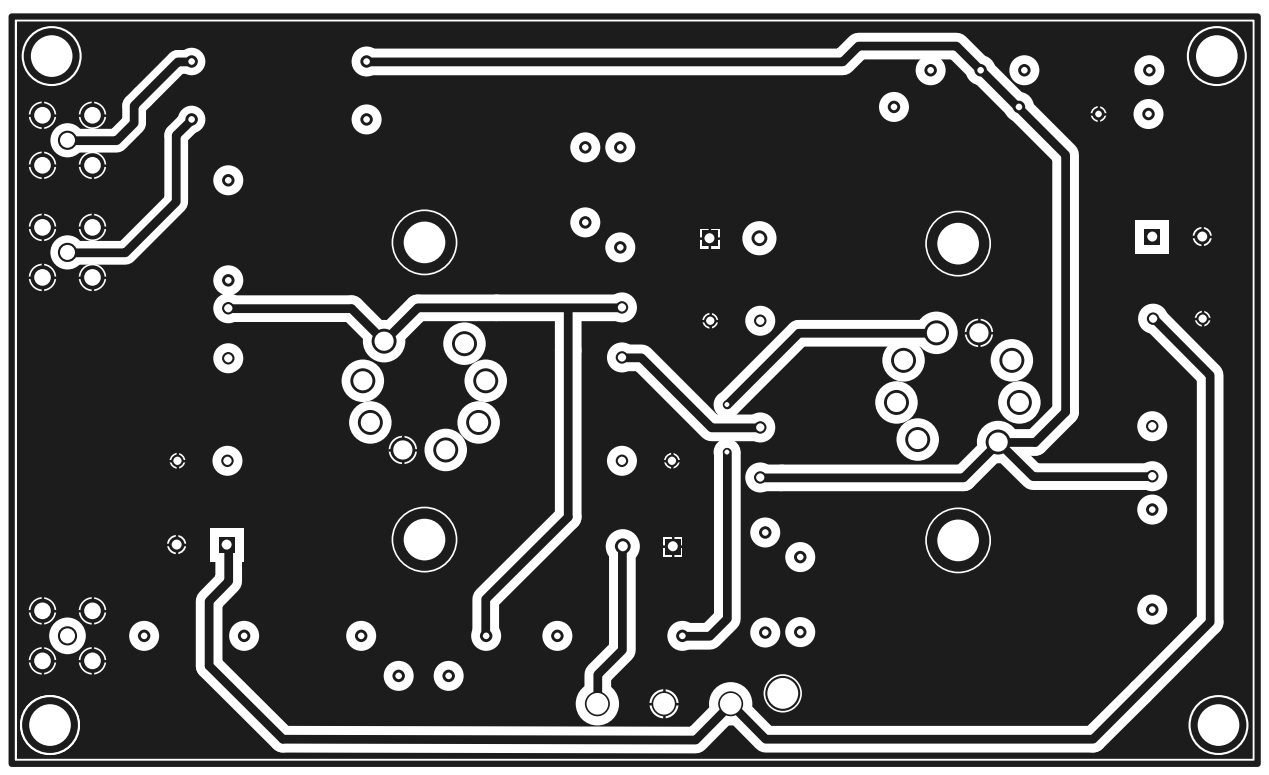

Piezo Driver PA88 Board V2 (Bottom Layer) Scale 1:1

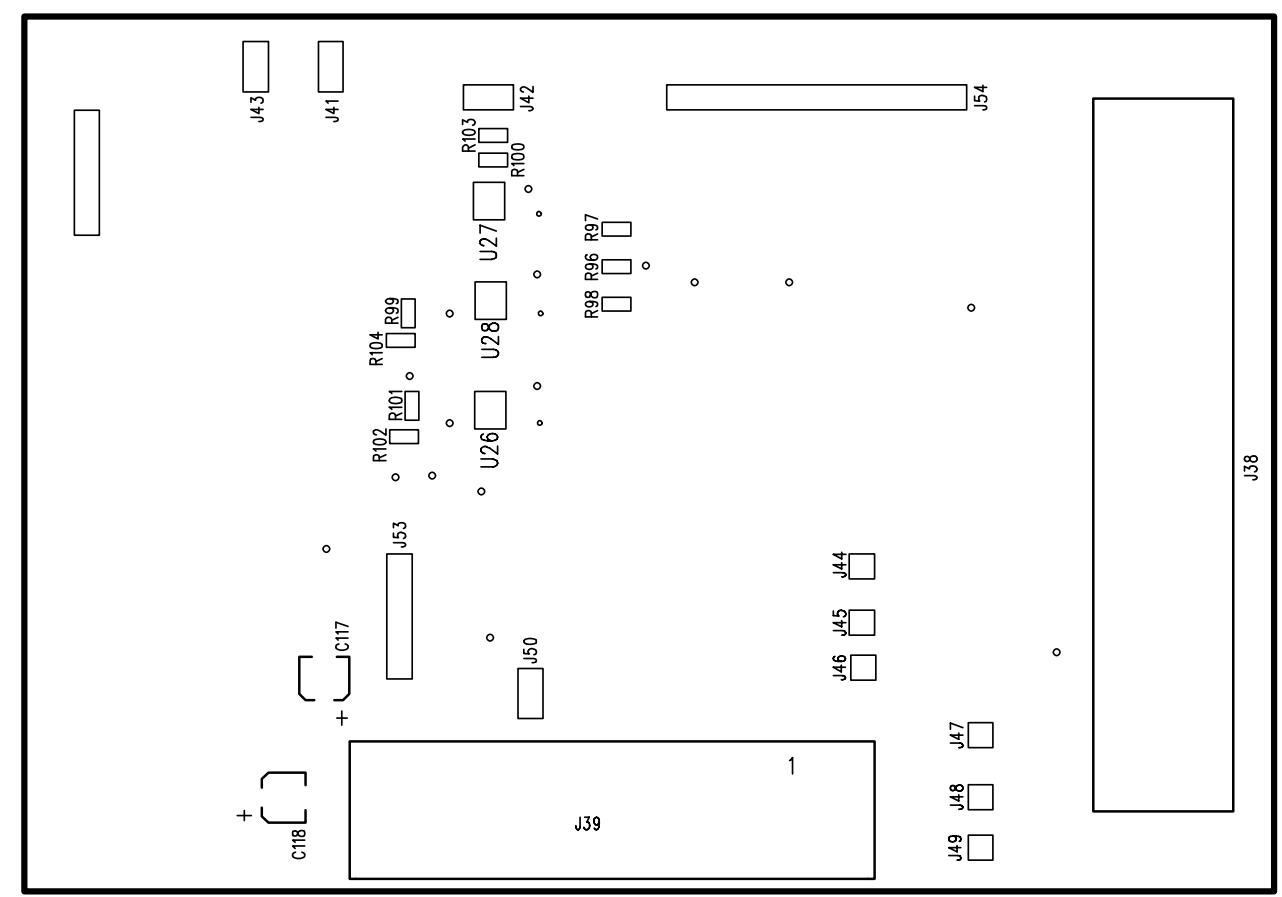

Breakout Board V2 (Silk Screen Layer) Scale 1:1 


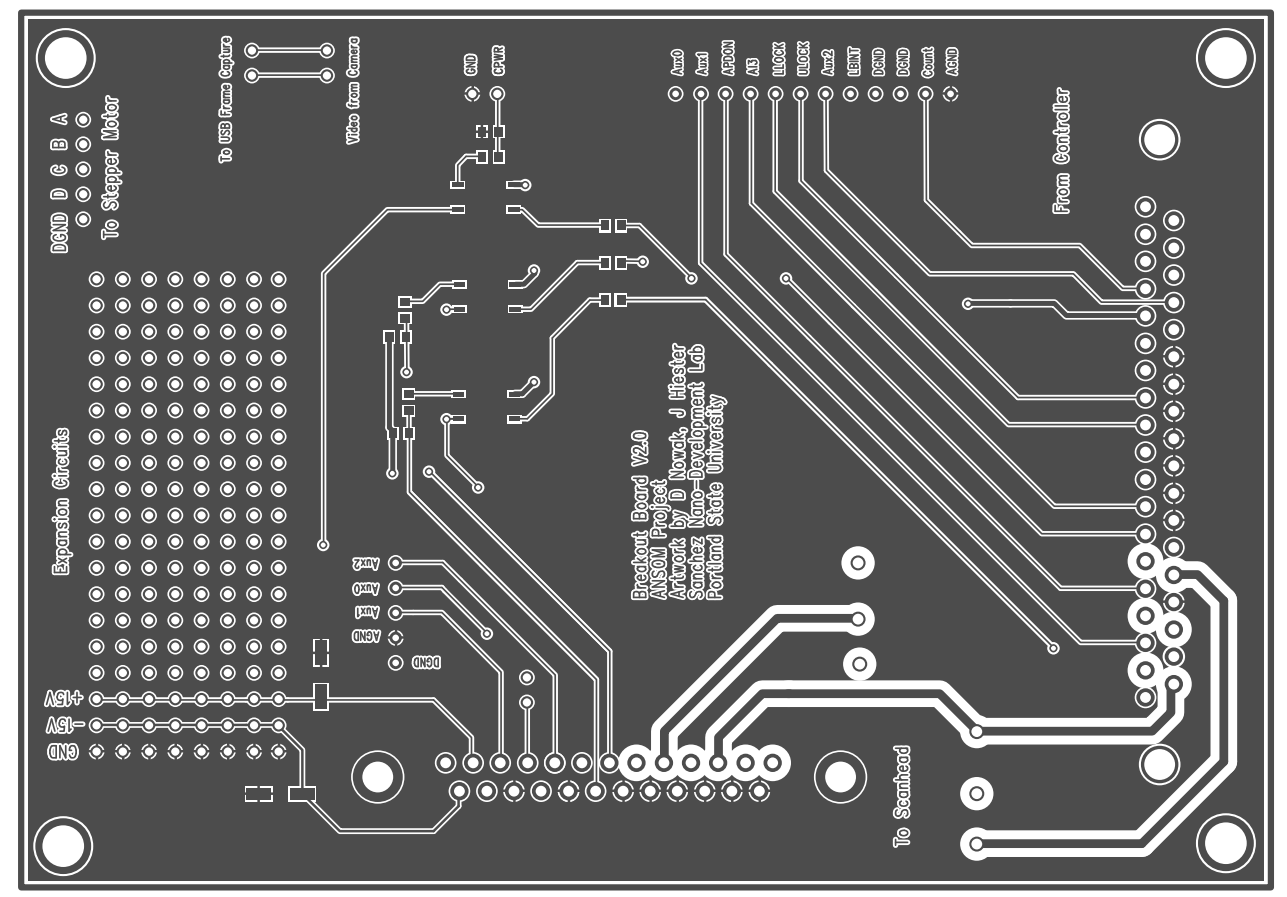

Breakout Board V2 (Top Layer) Scale 1:1

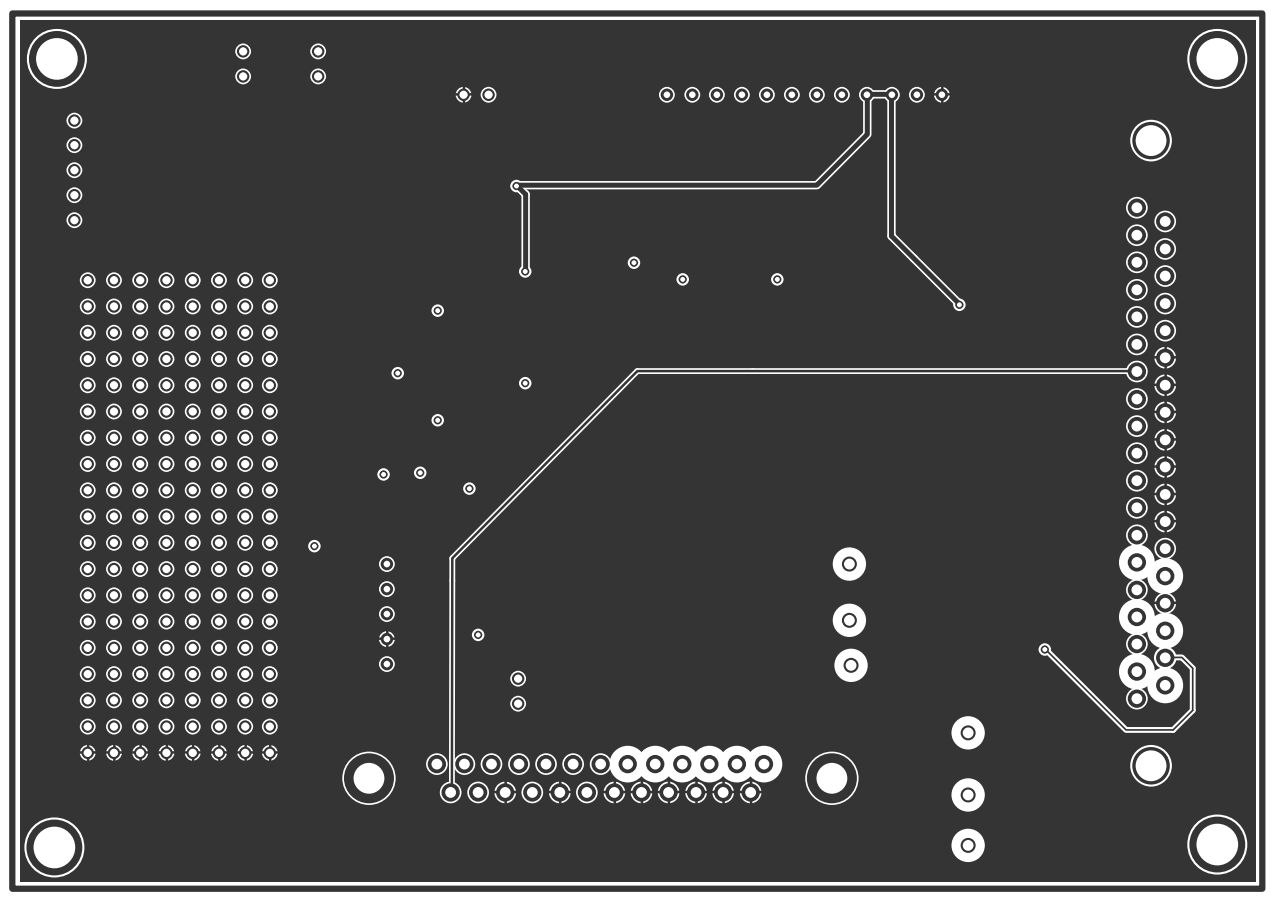

Breakout Board V2 (First Inner Layer) Scale 1:1 


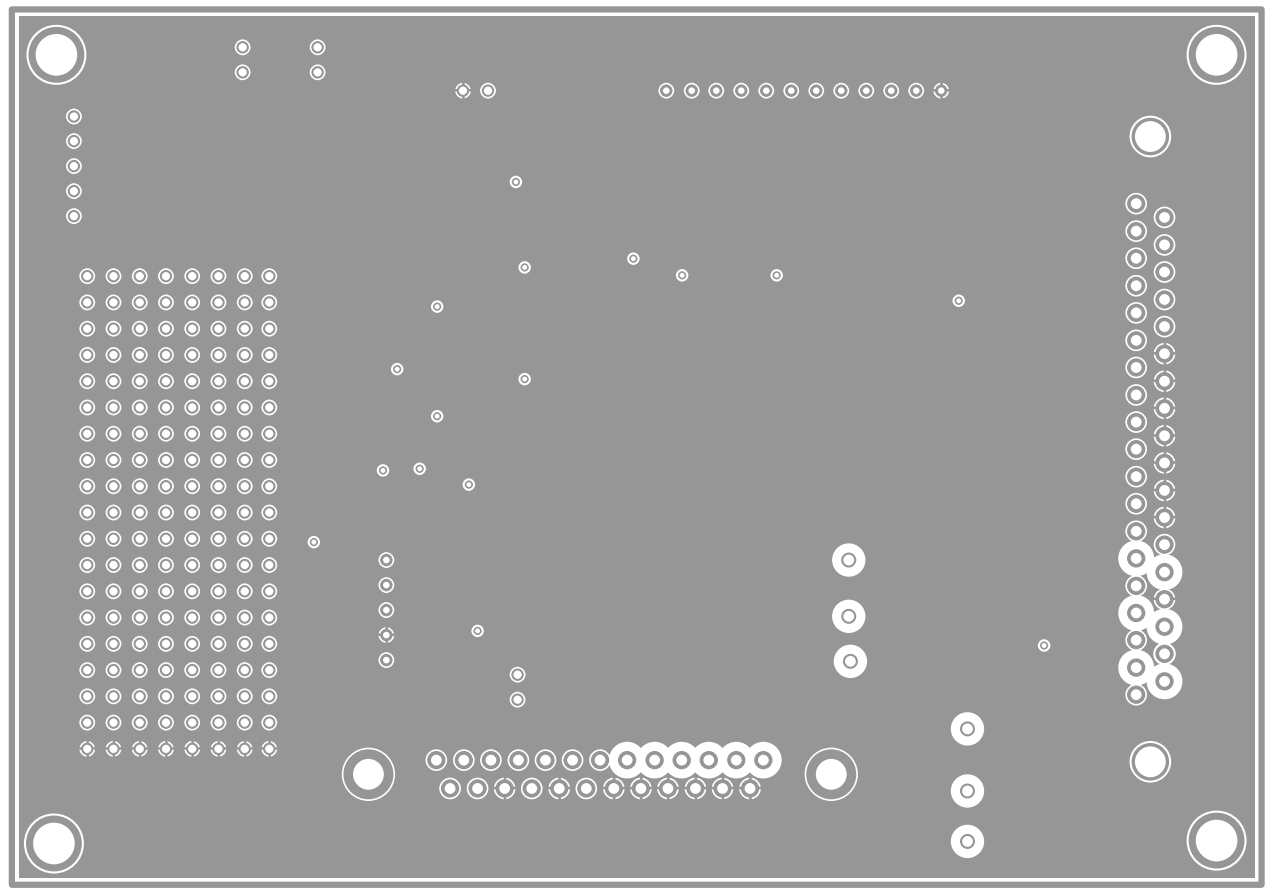

Breakout Board V2 (Second Inner Layer) Scale 1:1

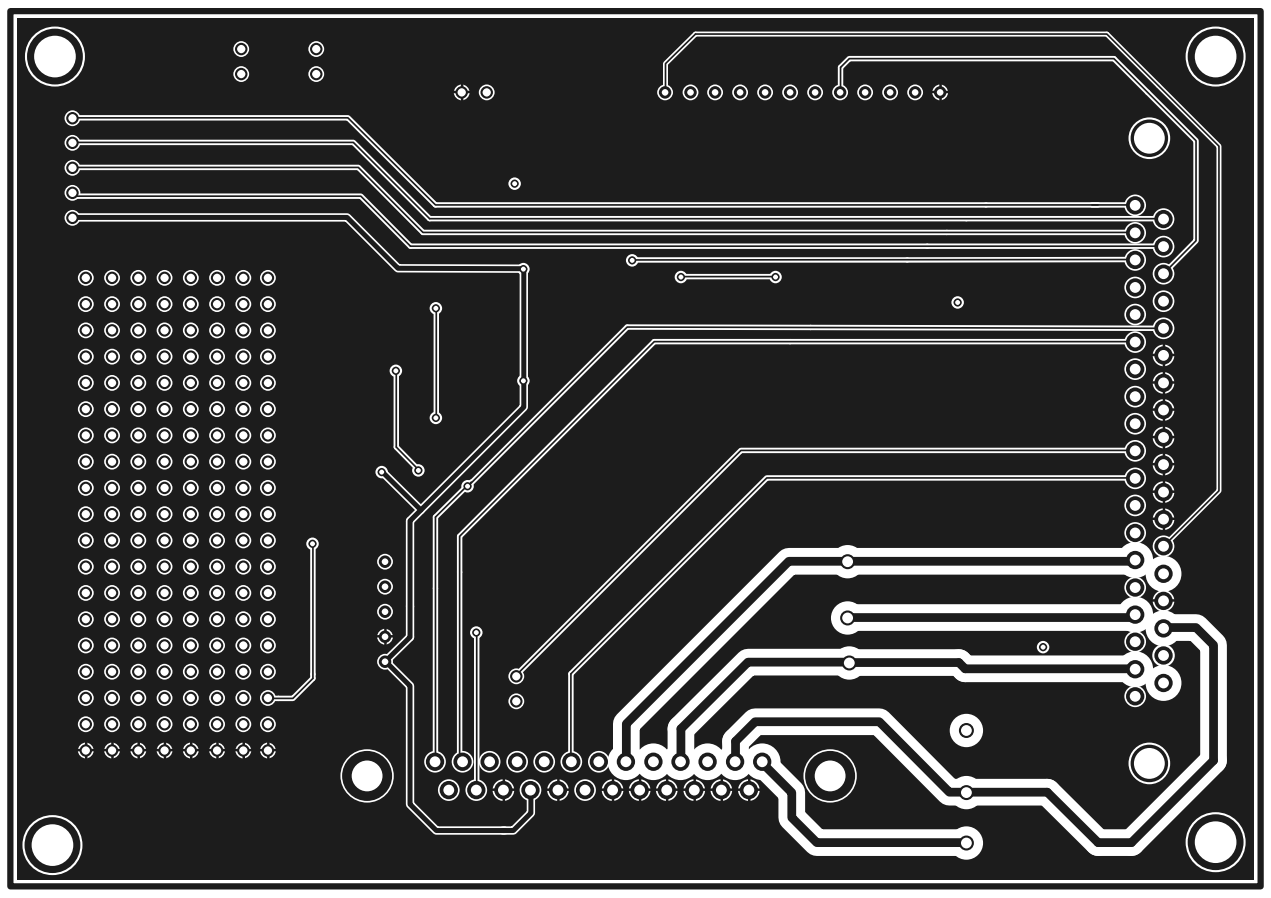

Breakout Board V2 (Bottom Layer) Scale 1:1 


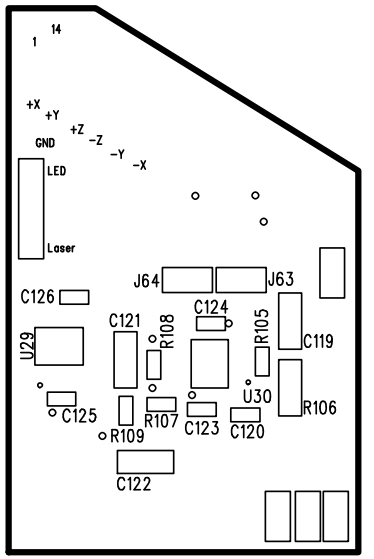

Scan Head Board V2

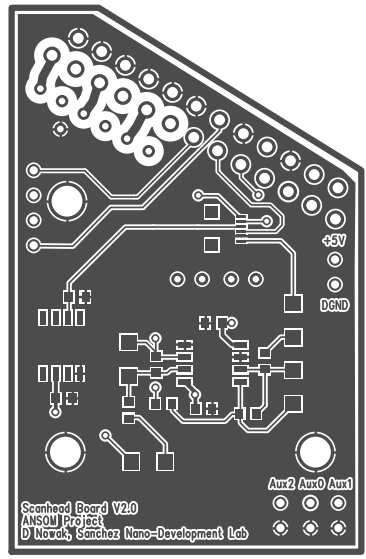

Scan Head Board V2

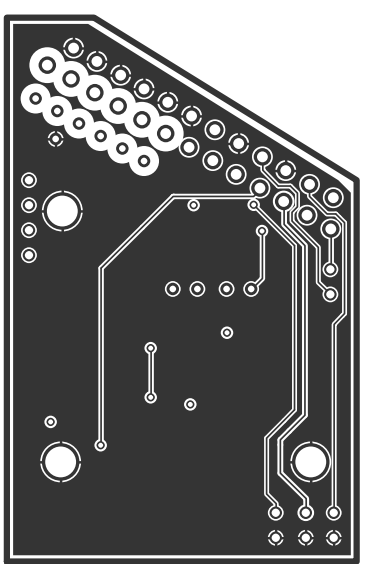

Scan Head Board V2

(Silk Screen Layer) Scale 1:1

(First Inner Layer) Scale 1:1

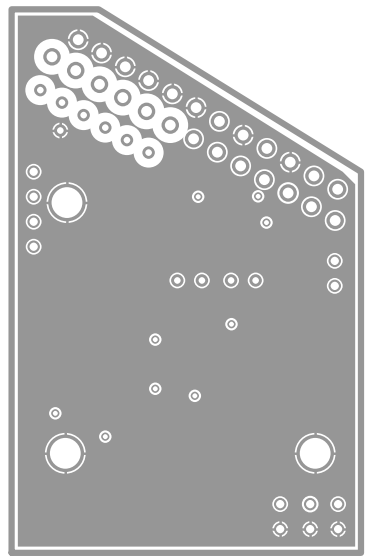

Scan Head Board V2

(Second Inner Layer) Scale 1:1

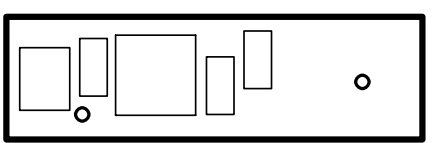

Mesfet Board V2

(Silk Screen Layer) Scale 2:1

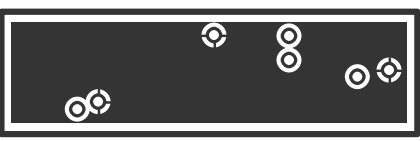

Mesfet Board V2

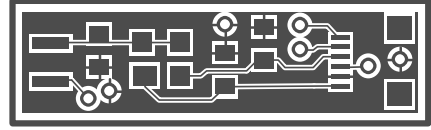

Mesfet Board V2

(Top Layer) Scale 2:1

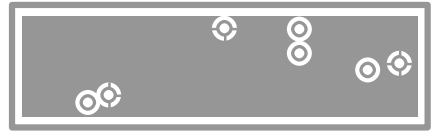

Mesfet Board V2

(First Inner Layer) Scale 2:1 (Second Inner Layer) Scale 2:1 


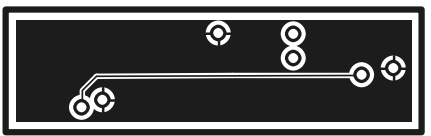

Mesfet Board V2

(Bottom Layer) Scale 2:1

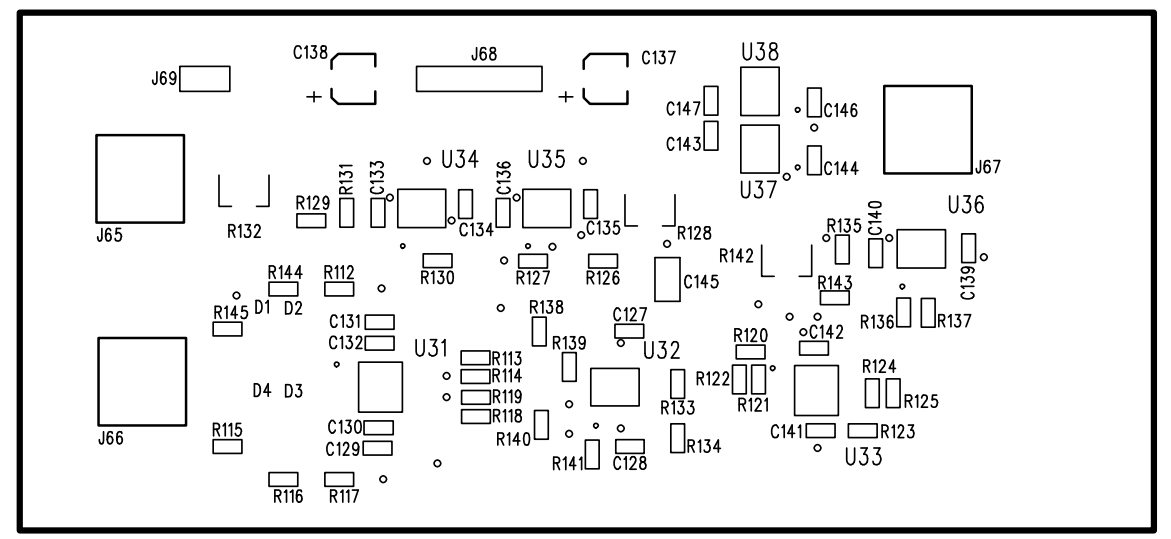

Phase Detection Board V2 (Silk Screen Layer) Scale 1:1

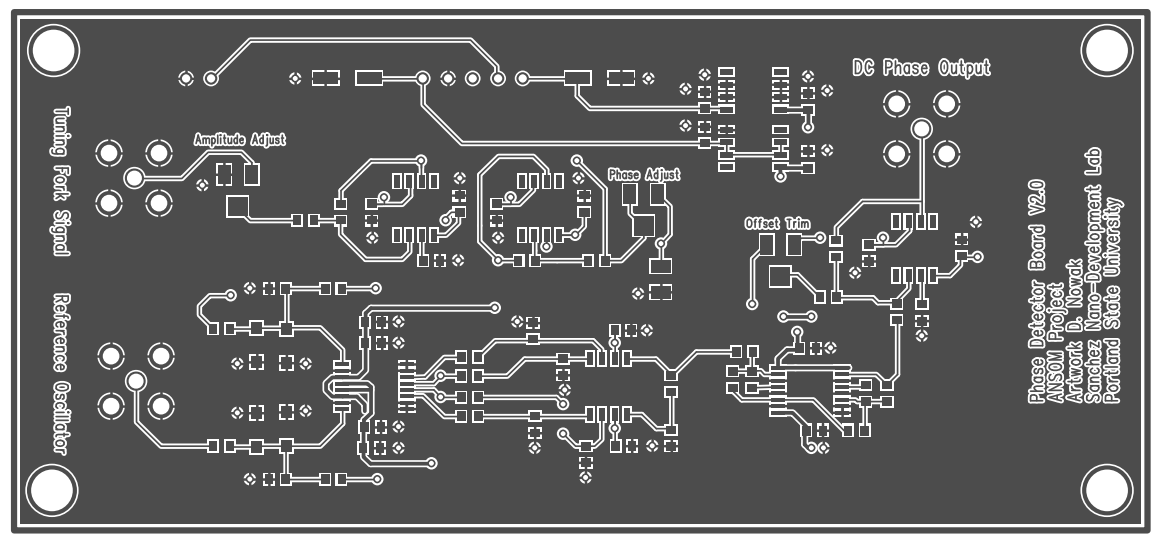

Phase Detection Board V2 (Top Layer) Scale 1:1 


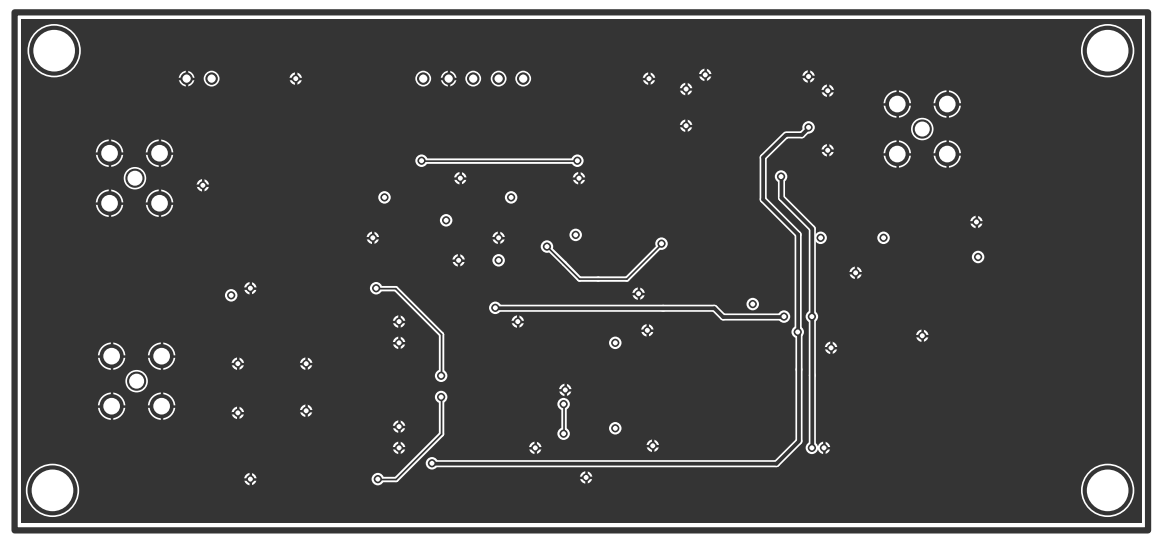

Phase Detection Board V2 (First Inner Layer) Scale 1:1

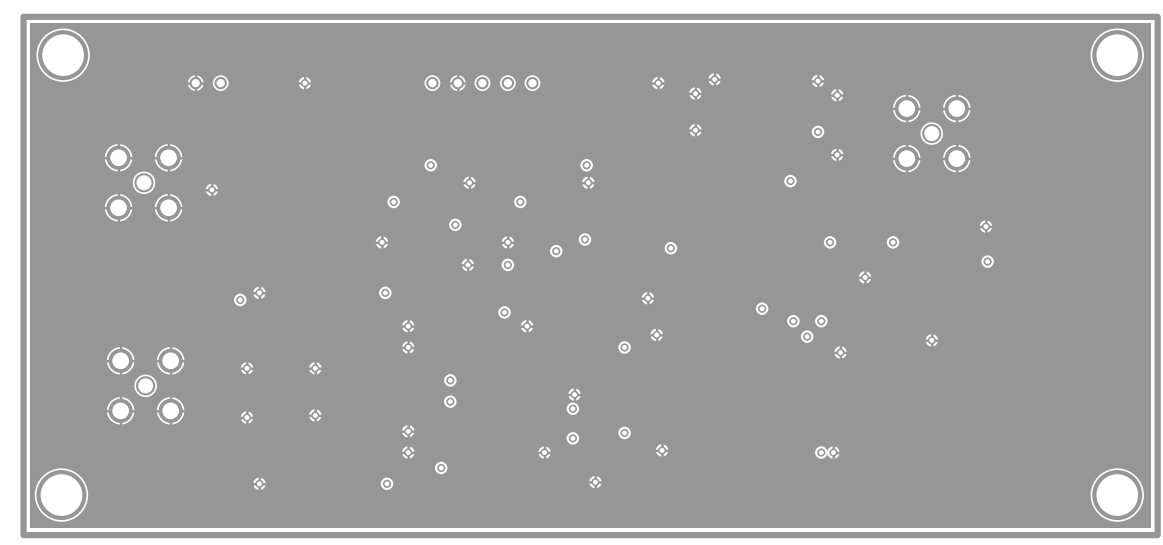

Phase Detection Board V2 (Second Inner Layer) Scale 1:1

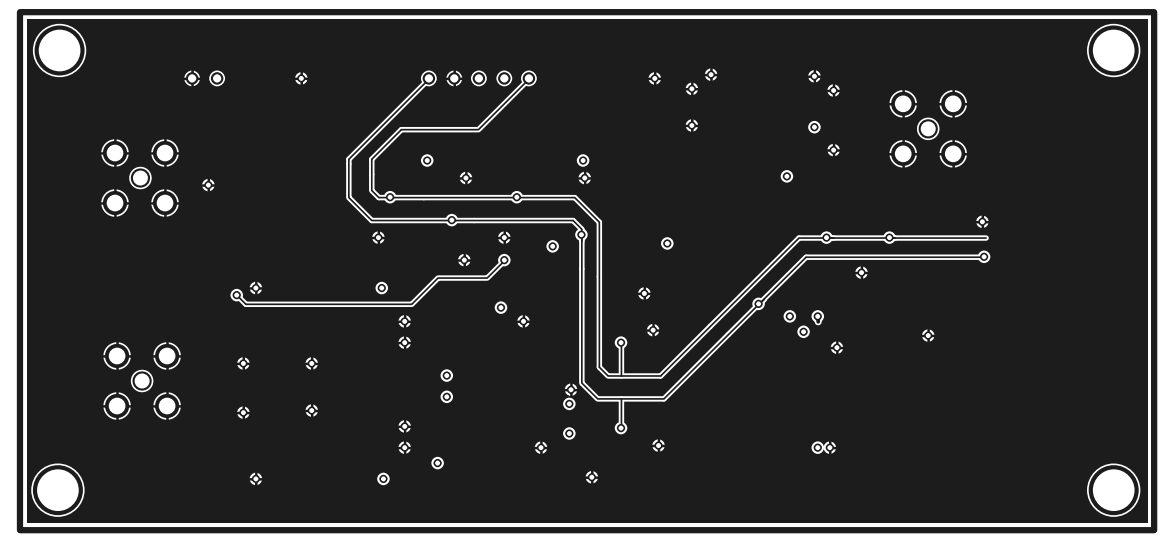

Phase Detection Board V2 (Bottom Layer) Scale 1:1 


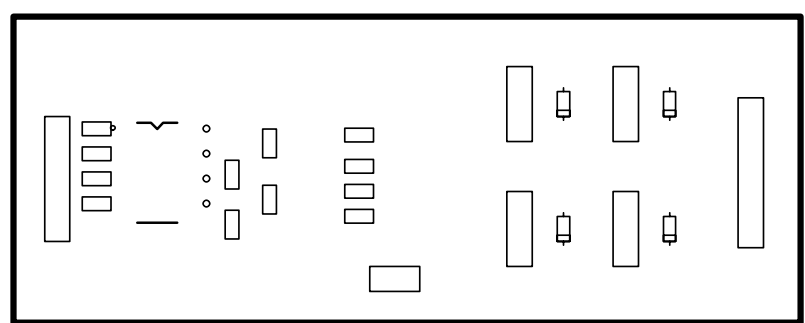

Stepper Motor Board V2 (Silk Screen Layer) Scale 1:1

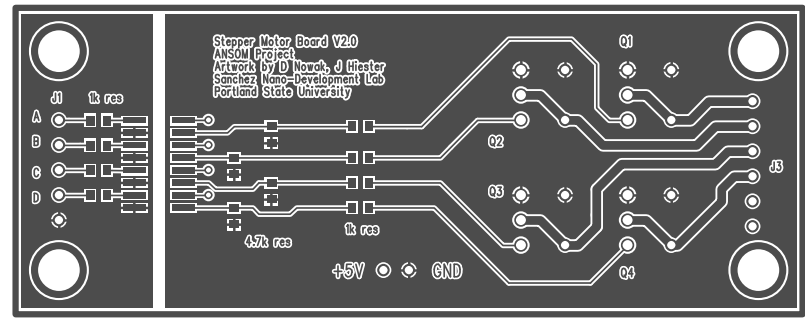

Stepper Motor Board V2 (Top Layer) Scale 1:1

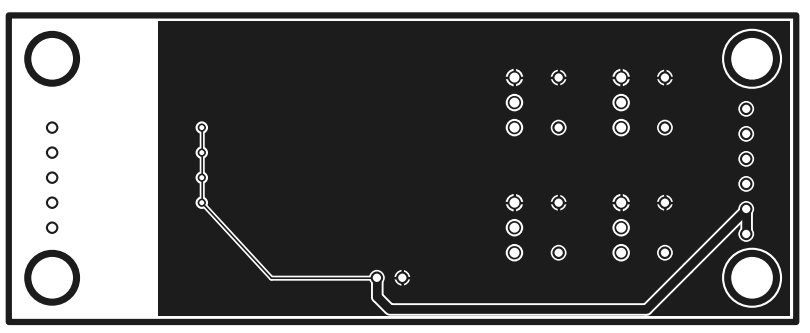

Stepper Motor Board V2 (Bottom Layer) Scale 1:1 


\section{C.4 Assembled Microscope Controller Prototype}

Microscope Controller Prototype
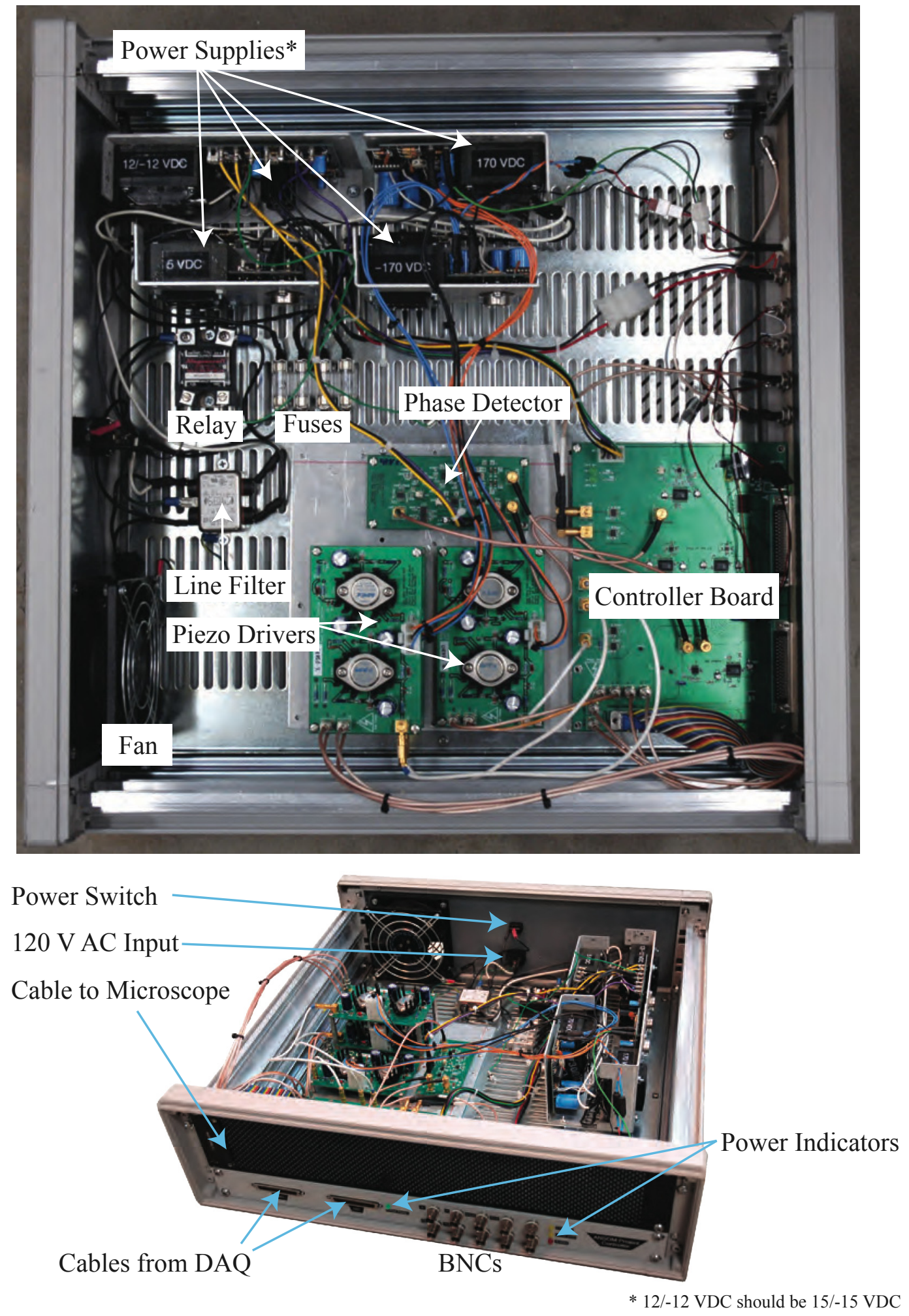


\section{APPENDIX D}

\section{Computer Programming Overview}

The graphical nature of LabVIEW programming can make code documentation a little tricky. More traditional text based programming languages are easier to document and present in a manuscript, but require a good understanding of syntax while keeping in mind the overall flow of the entire program. Either way, the complexity and amount of code necessary to document the complete ANSOM Project software would be difficult in the scope of this dissertation. Instead, the goal here is to present relevant tidbits of code that will help future developers understand the more complex, custom algorithms. The flow chart like design of the LabVIEW programming interface can easily be developed with a little programming experience. The overall project is outlined by a list of all vi's and FPGA DAQ definitions. This appendix then covers some of the important FPGA algorithms and the PID algorithm correction, followed by the Windows algorithms and finally the global constant file.

\section{D.1 ANSOM Project (Host) Files}

Table D.1 List of Windows LabVIEW files

ANSOM Entry

Ansom Entry.vi (requires Rcube software and IMAQ package)

Ansom Entry No Mono.vi (requires IMAQ)

Ansom Entry No Mono No Vision.vi

Ansom.rtm

First Level ANSOM Entry (Invoked from ANSOM entry programs.)

Oscillator Tune.vi

Z Control.vi

Stepper Motor Control.vi

Photon Counter.vi

Oscilloscope.vi

X-Y Piezo Offset.vi

Machine Vision.vi (requires IMAQ package)

Interaction Force.vi

Spectrum Analyzer.vi

Strip Chart.vi

Excitation Laser Power.vi

Image Processing.vi 
Scan Data Controls.vi

Second Level ANSOM Entry

Line Analyzer.vi

Background ANSOM Entry

DAQ to Phase Converter.vi

Write ANSOM Globals.vi

Read ANSOM Default Globals.vi

Panel to Graph Coords.vi

Write Global Parameters.vi

FPGA Initialize.vi

Intensity Graph ScalValues.vi

Read ANSOM Globals.vi

DAQ to Voltage Converter Arrays.vi

DAQ to Voltage Converter.vi

Hex Conversion.vi

AD9835 Command Generator.vi

Decimal to Hex Conversion.vi

$D L L ' s$

LabViewLayer.dll

lvStorage.dll

Note: Italicized names are project directories. This is a partial list of the all the required files. Some files are embedded with the LabVIEW installation and are not included in this list. A typical complication with a new LabVIEW installation involves setting up how the project locate these files. Searching for the files manually can be an option.

\section{D.2 FPGA (Embedded Target) Files and Dependencies}

Table D.2 FPGA Project files and FIFO structures for DMA transfers.

$\begin{array}{ll}\text { ANSOM(fpga).vi } & \text { Main Program } \\ \text { ANSOM_PID.vi } & \text { PID Algorithm* } \\ \text { ANSOM_PID_sub.vi } & \text { Sub routine for PID Algorithm* } \\ \text { DDS Commands FIFO } & \text { Host to Target - DMA U32 8197 } \\ \text { Data FIFO } & \text { Target to Host - DMA U32 4095 } \\ \text { Freq Sweep FIFO } & \text { Target to Host - DMA U32 1023 } \\ \text { *These VIs were modified from the NI provided PID toolkit. }\end{array}$




\section{D.3 FPGA DAQ Channel Descriptors}

Table D.3 Analog input channels for ANSOM Project.

\section{Analog Input}

Tuning Fork Voltage RMS

Tuning Fork Phase

(J19-1) Photo-Diode

PMT Voltage

(J19-3) AI4

(J19-5) AI5

(J19-7) AI6

(J19-3) AI7
DAQ

Connector0 / AI0

Connector0 / AI1

Connector0 / AI2

Connector0 / AI3

Connector0 / AI4

Connector0 / AI5

Connector0 / AI6

Connector0 / AI7
Description

RMS signal from the AD637 IC.

From Phase Detection Board.

For photo-diode laser power meter.*

(Not used - wired to breakout board)

Expansion connector J19 pin 3.

Expansion connector J19 pin 5.

Expansion connector J19 pin 7.

Expansion connector J19 pin 9.

Table D.4 Analog output channels for ANSOM Project.

$\quad$ Analog Output
Z-Piezo
X-axis ScanStage
Y-axis ScanStage
X-axis Offset
Y-axis Offset
X-offset Piezo
Y-offset Piezo
(J9-1) AO7

DAQ Connector0 / AO0

Connector0 / AO1 Connector0 / AO2 Connector0 / AO3 Connector0 / AO4 Connector0 / AO5 Connector0 / AO6 Connector0 / AO7
Description

Low voltage $Z$ piezo signal.

Low voltage $X$ raster scan signal.

Low voltage $Y$ raster scan signal.

Low voltage $\mathrm{X}$ scan offset signal.

Low voltage $Y$ scan offset signal.

Low voltage $X$ piezo-stack offset.

Low voltage Y piezo-stack offset.

Expansion point, connector J9 pin 1.

Table D.5 Digital input and output channels for ANSOM Project.

\begin{tabular}{|c|c|c|}
\hline Digital Input and Output & DAQ & Description \\
\hline APD Count & Connector0 / DIO0 & TTL pulses from APD or PMT \\
\hline APD On & Connector0 / DIO1 & APD interlock \\
\hline Light Box Interlock & Connector0 / DIO2 & $\begin{array}{l}\text { Interlock for ambient light levels } \\
\text { (Not currently used) }\end{array}$ \\
\hline Z UpperLimit Switch & Connector0 / DIO3 & $\begin{array}{l}\text { Interlock for scan head translation } \\
\text { stage upper limit. } \\
\text { (Not currently used) }\end{array}$ \\
\hline Z LowerLimit Switch & Connector0 / DIO4 & $\begin{array}{l}\text { Interlock for scan head translation } \\
\text { stage lower limit. } \\
\text { (Not currently used) }\end{array}$ \\
\hline Scanhead LED & Connector0 / DIO5 & $\begin{array}{l}\text { Toggles on/off illumination LED } \\
\text { on scan head. }\end{array}$ \\
\hline Z Resolution & Connector0 / DIO6 & Toggles $Z$ resolution \\
\hline
\end{tabular}




\begin{tabular}{|c|c|c|}
\hline Scanhead Laser & Connector0 / DIO7 & $\begin{array}{l}\text { Toggles on/off scan head laser. } \\
\text { (Not currently used) }\end{array}$ \\
\hline Scanhead Camera & Connector0 / DIO8 & Toggles on/off scan head camera. \\
\hline $1 \mathrm{~A}$ & Connector1 / DIO0 & Stepper Motor Phase 1A \\
\hline $1 \mathrm{~B}$ & Connector1 / DIO1 & Stepper Motor Phase 1B \\
\hline $2 \mathrm{~A}$ & Connector1 / DIO2 & Stepper Motor Phase 2A \\
\hline $2 \mathrm{~B}$ & Connector1 / DIO3 & Stepper Motor Phase 2B \\
\hline SClk AD9835 & Connector1 / DIO6 & SPI signal SClk to AD9835 IC \\
\hline SData AD9835 & Connector1 / DIO7 & SPI signal SData to AD9835 IC \\
\hline FSync AD9835 & Connector1 / DIO8 & SPI signal FSync to AD9835 IC \\
\hline FSelect AD9835 & Connector1 / DIO9 & SPI signal FSelect to AD9835 IC \\
\hline SDI AD5290 OSC & Connector1 / DIO11 & $\begin{array}{l}\text { SPI signal SDI to AD5290 IC for } \\
\text { tuning fork oscillator. }\end{array}$ \\
\hline Clk AD5290 OSC & Connector1 / DIO12 & $\begin{array}{l}\text { SPI signal Clk to AD5290 IC for } \\
\text { tuning fork oscillator. }\end{array}$ \\
\hline CS AD5290 OSC & Connector1 / DIO13 & $\begin{array}{l}\text { SPI signal CS to AD5290 IC for } \\
\text { tuning fork oscillator. }\end{array}$ \\
\hline CS AD5290 Scan & Connector1 / DIO16 & $\begin{array}{l}\text { SPI signal CS to AD5290 IC for } \\
\text { raster scan signal. }\end{array}$ \\
\hline Clk AD5290 Scan & Connector1 / DIO17 & $\begin{array}{l}\text { SPI signal Clk to AD5290 IC for } \\
\text { raster scan signal. }\end{array}$ \\
\hline SDI AD5290 Scan & Connector1 / DIO18 & $\begin{array}{l}\text { SPI signal SDI to AD5290 IC for } \\
\text { raster scan signal. }\end{array}$ \\
\hline (J10-9) Shutter & Connector1 / DIO21 & $\begin{array}{l}\text { Control logic for external laser } \\
\text { shutter.* }\end{array}$ \\
\hline$(\mathrm{J} 10-8) \mathrm{ELS}$ & Connector1 / DIO22 & $\begin{array}{l}\text { Control logic for external light } \\
\text { source.* }\end{array}$ \\
\hline$(\mathrm{J} 10-7)$ & Connector1 / DIO23 & Expansion (DIO) \\
\hline$(\mathrm{J} 10-6)$ & Connector1 / DIO24 & Expansion (DIO) \\
\hline$(\mathrm{J} 10-5)$ & Connector1 / DIO25 & Expansion (DIO) \\
\hline$(\mathrm{J} 10-4)$ & Connector1 / DIO26 & Expansion (DIO) \\
\hline (J10-3) & Connector1 / DIO27 & Expansion (DIO) \\
\hline$(\mathrm{J} 10-2)$ & Connector1 / DIO29 & Expansion (DIO) \\
\hline$(\mathrm{J} 10-1)$ & Connector1 / DIO31 & Expansion (DIO) \\
\hline Controller On & Connector1 / DIO39 & $\begin{array}{l}\text { Toggles on/off relay for HV } \\
\text { power supplies in controller. }\end{array}$ \\
\hline Filter Wheel (1) 1A & Connector2 / DIO0 & Stepper Motor Phase 1A* \\
\hline Filter Wheel (1) 1B & Connector2 / DIO1 & Stepper Motor Phase 1B* \\
\hline Filter Wheel (1) 2A & Connector2 / DIO2 & Stepper Motor Phase 2A* \\
\hline Filter Wheel (1) 2B & Connector2 / DIO3 & Stepper Motor Phase 2B* \\
\hline Filter Wheel (2) $1 \mathrm{~A}$ & Connector2 / DIO4 & Stepper Motor Phase 1A* \\
\hline Filter Wheel (2) 1B & Connector2 / DIO5 & Stepper Motor Phase 1B* \\
\hline Filter Wheel (2) 2A & Connector2 / DIO6 & Stepper Motor Phase 2A* \\
\hline Filter Wheel (2) 2B & Connector2 / DIO7 & Stepper Motor Phase 2B* \\
\hline
\end{tabular}




\section{D.4 FPGA (Embedded Target) Algorithms}

\section{Counters (for photon counting)}

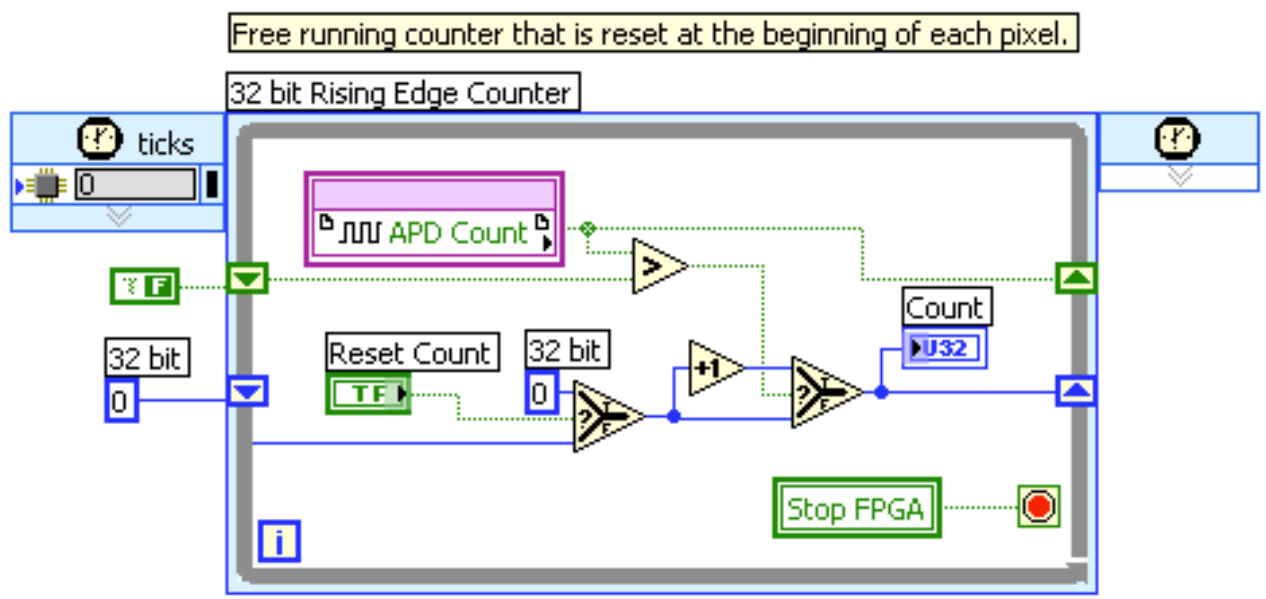

This free running counter is reset at the beginning of each new pixel.

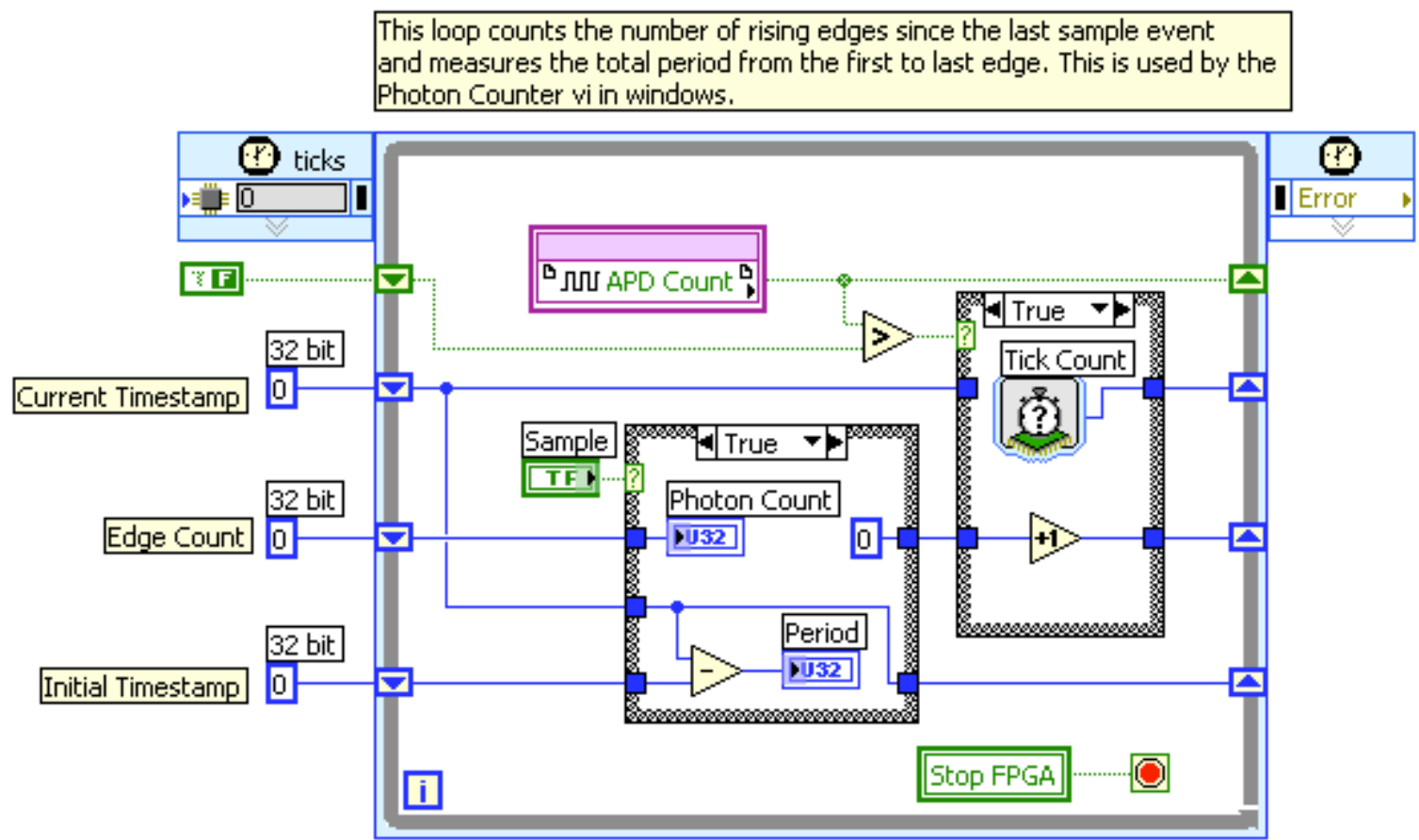

This counter runs until a sample is requested, at which time a counts per second is calculated from the period of time elapsed. 


\section{Serial Peripheral Interface (SPI)}
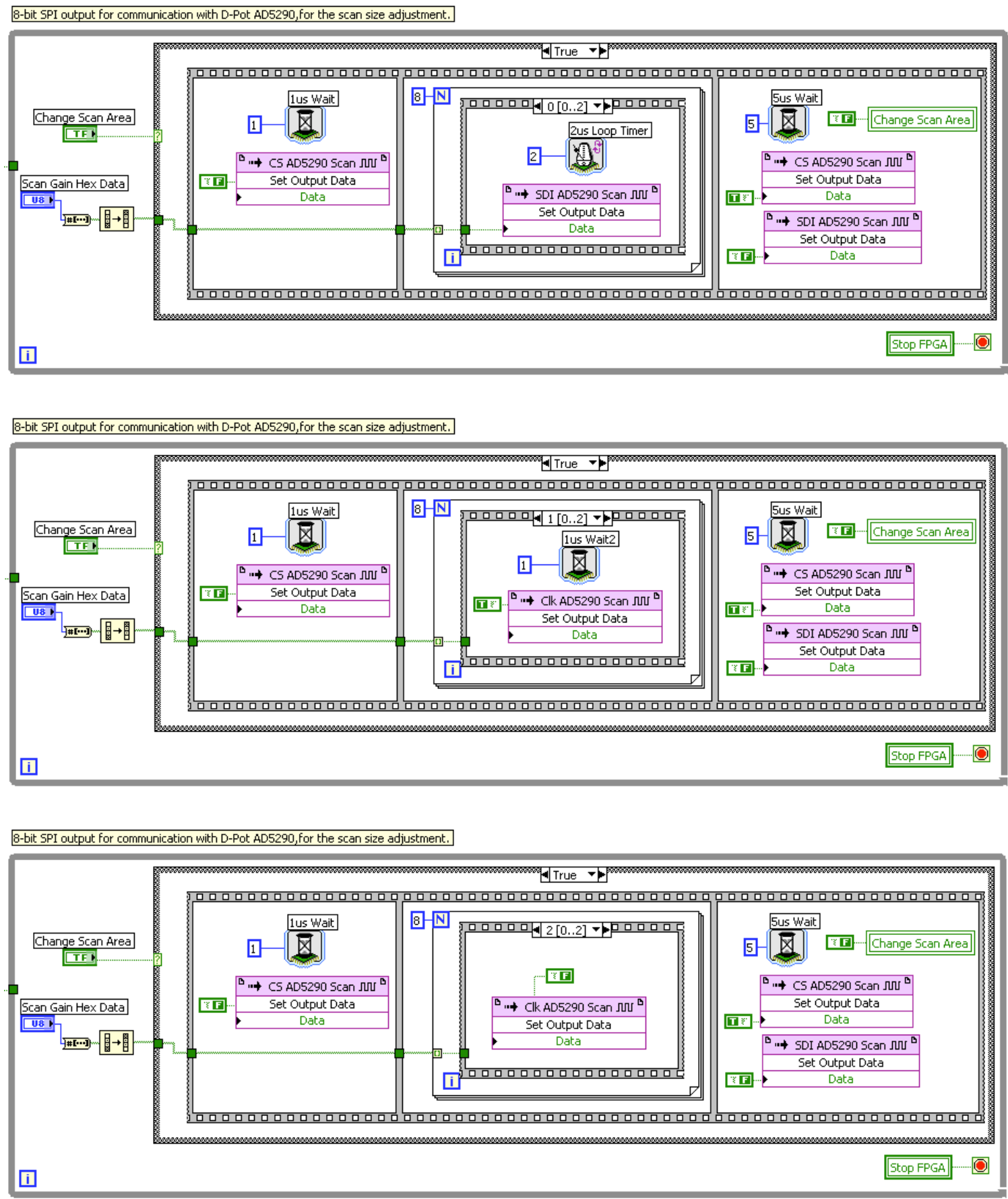

Controls the timing requirements for SPI communication. 


\section{Stepper Motor Algorithm}
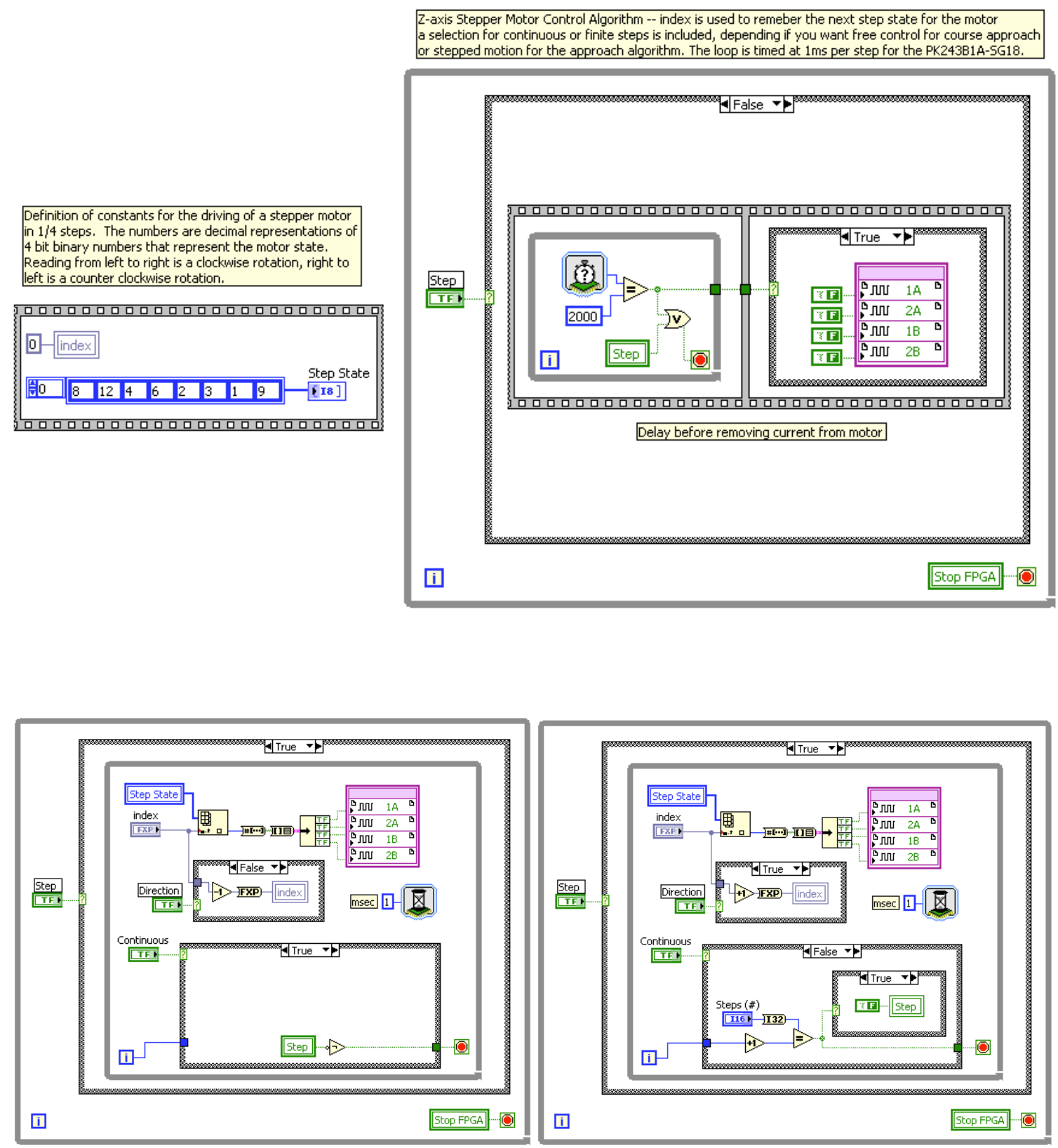

The stepper motor algorithm can be used as generic $1 / 4$ step logic for stepper motor movement. The only change needed is the delay time between steps that best suits the motor to be used. 


\section{Synchronized Data Collection}
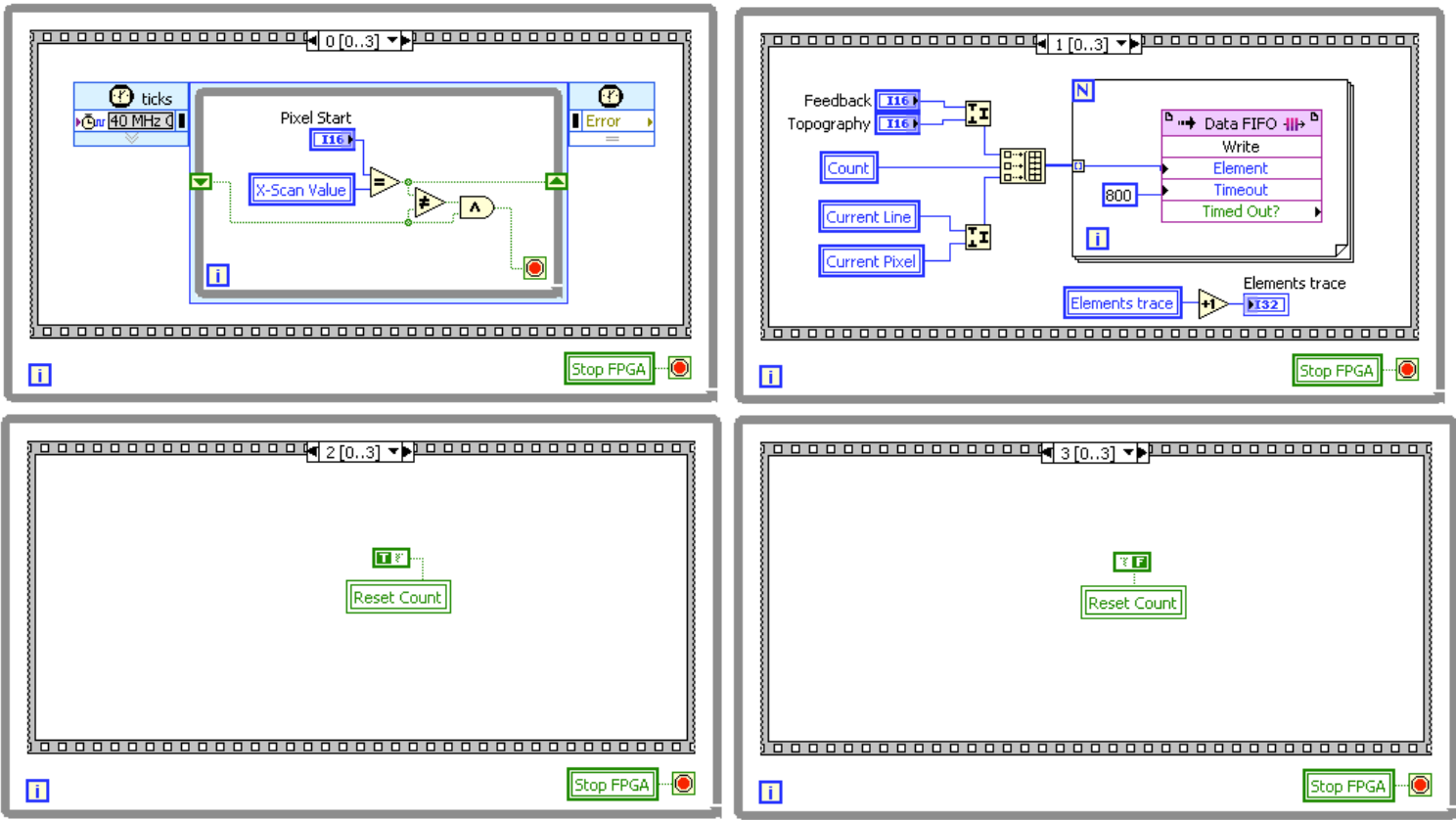

The collection of data is synchronized by a single cycle loop that checks to see if the condition for data recording has occurred. The loop is then stopped and the appropriate values are recorded to the DMA FIFO.

\section{Dynamic Variable Timing Control}

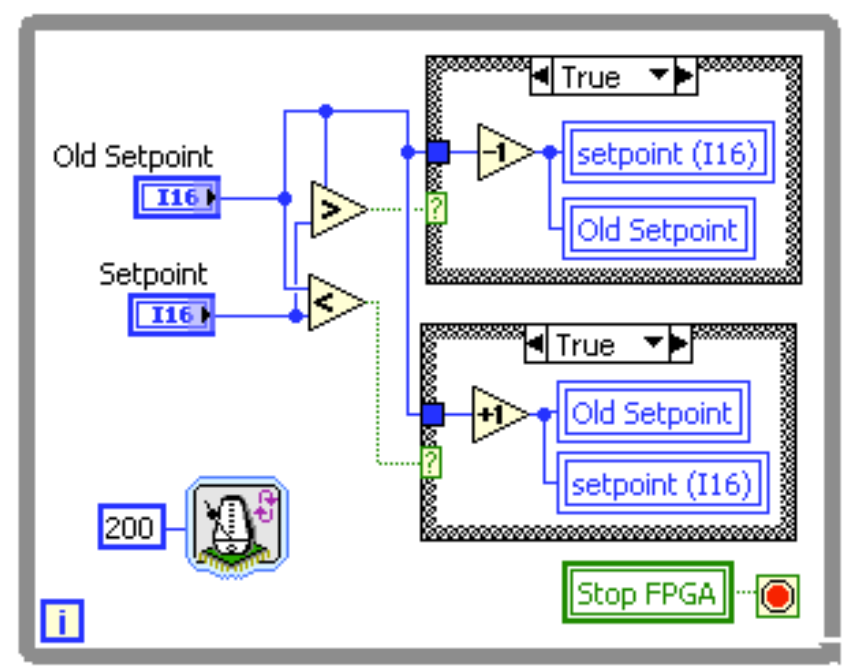

Dynamic timing of variables prevents damage to the microscope by softening any abrupt changes in scanning parameters. 


\section{D.5 FPGA PID Algorithm Correction}

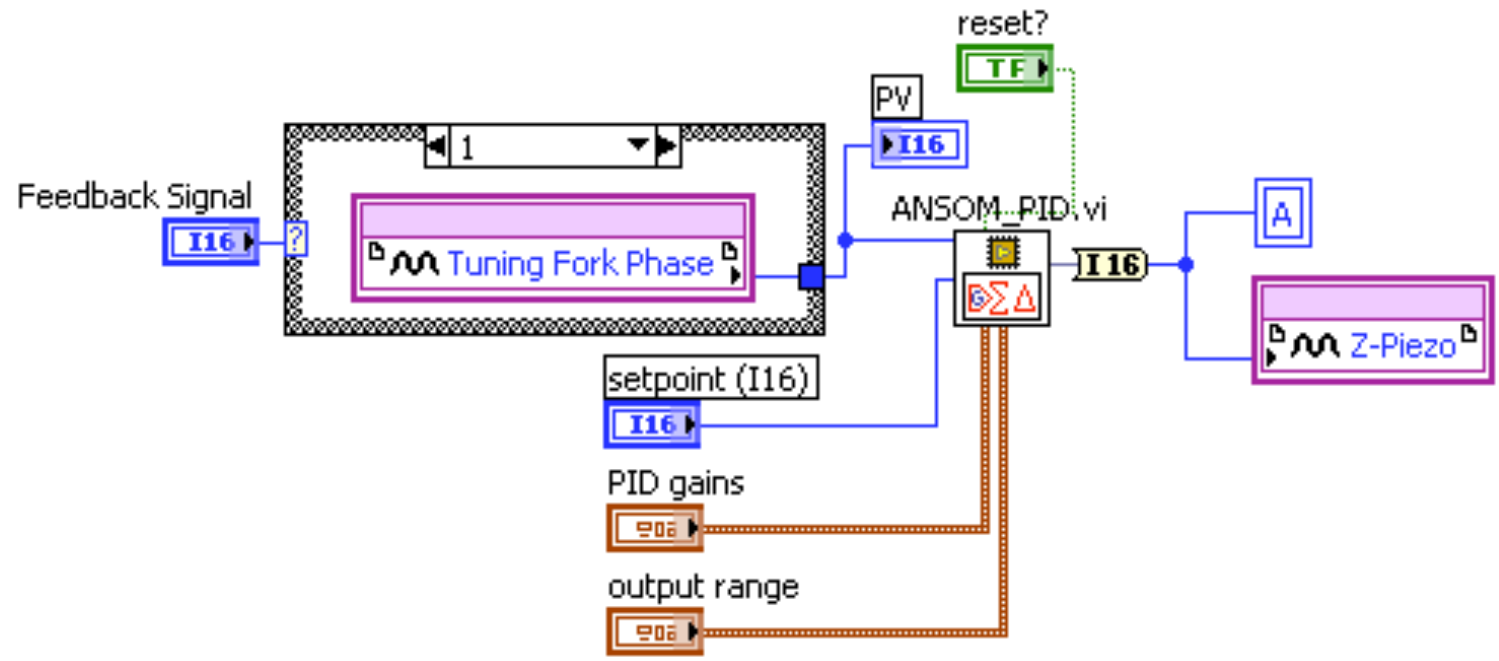

This is the FPGA code that interacts with the prepackaged National Instruments PID VI.

(Here the VI was renamed to ANSOM_PID.vi).

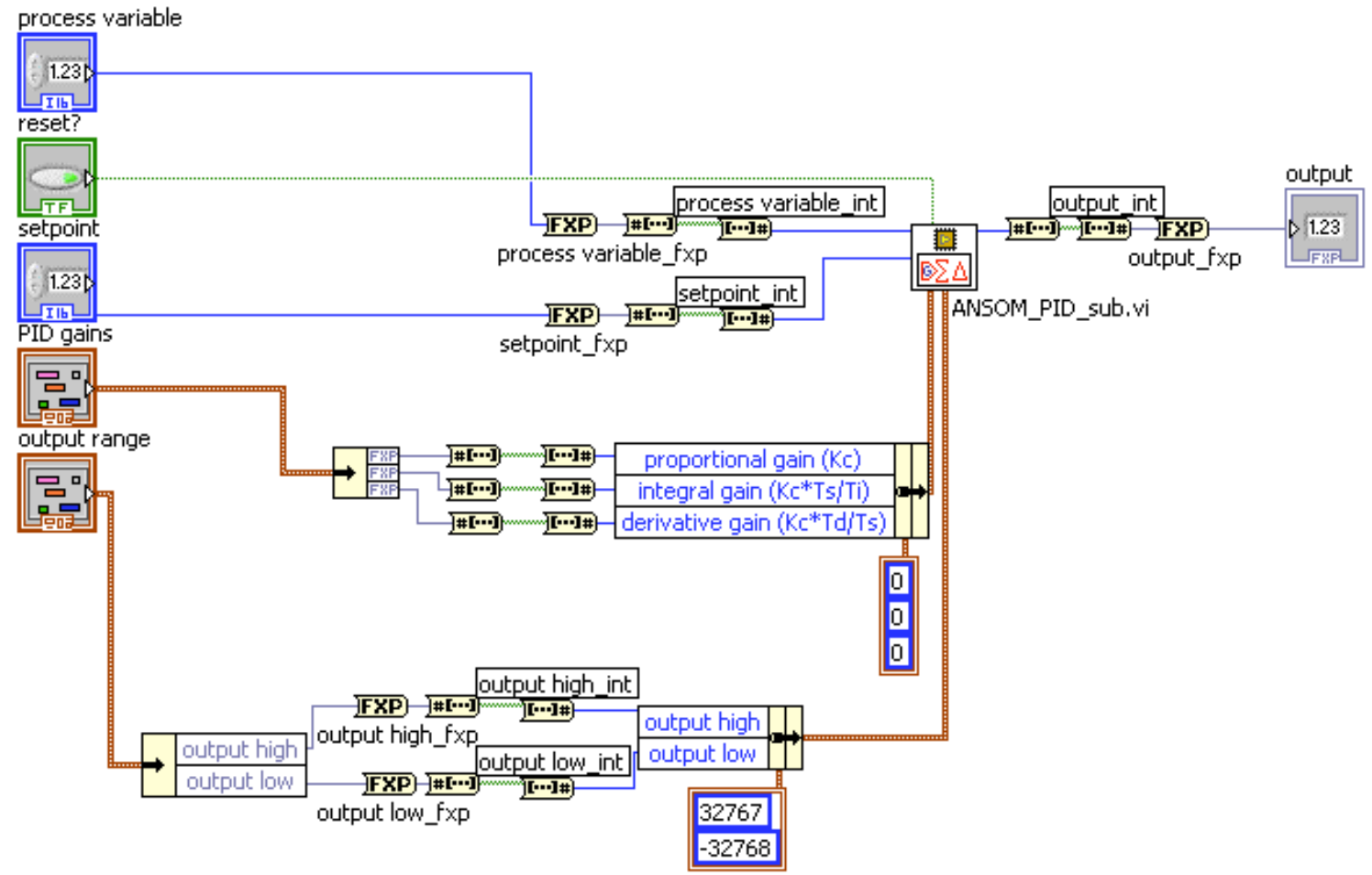

The LabVIEW Code for ANSOM_PID.vi 


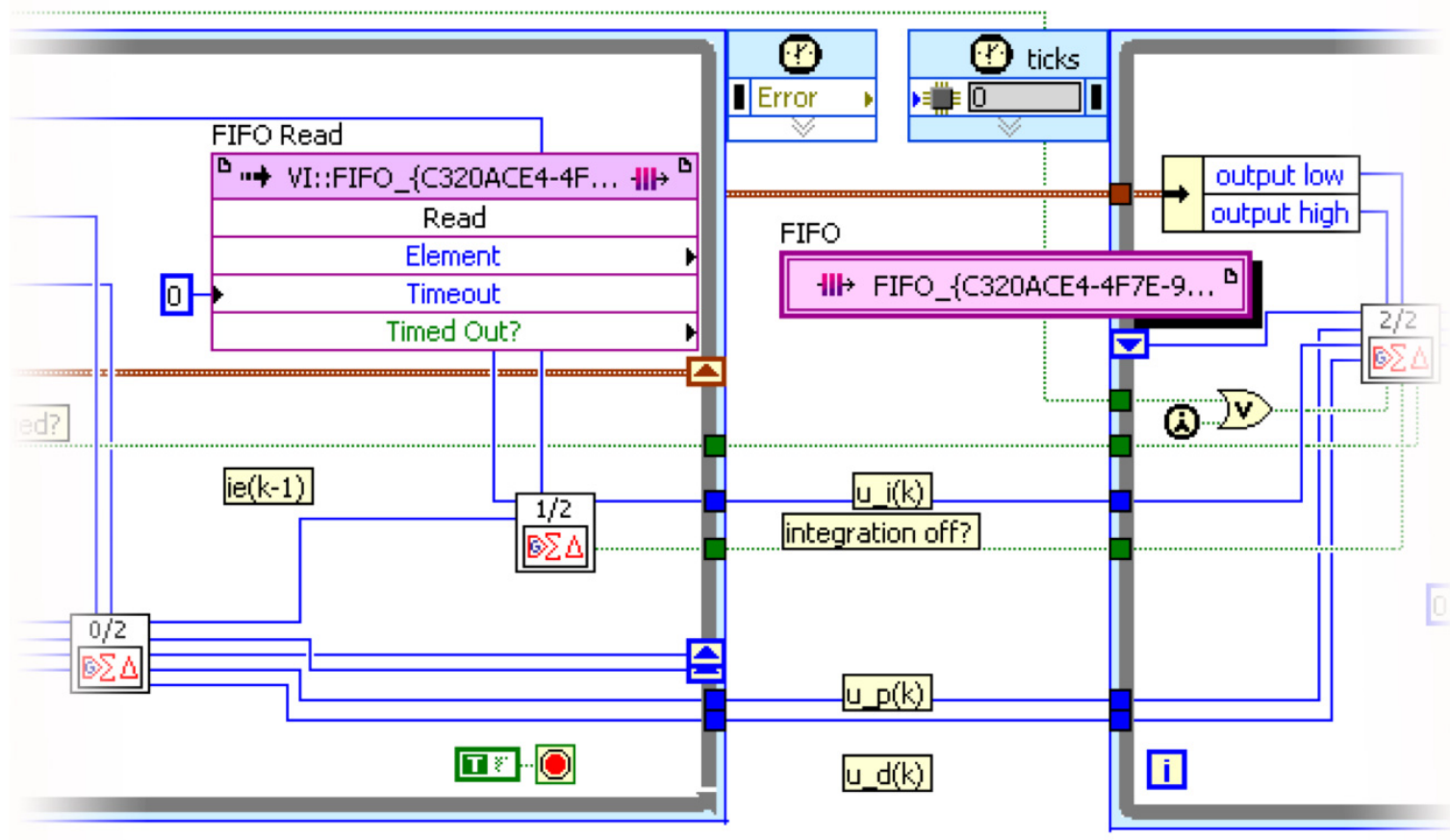

A section of the LabVIEW Code for ANSOM PID sub.vi. The PID library labeled $1 / 2$ is the integral term that needs to be adjusted for the correct integral action for the scanning probe microscope.

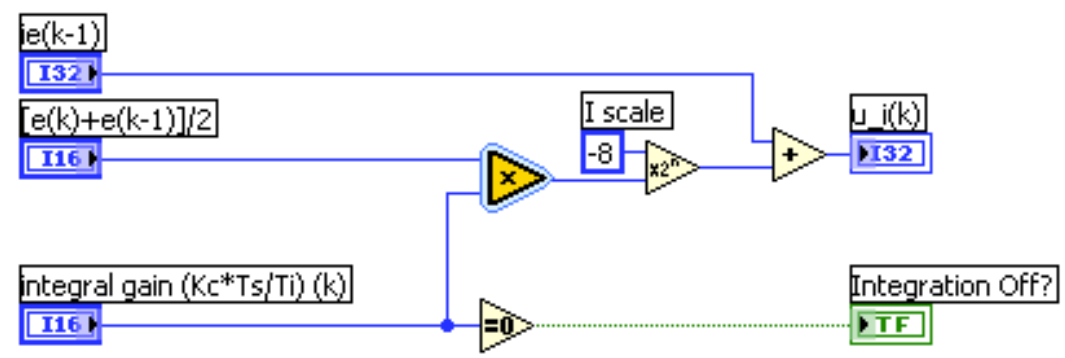

The LabVIEW code for the PID library labeled 1/2.

The I scale term is adjusted from -1 to -8 to improve the response of the integral term in the PID loop. 


\section{D.6 Host Algorithms}
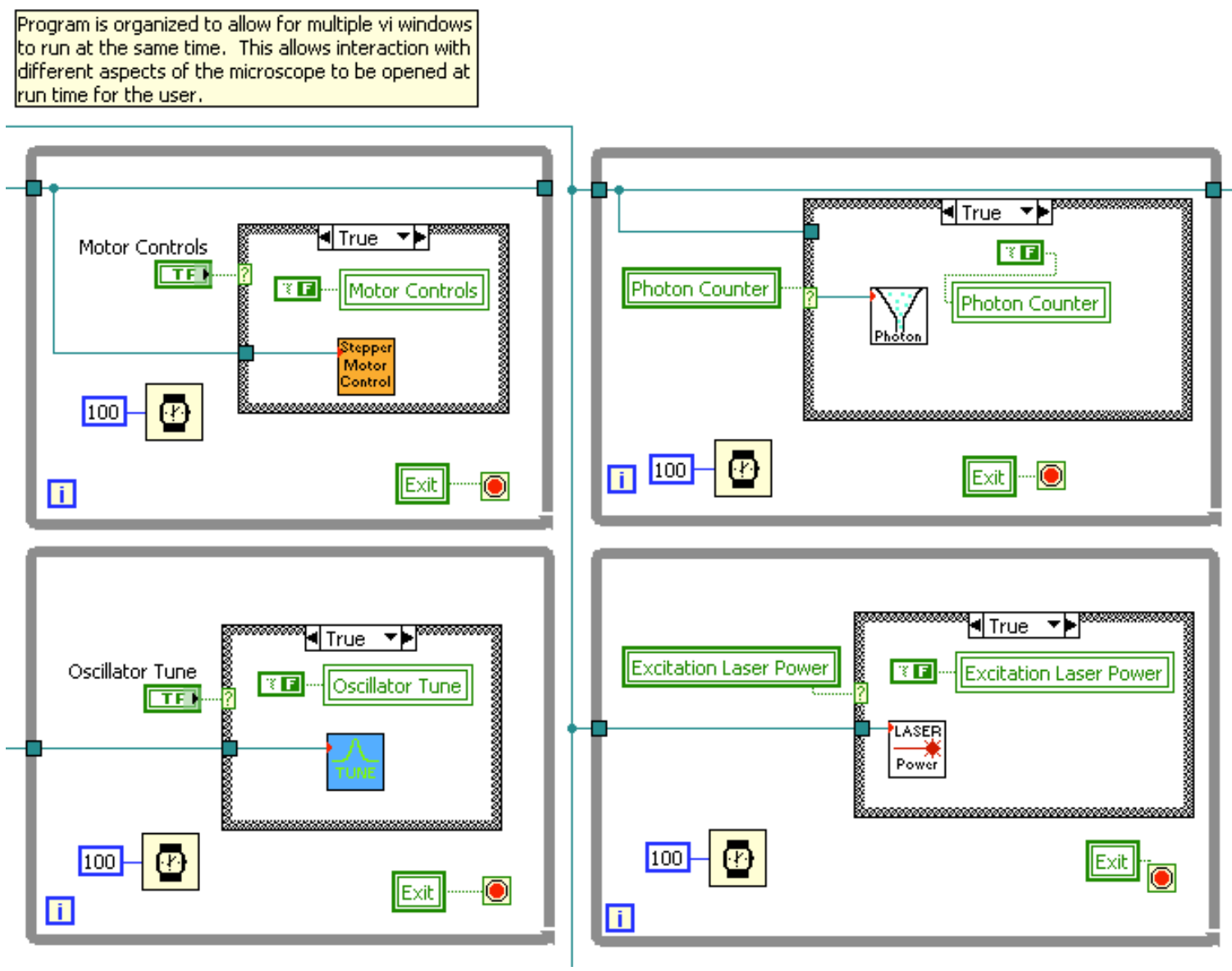

Using multiple While Loops with delay timers allow for the execution of multiple subVIs to create a multiple windowed GUI for the operation of the microscope.

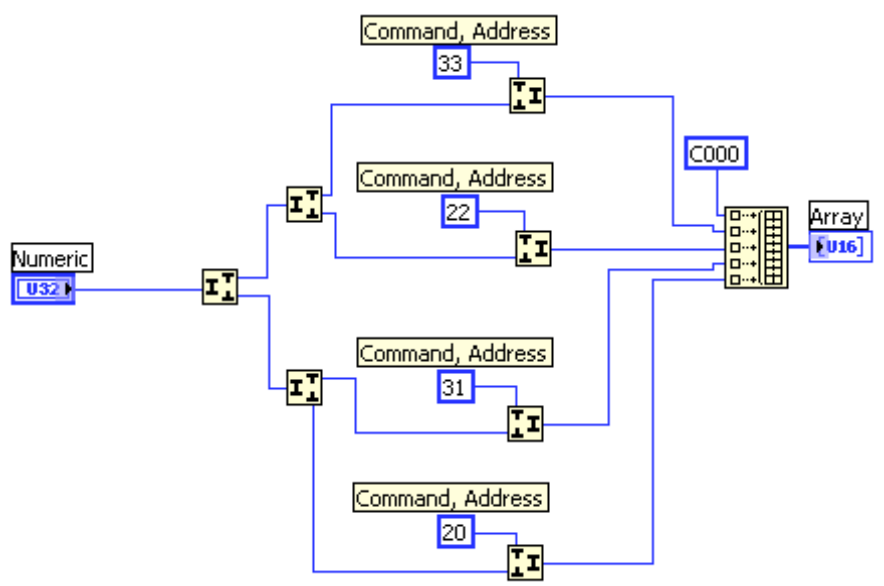

This algorithm builds the 32bit HEX words for the DDS IC. 


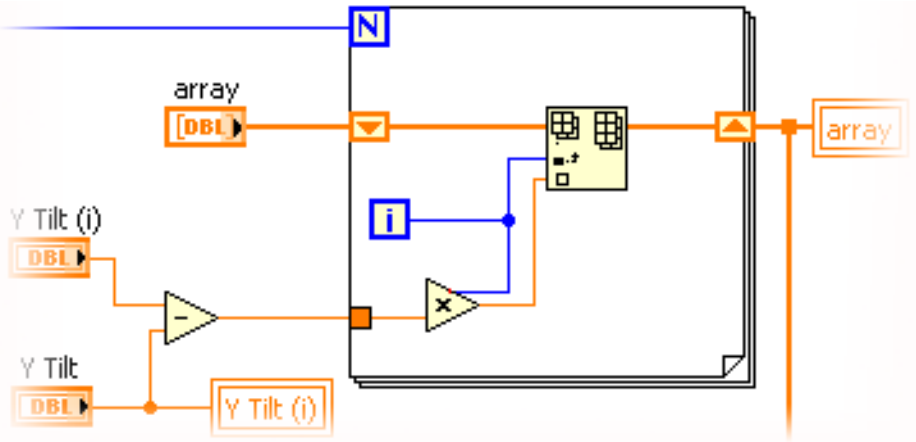

Using shift registers (arrows one points up and the other points down on the For Loop) to build arrays. This is much more efficient in CPU cycles than calling a local variable for the array in the for loop.

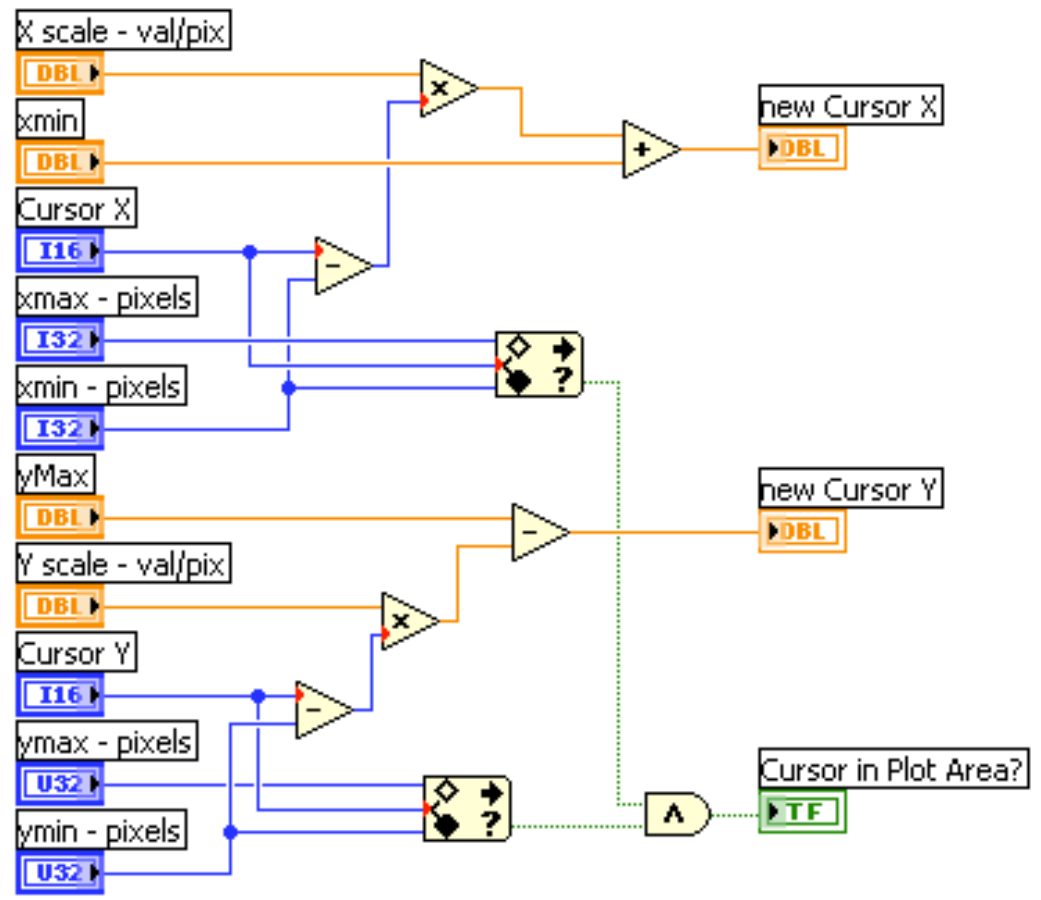

Routine that determines the location of a mouse click over a graph area. The goal is to determine what the actual coordinates of the click are. This allows the user to interact with graphs for real time microscope control. 


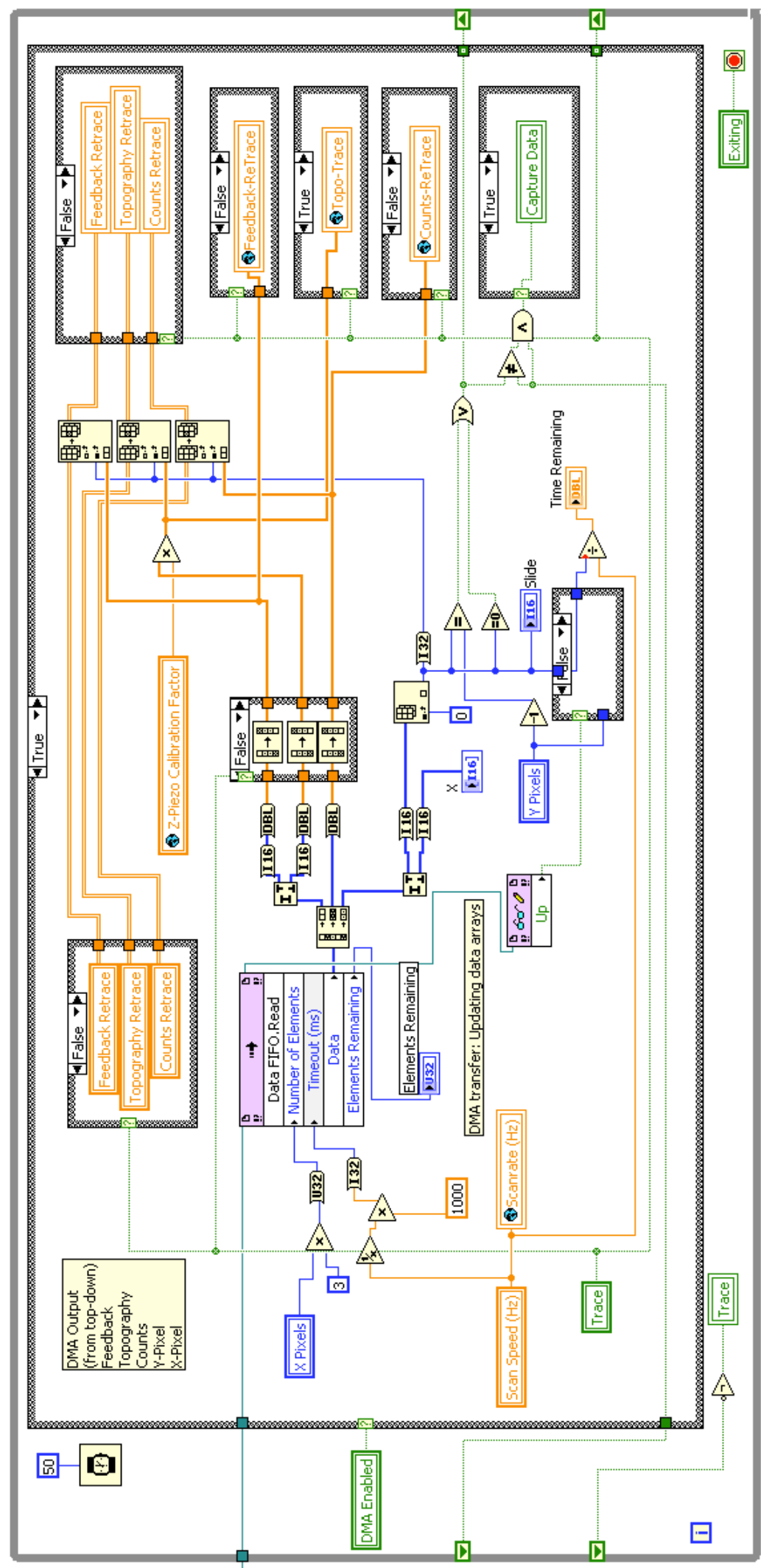

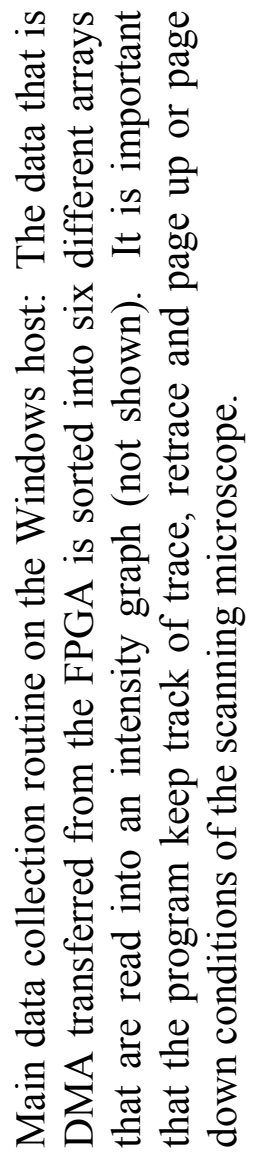




\section{D.7 Global Constants File}

Global Variable Settings ANSOM Project SPM v2 Software Sanchez Nano-Development Lab, Portland State University www.ansom.research.pdx.edu

$\begin{array}{ll}\text { Data Type } & \text { Feedback Trace } \\ \text { Date (m/d/y) } & 5 / 3 / 2010 \\ \text { Time (12h) } & 5: 42 \text { PM } \\ \text { Username } & \\ \text { Comments } & \\ \text { Pixels (x) } & 512.000000 \\ \text { Pixels (y) } & 512.000000 \\ \text { Scan area (x) } & 80.233000 \\ \text { Scan area (y) } & 80.233000 \\ \text { Scanrate (Hz) } & 0.500000 \\ \text { Proportional } & 0.400000 \\ \text { Integral } & 0.021000 \\ \text { Differential } & 0.000000 \\ \text { Output Gain } & 0.000000 \\ \text { Averages } & 0.000000 \\ \text { Bit Resolution } & 0.000000 \\ \text { Z-Bandwidth } & 0.000000 \\ \text { Z Polarity } & \text { Negative } \\ \text { HighRes Z } & \text { False } \\ \text { Feedback } & \text { Phase } \\ \text { Setpoint } & 0.000000 \\ \text { Drive Freq } & 0.000000 \\ \text { Drive Voltage } & 0.000000 \\ \text { Forward Speed } & 0.000000 \\ \text { Back Speed } & 0.000000 \\ \text { Motor Steps } & 3.000000 \\ \text { Step Delay } & 0.000000 \\ \text { Z Piezo Cal } & 0.000000 \\ \text { X Piezo Cal } & 0.000000 \\ \text { Y Piezo Cal } & 0.000000 \\ \text { X Scan-bed Cal } & 0.000000 \\ \text { Y Scan-bed Cal } & 0.000000 \\ \text { Laser Power } & 1671.600000 \\ \text { CW Laser Cal } & 0.420000 \\ \text { Pulsed Cal } & 0.028000 \\ \text { CW / Pulsed } & \text { CW } \\ \text { Wavelength } & 800 \\ \text { Whe } & \end{array}$




\section{APPENDIX E}

\section{Fabrication of Substrates for Nanometer Relocation of Samples}

(Adapted from: "Fabrication of a versatile substrate for finding samples on the nanometer scale", Journal of Microscopy, Vol. 230, Pt I 2008, pp. 32-41)

\section{Experimental Method}

The FIB system utilized in this study is a combination of two systems for comparison, an FEI / Micrion 2500 (Micrion hereafter in this article) and an FEI dualbeam Strata DB-237 (DB-237). Both systems utilize a liquid metal $\left(\mathrm{Ga}^{+}\right)$ion gun source. The Micrion FIB generates a $\mathrm{Ga}^{+}$beam that is accelerated to a maximum potential of 50 $\mathrm{kV}$ and the DB-237 to $30 \mathrm{kV}$. The beam current reaching the specimen ranges from 0.5 $\mathrm{pA}$ to $35 \mathrm{nA}$ and $1 \mathrm{pA}$ to $10 \mathrm{nA}$ respectively. For all the experiments the beam extraction current is in the range of 2.0 to $2.2 \mu \mathrm{A}$ to maintain the stability of the emitter [7] as well as to extend the lifetime of the Ga reservoir while maintaining a low energy spread. The secondary electrons liberated from the surface by the ions are collected for imaging.

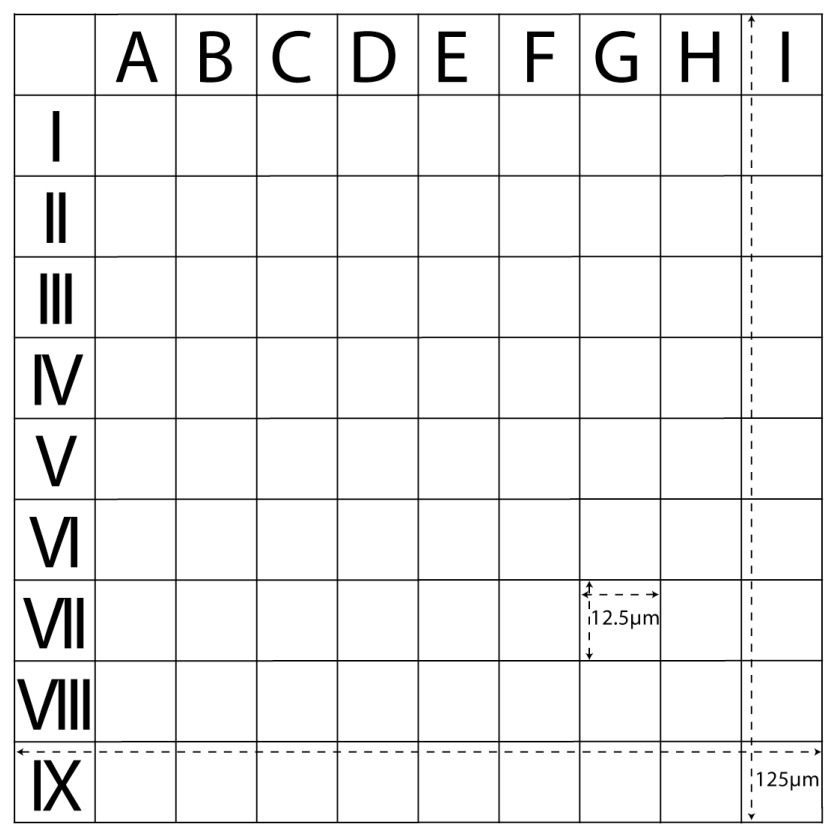

Figure 1. Grid pattern utilized by the FIB milling programs, the outer dimension is $(125 \times 125) \mu \mathrm{m}^{2}$, the inner grid dimension is $(12.5 \times 12.5)$ $\mu \mathrm{m}^{2}$. 
Beam currents are varied from $100 \mathrm{pA}-30 \mathrm{nA}$ for milling and reduced to $1-15 \mathrm{pA}$ for imaging in order to minimize damage by the ion beam, with spot sizes of $\sim 5-25 \mathrm{~nm}$ (current depending). The type of material and dose rate of the ion beam determines the milling rate of the FIB. The DB-237 has the added advantage of an additional electron beam that operates as a field emission scanning electron microscope (FESEM). This allows nondestructive, high-resolution sample imaging during the ion milling process.

Due to the lack of user friendly interface for the Micrion FIB system, an in-house Visual Basic ${ }^{\mathrm{TM}}$ (VB) (Microsoft) Program was developed to create complex patterns for the for Micrion system. Using this VB program, an alphanumeric grid was created and is transferred into the FIB's memory as a saved pattern, demonstrated in Figure 1. To demonstrate the reproducibility of this technique, gold nanoparticles $(\sim 25 \mathrm{~nm}$ in size) prepared by the citrate reduction method [1] are placed on 75 mesh Formvar ${ }^{\circledR}$ and carbon coated TEM grid made by Ted Pella (Prod No. 01802-F). By dipping the grid into the nanoparticle solution and allowing the sample to air dry for approximately 30 minutes, colloidal nanoparticles are left on the surface of the Formvar ${ }^{\mathbb{B}}$ coating.

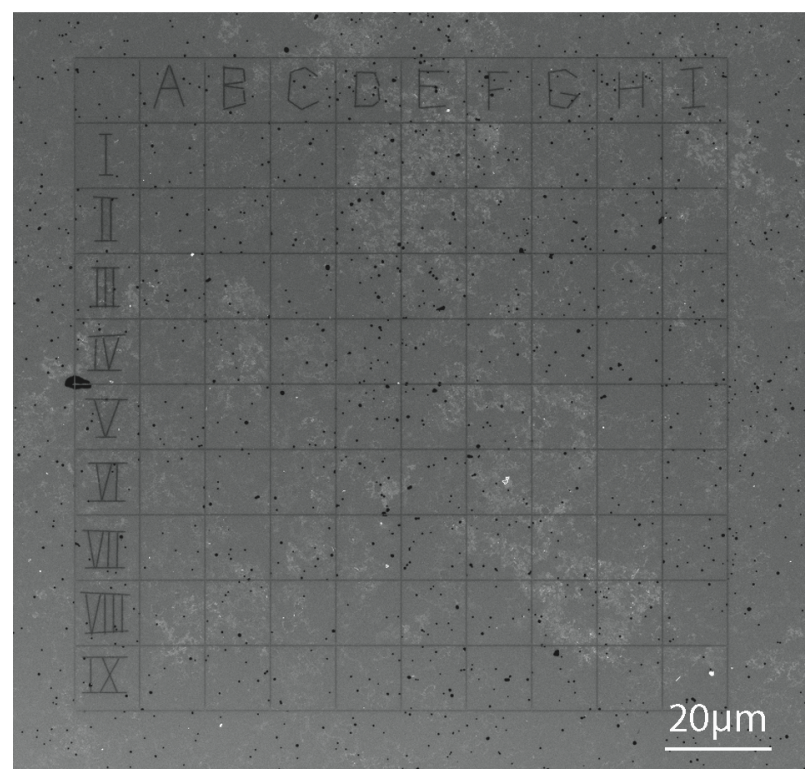

Figure 2: Ion induced secondary electron micrograph of a milled grid pattern after milling was completed with nanoparticles present. The black dots are caused by inhomogeneities in the carbon coating on the Formvar $^{\circledR}$ film.

Imaging and milling in the FIB requires one to use low beam currents, typically $10 \mathrm{pA}$ or less, and these beam currents are also suitable for milling. One should avoid imaging on one location of the grid for long periods of time especially at high magnifications since the ion beam will cut through eventually. This lends to a perfect fit with a dual beam system where the user can find the location of interest with the electron beam then mill the pattern of choice by only changing the angle at the eucentric height. 
Once selected, a portion of the Formvar ${ }^{\circledR}$ coating is milled in a serpentine mode (triangle wave scanning, as opposed to raster or saw tooth) with a dosage of $0.24 \mathrm{nC} / \mu \mathrm{m}^{2}$, dwell time of $1 \mathrm{~ms}, 1 \mathrm{~nm} \mathrm{X}$ and $\mathrm{Y}$ dwell spacing and a working distance of $21 \mathrm{~mm}$. Exceeding the $0.24 \mathrm{nC} / \mu \mathrm{m}^{2}$ dosage can cause the beam to mill through the Formvar ${ }^{\circledR}$ or substantially weaken the film. Since the film has a thickness range typically of $30-70 \mathrm{~nm}$ there is some room to increase or decrease this value of dosage if needed. Figures 2 and 3 demonstrate a typical grid milling. In terms of charging, the sample behaves well without additional coatings of carbon or other metals. Although not necessary in the Formvar ${ }^{\mathbb{R}}$ portion of this work, for ultra-precise alteration, a charge-neutralizing flood gun (found in FIB systems) could be used and for bulk glass substrates is preferred. Once the grid is fabricated, the sample is available for AFM as shown in figure 4, SEM, TEM, and optical studies.
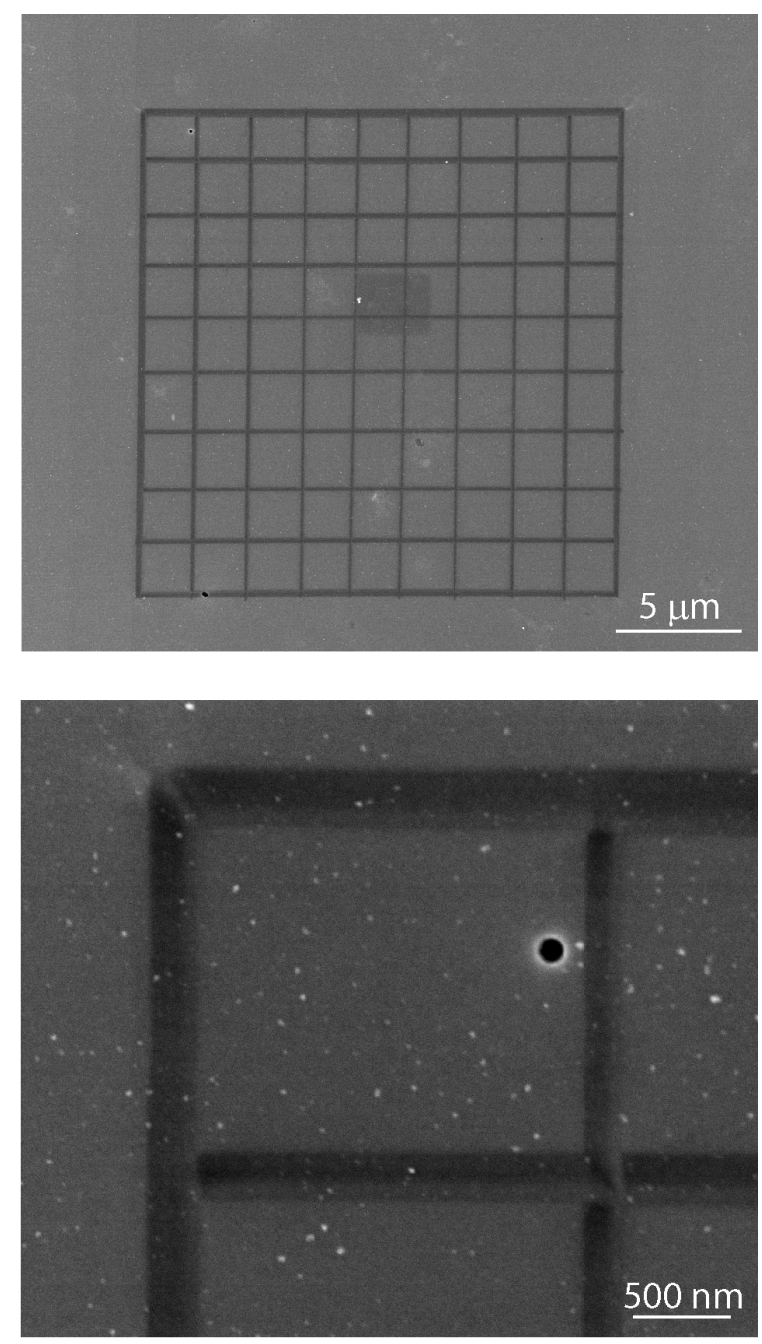

Figure 3: Bright field STEM micrographs acquired with the electron column on the FEI DB-237 Dual Beam in STEM mode, with acceleration of $30 \mathrm{kV}$, WD of $5 \mathrm{~mm}$. Grid is $(20 \times 20) \mu \mathrm{m}^{2}$ with inter grid spacing of $(2.2 \times 2.2) \mu \mathrm{m}^{2}$. 


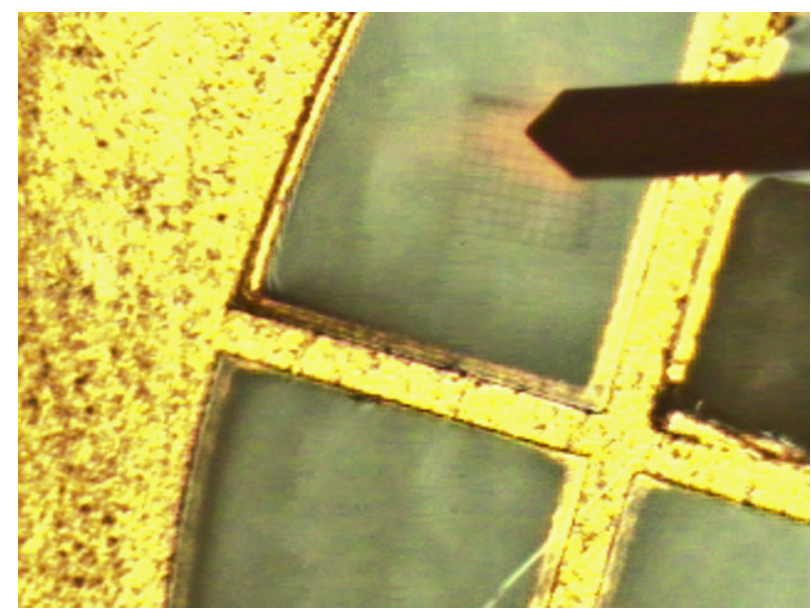

Figure 4: CCD video image captured with a Belkin (F5U208 - frame grabber) of a FIB-milled alphanumeric grid on the pre-existing Formvar $^{\circledR}$ coated TEM grids, showing one of many fabricated grids, during AFM imaging.

\section{Results}

In the field of biological optical imaging, fluorescence and Raman microscopy are commonly used methods. Once a region of interest (ROI) on a substrate is found, one is typically limited to the spatial diffraction limit of an optical system. Further inspection can be done with a higher resolution technique, such as an AFM, TEM, SEM or scanning transmission electron microscopy (STEM) (which typically shows higher resolution than normal secondary electron (SE) collection as demonstrated in Figure 3). The TEM grid used to demonstrate this method has a spacing of 284 microns ( 75 mesh); thus the grid pattern chosen fits the size ideally in order to subdivide the region further. As seen in Figure 1, the overall grid scale is 125 microns and the inner grid 12.5 microns.

\section{Optical Imaging}

In locating the grid optically it helps to use either epi-illumination (as shown in figure 4) or dark-field collimated illumination. As normal bright field microscopy has difficulty imaging the grid, the contrast is improved by using a dark field method. Optical microscopes suffer from poor depth-of-field especially at high magnifications; thus care is needed to keep the grids as flat as possible. Optical images were acquired with a Leica DFC 480 5-megapixel camera on a Leica DMRX under collimated darkfield illumination. The contrast of the grid is shown in Figure 5 (a-d). The microscope should be aligned with Köehler illumination in order to get a clearly visible grid. 

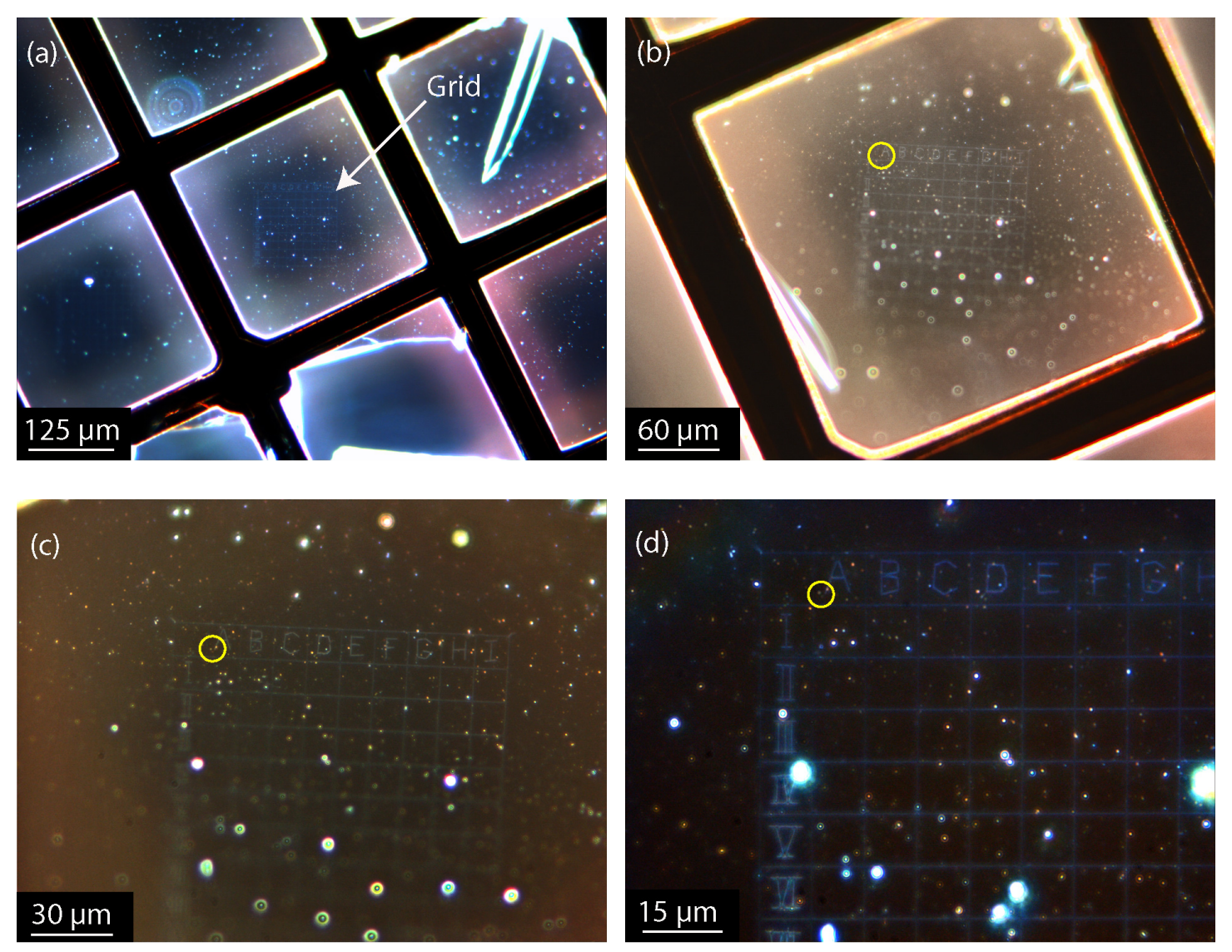

Figure 5: Optical micrographs of the milled grid. The following objectives were combined with a 0.9 numerical aperture (NA) condenser lens: (a) 10x 0.3 NA, (b) 20x 0.5 NA, (c) 40x 0.55 NA, (d) 63x 0.7 NA long working distance (LWD) respectively.

\section{Atomic Force Microscope Imaging}

In order to locate the milled grid pattern within the TEM grid with an AFM, the grid is mounted to the dull side of a silicon wafer. Mounting the grid on the silicon requires a very small drop of silver paint, then placing the grid next to the paint drop and sliding the grid so that the edge just makes contact. The paint is then allowed to dry for about 15 minutes. The silicon section containing the mounted grid is placed on a magnetic AFM mount via carbon tape. It is important to mention that this sample mounting serves for both AFM and SEM imaging. The user will need to take care in removing the grid for TEM and optical imaging in order to not damage the milled area by unnecessary flexing or bending of the TEM grid.

The area of interest is scanned with a Veeco/Digital Instruments Nanoscope III AFM using non-contact tapping mode cantilevers (SPI Supplies \#ACL10). The nanoscope was equipped with an optional video camera (figure 4) for viewing the sample area; this is critical for proper location of a ROI. 
(a)

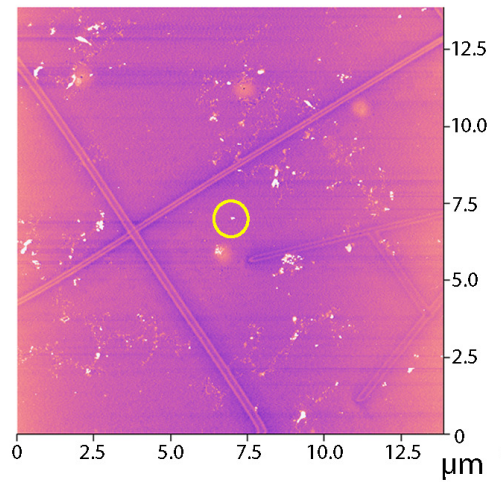

(b)

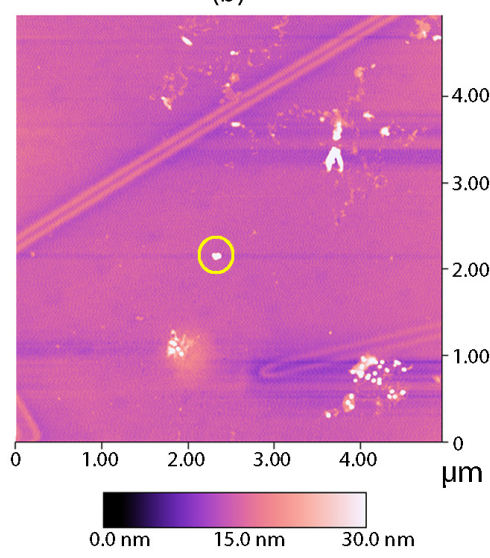

(d)
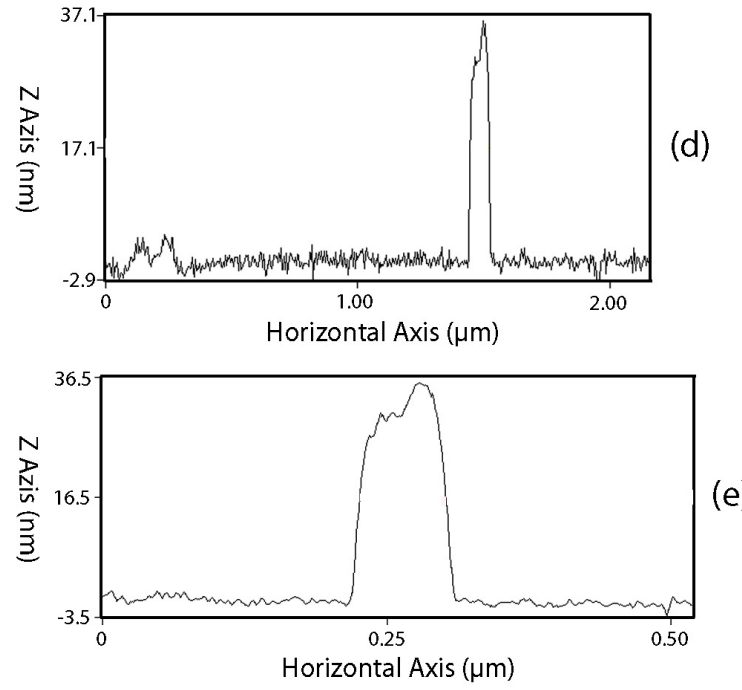

e) (c)

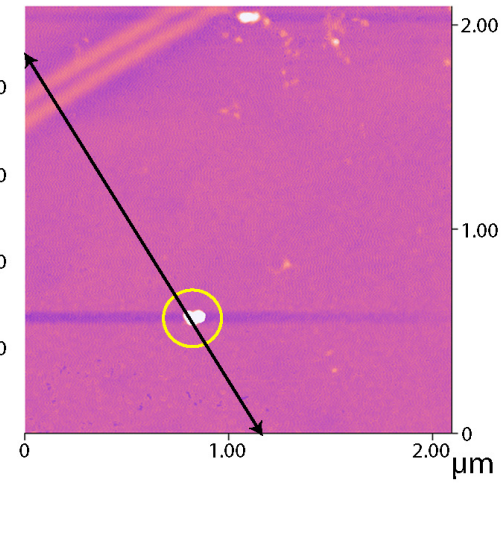

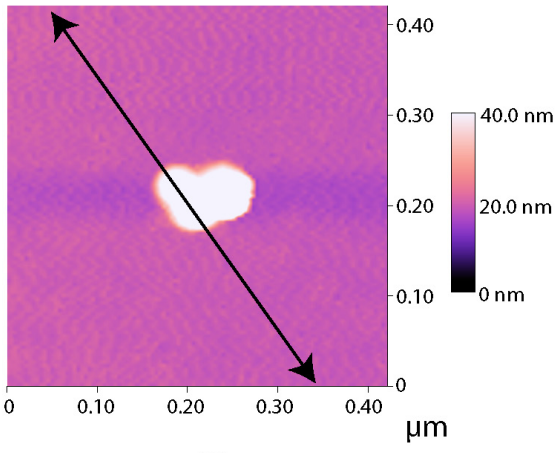

(f)

Figure 6. AFM surface topology of the milled grid with Au nanoparticles. Image (a) represents the ROI with a field of view (FOV) of $13.9 \mu \mathrm{m}$. The circled particle cluster is investigated at $5.6 \mu \mathrm{m}$ (b) and $2.1 \mu \mathrm{m}(\mathrm{c})$. Image (f) is a zoomed location of image (c) of the ROI with a FOV of $420 \mathrm{nms}$. Graph (d) shows a cross sectional analysis represented by the line in image (c). Graph (e) shows the cross section as labeled in image (f).

Scanning was performed in tapping mode with an integral gain of $\sim 1.5$ and a proportional gain of $\sim 2$ with 512 samples per line at a scan rate of $\sim 0.3 \mathrm{~Hz}$. Figure 6 shows an AFM surface topographic scan of the milled grid with $\mathrm{Au}$ nanoparticles present. Figure 6a represents the ROI with a field of view (FOV) of $13.9 \mu \mathrm{m}$. The circled particle cluster is investigated at $5.6 \mu \mathrm{m}$ (Figure 6b). Figure 6c shows a further zoomed in FOV of $2.1 \mu \mathrm{m}$. Figure $6 \mathrm{~d}$ is the cross-sectional line over the surface in Figure $6 \mathrm{c}$. Figure $6 \mathrm{f}$ is a zoomed location of image Figure $6 \mathrm{c}$ of the ROI with a FOV of $420 \mathrm{~nm}$. Figure 6e shows a cross sectional analysis across the line in Figure $6 f$. 


\section{SEM Imaging}

The same TEM grid was imaged under the FESEM (FEI Sirion) proving the ease in finding the same nanoparticle at the very same position. There is no need of any extra conductive coatings to image the TEM grid in the SEM. This analytical technique helps in imaging the samples in a less damaging approach than a FIB. Secondary electron imaging of the milled grid was accomplished with an accelerating voltage of $2 \mathrm{kV}$ at a 5 $\mathrm{mm}$ working distance. The grid is not detectable at higher acceleration voltages due to the larger interaction volume with the sample, which generates SEs from underneath the sample. Careful examination of the images show that the Formvar ${ }^{\circledR}$ can easily withstand the beam damage that is caused by contaminates in the SEM chamber. The black dots on the Formvar ${ }^{\circledR}$ film are locations where there are manufacturing defects caused by small bubbles in the film making process. There is no SE production in these bubbles.
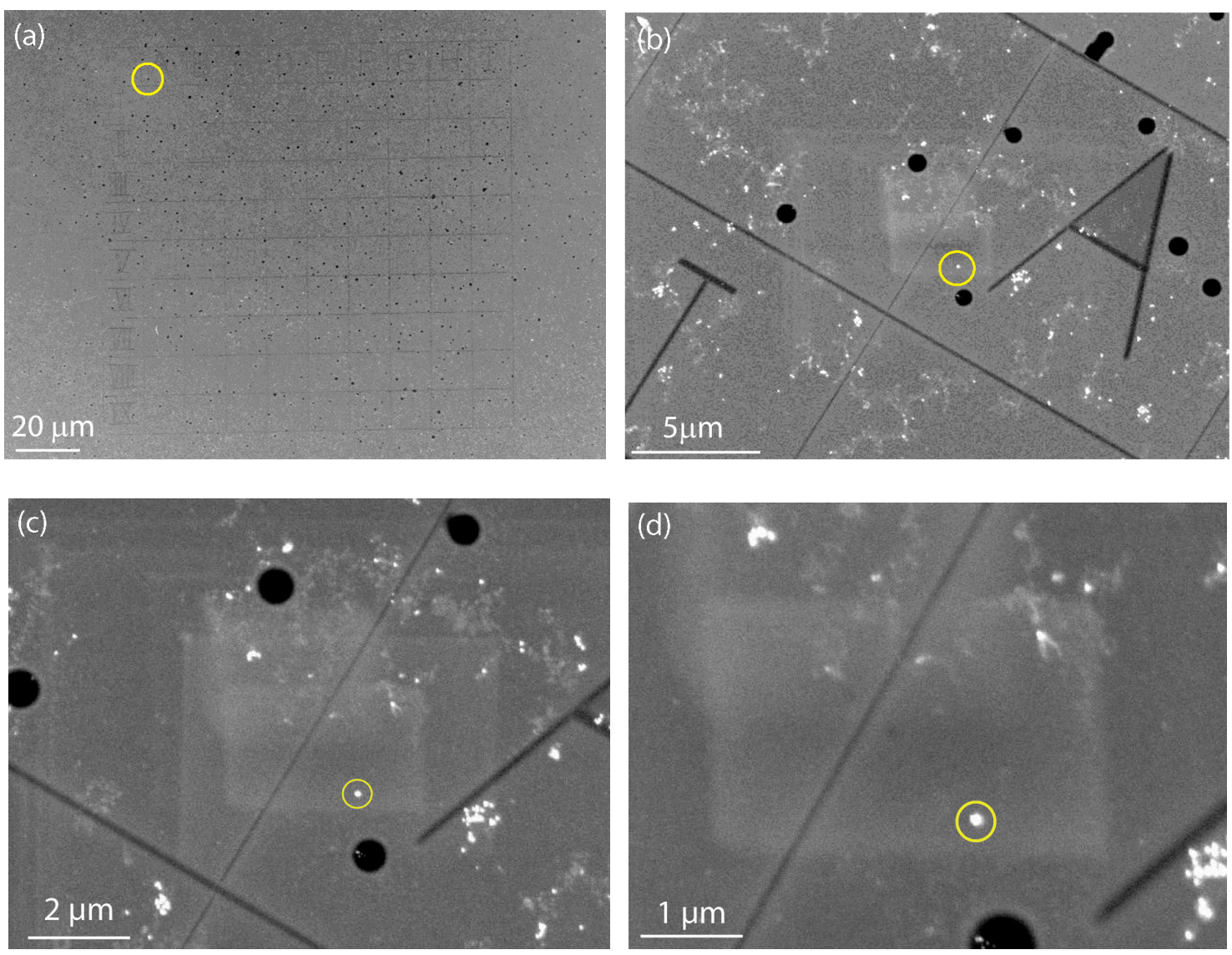

Figure 7: Scanning electron micrographs of the ROIs identified by the AFM. Images were acquired in SE imaging mode. The sample has a high tolerance to beam damage which is highly noticeable due to the low accelerating voltage used for imaging; (a) is at a magnification of 500x, (b) $4 \mathrm{kx}$ mag., (c) $7.5 \mathrm{kx}$ mag., (d) $15 \mathrm{kx}$ mag. 


\section{TEM Imaging}

TEM (FEI Tecnai F-20) images clearly depict the procedure one would employ to locate a nanoparticle and reduce the probability of not finding the particle for later purposes. We tried to image particles at the intersection of the first row and the first column (A1). As one increases the magnification, one easily addresses the nanoparticle of interest. Since the size of the patterned grid is optically resolvable, this situation would be very helpful in the cases of fluorescence, 2-photon, Raman and other imaging techniques requiring that the ROI be precisely located.
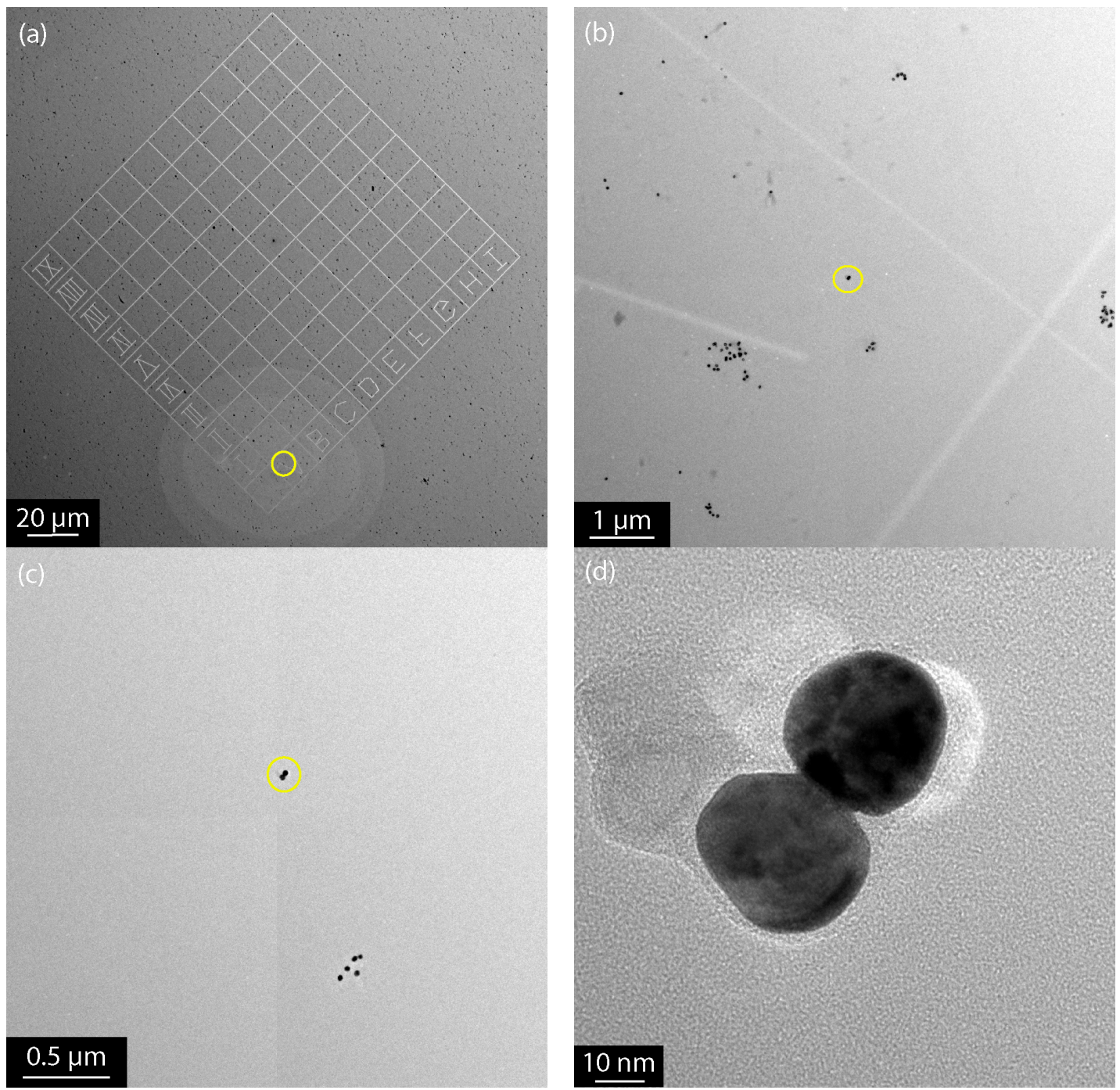

$0.5 \mu \mathrm{m}$

Figure 8: TEM micrographs of the ROI. Images are shown out-offocus to locate the milled grid. Beam damage was caused by two hours of imaging on the grid; (a) is at a magnification of 500x, (b) $10 \mathrm{kx}$ mag., (c) $40 \mathrm{kx}$ mag., (d) $1000 \mathrm{kx}$ mag. 
The TEM images were obtained at a $200 \mathrm{kV}$ acceleration voltage and a spot size of 5 (specific to FEI system). Due to the small milling depth of the FIB $(\sim 5 \mathrm{~nm})$ focusing on the grid with least contrast on the TEM causes the grid to disappear; only under or over focusing will resolve the grid locations. After all of the processing and imaging techniques the Formvar ${ }^{\mathbb{B}}$ was able to withstand TEM beam damage for over $2 \mathrm{hrs}$ of imaging. Figures 6a-d show the imaging at high resolution. The oval rings on the low magnification TEM image are a result of a corrected projection lens system in the TEM.

\section{Modification of Glass Coverslips}

In circumstances where is not necessary to use a TEM grid, bulk glass substrates, glass coverslips, can be used for creating the necessary locator grid. We used commercially available glass coverslips (VWR Scientific \# 48366-045). In bulk glass substrates, the process of milling and/or depositing with high beam currents suffers more from image drift due to charging of the substrate. This problem can be circumvented by use of a flood gun if needed. Electron flood guns cover the surface with low energy electrons to neutralize the positive charge build up due to $\mathrm{Ga}^{+}$ions, and stabilize the imaging, milling and depositing processes. Both milling and deposition were examined in order to determine the best method to see the grid optically. The depth of milling and level of deposition were verified with an AFM (Digital Instruments Nanoscope III). The optical attenuation of transmitted light was the method used to determine the effectiveness of the deposition or milling. The milling causes some degree of implantation that slightly colors the surface; this together with the milling causes diffraction and thus a reduction of the transmitted intensity is noticeable.

\section{Milling of a Bulk Dielectrics}

Microscope glass slides were cleaned with isopropyl alcohol (IPA). The substrate was then dried by blowing air over the surface, making sure no residue of IPA was left on the surface. Although not necessary, a thin layer of gold (few nanometers) was sputtercoated onto one half of the side of the glass substrate. This area with the thin film of gold facilitated focusing the FIB on the surface. The sample was then moved over to the non coated side where the millings took place. Millings were executed with a beam current of $2.87 \mathrm{nA}$ and a spot size of approximately a $100 \mathrm{~nm}$.

Since milling with $\mathrm{Ga}^{+}$ions on a plain glass substrate charged the surface with positive ions, charging became an issue. The problem was solved by an electron flood gun. The flood gun neutralized the positive ions with electrons and prepared the surface for stable imaging; however settings were altered on the neutralizer electron gun to maximize image stability that varied from location to location very slightly. It also helped to change the imaging mode to ion-collection mode (reverse detector polarity to ions instead of electrons). With the Micrion system it was determined that a neutralizing beam current of 25 microamps and beam energy of $\sim 90-100 \mathrm{eV}$, gave the most favorable results. It was also necessary to focus and deflect the beam to concentrate the electrons towards the substrate beam area. Once the flood gun stabilized the imaging, the same 
milling patterns as performed on the Formvar ${ }^{\circledR}$ millings were executed. Figure 9a demonstrates the grid pattern without a flood gun usage, which clearly demonstrates drift. Figure $9 \mathrm{~b}$ shows the results of milling with the flood gun on. The total milling time was approximately 10 minutes for one complete pattern.
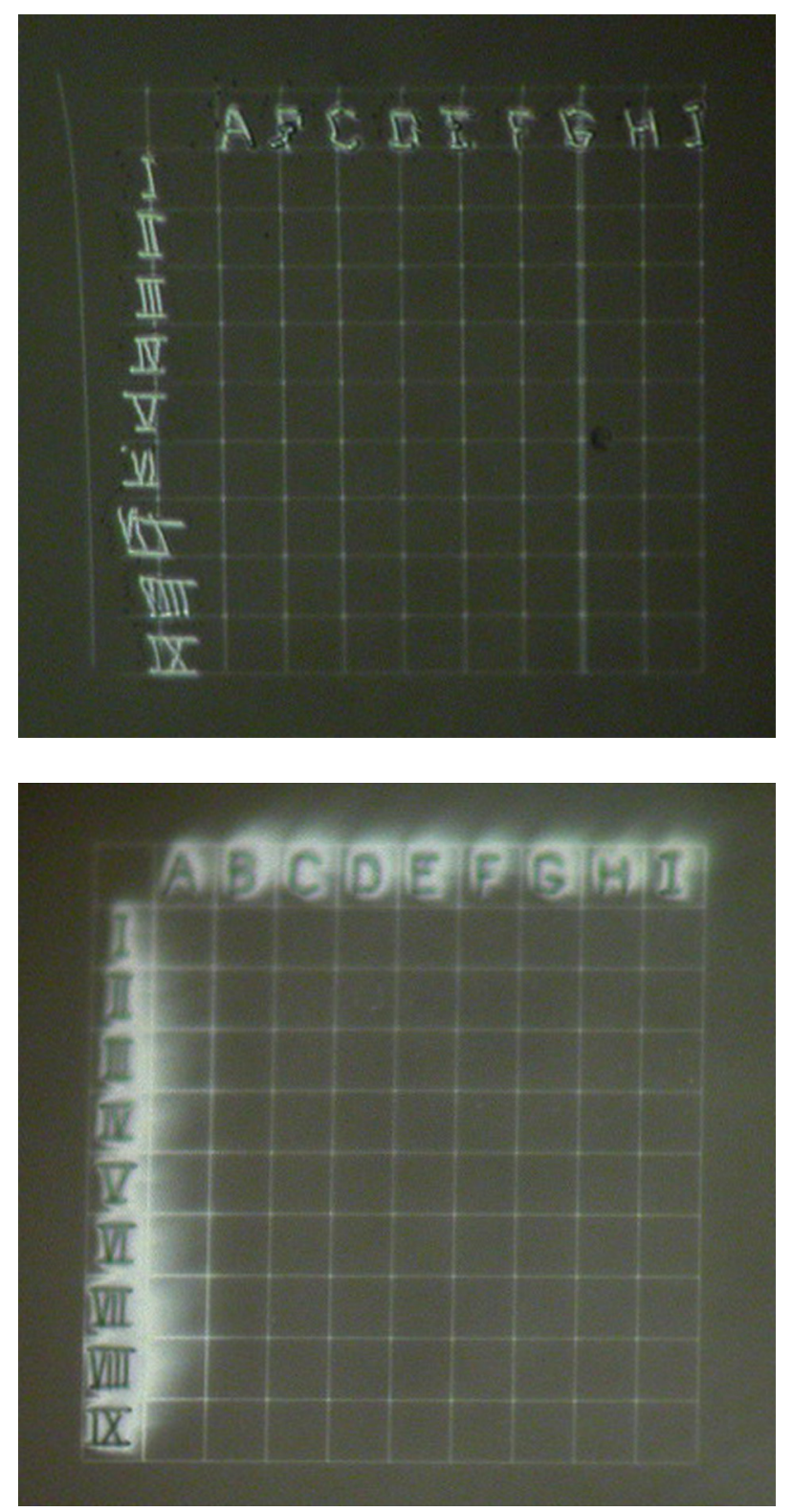

Figure 9: Optical micrograph of the milled grid on bulk glass (a) without an electron flood gun charge neutralizer, (b) with an electron flood gun in use.

Charging effects can clearly be seen in Figure 9a [taken by an Olympus BH2 series microscope at a total magnification of 500x (Infinity corrected Neo S series 50x with $0.8 \mathrm{NA}$ and $10 \mathrm{x}$ eyepiece)]. The milling stabilized when the low energy electron beam 
was incident upon the dielectric surface. Further analysis was performed by imaging the milled structure using an AFM (Nanoscope III). The depth profile of the milling was determined using the non-contact tapping mode AFM cantilever tips (SPI Supplies, \# ACL-10) and a scan rate of $0.3 \mathrm{~Hz}$. High momentum $\mathrm{Ga}^{+}$ions impinged on the substrate surface and milled the glass substrate to a depth of $168 \mathrm{~nm}$ for the letter A as shown in Figure $10 \mathrm{a}, \mathrm{b}$ and $6 \mathrm{~nm}$ for the vertical line. This is the same "A" alphabet letter which is shown in Figure 9b. Since the plotting line in Figure 10b is on a negative Z-axis, one can easily conclude that the surface was actually milled and was not either deformed or deposited.

(a)

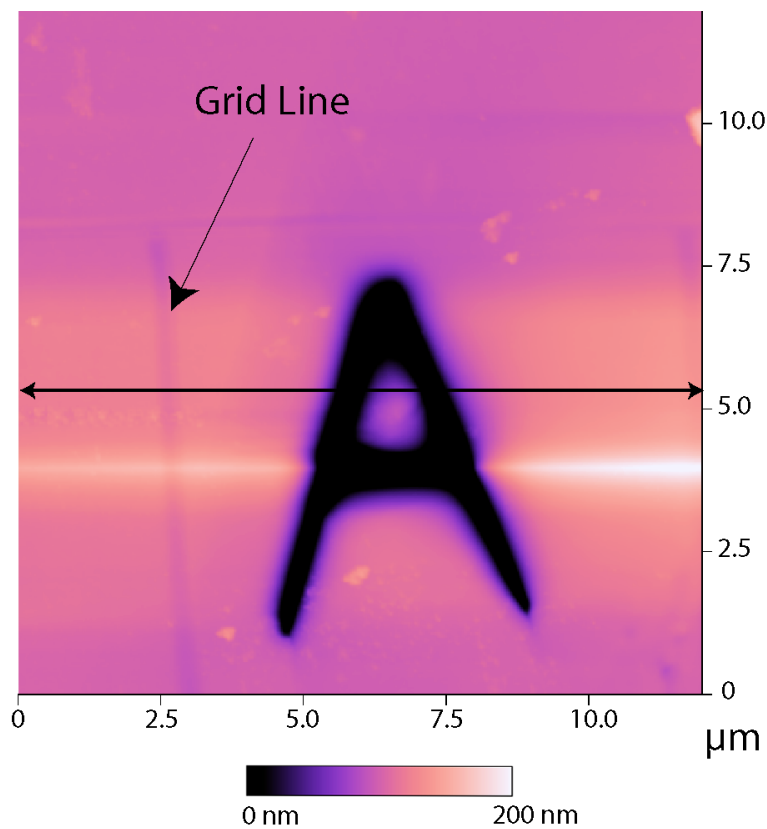

(b)

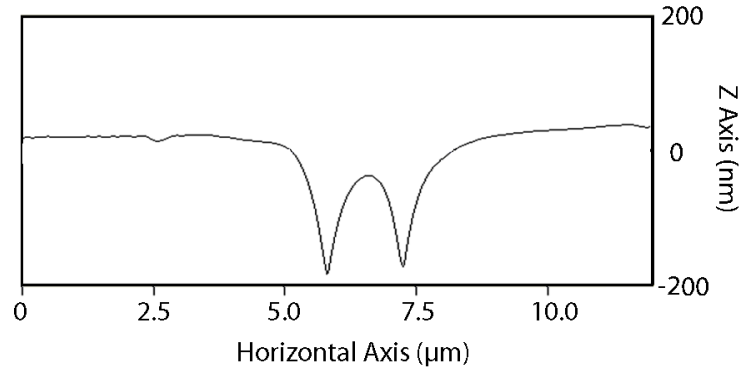

Figure 10: AFM topographic image of the milled bulk glass, (a) image of the letter "A" in the grid milling, (b) the cross-section of the milling depth. 


\section{Deposition on Glass Substrates}

Ion Beam-Assisted Deposition (IBAD) is a very important technique in the semiconductor industry for repairing chip electrical traces. FIB-assisted tungsten deposition of the grid patterns is an easy approach to locating a particular section of a biological sample or nano-scaled object. Typically there exists an optimum beam current for maximizing growth rate of deposited material. For our application, a beam current of $330 \mathrm{pA}$ with a corresponding spot size of $100 \mathrm{~nm}$ was used. Setting the electron gun at the earlier discussed values reduced sample charging and resulted in the proper millings/depositions without any drifting. The deposition optical attenuation was not determined with a grid pattern, but rather a square solid pad $(10 \times 10) \mu \mathrm{m}^{2}$ in order to facilitate the measuring process.

The timings needed for the beam to maximize the quality of ion beam-assisted deposition (IBAD) were evaluated. The optical system used to evaluate the attenuation is seen in Figure 7. The system consisted of a helium neon laser $(5 \mathrm{~mW})$, with an optical telescope, which was directed into an inverted Nikon TMD microscope. The sample was held above the high NA objective on a manually translatable stage where an optical power meter (Coherent Fieldmaster \#33-0506-000, with LM-2 VIS power head) was located for attenuation measurements.

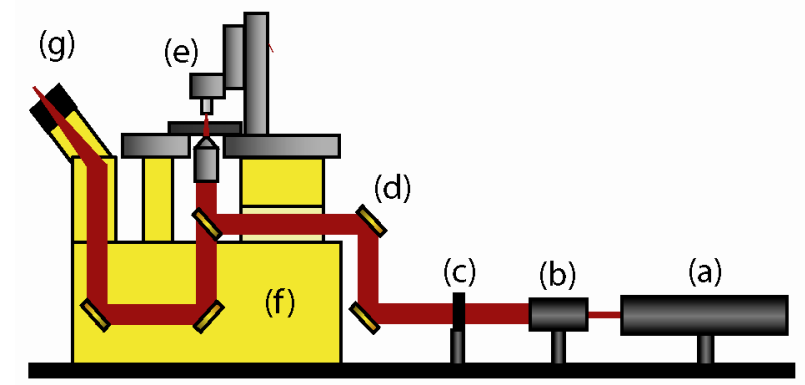

Figure 11: Laser attenuation optical setup, (a) $5 \mathrm{~mW}$ helium neon laser, (b) beam expander, (c) shutter, (d) periscope for alignment, (e) laser power meter, (f) Nikon inverted microscope with high NA objective, (g) viewing port for alignment.

The sample pad area was first located in the inverted microscope and the laser was carefully focused through the objective in order to have the entire area of focus $(\sim 500$ $\mathrm{nm}$ ) at the surface coincident with the user's view. Figure 11 shows the optical setup for measuring the light attenuation due to deposition of $\mathrm{W}$ on the glass surface. The laser power was measured next to the pad area in a clear region to obtain a baseline of initial intensity, $\mathrm{I}_{0}$, then the sample was moved so the laser was incident on a deposited pad and clearly within the pad dimensions $(10 \mathrm{x} 10) \mu \mathrm{m}^{2}$ on the surface. A series of pads were placed next to each other in order to easily move from sample to sample. The ratio of measured power (I) on the pad versus $\mathrm{I}_{\mathrm{o}}$ was taken for all pads. The parameters used in the process of IBAD such as dwell time and current play a vital role in the deposition process. Figure 12 shows the $\mathrm{W}$ thickness as a function of IBAD exposure time. Based on 
previous work ${ }^{8}$ with Pt organometallic deposition, the maximum growth rate occurs at a beam overlap of $-300 \%$ ( $\sim 100 \mathrm{~nm}$ spot size and $300 \mathrm{~nm}$ beam spacing) and a current of $330 \mathrm{pA}$. Initial base pressure of the FIB is $5.3 \times 10^{-7} \mathrm{mbar}$, and $1.2 \times 10^{-5} \mathrm{mbar}$ during deposition.

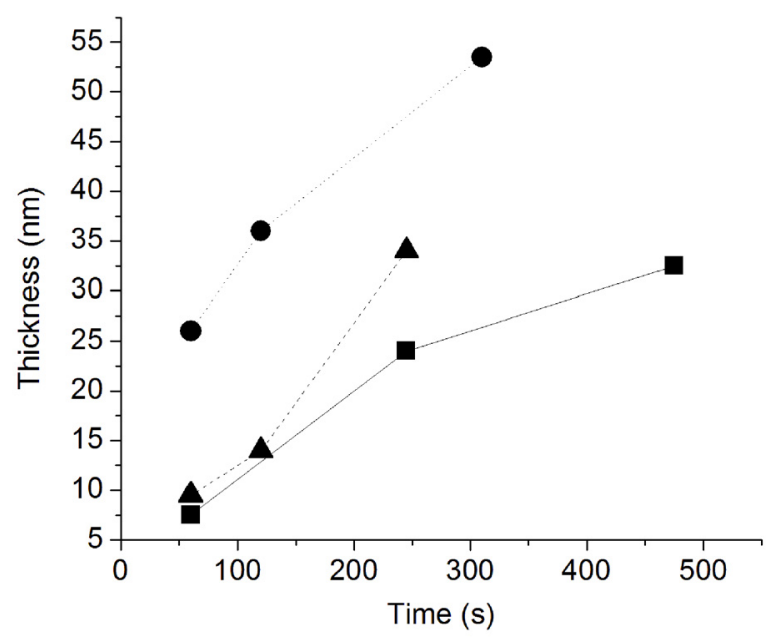

Figure 12: Thickness of the deposited tungsten as a function of beam exposure time; $(\bullet)$ represents a $1 \mu \mathrm{s}$ beam dwell time, and gives the fasted rate of growth, ( $\boldsymbol{\Delta})$ a $10 \mu$ s beam dwell time, ( $\boldsymbol{\square})$ a $100 \mathrm{~ns}$ beam dwell time.

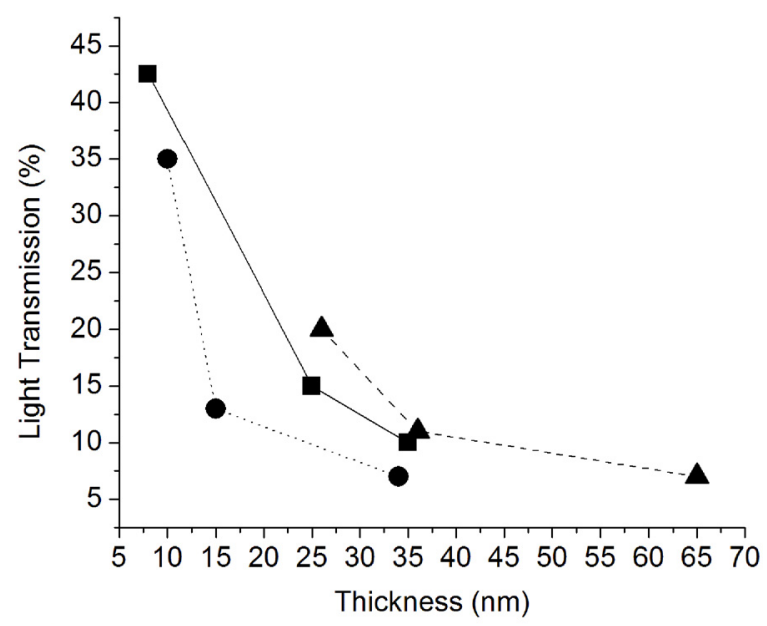

Figure 13: Light transmission of the deposited tungsten as a function of deposition thickness above the glass surface; $(\bullet)$ represents a $10 \mu \mathrm{s}$ beam dwell time, $(\mathbf{\Lambda})$ a $1 \mu$ s beam dwell time, (-) a $100 \mathrm{~ns}$ beam dwell time.

Initially when depositing $\mathrm{W}$ onto the surface, the $\mathrm{Ga}^{+}$beam mills into the surface; later it begins to build up a $\mathrm{W}$ deposition. This milling with deposition combination leads to attenuation that is a result of $\mathrm{W}$ in the material as well as above the surface. If one were to look at the thickness of pure $\mathrm{W}$ and compare it to IBAD deposition for the values 
shown in Figure 13, the values given by IBAD lead to higher attenuation because the actual thickness extends below the surface due to simultaneous milling. Figure 13 demonstrates the light transmission of the deposited tungsten as a function of deposition thickness above the glass surface; $(\bullet)$ represents a $10 \mu$ s beam dwell time, $(\boldsymbol{\Delta})$ a $1 \mu \mathrm{s}$ beam dwell time, (-) a $100 \mathrm{~ns}$ beam dwell time. The highest attenuation, and thereby the most visible lines, occur for the $10 \mu$ s beam dwell time at $\sim 30 \mathrm{~nm}$ thicknesses above the surface.

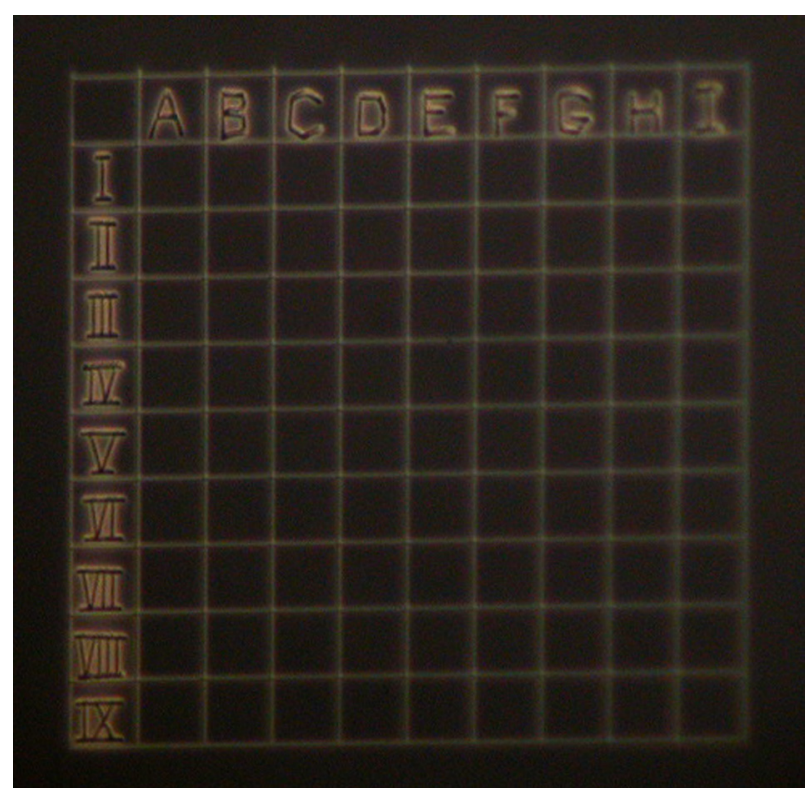

Figure 14: Optical Micrograph of deposited W to create a grid pattern on a glass substrate. The contrast is easily seen with dark and bright field microscopies.

\section{References:}

[1] Frens, G. (1973) Controlled nucleation for the regulation of the particle size in monodisperse gold suspensions, Nature (Phys. Sci.), 241, p. 20

[7] Orloff, J. et al. (1991) Experimental study of a focused ion beam probe size and comparison with theory. J. Vac. Sci. Technol., B, 9(5): p. 2609-12.

[8] Sánchez Erik J. et al. (2002) Ion and Electron Beam Assisted Growth of Nanometeric $\mathrm{Si}_{m} \mathrm{O}_{n}$ Structures for Near-Field Microscopy, J. Chem. Phys. 284, 423. 